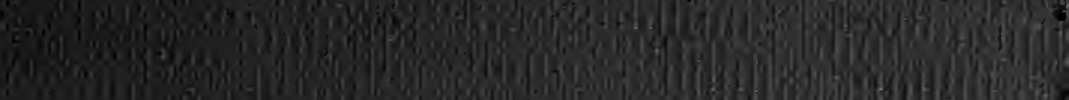

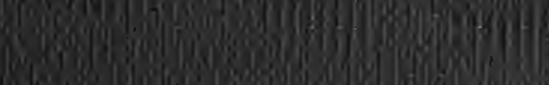

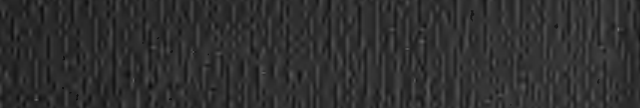

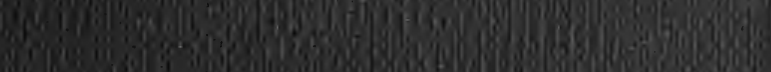
19,

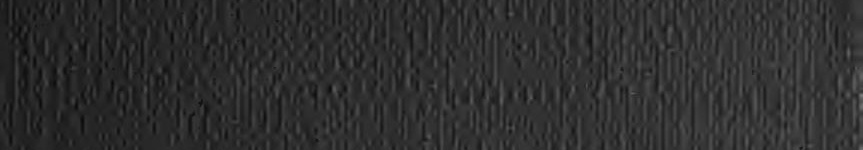




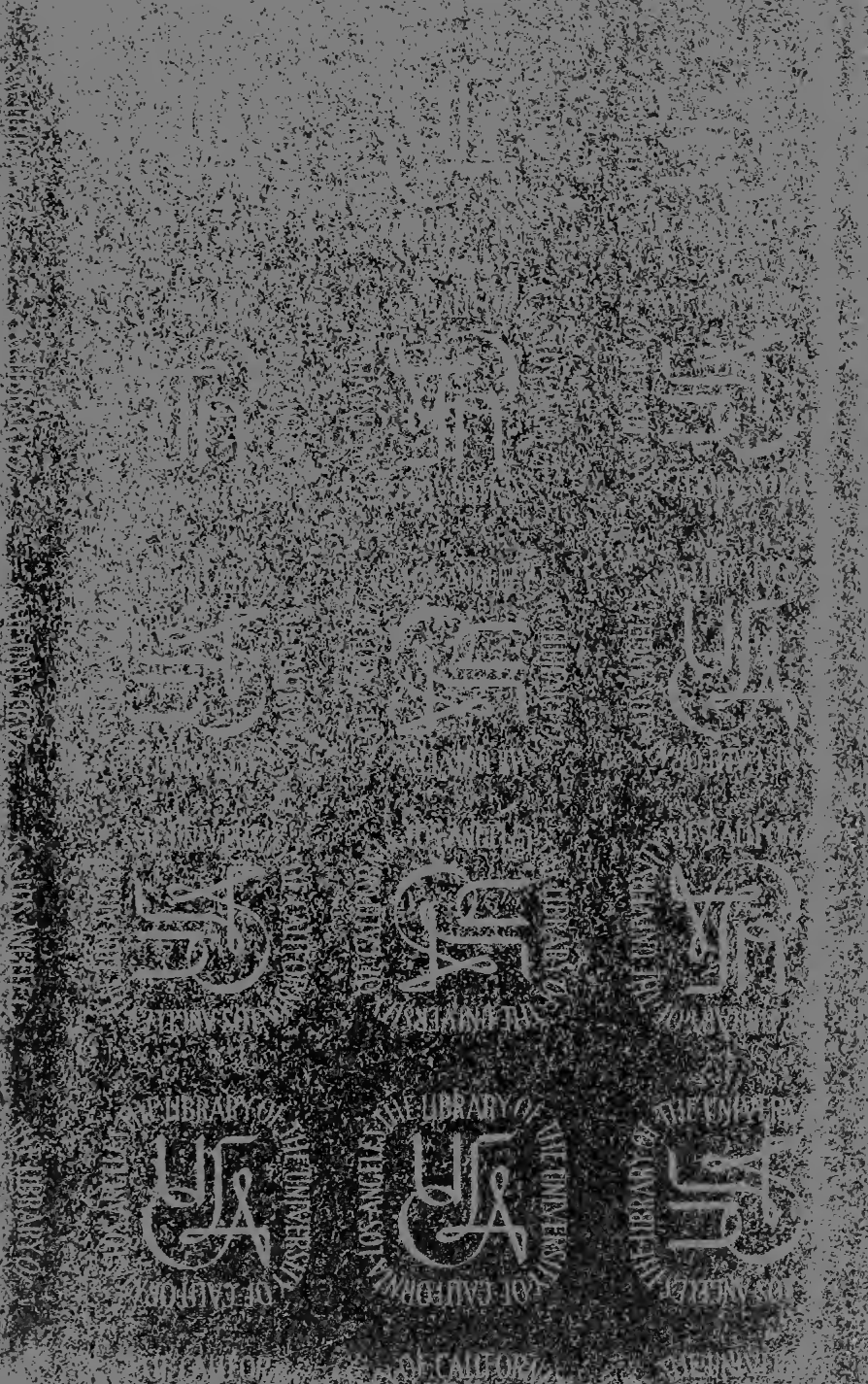

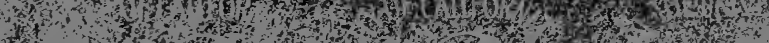

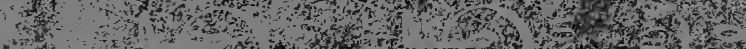

S 


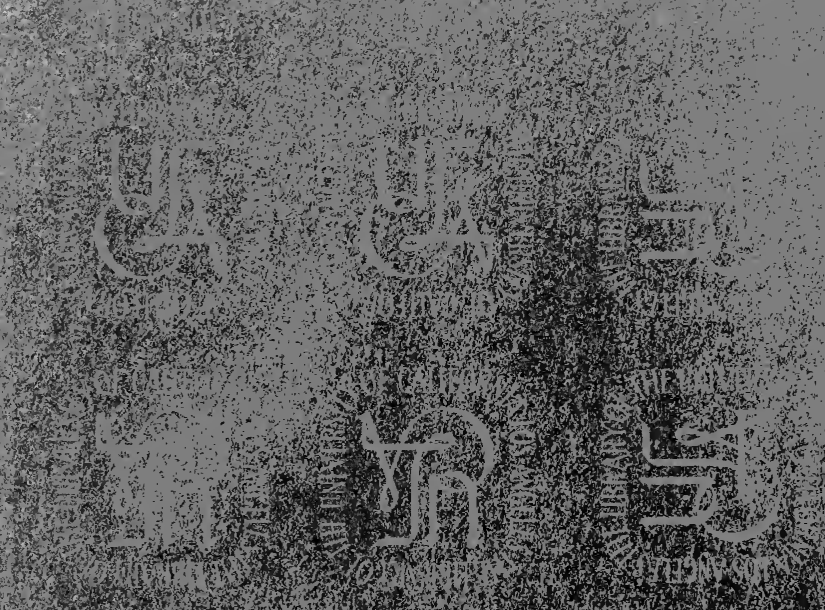

20
3 14. Af

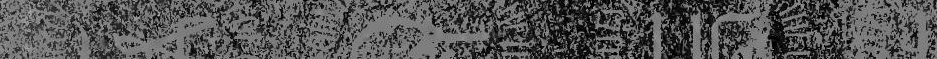

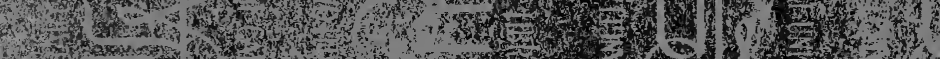

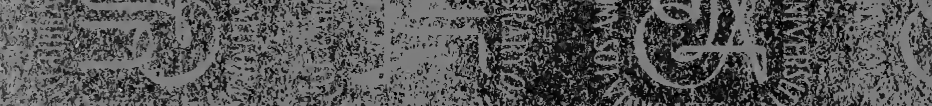
3
3

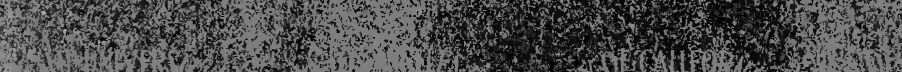

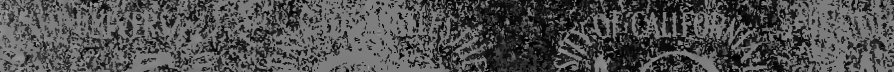

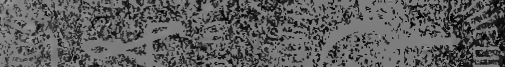

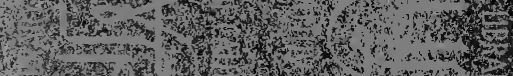

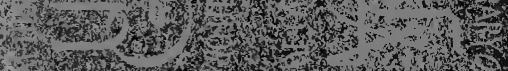

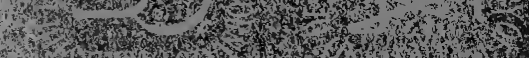

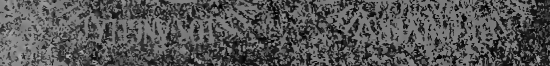

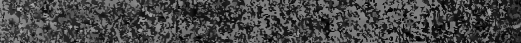

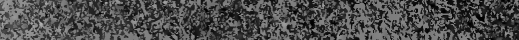

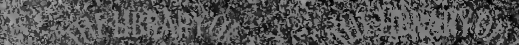
W.7.

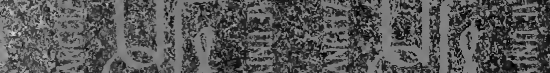

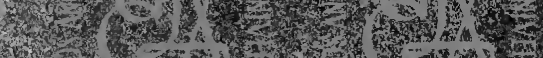

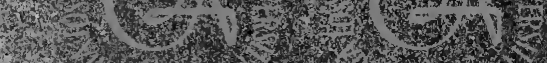

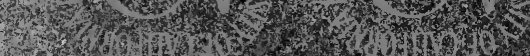

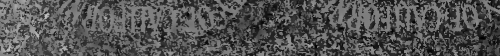

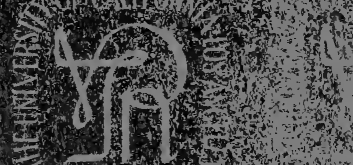

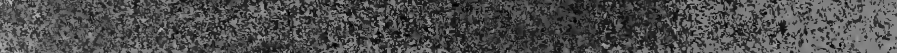
17. 3 H.

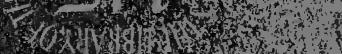

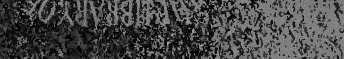
3) 3.17 W 

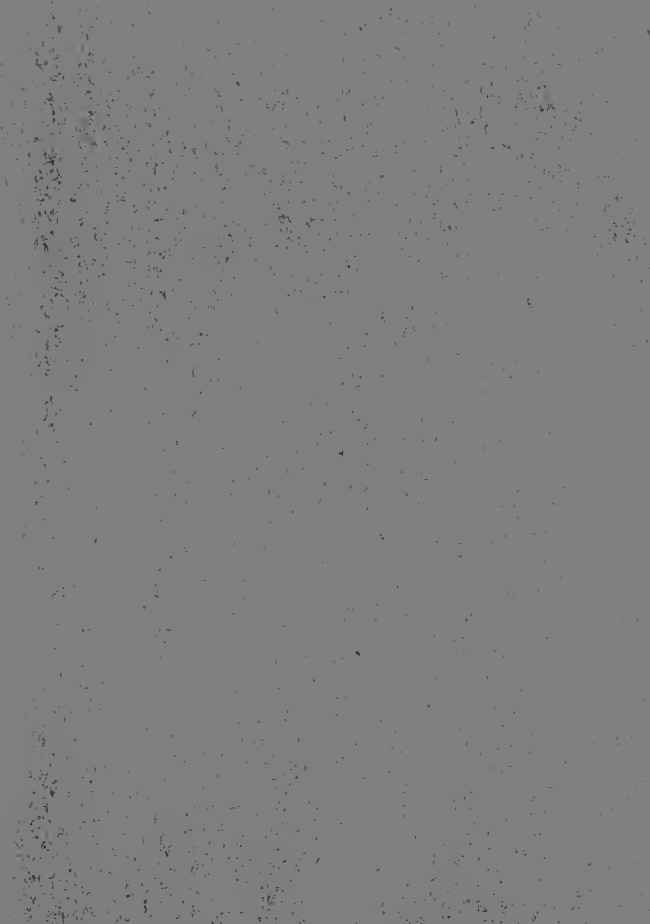

$\frac{1}{3}$

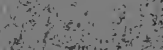

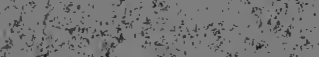

3
$\vdots$
$\vdots$
$\vdots$
$\vdots$
$\vdots$
$\vdots$

P

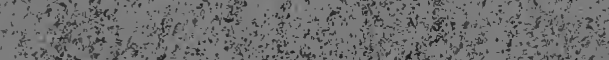

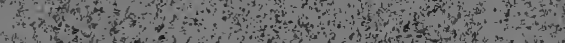

1 is

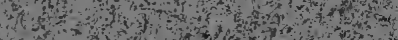

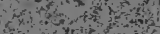

s.t.

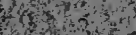

to $3 x$

tix

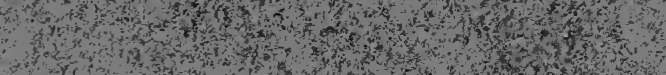

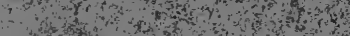

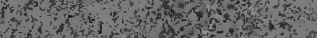

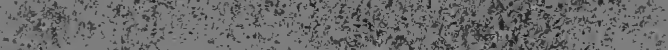

trotents

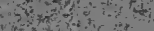

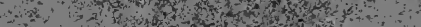

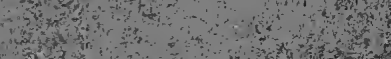






\title{
THE DETECTION OF POISONS AND POWERFUL DRUGS
}

\author{
AUTENRIETH-WARREN
}





\section{LABORATORY MANUAL}

FOR

\section{The Detection of Poisons}

AND

\section{Powerful Drugs}

BY

DR. WILHELM AUTENRIETH

PROFESSOR IN THE UNIVERSITY OF FREIBURG i. B.

AUTHORIZED TRANSLATION

BY

WILLIAM H. WARREN, Ph.D.

FIFTH AMERICAN EDITION WITH 25 ILLUSTRATIONS

PHILADELPHIA

P. BLAKISTON'S SON \& CO. 1012 WALNUT STREET

$$
38921
$$


Copyright, ig2i, By P. Blakiston's Son \& Co. 


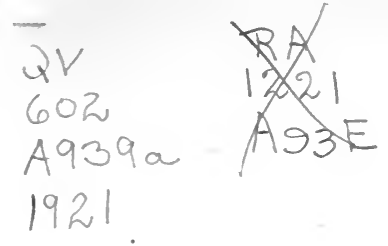

\section{AUTHOR'S PREFACE}

Additional matter in "Detection of Poisons" has made the fourth edition considerably larger than the third. The seven chapters now comprised in the book have been entirely revised but the first three chapters remain unchanged in arrangement. Chapter I treats of poisons volatile with steam. Organic poisons, especially the alkaloids, form the subject of Chapter II. Hydrastine and veronal, introduced into this chapter for the first time, have been incorporated into the Stas-Otto process. Chapter III deals with metallic poisons.

The toxic substances included in Chapter IV find no place in the three groups just mentioned. As they seldom appear in toxicological examinations, their significance is theoretical rather than practical. The following members of this group have been introduced for the first time, namely, cantharidin, cytisine, ergot, papaverine, pilocarpine, saponin substances, solanine, thebaine, and the toxalbumins, ricin, abrin and crotin.

Chapter V.deals with special qualitative and quantitative methods, such as the quantitative estimation of phosphorus in phosphorated oils; the electrolytic detection and estimation of arsenic; the biological test for arsenic; the destruction of organic matter and detection of arsenic by A. Gautier and G. Lockemann; Karl Th. Mörner's estimation of minute quantities of arsenic; methods of estimating alkaloids by $\mathrm{H}$. Matthes, $\mathrm{H}$. Thoms and A. H. Gordin. This chapter also includes A. J. J. Vandevelde's estimation of the toxic action of organic compounds by means of blood hæmolysis.

Chapter VI takes up the estimation of alkaloids and other active principles in raw materials (drugs) and in their preparations. Pharmacopœial as well as other estimations, such as that of nicotine in tobacco, caffeine in tea, coffee, cola preparations, etc., pilocarpine in jaborandum leaves, piperine in pepper, 
solanine in potatoes, and theobromine in cacao and its preparations have been included. The author has endeavored to treat these subjects as thoroughly as possible.

Chapter VII describes the methods employed in detecting carbon monoxide.in blood, in recognizing blood itself in stains and in differentiating human from animal blood.

The new edition, though more comprehensive in its scope than the last, has lost nothing in cleainess beçause of the rearrangement of subject-matter. Beginners will probably confine their attention to the first three chapters. Students of pharmacy will undoubtedly add Chapter VI which deals with drug assaying. The other chapters are designed more especially for those who wish to become better acquainted with toxicological procedures.

Descriptions of syntheses of organic drugs such as acetanilide, antipyrine, phenacetine, pyramidone, salicylic acid, sulphonal and veronal allow the student to review the methods employed in connection with laboratory work. Structural formulæ of alkaloids and their cleavage-products have been given only when they have been definitely determined or shown to be highly probable. By introducing this specific information the author hopes to stimulate the student's interest in alkaloidal chemistry which has become so important within recent years.

More advanced students will find in fine print brief statements about the poisonous action of the better known physiologically active substances as well as their distribution in and elimination from the human organism. Repeated references to larger treatises upon toxicology, especially to R. Kobert's "Intoxikationen," have been made. Numerous citations of literature enable the student to consult original articles for fuller information.

The translation of the third and fourth editions into English and Spanish and the proposed translation of the fourth edition into Italian indicate that colleagues in other countries have favorably received this work.

Freiburg in Baden.

Wilhelm Autenrieth. 


\section{TRANSLATOR'S PREFACE}

The fourth English edition of Professor Autenrieth's "Auffindung der Gifte" was a translation of the fourth completely revised German edition. The present, or fffth, English edition is also a translation of the same work, for a fifth German edition so far as the writer is aware has not yet appeared.

The last English edition, though adhering closely to the text of the German work, included a few subjects not found in the latter. Among these may be mentioned a fuller discussion of the "normal arsenic" question and the quantitative estimation of arsenic and antimony by the Gutzeit test. These subjects have been retained in the present edition. Owing to the prominence attained of late by wood (methyl) alcohol, due to ignorant or criminally careless substitution of this intoxicant for grain (ethyl) alcohol, this substance has been added to the list of volatile poisons. Aside from minor corrections of the text, the omission and correction of certain tests, the introduction of a few new tests of recent appearance in the literature, and the expansion of the index to include authors as well as subjects, no changes of importance have been made in the last edition of this work.

WiLliam H. WARREN.

Somerville, New Jersey. 



\section{CONTENTS}

PAGE

Author's Preface .. . . . . . . . . . . . . . . . . . v v

Translator's Preface . . . . . . . . . . . . . . . . . . vii

Introduction. . . . . . . . . . . . . . . . . . . I

\section{CHAPTER I}

Tests for Phosphorus and Other Poisons Volatile with Steam from * Acin Solution

Phosphorus ......................... . . 5

Scherer's test; Mitscherlich's test; Blondlot and Dusart's test;

(a) in the Fresenius-Neubauer apparatus. (b) in the HilgerNattermann apparatus; Detection of phosphorous acid; Phosphorus in phosphorated oils; Detection and quantitative estimation by the Mitscherlich-Scherer method; Metabolism in phosphorus poisoning.

\section{Further Examination of the Distillate}

Hydrocyantc Acid

Physiological action; Preliminary test; Detection; Quantitative estimation; Detection in presence of potassium ferrocyanide; Mercuric cyanide; Mercuric cyanide in presence of potassium ferrocyanide.

CARBolic ACID .

Action and fate in the organism; Detection; Quantitative estimations; I. Gravimetrically; 2. Volumetrically (Beckurts-Koppeschaar); 3. Volumetrically (J. Messinger-G. Vortmann); Estimation in urine; Carbolic acid in presence of aniline.

ChLOROFORM .

Behavior in the human organism; Distribution in the cadaver;

Detection; Quantitative estimation in cadaveric material.

Chloral Hydrate

Detection; Action and fate in the human organism; Quantitative estimation in blood and tissues.

IODOFORM

Detection.

NITROBENZENE

Toxic action; Detection.

Toxic action; Detection.

Carbon Disulphide

Toxic action; Detection; Quantitative estimation of carbon disulphide vapor in air. 
Pagk

Eтhyl Alcohol. . . . . . . . . . . . . . . . . . . . . . . . . . 49

Fate in the human organism; Detection.

MEthyl Alcohol. . . . . . . . . . . . . . . . . . . . 52

Physiological action; Detection.

ACETone. . . . . . . . . . . . . . . . . . . . . . 55

Occurrence in urine; Detection; Acetone in presence of ethyl alcohol;

Detection in urine.

Bitter Almond Water and Benzaldehyde . . . . . . . . . . . 57

Synopsis of Group I (Chapter I) . . . . . . . . . . . . . . . . . 59

\section{CHAPTER II}

Detection of those Organic Substances which are not Volatile with STEAM FROM ACID SOLUTION

Stas-Otto Process . . . . . . . . . . . . . . . . . . . . . . . . 63

A. Examination of Ether Extract of the Aqueous Tartaric Acid

Solution. . . . . . . . . . . . . . . . . . . 63

Picrotoxin. ...................... . . . 66

Detection in beer.

Colchicin . . . . . . . . . . . . . . . . . . . . 68

PICRIC ACID . . . . . . . . . . . . . . . . . . . . . . . . . . . . . 70

Action and elimination; Detection.
Acetanilme . . . . . . . . . . . . . . . . . . . . . . . 73

Action; Detection; Examination of acetanilide urine.

Phenacetine. . . . . . . . . . . . . . . . . . . . . . . . . . . 75

Preparation; Detection.
SALICyLIC ACID . . . . . . . . . . . . . . . . . . . . . . . . . . . . . 77

Detection; Quantitative estimation; Detection in urine.

Veronal. . . . . . . . . . . . . . . . . . . . . . . . . . . . . 79

Preparation; Physiological action; Detection in urine.

ANTIPYRINE . . . . . . . . . . . . . . . . . . . . . . 82

Preparation; Detection in urine.

Fate in human metabolism; Detection.

B. Examination of Ether Extract of the Aqueous Alraline Solution 85

Conine . . . . . . . . . . . . . . . . . . . . . . . . . . . 89

Nicotine. . . . . . . . . . . . . . . . . . . . . . . . . 90

Physiological action; Reactions.

ANILINE .

Veratrine. . . . . . . . . . . . . . . . . . . . . . 93

Preparation of crystalline and water soluble veratrine; Constitution;

Reactions.

STRYchnine

BRUCINE

Physiological action; Detection; Detection in presence of brucine.

Constitution; Reactions. 
Homatropine. . . . . . . . . . . . . . . . . . I05

Cocatne .. . . . . . . . . . . . . . . . . . . . I05

Constitution; Behavior in the animal organism; Detection.

Physostigurine .. . . . . . . . . . . . . . . . Iro

CoDEINE . . . . . . . . . . . . . . . . . . . . . . . III

NARCOtINE. . . . . . . . . . . . . . . . . II I

Constitution; Detection.

Hydrastine . . . . . . . . . . . . . . . . . . . . II6

QUININE .

Preparation; Constitution; Reactions.

Constitution; Detection.

Caffeine

ANTIPYRINE

Detection in urine.

PyRAMIDONE

Preparation; Behavior in the organism; Detection.

C. Examination of Exher Extract and of Chloroform Extract of the Solution Alkaline with Ammonia.

$\alpha$. Ether Extract . . . . . . . . . . . . . . . . . . 126

APOMORPHINE. . . . . . . . . . . . . . . . . . . . . . I 27

ß. Chloroform Extract. . . . . . . . . . . . . . . . . . . 130

Preliminary test for morphine; Purification of crude morphine.

Constitution; Detection; Behavior in the animal organism.

Synopsis of Group II (Chapter II). . . . . . . . . . . . . . . . . I40

\section{CHAPTER III}

\section{Examination for Metallic Poisons}

Destruction of Organic Matter . . . . . . . . . . . . . . . . . I48

Fresenius-v. Babo Method . . . . . . . . . . . . . . . . . 148

By free chloric acid . . . . . . . . . . . . . . . . . I5I

C. Mai's Method . . . . . . . . . . . . . . . . . . . . . ${ }_{152}$

Precipitation with Hydrogen Sul.Phide . . . . . . . . . . . . . . I52

Metalic Poisons I: Examination of that portion of the hydrogen sul-

phide precipitate soluble in ammonia-ammonium sulphide.

ARSENIC . . . . . . . . . . . . . . . . . . . . ${ }_{156}$

Marsh-Berzelius method; Fresenius-v. Babo method; Bettendorff's test; Gutzeit's test.

Antmony, Trn, Copper . . . . . . . . . . . . . . . . . . . . . . $\mathrm{I}_{3}$

Metallic Poisons II: Examination of that portion of the hydrogen sul-

phide precipitate insoluble in ammonium sulphide . . . . . . . 165

Mercury, Lead, Copper, Bismuth, Cadmium . . . . . . . . . . . . 165

Metallic Poisons III: Examination for Chromium and Zinc. . . . . . I68

ZrNc. . . . . . . . . . . . . . ..... . 168 
Metallic Poisons IV: Examination for Barium, Lead and Silver of the insoluble residue left on treatment with potassium chlorate and hydrochloric acid.

SyNopsis of Group III (Chapter III). . . . . . . . . . . . . . . . I II

The Action of Heavy Metals. . . . . . . . . . . . . . . . . . . 172

Fate, Distribution and Elimination of Metals in the Body . . . . 173

Normal Arsentc . . . . . . . . . . . . . . . . . . . . . . . . . . I74

CHAPTER IV

I. Examination for those Poisons which do not Belong in the Three MaIn Groups

Mineral Acids

HYdRochloric AcId. . . . . . . . . . . . . . . . . . . . . . . . ${ }_{1} 8_{3}$

NItrIC ACID . . . . . . . . . . . . . . . . . . . . . . . . . . . 184

SUlPHURIC ACID. . . . . . . . . . . . . . . . . . . . . . . . . . I86

SulphURous ACID. . . . . . . . . . . . . . . . . . . . . . . . . . . 188

Oxalic ACID . . . . . . . . . . . . . . . . . . . . . . . . . . . . . . . . . ${ }^{1} 89$

Toxic action; Distribution in the organism; Detection.

Detection of Free Alkalies

Potassium Hydroxide, Sodium Hydroxide, Ammonia . . . . . . . . . ${ }^{92}$

Detection of Potassium Chlorate, Santonin, Sulphonal, Trional

Potassium Chlorate . . . . . . . . . . . . . . . . . . . . . . . I94

Toxic action; Detection; Quantitative estimation; Behavior during

putrefaction; Detection in meat.

S4NTONIN

Constitution; Behavior in the organism; Detection.

Sulphonal. . . . . . . . . . . . . . . . . . . . . . 200

Preparation; Detection in urine; Detection of hæmatoporphyrin in urine.

TRIONAL .. . . . . . . . . . . . . . . . . . . . . . 203

2. Powerful Organic Substances Seldom Occurring in Toxicological Examinations

Cantharidin. 203

Constitution; Detection.

Cytisine.

Preparation; Toxic action; Detection.

Digrtalis Bodres. . . . . . . . . . . . . . . . . . . . . . . 207

Digitonin, Digitoxin, Digitalinum verum.

Ekgot . . . . . . . . . . . . . . . . . . . . . . . . 209

Alkaloids; Sclererythrin; Detection of ergot in flour; Detection and estimation of the alkaloids.

Meconic acid; Meconin; Selenious-sulphuric acid reagent for opium alkaloids. 
Constitution; Detection.

Pilocarpine

Ptomaines .

SAPONINS.

Physiological action; Detection in foaming beverages, such as beer, etc.; Detection of githagin in flour.

Hamolysis and Physiological Salt Solution.

Solanine

Toxic action; Detection.

Thebaine .

Constitution; Detection.

Toxalbumins.

Abrin, Ricin; Crotin; Coagulation of blood and defibrinated blood.

\section{CHAPTER V}

\section{Special Methods}

Quantitative Estimation of Phosphords in Phosphorated Oils

I. W. Straub's method; 2. A. Fränkel's and C. Stich's method. . . 23 I

Special Methods for Detecting Arsentc

Separation of arsenic as arsenic trichloride . . . . . . . . . . . 233

Electrolytic detection of arsenic. . . . . . . . . . . . . 233

Destruction of organic matter and detection of arsenic by A. Gautier and G. Lockemann . . . . . . . . . . . . . . . . . . . . .

Electrolytic estimation of minute quantities of arsenic by C. Mai and $\mathrm{H}$. Hurt

Quantitative estimation of arsenic and antimony by the Gutzeit method

Biological detection of arsenic by means of penicillium brevicaule

Detection of arsenic in organic arsenic compounds, i.e. Cacodylic acid; Arrhenal; Atoxyl. Their detection in urine.

Quantitative estimation of minute quantities of arsenic by Karl Th.

Mörner

Detection of Salicylic Acid in Foods and Beverages; In Wine, Meat Products, Milk

2. By precipitation with potassium bismuthous iodide and decomposition of the precipitate with alkali hydroxide-carbonate by $\mathbf{H}$. Thoms.

3. By the method of H. M. Gordin

Quantitative estimation of strychnine and quinine in presence of each other

Estimation of the toxicity of chemical compounds by blood hæmolysis by A. J. J. Vandervelde 


\section{CHAPTER VI}

Quantitative Estimation of Alkaloids and other Active Principles in Raw Materials and in their Preparations

Alralomal Estimations of Drugs and therr Pharmaceutical Preparations According to the German Pharmacopgia . . . . . 260 Estimation of alkaloid in aconite root . . . . . . . . . . . $26 \mathbf{r}$ Estimation of cantharidin in Spanish fly . . . . . . . . . . . ${ }_{2}^{26} 3$ Estimation of cinchona alkaloids . . . . . . . . . . . 264

r. In cinchona bark; 2 . In aqueous extract of cinchona and in alcoholic extract of cinchona.

Estimation of quinine in mixtures of cinchona alkaloids by the sulphate method

r. Cinchona bark; 2. Cinchona extract.

Estimation of colchicin in colchicum seeds and in colchicum corms Estimation of alkaloid in pomegranate bark. . . . . . . . . . . Estimation of caffeine in coffee, tea, cola nuts and Guarana paste

r. C. C. Keller's method; 2. A. Hilger-A. Juckenack's method. 3. A. Hilger-H. Göckel's method; 4. Socolof-Trillich-Göckel method; 5. E. Katz's method; 6. K. Dieterich's method.

Estimation of alkaloid in ipecacuanha root . . . . . . . . . 277

Estimation of nicotine in tobacco . . . . . . . . . . . . . . 279

I. R. Kissling's method; 2. C. C. Keller's method; 3. J. Toth's method.

Estimation of hydrastine in hydrastis extract . . . . . . . . . .

Estimation of berberine .................. . .

Estimation of hydrastine by the picrolonate method of H. Matthes and O. Rammstedt .

I. In fluid extract of hydrastis; 2 . In hydrastis root.

Estimation of morphine in opium and in its pharmaceutical preparations . . . . . . . . . . . . . . . . . . . . .

r. In opium; 2. In extract of opium; 3 . In wine of opium and in tincture of opium.

Estimation of pilocarpine in jaborandum leaves . . . . . . . . .

r. G. Fromme's method; 2. H. Matthes and O. Rammstedt's method.

Piperine and its estimation in pepper

r. J. König's method; 2. Cazeneuve and Caillot's method.

Estimation of santonin in wormseed ... . . . . . . . . . . .

r. K. Thæter's method; 2. J. Katz's method.

Estimation of solanine in potatoes.

r. O. Schmiedeberg and G. Meyer's method; 2. F. v. Morgenstern's method.

Estimation of alkaloid in nux vomica and its preparations

C. C. Keller's method . . . . . . . . . . . . . . . . . . . 293

Method of the German Pharmacopœia. . . . . . . . . . . . 294

r. In nux vomica; 2 . In extract of nux vomica; 3 . In tincture of nux vomica. 
PAgE

H. Matthes and O. Rammstedt's method. . . . . . . . . . . 296

I. In extract of nux vomica; 2. In tincture of nux vomica; 3 . In nux vomica.

Estimation of strychnine in mixtures of strychnine and brucine by

C. C. Keller-H. M. Gordin . . . . . . . . . . . . . . . 298

Estimation of theobromine and caffeine in cacao and in chocolate 298

Estimation of alkaloid in the leaves of atropa belladonna, hyo-

scyamus niger and datura strammonium . . . . . . . . . . 300

Estimation of alkaloid in extract of belladonna, according to the

German Pharmacopœia, in extract of hyoscyamus. . . . . . 30r

Assay of officinal extracts by E. Merck . . . . . . . . . . . . 302

Extract of belladonna; Extract of cinchona; Extract of strychnine.

\section{CHAPTER VII}

Detection of Carbon Monoxide Blood, Blood Stains and Human Blood

x. Recognition of carbon monoxide blood. . . . . . . . . . . 304

2. Detection of blood stains . . . . . . . . . . . . . . . . 307

Hæmatin . . . . . . . . . . . . . . . . 308

Spectroscopic detection of blood . . . . . . . . . . . 310

Other tests for blood . . . . . . . . . . . . . . . . $3{ }_{2}$

Schönbein-van Deen's test; Vitali's procedure in this test;

Schaer's procedure; Aloin test.

3. Biological detection of human blood . . . . . . . . . . . 3r4

\section{APPENDIX}

\section{Preparation of Reagents}

A. General alkaloidal reagents . . . . . . . . . . . 3 I $7^{7}$

B. Special reagents and solutions . . . . . . . . . . . 320

C. The indicator iodeosine . . . . . . . . . . . . . . . . . . 322

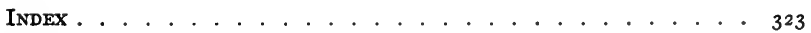





\section{INTRODUCTION}

Nearly all the common poisons and drugs may be placed in one of three groups. This classification, based upon the chemical behavior of these substances during isolation from mixtures, is as follows:

Group I.-The members of this group, when heated, volatilize without decomposition and distil with steam from an acid solution. Yellow phosphorus, hydrocyanic acid, carbolic acid, chloroform, chloral hydrate, iodoform, aniline, nitrobenzene, carbon disulphide and alcohol (ethyl and methyl) are the principal substances of this class.

Group II.-The members of this group are non-volatile, organic substances which do not distil with steam from an acid solution. But hot alcohol containing tartaric acid will extract them from extraneous matter. Alkaloids, many glucosides and bitter principles, as well as certain synthetic organic drugs, like acetanilide, phenacetine, antipyrine, pyramidone, sulphonal and veronal comprise this group.

Group III.-This group includes all poisonous metals.

In toxicological analysis, therefore, poisons are divided into three groups, each of which has its own special methods of procedure. A few poisons like mineral acids, caustic alkalies, oxalic acid and potassium chlorate cannot be conveniently placed in these three groups owing to differences in solubility and other peculiarities. Special tests for such substances must be made with a separate portion of material. Chapter IV contains a description of the methods used in identifying these substances.

The material must be thoroughly mixed and divided into three or four approximately equal portions, unless the analysis is to be limited to the detection of a single well-defined substance. One portion is tested for non-volatile, organic substances (Chapter II). The second portion is examined for 
volatile poisons (Chapter I) and the residue from this portion is used in testing for poisonous metals (Chapter III). The third portion is tested for substances considered in Chapter IV. The fourth portion is held in reserve in case additional material is needed to verify a doubtful result, or to replace a portion accidentally lost during analysis.

Occasionally it is advisable to depart from the general procedure and follow a special method, especially in detecting a single poison, or in estimating it quantitatively. For instance, pure ether would not be the best solvent to use in extracting strychnine quantitatively from an alkaline solution. A mixture of ether and chloroform, or better pure chloroform would be preferable, since strychnine is more soluble in the latter solvent than in pure ether. For the same reason chloroform should be used in the quantitative extraction of caffeine or antipyrine. When only a small quantity of material is available for analysis, tests for all three groups of poisons may be made with the same portion. In this case after removal of volatile poisons (Chapter I) the residue should be divided into two unequal portions. The larger portion should be tested for non-volatile, organic poisons (Chapter II). The smaller portion together with the residue left after extracting non-volatile, organic poisons should be tested for poisonous metals (Chapter III). It is advisable, however, even in such a case to reserve a portion of material for any contingencies.

Organs of the human body like liver, kidneys, spleen, heart, brain, stomach or intestines with contents should be cut into small pieces and then finely chopped before being examined chemically. An organ should first be cut into small pieces with sharp, clean scissors and then minced with a clean chopping knife in a new wooden bowl, or a small meat machine, which has been carefully cleaned, may be used. Material may be held with nickel plated tongs while being cut with scissors. Largely to eliminate the unpleasant odor of viscera and facilitate bringing them by hashing into a condition most favorable to the action of acids or solvents, it has been suggested that they be cooled $\left(-6^{\circ}\right.$ to $\left.-10^{\circ}\right)$. 


\section{DETECTION OF POISONS}

\section{CHAPTER I \\ VOLATILE POISONS}

Yellow Phosphorus and Other Poisons Volatile with Steam from Acid Solution

Scherer's Test.-This test should precede the distillation described on page 18 . The principle of the test is that moist phosphorus vapor and silver nitrate form black silver phosphide $\left(\mathrm{Ag}_{3} \mathrm{P}\right)$, metallic silver, phosphoric and sometimes phosphorous acid. Place the finely divided material in a small flask and. cover with water if a sufficient quantity is not present. Cut a V-shaped slit in the cork and place the latter loosely in the mouth of the flask so that the two strips of filter paper are freely suspended (Fig. I). Moisten one strip with silver nitrate and the other with lead acetate solution. ${ }^{1}$ Warm gently upon the water-bath ( 40 to $\left.50^{\circ}\right) .^{2}$ If the silver but not the lead paper is darkened, yellow phosphorus may be present. If both papers are darkened, hydrogen sulphide also is present. In the latter case yellow phosphorus may be present with hydrogen sulphide. In absence of hydrogen sulphide, darkening of the silver

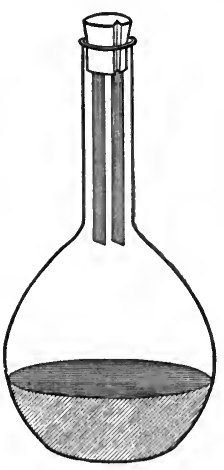

FIG. I. paper is not final proof of yellow phosphorus, for any volatile organic substance having reducing properties, as formalde-

'A more sensitive "lead paper" may be obtained by using alkaline lead solution prepared by adding excess of sodium hydroxide to the solution of a lead salt whereby $\mathrm{Pb}(\mathrm{OH})(\mathrm{ONa})$ and $\mathrm{Pb}(\mathrm{ONa})_{2}$ are formed.

${ }^{2}$ Temperatures in this book are expressed in Centigrade degrees. Tr. 
hyde (H.CHO), or formic acid (H.COOH), may give the same result.

Scherer's test is of value in proving the absence rather than the presence of yellow phosphorus. It is a good preliminary test, as it excludes phosphorus if the silver paper is unchanged.

Distillation.-Place a portion of finely divided and thoroughly mixed material in a large round-bottom flask and add enough distilled water for free distillation. Then add tartaric acid solution drop by drop until the mixture is acid after thorough shaking. Practice analyses ${ }^{1}$ usually require 20 to 30 drops of Io per cent. tartaric acid solution.

In examining animal material, as the stomach or intestines and contents, or organs, like liver, spleen and kidneys, it is often unnecessary to add•much water because enough is usually present. First chop the material in a wooden tray with a steel knife. In a medico-legal analysis the tray should be new. A meat machine which has been carefully cleaned may be used. Thin the material with a little distilled water, acidify with dilute tartaric or sulphuric acid and finally distil.

If Scherer's test is positive, begin distilling with the Mitscherlich apparatus (Fig. 2); but if negative, distil in the usual way with the Liebig condenser (see page I8). The distillate may contain:

Yellow phosphorus
Hydrocyanic acid
Carbolic acid
Chloroform
Chloral hydrate
Iodoform
Nitrobenzene

\author{
Aniline \\ Ethyl alcohol \\ Methyl alcohol \\ Acetone \\ Carbon disulphide \\ Benzaldehyde \\ Bitter almond water
}

${ }^{1}$ Laboratory practice in detecting poisons may be given by mixing small quantities (from 0.03 to 0.05 or 0.1 gram) of a poison with dry bread or biscuit crumbs, meal or meat. Finely chopped organs (liver, kidney, spleen, etc.), sausage meat, beer, wine or milk may be used. Drugs like morphine, codeine, quinine, acetanilide, phenacetine, antipyrine, caffeine, santonin, sulphonal, veronal, calomel, tartar emetic, subnitrate of bismuth, etc., may be mixed with powdered cane- or milk-sugar. The last kind of practice is especially suitable for students of pharmacy. 


\section{YELLOW PHOSPHORUS}

Mitscherlich Method of Detecting Yellow Phosphorus

The principle of this method is that yellow phosphorus volatilizes with steam and becomes luminous in contact with air. The phosphorescence is best seen in a dark room.

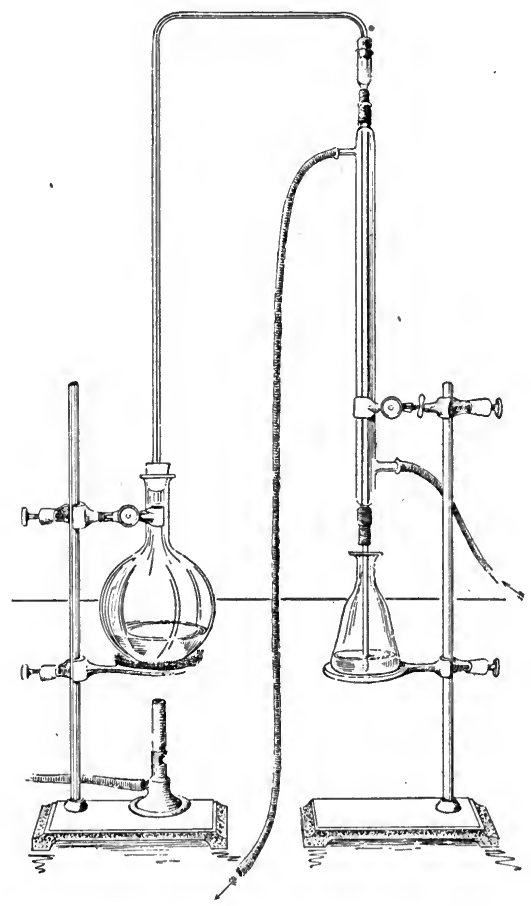

FIG. 2.-Mitscherlich Apparatus.

Procedure.-Arrange the apparatus as in Fig. 2. Support the condenser in a vertical position and connect the upper end with the flask by a glass tube about $8 \mathrm{~mm}$. internal diameter. This tube has two right-angle bends and each end passes through 
a cork. Have condenser and tube scrupulously clean to avoid interference with the phosphorescence.

Have the flask at most not more than a third full. This precaution is necessary because many materials, containing protein substances like albumin, albumose, etc., and starchy matter, when distilled in aqueous solution, cause more or less foaming which is liable to carry over solid matter into the receiver. Use as the receiver an Erlenmeyer flask containing a little distilled water ( 3 to $5 \mathrm{cc}$.) into which the end of the condenser dips. This precaution prevents loss of easily volatile substances like hydrocyanic acid and chloroform. Heat the flask upon a wire gauze of fine mesh, asbestos plate or sand-bath and bring the contents to boiling by raising the temperature gradually. There is some danger of burning or carbonizing organic matter on the bottom of the flask, if heat is applied too strongly or rapidly. When boiling begins, make the room as dark as possible and watch for phosphorescence in the tube and condenser. It usually appears as a luminous ring or band in the upper part of the condenser. When this is distinctly visible, the presence of yellow phosphorus is established. Phosphorescence during distillation with steam is very characteristic of yellow phosphorus and frequently is the only sure and unquestionable test for this element.

Phosphorescence is a process of oxidation by which phosphorus vapor is changed to phosphorous acid. Should it not appear immediately, distillation must be continued for some time, since certain substances like ethyl or methyl alcohol, ether, turpentine and many other ethereal oils either prevent the phenomenon entirely or seriously retard it. Considerable carbolic acid, creosote, chloroform, chloral hydrate, as well as hydrogen sulphide, may completely prevent phosphorescence.

K. Polstorff and J. Mensching ${ }^{1}$ have shown that mercuric chloride as well as other mercury compounds may also interfere with phosphorescence. Possibly mercuric chloride carried over by steam is reduced to metallic mercury by phosphorus vapor. In that case the metal should appear in the distillate. The

${ }^{1}$ Berichte der Deutschen chemischen Gesellschaft 19,1763 (1886). 
fact that both metallic mercury and phosphoric acid can be detected in the distillate favors the supposition that action takes place between phosphorus vapor and mercuric chloride.

Phosphorescence, however, often appears when these substances have passed over. But even when prolonged distillation fails to give a positive result, this must not be accepted as final proof of the absence of phosphorus until other tests have been made. Whatever the result, evaporate a portion of the distillate to dryness on the water-bath with excess of saturated chlorine water, or with a little fuming nitric acid. Phosphorus always imparts a strong odor to the distillate. Small drops of phosphorus appear if the quantity is large, and the solution contains phosphorous acid. Dissolve the residue from evaporation in 2 to $3 \mathrm{cc}$. of water and test in two separate portions for phosphoric acid.

I. Ammonium Molybdate Test.-Acidify the solution with a few drops of concentrated nitric acid. Add an equal volume of ammonium molybdate solution and warm to about $40^{\circ}$. Phosphoric acid precipitates yellow ammonium phospho-molybdate.

\section{Ammonium Magnesium} Phosphate Test.-Add magnesia mixture $^{1}$ to the second portion. Phosphoric acid gives a white crystalline precipitate of ammonium magnesium phosphate $\left(\mathrm{H}_{4} \mathrm{~N}\right)$ $\mathrm{MgPO}_{4} \cdot 6 \mathrm{H}_{2} \mathrm{O}$. Vigorous shaking favors precipitation. When only

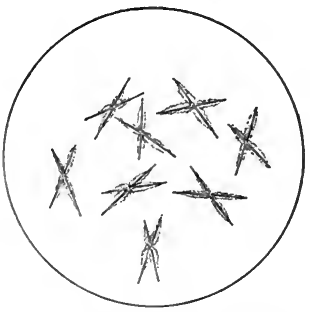

FIG. 3.-Ammonium magnesium phosphate crystals. traces of phosphoric acid are present, long standing is necessary before the precipitate appears. Always examine the precipitate with the microscope. It should consist of well-formed

${ }^{1}$ Magnesia mixture is a clear solution prepared by mixing equal volumes of magnesium chloride, ammonium chloride and ammonium hydroxide (about ro per cent.) solutions. It contains the readily soluble double chloride of ammonium and magnesium which is not decomposed by ammonium hydroxide. This reagent is prepared as needed and should be perfectly clear and colorless. 
crystals or at least should be crystalline. These crystals are transparent, acicular prisms (Fig. 3).

Notes.-A. Fischer ${ }^{1}$ states that substances interfering more or less with the detection of phosphorus by the Mitscherlich method are usually less troublesome if Hilger and Nattermann's procedure is used (see page 15). The essential feature of the latter process consists in allowing steam charged with phosphorus to pass into the air, or in admitting air into the apparatus.

\section{Detection of Phosphorus and Phosphorous Acid}

\section{(Blondlot ${ }^{2}$-Dusart ${ }^{3}$ )}

When the Mitscherlich method fails to show phosphorus, it is often necessary to test for phosphorous acid. This is the first product in the oxidation of phosphorus and is easily formed. The Blondlot-Dusart method shows the slightest trace of phosphorous $\left(\mathrm{H}_{3} \mathrm{PO}_{3}\right)$ and hypophosphorous $\left(\mathrm{H}_{3} \mathrm{PO}_{2}\right)$ acids as well as yellow phosphorus. The method consists in converting yellow phosphorus into phosphine $\left(\mathrm{PH}_{3}\right)$ by nascent hydrogen. The lower oxidation products of phosphorus, namely, hypophosphorous $\left(\mathrm{H}_{3} \mathrm{PO}_{2}\right)$ and phosphorous $\left(\mathrm{H}_{3} \mathrm{PO}_{3}\right)$ acids, ${ }^{4}$ when warmed with zinc and dilute sulphuric acid are reduced to phosphine by nascent hydrogen:

$$
\begin{aligned}
& \mathrm{H}_{3} \mathrm{PO}_{2}+{ }_{4} \mathrm{H}=\mathrm{PH}_{3}+{ }_{2} \mathrm{H}_{2} \mathrm{O}, \\
& \mathrm{H}_{3} \mathrm{PO}_{3}+6 \mathrm{H}=\mathrm{PH}_{3}+{ }_{3} \mathrm{H}_{2} \mathrm{O} .
\end{aligned}
$$

Phosphine, or hydrogen charged with phosphorus vapor, burns with a characteristic green flame (Dusart's reaction):

$$
{ }_{2} \mathrm{PH}_{3}+{ }_{4} \mathrm{O}_{2}=\mathrm{P}_{2} \mathrm{O}_{5}+{ }_{3} \mathrm{H}_{2} \mathrm{O} \text {. }
$$

The green flame is easily recognized by depressing a cold porcelain dish or plate upon the flame. Detection of phosphorus by the Blondlot-Dusart method depends upon these two facts.

A toxicological analysis usually deals with the detection of traces of yellow phosphorus. Hydrogen after acting in the nascent state upon the material is not directly examined for

1 Pflueger's Archiv, 97, 578 (1903).

2 Journal de Pharmacie et de Chimie (3), 40, 25.

3 Comptes rendus de l'Academie des Sciences, 43, I 126.

${ }_{4}$ Nascent hydrogen will not reduce ordinary, ortho-phosphoric acid $\left(\mathrm{H}_{3} \mathrm{PO}_{4}\right)$, and its derivatives, pyrophosphoric $\left(\mathrm{H}_{4} \mathrm{P}_{2} \mathrm{O}_{7}\right)$ and meta-phosphoric $\left(\mathrm{HPO}_{3}\right)$ acids, to phosphine. 
phosphorus but is first passed into dilute silver nitrate solution. Phosphorus and phosphine precipitate black silver phosphide ${ }^{1}$ $\left(\mathrm{Ag}_{3} \mathrm{P}\right)$ :

$$
\mathrm{PH}_{3}+{ }_{3} \mathrm{AgNO}_{3}=\mathrm{Ag}_{3} \mathrm{P}+{ }_{3} \mathrm{HNO}_{3} .
$$

Thus traces of yellow phosphorus may be concentrated in the silver precipitate from which nascent hydrogen will liberate phosphine:

$$
\mathrm{Ag}_{3} \mathrm{P}+{ }_{3} \mathrm{H}=\mathrm{PH}_{3}+3 \mathrm{Ag} \text {. }
$$

If hydrogen produces a black or gray precipitate in the silver solution, phosphorus is not necessarily present, as hydrogen sulphide, arsine, stibine and reducing organic compounds behave similarly with silver nitrate. A black precipitate therefore should always be examined for phosphorus by the Dusart reaction.

In the detection of yellow phosphorus, the Blondlot-Dusart method combines two distinct operations, namely:

I. Preparation of the silver phosphide precipitate.

2. Examination of this precipitate in the Dusart apparatus.

Procedure. I. Preparation of Silver Phosphide.-Thin the finely divided material with water in a capacious flask where hydrogen is being evolved from phosphorus-free zinc and pure dilute sulphuric acid ( $\mathrm{I}: 5)$. In testing for phosphorous acid alone (see page I4) use the filtrate from an aqueous extract of the material, or the filtrate from the residue left after the Mitscherlich distillation (see page 5). Nascent hydrogen should act for I. 5 to 2 hours, or even longer, and pass through neutral silver nitrate solution in the receiver at the end of the apparatus. If yellow phosphorus is present, the hydrogen will contain phosphorus and phosphine and cause a black precipitate of silver phosphide in the silver nitrate solution. Collect the precipitate upon a small ash-free paper, wash with a little cold water and examine in the Dusart apparatus as described elsewhere.

1 Phosphorus cannot be determined quantitatively as silver phosphide because this compound is partially decomposed by water. Phosphoric and phosphorous acids pass into solution:

(a) ${ }_{2} \mathrm{Ag}_{3} \mathrm{P}+5 \mathrm{O}+3 \mathrm{H}_{2} \mathrm{O}=6 \mathrm{Ag}+{ }_{2} \mathrm{H}_{3} \mathrm{PO}_{4}$
(b) ${ }_{2} \mathrm{Ag}_{3} \mathrm{P}+3 \mathrm{O}+3 \mathrm{H}_{2} \mathrm{O}=6 \mathrm{Ag}+{ }_{2} \mathrm{H}_{3} \mathrm{PO}_{3}$. 
If there is silver phosphide in the precipitate, the filtrate will contain phosphoric or phosphorous acid (see Note, page 9). To detect phosphoric acid, first add hydrochloric acid to remove excess of silver from this filtrate. Filter through paper previously well washed with acid and water and completely expel hydrochloric acid from the filtrate by evaporation upon the water-bath with concentrated nitric acid. Dissolve the residue in a little warm water and test for phosphoric acid with ammonium molybdate or magnesia mixture.

2. Examination of the Silver Precipitate $\left(\mathrm{Ag}_{3} \mathrm{P}\right)$ for Phosphorus.-Two forms of apparatus may be used for this purpose, namely:

(a) Fresenius-Neubauer ${ }^{1}$ Apparatus.-Generate hydrogen in flask A (Fig. 4) from pure phosphorus-free zinc and dilute

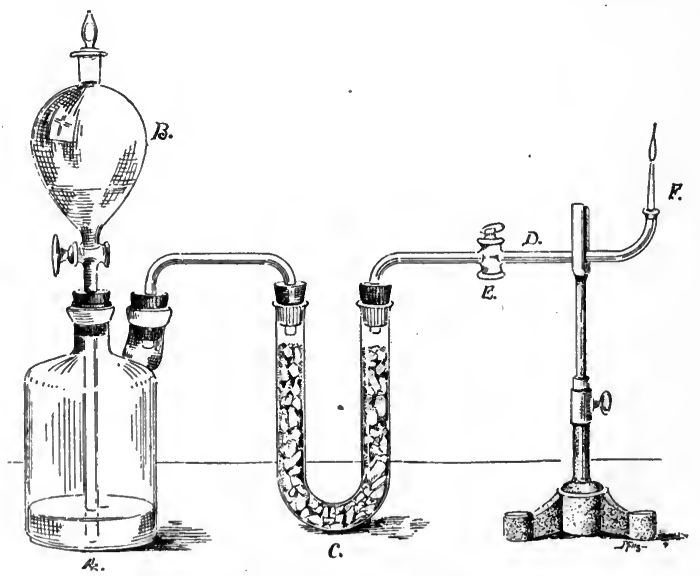

FIG. 4.-Fresenius-Neubauer Apparatus.

sulphuric acid. Fill U-tube $\mathrm{C}$ with pieces of pumice stone saturated with concentrated potassium hydroxide solution to absorb any hydrogen sulphide. Use hard glass for tube $\mathrm{D}$ and

${ }^{1}$ C. R. Fresenius, Qualitative chemische Analyse, XVI edition, page $52 \mathrm{I}$. 
have the tip $\mathrm{F}$ of platinum. The part marked $\mathrm{E}^{1}$ is a glass stop-cock or screw-tap. Reservoir B serves to hold liquid from A when cock $\mathrm{E}$ is closed. A platinum tip is essential, otherwise the flame instead of being colorless will always be yellow from sodium in the glass. The place where the platinum tip is fused into the glass should be cooled by wrapping cotton around the glass and keeping it moist.

Procedure.-Open E and let hydrogen from A pass for some time through the apparatus to expel air. Then close $\mathrm{E}$ and liquid in $\mathrm{A}$ will rise into $\mathrm{B}$. Now open $\mathrm{E}$ just enough to allow hydrogen to burn with a small flame which should be colorless in the dark. If there is no trace of green in the inner cone and a porcelain dish depressed upon the flame does not show an emerald-green coloration, hydrogen is phosphorus-free. It is well to repeat this test. To test the silver precipitate for phosphorus, wash it with the paper into $\mathrm{B}$ with a little water.

When the entire precipitate is in $\mathrm{A}$, close $\mathrm{E}$ until all the liquid has risen from $\mathrm{A}$ into $\mathrm{B}$. Then open $\mathrm{E}$, light the hydrogen and examine the flame in the dark. If the precipitate contains a trace of silver phosphide, the inner cone will be green and a porcelain dish depressed upon the flame will show an emeraldgreen coloration. Have the hydrogen flame small so that its color may be observed for some time.

(b) Hilger-Nattermann ${ }^{2}$ Apparatus.-Reduction takes place in a roo cc. flask closed by a rubber stopper with three holes, two of which are for right-angle tubes just passing through the stopper and the third for a thistle-tube going to the bottom of the flask (Fig. 5). Hydrogen from a Kipp generator enters the flask by one tube and leaves by the other. Attach to the latter a U-tube filled with pieces of pumice stone saturated with concentrated potassium hydroxide solution to absorb hydrogen sulphide. Connect the other end of the U-tube with a hard

${ }^{1}$ Fresenius and Neubauer use a screw pinch-cock instead of a gas-cock but by means of a short rubber connector they interpose an ordinary cock between the gas-flask $\mathrm{A}$ and the $\mathrm{U}$-tube $\mathrm{C}$.

${ }^{2}$ Forschungsbericht über Lebensmittel und ihre Beziehungen zur Hygiene, etc., $4,24 \mathrm{I} \sim 258$ ( 1897 ). 
glass tube tipped with platinum. ${ }^{1}$ Cut the paper containing the precipitate into small pieces and place in the flask which contains in addition a few pieces of phosphorus-free zinc and enough water to seal the thistle-tube. Light the hydrogen after it has passed through the apparatus for some time and been found free from air by the usual test. Seen in the dark the flame should be entirely colorless and burn without a green cone or a greenish glow. ${ }^{2}$ Hilger and Nattermann advise a spectroscopic examination of the flame to determine the purity of the

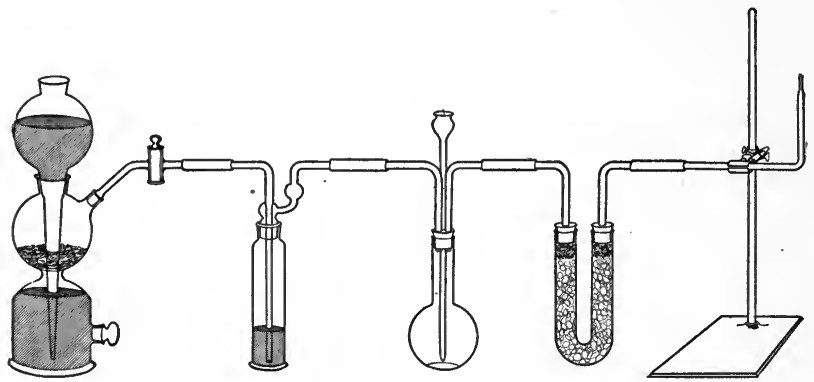

FIG. 5.-Hilger-Nattermann Apparatus.

zinc. Pure zinc gives a hydrogen spectrum which shows only an orange colored line in place of the yellow sodium line. The minutest trace of phosphorus will give three green lines lying to the right of the line $\mathrm{D}$. The color of two of these lines is more pronounced than that of the third. Having thus tested the purity of zinc and sulphuric acid, pour a few cc. of dilute sulphuric acid $(\mathrm{I}: 5)$ through the thistle-tube into the flask containing zinc and the silver precipitate. If the latter contains phosphorus, the flame will show, though not always at once, a green coloration which should be examined with the spectroscope.

1 Hilger and Nattermann use a platinum tipped blow-pipe instead of a glass tube tipped with the same metal. Cotton, which is kept moist and acts as a cooler, is wrapped around the blow-pipe below the tip.

${ }^{2}$ Zinc entirely free from phosphorus which will stand this test is difficult to obtain. 
The Mitscherlich method affords a distillate especially suitable for the Blondlot-Dusart test. If this imparts a green color to the hydrogen flame, there can be no question about the presence of phosphorus.

Although the Blondlot-Dusart test is very delicate, many chemists refuse to accept it as a substitute for the Mitscherlich test. Selmi states that animal material like brain, which contains organic phosphorus compounds, yields after putrefaction a distillate that often gives a black precipitate with silver nitrate solution. This will impart a green tinge to the hydrogen flame in the Blondlot-Dusart test.

Z. Halász, ${ }^{1}$ however, has failed to confirm Selmi's results. He examined two kinds of animal material by the Blondlot-Dusart method. First, he tested normal brains (man, calf, hog); second, the brain and other organs of rabbits that had been given poisonous doses of phosphorus by the mouth and subcutaneously. He examined these organs when fresh and also from week to week after more or less pronounced putrefaction had set in, but could not detect phosphorus in the brain in a single instance. These experiments disprove the earlier idea that phosphorus normally present in the brain is so changed during putrefaction that it can be detected by the Blondlot-Dusart reaction. He also failed to detect phosphorus in the brain of rabbits poisoned by this element, though he found it in other organs, as stomach and intestines, and in those rich in blood, as liver, lungs and kidneys. He could always detect small or large quantities of phosphorus in any organ which this element had directly reached, or by which it had been indirectly absorbed. If any compound containing phosphorus is really formed in the brain during putrefaction, Halász concluded that it is not volatile with steam and does not give the Blondlot-Dusart reaction. On the basis of these experiments Halâsz holds that the Blondlot-Dusart method of detecting phosphorus is just as reliable for forensic purposes as that of Mitscherlich.

\section{Procedure of Halász in the Blondlot-Dusart Method}

Make a thin mixture of very finely divided material and boiled water in a flatbottom flask where hydrogen is being generated from phosphorus-free zinc and dilute sulphuric acid. Warm upon the water-bath and pass the gas through an absorption-tube provided with several bulbs and containing neutral silver nitrate solution. Concentrated sulphuric acid and a little platinic chloride may be added toward the end to hasten the evolution of gas. Nascent hydrogen thus acts upon phosphorus in the animal material for $2-2.5$ hours. Finally wash the silver precipitate carefully with water and transfer it with the paper to the BlondlotDusart apparatus.

${ }^{1}$ Zeitschrift für anorganische Chemie 26,438 (rgor). 


\section{Detection of Phosphorous Acid}

The reduction of phosphorous acid to phosphine by zinc and dilute sulphuric acid takes place very slowly. Hilger and Nattermann state that even a few milligrams require the action of nascent hydrogen for ro to 14 days. Moreover careful manipulation is necessary because silver phosphide is quite unstable. Water decomposes this substance into metallic silver and phosphorous acid and the nitric acid present oxidizes the latter to phosphoric acid. Therefore when special attention must be given to phosphorous acid, Hilger and Nattermann recommend examining the silver precipitate (presumably $\mathrm{Ag}_{3} \mathrm{P}$ ) after 2 days, or at most 3 , for phosphorus by the Blondlot-Dusart method and the filtrate for phosphoric acid (see page ro).

\section{Detection of Phosphorus in Phosphorated Oils}

I. Straub's ${ }^{1}$ Test.-If a phosphorated oil is placed on the surface of copper sulphate solution, phosphorus will gradually pass from the oil to the aqueous solution and first form black copper phosphide. The latter, acting as a carrier of oxygen, oxidizes phosphorus still in the oil to phosphoric acid which dissolves in the water.

Shake ro cc. of phosphorated oil in a test-tube with $5 \mathrm{cc}$. of I per cent. copper sulphate solution. According to the amount of phosphorus a black or light brown coloration will appear in the emulsion at once, or in a few minutes, and at most after 2 hours. Phosphoric acid in the aqueous solution may be recognized by ammonium molybdate. At least 0.0025 per cent. of phosphorus may be detected in this way.

A practical, therapeutic application of this reaction may be made in acute phosphorus poisoning. Administration of copper sulphate solution may prevent absorption of free phosphorus still in the gastro-intestinal tract.

2. The Mitscherlich test is also applicable to phosphorated oils, even though the oil may contain only 0.0002 gram of phosphorus in Ioo grams. But phosphorescence will not appear unless air is admitted into the tube from time to time.

Phosphorus in oils cannot be determined quantitatively by the distillation method, for not more than 36 to $4 \mathrm{I}$ per cent. of

${ }^{1} \mathrm{~W}$. Straub, Münchener medizinische Wochenschrift 50, II45; Archiv der Pharmazie 24I, 335 (r903); and Zeitschrift für anorganische Chemie 35, 460 (I903). 
phosphorus will distil over. The quantitative method recommended by Straub (see page 23I) may be used in that case.

\section{Detection and Quantitative Estimation of Phosphorus}

(Mitscherlich-Scherer)

Acidify a weighed portion of material with dilute sulphuric acid and add a little ferrous sulphate. Distil in a gentle stream of carbon dioxide, using a large flask fitted with a two-hole stopper. Expel air completely from the apparatus by carbon dioxide before heating. Use as receiver a flask fitted with a

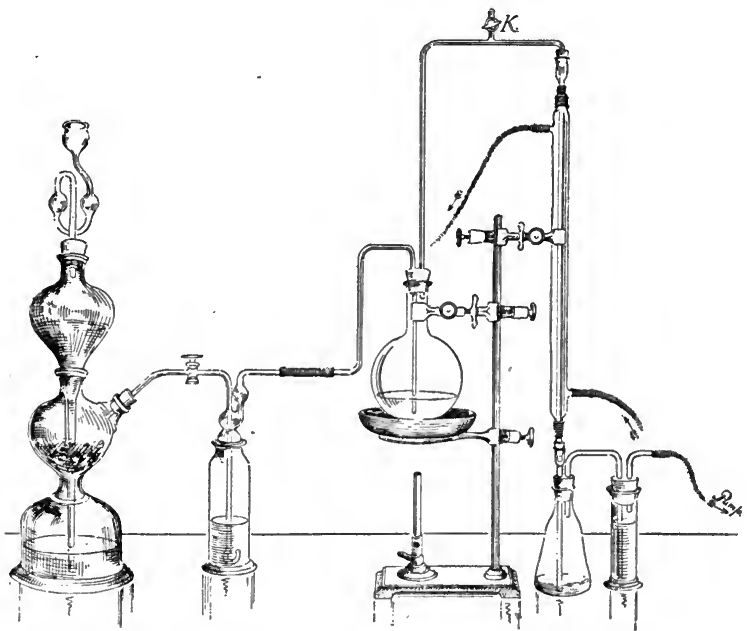

FIG. 6.-Hilger-Nattermann Apparatus for Detecting and Quantitatively Estimating Phosphorus.

two-hole stopper. Pass the end of the condenser through one hole and connect the other with a U-tube containing silver nitrate solution. Evaporate the distillate upon the waterbath with strong bromine water, or with concentrated nitric acid, to oxidize phosphorus or any phosphorous acid formed. Dissolve the residue in a little water and precipitate phos- 
phoric acid with magnesia mixture. Weigh the precipitate as magnesium pyrophosphate, $\mathrm{Mg}_{2} \mathrm{P}_{2} \mathrm{O}_{7}$. Heat the contents of the U-tube with concentrated nitric acid. Precipitate silver as silver chloride and filter. Concentrate the filtrate by evaporation and precipitate phosphoric acid with magnesia mixture as before. Combine this precipitate with the other. In distillation some phosphorus separates as globules in the first receiver and any carried over as vapor by carbon dioxide is retained by silver nitrate solution. As the steam distillation of phosphorus is very slow, the process should be carried on for at least 3 hours. Hilger and Nattermann recommend the apparatus in Fig. 6 not only for detecting phosphorus but for estimating it quantitatively. Air may be mixed with phosphorus vapor by means of stop-cock $\mathrm{K}$ and the characteristic phosphorescence will appear.

Remarks Upon the Mitscherlich Test.-Hilger and Nattermann state that 0.00006 gram of yellow phosphorus is the smallest quantity that can be detected by the Mitscherlich method. When $200 \mathrm{cc}$. of water containing 0.0003 gram of phosphorus were distilled, there was brilliant phosphorescence for 5 minutes. The degree of dilution seems to have no effect upon the result, at least not within limits occurring in practice. Hydrogen sulphide, always present in putrefying animal matter, has no apparent effect upon phosphorescence. Free phosphorus can be detected in putrid organic matter even after the lapse of considerable time. Putrefactive and digestive processes appear to prevent oxidation of phosphorus. Dragendorff detected phosphorus in an exhu med body several weeks after death. Neumann found free phosphorus in a human body fourteen days and Elvers eight weeks after death.

When an acid aqueous solution is distilled in the Mitscherlich apparatus, the flask residue always contains phosphoric $\left(\mathrm{H}_{3} \mathrm{PO}_{4}\right)$, phosphorous $\left(\mathrm{H}_{3} \mathrm{PO}_{3}\right)$ and hypophosphorous $\left(\mathrm{H}_{3} \mathrm{PO}_{2}\right)$ acids and red phosphorus. Distillation of a solution of 0.0644 gram of phosphorus gave only $7 I .33$ per cent. in the distillate. The residue contained:

$\begin{array}{lrr}\text { Phosphorus as phosphoric acid } & \left(\mathrm{H}_{3} \mathrm{PO}_{4}\right) & \text { I8.93 per cent. } \\ \text { Phosphorus as phosphorous acid } & \left(\mathrm{H}_{3} \mathrm{PO}_{3}\right) & 2.15 \text { per cent. } \\ \text { Phosphorus as hypophosphorous acid }\left(\mathrm{H}_{3} \mathrm{PO}_{2}\right) & 4.27 \text { per cent. } \\ \text { Phosphorus as red phosphorus } & \frac{2.98}{28.33} \text { per cent. }\end{array}$

Oxidation of phosphorus may be prevented by distilling in a current of carbon dioxide as in the Mitscherlich-Scherer method (see page I $_{5}$ ).

Metabolism in Phosphorus Poisoning.-Phosphorus has a very poisonous action upon the processes of metabolism. Present as a vapor in the blood and tissue fluids, it retards normal oxidative processes occurring in the animal organ- 
ism during metabolism. In phosphorus poisoning the usual course of chemical metabolism is wholly changed. Fat instead of being oxidized is deposited in the organs in large quantity (fatty degeneration of the liver). Different observers believe there is formation of fat from protein. During phosphorus poisoning the quantity of protein broken down is greatly increased. In human metabolism this applies to protein in both food and tissues. Yet the needs of the organism are not satisfied and the conclusion is that the changes are not as complete as in normal protein metabolism. This increase in the breaking down of tissues in phosphorus poisoning recalls similar changes which take place during respiration in insufficient oxygen. Accompanying these abnormal processes are certain nitrogenous and non-nitrogenous products of metabolism which either are not normally formed in the organism or appear merely as intermediate steps in the formation of the oxidative products of metabolism. Decomposition of the protein molecule goes in part only as far as the amino-acids. Consequently in phosphorus poisoning the urine almost always contains

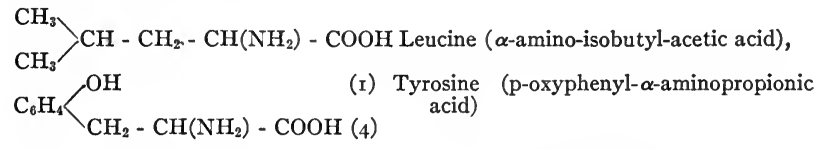

$\mathrm{CH}_{3}-\mathrm{CH}(\mathrm{OH})-\mathrm{COOH}$

Sarcolactic acid (dextro-lactic acid).

In acute phosphorus poisoning the following acids can be detected in the urine in greatly increased quantity:<smiles>CCCCC(O)CC(=O)OCC</smiles><smiles>CCCCO</smiles>
$\mathrm{CH}_{2}-\mathrm{CH}_{2}-\mathrm{COOH}(4)$

Para-oxyphenyl-acetic acid,

(I) Para-oxyphenyl-propionic acid (hydropara-cumaric acid).

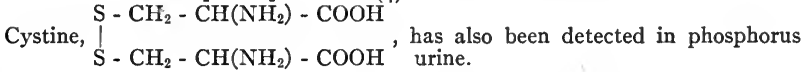

In phosphorus poisoning there is a marked decrease in the urea-content of the urine but a decided increase in total nitrogen. A considerable part of the nitrogen, that is to say, 25 per cent. or more of the total nitrogen, appears to leave the body as ammonia. The urine of adults usually contains from 2 to 5 per cent. of the total nitrogen as ammonia. The increase of ammonia may have some connection with the increase in formation of acid during phosphorus poisoning.

Peptone-like substances, the presence of which is attributed to profound disturbance of metabolism, frequently appear in the urine in phosphorus poisoning. Various observers believe there is no longer any doubt as to the appearance of geniune peptonuria. A glycosuria may also appear, or be easily induced by a diet rich in sugar. In accord with this observation is the fact that the liver of an animal poisoned by phosphorus is without the power to change glucose of the blood into glycogen and store up the latter. In phosphorus poisoning the alkalinity of the blood rapidly diminishes owing to the increased formation of acid. Since persons poisoned by phosphorus have icterus (jaundice), bile-pigment, or at least urobilin, can be readily detected in the urine. 
The amounts of oxygen and carbon dioxide, which the organism respectively takes up and gives off, show a marked diminution during phosphorus poisoning. Only 48 per cent. of carbon dioxide, as compared with roo per cent. under normal conditions, may be eliminated.

In brief the chief characteristics of phosphorus urine are a strong acid reaction; presence of protein (peptone-like substances); and frequently occurrence of the amino-acids mentioned above, as well as fat cylinders, cell detritus, free fat globules and blood-corpuscles.

\section{Further Examination of the Distillate}

When phosphorescence has been distinctly observed in the Mitscherlich apparatus, it is advisable to stop distillation and change the Liebig condenser to its customary position. This simpler method of distilling is shown in Fig. 7 and should always be used in toxicological analysis when there is no occasion to test for phosphorus.

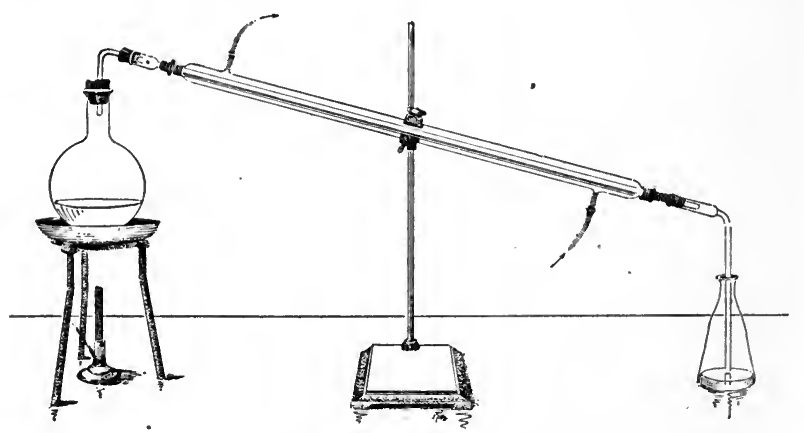

F1G. 7.-Distillation with Liebig Condenser.

Since the several poisons appearing here are not equally volatile with steam, it is best to collect the distillate in two or three fractions. The first will contain most of the easily volatile substances like hydrocyanic acid, chloroform, ethyl and methyl alcohol, acetone, iodoform and nitrobenzene. The others (second and third) will contain substances less easily volatile with steam like carbolic acid, aniline, chloral hydrate and carbon disulphide. This must not be understood to mean that the first part of the distillate will be free from substances that volatilize 
with difficulty, and the latter part free from those that volatilize easily. In the main such will be the separation, but either part of the distillate may contain traces of substances which will appear in larger quantity in the other part.

The proper procedure is to distil until 5 to Io $\mathrm{cc}$. of liquid have been collected. Divide the distillate into several portions and test for hydrocyanic acid, chloroform, ethyl and methyl alcohol, acetone, and, if necessary, also for iodoform and nitrobenzene. Use the second and third portions ( 10 to $20 \mathrm{cc}$.) to test for carbolic acid, aniline, chloral hydrate and carbon disulphide.

Several of these volatile substances have a characteristic odor, which makes it possible to recognize them with great certainty in the original material and especially in the distillate. First, test the distillate for each individual substance by its most characteristic reaction. Test for hydrocyanic acid by the Prussian blue or sulphocyanate reaction; for ethyl alcohol, acetone and acetaldehyde by Lieben's reaction; for methyl alcohol by one of the oxidation tests; for carbolic acid and aniline by Millon's reaction; for chloroform, chloral hydrate and iodoform by the phenylisocyanide reaction; for aniline with calcium hypochlorite solution; and finally, for carbon disulphide with lead acetate and potassium hydroxide solutions.

When there is reason to believe that a certain substance is present, confirm the result by making other characteristic tests. It is seldom necessary to examine the distillate for all the members of the group.

\section{HYDROCYANIC ACID, HCN}

Physiological Action.-In whatever way applied, hydrocyanic acid is absorbed, even from the skin. So rapid is the absorption of this poison that there is evidence of an intoxication after a few seconds, or a few minutes at most. Part of the poison thus absorbed passes from the body unchanged by way of the lungs. Another part, usually much less, is eliminated by the kidneys and passes into the urine. Sweat also is said to contain hydrocyanic acid.

Most of the absorbed hydrocyanic acid, though yariable in quantity, undergoes chemical change within the organism whatever be the form of its chemical combination. Hydrocyanic acid is supposed to combine with loosely bound sulphur of proteins and form sulphocyanic acid (HSCN) which is not nearly as toxic as hydrocyanic acid. (Antidote for hydrocyanic acid.) Hydrocyanic acid after the 
manner of the cyanohydrin reaction ${ }^{1}$ might combine chemically with carbohydrates of the blood and tissues. Finally, putrefactive changes as well as ferment action within the cadaver might convert hydrocyanic acid into ammonium formate. ${ }^{2}$ The last statement may explain the disappearance of hydrocyanic acid until only traces remain in the cadaver. Thus the possibility of making more than an approximately quantitative determination of hydrocyanic acid taken internally is precluded from the beginning. Yet there are instances where the poison has been found in the human cadaver after 44 days, and even after 100 and 180 days. After 48 days the author obtained enough hydrocyanic acid in the distillate from stomach and intestinal contents of a child $41 / 2$ years old to give the Prussian blue test in three different portions of the distillate after 3 to 4 hours.

Undoubtedly hydrocyanic acid has a very poisonous effect upon ferments for it kills certain vegetable and animal enzymes, or at least strongly retards their action. This acid interferes particularly with the action of that enzyme which causes transfer of oxygen from blood-corpuscles and thereby gives rise to oxidative processes (oxidation ferment, "respiration ferment"). Careful experiments in metabolism have shown that warm-blooded animals under the influence of hydrocyanic acid take up less than the normal amount of oxygen and consequently give off less carbon dioxide, even though relatively large quantities of oxygen are administered artificially. R. Kobert (Intoxikationen) regards hydrocyanic acid poisoning as an internal asphyxiation of the organs in presence of an excess of oxygen. The oxidative processes of the blood are checked and so little oxygen is used that the venous blood becomes arterial, that is to say, contains a large quantity of oxyhæmoglobin. As a result the color of the venous blood is bright red. This change of venous to arterial blood seems to be permanent in cold-blooded but usually only transitory in warm-blooded animals. The appearance of lactic acid in the blood and urine is due to the disturbing influence of the poison upon the oxidative processes of the organism. The processes of normal metabolism in warm-blooded animals finally oxidize lactic acid to carbon dioxide and water. Consequently the appearance of lactic acid in the blood is very transitory and it is not found in the urine at all. The occurrence of lactic acid in the blood and a decrease in its alkalinity are concurrent. As a result of very deficient oxidation during hydrocyanic acid poisoning, dextrose not infrequently appears in the urine.

The blood therefore in hydrocyanic acid poisoning is characteristically changed. Venous blood becomes bright red. And moreover blood which contains this acid cannot liberate oxygen from hydrogen peroxide, that is to say it has lost its catalytic power. $^{3}$ Such a compound as cyano-hæmoglobin appears to exist and

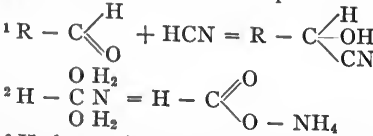

( $\mathrm{R}$ denotes any radical)

${ }^{3}$ Hydrocyanic acid poisons platinum black just as it does blood ferments. Put about 5 cc. of 3 per cent. hydrogen peroxide solution in each of two test-tubes. Add to one I or 2 drops of hydrocyanic acid (about I per cent. solution) and to both a trace of platinum black. Pure hydrogen peroxide at once gives off oxygen vigorously, whereas that containing hydrocyanic acid does not. 
its formation in the blood of a person poisoned by hydrocyanic acid would seem probable, yet for some unknown reason the union of this acid with hæmoglobin takes place either not at all or only with great difficulty.

In a chemical examination for hydrocyanic acid and potassium cyanide the contents of the stomach and intestines, organs rich in blood as liver, brain and heart, the blood itself and sometimes the urine are most important. Examine such material at once for hydrocyanic acid which may be recognized by its characteristic odor, provided putrefaction has not gone too far.

Preliminary Test.-A special test (Schönbein-Pagenstecher reaction) for hydrocyanic acid should precede distillation. Acidify a portion of the original material in a small flask with tartaric acid solution. Then suspend in the flask (see Fig. I) a strip of "guaiac-copper" paper ${ }^{1}$ without letting it touch the liquid. Gently warm the contents of the flask upon the waterbath. Neither hydrocyanic acid nor potassium cyanide is present, unless the paper is turned blue or bluish green. But the only conclusion to be drawn from a positive test is that hydrocyanic acid, or an easily decomposable cyanide, may be present. Further conclusions should not be drawn from a positive result, since other substances like ammonia, volatile ammonium compounds, hydrochloric acid and especially oxidizing agents like ozone, hydrogen dioxide, nitric acid and chlorine will turn the paper blue. Consequently, though very delicate, this test cannot be accepted as conclusive proof of the presence of hydrocyanic acid.

Mechanism of the Reaction.-Hydrocyanic acid has nothing directly to do with this reaction. But it forms ozone with copper sulphate and that turns the guaiaconic acid of guaiac resin blue. Cupric cyanide $(\alpha)$ is an intermediate product which furnishes ozonized oxygen as shown in $(\beta)$ :

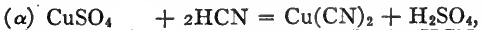

(B) $6 \mathrm{Cu}(\mathrm{CN})_{2}+{ }_{3} \mathrm{H}_{2} \mathrm{O}=6 \mathrm{CuCN}+6 \mathrm{HCN}+\mathrm{O}_{3}$.

1 Prepare "guaiac-copper" paper by saturating strips of filter paper with freshly prepared, Io per cent. alcoholic tincture of resin of guaiac. Dry these strips in air and moisten before using with very dilute aqueous copper sulphate solution ( $\mathrm{r}: \mathrm{r000}$ ). 
The actual chemical examination for hydrocyanic acid is made by adding tartaric acid solution to the finely divided material and distilling as described (see page I8). This acid volatilizes easily with steam and most of it will appear in the first part of the distillate. Therefore use the first 5 or Io cc. of distillate for the tests. Note cautiously the odor of the distillate, which is characteristic, and then proceed as follows:

I. Prussian Blue Test.-Add to the solution (distillate) a little potassium hydroxide solution; then I or 2 drops of ferrous sulphate solution and I drop of ferric chloride solution. Shake well and warm gently. Finally acidify with dilute hydrochloric acid. If much hydrocyanic acid is present, a precipitate of Prussian blue will appear immediately. But if the quantity is small, the solution will have merely a blue or bluish green color. After a long time (ro to 12 hours) a flocculent precipitate of Prussian blue will settle to the bottom of the test tube. The limit of delicacy is $\mathrm{I}: 5,000,000 .^{1}$

Mechanism of the Reaction.2-Hydrocyanic acid and potassium hydroxide form potassium cyanide which with ferrous sulphate produces ferrous cyanide $(\alpha)$. The latter combines with more potassium cyanide, forming potassium ferrocyanide $(\beta)$ which with ferric chloride precipitates Prussian blue $(\gamma)$, the ferric salt of hydroferrocyanic acid $\left(\mathrm{H}_{4} \mathrm{Fe}\left(\mathrm{CN}_{6}\right)\right.$.
(a) $\left.\mathrm{FeSO}_{4}+{ }_{2} \mathrm{KCN}=\mathrm{Fe}(\mathrm{CN})\right)_{2}+\mathrm{K}_{2} \mathrm{SO}_{4}$,
(в) $\mathrm{Fe}(\mathrm{CN})_{2}+{ }_{4} \mathrm{KCN}=\mathrm{K}_{4} \mathrm{Fe}(\mathrm{CN})_{6}$,
( $\gamma{ }_{3}{ }_{3} \mathrm{~K}_{4} \mathrm{Fe}(\mathrm{CN})_{6}+{ }_{4} \mathrm{FeCl}_{3}=\mathrm{Fe}_{4}\left[\mathrm{Fe}(\mathrm{CN})_{6}\right]_{3}+{ }_{2} \mathrm{KCl}$.

Prussian blue will not appear in presence of alkalies, since they decompose it as follows:

$$
\mathrm{Fe}_{4}\left[\mathrm{Fe}(\mathrm{CN})_{6}\right]_{3}+\mathrm{I}_{2} \mathrm{KOH}={ }_{3} \mathrm{~K}_{4} \mathrm{Fe}(\mathrm{CN})_{6}+{ }_{4} \mathrm{Fe}(\mathrm{OH})_{3} .
$$

Consequently test the final mixture with blue litmus paper to make sure it is acid.

2. Sulphocyanate Test.-Add to a portion of the distillate 3 or 4 drops of potassium hydroxide solution and then a little

${ }^{1}$ Link and Möckel, Zeitschrift für analytische Chemie r7, 455 (I878).

2 The reaction may be explained by saying that all the ferrous salt in presence of much potassium hydroxide is precipitated as ferrous hydroxide $(\alpha)$. The latter with potassium cyanide then forms ferrous cyanide $(\beta)$ and finally potassium ferrocyanide $(\gamma)$ :

$$
\begin{aligned}
& \text { (a) } \mathrm{FeSO}_{4}+{ }_{2} \mathrm{KOH}=\mathrm{Fe}(\mathrm{OH})_{2}+\mathrm{K}_{2} \mathrm{SO}_{4} \text {, } \\
& \text { (及) } \mathrm{Fe}(\mathrm{OH})_{2}+2 \mathrm{KCN}=\mathrm{Fe}(\mathrm{CN})_{2}+2 \mathrm{KOH} \text {, }
\end{aligned}
$$

With ferric chloride potassium ferrocyanide in presence of hydrochloric acid finally gives Prussian blue (see above). 
yellow ammonium sulphide solution. Evaporate to dryness upon the water-bath. Dissolve the residue in a little water, and acidify with dilute hydrochloric acid. Filter through double paper to remove sulphur, and add to the filtrate 2 or 3 drops of ferric chloride solution. If the distillate contained hydrocyanic acid, a reddish to blood-red color will appear. This is due to ferric sulphocyanate. The limit of delicacy is I : $4,000,000$.

Mechanism of the Reaction.-Hydrocyanic acid and potassium hydroxide form potassium cyanide which takes sulphur from yellow ammonium sulphide and becomes potassium sulphocyanate $(\alpha)$. The latter with ferric chloride forms ferric sulphocyanate $(\beta)$ :

( $\alpha) \mathrm{KCN}+\left(\mathrm{H}_{4} \mathrm{~N}\right)_{2} \mathrm{~S}_{x}=\mathrm{KSCN}+\left(\mathrm{H}_{4} \mathrm{~N}\right)_{2} \mathrm{~S}_{x-1}$,

(B) ${ }_{3} \mathrm{KSCN}+\mathrm{FeCl}_{3}=\mathrm{Fe}(\mathrm{SCN})_{3}+3 \mathrm{KCl}$.

3. Vortmann's ${ }^{1}$ Nitroprusside Test.-Add to a portion of the distillate a few drops of potassium nitrite solution; then 2 to 4 drops of ferric chloride solution and enough dilute sulphuric acid to give a bright yellow color. Heat to boiling, add sufficient ammonium hydroxide solution to remove excess of iron and filter. Add to the filtrate 1 or 2 drops of very dilute ammonium sulphide solution. If the solution contained hydrocyanic acid, a violet color will appear and pass through blue, green and yellow. The limit of delicacy is $I: 3$ I 2,000 .

Note.-This test is the reverse of the nitroprusside test for hydrogen sulphide and is due to conversion of hydrocyanic acid to potassium nitroprusside, $\mathrm{K}_{2} \mathrm{Fe}$ $(\mathrm{NO})(\mathrm{CN})_{5}$, which causes the color changes when ammonium sulphide is added. Very small quantities of hydrocyanic acid give a bluish green to greenish yellow color.

4. Silver Nitrate Test.-Acidify a portion of distillate with dilute nitric acid, and add silver nitrate solution in excess. If hydrocyanic acid is present, a white, curdy precipitate of silver cyanide $(\mathrm{AgCN})$ will appear. Excess of ammonium hydroxide solution will readily dissolve this precipitate. The limit of delicacy is $\mathrm{I}: 250,000$.

When a dilute aqueous solution of hydrochloric acid is distilled, the acid does not pass into the distillate. The precipitate, therefore, caused by silver nitrate solution cannot

1 Monatshefte für Chemie 7, 4I6 (r886). 
possibly be silver chloride, because at first nothing but pure water distils from a I per cent. or weaker solution of hydrochloric acid. To remove hydrochloric acid, if present, redistil the first distillate over borax. This will retain hydrochloric but not hydrocyanic acid. It is advisable to collect upon a filter, wash and dry the precipitate caused by silver nitrate solution. If silver cyanide is heated in a bulb-tube, it will form metallic silver and cyanogen gas. The latter may be recognized by its characteristic odor. ${ }^{1}$ The reaction is:

$$
{ }_{2} \mathrm{AgCN}={ }_{2} \mathrm{Ag}+(\mathrm{CN})_{2} \text {. }
$$

5. Picric Acid Test.-Make a portion of distillate alkaline with potassium hydroxide solution and heat gently (50 to $60^{\circ}$ ) with a few drops of picric acid solution. If hydrocyanic acid is present, the solution will become blood-red. This is due to formation of potassium isopurpurate.

Note.-This test is not as delicate, nor as characteristic of hydrocyanic acid as the other tests. If hydrogen sulphide is present, as it frequently is in distillates from animal matter, picric acid solution will produce a red color owing to formation of picramic acid.

6. Weehuizen's ${ }^{2}$ Test.--Add a few drops of phenolphthalin dissolved in dilute sodium hydroxide solution and then a little copper sulphate solution ( $1: 2000$ ) to a portion of the distillate. Even in a dilution of $\mathrm{I}: 500,000$ hydrocyanic acid will"produce a red color due to oxidation of phenolphthalin to phenolphthalein. Such oxidizing agents as hydrogen peroxide, nitric acid and ferric chloride do not give this test. Paper first moistened with alkaline phenolphthalin solution and then with very dilute copper sulphate solution may be used. These phenolphthalin-copper sulphate papers turn red even in air containing hydrocyanic acid.

Under the conditions of the test phenolphthalin is oxidized to phenolphthalein:

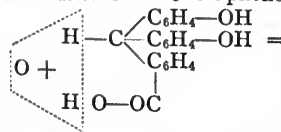

Phenolphthalin

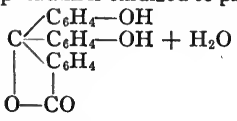

Phenolphthalein

${ }^{1}$ Owing to the very poisonous character of cyanogen gas, it is safer to ignite the gas at the mouth of the bulb-tube. Cyanogen gas burns with a purple flame. Tr.

${ }^{2}$ Pharmaceutisch Weekblad 42, 27I; and Pharmaceutische Zentralhalle 46, 256 (1905). 
On the other hand the phthalein heated with an alkaline hydroxide and zinc dust is reduced to the phthalin.

\section{Quantitative Estimation of Hydrocyanic Acid}

To determine hydrocyanic acid quantitatively, acidify a weighed portion of material with dilute sulphuric or tartaric acid and distil. Determine the quantity of hydrocyanic acid in the distillate either gravimetrically or volumetrically. If the former method is used, collect the precipitate of silver cyanide upon a weighed filter, wash and dry at $100^{\circ}$ to constant weight; or ignite the precipitate in a weighed porcelain crucible, and determine the quantity of metallic silver obtained. If hydrochloric acid is present in the distillate, redistil once over borax. The distillate will then be free from hydrochloric acid.

\section{Detection of Hydrocyanic Acid in Presence of Potassium Ferrocyanide}

When material contains non-poisonous potassium ferrocyanide, hydrocyanic acid will appear in the distillate from a solution acidified with tartaric acid. In an experiment, where 1 per cent. potassium ferrocyanide solution was distilled with 0.03 gram of tartaric acid, the distillate contained considerable hydrocyanic acid. Carbon dioxide, passed into hot, aqueous potassium ferrocyanide solution, will liberate hydrocyanic acid even at water-bath temperature $\left(75^{\circ}\right)$. To test for potassium ferrocyanide beforehand, shake some of the original material with water and filter. Test the filtrate with ferric chloride solution and dilute hydrochloric acid. If there is a precipitate of Prussian blue, potassium ferrocyanide is present. To detect free hydrocyanic acid, potassium or sodium cyanide ${ }^{1}$ with certainty, in presence of potassium ferrocyanide, add to the material acid sodium carbonate in not too small quantity and distil. Even long distillation over free flame by this method will liberate hydrocyanic acid only from simple cyanides and not from potassium ferrocyanide.

\section{Detection of Mercuric Cyanide}

When an aqueous solution of mercuric cyanide, which is exceedingly poisonous, is distilled with tartaric acid, the distillate will contain hydrocyanic acid only when a large quantity of mercuric cyanide is present. Distillation of roo cc. of I per cent. aqueous mercuric cyanide solution yields a distillate which gives the Prussian blue test distinctly. But, if the quantity of mercuric cyanide is less and the solution very dilute (for example, roo cc. of 0.0 r per cent. solution), there will not be a trace of hydrocyanic acid in the distillate, even though the solution is strongly acidified with tartaric acid. If, however, a few cc. of freshly prepared hydrogen sulphide water are added and distillation is resumed, mercuric cyanide will be completely decomposed and the distillate will contain hydrocyanic acid.

\section{Detection of Mercuric Cyanide in Presence of Potassium Ferrocyanide}

The method of detecting hydrocyanic acid from simple cyanides, in presence of potassium ferrocyanide, is not applicable to mercuric cyanide. Long distillation, even from saturated acid sodium carbonate solution, gives no trace of hydrocyanic acid. But distillation in presence of not too little acid sodium carbonate, after addition of a few cc. of freshly prepared, saturated hydrogen sulphide solu-

1 Mercuric cyanide is an exception. 
tion, liberates hydrocyanic acid from mercuric cyanide but not from potassium ferrocyanide. It is possible to detect hydrocyanic acid by this method, when very little mercuric cyanide is mixed with considerable potassium ferrocyanide. For example, o.01 gram of mercuric eyanide in roo cc. of ro per cent. potassium ferrocyanide solution can be detected. If potassium ferrocyanide is distilled directly with hydrogen sulphide without addition of acid sodium carbonate, the distillate will contain considerable hydrocyanic acid.

\section{CARBOLIC ACID}

\section{Action and Fate of Carbolic Acid in the Animal Body}

Concentrated carbolic acid coagulates and destroys the constituents of the human body, especially proteins and protoplasmic structures. It has therefore a very strong caustic action. But its action is not merely local, for after absorption OH it shows an affinity particularly for the central nervous system, I brain and spinal cord. The first indications of this in animals are strong stimulation, increased irritability as in the case of strychnine and paralysis. In man the period of stimulation is very slow in appearing. In chronic poisoning, after repeated small doses of carbolic acid, degeneration of the kidneys and liver is a result of absorption. The human organism absorbs carbolic acid very rapidly. Absorption from the skin, the gastro-intestinal tract, abrasions and the respiratory organs takes place readily. In the human organism the poison is converted by conjugation with sulphuric acid into phenylsulphuric acid:

$$
\mathrm{HO}-\mathrm{SO}_{2}-\mathrm{OH}+\mathrm{HO}-\mathrm{C}_{6} \mathrm{H}_{5}=\mathrm{HO}-\mathrm{SO}_{2}-\mathrm{OC}_{6} \mathrm{H}_{5}+\mathrm{H}_{2} \mathrm{O} \text {. }
$$

When the quantity of carbolic acid is very large, it is also converted into phenyl glycuronic acid by conjugation with glycuronic acid, HOOC-(CH.OH) ${ }_{4} \mathrm{CHO}$. Considerable carbolic acid is oxidized within the body to dihydroxy-benzenes, namely pyrocatechol $\left(\mathrm{C}_{6} \mathrm{H}_{4}(\mathrm{OH})_{2}(\mathrm{I}, 2)\right)$ and hydroquinol $\left(\mathrm{C}_{6} \mathrm{H}_{4}(\mathrm{OH})_{2}(\mathrm{r}, 4)\right)$. These enter into synthesis with sulphuric acid and appear in urine as ethereal salts of sulphuric acid. The dark color of "carbolic urine" is largely due to further oxidation of hydroquinol, whereby colored products (quinone?) are formed. In carbolic acid poisoning, urine often has a pronounced dark color (greenish to black). Urine in other cases is amber-yellow at first, but standing in air gives it a deeper color. When carbolic acid poisoning is suspected, the urine should be examined chemically. "Carbolic urine" differs from normal human urine in being nearly free from sulphuric acid," the so-called "preformed sulphuric acid." Consequently barium chloride solution, in presence of excess of acetic acid, gives only a slight precipitate of barium sulphate or none at all. Filter when there is a precipitate and warm the clear filtrate with a few cc. of concentrated hydrochloric acid. An abundant precipitate of barium sulphate will usually appear. The mineral acid decomposes phenyl-sulphuric acid into phenol and sulphuric acid which is then precipitated. Normal human urine

1 This is sulphuric acid present in urine as sulphates. It is also termed "preformed sulphuric acid," by which is meant that it enters the body as such. In this respect it differs from "ethereal," or "conjugate" sulphuric acids, which result from syntheses within the body. 
contains considerably more "sulphate sulphuric acid" (A-sulphuric acid) than "ethereal sulphuric acid" (B-sulphuric acid). The average proportion between the two being: $\mathrm{A}-\mathrm{SO}_{4}: \mathrm{B}-\mathrm{SO}_{4}=$ I0:I. Barium chloride solution, added to normal urine in presence of acetic acid, produces a heavy precipitate of barium sulphate.

\section{Distribution of Carbolic Acid in the Human Body After Poisoning}

C. Bischoff ${ }^{1}$ examined organs, removed from a man who died $\mathrm{r}_{5}$ minutes after taking $\mathrm{I}_{5} \mathrm{cc}$. of liquid carbolic acid, and found the poison distributed as stated in the table below. The organs in this case were perfectly fresh. Only a small portion of the stomach was received.

\begin{tabular}{rlc}
\multicolumn{1}{c}{ Weight } & \multicolumn{1}{c}{ Organ } & Phenol \\
242 grams & Contents of stomach and intestine & 0.17 gram \\
I I 2 grams & Blood & 0.028 gram \\
1480 grams & Liver & 0.637 gram \\
322 grams & Kidney & 0.201 gram \\
1445 grams & Brain & 0.314 gram
\end{tabular}

Bischoff distilled with steam until the distillate gave no further precipitate with bromine water. The results show how rapidly carbolic acid is absorbed, and how soon it is distributed throughout the body.

E. Baumann ${ }^{2}$ has published certain facts relating to the quantity of carbolic acid formed during putrefaction of protein substances. Baumann states that he. obtained from roo grams of fresh pancreas and 100 grams of moist fibrin, mixed with $250 \mathrm{cc}$. of water, after 6 days of putrefaction 0.073 to 0.078 gram of tribromophenol, corresponding to 0.0208 to 0.022 gram of phenol.

Urine gives a distinet test for carbolic acid ${ }_{5} 5$ minutes after the poison has been taken by the mouth, or hypodermically. This shows how rapidly carbolic acid is absorbed. Most of the carbolic acid absorbed is eliminated in 4 or 5 hours. Schaffer ${ }^{3}$ found the quantity of conjugate sulphuric acid in urine to increase in exact proportion to the quantity of carbolic acid taken.

\section{Tests for the Detection of Carbolic Acid}

Carbolic acid distils quite easily with steam. Yet, to remove the last traces, long-continued distillation is necessary. If fractional distillation is made, when carbolic acid is present, this substance will appear in the first and second fractions and even in the third. Usually carbolic acid can be recognized by its peculiar odor. When much carbolic acid is present, the distillate is milky. Colorless or reddish globules may be seen

${ }^{1}$ Berichte der Deutschen chemischen Gesellschaft r6, I337 (1883).

${ }^{2}$ Berichte der Deutschen chemischen Gesellschaft ro, 685 (r877) and Zeitschrift für physiologische Chemie I, 6I (r877-78).

${ }^{3}$ Journal für praktische Chemie, Neue Folge r8, 282 (1878). 
floating in the liquid. Excess of potassium or sodium hydroxide solution will dissolve carbolic acid and render the distillate perfectly clear. Pure, anhydrous carbolic acid melts at 40 to $42^{\circ}$ and distils at 178 to $182^{\circ}$.

Decomposition of protein substances produces phenol and especially para-cresol in small quantity. Traces of phenols can almost always be detected in distillates from animal matter in an advanced stage of decomposition. Millon's reagent, and usually bromine water, will give positive tests with such distillates.

I. Millon's Test.-Millon's reagent, ${ }^{1}$ heated with a solution containing only a trace of carbolic acid, produces a red color. An aqueous solution containing only $20 \mathrm{mg}$. of carbolic acid, diluted I : 100,000, will give a distinct red color. If the phenol solution is not very dilute, the color will appear even in the cold. Though a very delicate test, it is not characteristic of carbolic acid, because several other aromatic compounds behave similarly. This is true of derivatives of mon-acid phenols like the three cresols, salicylic acid, ${ }^{2}$ para-

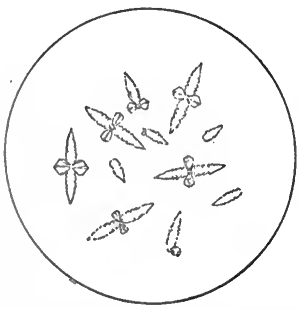

Fig. 8.-Tribromophenol Crystals. From a dilution of $1: 20,000$. hydroxy-benzoic acid, para-hydroxyphenyl-acetic acid, para-hydroxyphenyl-propionic acid (hydro-paracumaric acid $^{3}$ ) and tyrosine. Aniline heated with Millon's reagent also gives a dark red color.

2. Bromine Water Test.-Excess of bromine water produces a yellowish white, crystalline precipitate, even with very dilute carbolic acid solutions. It is a very delicate test for carbolic acid. Phenol diluted I : 50,000 yields, after some time, a precipitate made up in part of well-formed crystals (Fig. 8).

${ }^{1}$ For the preparation of this reagent see page $32 \mathrm{I}$.

${ }^{2}$ Traces of salicylic acid volatilize with steam, at least in such quantity that it can be detected with Millon's reagent.

${ }^{8}$ Para-hydroxy-phenyl-acetic acid and hydro-para-cumaric acid are formed in the putrefaction of proteins but are not volatile with steam. 
If there is a sufficient excess of bromine water to give the supernatant liquid a brownish red color, the precipitate consists only of tribromophenyl hypobromite, $\mathrm{C}_{6} \mathrm{H}_{2} \mathrm{Br}_{4} \mathrm{O}$. R. Benedikt ${ }^{1}$ regards this compound as a brom-phenoxy-tribromobenzene with the structure

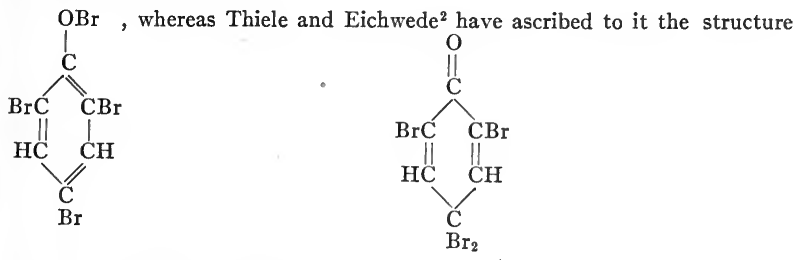

This reaction takes place so easily that carbolic acid may even be determined quantitatively as this tetrabromo-derivative (see page $3 \mathrm{I}$ ). It melts at I32$\mathrm{I}_{34^{\circ}}$ with evolution of bromine and crystallizes as lemon-yellow
leaflets from alcohol-free chloroform or ligroin. Heated with
alcohol, acetone, xylene, or aqueous sulphurous acid, this com-

3. Ferric Chloride Test.-Very dilute ferric chloride solution, added drop by drop, imparts a blue-violet color to aqueous carbolic acid solutions. Addition of dilute hydrochloric or sulphuric acid changes this color to yellow. This test is not as delicate as I and 2. It is entirely negative in presence of mineral acids. The limit of delicacy is about I : Iooo.

4. Hypochlorite Test.-Add a few cc. of ammonium hydroxide solution to a dilute, aqueous carbolic acid solution, and then 2 or 3 drops of freshly prepared calcium or sodium hypochlorite solution. Gentle warming will produce a blue color. Very dilute carbolic acid solutions after some time give only a green to blue-green color. F. A. Flückiger ${ }^{3}$ allows bromine vapor to come into contact with the phenol solution which has been mixed with a little ammonium hydroxide solution in a porcelain dish.

${ }^{1}$ Annalen der Chemie und Pharmazie r99, 127 (1879).

${ }^{2}$ Berichte der Deutschen chemischen Gesellschaft 33, 637 (I900).

${ }^{3}$ Pharmaceutische Chemie, page 287 (r879). 
5. Nitrite Test.-Mix a carbolic acid solution with a dilute alcoholic solution of ethyl nitrite, $\mathrm{C}_{2} \mathrm{H}_{5}-\mathrm{O}-\mathrm{N}=\mathrm{O},{ }^{1}$ or isoamylnitrite, $\mathrm{C}_{5} \mathrm{H}_{11}-\mathrm{O}-\mathrm{N}=\mathrm{O},{ }^{2}$ and add concentrated sulphuric acid from a pipette so that it forms a distinct under-layer. A red zone will appear at the contact surface of the two liquids. This is a very delicate test.

This test may also be made by adding the liquid under examination as an upper layer upon concentrated sulphuric acid containing a trace of red fuming nitric acid.

6. H. Melzer's Benzaldehyde Test. ${ }^{3}$-Add 2 cc. of concentrated sulphuric acid to I cc. of the solution (distillate) to be tested for carbolic acid, then I or 2 drops of benzaldehyde and heat. The mixture, at first yellowish brown, will become dark red. At the same time a red resinous substance will appear. unless the solution is too dilute. When cold add ro cc. of water and enough potassium hydroxide solution to give a distinct alkaline reaction. If carbolic acid is present, a violet-blue color will appear. To obtain this coloring-matter, acidify the solution, extract with ether and evaporate the solvent. Alkalies, added to alcoholic solutions of the coloring-matter, produce a blue color which acids discharge. This is a very delicate test. One cubic centimeter of 0.05 per cent. carbolic acid solution ( $=0.0005$ gram of carbolic acid), will still give the blue color very distinctly.

Note.-In absence of phenol concentrated sulphuric acid produces a dark brown color with benzaldehyde. According to A. Russanow ${ }^{4}$ the first condensation product between phenol and benzaldehyde in presence of concentrated sulphuric acid is para-dihydroxy-triphenyl-methane which crystallizes in yellowish needles:

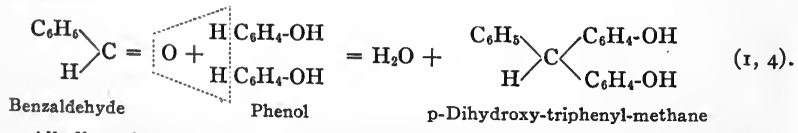

Alkalies dissolve the pure crystals without color but, if these solutions are exposed to air, oxidation takes place and a red or red-violet color appears. Prob-

'The officinal preparation is called "Spiritus Aetheris Nitrosi."

${ }^{2}$ Amylium nitrosum of pharmacists.

${ }^{3}$ Zeitschrift fur analytische Chemie 37,345 (I898).

4 Berichte der Deutschen chemischen Gesellschaft 22, I943 (1889). 
ably benzaurine, dihydroxy-triphenyl-carbinol, is first formed. This compound is a brick-red crystalline powder soluble in alkalies with a violet color.

\section{QUANTITATIVE METHODS OF ESTIMATING PHENOL}

\section{Gravimetric Estimation as Tribromophenol}

The principle of this method is based on the complete precipitation of phenol from aqueous solution as tribromophenyl hypobromite by an excess of saturated bromine water (see Test 2). The precipitate is practically insoluble in cold bromine water and the results are very satisfactory. ${ }^{1}$

Procedure.-Place the aqueous phenol solution in a large glass-stoppered flask. Add gradually, while shaking, saturated bromine water until the supernatant liquid has a red-brown color and bromine vapor is visible above the solution. Let stand 2-4 hours and shake frequently. Then collect the precipitate in a weighed Gooch crucible and dry in a vacuum desiccator over sulphuric acid to constant weight. On the basis of the following proportion calculate the weight of phenol corresponding to the weight of the precipitate:

$\mathrm{C}_{6} \mathrm{H}_{2} \mathrm{Br}_{4} \mathrm{O}: \mathrm{C}_{6} \mathrm{H}_{5}-\mathrm{OH}=$ Wt. of Ppt. found : $\mathrm{x}$ $409.86 \quad 94.05$

Since the ratio $\frac{94.05}{409.86}=0.2295$, the weight of phenol may be found by multiplying the weight of the precipitate by 0.2295 .

\section{Beckurts-Koppeschaar ${ }^{2}$ Volumetric Method}

Dilute sulphuric acid liberates hydrobromic acid from potassium bromide $(\alpha)$ and bromic acid from potassium bromate $(\beta)$. These two acids react according to $(\gamma)$ :
( $\alpha) \mathrm{KBr}+\mathrm{H}_{2} \mathrm{SO}_{4}=\mathrm{KHSO}_{4}+\mathrm{HBr}$,
( $\beta) \mathrm{KBrO}_{3}+\mathrm{H}_{2} \mathrm{SO}_{4}=\mathrm{KHSO}_{4}+\mathrm{HBrO}_{3}$,
(r) $5 \mathrm{HBr}+\mathrm{HBrO}_{3}={ }_{3} \mathrm{Br}_{2}+{ }_{3} \mathrm{H}_{2} \mathrm{O}$.

1 The following results were obtained by $\mathrm{F}$. Beuttel:

\begin{abstract}
Phenol taken
\end{abstract}
I. $0.103 \mathrm{grm}$.

2. $0.2072 \mathrm{grm}$.

3. 0.2072 grm.

${ }^{2}$ Archiv der Pharmazie 24, 570 (1886).
$\mathrm{C}_{6} \mathrm{H}_{2} \mathrm{Br}_{4} \mathrm{O} \quad$ Phenol found

$0.4538 \mathrm{grm}$.

$0.8806 \mathrm{grm}$.

0.8708 grm.

$0.0997 \mathrm{grm}$.

$0.2014 \mathrm{grm}$.

$0.2006 \mathrm{grm}$.
Per cent. found

96.2

98.6

98.6 
Therefore addition of dilute sulphuric acid to a mixture of potassium bromide and bromate solutions liberates bromine which will convert phenol into a mixture of tribromophenol and tribromophenyl hypobromite. The excess of free bromine and also the loosely bound bromine atom of tribromophenyl hypobromite will displace iodine from potassium iodide and finally all the phenol will be present as tribromophenol:

$$
\mathrm{C}_{6} \mathrm{H}_{2} \mathrm{Br}_{3} \mathrm{OBr}+{ }_{2} \mathrm{KI}=\mathrm{C}_{6} \mathrm{H}_{2} \mathrm{Br}_{3} \mathrm{OK}+\mathrm{KBr}+\mathrm{I}_{2}
$$

One molecule of phenol requires 6 atoms of bromine, as shown by the equation:

$$
\begin{aligned}
5 \\
5 \mathrm{KBr}+\mathrm{KBrO}_{3}+6 \mathrm{H}_{2} \mathrm{SO}_{4}+\mathrm{C}_{6} \mathrm{H}_{5} \mathrm{OH}=\underset{3}{ }=\mathrm{C}_{6} \mathrm{H}_{2} \mathrm{Br}_{3} \mathrm{OH}+{ }_{3} \mathrm{HBr}+6 \mathrm{KHSO}_{4}+
\end{aligned}
$$

The following standard solutions are required:

I. $0.0 \mathrm{I} \mathrm{n}$-potassium bromide solution, containing $\frac{5 \mathrm{KBr}}{\mathrm{I} 00}$ grams $=\frac{595.6^{\circ}}{100}=5.956$ grams $\mathrm{KBr}$ in $1000 \mathrm{cc}$.

2. $0.0 \mathrm{I}$ n-potassium bromate solution, containing $\frac{\mathrm{IKBrO}_{3}}{\mathrm{IOO}}$ grams $=\frac{167.17}{100}=1.67 \mathrm{I} 7$ grams $\mathrm{KBrO}_{3}$ in $1000 \mathrm{cc}$.

3. O.I n-sodium thiosulphate solution, containing o.I $\mathrm{Na}_{2} \mathrm{~S}_{2} \mathrm{O}_{3} \cdot 5 \mathrm{H}_{2} \mathrm{O}$ grams $=24.83$ grams in $1000 \mathrm{cc}$.

4. Potassium iodide solution, containing $\mathrm{I} 25$ grams of $\mathrm{KI}$ in $1000 \mathrm{cc}$.

Procedure.-Put about $25 \mathrm{cc}$. of aqueous phenol solution (distillate) into a flask having a tight glass stopper. Add 50 cc. each, of o.or n-potassium bromide and o.or n-potassium bromate solutions, then $5 \mathrm{cc}$. of pure concentrated sulphuric acid and shake vigorously for several minutes. The gradually increasing opalescence of the solution becomes more and more marked, as tribromophenol and tribromophenyl hypobromite are precipitated. The yellow color which soon appears shows excess of bromine. Open the flask in 15 minutes, add ro cc. of potassium iodide solution, shake and titrate free iodine in 5 minutes with o.I n-sodium thiosulphate solution. 
Calculation. $-\frac{6 \text { gram-atoms Br }}{100}=\frac{6 \times 79.96}{100}=4.7976$ grams of bromine are set free from a mixture of $1000 \mathrm{cc}$. of 0.0 r n-potassium bromide solution and roo cc. of $0.01 \mathrm{n}$-potasssium bromate solution. A mixture therefore of $50 \mathrm{cc}$. of each of the two solutions will give 0.2399 gram of bromine. This quantity of bromine can convert 0.04704 gram of phenol into tribromophenol:

$6 \mathrm{Br}: \mathrm{C}_{6} \mathrm{H}_{5} \mathrm{OH}$

$479.7694 .05=0.2399: \mathrm{x}(\mathrm{x}=0.04704)$

I cc. of $0.1 \mathrm{n}$-sodium thiosulphate solution corresponds to 0.012697 gram of iodine and this quantity of iodine to 0.007996 gram of bromine. But 0.007996 gram of bromine will convert $0.00157 \mathrm{gram}$ of phenol into tribromophenol:

$6 \mathrm{Br}: \mathrm{C}_{6} \mathrm{H}_{5} \mathrm{OH}$

$479.7694 .05=0.007996: x(x=0.00157)$

Consequently, for each cc. of 0.1 n-sodium thiosulphate solution used, subtract 0.00157 from 0.04704 gram of phenol. This determines the quantity of carbolic acid in the $25 \mathrm{cc}$. of distillate taken.

\section{Messinger-Vortmann ${ }^{1}$ Volum etric Method}

Excess of iodine (8 atoms of iodine to I molecule of phenol dissolved in 4 molecules of potassium hydroxide), added to an alkaline phenol solution at $50-60^{\circ}$, will produce a dark red, noncrystalline precipitate. One molecule of phenol requires 6 atoms of iodine.

$$
\begin{aligned}
& \text { I. } \mathrm{C}_{6} \mathrm{H}_{5} \mathrm{OH}+3 \mathrm{I}_{2}=\mathrm{C}_{6} \mathrm{H}_{2} \mathrm{I}_{3} \mathrm{OH}+{ }_{3} \mathrm{HI} \text {, } \\
& \text { 2. } 3 \mathrm{HI}+3 \mathrm{KOH}={ }_{3} \mathrm{H}_{2} \mathrm{O}+3 \mathrm{KI} \text {. }
\end{aligned}
$$

This red precipitate dissolves in hot potassium hydroxide solution with a red-brown color and appears as white 2, 4, 6tri-iodophenol, melting at $\mathrm{I}_{54}-\mathrm{I} 56^{\circ}$, on addition of an excess of dilute sulphuric acid. Messinger and Vortmann regard the red compound as di-iodophenyl hypoiodite $\left(\mathrm{C}_{6} \mathrm{H}_{3} \mathrm{I}_{2} \mathrm{OI}\right)$ which potassium hydroxide converts into the more stable isomeric triiodophenol:

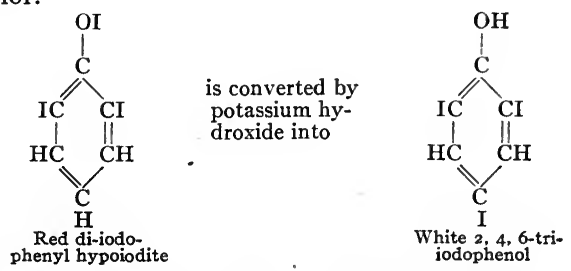

1 Berichte der Deutschen chemischen Gesellschaft 22, 2312 (1889); and 23, 2753 (r89o). See also Kossler and Penny, Zeitschrift für physiologische Chemie I $7, \mathrm{II}_{7}\left(\mathbf{1} 8 \mathbf{g}_{2}\right)$. 
- Procedure. ${ }^{1-}$ The reaction between the alkaline phenol solution and iodine is rather slow in the cold but is hastened at $5^{\circ}$ to $60^{\circ}$.

Place a measured volume of aqueous phenol solution ( 5 to ro cc.) in a small flask and add a measured volume of o.I n-potassium hydroxide solution until the mixture is strongly alkaline. Warm gently by dipping the flask in water at $60^{\circ}$ and add IO-I5 cc. more of O.I n-iodine solution than the volume of 0.1 n-potassium hydroxide solution used, or until the excess of iodine produces a strong yellow color. Agitation will cause a deep red precipitate to appear. Cool the solution, acidify with dilute sulphuric acid and dilute to a definite volume ( 250 to $500 \mathrm{cc}$.). Filter an aliquot portion (100 cc.) rapidly and determine excess of iodine with o.I n-sodium thiosulphate solution.

Calculation.-Each molecule of phenol requires 6 atoms of iodine. Therefore $\mathrm{I}$ atom of iodine $=\frac{\mathrm{C}_{6} \mathrm{H}_{5} \mathrm{OH}}{6}=\frac{94.05}{6}=$ I5.675 phenol. rooo cc. of o.I n-iodine solution, containing o.I gram-atom of iodine, correspond therefore to 1.5675 grams of phenol.

Note.-This method will not give satisfactory results, unless at least 3 molecules of sodium or potassium hydroxide are taken for I molecule of phenol.

\section{Estimation of Phenol in Urine}

In determining carbolic acid in urine, the regular occurrence of phenols must not be overlooked. After a mixed diet, the quantity of normal human urine passed in 24 hours will yield approximately 0.03 gram of phenols (phenol and more especially para-cresol).

In certain diseases where there is excessive bacterial decomposition within the organism, in the intestines for example, urine contains more of these phenols and, consequently, more conjugate sulphuric acids. Even external application of carbolic acid, for instance the use of carbolic acid water as a lotion, is sufficient to i ncrease the quantity of phenyl-sulphuric acid in urine.

\section{Detection of Carbolic Acid in Presence of Aniline}

Aniline closely resembles carbolic acid in behavior toward Millon's reagent and bromine water. But the two substances can be easily separated. Add potas-

${ }^{1}$ Use 0.5 to I per cent. carbolic acid solution for laboratory experiments. 
sium hydroxide solution in large excess and distil. The distillate will contain aniline alone. Or make the solution strongly acid with dilute sulphuric acid, and extract with ether which will dissolve only carbolic acid. Evaporate the ether extract at a moderate temperature and examine the residue.

\section{CHLOROFORM}

Behavior in the Human Organism. - When inhaled, chloroform first passes from the air into the blood-plasma which then transmits it to the red blood-corpuscles<smiles>ClC(Cl)Cl</smiles>
where it may accumulate in relatively large quantity. Air passed through blood will remove chloroform completely. Pohl (see Kobert's "Intoxikationen") states that blood may contain 0.62 per cent. of chloroform, three-fourths of which will be in the red blood-corpuscles. At the height of a harmless narcosis the blood contained only 0.035 per cent. of chloroform. Absorption of chloroform is rapid from all parts of the body. The stimulative action of chloroform on the mucous membranes of the respiratory passages explains such disturbances as coughing, secretion of saliva and reflex slowing of respiration and heart-beat, occurring at the beginning of narcosis. Dilatation of the blood-vessels of organs living after death is due to paralysis caused by even small doses of chloroform. A drop in blood-pressure accompanies paralysis of the brain and the heart's action is feebler and slower. Several researches regarding the effect of inhaled chloroform upon human and animal metabolism have shown an increase in the quantity of nitrogen in the urine after prolonged narcosis because more protein is decomposed. The amount of neutral sulphur and chlorine in the urine also increases. The increase of the latter is due in part at least to the conversion of chloroform into chloride. The acidity of the urine is also much higher. A final characteristic of chloroform urine is the high content of reducing substances. The increased protein decomposition in chloroform narcosis affects both reserve protein and that of the tissues. This may explain degeneration in red blood-corpuscles, glandular organs, the heart, etc., after frequent narcoses or one of long duration.

The temporary or permanent paralysis of isolated animal or vegetable cells, such as leucocytes, ciliated cells, yeast cells, algæ and spores, is evidence of the antiseptic action of chloroform when present in proper concentration in air or in a liquid. This explains the use of I per cent. aqueous chloroform solution as an antiseptic. Added to urine it acts as a preservative. Therefore it may be used in the study of the action of enzymes but not of bacteria, though all micro-organisms are not paralyzed or killed by chloroform water.

Pohl and Hans Meyer have studied the distribution of chloroform in the body and found that the red blood-corpuscles and the brain are most likely to show this poison. After chloroform has been inhaled, some will appear in the gastric juice but at most only traces in the urine. In but two out of 15 cases of chloroform narcosis was this poison found in the urine and then only in traces.

Kobert states that as a rule it is the exception to find chloroform itself in the cadaver, because part of the poison is converted into chloride in the human organism and part is quickly exhaled during respiration. Usually it is possible to detect chloroform in the breath of patients even 24 hours after narcosis. Büdinger states that the mucus of the respiratory passages retains chloroform. 


\section{Tests for the Detection of Chloroform}

Chloroform distils easily with steam and appears in the first fraction in largest quantity. When much chloroform is present, it will separate from the distillate as heavy, colorless globules, whereas a small quantity will remain in solution. This solution usually has the characteristic odor and sweetish taste of chloroform. The following tests should be applied to the first fraction.

I. Phenylisocyanide Test.-Add I or 2 drops of aniline to the chloroform solution (distillate), and then a few cc. of aqueous, or alcoholic potassium hydroxide solution. Gentle heat will produce phenylisocyanide $\left(\mathrm{C}_{6} \mathrm{H}_{5} \mathrm{NC}\right)$. The penetrating and very repulsive odor of this compound is easily recognized. $\mathrm{CHCl}_{3}+\mathrm{C}_{6} \mathrm{H}_{5} \cdot \mathrm{NH}_{2}+{ }_{3} \mathrm{KOH}=\mathrm{C}_{6} \mathrm{H}_{5} \cdot \mathrm{NC}+{ }_{3} \mathrm{KCl}+{ }_{3} \mathrm{H}_{2} \mathrm{O}$.

A. W. Hofmann states that this test will show with certainty I part of chloroform in 5000 to 6000 parts of alcohol.

Note.-Chloral, chloral hydrate, bromoform, iodoform and tetrachloromethane also give this test.

The fact that aniline boiled with potassium hydroxide solution gives a peculiar, faintly ammoniacal odor, even when chloroform is absent, must not be overlooked. There is small chance, however, of confusing this odor with the repulsive smell of phenylisocyanide. In doubtful cases warm some water, containing a drop of aniline and a trace of chloroform, with potassium hydroxide solution and compare the odor with that in question.

2. Schwarz's Resorcinol Test. ${ }^{1-D i s s o l v e, ~ a b o u t ~ 0 . I ~ g r a m ~ o f ~}$ resorcinol $\left(\mathrm{C}_{6} \mathrm{H}_{4} \backslash \mathrm{OH}(\mathrm{I})\right)$ in 2 cc. of water, add a few drops of sodium hydroxide solution and finally the liquid containing chloroform. This mixture heated to boiling will develop even in very dilute solution a yellowish red color attended by a beautiful yellowish green fluorescence.

Chloral, bromal, bromoform and iodoform also give this test.

3. Lustgarten's ${ }^{2}$ Naphthol Test.-Dissolve a few centigrams of $\alpha$ - or $\beta$-naphthol in I or 2 cc. of 33 per cent. aqueous potassium hydroxide solution. Warm to $50^{\circ}$ and add the solution to

1 Zeitschrift für analytische Chemie, 27, 668.

${ }^{2}$ Monatshefte für Chemie, 3, 7 55 (1882). 
be tested. Chloroform will produce an evanescent blue color which in contact with air will change to green and then to brown. This color is less stable when $\beta$-naphthol is used. Acidification of the blue solution will precipitate naphthol colored by a red dye-stuff. This precipitate is usually brick-red.

Chloral, bromal, bromoform and iodoform also give this test.

4. Fujiwara's Pyridine ${ }^{1}$ Test.-Mix 2 cc. of pyridine with $3 \mathrm{cc}$. of ro per cent. sodium hydroxide solution, heat to boiling and add $\mathrm{I}$ cc. of the liquid to be tested. Even a trace of chloroform will produce a bright, blue-red color.

Chloral, bromoform, iodoform and several other similar compounds also respond to this test.

One part of chloroform in I, 000,000 parts of water, 500,000 parts of ether, or 300,000 parts of ethyl alcohol can be detected by this test. It is equally sensitive toward the other substances mentioned.

5. Cyanide Test.--Seal the liquid to be tested for chloroform in a glass tube (pressure-tube) ${ }^{2}$ with a little solid ammonium chloride and alcoholic potassium hydroxide solution. Heat for several hours in a boiling water-bath. Cool the tube, remove the solution and test for hydrocyanic acid by the Prussian blue reaction. A positive test means that the distillate contained chloroform. The following reactions take place:

(a) $\mathrm{CHCl}_{3}+\mathrm{H}_{8} \mathrm{~N}+{ }_{3} \mathrm{KOH}=\mathrm{HCN}+{ }_{3} \mathrm{KCl}+{ }_{3} \mathrm{H}_{2} \mathrm{O}$,

(B) $\mathrm{HCN}+\mathrm{KOH}=\mathrm{KCN}+\mathrm{H}_{2} \mathrm{O}$.

6. Reduction Tests. (a) With Fehling's Solution.-Warm the liquid containing chloroform with Fehling's solution. A red precipitate of cuprous oxide will appear.

(b) With Ammoniacal Silver Nitrate Solution.-Add excess of ammonium hydroxide to silver nitrate solution and then the liquid containing chloroform. Heat will produce a black precipitate of metallic silver.

These reactions are not characteristic of chloroform, because many volatile organic substances, as formic acid and aldehydes

${ }^{1}$ Chemical Abstracts II, 3201 (r917).

${ }^{2}$ An ordinary citrate of magnesium bottle is a convenient apparatus for this test. Wrap a towel around the bottle, place it in the water-bath and gradually raise the temperature to boiling. Do not remove the bottle until it is cold. Tr. 
which may occur in distillates from animal material, reduce Fehling's and ammoniacal silver nitrate solutions.

\section{Quantitative Estimation of Chloroform in Cadavers}

(Ludwig-Fischer ${ }^{1}$ )

Mix a weighed portion of material with water and distil as long as there is any chloroform. To tell when this point is reached, apply the phenylisocyanide test to a few cc. of liquid collected at the end of distillation. Add some calcium carbonate to combine with free hydrochloric acid. Warm the distillate to about $60^{\circ}$ and draw washed air through it by suction. Pass this air through a combustion-tube heated to high temperature and then into silver nitrate solution acidified with nitric acid. Weigh the precipitated $\mathrm{AgCl}(\mathrm{N})$.

\section{Calculation:}

$$
{ }_{3} \mathrm{AgCl}: \mathrm{CHCl}_{3}=\mathrm{N}: \mathrm{x} \text {. }
$$

This method is based upon the fact that chloroform heated with steam above $200^{\circ}$ is decomposed into carbon monoxide, hydrochloric and formic acids:

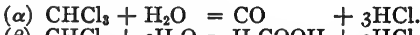
(B) $\mathrm{CHCl}_{3}+{ }_{2} \mathrm{H}_{2} \mathrm{O}=\mathrm{H} . \mathrm{COOH}+{ }_{3} \mathrm{HCl}$.

In a series of blank experiments $B$. Fischer has shown that the stomach, stomach contents and blood, of a person who has not taken chloroform, give no volatile chlorine compounds under these conditions. By this method $B$. Fischer found in the cadaver of a laborer, who had died during chloroform narcosis, the following quantities of chloroform:

\begin{tabular}{|c|c|c|}
\hline Weight & Organ & Chloroform \\
\hline 985 grams & $\begin{array}{l}\text { Stomach and contents and parts of } \\
\text { the intestine }\end{array}$ & O.I gram \\
\hline 780 grams & Lungs and blood from the heart & 0.055 gram \\
\hline $45 \mathrm{gre}$ & Portions of spleen, kidneys and liver & traces \\
\hline 480 grams & Brain & 0.07 gram \\
\hline
\end{tabular}

From these results it appears that most of the chloroform was in the brain and blood.

\section{CHLORAL HYDRATE}<smiles>OC(O)C(Cl)(Cl)Cl</smiles>

Chloral hydrate distils very slowly with steam from an acid solution. Therefore the complete distillation of a large quantity of chloral hydrate requires considerable time. Chloral hydrate appears as such in the distillate.

${ }^{1}$ Jahresbericht des chemischen Untersuchungsamtes der Stadt Breslau für die Zeit vom I April I894 bis 3r März 1895. 


\section{Tests for the Detection of Chloral Hydrate}

Chloral hydrate like chloroform will give the phenylisocyanide, resorcinol, naphthol and pyridine tests. But the distillate containing chloral hydrate does not have the characteristic chloroform odor which is also scarcely perceptible in very dilute aqueous chloroform solutions.

Jaworowski ${ }^{1}$ suggests the following tests to differentiate chloral hydrate from chloroform:

I. Test with Nessler's Solution.-Add a few drops of this reagent to an aqueous chloral hydrate solution and shake. It will produce a yellowish red precipitate, the color of which will change after a while to a dirty yellowish green. This is an aldehyde reaction.

2. Test with Sodium Thiosulphate.-Boil a few cc. of chloral hydrate solution with $0.2-0.3$ gram of solid sodium thiosulphate. This will give a turbid liquid of brick-red color. A few drops of potassium hydroxide solution will remove the turbidity and change the color to brownish red.

When the quantity of chloral hydrate is not too small, it may also be detected by the following procedure:

Decomposition of Chloral Hydrate.-Heat a portion of the distillate for 30 minutes under a reflux condenser with calcined magnesium oxide (MgO) upon a boiling water-bath. Magnesium formate and chloroform are produced by decomposition of chloral hydrate.

$$
{ }_{2} \mathrm{CCl}_{3} \cdot \mathrm{CH}(\mathrm{OH})_{2}+\mathrm{MgO}={ }_{2} \mathrm{CHCl}_{3}+\mathrm{Mg}(\mathrm{OOCH})_{2}+\mathrm{H}_{2} \mathrm{O} .
$$

Proceed as follows to detect these products:

Chloroform.-Distil a few cc. from the solution in the flask and test for chloroform by the phenylisocyanide, resorcinol, $\alpha$-naphthol and pyridine tests.

Formic Acid.-Filter the residue from the distillation, concentrate the filtrate to a few cc. by evaporation and divide into two parts for the following reduction tests:

(a) Reduction of Mercuric to Mercurous Chloride.-Add a few drops of mercuric chloride solution and warm. Formic

1 Pharmaceutische Zeitung für Russland 33, 373, und Zeitschrift für analytische Chemie, $37,60(1898)$. 
acid, if present, will produce a white precipitate of mercurous chloride (calomel):

$$
\mathrm{Mg}(\mathrm{OOCH})_{2}+{ }_{4} \mathrm{HgCl}_{2}={ }_{2} \mathrm{Hg}_{2} \mathrm{Cl}_{2}+\mathrm{MgCl}_{2}+{ }_{2} \mathrm{HCl}+{ }_{2} \mathrm{CO}_{2} \text {. }
$$

(b) Reduction of Silver Nitrate.-Warmed with silver nitrate solution, formic acid and its salts produce a black precipitate of metallic silver:

$$
\mathrm{Mg}(\mathrm{OOCH})_{2}+{ }_{4} \mathrm{AgNO}_{3}=4 \mathrm{Ag}+\mathrm{Mg}\left(\mathrm{NO}_{3}\right)_{2}+{ }_{2} \mathrm{HNO}_{3}+{ }_{2} \mathrm{CO}_{2} \text {. }
$$

\section{Detection of Chloral Hydrate in Powders or Solutions}

Extract a powder with cold water containing sulphuric acid, filter, extract the filtrate several times with ether and spontaneously evaporate the ether extracts in a shallow dish or on a clock-glass. Chloral hydrate imparts to the residue its characteristic pungent odor. The odor of chloroform is easily recognized by warming the residue with sodium hydroxide solution:

$$
\mathrm{CCl}_{3}-\mathrm{CH}(\mathrm{OH})_{2}+\mathrm{KOH}=\mathrm{CHCl}_{3}+\mathrm{H} \cdot \mathrm{COOK}+\mathrm{H}_{2} \mathrm{O}
$$

The phenylisocyanide, resorcinol, naphthol and pyridine tests, as well as that with Nessler's reagent, should be applied to the residue.

In the case of an aqueous solution of chloral hydrate, first acidify with dilute sulphuric acid and repeatedly extract with ether. Evaporate the ether extracts and examine the residue as already described.

Note.-Pure chloral hydrate forms transparent crystals which are dry, permanent and colorless. This compound has a pungent odor, its taste being caustic and faintly bitter. It dissolves with ease in water, alcohol and ether; and in 5 parts of chloroform. It melts at $58^{\circ}$.

\section{Action and Fate of Chloral Hydrate in the Human Organism}

Applied locally chloral hydrate acts as a strong stimulant. Taken internally it frequently stimulates the stomach. When it reaches the blood, it acts like chloroform in paralyzing the brain, spinal cord and heart but usually no previous stimulation is noticeable. There is marked decrease in blood-pressure due to paralysis of the blood-vessels. Death from chloral hydrate poisoning is occasioned by impaired circulation and respiration, in consequence of which the quantity of oxygen taken in and of carbon dioxide given off is considerably diminished. H. Meyer has shown that the narcotic action of chloral hydrate depends, as does that of all compounds of the alcohol and chloroform group, upon the affinity of the poison for lipoids, the fatty constituents of the nervous system. It is also 
held by the blood, especially by the red blood-corpuscles. Later it appears unchanged, most abundantly in the cells of the brain and spinal cord (Kobert, "Intoxikationen").

Only very little chloral hydrate taken internally passes as such into the urine. As shown by v. Mering and Musculus, ${ }^{1}$ the greater part by conjugation with glycuronic acid forms urochloralic acid $\left(\mathrm{C}_{8} \mathrm{H}_{11} \mathrm{Cl}_{3} \mathrm{O}_{7}\right)$ which is eliminated as such in the urine. This conjugated acid undergoes hydrolysis, when boiled with dilute acids, and gives trichlor-ethyl alcohol and free dextro-rotatory glycuronic acid:

$$
\underset{\substack{\text { Urochloralic } \\ \text { acid. }}}{\mathrm{C}_{8} \mathrm{H}_{11} \mathrm{Cl}_{3} \mathrm{O}_{7}}+\mathrm{H}_{2} \mathrm{O}=\underset{\substack{\text { Trichlor- } \\ \text { ethyl alcohol. }}}{\mathrm{CCl}_{3}-\mathrm{CH}_{2} \mathrm{OH}}+\mathrm{HOOC}_{\substack{\text { Glycuronic } \\ \text { acid. }}}^{(\mathrm{CH} . \mathrm{OH})_{4}-\mathrm{CHO}}
$$

Urochloralic acid is therefore trichlor-ethyl glycuronic acid. It is crystalline and with heat reduces silver solution as well as alkaline copper and bismuth solutions. Consequently chloral urine behaves much like sugar urine but differs from the latter in being strongly lævo-rotatory. The reduction of the aldehyde chloral, to its corresponding primary alcohol, trichlor-ethyl alcohol, is especially noteworthy as regards the behavior of chloral hydrate in the human organism.

\section{Quantitative Estimation of Chloral Hydrate in Blood and Tissues}

\section{$\left(\right.$ Archangelsky ${ }^{2}$ )}

Distil the material for $12-20$ hours with its own weight of 20 per cent. phosphoric acid, repeating the process if the distillate is turbid or yellow. To complete the decomposition of chloral hydrate into chloroform and formic acid, add $50 \mathrm{cc}$. of sodium hydroxide solution to the distillate and concentrate on the waterbath to about $20 \mathrm{cc}$. Neutralize the solution exactly and heat for 6 hours on the water-bath with an excess of mercuric chloride solution. Finally weigh the precipitated mercurous chloride. Satisfactory results were obtained by this method when known quantities of chloral hydrate were added to blood and organs. Using this method Archangelsky has shown that chloral hydrate is not uniformly distributed in the blood but is contained especially in the blood-corpuscles. When narcosis begins there is less chloral hydrate in the brain than in the blood. But later the percentage of the poison in the brain is higher than in the blood. Archangelsky has further shown how much chloral hydrate the blood must contain before narcosis can appear. A dog's blood must contain $0.03-0.05$ per cent. When the blood contains o.r 2 per cent., respiration ceases.

\section{IODOFORM}

Iodoform crystallizes in shining hexagonal leaflets or plates. It may also<smiles>[3H]C([3H])[3H]</smiles>
appear as a rather fine crystalline powder, lemon-yellow in color and having a penetrating odor somewhat like saffron. The melting-point of iodoform is approximately $120^{\circ}$. It is nearly insoluble in water; soluble in 50 parts of cold and in about ro parts of boiling alcohol; and soluble in 6 parts of ether. It is also freely soluble in chloroform.

${ }^{1}$ Berichte der Deutschen chemischen Gesellschaft 8, $66_{2}$ (1875); and v. Mering, Ibid., I $_{5}$, rorg ( 1882 ).

${ }^{2}$ Archiv für experimentelle Pathologie und Pharmakologie, 46, 347 (rgor). 


\section{Detection of Iodoform}

Iodoform distils quite easily with steam and gives a milky distillate having a characteristic odor. Extract this distillate with ether and carefully test the residue left by the spontaneous evaporation of the solvent. If much iodoform is present, it will form yellow hexagonal plates. Dissolve the ether residue in a little alcohol, and use this solution for the following tests:

I. Lustgarten's ${ }^{1}$ Test.-Gently warm a few drops of alcoholic iodoform solution in a test-tube with a little sodium phenolate $\left(\mathrm{C}_{6} \mathrm{H}_{5} . \mathrm{ONa}\right)$ solution. $^{2}$ If iodoform is present, a red substance will be deposited on the bottom of the tube. A few drops of dilute alcohol will dissolve this precipitate with a carmine-red color.

Also make the resorcinol, pyridine and phenylisocyanide tests (see pages 36 and 37 ).

\section{NITROBENZENE}

Nitrobenzene has a strong poisonous action. Administration of very small quantities of this compound has produced death in human beings. There are<smiles>C=CC=C[N+](=O)[O-]</smiles>
records in the literature of several cases where 20 drops, and even 7 to 8 drops, have caused fatal results. But on the other hand complete recovery has followed poisoning by much larger doses. Fatal poisonings have come also from inhaling nitrobenzene vapor. Within recent years nitrobenzene has been used to some extent as an abortifacient. Nitrobenzene poisons the blood and changes its appearance. The blood has a chocolate color and at the same time the red blood-corpuscles change their shape and go into solution. As a result the blood is incapable of uniting with oxygen. The blood of persons poisoned by nitrobenzene is said to contain less than I per cent. of oxygen so that death is caused by asphyxiation. Healthy blood contains about I 7 per cent. of oxygen by volume. There seems to be no methæmoglobin in blood containing nitrobenzene. Such blood examined spectroscopically shows the two oxyhæmoglobin bands and also a special absorption-band between $\mathrm{C}$ and $\mathrm{D}$ (Fihlene's nitrobenzene band). It is probable that the slight solubility of this poison necessitates a definite incubation period, for 2 to 3 hours usually elapse after nitrobenzene has been taken before

${ }^{1}$ Monatshefte für Chemie, 3, 715 (1882).

${ }^{2}$ Prepare sodium phenolate solution by mixing 20 grams of phenol with 40 grams of sodium hydroxide and 70 grams of water. 
signs of intoxication appear. A woman, who had taken ro drops of mirbane oil as an abortifacient, gave no indication of intoxication, that is to say, unconsciousness and cyanosis, for 8 hours after taking the poison.

Nitrobenzene not only profoundly changes the blood but it irritates and paralyzes the central nervous system (see R. Kobert, "Intoxikationen").

Some nitrobenzene passes into the urine. Although it has been stated that the organism does not convert nitrobenzene into aniline, Rossi ${ }^{1}$ found in the viscera of a person, who had died from supposed nitrobenzene poisoning, aniline which evidently had been formed as a result of putrefaction. Consequently in cases of fatal poisoning, tests for nitrobenzene should be made immediately after death. In nitrobenzene poisoning human urine contains a brown pigment but only rarely hæmoglobin or methæmoglobin. Urine containing nitrobenzene will reduce Fehling's solution. It is also unfermentable and distinctly lævo-rotatory. A conjugated glycuronic acid is possibly concerned in these reactions.

\section{Detection of Nitrobenzene}

In nitrobenzene poisoning the urine and all the organs have the odor of this compound. For the chemical tests the material should first be distilled with water. Nitrobenzene distils quite easily with steam and appears in the distillate as yellowish globules. These are heavier than water and have a characteristic odor. Vigorously agitate the globules, when separated as completely as possible from water, with granulated tin and a few cc. of concentrated hydrochloric acid, until there is no odor of nitrobenzene. Pour the acid solution from undissolved tin, and add an excess of potassium hydroxide solution to decompose the double chloride of aniline and tin. Extract free aniline with ether. Withdraw the aqueous liquid from the separating funnel, and evaporate the ether extract spontaneously in a small glass dish. Aniline, formed by reducing nitrobenzene, will remain as globules which usually have a red or brown color. Dissolve these globules by agitation with water, and use this solution for the hypochlorite and phenylisocyanide tests (see pages 45 and 36 ).

Mechanism of the Reaction.-Nitrobenzene is reduced by nascent hydrogen to aniline $(\alpha)$ which combines with the excess of hydrochloric acid forming aniline hydrochloride $(\beta)$. From the latter compound potassium hydroxide liberates aniline $(\boldsymbol{\gamma})$ :

\footnotetext{
${ }^{1}$ Chemical Abstracts 9, I34I (I9I5).
} 


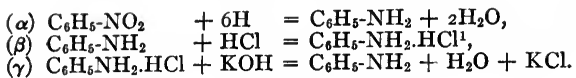

\section{ANIIINE}

Toxic Action.-Aniline is moderately toxic in its action. Doses of 1.5 to 2 grams, administered in the course of a day, have proved fatal to small dogs. It is not possible to state definitely the average lethal dose for human beings. Very<smiles>Nc1ccccc1</smiles>
serious results are said to have followed a dose of 3 or 4 grams of aniline. The lethal dose is certainly less than 25 grams, for that quantity of aniline was sufficient to kill a healthy man. Even inhalation of aniline vapor may cause severe or fatal intoxications.

Aniline produces methæmoglobin and therefore poisons the blood. The conversion of oxyhæmoglobin into methæmoglobin by aniline may be demonstrated by adding an aqueous aniline solution to blood in a test-tube. Aniline changes their form and partially decomposes red blood-corpuscles. Thereby the quantity of available oxygen in the blood is so diminished that it amounts to only 5 to 10 volumes instead of $x_{5}$ to 20 , the normal quantity. The number of red blood-corpuscles is diminished in aniline poisoning but not that of the white blood-cells.

R. v. Engelhardt has shown that aniline is partly changed in the human organism into aniline black, or into a similar compound insoluble in water. At the climax of aniline poisoning blue-black granules may be seen in every drop of blood and also in the urine. Aniline is oxidized in the system to para-aminophenol $\left(\mathrm{C}_{6} \mathrm{H}_{4}, \mathrm{OH}, \mathrm{NH}_{2}(\mathrm{r}, 4)\right)$. Like all phenols this compound forms an ethereal sul-

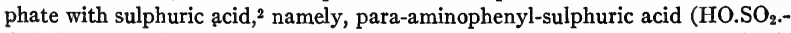
O. $\mathrm{C}_{6} \mathrm{H}_{4} \cdot \mathrm{NH}_{2}(\mathrm{r}, 4)$. This acid is eliminated through the kidneys as an alkali salt and then appears in the urine. A part of the para-aminophenol is also eliminated as a conjugate of glycuronic acid. ${ }^{3}$

The reduction of Fehling's solution by urine containing aniline is due to this conjugated acid. In severe cases of poisoning unchanged aniline has also been found in the urine. Usually urine that contains aniline has a very dark color. Besides the substances mentioned, a dark pigment has been detected

${ }^{1}$ Organic ammonium bases resemble ammonia in combining with acids to form salts. Trivalent nitrogen of the free base is changed to pentavalent nitrogen in the salt:

$$
\underset{\text { Aniline }}{\mathrm{C}_{6} \mathrm{H}_{5}-\mathrm{III}} \mathrm{N}_{\mathrm{H}}^{\mathrm{H}}+\mathrm{HCl}=\mathrm{C}_{6} \mathrm{H}_{5}-\underset{\text { Aniline hydrochloride }}{\stackrel{\mathrm{N}}{\mathrm{N}} /{ }_{-}^{\mathrm{H}}}
$$

${ }^{2}$ This conjugation takes place with elimination of water:

$\mathrm{H}_{2} \mathrm{~N} . \mathrm{C}_{6} \mathrm{H}_{4} \cdot \mathrm{OH}+\mathrm{HO} . \mathrm{SO}_{2} . \mathrm{OH}=\mathrm{H}_{2} \mathrm{O}+\mathrm{H}_{2} \mathrm{~N}_{2} \mathrm{C}_{6} \mathrm{H}_{4} \cdot \mathrm{O} \cdot \mathrm{SO}_{2} . \mathrm{OH}(1 ; 4)$

${ }^{3}$ Glycuronic acid, $\mathrm{C}_{6} \mathrm{H}_{10} \mathrm{O}_{7}=\underset{\mathrm{O}}{\mathrm{H}} / \mathrm{C}-(\mathrm{CH} . \mathrm{OH})_{4}-\mathrm{COOH}$, is closely related to glucose. It is an uncrystallizable syrup. If its aqueous solution is boiled, the acid is partly converted into the internal anhydride, glycurone $\left(\mathrm{C}_{6} \mathrm{H}_{8} \mathrm{O}_{6}\right)$, which crystallizes well. 
in urine in aniline poisoning as well as hæmoglobin, methæmoglobin and an abundance of urobilin (R. Kobert, "Intoxikationen").

\section{Detection of Aniline}

Aniline is a rather feeble base and part of it will pass over with steam, when the acid solution is distilled. There will be enough in the distillate for detection by the tests described below. In estimating aniline quantitatively in any kind of material the distillation must be as complete as possible. Mix the substance with water, make strongly alkaline with sodium hydroxide or carbonate solution and distil in a current of steam. Since 30 parts of water at $15^{\circ}$ dissolve I part of aniline, the distillate may contain considerable of this amine. When the quantity is large, oil-drops will appear. An aqueous aniline solution (aniline water) colors pine wood and elder pith intensely yellow. The following tests should be used for aniline:

I. Hypochlorite Test.-Add a few drops of aqueous calcium or sodium hypochlorite solution drop by drop to a portion of the distillate. A violet-blue or purple-violet color, gradually changing to a dirty red, will appear if aniline is present. Addition of a little dilute aqueous phenol solution containing some ammonia will produce a blue color which is quite stable. This test is sensitive $\mathrm{I}: 66,000 .^{1^{\circ}}$

2. Phenylisocyanide Test.-Heat a portion of the distillate with a few drops of chloroform and potassium hydroxide solution. The repulsive odor of phenylisocyanide will show the presence of aniline.

3. Bromine Water Test.-Bromine water added to a solution containing aniline will produce a flesh-colored precipitate. This test is sensitive $\mathrm{I}: 66,000$.

4. Chromic Acid Test. ${ }^{2}-$ Mix a trace of pure aniline with 4 to 5 drops of concentrated sulphuric acid in a porcelain dish and add a drop of aqueous potassium dichromate solution. After a few minutes the mixture beginning at the edge will take on a

${ }^{1}$ Test this experimentally with very little aniline. For example, dissolve a small drop in $30 \mathrm{cc}$. of water and take only $2-3 \mathrm{cc}$. of this dilute solution for the test.

${ }^{2}$ Beissenhirtz reaction, Annalen der Chemie und Pharmazie, 87, 376 (I853). 
pure blue color. Addition of I-2 drops of water produces at once a deep blue color. To apply this test to the distillate, first extract with ether, evaporate the ether solution and test an oily residue as described.

\section{CARBON DISULPHIDE}

Carbon disulphide, $\mathrm{CS}_{2}$, is a colorless liquid having a characteristic odor and a high index of refraction. It is only slightly soluble in water. There is some difference of opinion as regards the solubility of carbon disulphide in water.

$\begin{array}{lcl} & \text { I000 cc. of water dissolve } \\ 13^{-14^{\circ}} & 2.03 & \text { parts (Page) } \\ 15-16^{\circ} & 1.81 & \text { parts (Chancel; Parmentier) } \\ 15-16^{\circ} & 2-3 & \text { parts (Ckindi) } \\ 15-16^{\circ} & 3.5-4.5^{2} & \text { parts (Péligot) }\end{array}$

Carbon disulphide is miscible in all proportions with absolute alcohol, ether, ethereal and fatty oils.

Toxic Action.-Carbon disulphide administered internally has a very poisonous action upon the blood causing especially decomposition of red blood-corpuscles. Even inhalation of carbon disulphide vapor frequently occasions severe poisoning. Carbon disulphide was formerly considered a typical producer of methæmoglobin but recent investigations have not confirmed this opinion. It has a very injurious action upon the red blood-corpuscles and causes hæmolysis. R. Kobert (Intoxikationen) states that its power of dissolving lipoids is responsible for its injurious action upon the blood and the central nervous system. E. Harmsen ${ }^{1}$ has recently come to practically the same conclusion. He considers carbon disulphide a powerful blood poison because it decreases the number of red blood-corpuscles and the quantity of hæmoglobin and brings about a leucocytosis. ${ }^{2}$

\section{Detection of Carbon Disulphide}

Carbon disulphide distils very slowly with steam. Consequently the second or third fraction of the distillate should be used in testing for this substance. If $40 \mathrm{cc}$. are distilled from roo cc. of water containing 2 drops of carbon disulphide, the following Io cc. will give a distinct test. If the quantity of carbon disulphide is small, it will remain in solution. Such a solution does not have a strong odor. Carbon disulphide may be recognized by the following tests:

1 Vierteljahrsschrift für gerichtliche Medizin, 30, 442 (r905).

${ }^{2}$ Leucocytosis means a temporary increase in the number of white bloodcorpuscles (leucocytes) as compared with the number of red blood-corpuscles. Normally there are about 350 red to I white blood-corpuscle, whereas in leucocytosis the proportion is $20: \mathrm{I}$. 
r. Lead Acetate Test.-Add a few drops of lead acetate solution to the liquid containing carbon disulphide. It will cause neither a precipitate (distinction between $\mathrm{CS}_{2}$ and $\mathrm{H}_{2} \mathrm{~S}$ ) nor a color. Add excess of potassium hydroxide solution and boil. A black precipitate $(\mathrm{PbS})$ will appear. This is a very delicate test.

2. Sulphocyanate Test.-Heat an aqueous solution of carbon disulphide for a few minutes with concentrated ammonium hydroxide solution and alcohol. Ammonium sulphocyanate $\left(\mathrm{H}_{4} \mathrm{NSCN}\right)$ is formed together with ammonium sulphide. Concentrate this solution upon the water-bath to about I cc. and acidify with dilute hydrochloric acid. Add a drop of ferric chloride solution and a deep red color will appear. This test will show even traces of carbon disulphide, for example 0.05 gram in I cc. of water.

\section{Mechanism of the Reaction:}

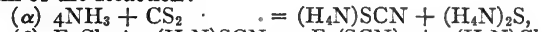

(B) $\mathrm{FeCl}_{3}+3\left(\mathrm{H}_{4} \mathrm{~N}\right) \mathrm{SCN}=\mathrm{Fe}(\mathrm{SCN})_{3}+3\left(\mathrm{H}_{4} \mathrm{~N}\right) \mathrm{Cl}$.

3. Xanthogenate Test.-Shake a few cc. of distillate for several minutes with 3 or 4 times its volume of saturated solution of potassium hydroxide in absolute alcohol. Faintly acidify the solution with acetic acid and add I or 2 drops of copper sulphate solution. If carbon disulphide is present, a brownish black precipitate of cupric xanthogenate will appear. This will soon change to a yellow, flocculent precipitate of cuprous xanthogenate, $\mathrm{CS}(\mathrm{SCu})\left(\mathrm{OC}_{2} \mathrm{H}_{5}\right)$. Vitali's procedure is somewhat different and consists in adding solid ammonium molybdate to the alkaline reaction-product and then in acidifying with dilute sulphuric acid. The appearance of a red color indicates carbon disulphide.

Mechanism of the Reaction.-Alcoholic potassium hydroxide acts like potassium alcoholate $\left(\mathrm{C}_{2} \mathrm{H}_{5}-\mathrm{OK}\right)$ and converts carbon disulphide into potassium xanthogenate

$$
\mathrm{CS}_{2}+\mathrm{C}_{2} \mathrm{H}_{5}-\mathrm{OK}=\mathrm{C} \stackrel{\angle \mathrm{SK}}{=} \underset{\mathrm{S}}{\mathrm{OC}_{2} \mathrm{H}_{5}}
$$

This compound treated with cupric salts gives first a brownish black precipitate of cupric xanthogenate:

$$
{ }_{2} \mathrm{C} \stackrel{/ \mathrm{SK}_{\mathrm{S}}^{\mathrm{S}}}{>\mathrm{OC}_{2} \mathrm{H}_{5}}+\mathrm{CuSO}_{4}=\left(\mathrm{S}=\mathrm{C}\left\langle\left\langle_{\mathrm{OC}_{2} \mathrm{H}_{5}}^{\mathrm{S}-}\right)_{2} \mathrm{Cu}+\mathrm{K}_{2} \mathrm{SO}_{4}\right.\right.
$$


The cupric salt then forms cuprous xanthogenate and.ethyl xanthogen disulphide:<smiles>CCOC(=S)SSC(=S)OC(=S)SC(=S)OCC</smiles>

\section{Quantitative Estimation of Carbon Disulphide in Air}

Inhalation of air containing carbon disulphide has frequently given rise to chronic poisoning. Persons thus affected have usually been laborers in rubber factories. Consequently experiments have been made to determine the maximum quantity of carbon disulphide air may contain without injury to health. The results of these investigations may be summarized as follows:

\begin{tabular}{lcl} 
& \multicolumn{2}{c}{$\mathrm{CS}_{2}$ in mgrs. } \\
per liter of air & Result \\
r. & $0.5^{-0.8}$ & No injurious effect. \\
2. & $\mathrm{I} .3$ & Slight uneasiness after several hours. \\
3. & 3.4 & Uneasiness in 30 minutes. \\
4. & 6.0 & Uneasiness in 20 minutes. \\
5. & ro.0 & Paralysis attended by after-effects last- \\
& & ing several days.
\end{tabular}

The exact danger limit for persons obliged to live for weeks at a time in an atmosphere containing carbon disulphide should be placed below $3 \mathrm{mg}$. per liter of air. Air in factories, where operatives work in presence of carbon disulphide vapor, should never exceed this limit. In rubber factories the air is said frequently to contain 2.5 to $3 \mathrm{mg}$. per liter. Since experiments have shown that 93 to 96 per cent. of the carbon disulphide breathed was exhaled unchanged, an exceedingly small quantity is capable of producing toxic symptoms.

Procedure.-Place a saturated alcoholic solution of potassium hydroxide in a Péligot absorption-tube and draw through this solution ro to 20 liters of air containing carbon disulphide vapor. A quantitative formation of potassium xanthogenate (see above) will take place.

Dilute the contents of the receiver at the end of the experiment with 96 per cent. alcohol and bring the volume to $50 \mathrm{cc}$. Measure an aliquot portion of this solution and dilute with water. Faintly acidify the solution with acetic acid and remove excess of acid with acid sodium carbonate. Add freshly prepared starch solution and o.r n-iodine solution until there is a permanent blue color.

Iodine converts potassium xanthogenate according to equation (I) into ethyl xanthogen-disulphide:

$$
\text { I. } \mathrm{I}_{2}+\frac{\text { KS.CS.OC } \mathrm{H}_{5}}{\text { KS.CS.OC } \mathrm{H}_{2} \mathrm{H}_{2}}=2 \mathrm{KI}+\text { I. S.CS.OC } \mathrm{OC}_{5}
$$


E. Rupp and L. Krauss 1 think the action of iodine upon potassium xanthogenate is expressed by equation (II):

II. ${ }_{2} \mathrm{KS} . \mathrm{CS} . \mathrm{OC}_{2} \mathrm{H}_{5}+\mathrm{H}_{2} \mathrm{O}+{ }_{2} \mathrm{I}=\mathrm{KS} . \mathrm{CS} . \mathrm{SK}+{ }_{2} \mathrm{C}_{2} \mathrm{H}_{5} \cdot \mathrm{OH}+{ }_{2} \mathrm{HI}+\mathrm{S}$. Both equations require the same quantity of iodine, namely, 2 atoms for 2 molecules of xanthogenate. A difference therefore in the mechanism of the reaction has no influence on the combining relations of the iodine and the method is applicable to the quantitative determination of xanthogenate.

I000 cc. of $0.1 \mathrm{n}$-iodine solution, containing 0.1 gram-atom of iodine, correspond to o.r gram-molecule of $\mathrm{CS}_{2}=7.6$ grams.

\section{ETHYL ALCOHOL 2}

Fate in the Human Organism.-Ethyl alcohol brought in contact with many different parts of the organism is very rapidly absorbed, but especially easily from an empty stomach. Although there is practically no absorption of non-<smiles>CCO</smilesvolatile aqueous liquids from the stomach, ethyl alcohol is freely absorbed. After absorption it passes into the blood and is then distributed to all organs (see chloral hydrate). Experiments upon dogs, colts and adult horses (see Kobert, "Intoxikationen") have shown that blood at the climax of narcosis contains 0.72 per cent. of ethyl alcohol. There is stupor even when 0.12 per cent. is present.

There is difference of opinion among toxicologists regarding alcoholic intoxication, as to whether the poison is distributed uniformly throughout the body, or accumulated in the brain in larger quantity than in other organs. The following percentages of ethyl alcohol, found in the organs of a man, who had died at the climax of severe acute ethyl alcohol poisoning, lend support to the latter view: liver 0.21 , brain 0.47 and blood 0.33 per cent. It appears from these results that the brain takes up an especially large quantity of ethyl alcohol.

Uncertainty concerning the subsequent fate of ethyl alcohol in the organism has finally been removed. Experiments have shown that ethyl alcohol is never eliminated unchanged through the skin. At most only I-I.5 per cent. passes off through the kidneys and only r.6-2 per cent. through the lungs. Strass$m^{m a n n}{ }^{3}$ found the quantity eliminated by the lungs somewhat higher $\left(5^{-} 6\right.$ per cent.) and by the kidneys $\mathrm{I}-2.5$ per cent. The remainder is completely oxidized in the human organism to carbon dioxide and water.

B. Fischer found the following quantities of ethyl alcohol in organs removed from a man who had probably died from drinking too much brandy:

\section{Weight}

$$
2720 \text { grams }
$$

2070 grams

1820 grams

1365 grams
Organ

Stomach and intestines

Heart, lungs and blood

Kidneys and liver

Brain

\section{Ethyl Alcohol \\ 30.6 grams \\ I0.85 grams \\ 7.8 grams \\ 4.8 grams}

${ }^{1}$ Berichte der Deutschen chemischen Gesellschaft 35, 4257 (I902).

${ }^{2}$ The word "alcohol," unqualified by an adjective, i.e., methyl, amyl, etc., means ethyl alcohol. Tr.

${ }^{3}$ Pflüger's Archiv, 49, 315 (189r). 


\section{Detection of Ethyl Alcohol}

Ethyl alcohol distils easily with steam and consequently most of it will be in the first fraction. If present in sufficient quantity, it can be recognized in the distillate by its odor. The following tests should be made:

I. Lieben's Iodoform Test. ${ }^{1}-$ Gently warm the liquid (40$50^{\circ}$ ), add a few cc. of aqueous iodo-potassium iodide solution, or a small crystal of iodine, and enough potassium hydroxide solution to give the liquid a distinct

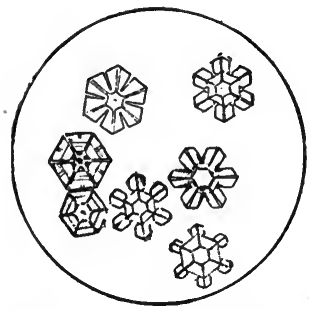

Fig. 9.-Iodoform Crystals. yellow to brownish color. If ethyl alcohol is present, a yellowish white to lemon-yellow precipitate of iodoform will appear immediately; or as the solution cools. If the quantity of ethyl alcohol is very small, a precipitate will form on long standing. When iodoform is deposited slowly, the crystals are very perfect hexagonal plates and stars (see Fig. 9).

Note.-This iodoform test is very delicate but not characteristic of ethyl alcohol, since other primary alcohols, except methyl alcohol, and many secondary alcohols, as well as their oxidation products, aldehydes and ketones, give iodoform under the same conditions. Acetic ether, aceto-acetic ether, lactic acid, etc., also give iodoform.

The correct explanation of the iodoform reaction is probably the following: Iodine and potassium hydroxide form potassium hypo-iodite (KOI) by reaction $(\alpha)$. This compound brings about the oxidation of ethyl alcohol to acetic aldehyde $(\beta)$ and at the same time substitutes iodine for hydrogen in the latter $(\gamma)$. Finally tri-iodo-acetic aldehyde is decomposed by the excess of potassium hydroxide into iodoform and potassium formate $(\delta)$ :
( $\alpha) 2 \mathrm{KOH}$ $+\mathrm{I}_{2}=\mathrm{KI}+\mathrm{H}_{2} \mathrm{O}+\mathrm{KOI}$,
(a) $\mathrm{CH}_{3} \cdot \mathrm{CH}_{2} \cdot \mathrm{OH}+\mathrm{KOI}=\mathrm{CH}_{3} \cdot \mathrm{CHO}+\mathrm{H}_{2} \mathrm{O}+\mathrm{KI}$,
(r) $\mathrm{CH}_{3} \cdot \mathrm{CHO}+{ }_{3} \mathrm{KOI}={ }_{3} \mathrm{KOH}+\mathrm{CI}_{3} \cdot \mathrm{CHO}$,

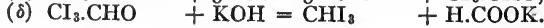

2. Berthelot's Test.-Shake the liquid containing ethyl alcohol with a few drops of benzoyl chloride and about $5 \mathrm{cc}$. of sodium hydroxide solution (Io per cent.), until the irritating

1 Annalen der Chemie und Pharmazie, Supplement Band, 7, 218. 
odor of benzoyl chloride has gone. The aromatic odor of ethyl benzoate will appear.

$$
\mathrm{C}_{6} \mathrm{H}_{5} \cdot \mathrm{COCl}+\mathrm{C}_{2} \mathrm{H}_{5} . \mathrm{OH}+\mathrm{KOH}=\mathrm{C}_{6} \mathrm{H}_{5} \cdot \mathrm{CO}_{0} . \mathrm{OC}_{2} \mathrm{H}_{5}+\mathrm{KCl}+\mathrm{H}_{2} \mathrm{O}
$$

Ten cc. of 0.5 per cent. ethyl alcohol will give a distinct odor of this ester.

3. Chromic Acid Test.-Warm the liquid containing ethyl alcohol with dilute sulphuric, or hydrochloric acid, and add I or 2 drops of very dilute potassium dichromate solution. The color of the liquid will change from red to green, and at the same time the odor of acetaldehyde will be recognized. This test is not characteristic of ethyl alcohol because many other volatile organic compounds behave similarly.

\section{Mechanism of the Reaction}

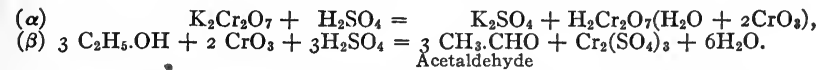

4. Ethyl Acetate Test.-Mix the liquid containing ethyl alcohol with the same volume of concentrated sulphuric acid. Add a very small quantity of anhydrous sodium acetate and heat. Ethyl acetate will be recognized by its odor.

(a) $\mathrm{C}_{2} \mathrm{H}_{6} . \mathrm{OH}+\mathrm{H}_{2} \mathrm{SO}_{4}=\mathrm{C}_{2} \mathrm{H}_{5} \mathrm{O}_{2} \mathrm{SO}_{2} \cdot \mathrm{OH}^{1}+\mathrm{H}_{2} \mathrm{O}$,

( $\beta$ ) $\mathrm{CH}_{3} . \mathrm{CO} . \mathrm{ONa}+\mathrm{C}_{2} \mathrm{H}_{5} \mathrm{O} . \mathrm{SO}_{2} . \mathrm{OH}=\mathrm{CH}_{3} \cdot \mathrm{CO}_{0} \mathrm{OC}_{2} \mathrm{H}_{5}+\mathrm{NaHSO}_{4}$.

5. Vitali's Test.-Thoroughly mix a few cc. of distillate in a glass dish with a small piece of solid potassium hydroxide and 2 or 3 drops of carbon disulphide. Let this mixture stand for a short time without warming. When most of the carbon disulphide has evaporated, add a drop of ammonium molybdate solution and then an excess of dilute sulphuric acid. If the distillate contains ethyl alcohol, a red color will appear. Potassium xanthogenate $\left(\mathrm{CS}\left(\mathrm{OC}_{2} \mathrm{H}_{5}\right)(\mathrm{SK})\right)$ is first formed. This compound gives a red color with ammonium molybdate. Acetone and acetaldehyde produce a similar color. This test is given distinctly by 5 per cent. aqueous ethyl alcohol solution.

1 The structural formula of ethyl sulphuric acid is 


\section{METHYL ALCOHOL}

$\mathrm{H}-\mathrm{l}_{\mathrm{O}}^{\mathrm{H}}-\mathrm{H}$

alcohol.

Pure methyl alcohol is a colorless liquid having an agreeable alcoholic odor and boiling at about $66^{\circ}$. It is miscible with water in all proportions. Combined chemically with other substances, it occurs in nature; for example, as methyl salicylate in oil of wintergreen. Methyl alcohol has many industrial applications. It is extensively used as a solvent and in making other compounds. The crude alcohol in considerable quantity furnishes a means of denaturing grain alcohol, since its disagreeable odor renders the latter obnoxious as a beverage but does not unfit it for many manufacturing processes. Methyl alcohol is also frequently employed as an adulterant of ethyl alcohol and as a substitute for the latter, especially in tinctures and varnishes. These uses, however, are not legitimate and should be condemned.

Toxic Action.-Methyl alcohol is a subtle and dangerous poison. Since it is used not only in adulterating spirituous liquors but in preparing Jamaica ginger, peppermint and lemon extracts, cologne, bay rum, Flofida water and witch hazel, as well as lacquers and varnishes, its deleterious effects are to be expected whenever such preparations are taken internally. The increasing diffculty of obtaining in the United States beverages that contain ethyl alcohol has led unscrupulous and ignorant persons to substitute in its place the more accessible but more deadly methyl alcohol.

In the case of animals a single dose of methyl alcohol has been found less toxic than the same quantity of ethyl alcohol. Also its action is slower but of longer duration. More marked gastric irritation and convulsive movements frequently have been observed. If administered repeatedly, methyl alcohol is much more toxic than ethyl alcohol. Von $\mathrm{Pohl}^{1}$ has shown that methyl alcohol unlike ethyl alcohol is incompletely oxidized in the organism. In consequence formic acid and possibly formaldehyde, both more toxic than methyl alcohol, are formed in the tissues.

In man methyl alcohol gives rise to marked muscular weakness and defective heart action. Nausea, vomiting, coma and a delirium that is much more intense and persistent than that attending intoxication by ethyl alcohol subsequently have been observed. Methyl alcohol frequently has proved lethal, which would not have been the case had the same quantity of ethyl alcohol been taken. Cases are on record where the victims have recovered only to be totally and permanently blinded because of optic neuritis and subsequent complete optic atrophy. There can be no doubt therefore that methyl alcohol is a very dangerous poison which should never be administered internally nor applied externally in a manner making possible its entrance into the body through any of the ordinary channels of absorption.

1 Therapeutische Monatshefte 1892 , page 327 . 


\section{Detection of Methyl Alcohol}

Methyl alcohol distils easily with steam and will appear mainly in the first fraction. When pure it may be recognized by its boiling-point but usually it is necessary to detect a small quantity in presence of vegetable or animal extractive matter and of other volatile liquids or solids.

Isolation of Methyl Alcohol from Mixtures.-It is not advisable to undertake the detection or estimation of methyl alcohol in mixtures containing other volatile products unless a preliminary purification has been made. Thorpe and Holmes ${ }^{1}$ bring the volume of $25 \mathrm{cc}$. of the liquid to $100-150 \mathrm{cc}$. with water in a separatory funnel, saturate with sodium chloride and shake well for 5 minutes with $50-80 \mathrm{cc}$. of petroleum ether boiling below $60^{\circ}$. Withdraw the under layer after 30 minutes and, if necessary, extract again. Shake the petroleum ether extracts successively a second time with $25 \mathrm{cc}$. of saturated salt solution. Mix the original solution with the brine washing in a distilling flask, neutralize any acidity with sodium hydroxide solution and collect $25-50 \mathrm{cc}$. of distillate. Bring the volume of the distillate to roo cc. and use this solution for tests.

Several tests depend upon first oxidizing methyl alcohol to formaldehyde and then detecting the latter by means of a color change.

I. Copper Oxidation Test. ${ }^{2}$-Dilute the solution, if necessary, until the total alcoholic strength does not exceed Io per cent. Immerse in cold water the test-tube containing $3 \mathrm{cc}$. of liquid and insert 3-4 times a copper spiral heated to redness, reheating each time. Filter, expel by boiling any odor of acetic aldehyde, cool and add one drop of 0.5 per cent. aqueous resorcinol solution. Add this mixture from a pipettę to form an upper layer on 2 cc. of concentrated sulphuric acid and gently rotate the test-tube for 3 minutes. If a rose-red ring does not appear at the contact-surface of the two liquids, less than 2 per cent. of methyl alcohol is present.

2. Permanganate Oxidation Test.-The following methods have been proposed:

(a) Scudder-Biggs ${ }^{3}$ Method.-Add $0.5 \mathrm{cc}$. of concentrated

${ }^{1}$ Journal of the Chemical Society 83,3 I4 (I903).

${ }^{2}$ Mulliken and Scudder: American Chemical Journal 21, 266 (r899).

${ }^{3}$ Journal of the American Chemical Society 28, I 202 (Igo6). 
sulphuric acid and $5 \mathrm{cc}$. of saturated potassium permanganate solution to ro cc. of liquid, keeping the temperature at $20-25^{\circ}$. After 2 minutes discharge the color by means of sulphurous acid, expelling the latter and any acetic aldehyde by heat. Complete the test with resorcinol and sulphuric acid as in the copper wire test. In presence of ethyl alcohol, less acetic aldehyde is said to be produced by permanganate than by copper.

(b) United States Pharmacopoeia ${ }^{1}$ Method.-This method is employed to test for methyl alcohol in ethyl alcohol. The liquid should contain not more than Io per cent. of ethyl alcohol by volume. Add 2. cc. of potassium permanganate solution (3 grams of $\mathrm{KMnO}_{4}$ in Ioo cc. of distilled water) and $0.3 \mathrm{cc}$. of sulphuric acid to $5 \mathrm{cc}$. of the liquid. Dissolve the precipitated manganese dioxide after 5 minutes by adding sulphurous acid drop by drop and shaking. Then add I cc. of sulphuric acid, 5 cc. of fuchsin-sulphurous acid solution, ${ }^{2}$ and mix thoroughly. If methyl alcohol is present, the liquid will be colorless after ro minutes standing.

(c) Denigés ${ }^{3}$-Simmonds ${ }^{4}$ Method. - The total alcoholic strength of the liquid should not exceed ro per cent. by volume. Add to $5 \mathrm{cc}$. of the liquid $2.5 \mathrm{cc}$. of potassium permanganate solution ( 2 per cent.) and $0.2 \mathrm{cc}$. of concentrated sulphuric acid. After 3 minutes follow with $0.5 \mathrm{cc}$. of oxalic acid solution (9.6 grams of crystals in Ioo cc.). When shaken, the mixture becomes clear and nearly colorless. Then run in I cc. of concentrated sulphuric acid, mix well and finally add 5 cc. of Schiff's reagent. $^{5}$ In a few minutes a violet color will appear, if more than traces of methyl alcohol are present, otherwise it may take 20-30 minutes.

3. Hinkel's Test. ${ }^{6}$ - Add to I cc. of liquid 0.8 gram of am-

1 United States Pharmacopoeia, Ninth Decennial Revision, page 36.

${ }^{2}$ Add ro cc. of hydrochloric acid to a solution of 0.5 gram of fuchsin and 9 grams of sodium bisulphite in $500 \mathrm{cc}$. of distilled water.

- Comptes rendus de l'Academie des Sciences I50, 832 (I9ro).

- Analyst 37, I6 (I9I2).

Dissolve 0.2 gram of rosaniline base in $20 \mathrm{cc}$. of cold saturated sulphurous acid solution. If the color is not discharged in 24 hours, add ro cc. more of sulphurous acid. Repeat, if necessary, until there is no color and dilute to $200 \mathrm{cc}$.

B Analyst 33, 417 (1908). 
monium persulphate $\left(\mathrm{H}_{4} \mathrm{~N} . \mathrm{SO}_{4}\right)$ and $3 \mathrm{cc}$. of dilute sulphuric acid ( $1: 5$ ). Dilute the mixture to $20 \mathrm{cc}$. with water and distil, collecting in test-tubes 5 separate $2 \mathrm{cc}$. portions. Reject the first two portions, which contain any acetic aldehyde, and add to the last three a few drops of 0.5 per cent. morphine hydrochloride solution. Finally run into each test-tube $3 \mathrm{cc}$. of concentrated sulphuric acid as an under layer. A violet ring will appear at the contact-surface of the two liquids, if formaldehyde is present. This test will not show with certainty less than 5 per cent. of methyl alcohol in ethyl alcohol.

\section{Quantitative Estimation of Methyl Alcohol}

The method of Leach and Lythgoe, ${ }^{1}$ requiring the use of the Zeiss immersion refractometer, furnishes a rapid means of estimating methyl alcohol in presence of ethyl alcohol. This instrument gives a reading of $\mathrm{I} 4.5$ for distilled water at $20^{\circ}$, a maximum reading of IOI for 75 per cent. ethyl alcohol and 9I for roo per cent.; and a maximum reading of 39.8 for 50 per cent. methyl alcohol, I4.9 for 9I per cent. and 2 for Ioo per cent. $^{2}$

\section{ACETONE}

Human urine almost always contains a very small quantity of acetone as a physiological constituent. Under pathological conditions, especially in diabetes<smiles>CC=O</smiles>
mellitus (diabetic acetonuria), urine contains much more. It is also present in urine in protracted high fever, digestive disturbances, severe forms of carcinoma (carcinomatous acetonuria), etc. Finally, acetone has been found in urine in considerable quantity in various intoxications (toxic acetonuria), for example, in poisoning by phosphorus, carbon monoxide, atropine, curare, antipyrine, pyrodine, sulphuric acid, extract of male fern; in chronic lead poisoning; and in chronic morphinism after discontinuance of the drug (see R. Kobert, "Intoxikationen").

Acetone is not poisonous nor in the least corrosive. Man and animals can tolerate considerable quantities of acetone taken internally. It seems to produce no effect, though it may possibly possess very feeble narcotic properties. Archangelsky found dogs to show signs of narcosis when the blood contained 0.5 per cent. of acetone. Even smaller doses produce narcosis in rabbits and have an injurious action upon the blood and kidneys.

1 Journal of the American Chemical Society 27, 964 (1905).

2 Tables giving the percentages of methyl alcohol corresponding to the different readings will be found in the original communication of the authors. 
Distillates from human urine, as well as from blood and various organs, as liver, spleen, kidneys, brain, etc., often contain acetone, or more correctly perhaps, substances like acetone. This is especially the case when cadaveric material has begun to putrefy.

Acetone is a clear, colorless liquid boiling at $56^{\circ}$. It has a peculiar, fruity odor and is neutral in reaction. It is miscible in all proportions with water, ethyl alcohol and ether. It distils easily with steam.

\section{Detection of Acetone}

I. Lieben's Iodoform Test.-Add a few cc. of aqueous iodopotassium iodide solution, or a small crystal of iodine, to an aqueous solution of acetone and then potassium hydroxide solution drop by drop until the color is yellow. Iodoform immediately separates, even in the cold, as a yellowish white precipitate which is usually amorphous. Acetone differs from ethyl alcohol in giving iodoform, when ammonium hydroxide solution is substituted for potassium or sodium hydroxide solution (Gunning's acetone test).

Acetaldehyde resembles acetone in giving iodoform in the cold and under conditions the same as those stated above.

Note.-Potassium hypo-iodite $(\alpha)$ probably converts acetone into tri-iodoacetone $\left(\mathrm{CH}_{3} . \mathrm{CO} . \mathrm{CI}_{3}\right)(\beta)$ and this compound is then decomposed by potassium hydroxide into iodoform and potassium acetate $(\gamma)$ :
$(\alpha) 6 \mathrm{KOH}$
$+3 \mathrm{I}_{2}={ }_{3} \mathrm{KI}+3 \mathrm{KOI}+{ }_{3} \mathrm{H}_{2} \mathrm{O}$
(a) $\mathrm{CH}_{3} \cdot \mathrm{CO} \cdot \mathrm{CH}_{3}+{ }_{3} \mathrm{KOI}=\mathrm{CH}_{3} \cdot \mathrm{CO} \mathrm{CI}_{3}+{ }_{3} \mathrm{KOH}$,
$(\gamma) \mathrm{CH}_{3} \cdot \mathrm{CO}^{-\mathrm{CI}_{3}}+\mathrm{KOH}=\mathrm{CHI}_{3}$
$+\mathrm{CH}_{3}$.CO.OK.

2. Legal's Test.-Add a few drops of freshly prepared sodium nitroprusside solution to a liquid containing acetone, and then potassium hydroxide solution. A red or reddish yellow color will appear. This color soon changes to yellow. Add an excess of acetic acid to the solution. The solution will now have a carmine to purplish red color, according to the quantity of acetone present. Heat will change this color to violet.

Ethyl alcohol does not give Legal's test, though acetaldehyde does. The red color caused by aldehyde fades upon addition of acetic acid, and changes to green with heat. Le Nobel states that ammonium hydroxide, or ammonium carbonate solution, may be substituted for potassium hydroxide solution in Legal's test, but under these conditions the red color is very slow to appear. Le Nobel's modification, however, eliminates the possibility of confusing acetone with acetaldehyde. 
3. Penzoldt's Test.-Prepare a hot, saturated, aqueous ortho-nitro-benzaldehyde $\left(\mathrm{C}_{6} \mathrm{H}_{4} \cdot \mathrm{NO}_{2} \cdot \mathrm{CHO}(\mathrm{I}, 2)\right)$ solution and allow it to cool. Add this solution to the liquid containing acetone, and also some sodium hydroxide solution. At first the color of the mixture is yellow. It then becomes green, and a blue precipitate of indigotin is formed in Io to ${ }_{5}$ minutes. When indigotin is present in traces only, shake the solution with chloroform. This solvent will dissolve the coloring matter and become blue.

4. Reynolds' Test.-Acetone will dissolve freshly precipitated mercuric oxide, and this test is based upon this property. Add mercuric chloride solution to the distillate, and an alcoholic potassium hydroxide solution. Shake thoroughly and filter. Add ammonium sulphide solution to the clear filtrate as an upper layer. If acetone is present, there will be a black zone ( $\mathrm{HgS})$ where the two solutions meet.

Detection of Acetone in Urine.-Acidify 200 to $500 \mathrm{cc}$. of urine with a few drops of sulphuric acid and distil. Collect 20 to $30 \mathrm{cc}$. of distillate. This will contain the entire quantity of acetone in the urine. Acetone thus obtained may possibly be derived from aceto-acetic acid which is often present in human urine, especially in a severe case of diabetes mellitus. Distillation decomposes this acid into acetone and carbon dioxide.

$$
\mathrm{CH}_{3} \cdot \mathrm{CO} \cdot \mathrm{CH}_{2} \cdot \mathrm{CO} \cdot \mathrm{OH}=\mathrm{CH}_{3} \cdot \mathrm{CO} \cdot \mathrm{CH}_{3}+\mathrm{CO}_{2} \text {. }
$$

Detection of Ethyl Alcohol and Acetone in Mixtures.-Ethyl alcohol may be detected in presence of acetone by Berthelot's test. On the other hand, acetone may be distinguished from ethyl alcohol by Legal's or Penzoldt's test.

\section{BITTER ALMOND WATER AND BENZALDEHYDE}

Bitter almond water (Aqua Amygdalæ Amaræ of the Pharmacopœia) contains hydrocyanic acid. Only a small portion of this acid, however, is free so that it can be precipitated by silver nitrate solution. The greater part is combined as the cyanohydrin of benzaldehyde, $\mathrm{C}_{6} \mathrm{H}_{5} . \mathrm{C} \underset{\mathrm{CN}}{-} \underset{\mathrm{OH}}{\mathrm{H}}$, which does not react with silver nitrate. But potassium hydroxide solution will decompose this compound.

$$
\mathrm{C}_{6} \mathrm{H}_{5} \mathrm{CH}(\mathrm{OH}) \mathrm{CN}+\mathrm{KOH}=\mathrm{KCN}+\mathrm{H}_{2} \mathrm{O}+\mathrm{C}_{6} \mathrm{H}_{5} . \mathrm{CHO} .
$$


Pure benzaldehyde, also called hydrocyanic acid-free oil of bitter almonds, is not poisonous. It is oxidized to benzoic acid in the body and eliminated in the urine partly as that acid and.partly as hippuric acid after conjugation with glycocoll (amino-acetic acid):

$\begin{array}{ll}(\alpha) \mathrm{C}_{6} \mathrm{H}_{5}-\mathrm{CHO}+\mathrm{O} & =\mathrm{C}_{6} \mathrm{H}_{5}-\mathrm{COOH}, \\ (\beta) \mathrm{C}_{6} \mathrm{H}_{6}-\mathrm{COOH}+\mathrm{H}_{2} \mathrm{~N}-\mathrm{CH}_{2}-\mathrm{COOH} & =\mathrm{C}_{6} \mathrm{H}_{5}-\mathrm{CO}-\mathrm{NH}-\mathrm{CH}_{2}-\mathrm{COOH} .\end{array}$ Benzoic acid Glycocoll furnished Hippuric acid

Ordinary commercial oil of bitter almonds contains hydrocyanic acid and is poisonous in proportion to the quantity of this acid present.

Test for hydrocyanic acid by shaking about 2 cc. of oil of bitter almonds with $20 \mathrm{cc}$. of potassium hydroxide solution and making the Prussian blue test. When oil of bitter almonds is mixed with other material, distil with steam from a solution acidified with tartaric, or dilute sulphuric acid, and test the first part of the distillate for hydrocyanic acid. If benzaldehyde is present, the distillate at the same time will be milky and have the characteristic odor of that compound. Distil until the drops of water are perfectly clear. Benzaldehyde may be detected with certainty, and at the same time distinguished from nitrobenzene which has a somewhat similar odor, by adding a few drops of potassium hydroxide solution to the milky distillate, to combine with any hydrocyanic acid, and extracting with ether. The ether upon evaporation will deposit benzaldehyde as globules, which can be positively identified by conversion into benzoic acid. Heat the globules for a few minutes in a small flask, attached to a reflux condenser, with about ro cc. of potassium dichromate solution and a little dilute sulphuric acid. Cool, extract with ether and evaporate the ether solution in a glass dish. When the material contains benzaldehyde, this residue will consist of benzoic acid. This substance may be further identified by its melting-point ( $\left(20-\mathrm{I} 2 \mathrm{I}^{\circ}\right.$ ), its property of subliming and the test with ferric chloride solution. ${ }^{1}$

${ }^{1}$ Dissolve the residue in a small quantity of water, and neutralize benzoic acid by heating the solution to boiling with excess of calcium carbonate. Filter and add a few drops of ferric chloride solution. If benzoic acid is present, a fleshcolored precipitate of basic ferric benzoate will appear. $\mathrm{Tr}$. 


\section{SYNOPSIS OF GROUP I}

\section{Scherer's Test for Phosphorus Precedes Distillation}

The material to be examined must.first be rendered uniform by grinding or chopping. Add sufficient water to thin the mass, acidify with tartaric acid and distil. If the preliminary test for phosphorus (Scherer's) is positive, distil in the Mitscherlich apparatus; otherwise distil as usual with a Liebig condenser. It is advisable to collect the distillate in two or three fractions. Test the first 5 to ro cc. of distillate for hydrocyanic acid, chloroform, ethyl and methyl alcohol, acetone and possibly also for nitro-benzene and iodoform. Use the remainder of the distillate in testing for carbolic acid, chloral hydrate and carbon disulphide.

Phosphorus.-Phosphorescence in Mitscherlich apparatus during distillation in a dark room. Evaporate distillate with strong chlorine water, or a little fuming nitric acid, and test the residue for phosphoric acid. As an alternative procedure, examine the original material, or at least the Mitscherlich distillate, for phosphorus by the Blondlot-Dusart method.

Hydrocyanic Acid.-Odor. Schönbeịn's preliminary test. Prussian blue test. Sulphocyanate test. Nitroprusside test. Silver nitrate test. Alkaline phenolphthalin test.

Carbolic Acid.-Odor. Red color with Millon's reagent. Yellowish white precipitate with bromine water. Violet color with ferric chloride solution.

Chloroform.-Separation of colorless globules, when the quantity is large. Odor. Phenylisocyanide test, when heated with aniline and potassium hydroxide solution. Reduces silver nitrate and Fehling's solutions with heat. Red color with resorcinol and potassium hydroxide solution. Blue color with naphthol and potassium hydroxide solution. Blue-red color with pyridine and sodium hydroxide solution.

Chloral Hydrate.-Gives chloroform reactions. Brick-red precipitate with Nessler's solution which in time becomes yellowish green. Gives chloroform and magnesium formate, 
when heated with magnesium oxide and water. Test for formate with silver nitrate or mercuric chloride solution.

Iodoform.-Odor. Distillate milky and yellowish white. Ether extract of distillate leaves crystals upon evaporation, gives chloroform reactions.

Nitrobenzene.-Yellowish globules with characteristic odor. Reduced to aniline, when shaken with tin and hydrochloric acid. Test for aniline.

Aniline.-Violet color with calcium hypochlorite solution. Phenylisocyanide test, when heated with chloroform and potassium hydroxide solution. Flesh-colored precipitate with bromine water. Dark red color on warming with Millon's reagent.

Carbon Disulphide.-Black precipitate, or only black coloration $(\mathrm{PbS})$, when heated with lead acetate and potassium hydroxide solutions. Formation of ammonium sulphocyanate by evaporation with concentrated ammonium hydroxide solution and detection with ferric chloride solution. Formation of potassium xanthogenate, when shaken with alcoholic solution of potassium hydroxide and detection with copper sulphate solution.

Ethyl Alcohol.-Iodoform test. Odor of ethyl benzoate, when shaken with benzoyl chloride and sodium hydroxide solution. Green color and aldehyde odor, when heated with potassium dichromate and hydrochloric acid. Vitali's test.

Methyl Alcohol.-Oxidation tests with copper, potassium permanganate and ammonium persulphate.

Acetone.-Gives iodoform, even in the cold, with iodine and potassium hydroxide or ammonium hydroxide solution. Legal's test. Indigotin test. Reynolds' test. 


\section{CHAPTER II}

\section{NON-VOLATILE POISONS ${ }^{1}$}

\section{Alkaloids, Glucosides and Synthetic Compounds Non-volatile with Steam from Acid Solution}

Put a portion of finely chopped material into a large flask, and thoroughly mix with two or three times as much absolute ethyl alcohol. ${ }^{2}$ Add enough tartaric acid solution to give the mixture a distinct acid reaction after shaking. Laboratory experiments usually require 20 to 30 drops of ro per cent. tartaric acid solution. Avoid a large excess of tartaric acid, since it may act as an objectionable impurity in the ether extract, owing to its solubility in that solvent. Connect the flask with a glass tube ( 80 to $100 \mathrm{~cm}$. long) serving as a reflux cooler. Frequently shake and heat Io to ${ }_{5}$ minutes upon the water-bath. In the extıaction of a large quantity of material from a cadaver, connect the flask with an upright Liebig condenser used as a reflux cooler (Fig. IO). Cool the flask contents and filter to remove fat and other insoluble matter. Wash the residue with ethyl alcohol. Evaporate the filtrate, which must have an acid reaction, to a thin syrup in a glass dish upon the water-bath. Thoroughly mix this residue with Ioo cc. of cold water. Usually this causes an abundant separation of fat and resinous matter, especially when parts of a cadaver are examined. Filter and evaporate the filtrate to dryness, or to a syrup, upon the water-

1 The isolation of these toxic substances from cadaveric material, food, etc., is necessary before tests establishing their presence can be made. Mixtures used for laboratory practice, consisting of dog biscuit, meat, comminuted organs (liver, kidneys, spleen), sausage meat, etc., with any of the poisons of this group, should be examined according to the method outlined above.

${ }^{2}$ Commercial ethyl alcohol usually contains basic compounds, the presence of which is objectionable. They should be removed by adding tartaric acid to the ethyl alcohol and distilling. Ethyl alcohol should not be used in toxicological analysis, unless an actual test has shown it to be free from such impurities. $\operatorname{Tr}$. 
bath. Thoroughly mix this residue with absolute ethyl alcohol. As a result of this treatment, a whitish substance, which is more or less viscous or slimy, usually remains undissolved. This residue. which consists chiefly of protein substances (albumin, albumoses and peptones), dextrin-like compounds and in part

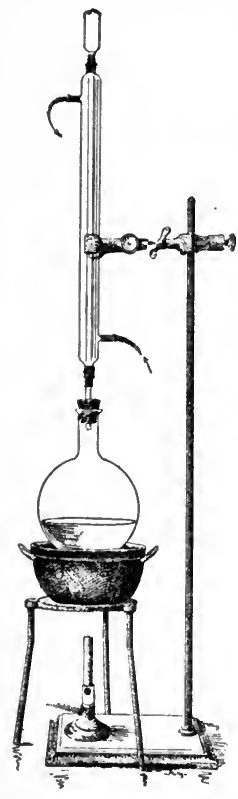

Frg. 10.-Liebig Condenser as Reflux Cooler. also of inorganic salts, frequently becomes granular upon standing. Tartrates of the alkaloids and other organic poisons are dissolved. The larger the quantity of absolute ethyl alcohol used, the more complete the precipitation of those substances which interfere more or less with the detection of organic poisons. Again evaporate the filtered alcoholic solution upon the water-bath, and dissolve the residue in about $50 \mathrm{cc}$. of water. If the solution is not perfectly clear, filter through a moistened paper.

The result of this procedure is a solution containing alkaloidal tartrates and other organic substances belonging to this group. This solution should have an acid reaction and be practically free from protein substances, fat, resinous bodies and coloring matter. If the solution fulfils these requirements, it is ready to be examined for organic poisons according to the "Stas-Otto" method. The utmost care must be taken in preparing this solution, because definite conclusions cannot be drawn from the uncertain tests given by impure material.

For the purpose of isolating alkaloids from viscera free from ptomaines and other impurities, Magnin and Zappi ${ }^{1}$ suggest the following procedure. Finely comminute the material and macerate in water acidulated with a few drops of dilute sul-

${ }^{1}$ Chemical Abstracts 9, 2748 (1915). 
phuric acid ( $\mathrm{I}: 4)$. Warm at $40^{\circ}$ for $\mathrm{I}-2$ hours to facilitate extraction and set aside for $18-20$ hours. Filter, add an equal volume of $90-92$ per cent. ethyl alcohol to the filtrate, then Io cc. of 30 per cent. aluminium sulphate solution and finally ro cc. of 15 per cent. potassium hydroxide solution. After 2-3 hours filter and concentrate the filtrate in vacuo to a sirupy consistency at a temperature not exceeding $45-50^{\circ}$. Treat the residue with 20 times its volume of $90-92$ per cent. ethyl alcohol, set aside for I8-20 hours, filter and again concentrate in vacuo as above. Dissolve the residue in water and use the solution for the extraction of alkaloids. Ptomaines and other impurities are almost completely eliminated by this procedure.

When the material is a powder mixed with cane- or milksugar, it is usually possible, after the aqueous solution has been acidified with tartaric acid, to extract dilectly with ether and continue according to the Stas-Otto method.

Frequently in suspected poisoning an examination of beer, wine, black coffee, infusion of tea, food, etc., is necessary. In such cases the process outlined above may of ten be considerably shortened. Acidify the material with aqueous tartaric acid solution, if necessary, and evaporate in a glass dish upon the water-bath. Treat the residue thoroughly with absolute ethyl alcohol and filter. Evaporate the filtrate upon the waterbath and dissolve the residue in tepid water. Filter this solution, if necessary, and then examine according to the Stas-Otto process.

\section{STAS-OTTO PROCESS}

\section{A. Examination of Ether Extract of Tartaric Acid Solution}

Thoroughly extract the acid aqueous solution (see process of preparation described above) two or three times with ether, using each time about the same quantity of solvent. Employ a separating funnel for this purpose (Fig. Ir). Pour the combined ether extracts into a dry flask loosely stoppered. If the solution stands for $\mathrm{r}$ or 2 hours at rest, a few drops of water usually settle out. Decant the ether solution and pour through 
a dry filter. Slowly evaporate this solution in a small glass dish upon a water-bath previously heated slightly above $35^{\circ}$. Do not have gas burning during this operation! Examine the residue as described below. An excellent method of evaporating ether consists in setting a small glass dish $(8$ to $10 \mathrm{~cm}$. in

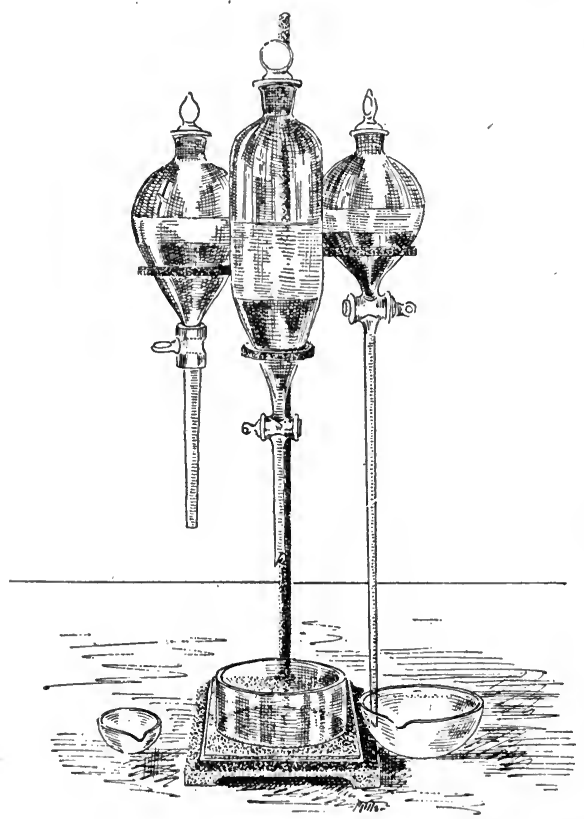

FIG. II.-Separating Funnels and Glass Crystallizing Dishes.

diameter) upon a hot water-bath and dropping the filtered ether extract into it as fast as the solvent evaporates. Thus a large quantity of extract may be evaporated in a small dish. The advantage of this method is the ease with which the residue can be removed for the various tests. The residue is usually quite small and it is not advisable to have it distributed over too large a surface. 
Examine the residue from the ether extract for the following substances:

\section{Picrotoxin}

Colchicin

Picric Acid
Caffeine

Acetanilide

Phenacetine
Antipyrine

Salicylic Acid

Veronal

Evaporation of the ether extract, even in the absence of members of the group, usually leaves a more or less viscous residue, containing tartaric and lactic acids as well as fatty, resinous and colored substances. This is especially so in analyses of cadaveric material. Moreover ether extracts certain metallic salts from aqueous solutions, for example, mercuric cyanide $^{1}$ and chloride.

First, note the general appearance and taste of the residue. Then examine it with a microscope. Very definite conclusions as to the presence or absence of certain substances can frequently be drawn. A very bitter residue should be examined carefully for picrotoxin and colchicin. If there is a pronounced yellow color, the examination should include picric acid also. Veronal is colorless and has a very bitter taste. A tasteless, or only faintly bitter, residue probably does not contain these substances and should be examined for acetanilide, antipyrine, caffeine, phenacetine and salicylic acid.

The residue from evaporation of the ether extract may contain the following substances:

Picrotoxin.-Usually a thick syrup which gradually solidifies and becomes crystalline. Tastes intensely bitter.

Colchicin.-Yellowish, amorphous residue which does not become crystalline. Tastes intensely bitter. Dissolves in water with a yellowish color, which increases in intensity on addition of a few drops of dilute hydrochloric acid.

${ }^{1}$ Ether to some extent will extract mercuric cyanide from a tartaric acid solution which is not too dilute. For instance, it will remove appreciable quantities from roo cc. of 0.1 per cent. mercuric cyanide solution, but the extraction will not be complete. The solution after five extractions will still give a distinct test for mercury. Ether will not remove even a trace of mercuric cyanide from o.or per cent. solution. To test for cyanide, add ammonium sulphide solution to the ether residue. This will precipitate mercuric sulphide and the filtrate will contain ammonium sulphocyanate (see hydrocyanic acid, page 22). 
Picric Acid.-Usually appears as a syrup which gradually solidifies and becomes crystalline. Tastes very bitter. Residue intensely yellow, giving yellow aqueous solutions not intensified by hydrochloric or sulphuric acid.

Acetanilide.-Leaflets or flattened needles. Has a faint, burning taste but is not bitter.

Phenacetine.-Inodorous and tasteless leaflets and small needles.

Antipyrine.-Residue a syrup which is rarely crystalline. Tastes mildly bitter. Very easily soluble in water.

Caffeine.-Residue composed of shining needles frequently in radiating clusters. Tastes mildly bitter.

Salicylic Acid.-Crystallizes frequently in long needles. Tastes harsh and at the same time sweet and acid.

Veronal.-Crystalline needles having an agreeable, bitter taste.

\section{PICROTOXIN}

Picrotoxin, $\mathrm{C}_{30} \mathrm{H}_{34} \mathrm{O}_{13}$, the poisonous principle of Cocculus indicus, the fruit of Menispermum Cocculus, crystallizes from hot water in long colorless needles melting at $199-200^{\circ}$. It dissolves with difficulty in cold water but more readily in hot water or ethyl alcohol. It is slightly soluble in ether but freely soluble in chloroform, amyl alcohol and glacial acetic acid. Its alcoholic solution is neutral and lævo-rotatory. Picrotoxin has a very bitter taste. It is not as readily soluble in acids as in pure water, but is soluble in caustic alkalies and aqueous ammonia, forming unstable, salt-like compounds which do not crystallize. Picrotoxin behaves toward strong bases as if it were a weak acid. Heated to boiling with twenty times its volume of benzene, it is decomposed into picrotoxinin and picrotin. The former passes into solution but picrotin is almost completely insoluble:

$$
\underset{\text { Picrotoxin }}{\mathrm{C}_{30} \mathrm{H}_{34} \mathrm{O}_{13}}=\underset{\text { Picrotoxinin }}{\mathrm{C}_{15} \mathrm{H}_{16} \mathrm{O}_{6}}+\underset{\text { Picrotin }}{\mathrm{C}_{15} \mathrm{H}_{18} \mathrm{O}_{7}}
$$

Chloroform brings about this cleavage even more easily. On the other hand, if picrotoxinin and picrotin in molecular proportions are dissolved in hot water, picrotoxin crystallizes out as the solution cools. Treated with bromine direct or dissolved in water or ether, picrotoxin is first split into picrotoxinin and picrotin. The former is immediately converted into monobromo-picrotoxinin, $\mathrm{C}_{15} \mathrm{H}_{15} \mathrm{BrO}_{6}$, but picrotin remains almost unchanged. Monobromo-picrotoxinin is soluble with difficulty in water but is reduced by zinc dust and acetic acid to picrotoxinin. Picrotin is almost non-toxic, whereas picrotoxinin has a very poisonous action. Picrotoxin is a powerful convulsive poison, standing in its action between cicutoxin and strychnine. 
R. Meyer and P. Bruger ${ }^{1}$ regard picrotoxin as a complex of the two compounds, picrotin and picrotoxinin, crystallizing together in definite but not molecular proportion, and not as a molecularly constituted chemical compound.

\section{Detection of Picrotoxin}

I. Fehling's Test.-Dissolve picrotoxin in a small test-tube, using ro-20 drops of very dilute sodium hydroxide solution. Add a few drops of Fehling's solution ${ }^{2}$ and warm but do not shake. A red or yellowish red precipitate forms and settles to the bottom. If the ether residue, not too little of which should be taken, fails to give a clear solution in very dilute sodium hydroxide solution, filter through moistened paper and examine the filtrate with Fehling's solution.

2. Ammoniacal Silver Test.-Warm picrotoxin with aqueous silver nitrate solution containing a slight excess of ammonium hydroxide solution. The reducing action' of picrotoxin will produce a black precipitate of metallic silver, or a dark brown color when only traces are present.

3. Oxidation Test.-Picrotoxin, treated with a little concentrated sulphuric acid in a porcelain dish, first becomes orange-red and then dissolves when stirred forming a reddish yellow solution. A drop of potassium dichromate solution will produce a red-brown color around the margin of the drop. If the two liquids are thoroughly mixed, there is an immediate dirty brown color which passes into green on long'standing.

A green color alone is without significance, since many organic substances capable of reducing chromic acid to chromic oxide produce the same result.

4. H. Melzer's Test. ${ }^{3}$ - Put some picrotoxin upon a watchglass and add $\mathrm{I}$ or 2 drops of a mixture of benzaldehyde and absolute ethyl alcohol. Careful addition of a drop of concentrated sulphuric acid will produce a distinct red color. If the watch-glass is tilted, red streaks will run from the substance through the liquid.

Use a freshly prepared, 20 per cent. solution of benzaldehyde in absolute ethyl alcohol. Benzaldehyde alone gives a yellowish brown color with concentrated

${ }^{1}$ Berichte der Deutschen chemischen Gesellschaft 3I, $295^{8}$ (I898).

${ }^{2}$ Fehling's solution heated by itself should not give a precipitate of cuprous. oxide.

${ }^{3}$ Zeitschrift für analytische Chemie $37,35^{\mathrm{I}}$ and 747 (1898). 
sulphuric acid. Ethyl alcohol is added as a diluent to diminish this color as much as possible. Under these conditions the solution has a light yellow color, and the dark red tint caused by picrotoxin is very clearly defined. This red color is unstable and, beginning at the margin, gradually fades into a pale pink or violet. H. Kreis ${ }^{1}$ has found that cholesterine and phytosterine ${ }^{2}$ give similar colors with Melzer's reagent.

5. Langley's Test.-Mix picrotoxin with about 3 times the quantity of potassium nitrate, and moisten the mixture with the smallest possible quantity of concentrated sulphuric acid. Then add strong sodium hydroxide solution in excess and an intense red color will appear.

\section{Detection of Picrotoxin in Beer}

First, neutralize the beer with magnesium oxide. Then evaporate $500 \mathrm{cc}$. or more to a syrup upon the water-bath. Digest this residue with 4 or 5 times its volume of ethyl alcohol and evaporate the alcoholic extract. Dissolve the residue in hot water and filter the solution through a moistened paper. Acidify the filtrate with dilute sulphuric acid and extract repeatedly with ether, or better with chloroform. Evaporate these extracts and test the residue for picrotoxin.

Should the residue from the ether or chloroform be too impure, dissolve it again in hot water, filter, evaporate and extract with ether or chloroform. To purify picrotoxin further, precipitate colored substances from its aqueous solution with lead acetate, filter and remove lead from the filtrate by hydrogen sulphide. The filtrate from lead sulphide upon evaporation, or extraction with ether or chloroform, will give nearly pure picrotoxin. The very bitter taste of picrotoxin as well as its strong tendency to crystallize are additional characteristics of this substance.

\section{COLCHICIN}

Colchicin, $\mathrm{C}_{22} \mathrm{H}_{25} \mathrm{NO}_{6}$, an alkaloid occurring in all parts of the meadow saffron, Colchicum autumnale, is a yellowish, amorphous powder which is poisonous and very bitter to the taste. It is freely soluble in water, ethyl alcohol, and chloroform, less so in ether and benzene, and almost insoluble in petroleum ether. Solutions of colchicin have a more or less yellowish color which becomes more pronounced upon addition of acids or alkalies. These solutions have very faint basic properties. Consequently ether or chloroform, but not benzene nor petroleum ether, will extract colchicin from an acid, aqueous solution. Upon evaporation of the solvent, colchicin will appear as a yellowish, sticky residue resembling a resin or varnish. Heated with water containing sulphuric acid, colchicin splits into colchicein and methyl alcohol. Boiling the alkaloid $1.5^{-2}$ hours with 60 parts of I per cent. hydrochloric acid will produce the same result:

1 Chemiker-Zeitung 33, 2 I (r899).

$$
\underset{\text { Colchicin }}{\mathrm{C}_{22} \mathrm{H}_{25} \mathrm{NO}_{6}}+\mathrm{H}_{2} \mathrm{O}=\underset{\text { Colchicein }}{\mathrm{C}_{21} \mathrm{H}_{23} \mathrm{NO}_{6}}+\underset{\text { Methyl Alcohol }}{\mathrm{CH}_{3} . \mathrm{OH}}
$$

${ }^{2}$ A substance very similar to cholesterine, and named paracholesterine or phytosterine, is found in the seeds of certain plants. (Perkin and Kipping, Organic Chemistry, page 608.) 
On the other hand, colchicin is formed when colchicein is heated to $100^{\circ}$ with sodium methylate $\left(\mathrm{CH}_{3} . \mathrm{ONa}\right)$ and methyl iodide $\left(\mathrm{CH}_{3} \mathrm{I}\right)$. Since colchicein on treatment with hydriodic acid yields three molecules of methyl iodide, colchicein as well as colchicin contains three methoxyl groups. Heated with strong hydrochloric acid, colchicein loses acetic acid and passes into trimethyl-colchicinic acid. Consequently colchicein and colchicin contain an acetyl group $\left(\mathrm{CH}_{3} \mathrm{CO}-\right)$. The formula of colchicin, that is to say, of methyl-colchicein, may be written as follows:<smiles>COC(=O)C[14C](OC)(OC)OC</smiles>

\section{Detection of Colchicin}

Aqueous colchicin solutions, especially in presence of dilute mineral acids, have a yellow color. Unless the ether residue has this characteristic, colchicin is absent.

I. Tannic Acid Test.-This reagent will precipitate colchicin from aqueous solution, if not too dilute, as white flocks. This test, however, is not characteristic of colchicin.

2. Nitric Acid Test.-Nitric acid (Sp. gr. I.4 $=66$ per cent.) dissolves colchicin with a dirty violet color which soon changes, when stirred, to brownish red and finally to yellow. Addition of dilute sodium or potassium hydroxide solution, until the reaction is alkaline, produces an orange-yellow or orange-red color.

3. Sulphuric Acid Test.-Concentrated sulphuric acid dissolves colchicin with an intense yellow color. A drop of nitric acid added to such a solution produces a green, blue, violet and finally a pale yellow tone. Excess of potassium hydroxide solution will now bring out an orange-red color. Erdmann's reagent (see page 320) dissolves colchicin with a blue to violet color.

4. Hydrochloric Acid Test.-Concentrated hydrochloric acid dissolves colchicin with an intense yellow color. Add two drops of ferric chloride solution and heat the mixture $2-3$ minutes in a test-tube. The color deepens and the solution on cooling, especially if diluted with the same volume of water, becomes green or olive-green. Finally shake the solution with a few drops of chloroform. This solvent becomes yellowish brown, or garnet-red, and the aqueous solution retains its green color. Zeisel's reaction. 


\section{Purification of the Residue Containing Colchicin}

To isolate as pure colchicin as possible from the yellow residue, extract with warm water. Filter the solution and, when cold, extract it first with petroleum ether. This will remove fatty, resinous and colored impurities but not colchicin. Then extract with chloroform. Or precipitate colchicin from aqueous solution, which must not be too dilute, with tannic acid. Collect this precipitate upon a filter and wash with cold water. Mix the moist precipitate with freshly precipitated, washed lead hydroxide. Dry the mixture, grind to a powder and extract with chloroform. Evaporation of the solvent will leave nearly pure colchicin.

\section{PICRIC ACID}

Picric acid, or 2,4,6-trinitrophenol, crystallizes from water in light yellow leaflets and from ether in lemon-yellow, rhombic prisms. It melts at $122.5^{\circ}$. Though soluble in cold water with difficulty, picric acid dissolves freely in hot water, as well as in ethyl alcohol, ether and benzene. Aqueous solutions have an<smiles>O=[N+]([O-])C1=CC([N+](=O)[O-])CC([N+](=O)[O-])C1O</smiles>
acid reaction, a very bitter taste and dye animal fibers fast yellow. Material containing picric acid has a yellow or yellowish green color.

Physiological Action and Elimination.-Picric acid is quite an active poison. Taken internally it produces a striking yellow pigmentation first of the conjunctiva and then of the entire skin, usually designated as "picric acid icterus." Picric acid and its salts like most nitro-compounds decompose the red blood-corpuscles forming methæmoglobin. Consequently it is a blood-poison. At the same time it irritates the central nervous system and causes convulsions. Finally it exercises its power of precipitating proteins in acid solution. This is especially noticeable in those organs of the body, for example, the stomach and

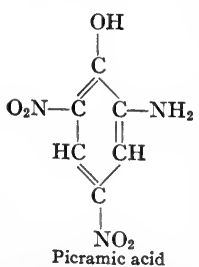
kidneys, which, owing to necrotic tissue changes, have an acid or only a faintly alkaline reaction. The organism reduces picric to picramic acid which does not so readily precipitate protein. By thus changing picric acid the organism rids itself of the poison. In picric acid poisoning the urine has a marked red color owing to formation of picramic acid. Some picric acid passes into the urine unchanged. Elimination is slow. In one case (see $R$. Kobert, "Intoxikationen"), after administration of a single be be recognized for 6 days. The urine was ruby-red, clear, acid and free from albumin and bile-constituents. Picric acid was also easily detected in the fæces. 


\section{Detection of Picric Acid}

Material containing picric acid has a more or less yellow or yellowish green color. Aqueous, alcoholic and ethereal solutions show the same color. Finely divided animal material should be extracted several hours under a return-condenser with ethyl alcohol containing hydrochloric acid to decompose compounds of picric acid with albumins and thus bring the acid into solution. Filter and evaporate such an alcoholic extract upon the water-bath. Treat the residue, which is yellow, yellowish green, or frequently yellowish red or reddish brown, with warm water and filter the extract. The filtrate itself may be tested directly for picric acid, or it may first be extracted as usual with considerable ether. The following tests may then be applied to the residue left on evaporating the ether extract:

I. Isopurpuric Acid Test.-Gently heat $\left(50-60^{\circ}\right)$ an aqueous solution of picric acid with a few drops of saturated, aqueous potassium cyanide solution $(\mathrm{r}: 2)$. The solution will become red owing to formation of potassium isopurpurate. One milligram of picric acid, dissolved in $5 \mathrm{cc}$. of water, will give a distinct test.

Isopurpuric acid does not exist in the free state but is present in this test as the potassium salt. Nietzki and $\mathrm{Petri}^{1}$ regard isopurpuric acid $\left(\mathrm{C}_{8} \mathrm{H}_{3} \mathrm{O}_{6} \mathrm{~N}_{5}\right)$ as a dicyano-picramic acid $=5$-oxy-6-amino-2,4-dinitro-isophthalic nitrile; whereas Borsche ${ }^{2}$ considers it a dicyano-dinitro-oxy- $\beta$-phenyl hydroxylamine:
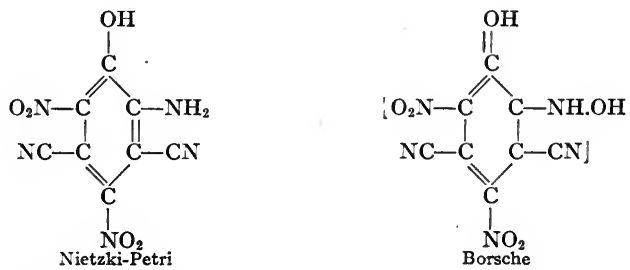

1 Berichte der Deutschen chemischen Gesellschaft 33, I 788 (I900).

2 Ibid., 33, 2718 and 2995 (1900). 
2. Picramic Acid Test.- $(\alpha)$ Heat picric acid solution with a few drops of sodium hydroxide solution and glucose. Picramic acid, formed by reduction of picric acid, colors the solution deep red. Avoid excess of sodium hydroxide solution, otherwise there will be a red color due solely to the action of the alkali upon glucose.

$(\beta)$ The test may also be made by warming picric acid solution with a few drops of sodium hydroxide and ammonium sulphide solutions. This will reduce picric acid and produce a red color.

In both reactions $(\alpha$ and $\beta$ ) picric acid is reduced to picramic acid, 2-amino-4,6-dinitro-phenol:
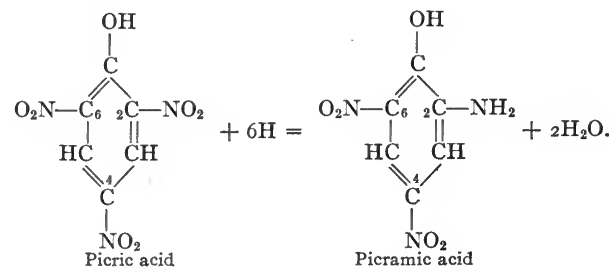

The presence of fat and other impurities materially influence this test.

3. Dyeing Test.-Dissolve the substance containing picric acid in hot water and put white threads of wool, silk and cotton in the solution. In a few hours ( 12 to 24 ) remove the threads and thoroughly rinse in pure water. If picric acid is present. the wool and silk will be dyed yellow but not the cotton. In other words, picric acid is not fast upon vegetable fibers like cotton. Picric acid, diluted I : I00,000, will still produce a yellow color upon wool.

4. Ammoniacal Copper Test.-Add a few drops of ammoniacal copper sulphate solution (copper sulphate solution and an excess of ammonia) to an aqueous picric acid solution. A yellowish green precipitate, consisting of hexagonal needles with a polarizing action upon light, will appear. Picric acid, diluted I : $80, \infty 00$, will give this test. 


\section{ACETANILIDE}

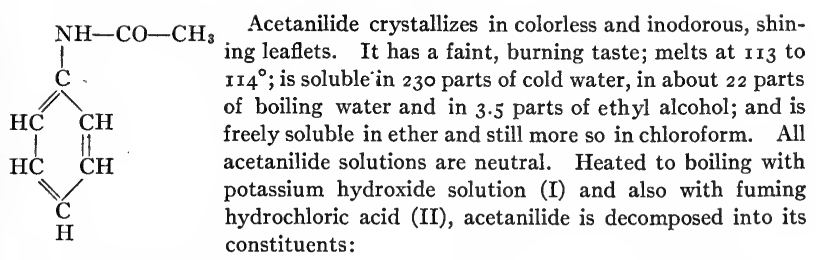

I. $\mathrm{C}_{6} \mathrm{H}_{5} \cdot \mathrm{NH} . \mathrm{CO} \cdot \mathrm{CH}_{3}+\mathrm{KOH}=\mathrm{C}_{6} \mathrm{H}_{5} \cdot \mathrm{NH}_{2}+\mathrm{CH}_{3}$ CO.OK.

II. $\mathrm{C}_{6} \mathrm{H}_{5} \cdot \mathrm{NH} \cdot \mathrm{CO} \cdot \mathrm{CH}_{3}+\mathrm{HCl}+\mathrm{H}_{2} \mathrm{O}=\mathrm{C}_{6} \mathrm{H}_{5} \cdot \mathrm{NH}_{2} \cdot \mathrm{HCl}+\mathrm{CH}_{3} \cdot \mathrm{COOH}$.

Physiological Action.-Being an aniline derivative, acetanilide has the poisonous properties of that amine though in less degree. R. Kobert " Intoxikationen") refers to several instances of acetanilide poisoning which did not terminate fatally. In one case a student took a teaspoonful of the drug. There was stupor, uneasiness, marked cyanosis and lowering of the pulse. A purgative and restorative (stimulant) were used but there was considerable exhaustion for several days. The picture was nearly the same in the case of a man who took 2 grams of antifebrine daily for 2 days in succession.

Preparation.-Boil aniline and glacial acetic acid together for several hours under a return-condenser:

$$
\mathrm{C}_{6} \mathrm{H}_{5}-\mathrm{NH}_{2}+\mathrm{CH}_{3}-\mathrm{COOH}=\mathrm{C}_{6} \mathrm{H}_{5}-\mathrm{NH}-\mathrm{CO}-\mathrm{CH}_{3}+\mathrm{H}_{2} \mathrm{O} \text {. }
$$

\section{Detection of Acetanilide}

Ether or chloroform will extract acetanilide completely from an acid aqueous solution.

I. Indophenol Test.-Boil acetanilide with about $4 \mathrm{cc}$. of fuming hydrochloric acid and evaporate to a few drops (about Io). Cool and add $4 \mathrm{cc}$. of saturated, aqueous carbolic acid solution. A few drops of calcium hypochlorite solution will produce a violet-red color. In time the color will become deeper, especially if the mixture is shaken. Then carefully add ammonium hydroxide solution as a surface-layer which will take on a permanent indigo-blue color.

The indigo-blue color is characteristic of acetanilide only when preceded by the red-violet color, since a mixture of aqueous phenol and hypochlorite solution gives a blue color with ammonia (see carbolic acid).

Phenacetine also gives the indophenol test.

2. Phenylisocyanide Test.-Boil acetanilide with $5^{-6}$ cc. of alcoholic potassium hydroxide solution. Cool, add 2 or 3 
drops of choloroform and again heat. The offensive odor of phenylisocyanide will be developed.

Potassium hydroxide decomposes acetanilide into aniline and potassium acetate (see Reaction I above). The former with chloroform gives phenylisocyanide.

3. Calcium Hypochlorite Test.-Boil acetanilide a few minutes with alcoholic potassium hydroxide solution as in test 2 . Dilute with water and extract aniline with ether. This solvent upon evaporation will deposit aniline as an oily liquid. Dissolve the latter in water and test with calcium hypochlorite.

\section{Examination of Acetanilide Urine ${ }^{1}$}

Scarcely more than traces of unaltered acetanilide appear in urine even after large doses. The most essential change occurring in the body is oxidation of the benzene ring which produces aceto-para-aminophenol. This like most phenols forms a conjugate sulphuric acid and appears in the urine as a salt of aceto-paraaminophenyl sulphuric acid:
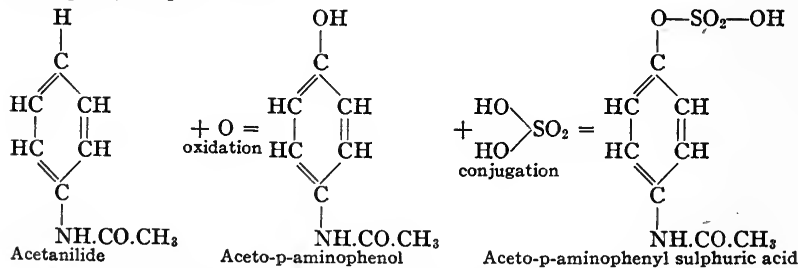

To some extent also, a conjugate glycuronic acid of aceto-para-aminophenol is formed. These compounds, heated with concentrated hydrochloric acid, give para-aminophenol which can be detected by the indophenol test previously described.<smiles>CC(=O)Nc1ccc(OS(=O)(=O)O)cc1</smiles>

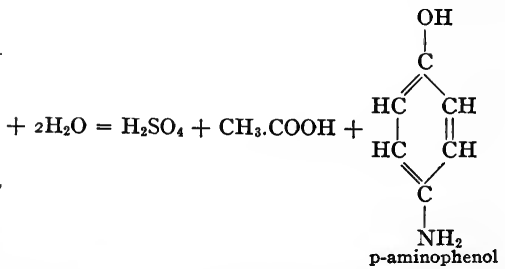

Such urine, boiled a few minutes with concentrated hydrochloric acid, will usually give the indophenol test. But the test will be more certain, if para-

${ }_{1}^{1}$ To study the behavior of acetanilide in the body, take at night 0.3 gram of this substance at a dose twice in the course of 3 hours and examine the urine passed in the next 12 hours. 
aminophenol is first isolated. Boil a larger quantity of urine ( 300 to $500 \mathrm{cc}$.) a few minutes with about ro cc. of concentrated hydrochloric acid. Then add an excess of sodium carbonate and repeatedly extract the cool urine with large quantities of ether. Distil or evaporate the ether. Para-aminophenol usually appears as a reddish or brownish oil. An aqueous solution of this substance will give the indophenol test.

\section{PHENACETINE}

Phenacetine, or p-aceto-phenetidine, crystallizes in shining leaflets, which are without color, odor or taste, and melts at $\mathrm{r}_{34}$ to $\mathrm{r}_{35^{\circ}}$. Phenacetine is soluble in about r400 parts of cold water, 70 parts of boiling water,
chloroform. Its solutions are neutral. Concentrated sul-
phuric acid dissolves it without color. Phenacetine is

Preparation.-The gradual addition of crystallized phenol to cold dilute nitric acid (sp. gr. $r . r r=17.5$ per cent.) results in the formation of a mixture of 0 - and p-nitro-phenol. Since the ortho-compound is volatile with steam, complete separation of the two products is possible by steam distillation. The residual p-nitro-phenol is converted into its sodium salt which is heated in sealed tube with ethyl bromide and thus changed to p-nitro-phenetol. The latter is reduced by means of nascent hydrogen from tin and hydrochloric acid to p-amino-phenetol, or p-phenetidine, which is then boiled with glacial acetic acid and converted into aceto-p-phenetidine, or phenacetine:
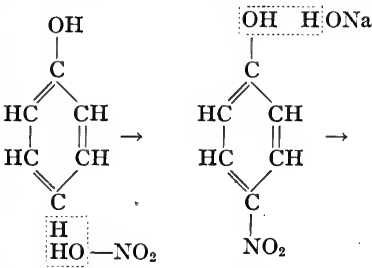

Phenol

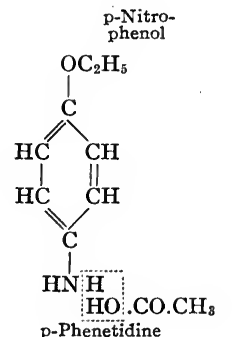

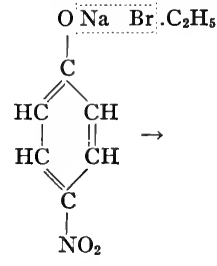

$\mathrm{Na}$ salt of pnitro-phenol

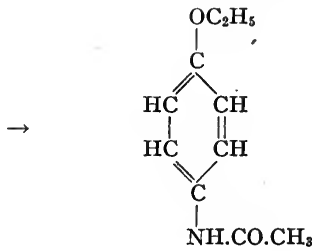

Phenacetine 


\section{Detection of Phenacetine}

The extraction of phenacetine by ether or chloroform from an aqueous tartaric acid solution is complete.

r. Oxidation Test.-Boil phenacetine for several minutes with 3 cc. of concentrated hydrochloric acid. Dilute with Io cc. of water and filter when cold. A few drops of chromic acid solution added to the filtrate will gradually produce a ruby-red color. Strong chlorine water may be substituted for chromic acid.

2. Indophenol Test.-Boil phenacetine I or 2 minutes with about 2 cc. of concentrated hydrochloric acid. Dilute with water and add a few cc. of aqueous carbolic acid solution. Filter the solution when cold. If a few drops of freshly prepared calcium hypochlorite solution are added, the filtrate will have a fine carmine-red color. Addition of ammonium hydroxide solution in excess will change this color to violet-blue. Freshly prepared chlorine water, or 3 per cent. chromic acid solution, may be substituted for hypochlorite solution as an oxidizing agent.

3. Autenrieth-Hinsberg Test. ${ }^{1-(a)}$ With Dilute Nitric Acid. Heat phenacetine to boiling with a few cc. of dilute nitric acid (Io to 12 per cent.). It is soluble and gives an intense yellow to orange-red color. As the solution cools, if sufficiently concentrated, nitro-phenacetine ${ }^{2}$ will crystallize in long, yellow needles which melt at $103^{\circ}$. This test is delicate, and characteristic of phenacetine, especially when nitro-phenacetine can be obtained in crystals and its melting-point determined. It serves to distinguish phenacetine from acetanilide and antipyrine, both of which give colorless solutions when warmed with dilute nitric acid.

(b) With Concentrated Nitric Acid.-A few drops of concentrated nitric acid poured upon phenacetine produce a yellow

1 Archiv der Pharmacie 229, 456 (189r).

2 The structural formula of mono-nitro-phenacetine is as follows:

$$
\mathrm{C}_{6} \mathrm{H}_{3} \begin{array}{ll}
\mathrm{OC}_{2} \mathrm{H}_{5} & \text { I } \\
\mathrm{NO}_{2} & 3, \\
\mathrm{NH}\left(\mathrm{C}_{2} \mathrm{H}_{3} \mathrm{O}\right) & 4
\end{array}
$$


to orange-red color. Part of the phenacetine is dissolved with the same color and heat completes the solution. Nitro-phenacetine crystallizes as the solution cools.

\section{SALICYLIC ACID}

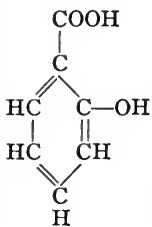

Salicylic acid, or ortho-oxy-benzoic acid, crystallizes in long, white needles soluble in about 500 parts of cold and in 15 parts of boiling water; and freely soluble in ethyl alcohol, ether, chloroform and carbon disulphide. It has a peculiar taste which is sweetish, acidulous and rather acrid. It melts at $157^{\circ}$. Heated carefully, salicylic acid will sublime in fine needles without decomposition. A little of the acid may show this behavior even upon the water-bath. If heated quickly, salicylic acid is decomposed in part into phenol and carbon dioxide.

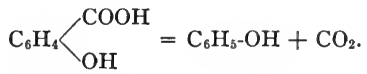

Concentrated sulphuric acid dissolves pure salicylic acid without color and without decomposition. The lead and silver salts of this acid are soluble in water with difficulty. Consequently lead acetate will precipitate lead salicylate, $\left(\mathrm{C}_{6} \mathrm{H}_{4}<\begin{array}{c}\mathrm{COO} \\ \mathrm{OH}\end{array}\right)_{2} \mathrm{~Pb}$, from neutral solutions. This salt is white, crystalline and soluble in hot water. It crystallizes unchanged as the hot solution cools. Silver nitrate precipitates white silver salicylate.

R. Schmitt's Method of Preparation.-Dry sodium phenolate is kept cool and saturated in an autoclave under pressure with carbon dioxide $(\alpha)$. The sodium phenyl-carbonate undergoes molecular rearrangement, when heated at $120-130^{\circ}$, and becomes isomeric sodium salicylate $(\beta)$ :

$(\alpha)$

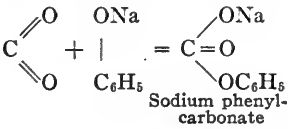

Molecular

$(\beta)$. rearrangement gives:

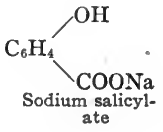

\section{Detection of Salicylic Acid}

I. Ferric Chloride Test.-Addition of ferric chloride solution to a solution of salicylic acid or salicylates produces a blueviolet color. If the solution is very dilute, the color is more of a red-violet. Hydrochloric acid changes the violet color to yellow. An excess of the reagent affects the delicacy of the test.

This test fails in presence of mineral acids, caustic alkalies and alkaline carbonates. 
2. Millon's Test.-If an aqueous salicylic acid solution is warmed with Millon's reagent, a deep red color will appear.

3. Bromine Water Test.-This reagent in excess produces a yellowish white, crystalline precipitate even with very dilute salicylic acid solutions. The compound thus formed is tribromo-phenyl hypobromite (see page 28 ).

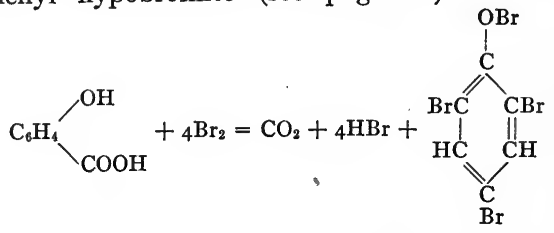

4. Melting-Point Test.-If the quantity of salicylic acid is not too small, dissolve the ether residue in very little hot water, shake the hot solution with a little animal charcoal and filter. Cool the filtrate, dry the crystals and determine the meltingpoint $\left(\mathrm{I} 57^{\circ}\right)$.

\section{Separation of Salicylic Acid from Simple Phenols}

When phenols like carbolic acid or the cresols are present, the above tests from I to 3 prove nothing as far as salicylic acid is concerned. If these compounds are present, add sufficient sodium carbonate solution to render the ether residue alkaline and extract the solution, with ether. This solvent will take up the phenols and salicylic acid will remain in the water as the sodium salt. Withdraw the aqueous solution from the separating funnel, acidify with dilute hydrochloric or sulphuric acid and extract salicylic acid with ether.

Directions are given elsewhere (see page 250) for the detection of salicylic acid in beer, milk, urine, fruit juices, meat and meat preparations, as well as in maltol.

\section{Quantitative Estimation of Salicylic Acid as Tribromo-phenyl Hypo- bromite}

Place the aqueous solution of salicylic acid in a glass-stoppered flask, add an excess of saturated bromine water and shake. The acid is completely precipitated as tribromo-phenyl hypo- 
bromite. At the end the solution should be reddish brown. It should stand I 2-24 hours, and be shaken frequently. Collect the precipitate of tribromo-phenyl hypobromite in a weighed Gooch crucible and dry to constant weight in a vacuum desiccator over sulphuric acid. The quantity of salicylic acid may be calculated from the weight of precipitate as follows:

$$
\underset{409.86}{\mathrm{C}_{6} \mathrm{H}_{2} \mathrm{Br}_{4} \mathrm{O}: \mathrm{C}_{7} \mathrm{H}_{6} \mathrm{O}_{3}}=\text { Wt. of precipitate: } \mathrm{x}
$$

\section{Detection of Salicylic Acid in Urine ${ }^{1}$}

Salicylic acid forms a conjugate with glycocoll, supplied by the organism, and is changed in the human body, in part at least, into salicyluric acid,

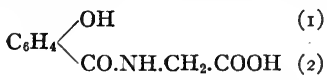

This is eliminated in urine with unaltered salicylic acid. Such urine gives a violet color with ferric chloride solution. Both salicylic and salicyluric acids give this test. To decompose salicyluric acid into its constituents, heat the acid half an hour with fuming hydrochloric acid under a return-condenser.

To isolate unchanged salicylic acid, acidify 500 to $1000 \mathrm{cc}$. of urine with hydrochloric acid and repeatedly extract with ether. Remove the ether from the aqueous solution in a separating funnel and shake vigorously with excess of sodium carbonate solution. Salicylic acid passes into the aqueous solution. Withdraw the aqueous solution, which is alkaline, acidify with dilute hydrochloric acid and extract with ether which upon evaporation usually deposits the acid in a crystalline condition. Purify the residue by recrystallization from water, using animal charcoal to remove color. Salicylic acid is rapidly taken up by all mucous surfaces and quickly absorbed. Elimination by way of the urine usually begins within the first half hour and is complete in 3 days.

\section{VERONAL}

Veronal, or barbital (name adopted by the Federal Trade Commission for the substance formerly sold under the protected name "veronal"), is C-diethylbarbituric acid, C-diethyl-malonyl-urea, $\mathrm{C}_{8} \mathrm{H}_{12} \mathrm{O}_{3} \mathrm{~N}_{2}$. It crystallizes from hot water in large, colorless, spear-shaped crystals melting at $19 \mathrm{I}^{\circ}$ (corrected) and $\begin{aligned} & \mathrm{C}_{2} \mathrm{H}_{5} \\ & \mathrm{C}_{2} \mathrm{H}_{6}\end{aligned}>\mathrm{C}\left\langle\begin{array}{l}\mathrm{CO}-\mathrm{NH} \\ \mathrm{CO}-\mathrm{NH}\end{array}>\begin{array}{l}\text { is soluble in } \mathrm{I}_{15} \text { parts at } 100^{\circ} \text {. Veronal is also freely soluble in } \\ \text { hot ethyl alcohol and in acetone. It dissolves with dif- }\end{array}\right.$ ficulty in cold ether. An aqueous veronal solution has a bitter taste and shows a very faint acid reaction with sensitive blue litmus paper. Veronal readily dis-

${ }^{1}$ To study the behavior of salicylic acid in this connection, take I to $\mathrm{r} .5 \mathrm{grams}$ of sodium salicylate at night in the course of several hours and examine, as described, the urine passed in the next 12 hours. 
solves in caustic alkalies, ammonia and in calcium or barium hydroxide solution. From such solutions, provided they are not too dilute, acids reprecipitate veronal in a crystalline condition. Of the veronal salts the sodium salt, $\mathrm{C}_{8} \mathrm{H}_{11} \mathrm{O}_{5}$ $\mathrm{N}_{2} \mathrm{Na}$, crystallizes best. It may be prepared by dissolving veronal in the calculated quantity of caustic soda solution free from carbonate, and then evaporating this solution with exclusion of carbon dioxide, or adding ethyl alcohol until turbidity appears. In both cases the sodium salt of veronal separates as splendid shining crystals.

\section{Preparation by E. Fischer and A. Dilthey ${ }^{1}$}

(a) From diethyl-ethylmalonate by condensation with urea in presence of sodium ethylate:

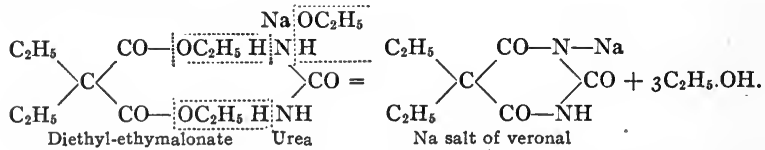

Dissolve metallic sodium ( 32 parts) in absolute ethyl alcohol (600 parts) and when cold add diethyl-ethylmalonate ( 100 parts). Dissolve in this mixture with heat finely powdered urea (40 parts). Heat 4-5 hours in an autoclave at 105$108^{\circ}$. The sodium salt of veronal is precipitated even from the hot solution as a colorless, crystalline mass. Cool, filter with suction and wash with ethyl alcohol. Dissolve the crystals in water and acidify with concentrated hydrochloric acid. Veronal thus precipitated is pure when recrystallized from water.

(b) From diethyl-malonyl chloride by condensation with urea:

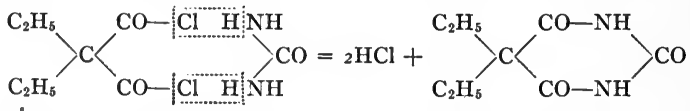

Heat diethyl-malonyl chloride (3 parts) on the water-bath for 20 hours with finely powdered, dry urea ( 2 parts). Considerable hydrochloric acid is given off toward the end and a solid mass finally remains which yields pure veronal upon crystallization from hot water. The yield is 70 per cent. of the theoretical amount.

Physiological Action.- - Veronal does not cause decomposition of the blood and in the usual medicinal doses (0.5-I gram) does not appear to act strongly upon the heart. Cumulative action has been noted only in rare instances. In large doses, however, veronal may cause serious intoxication with fatal termination. Death resulted in the case of a man in Holzminden who had taken ro grams of veronal by accident. There are also on record two other suicidal cases, one from II grams and the other from 15 grams of this hypnotic. There was loss of consciousness and contraction of the pupils in the second case. Atropine caused dilatation of the pupils but otherwise was of no avail. Death ensued in 20 hours.

${ }^{1}$ Annalen der Chemie und Pharmazie, 335, 334 (I904). 


\section{Detection of Veronal}

In examining cadaveric material (liver, spleen and kidneys) from a man, who had taken veronal, thinking it was a remedy for tape-worm (kamala), G. and H. Frerichs ${ }^{1}$ isolated small quantities of this drug. Following the Stas-Otto process, they extracted the aqueous tartaric acid solution with ether and evaporated the ether extract. They recrystallized the residue from a hittle hot water, using animal charcoal to remove color, and identified the crystals obtained as veronal by the following tests:

I. The aqueous solution of the crystals had a faintly acid reaction.

2. The crystals were soluble in sodium hydroxide solution and ammonia and were reprecipitated when the solutions were acidified with dilute hydrochloric acid.

3. The melting-point of the crystals was $187-188^{\circ}$. On mixing the substance with pure veronal, they obtained the same melting-point. ${ }^{2}$

4. Presence of nitrogen was shown by fusing the crystals in a dry test-tube with metallic sodium, cooling the melt, dissolving in water and testing for sodium cyanide by the Prussian blue reaction (see page 22 ).

5. The crystals were heated in a dry test-tube and sublimed. They were then compared with clystals known to be pure veronal and found identical.

\section{Detection of Veronal in Urine}

E. Fischer and J. v. Mering, ${ }^{3}$ and also B. Molle and H. Kleist, ${ }^{4}$ have found that most of the veronal leaves the human body unchanged and is present in the urine to the extent of 70-90 per cent. Consequently in veronal poisoning the urine should be examined first. Concentrate a considerable quantity on

1 Archiv der Pharmazie 244, 80́-90 (I906).

2 A mixture of two organic substances, having the same melting-point but not being identical, will show a melting-point lower than that of either substance taken by itself. But obviously a mixture of identical substances will show no depression of melting-point whatever.

${ }^{3}$ Die Therapie der Gegenwart 45, I904.

4 Archiv der Pharmazie 242, 401 (I904). 
the water-bath ${ }^{1}$ to one-fifth its volume and extract several times with ether, using a large volume at each extraction because veronal is not very soluble in this solvent. The residue left after distilling the ether is usually quite dark in color. Dissolve in as little hot water as possible, boil the solution I 5 minutes with animal charcoal and filter. Cool the nearly colorless filtrate with ice and veronal will crystallize in colorless needles melting at $\mathrm{I}_{9 \mathrm{I}^{\circ}}$ (corrected).

E. Fischer and v. Mering recovered from 5 days urine, after administration of 4 grams of veronal during 2 days, 2.49 grams $=62$ per cent. of the quantity used. The method therefore is not absolutely quantitative, nor is the elimination of veronal complete after 5 days. The crystals obtained should be proved conclusively to be veronal by the tests described.

Molle and Kleist first add lead acetate solution to the urine as long as it causes a precipitate, filter, remove lead from the filtrate by hydrogen sulphide and filter from lead sulphide. Hydrogen sulphide is expelled with heat and the urine, after dilution with twice its volume of water, is boiled with animal charcoal. The filtrate, after concentration on the water-bath to a small volume, is cooled, saturated with sodium chloride and extracted 3 times with ether. The filtered ether solution on distillation leaves nearly pure veronal.

\section{ANTIPYRINE}

Antipyrine, or $\mathrm{x}$-phenyl-2, 3-dimethyl-isopyrazolone, $\mathrm{C}_{11} \mathrm{H}_{12} \mathrm{ON}_{2}$, forms monoclinic, tabular crystals having a faintly bitter taste and melting at $\mathrm{Ir}_{3}{ }^{\circ}$.

(3)<smiles>CCCCCCCC</smiles>

(2) One part of antipyrine is soluble in less than $I$ part of cold water, in about $x$ part of eth yl alcohol,

(r) I part of chloroform and in about 50 parts of ether. An aqueous antipyrine solution has a neutral reaction, although this compound is a base and forms crystallizable salts with acids.

Preparation.-Antipyrine is formed directly by heating $\beta$-phenyl-methylhydrazine and aceto-acetic ester:<smiles>CCNNC(C=O)C(=O)OCC</smiles>

(3)<smiles>CCCCOC(CC)CO</smiles>

\section{Detection of Antipyrine}

Ether extracts only small quantities of antipyrine from a solution containing much tartaric acid. Ether, or better chloroform, extracts by far the greater part of the antipyrine

${ }^{1}$ Fischer and v. Mering evaporated the urine under diminished pressure. 
when the solution has been made alkaline. Antipyrine differs from most alkaloids in being more soluble in water. To detect antipyrine, dissolve in a little water the residue left on evaporating. the ether solution, filter and apply the following tests:

I. Ferric Chloride Test.-Add I or 2 drops of ferric chloride solution to an aqueous antipyrine solution. It will produce a deep red color which can be seen even in a dilution of $\mathrm{I}: 100,000$.

2. Tannic Acid Test.-Tannic acid solution produces an abundant, white precipitate, when added to an aqueous antipyrine solution.

3. Fuming Nitric Acid Test.-Dissolve antipyrine in a few drops of water and add $\mathrm{I}$ or 2 drops of fuming nitric acid. The solution will be green. If this solution is heated to boiling, another drop of nitric acid will produce a red color. Two cc. of antipyrine solution ( $\mathrm{I}: 200$ ) will give this test distinctly.

4. Nitroso-antipyrine Test.-Add a few drops of potassium or sodium nitrite solution to an aqueous antipyrine solution and then dilute sulphuric acid. A green or blue color will appear. A few drops of acetic acid may be substituted for sulphuric acid but the solution must be heated. If the antipyrine solution has been concentrated, green crystals of nitrosoantipyrine $\left(\mathrm{C}_{11} \mathrm{H}_{11}(\mathrm{NO}) \mathrm{ON}_{2}\right)$ will separate after some time.

\section{Detection of Antipyrine in Urine}

Part of the antipyrine passes unchanged into the urine but some is also present as oxy-antipyrine-glycuronic acid, a direct test for which may be made by means of ferric chloride solution. When the urine is highly colored, render a considerable quantity alkaline with sodium hydroxide solution or ammonia and extract with chloroform. Dissolve the residue left by chloroform in a little water and test for antipyrine with ferric chloride solution and with fuming nitric acid.

\section{CAFFEINE}

Caffeine (theine) or 1,3,7-trimethyl-2,6-dioxy-purine $\left(\mathrm{C}_{8} \mathrm{H}_{10} \mathrm{O}_{2} \mathrm{~N}_{4} \cdot \mathrm{H}_{2} \mathrm{O}\right)$, crystallizes in white, shining needles. It is soluble in 80 parts of water, giving

(I) $\mathrm{CH}_{3}-\mathrm{N}-\mathrm{CO}(6)$

(2)

(3) in ether. In crystallizing from hot water caffeine combines with I molecule of water, a part of which it loses upon exposure to air and all when dried at $100^{\circ}$.

a colorless solution with a neutral reaction and a faint, bitter taste. Caffeine is quite easily soluble in hot water $(x: 2)$. It requires for solution nearly $5^{\circ}$ parts of ethyl alcohol, only 9 parts of chloroform and is only slightly soluble 
Caffeine is only very slightly soluble in absolute ethyl alcohol, benzene and petroleum ether. It melts at $230^{\circ}$, but somewhat above $100^{\circ}$ begins to volatilize in small quantity and at $180^{\circ}$ to sublime without leaving a residue. Concentrated sulphuric and nitric acids dissolve it without color. Caffeine is a very weak base and its salts are decomposed by water. Therefore, caffeine can be extracted at least partially by ether, or better by chloroform, from an aqueous tartaric acid solution. The relation existing between caffeine and uric acid is quite apparent when the products, formed by oxidizing these two substances with potassium chlorate and hydrochloric acid, are compared. Oxidation of uric acid yields alloxan and urea; caffeine gives dimethyl-alloxan and monomethylurea.

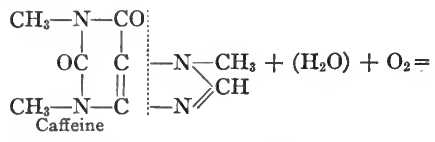

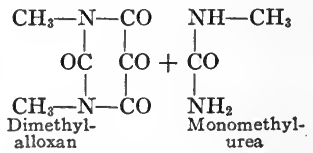

Fate of Caffeine in Human Metabolism.-Only a very small part of the caffeine taken into the body passes through unchanged and appears in the urine. About Io per cent. appears in the urine as decomposition products. The remainder may be changed into normal end-products of human metabolism. Most of the nitrogen of caffeine is eliminated as urea. A very important fact is the cleavage of methyl groups with formation of the first decomposition products of caffeine, namely, dimethyl- and monomethyl-xanthines. Of the monomethyl-xanthines, 7-monomethyl-xanthine is formed especially. Of the dimethyl-xanthines, paraxanthine $=1,7$-dimethyl-xanthine is found. Both of these compounds appear in urine after administration of caffeine. Paraxanthine is isomeric with theophylline, or 1,3-dimethyl-xanthine, and with theobromine, or 3,7-dimethyl-xanthine.

The structural formulæ of these cleavage-products of caffeine in animal metabolism are as follows:<smiles>CNC(=O)Nc1nccn1C</smiles>

(I)

(3)<smiles>CN1CN(C)c2nc[nH]c2C1=O</smiles>

(I)

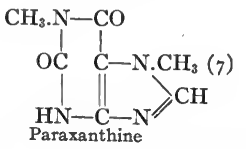

(3)<smiles>Cn1cnc2c1[nH]c(=O)n2C</smiles>

\section{Detection of Caffeine}

Ether will extract more caffeine from an aqueous alkaline solution than from an aqueous tartaric acid solution. Since 
caffeine dissolves with some difficulty in ether, but more easily in chloroform, the latter solvent is usually employed after the solution has been made alkaline with ammonia. After distillation of solvent, caffeine appears in concentric clusters of long shining needles. In an analysis by the Stas-Otto method caffeine will appear in all three extracts.

I. Oxidation Test.-Pour a few cc. of saturated chlorine water ${ }^{1}$ over caffeine and evaporate the solution to dryness upon the water-bath. A reddish brown residue will remain. If a few drops of ammonium hydroxide solution are added, a fine purple-red color will immediately appear. This test may be made by covering the dish containing the residue with a glass plate moistened with a drop of strong ammonia. Or two matched watch-glasses may be used, the material containing caffeine being evaporated to dryness with chlorine water upon one glass which is then placed for a short time upon the other glass containing a drop of strong ammonia.

This test, known as the murexide reaction, is also given by xanthine, theobromine, I- and 7 -monomethyl-zanthine and paraxanthine, especially if made as described by E. Fischer. ${ }^{2}$ Heat the material to boiling in a test-tube with strong chlorine water, or with hydrochloric acid and a little potassium chlorate, evaporate the liquid to dryness in a dish and moisten the residue with ammonia.

2. Tannic Acid Test.-This reagent, added to an aqueous caffeine solution, causes a heavy white precipitate which is soluble in an excess of the acid. This test is not characteristic of caffeine.

\section{B. Examination of Ether Extract of Alkaline Solution}

(Most of the alkaloids appear here)

Add enough sodium hydroxide solution to the acid solution separated from ether to make it strongly alkaline. The alkali

1 A convenient method of preparing a saturated, aqueous chlorine solution is to heat potassium chlorate with hydrochloric acid and pass the chlorine into a small quantity of water.

2 Berichte der Deutschen chemischen Gesellschaft 30, 2236 (1897). 
will liberate alkaloids from their salts and combine with morphine and apomorphine, if present. Thoroughly extract this alkaline solution with about the same quantity of ether. This solvent will dissolve all alkaloids except morphine, apomorphine and narceine. Separate the ether from the aqueous solution and again extract with a fresh quantity of ether. In certain cases 3 or 4 such extractions may be required. Pour the ether extracts into a dry flask, stopper loosely and set aside for I or 2 hours. A few drops of water always settle to the bottom of the flask. Carefully decant the ether and pour through a dry filter. Evaporate the filtrate with gentle heat in a glass dish (8 to $10 \mathrm{~cm}$. in diameter). Let the last part of the ether solution evaporate spontaneously. If small globules having a strong odor appear, the residue must be examined for coniine and nicotine. If there is no trace of these volatile alkaloids, gently heat the residue upon the water-bath to expel water left by evaporation of the ether. Remove the dish from the waterbath as soon as this has been accomplished. It is not advisable to heat the residue too long, as it tends to become viscous. This residue, obtained by extracting the alkaline solution with ether, may contain any alkaloid except morphine, apomorphine and narceine. It should be examined for

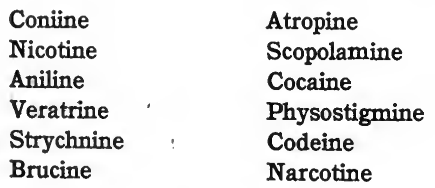

Hydrastine

Pilocarpine

Quinine

Caffeine

Antipyrine

Pyramidone.

First, note the general appearance of the residue and then examine with the microscope. Taste it cautiously. Certain alkaloids may be recognized beforehand by this test. Special tests should then be made at once. The various alkaloids appear in the residue as follows:

Strychnine.-Fine needles having an exceedingly bitter taste.

Brucine.-Usually a white, amorphous powder having a very bitter taste. 
Veratrine.-Usually an amorphous powder having a sharp, burning taste.

Atropine and Quinine.-A varnish which is resinous and sticky. Rarely crystalline or in the form of a powder.

Codeine.-A thick, viscous syrup which after a time becomes solid, especially if stirred with a glass rod, and frequently crystalline.

Caffeine.-Long, silky needles having a faintly bitter taste. These are frequently concentrically arranged.

Antipyrine.-A syrup which gradually becomes crystalline, especially if stirred. It has a mild, bitter taste and dissolves very easily in water. ${ }^{1}$

Pyramidone-- Usually as fine needles which have a faintly bitter taste. It is easily soluble in water.

Frequently ether leaves only a slight, tasteless residue. In that case alkaloids are absent. Such residues often consist of fat, resinous matter, or traces of nitrogenous substances (Peptones and their cleavage-products? Creatinine?). Parts of a cadaver, even when quite fresh, usually give small residues at this point. Alkaloids may be absent and every step in the process may have been performed with the greatest care. To be quite sure that alkaloids are absent, dissolve a portion of the residue in water containing a drop of dilute hydrochloric acid. Filter, if necessary, and distribute this solution upon several watch-glasses. Test with the following alkaloidal reagents:

Mercuric Chloride, Iodo-Potassium Iodide, Potassium Mercuric Iodide, Potassium Bismuthous Iodide,
Picric Acid, Tannic Acid, Phospho-Molybdic Acid, Phospho-Tungstic Acid.

Unless these reagents give distinct and characteristic precipitates, alkaloids are absent. It is advisable in every instance

1 Most of the alkaloids are only slightly soluble in cold water. Some cannot be detected satisfactorily by purely chemical means. Others have no characteristic tests. Such substances should not be selected for laboratory practice. They may cause beginners to think that the experienced toxicologist relies upon similar uncertain methods when he seeks to identify an alkaloid in an actual analysis. 
to make this preliminary test for alkaloids. Only a small portion of material is required and these general reagents show even traces of alkaloids.

To exclude mistakes and oversights in toxicological analysis, dissolve the ether residue, should it be very small, in a few cc. of very dilute hydrochloric acid (about I per cent. of $\mathrm{HCl}$ ). Evaporate this solution upon the water-bath and dissolve the residue in a little water. Inject this solution from a hypodermic syringe into the lymph-sac on the back of a small but lively frog. If the frog shows no sign of poisoning in the course of several hours, it is quite likely that the residue does not contain any very poisonous alkaloid.

In making special tests for alkaloids, distribute the residue upon several watch-glasses, using a platinum or nickel spatula or a small penknife. Or dissolve the residue in a little hot ethyl alcohol, filter the solution, distribute upon watch-glasses and evaporate at a gentle heat. R. Mauch ${ }^{1}$ dissolves the residue in 75 per cent. aqueous chloral hydrate solution and uses this solution in testing for alkaloids. (The details of this method will be found on page 25r.)

\section{Purification of the Alkaloidal Residue}

If alkaloids are contaminated with greasy, resinous or fatty substances, many of the tests will either fail entirely or give uncertain results. In this case the residue must be purified in one of two ways.

I. Thoroughly mix the residue with cold water containing hydrochloric acid. Filter to remove insoluble matter (fatty or resinous substances), add sodium hydroxide solution to the filtrate until alkaline and extract with ether. The alkaloids obtained by evaporating the solvent are usually quite pure.

2. Or dissolve the residue in hot amyl alcohol, extract this solution with a few cc. of very dilute sulphuric acid and withdraw the acid solution from the separating funnel. Amyl alcohol will retain greasy and colored impurities, and the alkaloids will be in the aqueous solution as sulphates. Add sodium

1 Richard Mauch (Mittheilungen aus dem Institut des Herrn Prof. Dr. E. Schaer in Strassburg), "Festgabe des Deutschen Apotheker-Vereins," Strassburg,
I897. 
hydroxide solution in excess and extract with ether. This method of purifying the alkaloidal residue is especially recommended, when there is considerable coloring matter.

W. H. Warren and R. S. Weiss ${ }^{1}$ have suggested picrolonic $\operatorname{acid}^{2}$ as a means of purifying alkaloids. An alkaloid like strychnine, whose picrolonate is very insoluble, may be precipitated from aqueous solution and thus separated from other substances which prevent purification. The precipitated picrolonate may be collected on a filter, washed with water and then warmed with dilute sulphuric acid which discharges the bright yellow color of the picrolonate causing the alkaloid to pass into solution and precipitating pale yellow picrolonic acid. By extracting with acetic ether, in which picrolonic acid is especially soluble, the aqueous solution of the alkaloid is left colorless. Neutralization with sodium hydroxide solution and extraction with ether will give a very pure alkaloid.

\section{CONIINE}

Coniine, $\alpha$-normal-propyl-piperidine, $\mathrm{C}_{8} \mathrm{H}_{17} \mathrm{~N}$, occurs in all parts of spotted hemlock (Conium maculatum) together with n-methyl-coniine, conhydrine,<smiles>CCCC1CCCCN1</smiles>
$\gamma$-coniceine and pseudo-conhydrine. It is a colorless, oily, very poisonous liquid which becomes yellowish or brown in contact with air and is partially resinified. It is slightly soluble in cold but even less soluble in hot water. Coniine is miscible with ethyl alcohol, ether, chloroform and benzene in all proportions. The unpleasant, narcotic odor of this alkaloid, sometimes said to resemble that of mouse urine, is more intense than the odor of nicotine. Coniine as it occurs in nature is dextro-rotatory, ${ }^{3}(\alpha) \mathrm{D}=+\mathrm{r} 8.3^{\circ}$, and rather a strong base. Heated with acetic anhydride, it forms acetyl-coniine:

$$
\mathrm{CH}_{3} \cdot \mathrm{CO} \text {. O.CO. } \mathrm{CH}_{3} \mathrm{CH}_{16} \mathrm{C} \mathrm{C}_{16} \mathrm{~N} \cdot \mathrm{CO} \cdot \mathrm{CH}_{3}+\mathrm{CH}_{3} \cdot \mathrm{COOH} \text {; }
$$

Shaken with benzoyl chloride and sodium hydroxide solution, it forms benzoylconiine:

$$
\underset{\mathrm{C}_{6} \mathrm{H}_{6} \cdot \mathrm{CO} C \mathrm{Cl}}{\mathrm{C}_{1} \mathrm{H}_{16} \mathrm{~N}}+\mathrm{NaOH}=\mathrm{C}_{8} \mathrm{H}_{16} \mathrm{~N} \cdot \mathrm{CO} \cdot \mathrm{C}_{6} \mathrm{H}_{5}+\mathrm{NaCl}+\mathrm{H}_{2} \mathrm{O} ;
$$

and with nitrous acid nitroso-coniine:

$$
\underset{\mathrm{NO}}{\mathrm{C}_{8} \mathrm{H}_{16} \mathrm{OH}}=\mathrm{C}_{8} \mathrm{H}_{16} \mathrm{~N} . \mathrm{NO}+\mathrm{H}_{2} \mathrm{O} \text {. }
$$

All these reactions show that coniine is a secondary base.

1 The Journal of Biological Chemistry, 3, 330 (1907).

${ }^{2}$ For the preparation of this reagent, see page 320 .

${ }^{3}$ The optical activity of this alkaloid is occasioned by the presence of the asymmetric carbon atom marked with an asterisk in the structural formula. 


\section{Detection of Co niine}

The alkaloidal reagents especially delicate with coniine are: iodo-potassium iodide $(\mathrm{x}: 8000)$, phospho-molybdic acid $(\mathrm{r}: 5000)$, potassium mercuric iodide $(\mathrm{r}: 8000)$ and potassium bismuthous iodide $(\mathrm{x}: 5000)$. Gold and platinum chlorides fail to precipitate coniine when the concentration is less than I: I00; whereas they will precipitate nicotine when the concentration of the solution is as low as I:I0,000 and $I: 5000$. When coniine is present, the residue left by the ether solution has the characteristic odor of this alkaloid. The two following tests should then be made:

I. Solubility Test.-Dissolve a drop of coniine in just enough cold water to give a clear solution. Gently heat the solution and it will become milky, because coniine is more easily soluble in cold than in hot water. A coniine solution which is milky when hot becomes clear on cooling. Aqueous coniine solutions have an alkaline reaction. Test the solution with red litmus paper.

2. Crystallization Test.-Put a little coniine upon a watchglass, or glass slide, and add I or 2 drops of hydrochloric acid. Evaporate to dryness and coniine hydrochloride $\left(\mathrm{C}_{8} \mathrm{H}_{17} \mathrm{~N} . \mathrm{HCl}\right)$ will remain. Immediately after evaporation examine this. residue with a microscope magnifying about 200 times. The colorless or faintly yellow crystals are needle-like, or columnar and frequently grouped in star-shaped clusters. They show the play of color characteristic of doubly refractive substances.

\section{NICOTINE}

Nicotine, $\mathrm{C}_{10} \mathrm{H}_{14} \mathrm{~N}_{2}$, is a colorless hygroscopic liquid which soon turns yellow and then brown upon exposure to air and in time becomes resinous. It is miscible<smiles>CN1CCCC1c1cccnc1</smiles>
with water in all proportions (distinction from coniine) and freely soluble in ethyl alcohol, ether, amyl alcohol, benzene and petroleum ether. Ether extracts nicotine from aqueous solution. It has a sharp, burning taste and strong odor of tobacco especially when warm. Chemically pure nicotine is said to be almost inodorous. The so-called tobacco odor is developed after the alkaloid has been for some time in contact with air. The free alkaloid is strongly lævo-rotatory, $[\alpha] \mathrm{D}=-\mathrm{r} 6 \mathrm{r} .55^{\circ}$, but its salts are dextrorotatory. 
Constitution.-Nicotine is a rather strong di-acid, ditertiary base and forms well-crystallized salts with one or two equivalents of acid. Like ditertiary bases it combines with two molecules of methyl iodide ${ }^{1}$ forming a di-iodo-methylate, $\mathrm{C}_{10} \mathrm{H}_{14} \mathrm{~N}_{2 \cdot 2} \mathrm{CH}_{3} \mathrm{I}$. Oxidized with chromic acid, nitric acid or potassium permanganate, nicotine is converted into nicotinic acid, or $\beta$-carboxy-pyridine. This shows that nicotine is a pyridine derivative having a side-chain in the $\beta$ position with respect to the pyridine nitrogen.<smiles>CN1CCCC1c1ccncc1</smiles>

gives on oxidation<smiles>O=C(O)c1ccccc1</smiles>

This formula for nicotine proposed by Pinner was confirmed several years later by Amé Pictet's synthesis of this alkaloid.

Physiological Action.-Nicotine is one of the most powerful poisons and scarcely inferior to hydrocyanic acid in toxicity and rapidity of action. It appears to be toxic to all classes of animals. It is absorbed from the tongue, the eye and the rectum even in a few seconds and from the stomach somewhat more slowly. Absorption of nicotine is also possible from the outer skin. Elimination takes place through the lungs and kidneys. In concentrated form nicotine is a local irritant, though, owing to the rapidity of its toxic action, it does not behave like a true corrosive nor does it cause inflammation of the mucous lining of the stomach even after a lethal dose. Nicotine, after causing stimulation for a brief period, then paralyzes the central nervous system and spinal cord, finally affecting various organs such as the heart, eyes and intestinal tract. Its poisonous influence probably extends to all parts of the brain, medulla oblongata and spinal cord. Huchard states that nicotine causes a general convulsion of the circulatory system which is apparent in chronic nicotine poisoning. In chronic tobacco poisoning the general condition of health is disturbed and quite frequently the eyes are affected. In acute nicotine poisoning death ensues from paralysis of the respiratory center. An action upon the heart is also always in evidence even in non-fatal cases.

${ }^{1}$ In methyl iodide-as well as in other alkyl haloids-we have an excellent means of recognizing the tertiary nature of a nitrogen base. Like trimethylamine, tertiary cyclic amines, as pyridine and quinoline, also give similar iodomethylates which are ammonium iodides with quinquivalent nitrogen:

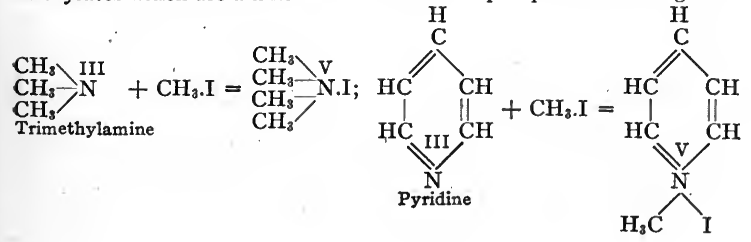




\section{Detection of Nicotine}

Ether or low-boiling petroleum ether will extract nicotine from an aqueous alkaline solution. Spontaneous evaporation of the solvent leaves the alkaloid as an oily liquid having the odor of tobacco and a strong alkaline reaction. General alkaloidal reagents will precipitate nicotine from quite dilute solutions, in which respect this alkaloid is very different from coniine. Phospho-molybdic acid and potassium bismuthous iodide precipitate nicotine even in a dilution of $1: 40,000$; potassium mercuric iodide in $1: 15,000$; gold chloride in $\mathrm{x}: \mathrm{x0}, 000$; and platinum chloride in $\mathbf{I}: 5000$.

I. Crystallization Test.-Evaporate nicotine on a watchglass with a few drops of concentrated hydrochloric acid. This will yield a yellow, varnish-like residue which microscopic examination will show to be entirely amorphous (distinction between nicotine and coniine). If kept for a long time in a desiccator over sulphuric acid, it will become indistinctly crystalline.

2. Roussin's Test.-Dissolve a trace of nicotine in ether, using a dry test-tube. Add to this solution about the same volume of ether containing iodine. Stopper and set the testtube aside. The mixture will become turbid and deposit a brownish red resin which will gradually become crystalline. After some time, ruby-red needles with a dark blue reflex will crystallize from the ether. These are "Roussin's crystals." If nicotine is old or resinous, it will not as a rule give these crystals.

3. Melzer's Test. ${ }^{1}$ - If a drop of nicotine is heated to boiling with $2-3$ cc. of epichlorohydrin, ${ }^{2}$ the mixture becomes distinctly red. This test applied to conüne causes no color.

${ }^{1}$ Zeitschrift des allgemeinen Oesterreichen Apotheker-Vereins 54, 65.

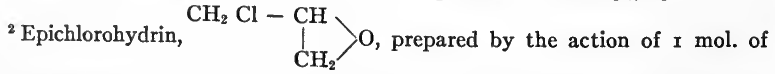
caustic alkali on $\alpha$-dichlorohydrin, $\mathrm{CH}_{2} \mathrm{Cl}-\mathrm{CH}(\mathrm{OH})-\mathrm{CH}_{2} \mathrm{Cl}$, or $\alpha$, $\beta$-dichlorohydrin, $\mathrm{CH}_{2}(\mathrm{OH})-\mathrm{CHCl}-\mathrm{CH}_{2} \mathrm{Cl}$, is a colorless liquid insoluble in water and freely soluble in alcohol and ether. It has an odor like chloroform and a burning, sweetish taste. 
4. Schindelmeiser's Test. ${ }^{1}-$ If nicotine that is not resinous is treated first with a drop of formaldehyde solution free from formic acid and then with a drop of concentrated sulphuric acid, the mixture takes on an intense rose-red color. If nicotine and formaldehyde are in contact for several hours, the solid residue obtained gives even a finer color reaction with a drop of nitric acid. Only a little formaldehyde should be used; otherwise the solution becomes green after a while and decomposition takes place.

Under the same conditions, trimethylamine, piperidine, pyridine, picoline, quinoline and aniline gave no color. Nor did extracts from putrefying horseflesh and the entrails of animals, poisoned by arsenic or mercury, give the test, at least not when these extracts were prepared according to the Stas-Otto method.

5. Physiological Test.-When very small quantities of nicotine are present, the physiological test should accompany the chemical tests. A very characteristic picture is given by frogs after administration of small doses of nicotine. First there is stimulation, then paralysis of the brain and respiratory muscles and apparent curare-action (tetanic convulsions). The toxic action of pure nicotine should be studied first. The experiment with a frog's heart, which shows temporary cessation of diastole, is also very characteristic.

\section{ANILINE}

Aniline, $\mathrm{C}_{6} \mathrm{H}_{5} \cdot \mathrm{NH}_{2}$, upon evaporation of the ether extract from the alkaline solution, will usually appear as reddish or brownish globules. Dissolve some of this residue in water and apply the aniline tests already described on page 45. A further test for aniline consists in mixing some of the residue with a few drops of concentrated sulphuric acid, and adding a few drops of potassium dichromate solution. If aniline is present, an evanescent blue color will appear.

\section{VERATRINE}

Pure officinal veratrine is an intimate mixture of two isomeric alkaloids having the composition $\mathrm{C}_{52} \mathrm{H}_{49} \mathrm{NO}_{9}$. These are cevadine, also called crystallized vera-

${ }^{1}$ Pharmazeutische Zentral-Halle: 40, 703 (I899). 
trine, which is nearly insoluble in water; and amorphous veratridine which is soluble in water. Even small quantities of the crystalline alkaloid will render veratridine insoluble in water. On the other hand, veratridine will prevent cevadine from crystallizing. Consequently the crystalline base cannot be isolated by recrystallizing officinal veratrine from ethyl alcohol or from any other solvent; nor can the water-soluble alkaloid be obtained by simple extraction with water.

Separation of Cevadine and Veratridine.-E. Schmidt uses the following method to isolate the crystalline and the water-soluble veratrine from officinal veratrine. Place the officinal preparation in a beaker and dissolve in strong ethyl alcohol. Heat this solution to $60-70^{\circ}$ and add enough warm water to produce a permanent turbidity. Cautiously add just enough ethyl alcohol to clear the solution and allow evaporation to take place slowly at $60-70^{\circ}$. A white, crystalline precipitate will presently appear. Filter with suction, wash the precipitate with a little dilute ethyl alcohol and recrystallize from hot ethyl alcohol. This is crystalline veratrine. Clear the filtrate from the crystalline precipitate by adding a little ethyl alcohol and evaporate at $60-70^{\circ}$. This will give a second crop of crystals. By repeating this process several times one may obtain in a crystalline condition about one-third of the veratrine taken. Finally evaporate the filtrate from the crystalline deposit at the given temperature until there is no longer any odor of ethyl alcohol. A considerable quantity of a resinous mass which is a mixture of both alkaloids will separate. The aqueous filtrate from this deposit will contain veratridine which may be obtained by rapidly evaporating the solution in vacuo over sulphuric acid.

Properties of Officinal Veratrine.-Veratrine appears as a white, amorphous powder which is crystalline under the microscope. It has a sharp, burning taste and the minutest quantity introduced into the nostrils excites protracted sneez-. ing. It is almost insoluble in boiling water and the aqueous extract always has a faintly alkaline reaction; fairly soluble in ether ( $r$ : ro), benzene, petroleum ether and amyl alcohol; and freely soluble in ethyl alcohol $(r: 4)$ and chloroform ( $r: 2)$. All these solutions have a strong alkaline reaction. Officinal veratrine melts at $\mathrm{r}_{50-\mathrm{r}} 55^{\circ}$ to a yellowish liquid which solidifies to a transparent, resinous mass. If the veratrine solution is faintly acid, ether will extract a very little of the alkaloid. Under the same conditions, chloroform and amyl alcohol will extract more. The alkaloid is usually deposited from ether as a white, amorphous powder. Phospho-molybdic acid, iodo-potassium iodide, tannic acid and potassium mercuric iodide give distinct precipitates with an aqueous veratrine solution containing hydrochloric acid and diluted r:5000. Chlorides of gold and platinum and picric acid fail to show the alkaloid in this dilution.

Constitution.-Heated with saturated barium hydroxide, or alcoholic potassium hydroxide solution, crystallized veratrine (cevadine) is hydrolyzed into angelic acid and cevine:

$$
\underset{\text { Cevadine }}{\mathrm{C}_{32} \mathrm{H}_{49} \mathrm{NOO}_{9}}+\mathrm{H}_{2} \mathrm{O}=\underset{\substack{\text { Angelic } \\ \text { acid }}}{\mathrm{C}_{5} \mathrm{H}_{8} \mathrm{O}_{2} 1}+\underset{\text { Cevine }}{\mathrm{C}_{27} \mathrm{H}_{43} \mathrm{NO}_{8}}
$$

${ }^{1}$ Angelic acid (I) and tiglic acid (II) are stereo-isomers:<smiles>CC(C)=C(C)CCCCCCCCCCCCCC(C)=C(C)C(=O)O</smiles> 
M. Freund ${ }^{1}$ has shown that cevadine takes up only one acetyl or benzoyl group, whereas cevine takes up two. The following formulæ show these relationships:

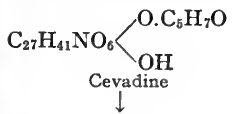<smiles>CCCOC(=O)OC(=O)OCC</smiles><smiles>CCC(C)OC(=O)O</smiles>

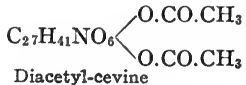

By means of hydrogen peroxide $M$. Freund has converted cevine into cevine oxide, $\mathrm{C}_{27} \mathrm{H}_{43} \mathrm{NO}_{9}$, which crystallizes well and contains one more atom of oxygen. $\mathrm{V}$

This compound must belong to the class of the amino-oxides, $R \equiv N=O$, for sulphurous acid easily converts it into cevine.

\section{Detection of Veratrine}

I. Concentrated Sulphuric Acid Test.-Pour a few drops of concentrated sulphuric acid upon a trace of veratrine. The alkaloid will have an intense yellow color and, if stirred, will give a solution of the same color. Gradually, this color will change to orange, then to blood-red and finally to cherry-red. Gentle heating will hasten this color change and veratrine, dissolved in concentrated sulphuric acid, will give a fine cherry-red solution almost immediately.

Fröhde's and Erdmann's reagents give color changes similar to those caused by sulphuric acid.

2. Concentrated Hydrochloric Acid Test.-If a trace of veratrine is dissolved in I or $2 \mathrm{cc}$. of cold concentrated hydrochloric acid, the solution will be colorless. When this solution is heated Io to $I_{5}$ minutes in a boiling water-bath, a cherry-red color will appear. This color will last for a day and even $\mathbf{0 . 2}$ $\mathrm{mg}$. of veratrine will produce it.

3. Concentrated Nitric Test.-Concentrated nitric acid dissolves veratrine with a yellow color.

4. Weppen's Test.-Thoroughly mix in a mortar I part of veratrine with about 5 parts of finely powdered cane-sugar. Add a few drops of concentrated sulphuric acid to some of

${ }^{1}$ Berichte der Deutschen chemischen Gesellschaft 37, I946 (1904). . 
the mixture upon a watch-glass. At first a yellow color will appear and later, beginning at the margin this will change to grass-green and finally to blue. Breathing upon the mixture will cause the color to change more quickly. Too great an excess of cane-sugar must be avoided.

E. Laves ${ }^{1}$ substitutes an aqueous furfural solution for canesugar in this test. Mix in a test-tube 3 or 4 drops of I per cent. aqueous furfural solution with I cc. of concentrated sulphuric acid. Add 3 to 5 drops of this solution to the substance to be tested so that it just touches the edge of the liquid. If veratrine is present, a dark streak will gradually run from the substance into the liquid. At the starting-point it will appear blue or blue-violet and farther away green. If substance and liquid are stirred with a glass rod, the liquid will become dark green. After some time, or more quickly when warmed, the color will become blue and finally violet.

5. Grandeau's Test.-Direct addition of $\mathbf{I}-2$ drops of bromine water to the yellow solution of veratrine in concentrated sulphuric acid produces an immediate purple color almost identical with that appearing when the solution of the alkaloid in concentrated sulphuric acid stands a long time or is gently warmed.

6. Vitali's Test.-Dissolve veratrine in a few drops of fuming nitric acid and evaporate the solution to dryness upon the water-bath in a porcelain dish. A yellowish residue will remain. If this is cooled and then moistened with an alcoholic potassium hydroxide solution, the color will change to orange-red or red-violet and stirring will produce a solution having the same color.

Atropine, hyoscyamine, scopolamine, as well as strychnine, respond to this test in a very similar manner.

\section{STRYCHNINE}

Strychnine, $\mathrm{C}_{21} \mathrm{H}_{22} \mathrm{~N}_{2} \mathrm{O}_{2}$, occurs with brucine chiefly in nux vomica and Ignatius beans, constituting the larger part of the mixed alkaloids. The former contains 2.93-3.I4 per cent. of these two alkaloids and the latter 3.1 1-3.22 per

${ }^{1}$ Pharmaceutische Zeitung, 37, 338. 
cent. The free base strychnine forms colorless, shining prisms belonging to the rhombic system which melt at $268^{\circ}$. The alkaloid dissolves in 6600 parts of cold and 2500 parts of hot water, giving alkaline solutions having a very bitter taste. It is nearly insoluble in absolute ethyl alcohol and in absolute ether. It dissolves in 160 parts of cold and 12 parts of boiling ethyl alcohol ( 90 per cent. by volume; ; it is also soluble in commercial ether and in benzene; but most readily in chloroform $\left(6\right.$ parts at $\left.15^{\circ}\right)$. Strychnine diluted with water $\dot{r}: 600,000$ can be recognized by its bitter taste.

Strychnine is a monacid base combining with one equivalent of acid and forming salts which are usually crystalline. These salts have a very bitter taste and are very poisonous. The best known strychnine salt, and one used medicinally, is the nitrate, $\mathrm{C}_{21} \mathrm{H}_{22} \mathrm{~N}_{2} \mathrm{O}_{2} \cdot \mathrm{HNO}_{3}$. The combination of one molecule of strychnine with one molecule of an alkyl haloid, for example, methyl iodide, to form strychnine iodo-methylate, $\mathrm{C}_{21} \mathrm{H}_{22} \mathrm{~N}_{2} \mathrm{O}_{2} . \mathrm{CH}_{3}$.I , shows that the alkaloid is a tertiary base. Sodium methylate $\left(\mathrm{CH}_{3} . \mathrm{ONa}\right)$ in alcoholic solution converts strychnine into strychnic acid which is probably an imino-carboxylic acid. Strychnic acid loses a molecule of water, when its solutions are boiled in presence of mineral acids, and is changed to strychnine. Because of this behavior Tafel regards strychnine as an inner anhydride of strychnic acid, one containing a group of the character of an acid imide:

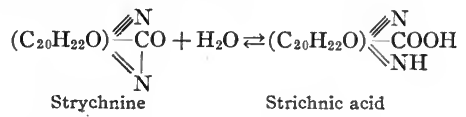

On the basis of Tafel's strychnine formula, strychnine iodo-methylate would be expressed as follows:

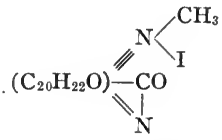

Physiological Action.- Strychnine increases reflex irritability of the brain and spinal cord. Even the slightest stimulus, especially if acoustic, optical, or tactile, may cause powerful reflexes after large doses of this alkaloid. Convulsions may follow each stimulus, if the dose is sufficient. Very large doses of strychnine cause curare-like paralysis of the peripheral ends of motor nerves in frogs and other warm-blooded animals. It may also affect the muscles of the heart. Strychnine diminishes the motile power of leucocytes and then arrests their motion. The poison also affects plant protoplasm, at least that of Mimosa pudica, in that the plant's motor organs lose their elasticity and flexibility.

Aside from the saliva, bile and milk, the urine is the main channel through which strychnine is eliminated from the organism. Human urine may contain even the unaltered alkaloid. Elimination begins during the first hour, is slight after 2 days but is not complete until much later. More unaltered strychnine is eliminated after large than after small doses. In the former case 70-75 per cent. of the alkaloid may remain undecomposed. The liver, kidneys, brain and spinal cord may store up unchanged strychnine. (See R. Kobert, "Intoxikationen.") 


\section{Detection of Strychnine}

Sodium and potassium hydroxide, ammonia and alkaline carbonates precipitate the free base strychnine from aqueous solutions of its salts as a white crystalline solid:

$$
\mathrm{C}_{21} \mathrm{H}_{22} \mathrm{~N}_{2} \mathrm{O}_{2} \cdot \mathrm{HNO}_{3}+\mathrm{NaOH}=\mathrm{C}_{21} \mathrm{H}_{22} \mathrm{~N}_{2} \mathrm{O}_{2}+\mathrm{H}_{2} \mathrm{O}+\mathrm{NaNO}_{3} \text {. }
$$

Ether will extract strychnine from an alkaline solution and deposit the alkaloid on evaporation in fine crystalline needles. Chloroform takes up the alkaloid more freely, since strychnine is considerably more soluble in this solvent than in ether. Even very dilute solutions of strychnine salts give precipitates with most of the alkaloidal reagents. Tannic acid, potassium mercuric iodide and phospho-tungstic acid produce white precipitates; gold chloride and phospho-molybdic acid yellow precipitates; and iodo-potassium iodide brown precipitates. To obtain tests with these reagents, the residue from ether should first be dissolved in very dilute hydrochloric acid.

Concentrated sulphuric acid, Erdmann's and Fröhde's reagents dissolve perfectly pure, brucine-free strychnine without color.

Strychnine is soluble in concentrated nitric acid with a yellowish color. Potassium dichromate, added to solutions of strychnine salts, precipitates strychnine dichromate, $\left(\mathrm{C}_{21} \mathrm{H}_{22} \mathrm{~N}_{2} \mathrm{O}_{2}\right)_{2}$.$\mathrm{H}_{2} \mathrm{Cr}_{2} \mathrm{O}_{7}$, in the form of fine yellow crystalline needles which upon recrystallization from hot water appear as shining orangeyellow needles.

Potassium ferricyanide, added to solutions of strychnine salts, precipitates golden-yellow, crystalline strychnine ferricyanide $\left(\mathrm{C}_{21} \mathrm{H}_{22} \mathrm{~N}_{2} \mathrm{O}_{2}\right)_{2} \cdot \mathrm{H}_{3} \mathrm{Fe}(\mathrm{CN})_{6}+6 \mathrm{H}_{2} \mathrm{O}$.

\section{Special Reactions}

I. Sulphuric Acid-Dichromate Test.-Dissolve a very little strychnine in 2 or 3 drops of concentrated sulphuric acid upon a watch-glass. The solution should be colorless. Add a fragment of potassium dichromate and hold it firmly in one place upon the glass. Intense blue or blue-violet streaks will come from the potassium dichromate, if the watch-glass is tilted up and down. If the entire mixture is stirred, the sul- 
phuric acid will have a beautiful evanescent blue or blue-violet color.

This test may also be made by scattering upon the surface of the solution of strychnine in concentrated sulphuric acid a few particles of coarsely powdered potassium dichromate and mixing well with a glass rod. In this way the blue to blue-violet color reaction is given very beautifully. The blue color is not permanent. It soon changes to red and finally to dirty green. ${ }^{1}$

Strychnine chromate and ferricyanide give this test especially well. To prepare the former salt, pour a very dilute potassium dichromate solution over strychnine upon a watch-glass. When the two substances have been in contact for some time, pour the remaining liquid from the strychnine chromate. Wash the precipitate once with a little water. Put some strychnine chromate upon the end of a glass rod and draw it through a few drops of concentrated sulphuric acid upon a watch-glass. This will produce violet and blue streaks in the acid.

Mandelin's reagent, ${ }^{2}$ that is to say, vanadic-sulphuric acid, gives this strychnine test very well. The blue or violet color given by this reagent with strychnine is more permanent than that produced by potassium dichromate. The color finally changes to orange-red.

Other oxidizing agents may be substituted for potassium dichromate, as potassium permanganate, lead peroxide, manganese dioxide, potassium ferricyanide (see above), cerium oxide and vanadic acid (Mandelin's reagent). But neither potassium nitrate nor nitric acid can be used, as these reagents even prevent this test. Consequently strychnine nitrate does not give the test.

2. Physiological Test.-Dissolve the ether residue in a little very dilute hydrochloric acid. Evaporate the filtered solution to dryness upon the water-bath. Dissolve the residue in pure water (about I cc.) and inject this solution into the lymph-sac on the back of a lively frog. Keep the experimental frog in a large, loosely covered beaker. Toxic symptoms will appear in 5 to 30 minutes, depending upon the quantity of strychnine.

1 According to Tafel (Annalen der Chemie und Pharmazie, 268, 233 (1892), this color reaction is characteristic of many anilides and is due to the presence of the group $-\mathrm{CO}-\mathrm{N}=$.

${ }^{2}$ See page $32 \mathrm{I}$ for the preparation of this reagent. 
Strychnine does not increase reflex irritability for all kinds of stimuli but only for tactile, optical and especially for acoustic stimuli. When the dose of strychnine is sufficiently large, each kind of stimulus mentioned will produce convulsions like those caused by tetanus. For example, if the beaker containing the "strychnine frog" is gently tapped, this slight acoustic stimulus is sufficient to produce convulsions.

\section{Detection of Strychnine in Presence of Brucine}

More than traces of brucine prevent detection of strychnine with concentrated sulphuric acid and potassium dichromate. Under certain conditions Mandelin's reagent will show strychnine more or less distinctly in presence of brucine. Dissolve the ether residue in concentrated sulphuric acid, if brucine is present, and add a trace of concentrated nitric acid. A red color indicates brucine. When the color has changed to yellow, add a fragment of potassium dichromate and stir. The mixture will become blue or reddish violet, if strychnine is present.

Solid potassium permanganate, stirred with concentrated sulphuric acid alone, will give a dark green solution which is yellowish green in a thin layer and assumes with time a red to violet color on the margin.

The same procedure used to estimate these two alkaloids quantitatively will permit detection of strychnine even in presence of considerable brucine. Dissolve the residue containing brucine in about 2 cc. of dilute sulphuric acid, add 2 drops of concentrated nitric acid and let the mixture stand 4 hours. Render alkaline with excess of sodium hydroxide solution and extract thoroughly with ether. The residue from ether will be brucine-free or nearly so. Strychnine thus treated will give very satisfactory tests with concentrated sulphuric acid and potassium dichromate and with Mandelin's reagent.

\section{BRUCINE}

Brucine, $\mathrm{C}_{23} \mathrm{H}_{26} \mathrm{~N}_{2} \mathrm{O}_{4}$, crystallizes in transparent, monoclinic prisms or shining leaflets. Crystals from water contain either 4 or 2 molecules and from ethyl alcohol 2 molecules of water of hydration. It melts in its water of hydration only a few degrees above $100^{\circ}$, whereas the anhydrous base melts at $178^{\circ}$. Brucine is more readily soluble than strychnine both in water and in ethyl alcohol and therefore remains dissolved in the mother-liquors from the preparation of strychnine. 
It is also more soluble than strychnine in ether. Brucine solutions have a very bitter taste and a strong alkaline reaction. Benzene, but especially chloroform and amyl alcohol, are excellent solvents for brucine. Brucine differs from strychnine in being deposited usually amorphous by evaporation of its ether solution.

Brucine is a monacid, tertiary base and as such forms addition-products with one molecule of an alkyl iodide. For example, with methyl iodide it gives brucine iodo-methylate, $\mathrm{C}_{23} \mathrm{H}_{26} \mathrm{NO}_{4} \cdot \mathrm{N} \mathrm{CH}_{3} \mathrm{I}$. With one equivalant of acid brucine gives in part crystalline salts. Brucine nitrate, $\mathrm{C}_{23} \mathrm{H}_{26} \mathrm{~N}_{2} \mathrm{O}_{4} \cdot \mathrm{HNO}_{3}$. ${ }_{2} \mathrm{H}_{2} \mathrm{O}$, crystallizes in rectangular prisms.

.Brucine may be shown by Zeisel's method' to contain two methoxyl groups $\left(-\mathrm{OCH}_{3}\right)$.

Heated in sealed tube to $80^{\circ}$ with sodium and ethyl alcohol, until solution is complete, brucine is converted into brucic acid, $\mathrm{C}_{23} \mathrm{H}_{28} \mathrm{~N}_{2} \mathrm{O}_{5} \cdot \mathrm{H}_{2} \mathrm{O}$, which contains an imino-group $(=\mathrm{NH})$ in its molecule since it forms a nitrosamine. Tafel and Moufang ${ }^{2}$ express the relationship between brucine and brucic acid as follows:

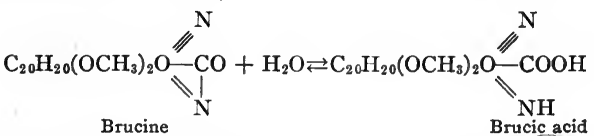

Heated with water, brucic acid is converted into brucine. Consequently. brucic acid is related to brucine as strychnic acid is to strychnine.

\section{Detection of Brucine}

Ether, benzene or chloroform will extract brucine from an alkaline solution. Evaporation of the ether extract usually leaves the alkaloid in an amorphous condition. The sensitiveness of the alkaloidal reagents toward brucine is as follows:

Iodo-potassium iodide $(\mathrm{r}: 50,000), \quad$ Potassium bismuthous iodide ( $\mathrm{I}: 5000$ ), Potassium mercuric iodide ( $\mathrm{I}: 30,000)$, Phospho-molybdic acid ( $\mathrm{I}: 2000)$, Gold chloride ( $1: 20,000)$,

Tannic acid $(\mathrm{I}: 2000)$,

Platinic chloride ( $\mathrm{r}: \mathrm{ro00})$.

${ }^{1}$ Many alkaloids contain one or more, sometimes three or more, methoxyl groups $\left(-\mathrm{OCH}_{3}\right)$ united with a benzene nucleus. The determination of the number of such groups in the molecule is of the greatest importance as a step in establishing the constitution of an alkaloid, because in this way some of the car bon, oxygen and hydrogen atoms are at once disposed of. The method employed for this purpose depends on the fact that all substances containing methoxy] groups are decomposed by hydriodic acid, yielding methyl iodide and a hydroxyl compound. By estimating the methyl iodide obtained from a given quantity of a compound of known molecular weight, it is easy therefore to determine the number of methoxyl groups in the molecule. This method was first applied by Zeisel and is of general application. (Perkin and Kipping, "Organic Chemistry," page 498.)

2 Annalen der Chemie und Pharmazie 304, 28 (I899). 
I. Nitric-Acid-Stannous Chloride Test.-Concentrated nitric acid dissolves brucine and its salts with a blood-red color. This color, however, is slightly stable and soon changes to yellowish red and finally, especially with heat, to yellow. Add a few drops of freshly prepared, dilute stannous chloride solution to this yellowish red or yellow solution. An intense violet color will appear. Heat usually changes this violet color again to yellowish red, but addition of a few more drops of stannous chloride solution will cause the violet color to reappear. The smaller the quantity of nitric acid, the more likelihood that this test will give a good result. Colorless ammonium sulphide solution may be substituted for stannous chloride.

2. R. Mauch's Modification of Nitric Acid-Stannous Chloride Test.-An excellent result can be obtained with this test in the following manner. Dissolve brucine in 60 per cent. aqueous chloral hydrate solution and put about $0.5 \mathrm{cc}$. of this solution into a test-tube. Add very little dilute nitric acid and thoroughly mix the two solutions. Add this mixture to 3 times its volume of concentrated sulphuric acid so that the former is on the surface. A yellowish red to deep red zone, depending upon the quantity of brucine, will appear immediately. When the upper layer becomes yellow introduce by a pipette a little stannous chloride solution ${ }^{1}$ as a top layer. A brilliant, intensely violet zone will appear between the two upper layers. The intensity of this color will gradually increase, especially if the test-tube is gently tilted to and fro.

ATROPINE<smiles>CCCC(CO)C(=O)OC1CC2CCC(C1)N2C</smiles>

Atropine, $\mathrm{C}_{17} \mathrm{H}_{23} \mathrm{NO}_{3}$, crystallizes in shining pointed needles which melt at $115^{\circ}$ and dissolve in 600 parts of water, $5 \circ$ parts of ether and 3.5 parts of chloroform. It'is also soluble in ethyl alcohol, amyl alcohol and benzene. The aqueous solution of the alkaloid is alkaline and has a lasting, unpleasant, bitter taste. Unlike the optically active hyoscyamine, atropine is inactive.

${ }^{1}$ Prepare stannous chloride solution by dissolving I part of stannous chloride in 9 parts of hydrochloric acid having a specific gravity of 1.12 (about 24 per cent. $\mathrm{HCl}$ ). 
Constitution.-Heated with hydrochloric acid at $120-130^{\circ}$, atropine is decomposed into tropic acid and tropine:<smiles>CCCCCCC(CO)C(=O)OC1CC2CCC3CC1CC32</smiles><smiles>CCC(CO)C(=O)O</smiles>

Heated with barium hydroxide solution, atropine yields atropic acid which is unsaturated and differs from tropic acid by one molecule of water:

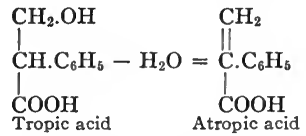

Since the structure both of tropine and tropic acid has been determined by synthesis as well as by decomposition, that of atropine is also known. Nitrogen in atropine is in the tertiary condition. Hyoscyamine is the stereo-isomer of atropine. The former, heated at $110^{\circ}$ out of contact with air, or allowed merely to stand in alcoholic solution with addition of a few drops of an alkaline hydroxide solution, is changed to inactive atropine. Atropine most likely is the racemic form, whereas hyoscyamine is the lævo-rotatory modification of this isomeric base. The degree of rotation of hyoscyamine is $[\alpha] \mathbf{D}=-20.97^{\circ}$. Toward alkaloidal reagents and when heated with concentrated sulphuric acid, hyoscyamine behaves like atropine. It also resembles the latter in giving Vitali's reaction (see below).

Putrefaction.-Ipsen ${ }^{1}$ has found atropine very resistant in presence of putrefying material. Even after 2 years he could detect the alkaloid which had been exposed to the influences of decomposition. He experimented with 0.05 gram of atropine sulphate in respectively $300 \mathrm{cc}$. of blood, urine and beer and with pure atropine in $300 \mathrm{cc}$. of blood.

\section{Detection of Atropine}

Ether, benzene or chloroform will extract atropine from a solution alkaline with sodium hydroxide or carbonate solution. In a special search for atropine use sodium carbonate solution and extract with chloroform which is a better solvent than ether.

1 Vierteljahrsschrift für gerichtliche Medizin und öffentliches Sanitätswesen, 3 I, 308 . 
Evaporate the solvent and test the residue, which is usually amorphous, as follows:

I. Vitali's Test.-Dissolve the alkaloid in a few drops of fuming nitric acid, and evaporate the solution in a porcelain dish to dryness upon the water-bath. Moisten the yellowish residue when cold with a few drops of a solution of potassium hydroxide in absolute ethyl alcohol. An evanescent violet color will appear, if atropine is present.

Hyoscyamine and scopolamine also give Vitali's test. Strychnine and veratrine behave similarly. This test therefore is characteristic of the atropine alkaloids only in the absence of the two latter alkaloids.

2. Para-Dimethylamino-Benzaldehyde Test. ${ }^{1}$ - The required reagent is prepared by dissolving 2 grams of para-dimethylamino-benzaldehyde, $\mathrm{C}_{6} \mathrm{H}_{4} \overbrace{\mathrm{N}\left(\mathrm{CH}_{3}\right)_{2}(4)}^{\mathrm{CHO}}$, in 6 grams of concentrated sulphuric acid and carefully adding 0.4 gram of water. The dark yellow solution obtained keeps well for two weeks. Place a trace of atropine on a watch-glass, add I drop of the reagent and warm gently. A very intense red-violet color is produced. This is an exceedingly delicate test.

Hyoscyamine and scopolamine also give this test. The color in the cold with morphine and codeine is a clear red; with quinine red; with physostigmine and veratrine green; and with narcotine and papaverine orange.

3. Odor Test.-Heat a little atropine in a dry test-tube until a white vapor appears. An agreeable odor will arise at the same time. Then add about I cc. of concentrated sulphuric acid, and heat until the acid begins to darken. Dilute at once with about 2 cc. of water. During the foaming there will be an intense, sweetish odor like that of honey. By this test, which was formerly the only method of identifying atropine, o.oI gram of the alkaloid can be detected.

4. Physiological Test.-Atropine acts in a very characteristic manner upon the pupil of the eye, and this behavior can be employed as a test. One drop of an atropine solution diluted I : I30,000 will produce a noticeable enlargement of the pupil.

${ }^{1}$ Chemical Abstracts II, 1518 (1917). 
Dissolve a small portion of the ether residue in 4 or 5 drops of very dilute sulphuric acid, and introduce a drop of this solution into a dog's or a cat's eye. The enlargement of the pupil often persists for several hours. The utmost care should be taken in performing this test, if applied to the human eye.

The following alkaloidal reagents are especially sensitive toward atropine: iodo-potassium iodide, phospho-molybdic

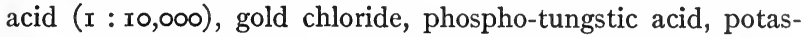
sium mercuric iodide, potassium bismuthous iodide. Picric acid, added to solutions of atropine salts that are not too dilute, will precipitate atropine picrate as yellow leaflets. Platinic chloride gives monoclinic prisms.

\section{HOMATROPINE}

Homatropine, $\mathrm{C}_{16} \mathrm{H}_{21} \mathrm{NO}_{3}$, is the tropyl ester of phenyl-glycolic or mandelic acid. The hydrochloride of this base is obtained by heating a mixture of tropine,<smiles>CCC(O)C(=O)OC1CC2CCC(C1)N2C</smiles>
mandelic acid and hydrochloric acid, the latter acting as a dehydrating agent.

The hydrobromide of homatropine $\left(\mathrm{C}_{16} \mathrm{H}_{21^{-}}\right.$ $\mathrm{NO}_{3} \cdot \mathrm{HBr}$ ) is used in medicine as a substitute for atropine. Its action on the pupil is nearly as strong as that of the natural alkaloid and its effect disappears in $\mathrm{r}_{2-24}$ hours, whereas that of atropine often lasts 8 days. Moreover it is less toxic than atropine. Homatropine is a strong tertiary base which forms neutral salts with acids. This alkaloid does not give Vitali's test. It melts at $92-96^{\circ}$; hyoscyamine at $108^{\circ}$; and atropine at $\mathrm{r} 15.5^{\circ}$.

Officinal homatropine hydrobromide may be distinguished from the hydrobromides of atropine and hyoscyamine by warming the substance in a test-tube with a little chloroform. This solvent dissolves the latter two salts in every proportion but homatropine hydrobromide is insoluble. An alternative procedure consists in dissolving the given salt in a little water, precipitating the base with sodium carbonate solution, and extracting with ether. Dehydrate the ether extract with potassium carbonate and evaporate slowly in a moderately warm place. This method will give crystals of the alkaloid. Dry these crystals in vacuo over concentrated sulphuric acid and determine their melting-point.

\section{COCAINE}

Cocaine, $\mathrm{C}_{17} \mathrm{H}_{21} \mathrm{NO}_{4}$, crystallizes from ethyl alcohol in large, colorless, monoclinic prisms which melt at $98^{\circ}$. It has a bitterish taste and, placed upon the<smiles>CCCOC(C(=O)OC)C(C)C1CCC2CCC2N1C</smiles>
tongue, causes temporary, local anæsthesia. The alkaloid is only slightly soluble in water ( $r: 700$ ), but easily soluble in ethyl alcohol, ether, chloroform, benzene and acetic ether. Its solutions are strongly alkaline and lævorotatory. Dilute acids easily dissolve cocaine and in most cases form readily 
crystallizable salts. The fixed alkalies, ammonia and alkaline carbonates precipitate the free base from solutions of its salts.

Constitution.-Cocaine is a monacid, tertiary base, since it adds a molecule of $\mathrm{CH}_{3} \mathrm{I}$. On distillation with barium hydroxide, this alkaloid loses methyl amine $\left(\mathrm{CH}_{3} . \mathrm{NH}_{2}\right)$, thus proving the attachment of a methyl group to nitrogen. Cocaine must therefore contain the group $=\mathrm{N}-\mathrm{CH}_{3}$. This base is also the methyl ester of an acid and at the same time the benzoyl derivative of an alcoho,1 for it is decomposed into benzoyl-ecgonine and methyl alcohol when heated with water. If mineral acids, barium hydroxide or alkalies are used instead of water, the primary product, benzoyl-ecgonine, is further decomposed into ecgonine, benzoic acid and methyl alcohol. Taking the structural formula proposed by Willstätter, we may express this reaction as follows:

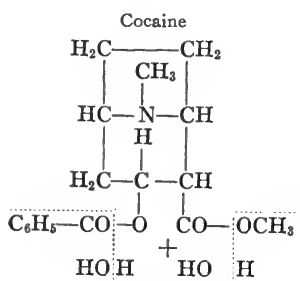

Water

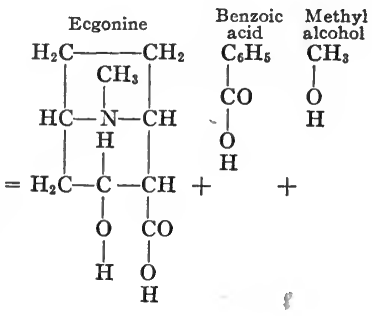

Ecgonine (I) heated with phosphorus oxychloride loses a molecule of water and passes into anhydro-ecgonine (II). The latter heated to $280^{\circ}$ with fuming hydrochloric acid loses carbon dioxide and is converted into tropidine (III). Tropidine heated with a caustic alkaline solution adds a molecule of water and passes into tropine (IV), the basic cleavage-product of atropine. Evaporation of tropine with tropic acid in dilute hydrochloric acid solution yields atropine (V). Thus it is possible to start with the alkaloid cocaine and synthesize the alkaloid atropine. The series of changes involved is as follows:<smiles>CN1C2CC(CC2C(=O)O)C1C(=O)O</smiles><smiles>CCCC(CO)C(=O)OC1CC2CC(O)CC(C1)N2C</smiles> 
<smiles>CCCC(OC(=O)C1CC2CCC(C2)[N+]1(C)CO)C(CC)CO</smiles>

Behavior in the Animal Organism.-Experiments upon dogs and rabbits show that the former animal eliminates through the kidneys not more than 5 per cent. of the cocaine as such and the latter none at all. As the urine of these animals also contains no ecgonine, the supposition is that the alkaloid is profoundly changed in the animal organism. The same is true of the human organism. Proells $^{1}$ was able to detect cocaine in cadaveric material at most after I4 days. In the living organism the alkaloid is said to be changed rapidly into ecgonine.

\section{Detection of Cocaine}

Ether, chloroform or benzene will extract cocaine from an alkaline aqueous solution. Most of the alkaloidal reagents will precipitate cocaine even from very dilute solutions of its salts: The reagents especially sensitive are: iodo-potassium iodide, phospho-molybdic and phospho-tungstic acids, potassium mercuric iodide, potassium bismuthous iodide, gold and platinum chlorides, and picric acid.

Pure concentrated sulphuric and nitric acids, as well as Erdmann's, Froehde's and Mandelin's reagents, dissolve cocaine without color.

I. Precipitation Test.-If I or 2 drops of potassium hydroxide solution are added to an aqueous solution of a cocaine salt not too dilute, it will become milky. First, resinous globules and later fine, crystalline needles of the free base, cocaine (meltingpoint $98^{\circ}$ ), separate from solution:

$$
\mathrm{C}_{17} \mathrm{H}_{21} \mathrm{NO}_{4} \cdot \mathrm{HCl}^{2}+\mathrm{KOH}=\mathrm{C}_{17} \mathrm{H}_{21} \mathrm{NO}_{4}+\mathrm{H}_{2} \mathrm{O}+\mathrm{KCl} \text {. }
$$

In applying this test to the ether residue, dissolve a considerable quantity in a few drops of dilute hydrochloric acid and add potassium hydroxide solution drop by drop until alkaline and

1 Apotheker-Zeitung $16,779,788$.

${ }^{2}$ Cocaine hydrochloride crystallizes from a concentrated aqueous solution in fine prisms containing 2 molecules of water which are easily given off. This salt crystallized from ethyl alcohol is anhydrous and has the formula $\mathrm{C}_{17} \mathrm{H}_{21} \mathrm{NO} 4 . \mathrm{HCl}$. The anhydrous compound is the officinal salt. 
cool well by setting in ice. Special care must be taken to have the alkaloid pure enough when dry for a melting-point determination.

This test is not characteristic of cocaine (except the meltingpoint which, however, requires considerable pure material), because most of the alkaloids are precipitated by potassium hydroxide solution in much the same way.

2. Potassium Pemanganate Test.-Add saturated potassium permanganate solution drop by drop to a concentrated aqueous solution of a cocaine salt. This reagent will give a violet, crystalline precipitate of cocaine permanganate. In applying this test to the ether residue, dissolve a considerable quantity in 2 drops of dilute hydrochloric acid and evaporate the solution upon the water-bath. Dissolve the residue in as little water as possible and add potassium permanganate solution.

3. Chromic Acid Test.-Add a few drops of a 5 per cent. chromic acid solution, or potassium dichromate solution of corresponding concentration ( 7.5 per cent.) to a solution of a cocaine salt. Each drop will produce a precipitate which will immediately disappear if the solution is shaken. Then add to the clear solution about I cc. of concentrated hydrochloric acid which will produce an orange-yellow precipitate more or less crystalline.

4. Detection of Benzoyl Group.-This test requires at least 0.2 gram of cocaine. First, digest the cocaine a few minutes in a test-tube with 2 cc. of concentrated sulphuric acid upon a boiling water-bath. Cool and dilute with a little water, all the while keeping the mixture cold. A white crystalline precipitate of benzoic acid will appear. Collect and dry this precipitate upon a filter. Benzoic acid may be recognized by subliming the precipitate, or, if the quantity is sufficient, by determining the melting-point $\left(120^{\circ}\right)$.

Benzoic acid may also be extracted with ether. Mix the residue, obtained by evaporating the solvent, with I cc. of absolute ethyl alcohol and the same quantity of concentrated sulphuric acid. The characteristic odor of ethyl benzoate, $\mathrm{C}_{6} \mathrm{H}_{5} \cdot \mathrm{CO} . \mathrm{OC}_{2} \mathrm{H}_{5}$, will be recognized. 
5. Reichard's ${ }^{1}$ Test.-Addition of a concentrated aqueous solution of sodium nitroprusside, $\mathrm{Na}_{2} \mathrm{Fe}(\mathrm{CN})_{5} \mathrm{NO}_{2} \mathrm{H}_{2} \mathrm{O}$, drop by drop to a cocaine salt solution, containing at least $4 \mathrm{mg}$. of cocaine per cc., causes an immediate turbidity which appears under the microscope as well-formed reddish crystals. These crystals will dissolve, if the liquid is warmed, and appear again if the solution is well-cooled. Morphine does not give this test.

6. Denigès ${ }^{2}$ Test.-If an equal volume of 5 per cent. sodium perchlorate $\left(\mathrm{NaClO}_{4}\right)$ solution is added to 0.5 per cent. solution of a cocaine salt, a precipitate consisting of very fine, long needles is produced. This precipitation may be observed under the microscope, when the quantity of material is very small.

7. Pisani's ${ }^{3}$ Test.-Heat cocaine or its hydrochloride with a few drops of concentrated sulphuric acid containing 2 per cent. of formamide (H.CO. $\mathrm{NH}_{2}$ ). A wine-red color, increasing in intensity as the temperature rises, is produced. This color soon disappears and a brownish gray precipitate remains. This test will detect $0.00 \mathrm{I}$ gram of cocaine.

Atropine, quinine, cinchonine, brucine, strychnine, morphine, apomorphine, codeine and narcotine do not give this test. Papaverine gives a wine-red coloration which changes to yellow, reddish brown and orange.

8. Physiological Test.-Dissolve the material (the residue from the ether extract) in a few drops of dilute hydrochloric acid and evaporate the solution to dryness upon the waterbath. Dissolve the residue in a little pure water and apply this solution to the tongue. Cocaine produces a temporary anesthesia.

R. Kobert ("Intoxikationen") has found small frogs sufficiently sensitive for use in the physiological test for cocaine. The effects to be observed are dilatation and fixedness of the pupil, enlargement of the palpebral fissure and also stimulation

${ }^{1}$ C. Reichard, Chemiker-Zeitung 28, 299 (rgo4). Pharmazeutische Zeitung r904, Nr. 29. Pharmazeutische Zentralhalle 45, 645 (r904).

${ }^{2}$ Chemical Abstracts 9, 234 (I915).

${ }^{3}$ Chemical Abstracts 9, 5 rr (ror 5$)$. 
of the nervous system. Administer the same quantity of cocaine hydrochloride to animals for comparison.

\section{PHYSOSTIGMINE}

Physostigmine, $\mathrm{C}_{15} \mathrm{H}_{21} \mathrm{~N}_{3} \mathrm{O}_{2}$, also called eserine, occurs in the Calabar bean, the seed of Physostigma venenosum. This alkaloid is deposited from benzene solution upon spontaneous evaporation of the solvent in large, apparently rhombic crystals melting at $105^{\circ}$. Though but slightly soluble in water, it dissolves freely in ethyl alcohol, ether, benzene or chloroform. Physostigmine solutions are strongly alkaline, almost tasteless and lævo-rotatory. It is a strong monacid tertiary base, forming salts with acids that easily undergo decomposition and crystallize with difficulty. Light and heat cause acid and alkaline solutions of this alkaloid to turn red. Owing to this tendency of physostigmine to undergo decomposition, care must be taken during its isolation to keep it from light and air and also to avoid rise of temperature. Exclusion as far as possible of free mineral acids and caustic alkalies is also desirable.

\section{Detection of Physostigmine}

Concentrated sulphuric and nitric acids dissolve physostigmine with a yellow color which soon changes to olive-green. The alkaloid evaporated upon the water-bath with fuming nitric acid leaves a residue having a green margin. Water, ethyl alcohol and sulphuric acid dissolve this residue with a green color.

I. Ammonia Test.-If a small quantity of a physostigmine salt is evaporated to dryness upon the water-bath with ammonium hydroxide solution, a blue or blue-green residue will remain. This will dissolve in ethyl alcohol with a blue color. Excess of dilute mineral acid, or acetic acid added to this solution will change the color to red. The solution is also strongly fluorescent. Examined spectroscopically, the blue alkaline solution shows one absorption-band in the red; and the red acid solution one absorption-band in the yellow.

A drop of concentrated sulphuric acid, added to the blue residue from evaporation with ammonia, will give a green solution. The green color diluted with ethyl alcohol will change to red. If the alcohol is evaporated, the green color will reappear.

2. Rubreserine Test.-If an aqueous solution of a physostigmine salt is shaken for some time with an excess of potassium or sodium hydroxide solution, a red coloring-matter, rubreserine $\left(\mathrm{C}_{18} \mathrm{H}_{16} \mathrm{~N}_{2} \mathrm{O}_{2}\right)$, is formed. This compound separates as red needles which become greenish blue on further oxidation owing to formation of eserine blue.

Barium hydroxide solution may be substituted for the caustic alkali. This reagent first produces a white precipitate which soon becomes red on being shaken. Sometimes this change occurs even in the cold but invariably takes place with heat.

3. Physiological Test.-The marked action of physostigmine in causing contraction of the pupil is very characteristic. It is advisable to use the cat's eye for this test. Even $0.1 \mathrm{mg}$. of this alkaloid will produce noticeable contraction. 


\section{CODEINE}

Codeine, $\mathrm{C}_{17} \mathrm{H}_{18}\left(\mathrm{CH}_{3}\right) \mathrm{NO}_{3}$, the methyl ether of morphine, crystallizes from water, or from ether containing water, in colorless, transparent octahedrons

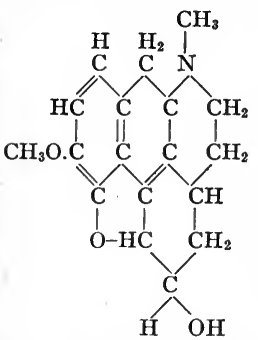
which are often very large. These crystals are quite easily soluble in water. One part of the free base is soluble at $15^{\circ}$ in 80 parts of water and at $100^{\circ}$ in 15 parts. Codeine differs from most of the other alkaloids, morphine, for example, in its relatively high 'solubility in water. Ethyl alcohol, ether, amyl alcohol, chloroform and benzene also dissolve codeine freely. It is, however, practically insoluble in petroleum ether. Aqueous codeine solutions are strongly alkaline and bitter. Pure codeine does not reduce iodic acid, nor does it immediately produce a blue color or a blue precipitate in a mixture of potassium ferricyanide and ferric chloride solutions. A pure codeine solution is also not colored blue by ferric chloride solution alone. (Difference between morphine and codeine.) Phospho-molybdic acid, iodopotassium iodide, potassium bismuthous iodide and potassium mercuric iodide give precipitates even with very dilute codeine solutions. On the other hand, tannic and picric acids, gold and platinum chlorides are less sensitive.

\section{Detection of Codeine}

I. Sulphuric Acid Test.-Concentrated sulphuric acid dissolves codeine without color. After long contact or upon application of gentle heat, the solution will have a reddish to bluish violet color. The solution of codeine in concentrated sulphuric acid, heated to about $150^{\circ}$ and then cooled, is colored deep red by a drop of concentrated nitric acid.

2. Nitric Acid Test.-Cold nitric acid (25 per cent.) will convert codeine into nitro-codeine $\left(\mathrm{C}_{18} \mathrm{H}_{20}\left(\mathrm{NO}_{2}\right) \mathrm{NO}_{3}\right)$. At the same time the acid will dissolve the alkaloid with a yellow color which soon changes to red. Concentrated nitric acid dissolves codeine with a reddish brown color.

3. Oxidation Test.-Mix a little codeine upon a watch-glass with four times the quantity of finely powdered potassium arsenate $\left(\mathrm{KH}_{2} \mathrm{AsO}_{4}\right)$. Add a few drops of concentrated sulphuric acid and then warm gently over a small flame. The acid will have a deep blue or blue-violet color, if the codeine is not quite pure. Excess of potassium arsenate does not affect the test. 
If water or sodium hydroxide solution is added, the blue color will change to orange-yellow.

A trace of ferric chloride solution may be substituted for potassium arsenate. Sulphuric acid containing $\mathrm{I}$ drop of ferric chloride solution to ro cc. of acid is prescribed by the German Pharmacopœia for detecting the alkaloid in codeine phosphate.

4. Froehde's Test.-This reagent dissolves codeine with a yellowish color which soon changes to green and finally to blue. Gentle warming of the solution over a very small flame will hasten this change of color.

R. Mauch warms 2 or 3 drops of a chloral hydrate solution of codeine with I drop of Froehde's reagent. An intense blue color finally appears.

5. Formalin-Sulphuric Acid Test. ${ }^{1}$ - Concentrated sulphuric acid containing formalin dissolves codeine with a reddish violet color which changes to blue-violet. This color is persistent. The spectrum shows an absorption of orange and yellow.

6. Furfural Test. ${ }^{2}$-Dissolve codeine in a few drops of concentrated sulphuric acid and warm very gently with a drop of cane-sugar solution which must not be in excess. This will produce a purple-red color.

This test may also be made by mixing a drop of sugar solution with codeine, dissolved in about 5 drops of $50-60$ per cent. aqueous chloral hydrate solution, and then adding $\mathrm{I}-2 \mathrm{cc}$. of concentrated sulphuric acid as an under layer. A carmine-red ring will appear at the zone of contact. The color is quite permanent and increases in intensity upon standing. If the sulphuric acid and chloral hydrate solution are thoroughly mixed, the entire liquid will be red. After a time the shade of color will be more of a red-brown.

7. Pellagri's Test.-Both codeine and morphine give this test. Dissolve codeine in concentrated hydrochloric acid and

${ }^{1}$ See preparation of reagents, page 321 .

2 This test depends upon furfural formed by the action of concentrated sulphuric acid upon cane-sugar. Very dilute aqueous furfural solution ( $r: 1000$ ) may be substituted for cane-sugar. Excess of furfural unlike cane-sugar does not interfere with the test. $\mathrm{Tr}$. 
add at the same time 3-4 drops of concentrated sulphuric acid. Expel hydrochloric acid upon the water-bath and heat the residue about ${ }_{5} 5$ minutes. Dissolve the dirty red or violet residue in 2-3 cc. of water, add a few drops of hydrochloric acid and neutralize with acid sodium carbonate. Then add alcoholic solution of iodine drop by drop ( 2 to 4 drops) and shake thoroughly for several minutes. An emerald-green solution indicates codeine. Extract the green solution with ether. The color of the ether will be red, whereas that of the aqueous solution will remain green. This is a test for apomorphine (see page 128 ) formed from codeine by the mineral acid.

$$
\mathrm{C}_{17} \mathrm{H}_{18}\left(\mathrm{CH}_{3}\right) \mathrm{NO}_{3}+\mathrm{HCl}=\underset{\text { Apomorphine }}{\mathrm{C}_{17} \mathrm{H}_{17} \mathrm{NO}_{2}}+\mathrm{CH}_{3} \mathrm{Cl}+\mathrm{H}_{2} \mathrm{O} \text {. }
$$

8. Mecke's Test.-The reagent, consisting of selenious acid and concentrated sulphuric acid, ${ }^{1}$ dissolves codeine with a blue color quickly changing to emerald-green and finally becoming a permanent olive-green.

\section{NARCOTINE}

Narcotine, $\mathrm{C}_{22} \mathrm{H}_{23} \mathrm{NO}_{7}$, crystallizes in shining prisms or in tufts of needles which are nearly insoluble in cold water but readily soluble in boiling ethyl alcohol or chloroform. Separation of alkaloid from the cold alcoholic solution is almost complete. At $15^{\circ}$ narcotine dissolves in 170 parts of ether; 31 parts of acetic ether; and 22 parts of benzene. Solutions of narcotine are not alkaline nor bitter. In these respects narcotine is very

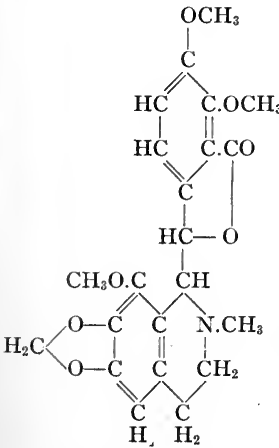
different from the other opium alkaloids. Salts of narcotine do not crystallize, their stability is slight and their solutions react acid. Salts with volatile acids are decomposed, when their solutions are evaporated, with separation of narcotine. Sodium acetate precipitates free narcotine from its solution in hydrochloric acid.

Constitution.--Narcotine is a monacid, tertiary base and as such combines with $\mathrm{I}$ mol. of $\mathrm{CH}_{3}$.I, forming narcotine methyl iodide $\left(\mathrm{C}_{22} \mathrm{H}_{23} \mathrm{NO}_{7}\right.$. $\mathrm{CH}_{3} \mathrm{I}$ ). This compound is formed at ordinary temperatures but the reaction is hastened by heat. Narcotine heated with hydriodic acid loses 3 methyl groups which form $\mathrm{CH}_{3} \mathrm{I}$. The alkaloid must therefore contain 3 methoxyls, ${ }_{3}\left(\mathrm{CH}_{3} \mathrm{O}-\right)$, in the molecule. Heated with water to $140^{\circ}$, with dilute sulphuric acid, or even with

${ }^{1}$ See preparation of reagents, page 322 . 
barium hydroxide solution narcotine is hydrolyzed into nitrogen-free opianic acid and into the basic and consequently nitrogenous hydrocotarnine:

$$
\underset{\text { Narcotine }}{\mathrm{C}_{22} \mathrm{H}_{23} \mathrm{NO}_{7}}+\mathrm{H}_{2} \mathrm{O}=\underset{\substack{\text { Opianic } \\ \text { acid }}}{\mathrm{C}_{10} \mathrm{H}_{10} \mathrm{O}_{5}}+\underset{\text { Hydrocotarnine }}{\mathrm{C}_{12} \mathrm{H}_{15} \mathrm{NO}_{3}}
$$

By oxidative cleavage, that is, by treatment of narcotine with such oxidizing agents as nitric acid, manganese dioxide and sulphuric acid, lead dioxide and ferric chloride, cotarnine and opianic acid are the products:

$$
\underset{\text { Narcotine }}{\mathrm{C}_{22} \mathrm{H}_{23} \mathrm{NO}_{7}}+\left(\mathrm{H}_{2} \mathrm{O}+\mathrm{O}\right)=\underset{\substack{\text { Opianic } \\ \text { acid }}}{\mathrm{C}_{10} \mathrm{H}_{10} \mathrm{O}_{5}}+\underset{\text { Cotarnine }}{\mathrm{C}_{12} \mathrm{H}_{15} \mathrm{NO}_{4}}
$$

Evidently these cleavage-products show that this alkaloid is made up of two complexes, one nitrogen-free and the other containing nitrogen. The chemical constitution of these cleavage-products has been determined and is expressed by the following formulæ:<smiles>COc1ccc(C=O)c(C(=O)O)c1O</smiles><smiles></smiles><smiles>CCCc1c2c(CCNC)c(C=O)c(OC)c1OCO2</smiles>

On the basis of these results, Roser and Freund have proposed the structural formula for narcotine given above. They consider the constitution of this alkaloid as definitely settled. If the formula of narcotine is compared with that of hydrastine (see page II6), a great similarity in structure will be seen. In fact narcotine is a methoxylized hydrastine.

\section{Detection of Narcotine}

Narcotine is so feebly basic that chloroform will extract the alkaloid completely from an aqueous tartaric acid solution. Consequently its separation from the rest of the opium alkaloids as well as from other alkaloids is easy. Naturally ether or chloroform will also extract narcotine from an aqueous alkaline 
solution. The alkaloid as it comes from its ether solution is usually a slightly colored, varnish-like residue which hardens after a time to a mass of radiating crystals. Narcotine is precipitated from its hydrochloric or sulphuric acid solution by iodo-potassium iodide, phospho-molybdic acid, potassium mercuric iodide, potassium bismuthous iodide even in considerable dilution ( 1 : 5000).

I. Sulphuric Acid Test.-Dissolved with stirring in concentrated sulphuric acid, narcotine produces a greenish yellow color which gradually changes to reddish yellow and finally after several days to raspberry-red.

2. Dilute Sulphuric Acid Test.-A solution of narcotine in dilute sulphuric acid ( $1: 5$ ), evaporated on the water-bath in a porcelain dish or over a very small flame, has a reddish yellow color, changing with stronger heat to crimson-red. As the acid begins to evaporate, blue-violet streaks radiate from the margin and finally the entire liquid has a dirty red-violet color (Dragendorff's reaction). The same color changes appear, if the yellowish solution of narcotine in concentrated sulphuric acid is heated very carefully.

3. Froehde's Test.- This reagent dissolves narcotine with a greenish color. If concentrated Froehde's reagent is used, the green color changes immediately to cherry-red, especially upon application of gentle heat. This color is quite persistent.

4. Couerbe's Test.-Dissolve narcotine in cold concentrated sulphuric acid and mix a trace of nitric acid with this solution after $\mathrm{r}-2$ hours. A red color will appear and gradually become more and more pronounced.

Erdmann's reagent gives the same color change.

5. Wangerin's Test. ${ }^{1}$-Place a mixture of 0.01 gram of narcotine with 20 drops of pure concentrated sulphuric acid and I-2 drops of I per cent. cane-sugar solution upon a watchglass and heat upon the water-bath with stirring about I minute. At first the solution has a greenish yellow color which passes through yellow, brownish yellow, brown and brown-violet into an intense blue-violet.

${ }^{1}$ Pharmazeutische Zeitung, 48, 607 (rgc3). 
The intensity of this color increases somewhat upon standing and the blue-violet color persists several hours.

Applied to apomorphine, atropine, brucine, quinine, codeine, caffeine, hydrastine, morphine, physostigmine, pilocarpine and strychnine, this test gives solutions that are colorless or nearly so. Only the morphine solution after a while has a pale pink color. Coniine and narcotine have a light yellow color; narceine chestnut-brown; and picrotoxin salmon color to pale pink.

Colchicin, digitalin and veratrine behave toward this reagent as toward pure concentrated sulphuric acid without the addition of the small quantity of sugar.

In this test I-2 drops of $\mathrm{I}$ per cent. aqueous furfural solution may be substituted for the sugar solution. From yellow, brown, olive and other colors there finally emerges a deep, clear, dark blue. The brilliancy of this color increases somewhat on standing. After several hours there is a gradual change to a pure green color. For the detection of traces of narcotine (0.00I gram) use a I per cent. sugar solution.

6. Selenious Acid-Sulphuric Acid Test.-This reagent dissolves narcotine with a greenish steel-blue color which after a time becomes cherry-red. Heat immediately discharges the cherry-red color.

\section{HYDRASTINE}

Hydrastine, $\mathrm{C}_{21} \mathrm{H}_{21} \mathrm{NO}_{6}$, occurs together with berberine, $\mathrm{C}_{20} \mathrm{H}_{17} \mathrm{NO}_{4}$, and canadine, $\mathrm{C}_{20} \mathrm{H}_{21} \mathrm{NO}_{4}$, in hydrastis root, the root of Hydrastis canadensis, to the $\mathrm{H}_{2} \mathrm{C}\left\langle\mathrm{C}_{\mathrm{C}}\right.$ amount of I.5 per cent. and more. The fluid extract prepared from this root and used in medicine contains 2-2.5 per cent. of hydrastine.

Preparation.-Extract hydrastis root with hot 3 water containing acetic acid. Filter the solution, evaporate to a thin extract and add 3 vols. of dilute sulphuric acid $(\mathrm{r}: 5)$. Nearly all the berberine separates out in fine yellow crystals as acid sulphate, $\mathrm{C}_{20} \mathrm{H}_{17} \mathrm{NO}_{4} \cdot \mathrm{H}_{2} \mathrm{SO}_{4}$. Precipitate hydrastine from the mother-liquor of berberine sulphate by means of ammonium hydroxide solution and purify the alkaloid by crystallization from acetic ether or ethyl alcohol. Hydrastine crystallizes from ethyl alcohol in rhombic prisms melting at $132^{\circ}$. It is nearly insoluble in water but freely soluble in hot ethyl alcohol, benzene or chloroform. This alkaloid has a bitter taste and its solutions are alkaline. Hydrastine solutions are optically active. In chloroform this alkaloid is lævo-rotatory, whereas in dilute hydrochloric acid it is dextrorotatory. 
Constitution.-The constitution of hydrastine is entirely analogous to that of narcotine (see page I13). On oxidation with dilute nitric acid hydrastine gives opianic acid and hydrastinine:

$$
\underset{\text { Hydrastine }}{\mathrm{C}_{21} \mathrm{H}_{21} \mathrm{NO}_{6}}+\left(\mathrm{H}_{2} \mathrm{O}+\mathrm{O}\right) \underset{\text { Opianic acid }}{=} \underset{\mathrm{C}_{10} \mathrm{H}_{10} \mathrm{O}_{5}}{\mathrm{H}_{11}}+\underset{\text { Hydrastinine }}{\mathrm{C}_{11} \mathrm{H}_{13} \mathrm{NO}_{3}}
$$

Hydrastine is a monacid base which is shown to be a tertiary base by its behavior toward alkyl iodides, for example, with $\mathrm{CH}_{3} \mathrm{I}$ it forms hydrastine methyl iodide, $\mathrm{C}_{21} \mathrm{H}_{21} \mathrm{NO}_{6} \cdot \mathrm{CH}_{5} \mathrm{I}$, which crystallizes in needles. Hydrastine contains two methoxyl groups, because when heated with hydriodic acid according to Zeisel's method two such groups are removed.

Since the chemical nature of opianic acid has long been known, the only problem is the explanation of the nature of hydrastinine, the other cleavageproduct. The constitution of hydrastinine, as well as that of many other alkaloids, has been determined by A. W. Hofmann's method of exhaustive methylation. ${ }^{1}$ Hydrastinine (I) is a secondary base which forms, when heated with an excess of $\mathrm{CH}_{5} \mathrm{I}$, hydrastinine hydriodide and trimethyl-hydrastyl-ammonium iodide (II). Heated with alkalies, this ammonium iodide is decomposed into trimethylamine, hydriodic acid and nitrogen-free hydrastal (III). The latter on oxidation gives hydrastic acid (IV) which was recognized as the methylene ether of nor-meta-hemipinic acid (V):

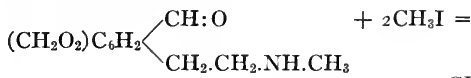

Hydrastinine

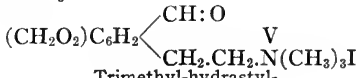

Trimethyl-hydrastylammonium iodide

(II)

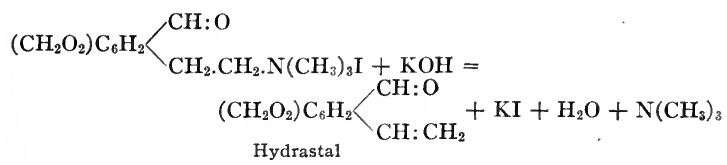

1 When the nitrogen of an organic base becomes quinquevalent, it is more subject to change. Hofmann (Liebig's Annalen, 78, 263 (1851) showed, for example, that tetra-ethyl-ammonium hydroxide breaks up on heating into triethylamine, ethylene and water:

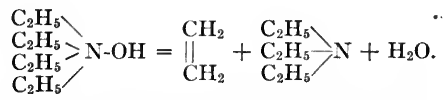

Nitrogen in alkaloids on treatment with an alkyl haloid (e.g., $\mathrm{CH}_{3} \mathrm{I}$ ) combines with it in many instances, forming compounds having a structure analogous to that of tetra-ethyl-ammonium hydroxide. This process is called "exhaustive methylation." Upon decomposition these derivatives yield products which often throw light upon the structure of the alkaloid. 
(III)

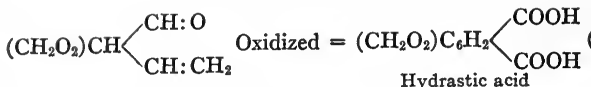

Hydrastic acid and nor-meta-hemipinic acid are identical. The latter has the structure (V):

(V)

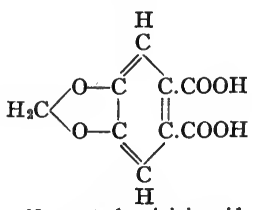

Nor-meta-hemipinic acid

From these and other relations it has been determined that cotarnine is a methoxy-hydrastinine:

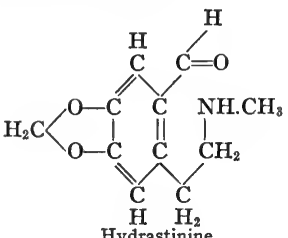

Hydrastinine

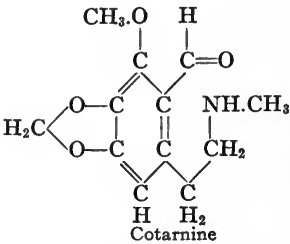

The alkaloid narcotine is a methoxy-hydrastine (see page r44).

\section{Detection of Hydrastine}

I. Concentrated Sulphuric Acid dissolves hydrastine without color but upon being gently warmed the solution becomes violet.

2. Froehde's Reagent dissolves hydrastine with a green color which gradually changes to brown.

3. Mandelin's Reagent dissolves hydrastine with a rosered color which immediately changes to orange-red and gradually fades.

4. Fluorescence Test.-Dissolve hydrastine in dilute sulphuric acid, shake vigorously and add drop by drop very dilute potassium permanganate solution. Hydrastinine is formed and the solution shows a beautiful blue fluorescence.

The ether extract of the alkaline solution on evaporation leaves hydrastinine in a crystalline condition. 


\section{QUININE}

Quinine, $\mathrm{C}_{20} \mathrm{H}_{24} \mathrm{~N}_{2} \mathrm{O}_{2}$, is precipitated amorphous and anhydrous from solutions of its salts by caustic alkalies, alkaline carbonates or ammonia. On standing,<smiles>CCC1CCN(Cc2ccnc3cccc(O)c23)CC1</smiles>
however, it gradually becomes crystalline, forming a hydrate with 3 molecules of water of hydration. There are also other hydrates of quinine. Anhydrous quinine melts at $173^{\circ}$; the trihydrate at $57^{\circ}$. An ether solution on evaporation usually deposits this alkaloid as a resinous, or varnish-like, amorphous residue. Quinine is soluble in about 2000 parts of cold and 700 parts of boiling water; and freely soluble in ethyl alcohol, ether or chloroform. Solutions of quinine in sulphuric, acetic or tartaric acid exhibit a beautiful blue fluorescence. In the case of the sulphate this fluorescence is distinctly visible in a dilution of $I: 100,000$.

Hydrochloric, hydrobromic and hydriodic acid do not give fluorescent solutions of quinine. These acids even discharge the fluorescence, if added to a fluorescent quinine solution.

Constitution.-Quinine is a diacid, ditertiary base, the salts of which with $\mathrm{I}$ and 2 equivalents of acid are usually crystalline. The salts with I equivalent of acid are the more stable. Quinine hydrochloride, $\mathrm{C}_{20} \mathrm{H}_{24} \mathrm{~N}_{2} \mathrm{O}_{2} \cdot \mathrm{HCl}_{2} \mathrm{H}_{2} \mathrm{O}$, used in medicine, crystallizes in long delicate tufts of needles. The ditertiary character of quinine is shown by the fact that it unites with 2 molecules of methyl iodide, for example, to form quinine dimethyliodide, $\mathrm{C}_{20} \mathrm{H}_{24} \mathrm{~N}_{2} \mathrm{O}_{2.2} \mathrm{CH}_{3} \mathrm{I}$. Quinine must contain an hydroxyl group, since it can form a mono-benzoyl and a mon-acetyl-quinine. Moreover one methoxyl group has been found in the quinine molecule. The difference empirically between cinchonine, $\mathrm{C}_{19} \mathrm{H}_{22} \mathrm{~N}_{2} \mathrm{O}$, and quinine, $\mathrm{C}_{20} \mathrm{H}_{24} \mathrm{~N}_{2} \mathrm{O}_{2}$, is $\mathrm{CH}_{2} \mathrm{O}$. Every investigation of these substances has shown that quinine is a methoxy-cinchonine. For example, on oxidation with chromic acid, cinchonine gives cinchonic acid which was recognized as quinoline $\gamma$-carboxylic acid; whereas quinine under the same conditions gives quinic acid, or p-methoxy-cinchonic acid:<smiles>CCOC(=O)c1ccccc1</smiles>

Cinchonic acid<smiles></smiles>

Quinic acid

Both alkaloids on oxidation also give the nitrogenous compounds meroquinene, cincholoiponic acid and loiponic acid. Consequently there is no doubt that cinchonine and quinine contain two nitrogenous nuclei, one of which 
is a quinoline complex. The second nucleus is connected with the latter in the $\boldsymbol{\gamma}$-position, as the formation of cinchonic and quinic acids shows. Meroquinene, cincholoiponic acid and loiponic acid, derived by oxidation with chromic acid from the so-called "second half" of the cinchonine and quinine molecules, form a continuous series of oxidation products, since meroquinene can be oxidized to cincholoiponic acid and the latter to loiponic acid. The following formulæ best explain the chemical behavior of these three compounds:
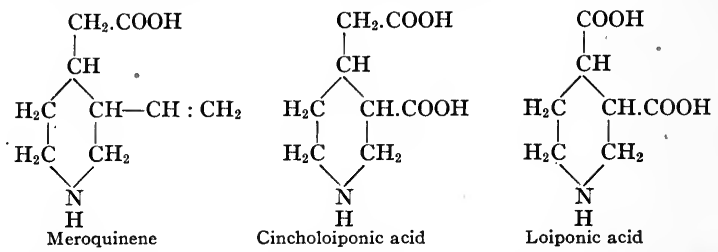

The structural formula already given for quinine was proposed by W. Koenigs ${ }^{1}$ and is based on the results of his own experiments as well as on those of W. V. Miller and of Skraup. Cinchonine has hydrogen in place of the methoxyl group in the quinoline nucleus; otherwise the two alkaloids are identical in structure.

\section{Detection of Quinine}

Ether, benzene or chloroform will extract quinine from an aqueous alkaline solution. Ether on evaporation deposits the alkaloid as a resinous, amorphous varnish in which its presence may be recognized by the following tests:

I. Fluorescence Test.-Dissolve the residue from the ether extraction of the alkaline solution in a little dilute sulphuric acid. If quinine is present, this solution will exhibit blue fluorescence.

2. Thalleioquin Test.-Dissolve quinine in a few drops of very dilute acetic acid and add 5-Io drops of saturated chlorine water. The colorless solution has a faint, blue fluorescence. Excess of ammonium hydroxide solution will produce an emerald-green color. A solution containing considerable quinine will give a green precipitate. This precipitate (thalleioquin) is always an amorphous substance, the composition of which has not been determined. It is soluble in ethyl alcohol and chloroform but not in ether.

1 Meroquinene and the Structure of the Cinchona. Alkaloids; Annalen der Chemie und Pharmazie 347, 147 (1906). 
E. Polacci recommends the following procedure for the thalleïoquin test. Gradually heat quinine (about 0.0 I gram) to boiling with a little lead dioxide $\left(\mathrm{PbO}_{2}\right), 2-3 \mathrm{cc}$. of water and 2 drops of dilute sulphuric acid. Let the solution settle and either decant or filter. Finally, carefully add $5^{-6}$ drops of ammonium hydroxide solution as a top layer. A beautiful green ring will appear at the zone of contact.

Interferences with the Thalleioquin Test.-Antipyrine interferes with this test. Mixtures of I per cent. solutions of antipyrine and quinine give finally a beautiful red instead of a green color. This interference does not cease until these two substances are in the proportion of 0.25 parts of antipyrine to 5 parts of quinine. Caffeine also interferes with the thalleioquin test, when the proportion is 2 parts of quinine to 3 parts of caffeine. Other compounds like urea prevent the appearance of this color, whereas morphine, pilocarpine, cocaine, atropine, codeine, strychnine, carbolic acid and chloral hydrate have no effect upon the thalleioquin test.

H. Fühner ${ }^{1}$ has shown that the thalleioguin reaction is connected with the p-oxyquinoline complex. Chlorine passed into a solution of pure p-oxy-quinoline cooled with ice produces a white crystalline precipitate. This substance crystallizes from petroleum ether in colorless prisms or tabular crystals melting at $58^{\circ}$. Structurally it is 5,5 -dichloro-6-keto-quinoline. Solutions of this dichloro-keto-quinoline and of its hydrochloride are colored a pure green or blue by ammonium hydroxide. Fühner thinks 5,6-quinoline quinone is probably formed and gives the green color with ammonia.

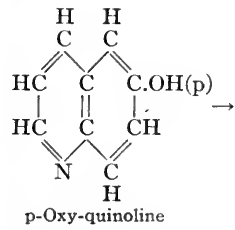

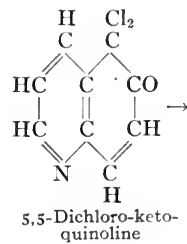

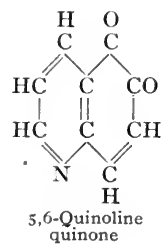

3. Herapathite Test.-Mix 30 drops of acetic acid, 20 drops of absolute ethyl alcohol and I drop of dilute sulphuric acid. Add 20 drops of this mixture to 0.01 gram of quinine and heat to boiling. Finally add I drop of an alcoholic solution of iodine ( $\mathrm{I}: \mathrm{IO}$ ) or 2 drops of o.I n-iodine solution. When the solution has stood for some time, green leaflets with a metallic luster will

1 Berichte der Deutschen chemischen Gesellschaft 38, 27 r3 (r905). 
form. This is an iodine compound of quinine called "Herapathite," having the constant composition

$$
{ }_{4} \mathrm{C}_{20} \mathrm{H}_{24} \mathrm{~N}_{2} \mathrm{O}_{2} \cdot 3 \mathrm{H}_{2} \mathrm{SO}_{4} \cdot 2 \mathrm{HI} \cdot 3 \mathrm{H}_{2} \mathrm{O} \text {. }
$$

This substance can be recrystallized from boiling ethyl alcohol. Herapathite crystals are pale olive-green by transmitted light but by reflected light they have a beautiful, cantharidingreen, metallic luster.

Caustic alkalies, ammonia, sulphurous acid and hydrogen sulphide decompose herapathite. A. Christensen recommends keeping on hand the following reagent for the herapathite test:

$\begin{array}{ll} & \text { Parts } \\ \text { Iodine } & \text { I } \\ \text { Hydriodic acid (50\%) } & \text { I } \\ \text { Sulphuric acid } & 0.8 \\ \text { Ethyl alcohol }(70 \%) & 50\end{array}$

Add a few drops of this reagent to the alcoholic solution to be tested for quinine.

4. Hirschsohn's Test. ${ }^{1}$ - If I drop each of 2 per cent. hydrogen dioxide and so per cent. copper sulphate solution are added to a neutral solution of quinine hydrochloride or sulphate at boiling temperature, a more or less intense raspberry-red color will appear. This color soon passes through blue-violet into blue and after a time into green. A quinine solution (I : Io,000) will still give a distinct red-violet color.

Excess of acid as well as of ethyl alcohol interferes with this test. The behavior of a solution of aloes toward this test is similar to that of quinine.

Of the alkaloidal reagents potassium bismuthous iodide is especially recommended as a precipitant of quinine. With quinine sulphate solutions this reagent produces precipitates having an intense yellowish red color. Shaken with sodium hydroxide solution this precipitate is decomposed and unaltered quinine can be obtained by extraction with ether and evaporation of the ether solution. H. Thoms ${ }^{2}$ has made use of this reaction in the quantitative separation of quinine from mixtures.

\section{CAFFEINE}

Since caffeine (see page 84 ) is a weak base, ether will extract only a little of the alkaloid from the tartaric acid solution. The

${ }^{1}$ Pharmazeutische Zentral-Halle $43,3 \epsilon_{7}$ ( $r_{902}$ ).

${ }^{2}$ Berichte der Deutschen pharmazeutischen Gesellschaft 16, I30 (1906). 
greater part will be in the ether extract of the alkaline solution. Ether usually deposits caffeine in white, shining needles arranged in clusters. Caffeine dissolves in ether with some difficulty and the alkaline solution should be extracted several times. For the tests characteristic of this alkaloid see page 85 .

\section{ANTIPYRINE}

Most of the antipyrine (see page 82) is obtained by extracting the alkaline solution with ether. It is usually purer from the acid than from the alkaline solution and frequently appears in crystalline leaflets. Antipyrine differs from most alkaloids in having only a faintly bitter taste and in being freely soluble in water. To identify antipyrine, dissolve the ether residue in a little water and divide the solution into two equal parts. Test one portion with ferric chloride solution and the other with fuming nitric acid.

Detection of Antipyrine in Urine.-The color of urine after administration of antipyrine is intensely yellow to blood-red. Part of the antipyrine in the organism appears in the urine as oxy-antipyrine-glycuronic acid and another part is unchanged and can usually be detected directly in urine by ferric chloride solution. A safer procedure is to add excess of ammonia to a considerable quantity of urine and extract with chloroform. Evaporate the solvent, dissolve the residue in a little water and test the filtered solution for antipyrine with ferric chloride solution and with fuming nitric acid.

Antipyrine is easily absorbed. The urine may show a reddish color, even an hour after the drug has been taken, and give a test with ferric chloride solution. The red color disappears in about 24 hours but the elimination of antipyrine is not complete in that time. Its detection is still possible after 36 hours. A convenient procedure is to add to the urine as an upper layer very dilute ferric chloride solution. A red ring will appear if the urine contains antipyrine. Jonescul states that antipyrine in the human organism passes unchanged into the urine. Only a small portion-and large doses of the drug must have been taken-is eliminated in conjugation with sulphuric acid. Conjugation with glycuronic acid $^{2}$ (see above) according to Jonescu does not occur in the human organism.

${ }^{1}$ Berichte der Deutschen pharmazeutischen Gesellschaft 16, I33 (I906).

${ }^{2}$ Glycuronic acid, $\mathrm{C}_{6} \mathrm{H}_{10} \mathrm{O}_{7}={ }_{\mathrm{O}}^{\mathrm{H}} \mathrm{C}(\mathrm{CH} . \mathrm{OH})_{4} \mathrm{COOH}$, may be regarded as a derivative of glucose. Possibly it occurs in normal urine in small quantity as a conjugated acid. After administration of various alcohols, aldehydes, ketones, phenols (chloral hydrate, camphor, phenol, thymol, menthol, borneol), there takes place in the animal organism - often after oxidation or reduction-a conjugation of these substances with glycuronic acid. 


\section{PYRAMIDONE}

Pyramidone, or 4-dimethyl-amino-antipyrine, $\mathrm{C}_{13} \mathrm{H}_{17} \mathrm{~N}_{3} \mathrm{O}$, has been extensively used in medicine of late as an antipy retic and anodyne. It is a white,

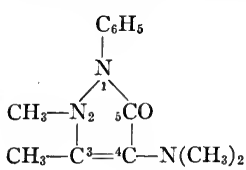
crystalline powder, nearly tasteless and readily soluble in water. It melts at $108^{\circ}$. Its aqueous solution has a neutral reaction. Ether removes only traces of pyramidone from acid solution, but extracts it easily and completely from alkaline solution. Ether usually deposits this substance in fine needles. Pyramidone is also freely soluble in ethyl alcohol, ether, chloroform or benzene. It is a strong reducing agent and in this respect differs from antipyrine. For example, pyramidone will reduce gold chloride even in the cold, whereas antipyrine and tolypyrine require heat.

Preparation.-Antipyrine dissolved in concentrated acetic acid is converted by treatment with potassium nitrite into nitroso-antipyrine which appears as green crystals. This compound dissolved in ethyl alcohol may be reduced by zinc and acetic acid to amino-antipyrine. The latter, dissolved in methyl alcohol and treated with methyl iodide and potassium hydroxide, is converted into dimethylamino-antipyrine, or pyramidone.

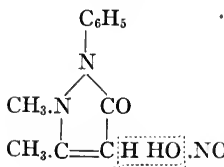

Antipyrine

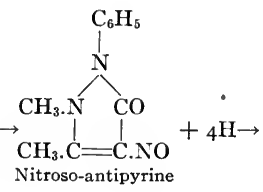

$\left.\mathrm{CH}_{3} \cdot \underset{\text { Pyramidone }}{\mathrm{C}}=\mathrm{CH}_{3}\right)_{2}$

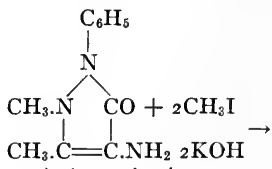

Amino-antipyrine

Behavior in the Organism.-Human urine, if neutral or faintly acid, usually has a bright purplish red color after administration of pyramidone. After standing for some time it will deposit a sediment consisting of red needles soluble in ether or chloroform but especially in acetic ether. Jaffé ${ }^{1}$ recognized this compound as rubazonic acid, a pyrazolone derivative. Isolation of rubazonic acid from urine may be brought about as follows. Acidify fresh urine with hydrochloric acid and let it stand in an open dish. The acid will appear as small red. particles. Ferric chloride solution produces a blue-violet color in the acid liquid filtered from rubazonic acid. This filtrate contains most of the product formed from pyramidone in animal metabolism, namely, crystalline antipyryl-urea melting at about $245^{\circ}$.

${ }^{1}$ Berichte der Deutschen chemischen Gesellschaft 34, 2737 (I90I); and 35, 289I (I902). 


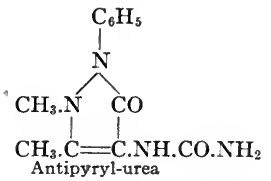

\section{Detection of Pyramidone}

I. Ferric Chloride Test.-Ferric chloride solution added to pyramidone produces a blue-violet color which soon changes to reddish violet and then disappears.

2. Fuming Nitric Acid Test.-A few drops of fuming nitric acid, added to a solution containing pyramidone, give a blue to blue-violet color.

3. Bromine Water Test.-This reagent imparts a grayish color to pyramidone solutions. With concentrated solutions it produces an inky color.

4. Iodine Test.-Tincture of iodine colors an aqueous pyramidone solution blue.

5. Guglialmelli's ${ }^{1}$ Test.-Either of the following two reagents may be used, but (b) usually gives the better result:

(a) Arseno-tungstic Solution.-Dissolve 25 grams of sodium tungstate $\left(\mathrm{Na}_{2} \mathrm{WO}_{4} \cdot 2 \mathrm{H}_{2} \mathrm{O}\right)$ in $200 \mathrm{cc}$. of cold distilled water, adding 20 grams of pure arsenic trioxide $\left(\mathrm{As}_{2} \mathrm{O}_{3}\right)$ and boiling the solution for $\mathrm{I} .5$ hours under a reflux-condenser. Filter the resulting light, bluish green solution when cold and bring the volume to $25^{\circ} \mathrm{cc}$.

(b) Arseno-tungsto-molybdic Solution.-Boil in the same manner ro grams of sodium tungstate, 2 grams of sodium molybdate $\left(\mathrm{Na}_{2} \mathrm{MoO}_{4} \cdot \mathrm{I}_{2} \mathrm{H}_{2} \mathrm{O}\right)$ and 10 grams of pure arsenic trioxide for $\mathbf{I}^{-2}$ hours with $75 \mathrm{cc}$. of distilled water. Bring the cold solution to a volume of roo cc.

Added to aqueous pyramidone solutions, these reagents produce white spots. Those from reagent $(a)$ are soluble in alkali with an intense blue color; whereas those from reagent (b) give an intense indigo color. Pyramidone produces these colors in a dilution of $\mathrm{I}: 750,000$.

These reagents produce white spots with antipyrine solutions but they are soluble in alkali without color.

${ }^{1}$ Chemical Abstracts I 2, 664 (1918). 
6. Palet's ${ }^{1}$ Test.-A few drops of a freshly prepared solution of potassium ferricyanide and ferric chloride, added to an aqueous pyramidone solution, produce the characteristic blue color and precipitate of Prussian blue. The reaction is very sensitive. Morphine gives the same test (see page I35).

With antipyrine the reagent gives a blood-red color and precipitate and is negative with phenacetine, acetanilide and caffeine. If pyramidone and antipyrine are together, a little hydrochloric acid should first be added.

\section{Extraction of the Ammoniacal Solution with Ether and Chloroform}

( $\alpha$ ) Ether Extract.-Apomorphine and traces of morphine. ${ }^{2}$

( $\beta$ ) Chloroform Extract.-Morphine and narceine. (It may also contain antipyrine and caffeine. ${ }^{3}$ )

The aqueous alkaline solution (see page 86 ), separated from ether, must be tested further for the substances under $a$ and $\beta$.

Apomorphine may be recognized by the green color of the aqueous acid solution. Excess of sodium hydroxide solution causes oxidation, especially if the solution is exposed for any length of time to air, and gradually changes the color to deep purple-red. Moreover, the ether extracts, both of the acid and alkaline solutions, are red or violet-red when apomorphine is present. Solutions, examined by the Stas-Otto method, not having these characteristics, need not be tested for apomorphine. In that case proceed at once with the morphine and narceine tests.

To extract apomorphine, morphine and narceine with the proper solvent, the aqueous solution separated from ether, which is alkaline from sodium hydroxide solution (see page 86 ), must be rendered alkaline with ammonium hydroxide solution. First acidify the solution with dilute hydrochloric acid (test with blue litmus paper) and then add ammonium hydroxide solution until alkaline.

${ }^{1}$ Chemical Abstracts, 13, 216 (1919).

${ }^{2}$ Ether dissolves traces of freshly precipitated, amorphous morphine.

${ }^{8}$ Antipyrine and caffeine, though freely soluble in chloroform, dissolve with difficulty in ether. The latter solvent frequently fails to extract these substances completely from aqueous solution. They will then appear in the chloroform
extract. 
( $\alpha$ ) If there is any indication of apomorphine, first extract the ammoniacal solution repeatedly with ether and then several times with hot chloroform for the morphine and narceine tests.

$(\beta)$ If there is no indication of apomorphine, extract the ammoniacal solution several times direct with hot chloroform (see below).

\section{APOMORPHINE}

Constitution.-Apomorphine, $\mathrm{C}_{17} \mathrm{H}_{17} \mathrm{NO}_{2}$, is a monacid, tertiary base with two phenol hydroxyl groups. According to R. Pschorr ${ }^{1}$ it has the structural formula here given.

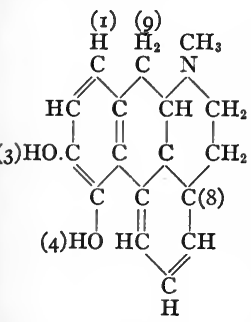

Properties.-Apomorphine is an amorphous base readily soluble in ethyl alcohol, ether, benzene or chloroform and colored green in contact with air. Aqueous and alcoholic apomorphine solutions, originally colorless, soon turn green in the air from oxidation. Solutions of apomorphine thus changed by oxidation are emerald-green. Ether and benzene solutions are purplish violet; those in chloroform blueviolet. Being phenolic in character, apomorphine resembles morphine in its solubility in sodium hy. droxide solution. Alkaline solutions of the alkaloid absorb oxygen from the air and become brown or even black in color. Apomorphine differs from morphine in being more soluble in water and in ethyl alcohol, but especially in being soluble in ether, benzene and cold chloroform, in which morphine is almost insoluble.

Formation and Preparation.-Sulphuric, hydrochloric, phosphoric and oxalic acids, the alkalies and zinc chloride have mainly a dehydrating action upon morphine and convert it into apomorphine:

$$
\underset{\text { Morphine }}{\mathrm{C}_{17} \mathrm{H}_{19} \mathrm{NO}_{3}}=\mathrm{H}_{2} \mathrm{O}+\underset{\text { Apomorphine }}{\mathrm{C}_{17} \mathrm{H}_{17} \mathrm{NO}_{2}}
$$

Codeine, the methyl ether of morphine, also gives apomorphine when heated at $140^{\circ}$ with concentrated hydrochloric acid.

$$
\mathrm{C}_{17} \mathrm{H}_{18} \mathrm{NO}_{\text {Codeine }}\left(\mathrm{OCH}_{3}\right)+\mathrm{HCl}=\mathrm{H}_{2} \mathrm{O}+\mathrm{CH}_{3} \mathrm{Cl}+\underset{\text { Apomorphine }}{\mathrm{C}_{17} \mathrm{H}_{17} \mathrm{NO}_{2}}
$$

Apomorphine is prepared by heating morphine (I part) with concentrated hydrochloric acid (20 parts) for 3 hours in an autoclave at $130-150^{\circ}$.

${ }^{1}$ Berichte der Deutschen chemischen Gesellschaft 39, 3I24 (1906); and 40, I984 (1907). 


\section{( $\alpha$ ) Detection of Apomorphine in the Ether Extract}

Ether will not extract apomorphine from a solution containing tartaric acid but will dissolve its colored oxidation products. This solvent behaves similarly toward solutions of this alkaloid in sodium or potassium hydroxide solutions. Ether or chloroform will extract apomorphine only from a solution alkaline with ammonium hydroxide. Ether solutions of apomorphine usually deposit a greenish residue. A characteristic of this alkaloid is its strong reducing action. For example, it will reduce iodic acid with liberation of iodine and produce a purple color with gold chloride. Apomorphine gives the following tests:

I. Sulphuric and Nitric Acids.-Concentrated sulphuric acid dissolves apomorphine without color. Addition of a drop of concentrated nitric acid to such a solution produces an evanescent violet color that soon changes to blood-red and finally to yellowish red. With concentrated nitric acid alone this alkaloid gives a violet-red color that soon becomes red-brown and finally brownish red.

2. Pellagri's Test.-Dissolve apomorphine in dilute hydrochloric or sulphuric acid and first add acid sodium carbonate in excess. Then add drop by drop $\mathrm{I}-3$ drops of an alcoholic iodine solution and shake for several minutes. The solution will have a blue-green or emerald-green color. Extract with a little ether and the solvent will become violet, whereas the aqueous solution will remain green.

3. Froehde's Test.-This reagent dissolves pure apomorphine with a green color. If the alkaloid has been acted upon by air to any extent, the color is violet.

4. Wangerin's ${ }^{1}$ Test.- Prepare a fresh solution of apomorphine hydrochloride (about I per cent.). Add 4 drops of potassium dichromate solution ( 0.3 per cent.) to I cc. of this solution and shake for about I minute. The solution will have an intense dark green color. Then add ro cc. of acetic ether and shake again. This solvent will become violet. Finally add

${ }^{1}$ Pharmazeutische Zeitung 47, 599 and 739-740 (r9o2). 
from a pipette about 5 drops of stannous chloride solution ${ }^{1}$ (I per cent.) and shake well. The color of the acetic ether layer will change to green and, upon further addition of a few drops of potassium dichromate solution, the acetic ether will again become violet. If Io cc. of chloroform are substituted for acetic ether in this test, the oxidation product of apomorphine will impart the same violet color to the chloroform. But if stannous chloride solution is added carefully, the color will change to pure indigo-blue and persist upon further agitation with potassium dichromate solution.

5. E. Schmidt's Tests. ${ }^{2}-(a)$ A drop of very dilute ferric chloride solution ( $\mathrm{I}: \mathrm{I00}$ ) will color ro cc. of an aqueous apomorphine hydrochloride solution blue even in a dilution of I : Io,o00.

(b) Shake ro cc. of the same apomorphine hydrochloride solution with I cc. of chloroform. Then render alkaline with sodium hydroxide solution and at once shake with air. The aqueous solution becomes evanescent violet in color and the chloroform blue.

6. Palet's Test. ${ }^{3}-$ Add I-2 drops of apomorphine solution to $\mathrm{I}-2$ cc. of Guglialmelli's reagents. ${ }^{4}$ After shaking the mixture for 2-3 minutes, add 5-10 cc. of a cold, saturated solution of pure sodium carbonate. An indigo-blue color, varying in intensity with 'concentration and time, appears. Divide the liquid after 5 minutes into 3 portions. These treated respectively with amyl alcohol, benzene and acetic ether give rise to an intense blue, dark violet and violet color. Further addition of $\mathrm{I}-2$ drops of ro per cent. stannous chloride solution to the acetic ether extract changes the violet color to emeraldgreen as in Wangerin's test. Apomorphine diluted I:500,000 gives a distinct blue color, and a slightly positive result when

1 Prepare this reagent as follows:

Crystallized stannous chloride $\left(\mathrm{SnCl}_{2.2} \mathrm{H}_{2} \mathrm{O}\right)$

Hydrochloric acid (25 per cent.)

I gram

Water

$50 \mathrm{cc}$.

$50 \mathrm{cc}$.

${ }^{2}$ Apotheker-Zeitung 23, 657 (I908).

${ }^{3}$ Chemical Abstracts r 2, 6or (rgr 8 ).

${ }^{4}$ See page 125 for the preparation of these reagents. 
diluted I : I,000,000. In all cases the intensity of the color increases on standing.

Morphine gives the same blue color which is not extracted by solvents. Narcotine and narceine do not give this reaction.

\section{( $\beta$ ) Examination of the Chloroform Extract}

Preliminary Morphine Test.-As a preliminary test for morphine, acidify a small portion of the aqueous alkaline solution separated from ether (see page 85) with dilute sulphuric acid, add iodic acid solution and extract' with a little chloroform. If the latter has a violet color from dissolved iodine, morphine may be present. But a final conclusion regarding the presence of morphine must not be drawn from a positive test, since there are many other organic substances besides this alkaloid that will reduce iodic acid. ${ }^{1}$ This is a delicate preliminary test for morphine and that is its only value. If it is negative, morphine is probably absent.

To detect morphine and narceine positively, render the aqueous, solution alkaline with ammonium hydroxide and extract at once as already directed (see page I26) with considerable hot chloroform ${ }^{2}$ in a capacious flask. Separate the two liquids as usual in a separatory funnel. Several extractions of the aqueous solution with fresh portions of hot chloroform are necessary because of the slight solubility of morphine even in boiling chloroform. Should the chloroform and the aqueous solution form a refractory emulsion that will not separate, add a few drops of ethyl alcohol, set the flask on a warm but not boiling water-bath and carefully turn the flask from time to time. This procedure usually causes the immediate separation of the two liquids. Place the combined chloroform extracts in a dry flask, add a few crystals of dry sodium chloride or anhydrous sodium sulphate to remove adherent water, pour the chloroform when clear through a dry filter and evaporate in not too large a glass

${ }^{1}$ In testing animal matter that contained no morphine, the author has repeatedly obtained extracts that strongly reduced iodic acid.

${ }^{2}$ C. Kippenberger (Zeitschrift für analytische Chemie 39, 20r, 290) uses chloroform, containing ro per cent. of ethyl alcohol by volume, to extract morphine. 
dish placed upon a warm water-bath. The chloroform may also be filtered directly into the dish as fast as it evaporates. If the residue is bitter and can be scraped together with a platinum spatula or a pocket-knife, test for morphine and narceine. ${ }^{1}$ In testing for morphine use Froehde's, Husemann's and Pellagri's tests as well as those given by formalin-sulphuric acid and iodic acid. The presence of morphine is not established unless all these morphine tests give positive results. If the quantity of the residue from chloroform permits, test for morphine with ferric chloride solution. This test is very characteristic of morphine but requires more than traces for a satisfactory result.

\section{Purification of Impure Morphine}

When the chloroform residue is too impure, especially if red or brown, it must be purified. Dissolve in hot amyl alcohol and shake the solution thoroughly with several portions of hot water containing a few drops of dilute sulphuric acid. The acid dissolves the morphine, whereas the amyl alcohol retains most of the coloring matter. Add ammonium hydroxide solution in 'excess to the acid solution and extract several times with hot chloroform. The morphine obtained by evaporation of the chloroform should be nearly pure.

\section{MORPHINE}

Morphine, $\mathrm{C}_{17} \mathrm{H}_{19} \mathrm{NO}_{3}$, crystallizes from dilute ethyl alcohol in shining prisms which are colorless and transparent and but slightly soluble in water ( $1: 5000$

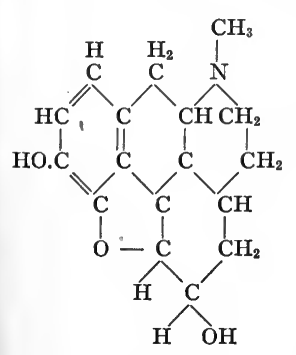

at $15^{\circ}$; and $1: 500$ at $\left.100^{\circ}\right)$. These solution. are very bitter and have an alkaline reactions Crystalline morphine is insoluble in ether and benzene. The amorphous alkaloid is soluble in amyl alcohol, hot chloroform and acetic ether. Solutions of the hydroxides of ammonia, potassium or sodium and sodium carbonate solution precipitate free morphine from solutions of morphine salts.

Constitution.-Morphine is a monacid, tertiary base whose nitrogen is in union with three atoms of carbon. The three oxygen atoms have different functions. One is a phenolic hydroxyl and gives to morphine the ${ }^{1}$ Antipyrine and caffeine may also be in this residue (see above). 
character of a monatomic phenol. Conseqeuntly when sodium hydroxide solution is added drop by drop to a morphine salt solution, there is first a precipitate of crystalline morphine (a) which is freely soluble in excess of alkali $(\beta)$ but is again precipitated on addition of ammonium chloride solution $(\gamma)$ :

(a) $\mathrm{C}_{17} \mathrm{H}_{18} \mathrm{NO}_{2}(\mathrm{OH}) \cdot \mathrm{HCl}+\mathrm{NaOH}=\mathrm{C}_{17} \mathrm{H}_{18} \mathrm{NO}_{2}(\mathrm{OH})+\mathrm{H}_{2} \mathrm{O}+\mathrm{NaCl}$,

(ק) $\mathrm{C}_{17} \mathrm{H}_{18} \mathrm{NO}_{2}(\mathrm{OH})+\mathrm{NaOH}=\mathrm{C}_{17} \mathrm{H}_{18} \mathrm{NO}_{2}(\mathrm{ONa})+\mathrm{H}_{2} \mathrm{O}$,

( $\gamma) \mathrm{C}_{17} \mathrm{H}_{18} \mathrm{NO}_{2}(\mathrm{ONa})+\left(\mathrm{H}_{4} \mathrm{~N}\right) \mathrm{Cl}=\mathrm{C}_{17} \mathrm{H}_{18} \mathrm{NO}_{2}(\mathrm{OH})+\mathrm{NH}_{3}+\mathrm{NaCl}$.

Hydrogen of this phenolic hydroxyl may be replaced also by alkyl groups and acid radicals. In codeine this hydrogen is replaced by methyl. A second oxygen atom of morphine is alcoholic and the third is indifferent. The latter like the oxygen of an ether is combined with two carbon atoms and forms a socalled bridge-oxygen atom.

Of the I 7 carbon atoms of morphine I 4 belong to the phenanthrene nucleus, ${ }^{1}$ since the nitrogen-free cleavage-products of morphine and codeine, namely, morphol and morphenol, have been identified as phenanthrene derivatives. R. Pschorr has synthesized morphol which is 3,4-dioxyphenanthrene. Morphenol contains two hydrogen atoms less and may be converted into morphol by reduction with nascent hydrogen. These two phenanthrene derivatives have the following structural formulæ:
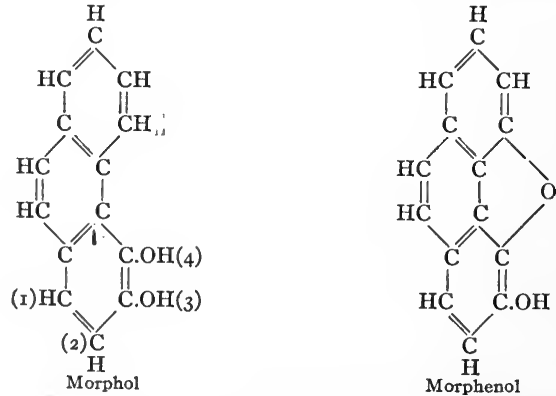

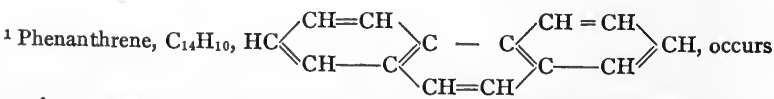

in coal-tar together with anthracene. It forms colorless crystals which melt at $99^{\circ}$ and boil at $340^{\circ}$. It is readily soluble in ether or benzene and with difficulty in ethyl alcohol. Phenanthrene solutions exhibit bluish fluorescence. 
By distillation over zinc dust morphenol may be reduced to phenanthrene.

The structural formula of morphine written above was proposed by R. Pschorr ${ }^{1}$ and seems to explain most satisfactorily the reactions of this alkaloid.

Morphine is easily oxidized. This may be brought about in alkaline solution by atmospheric oxygen. Potassium permanganate or ferricyanide and ammoniacal copper solution may also be used. As a result the non-toxic oxy-dimorphine, also called pseudomorphine, which is soluble in caustic alkali, is formed:

$$
\underset{\text { Morphine }}{\mathrm{C}_{17} \mathrm{H}_{19} \mathrm{NO}_{3}}+\mathrm{O}=\underset{\text { Oxydimorphine }}{\left(\mathrm{C}_{17} \mathrm{H}_{18} \mathrm{NO}_{3}\right)_{2}}+\mathrm{H}_{2} \mathrm{O} .
$$

\section{Detection of Morphine}

r. Nitric Acid Test.-Concentrated nitric acid dissolves morphine with a blood-red color which gradually changes to yellow. Stannous chloride or ammonium sulphide solution will not restore the violet color of a solution that has become yellow. (Distinction from brucine.)

2. Husemann's Test.-Dissolve morphine upon a watchglass in a few cc. of concentrated sulphuric acid. The solution is colorless. Heat for 30 minutes upon the water-bath, or over a small flame for a very short time until white fumes arise. A reddish or brownish color appears. Cool and add I-2 drops of concentrated nitric acid. A fugitive, reddish violet color appears and soon changes to blood-red or yellowish red. This color gradually disappears.

A preferable procedure is to dissolve morphine in cold concentrated sulphuric acid and add a trace of concentrated nitric acid after the solution has stood in a desiccator 24 hours. A small crystal of potassium nitrate or chlorate may be substituted for nitric acid.

Frequently impure morphine is obtained from the chloroform extract of a solution prepared from animal material.; Such a residue gives a more or less

1 Berichte der Deutschen chemischen Gesellschaft 40, 1984 (1907). 
highly colored solution with sulphuric acid. Heat usually intensifies the color. But even under these conditions it is possible to detect the red color caused by nitric acid or potassium nitrate.

3. Pellagri's Test.-Proceed as described for codeine. (See page I I2.), Avoid excess of alcoholic iodine solution, otherwise the latter may mask the green color.

4. Froehde's Test.-This reagent dissolves morphine with a violet color which passes through blue to dirty green and finally to faint red. These colors vanish on addition of water.

5. Formaldehyde-Sulphuric Acid Test.-The solution used for this test is called Marquis' reagent. ${ }^{1}$ With a trace of morphine it produces a purple-red color which changes to violet and finally becomes pure blue. This blue solution, kept in a test-tube and only slightly exposed to air, retains its color for some time. Codeine and apomorphine give the same violet color. Narcotine also gives violet solutions but they become olive-green and finally yellow. Oxy-dimorphine gives a green color.

6. Iodic Acid Test.-Shake a solution of morphine in dilute sulphuric acid with a few drops of iodic acid and chloroform. Morphine will liberate iodine which will dissolve in chloroform with a violet color.

Obviously this delicate test is conclusive for morphine only in the absence of other reducing substances.

7. Ferric Chloride Test.-Add I-2 drops of neutral ferric chloride solution to a neutral solution of a morphine salt. A blue color appears. In testing the chloroform residue, dissolve in a little very dilute hydrochloric acid. Evaporate this solution to dryness upon the water-bath, dissolve the residue in pure water and add a drop of ferric chloride solution.

8. Lloyd's Test.-Lloyd has found that a mixture of morphine, hydrastine and concentrated sulphuric acid alone without potassium dichromate will produce the same violet color given by the latter with a solution of strychnine in concentrated sul-

${ }^{1}$ Mix 2-3 drops of 40 per cent. formaldehyde solution with $5 \mathrm{cc}$. of concentrated sulphuric acid and use a few drops of this mixture for the morphine test. 
phuric acid. Lloyd's reaction is of value in the detection of morphine or hydrastine only when more than traces of both alkaloids are present. A. Wangerin ${ }^{1}$ considers these reactions characteristic only when $0.005-0.01$ gram of morphine and $0.002-0.01$ gram of hydrastine are present.

Make an intimate mixture of about these quantities of both alkaloids upon a watch-glass. Add 5 drops of pure concentrated sulphuric acid and stir the mixture for Io minutes over a white background. In the center the color-tone is a clear red-violet and more or less of a blue-violet in the thinner marginal region. Apomorphine hydrochloride, treated in the same way with hydrastine and concentrated sulphuric acid, gives almost the same reaction as morphine.

9. Prussian Blue Test.-Add a few drops of a dilute mixture of ferric chloride and potassium ferricyanide solutions to a morphine salt solution. A deep blue color appears. Considerable morphine produces a precipitate of Prussian blue.

Potassium ferricyanide oxidizes morphine to oxy-dimorphine:

$$
\underset{\text { Morphine }}{2 \mathrm{C}_{17} \mathrm{H}_{19} \mathrm{NO}_{3}}+{ }_{2} \mathrm{KOH}+\underset{\substack{\text { Potassium } \\ \text { ferricyanide }}}{\mathrm{K}_{6} \mathrm{Fe}_{2}(\mathrm{CN})_{12}}={ }_{2} \mathrm{H}_{2} \mathrm{O}+\underset{\text { Oxy-dimorphine }}{\left(\mathrm{C}_{17} \mathrm{H}_{18} \mathrm{NO}_{3}^{\prime}\right)_{2}}+\underset{\substack{\text { Potassium } \\ \text { ferrocyanide }}}{2 \mathrm{~K}_{4} \mathrm{Fe}(\mathrm{CN})_{6}}
$$

Potassium ferrocyanide then forms Prussian blue with ferric chloride.

Io. Silver Test.-Warm a morphine salt solution with silver nitrate and excess of ammonium hydroxide solution. Morphine produces a gray precipitate of metallic silver.

II. Bismuth Test.-Dissolve morphine in concentrated sulphuric acid and sprinkle a little bismuth subnitrate on the surface of the solution. A dark brown color appears.

12. G. Fleury's Test. ${ }^{2}-$ Dissolve morphine in a little very dilute sulphuric acid (about 0.05 normal), add some lead dioxide $\left(\mathrm{PbO}_{2}\right)$ and shake for 6-8 minutes. A pale rose color appears. Addition to the filtrate of ammonium hydroxide solution in excess produces a brown color which persists for

1 Pharmazeutische Zeitung 46, 57 (1903).

${ }^{2}$ Annales de Chimie analytique appliquée 6, 4r7 (1907). 
several hours. When the quantity of substance is very small, stir on a porcelain color-plate for 6-8 minutes with a drop of dilute sulphuric acid and a minute particle of lead dioxide. When the insoluble matter has settled, tilt the porcelain plate so that the clear solution runs up the side. A drop of ammonium hydroxide solution now gives a brown color.

I3. Dan Radulescu's Test. ${ }^{1}$ - Add a small particle of sodium nitrite to a very dilute morphine salt solution, then a dilute acid and render alkaline with concentrated potassium hydroxide solution before all the gas has escaped. The solution when concentrated has a pale rose to a deep ruby-red color. Acids discharge but alkalies restore this color. This reaction is said to be characteristic of morphine bases and especially adapted for the detection of morphine in mixtures.

14. Diazonium Test. ${ }^{2}$ - In presence of alkalies, morphine and its salts form dyestuffs with diazonium compounds. The reagent is a solution of diazotized sulphanilic acid.

Diazonium Reagent.-Dissolve 0.2 gram of sulphanilic acid $\left(\mathrm{C}_{6} \mathrm{H}_{4}\left\langle\begin{array}{l}\mathrm{NH}_{2}(\mathrm{r}) \\ \mathrm{SO}_{3} \mathrm{H}(4)\end{array}\right)\right.$ in $80 \mathrm{cc}$. of water, cooling with ice. Then add ro cc. o.I $\mathrm{n}-\mathrm{HCl}$ and ro cc. O.I $\mathrm{n}-\mathrm{NaNO}_{2}$.

$$
\mathrm{C}_{6} \mathrm{H}_{4}\left\langle{ }_{\mathrm{SO}_{3} \mathrm{H}}^{\mathrm{NH}_{2}}+\mathrm{HCl}+\mathrm{NaNO}_{2}=\mathrm{C}_{6} \mathrm{H}_{4}\left\langle\begin{array}{l}
\mathrm{N}=\underset{\mathrm{O}}{\mathrm{N}} \\
\mathrm{SO}_{2^{-}} \mathrm{O}
\end{array}+\mathrm{NaCl}+{ }_{2} \mathrm{H}_{2} \mathrm{O} .\right.\right.
$$

Dissolve 0.243 gram of morphine sulphate in roo cc. of water. Render 5 cc. of this solution alkaline with sodium carbonate or bicarbonate and add an equal volume of diazonium reagent. A red color, changing to orange on addition of acid, immediately appears. The color diluted $\mathrm{x}: \mathrm{x}, 000$ is faint red; $\mathrm{I}: \mathrm{x}, 0,000$ distinctly yellow; and $\mathrm{x}: 2000^{3}$ intensely dark red in a thin layer.

No opium alkaloid except morphine gives this reaction; not even the synthetic derivatives of this alkaloid (dionine, peronine and heroine). Other alkaloids

${ }^{1}$ Chemisches Zentralblatt 1906, I, 1378.

2 Lautenschlaeger: Chemical Abstracts 13, 2252 (I919).

${ }^{3}$ If the above proportions are used and the test is made as directed, the dilution will be $\mathrm{r}: 2000$. 
giving colors are: emetine and physostigmine (red); sparteine and piperidine (yellow); coniine and nicotine (bright yellow). But the morphine color above is stable in acid solution.

General Alkaloidal Reagents.-The reagents of this class especially sensitive toward solutions of morphine salts are:

Iodo-potassium iodide, Phospho-tungstic acid, Potassium mercuric iodide,
Potassium bismuthous iodide, Phospho-molybdic acid, Gold chloride.

Plantinic chloride after some time causes a granular orangeyellow precipitate. Tannic acid causes no precipitate, or at most only a very slight cloudiness which becomes somewhat more pronounced with time.

Behavior of Morphine in the Animal Organism.-The mucous lining of the stomach, rectum or respiratory passages as well as open wounds absorb morphine. The alkaloid injected hypodermically acts more rapidly and more potently than when absorbed from the stomach. Marquis ${ }^{1}$ found that morphine disappears very quickly from the blood but is firmly retained by certain organs like the brain. Some absorbed morphine is conjugated with glycuronic acid and some is oxidized but the rest of the alkaloid is eliminated unchanged. Faust has found that morphine is transformed or destroyed only in men and animals habituated to the poison but is eliminated unchanged nearly quantitatively in the fæces in the case of organisms not immunized. Morphine appears in the urine only in very small quantity after medicinal doses. In men and dogs a not insignificant quantity of the morphine taken is eliminated by the glands of the gastro-intestinal tract, even when the alkaloid has been subcutaneously injected.

Marquis found that more than 30 per cent. of intravenously injected morphine is deposited in the liver in the course of 15 minutes. The alkaloid is present at first in this organ in the free state and then is soon combined or transformed. The conjugation of morphine in the brain also begins very soon. Free morphine is also rapidly changed in the blood, spleen, kidneys and in the mucous lining of the intestines. Marquis states that always in acute and even more so in chronic morphine poisoning a large quantity of the poison leaves the blood and is stored in the salivary glands, mucous lining of the stomach and large intestine, kidneys, spleen, liver and is withdrawn by these organs from the brain and spinal cord.

Morphine is quite resistant to putrefaction. The author ${ }^{2}$ detected this alkaloid positively in animal material containing morphine (stomach and intestines together with contents) which had stood for 15 months in a glass vessel and had completely putrefied in presence of insufficient air. Doepmann ${ }^{3}$ obtained the

${ }^{1}$ Arbeiten des Dorpater Instituts, ed Kobert, ${ }_{4}$ (I896).

${ }^{2}$ Berichte der Deutschen Pharmazeutischen Gesellschaft Ir, 494 (Igor).

${ }^{3}$ Chemical Abstracts 9, I663 (I9r5). 
same result by mixing definite quantities of morphine hydrochloride with lean horse meat and allowing putrefaction to take place from one to eleven months.

\section{NARCEINE}

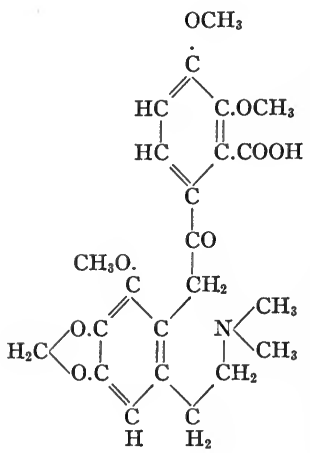

Narceine, $\mathrm{C}_{23} \mathrm{H}_{27} \mathrm{NO}_{3.3} \mathrm{H}_{2} \mathrm{O}$, crystallizes from water or etbyl alcohol in prisms which melt at I $65^{\circ}$ when air-dried. The alkaloid has a faintly bitter taste. Though only slightly soluble in cold water, it is freely soluble in hot. When a hot saturated aqueous solution of narceine is cooled, it solidifies to a crystalline mass. Narceine is insoluble in ether, benzene or petroleum ether and is soluble only with difficulty in cold ethyl alcohol, amyl alcohol or chloroform. In detecting narceine it is important to know that it is not extracted by ether, benzene or petroleum ether from a solution rendered alkaline by potassium or sodium hydroxide solution. It is, however, extracted by hot chloroform or amyl alcohol from an aqueous solution rendered alkaline by ammonium hydroxide solution.

Constitution.-Narceine is a weak tertiary base in which two methyl groups are attached to nitrogen. By means of Zeisel's method it may be shown that the molecule also contains three methoxyl groups. Narceine, being soluble in caustic alkalies and forming esters with alcohols, must contain a carboxyl group. The alkaloid must also contain a carbonyl group (CO), since it forms a hydrazone with phenyl-hydrazine. The narceine formula above may therefore be resolved into:

$$
\mathrm{C}_{23} \mathrm{H}_{27} \mathrm{NO}_{8}=\mathrm{C}_{16} \mathrm{H}_{11} \mathrm{ON}\left(\mathrm{CH}_{3}\right)_{2}\left(\mathrm{OCH}_{3}\right)_{3}(\mathrm{CO})(\mathrm{COOH}) \text {. }
$$

The narceine molecule contains neither an alcoholic nor a phenolic hydroxyl group, since it forms no acetyl derivative with acetic anhydride. There is a close relationship between narceine and narcotine. By heating narcotine iodo-methylate with sodium hydroxide solution Roser converted this compound into a base called pseudo-narceine. Freund has recently shown that Roser's pseudo-narceine is identical with the opium alkaloid narceine and explains the conversion of narcotine into narceine by saying that the iodo-methylate loses I molecule of hydriodic acid and takes up I molecule of water: 


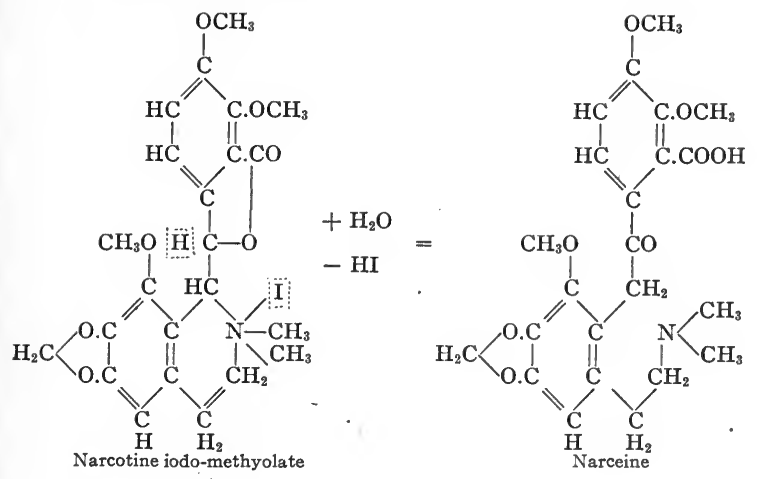

All the reactions and transformations of narceine can easily be explained on the basis of this structural formula.

\section{Detection of Narceine}

I. Sulphuric Acid Test.-Concentrated sulphuric acid dissolves narceine with a grayish brown color, which gradually changes to blood-red. This reaction takes place at once with heat.

2. Dilute Sulphuric Acid Test.-Narceine, warmed in a porcelain dish upon the water-bath with dilute sulphuric acid until a certain concentration is reached, gives rise to a fine violet color which changes after long heating to cherry-red.

3. Froehde's Test.-At first a solution of narceine in this reagent has a brownish green color which gradually changes to green and finally to red. Gentle heat hastens this reaction.

4. Iodine Test.-Aqueous iodine solution (iodine water) or iodine vapor colors solid narceine blue.

Morphine interferes with or entirely prevents this reaction.

5. Erdmann's Test.-This reagent, as well as concentrated nitric acid, dissolves narceine with a yellow color which heat changes to dark orange.

6. Chlorine-Ammonia Test.-Pour a few drops of chlorine water upon narceine and add, while stirring, a few drops of 
ammonium hydroxide solution. A deep red color immediately appears.

7. Resorcinol-Sulphuric Acid Test. ${ }^{1-M i x}$ thoroughly upon a watch-glass resorcinol (0.01 to $0.02 \mathrm{gram}$ ) with ro drops of pure concentrated sulphuric acid. Add a trace of narceine (about 0.002 to 0.005 gram) and, while stirring, warm the intensely yellow solution upon a boiling water-bath. A carminered to cherry-red color appears. As the solution cools, this color begins at the margin to change gradually to more of a blood-red and finally after several hours to orange-yellow.

8. Tannin-Sulphuric Acid Test.-Mix narceine ( 0.002 to o.or gram) with tannin (o.or to 0.02 gram) and Io drops of pure concentrated sulphuric acid. Heat with constant stirring upon the water-bath and the color of the solution, which is yellowish brown at first, soon becomes pure green. If heat is applied for some time, the green color changes to blue-green and finally through a more or less blue tone to a dirty green.

Tannin-sulphuric acid gives a similar color test with narcotine and hydrastine which closely resemble narceine in constitution.

Of the general alkaloidal reagents potassium zinc iodide ${ }^{2}$ precipitates narceine even in a dilution of $I:$ rooo. It is a white, filiform precipitate which after a time becomes blue. This blue color appears immediately, if a trace of iodine solution is added to the reagent.

Of the other general reagents iodo-potassium iodide, potassium mercuric iodide, potassium bismuthous iodide and phospho-molybdic acid are characterized by considerable delicacy toward narceine.

\section{SYNOPSIS OF GROUP II}

\section{Stas-Otto Method}

\section{A. Ether Extract of Acid Solution may Contain :} heat.

Picrotoxin.-Very bitter. Reduces Fehling's solution with

Melzer's test: red streaks radiating from picrotoxin with alcoholic benzaldehyde + conc. $\mathrm{H}_{2} \mathrm{SO}_{4}$.

${ }^{1}$ A. Wangerin, Pharmaceutische Zeitung, 47, 9r6 (r9o2).

${ }^{2}$ See page 3 I 9 for the preparation of this reagent. 
Conc. $\mathrm{H}_{2} \mathrm{SO}_{4}$ : soluble with yellow or orange-red color; drop of $\mathrm{K}_{2} \mathrm{Cr}_{2} \mathrm{O}_{7}+\mathrm{Aq}$ has brown margin.

Langley's test: picrotoxin +3 parts $\mathrm{KNO}_{3}$, moistened with conc. $\mathrm{H}_{2} \mathrm{SO}_{4}$, red with excess of saturated $\mathrm{NaOH}+\mathrm{Aq}$.

Colchicin.-Very bitter. Yellowish and amorphous. Dilute mineral acids render aqueous solutions intensely yellow.

Conc. $\mathrm{HNO}_{3}$ : soluble with dirty violet color changing to brownish red and finally to yellow; excess of $\mathrm{KOH}+\mathrm{Aq}$ renders orange-yellow or orange-red.

Zeisel's test: boil yellow colchicin solution in conc. $\mathrm{HCl}$ in testtube ${ }^{2-} 3$ minutes with 2 drops of $\mathrm{FeCl}_{3}+\mathrm{Aq}$. Green or olivegreen when cold, especially if diluted with equal volume of water.

Picric Acid.-Very bitter. Yellow. Material and extracts more or less intensely yellow.

Isopurpuric acid test: aqueous picric acid, gently warmed with a few drops of saturated $\mathrm{KCN}+\mathrm{Aq}$, gives red color.

Picramic acid test: aqueous picric acid, warmed with few drops of $\left(\mathrm{H}_{4} \mathrm{~N}\right)_{2} \mathrm{~S}+\mathrm{Aq}$, becomes red.

Dyeing test: aqueous picric acid dyes wool and silk intense yellow but not cotton.

Acetanilide.-Faint, burning taste.

Indophenol test: heat with a few cc. of conc. $\mathrm{HCl}$ and evaporate to about 20 drops. Cool, add aqueous phenol solution and then calcium hypochlorite solution drop by drop. Mixture, shaken with excess of ammonia, becomes dirty red to blue-violet and blue.

Phenylisocyanide test: boil with $\mathrm{KOH}+\mathrm{Aq}$ and then add a little chloroform. Odor of phenylisocyanide.

Isolation of aniline: boil several minutes with alcoholic $\mathrm{KOH}$, dilute with water and extract with ether. Evaporation of solvent leaves oily drops of aniline. Dissolve in water and test with calcium hypochlorite.

Phenacetine.-Tasteless. Gives indophenol but not phenylisocyanide test.

Conc. $\mathrm{HNO}_{3}$ : yellow color when cold. Dil. $\mathrm{HNO}_{3}$ dissolves with yellow or orange-yellow color, if heated. Yellow nitrophenacetine crystallizes as saturated solution cools. 
Salicylic Acid.-Sweet, acidulous, harsh taste.

$\mathrm{FeCl}_{3}+\mathrm{Aq}$ : aqueous solutions colored blue-violet; if dilute, more of a red-violet.

Millon's test: red color upon warming.

$\mathrm{Br}_{2}+\mathrm{Aq}:$ yellowish white, crystalline precipitate.

Veronal.-Bitter. Crystalline.

Dissolve ether residue in very little $\mathrm{NaOH}+\mathrm{Aq}$ or $\left(\mathrm{H}_{4} \mathrm{~N}\right)-$ $\mathrm{OH}+\mathrm{Aq}$, filter and acidify filtrate with dil. $\mathrm{HCl}$. Veronal crystallizes. Wash with a little cold water, dry and determine melting-point $\left(\mathrm{r} 87-\mathrm{r} 88^{\circ}\right)$. The crystals mixed with pure veronal should have same melting-point.

Antipyrine.-Mild, bitter taste. Examine aqueous solution of ether residue for antipyrine.

$\mathrm{FeCl}_{3}+\mathrm{Aq}$ : red color.

$\mathrm{HNO}_{3}$ : green color with $\mathrm{I}-2$ drops of fuming acid. Heat and a few more drops of fuming acid change green color to red.

Most of the antipyrine in ether extract of alkaline solution (see B).

Caffeine.-Faintly bitter.

$\mathrm{Cl}_{2}+\mathrm{Aq}$ : evaporated upon water-bath with saturated $\mathrm{Cl}_{2}+\mathrm{Aq}$, gives red-brown residue which turns purplish red moistened with very little $\left(\mathrm{H}_{4} \mathrm{~N}\right) \mathrm{OH}+\mathrm{Aq}$.

Most of the caffeine in ether extract of alkaline solution (see B).

Cantharidin. ${ }^{1}$ - Rhombic leaflets from ether solution.

Physiological test: triturate residue with few drops of almond oil and test mixture as vesicant by applying to upper part of the arm.

\section{B. Ether Extract of Alkaline Solution may Contain :}

Coniine.-Yellow oil drops with penetrating odor.

- Cold saturated aqueous solution becomes milky when warmed.

${ }^{1}$ Cantharidin is taken up in Chapter IV of this book upon page 203. Ether extracts this compound from acid solution but it dissolves with difficulty in this. solvent (0.11 : roo at $\left.18^{\circ}\right)$. 
Spontaneous evaporation with a drop of $\mathrm{HCl}$ gives coniine hydrochloride as doubly refractive crystals which are needle or prism-shaped and sometimes in star-like clusters.

Physiological test: paralysis of peripheral nerves.

Nicotine.-Liquid. Remains dissolved in residual water upon evaporation of ether and has faint tobacco odor.

Melzer's test: red color, heated with ${ }^{2-3}$ cc. of epichlorohydrin.

Schindelmeiser's test: nicotine, after standing several hours with a drop of formaldehyde solution, gives an intense red color with a drop of conc. $\mathrm{HNO}_{3}$.

Roussin's test: ether solution of iodine after some time produces ruby-red, crystalline needles.

Aniline.-Yellow, reddish or brownish oil drops from evaporation of ether extract. (See page 6o, "Synopsis of Group I," for further details.)

Veratrine.-Conc. $\mathrm{H}_{2} \mathrm{SO}_{4}$ : soluble with yellow color, gradually changing to orange, then to red and finally to cherry-red. Gentle heat hastens these changes. Solution at first shows greenish yellow fluorescence.

Froehde: same color charges as with conc. $\mathrm{H}_{2} \mathrm{SO}_{4}$.

Conc. $\mathrm{HCl}$ : very stable red color when heated in test-tube upon water-bath.

Weppen's test: mixed with 6 times the quantity of canesugar $+\mathrm{a}$ few drops of conc. $\mathrm{H}_{2} \mathrm{SO}_{4}$, gradually becomes green and finally blue. Conc. $\mathrm{H}_{2} \mathrm{SO}_{4}$ containing furfural may be used instead.

Vitali's test: same as for atropine (see below).

Strychnine.-Fine, crystalline needles having a very bitter taste upon evaporation of ether extract.

Oxidation test: colorless solution in conc. $\mathrm{H}_{2} \mathrm{SO}_{4}$ becomes evanescent blue or blue-violet with a little solid $\mathrm{K}_{2} \mathrm{Cr}_{2} \mathrm{O}_{7}$. Same color given by Mandelin's reagent but more permanent.

Brucine.-Conc. $\mathrm{HNO}_{3}$ : dissolves with blood-red color soon changing to reddish yellow and yellow. Dilution of yellow solution in a test-tube with a little water and addition drop by drop of dilute $\mathrm{SnCl}_{2}+\mathrm{Aq}$ changes yellow to violet. 
Careful addition of solution in dil. $\mathrm{HNO}_{3}$ to conc. $\mathrm{H}_{2} \mathrm{SO}_{4}$ as upper layer produces red or yellowish red zone.

Atropine.-Vitali's test: evaporated upon water-bath in porcelain dish with a little fuming $\mathrm{HNO}_{3}$, gives yellowish residue which becomes violet when moistened with alcoholic $\mathrm{KOH}$. Hyoscyamine and scopolamine also give this test. Strychnine and veratrine behave similarly.

Para-Dimethylamino-Benzaldehyde test: intense red-violet color. Hyoscyamine and scopolamine also give this test.

Physiological test: enlargement of pupil of eye caused by a single drop of solution I : I30,000.

Cocaine.-Free base, precipitated by $\mathrm{KOH}+\mathrm{Aq}$ from not too dilute cocaine salt solution, forms oil drops soon becoming solid and crystalline.

Benzoyl group: heat 5 minutes in a test-tube upon boiling water-bath with I cc. conc. $\mathrm{H}_{2} \mathrm{SO}_{4}$. Odor of methyl benzoate upon addition of $2 \mathrm{cc}$. of water. Upon cooling, benzoic acid separates. This acid, washed and dried, recognized by meltingpoint $\left(120^{\circ}\right)$ and by tendency to sublime.

Physiological test: anesthesia of the tongue.

Codeine.-Conc. $\mathrm{H}_{2} \mathrm{SO}_{4}$ : soluble without color. Reddish or more bluish upon long standing, or at once upon gentle warming.

Oxidation: deep blue or blue-violet, when warmed with conc. $\mathrm{H}_{2} \mathrm{SO}_{4}$ and $\mathrm{KH}_{2} \mathrm{AsO}_{4}$, or with a little $\mathrm{FeCl}_{3}+\mathrm{Aq}$.

Froehde: yellowish color soon changing to green and to blue upon gentle warming.

Sugar test: purple-red color upon gently warming with conc. $\mathrm{H}_{2} \mathrm{SO}_{4}$ and a little cane-sugar. Due to fuifural formed.

Formalin test: dissolves in conc. $\mathrm{H}_{2} \mathrm{SO}_{4}$ containing formaldehyde with reddish violet color soon changing to permanent blueviolet.

Pellagri's test: given by codeine (see apomorphine, page r46).

Hydrastine.-Froehde: dissolves with fairly permanent bluecolor later changing to brown.

Mandelin: dissolves with reddish color gradually changing to orange-red.

Fluorescence: intense blue fluorescence (characteristic) upon 
shaking dil. $\mathrm{H}_{2} \mathrm{SO}_{4}$ solution with very dilute $\mathrm{KMnO}_{4}+\mathrm{Aq}$ added carefully drop by drop.

Quinine.-Amorphous varnish having very bitter taste from ether.

Fluorescence: blue fluorescence in dil. $\mathrm{H}_{2} \mathrm{SO}_{4}$.

Thalleïoquin test: emerald-green color, upon adding I cc. saturated $\mathrm{Cl}_{2}+\mathrm{Aq}$ to dilute acetic acid solution and then at once excess of $\left(\mathrm{H}_{4} \mathrm{~N}\right) \mathrm{OH}+\mathrm{Aq}$ drop by drop.

Herapathite test: heat to boiling with Io drops of mixture (3o drops acetic acid +20 drops absolute alcohol + I drop dil. $\mathrm{H}_{2} \mathrm{SO}_{4}$ ) and add $\mathrm{I}$ drop alcoholic iodine solution ( $\mathrm{I}: \mathrm{IO}$ ). Shining, olive-green leaflets, appearing cantharides-green by reflected light.

Antipyrine.-Freely soluble in water. Neutral. Mildly bitter.

Dissolve ether residue in little water and test for antipyrine as directed in A (see page I42).

Pyramidone.-Fine needles from ether. Freely soluble in water. Neutral.

$\mathrm{FeCl}_{3}+\mathrm{Aq}$ : aqueous solution blue-violet or more red-violet. $\mathrm{HNO}_{3}$ : fuming acid renders aqueous solution blue to blueviolet.

Caffeine.-Concentric clusters of shining needles from ether. Mild, bitter taste. Fairly soluble in water. Neutral.

Apply tests described under A (see page I42).

Physostigmine. $-\left(\mathrm{H}_{4} \mathrm{~N}\right) \mathrm{OH}+\mathrm{Aq}$ : evaporated with $\left(\mathrm{H}_{4} \mathrm{~N}\right)-$ $\mathrm{OH}+\mathrm{Aq}$, gives blue residue soluble in alcohol with same color.

Physiological test: causes contraction of pupil of eye.

Narcotine.-Not bitter. Neutral.

Froehde: soluble with green color. A concentrated reagent (0.05 gram $\left(\mathrm{H}_{4} \mathrm{~N}\right)_{2} \mathrm{MoO}_{4}$ to I cc. conc. $\mathrm{H}_{2} \mathrm{SO}_{4}$ ) gives greenish color at first which gradually changes to cherry-red and to a blue from margin toward center.

Erdmann: soluble with fine red color.

Papaverine.-Tasteless, colorless, neutral prisms.

Conc. $\mathrm{H}_{2} \mathrm{SO}_{4}$ : pure alkaloid soluble without color. Heat produces dark violet color. 
Froehde: soluble with green color, soon changing when warmed to blue, violet and finally cherry-red.

$\mathrm{HNO}_{3}-\mathrm{H}_{2} \mathrm{SO}_{4}$ test: conc. $\mathrm{H}_{2} \mathrm{SO}_{4}$ containing $\mathrm{HNO}_{3}$, or conc. $\mathrm{HNO}_{3}$ itself, gives a dark red solution.

Thebaine.-Tasteless, colorless, alkaline prisms.

Conc. $\mathrm{H}_{2} \mathrm{SO}_{4}$ : soluble with deep red color. Froehde and Erdmann behave similarly.

\section{Ether Extract ${ }^{1}$ of Ammonia Solution may Contain :}

Apomorphine.-Residue amorphous and usually green. $\mathrm{H}_{2} \mathrm{SO}_{4}-\mathrm{HNO}_{3}$ test: solution in conc. $\mathrm{H}_{2} \mathrm{SO}_{4}$ colored evanescent violet, then reddish yellow or orange by drop of conc. $\mathrm{HNO}_{3}$.

Froehde: soluble with green or violet color.

Pellagri's test: dissolve in dil. $\mathrm{HCl}$, add excess of $\mathrm{NaHCO}_{3}$, shake well and add 2 drops alcoholic iodine solution. Blue or emerald-green color soluble in ether with violet color.

Wangerin's test: $\mathrm{I}-2$ drops $\mathrm{K}_{2} \mathrm{Cr}_{2} \mathrm{O}_{7}+\mathrm{Aq}$ (0.3 per cent.), added to apomorphine hydrochloride solution, gradually produces dark green color. Chloroform added becomes violet. Addition of dil. $\mathrm{SnCl}_{2}+$ Aq produces pure indigo-blue color.

\section{Chloroform Extract of Ammonia Solution may} Contain :

Morphine.-Very bitter. Usually amorphous. Rarely crystalline.

Froehde: soluble with violet color gradually changing to dirty green and finally to pale red.

Formaldehyde- $\mathrm{H}_{2} \mathrm{SO}_{4}$ : soluble with purple-red color later becoming blue-violet and almost pure blue.

Husemann's test: dissolve in conc. $\mathrm{H}_{2} \mathrm{SO}_{4}$, heat over very small flame until abundant white fumes appear, cool and add I drop conc. $\mathrm{HNO}_{3}$. Very evanescent, red-violet color which soon changes to blood-red or reddish yellow.

\footnotetext{
1 Unless the tartaric acid and alkaline solutions, as well as their ether extracts, behave as described on page 126 , that is to say, have a green or red color, omit this extraction.
} 
Pellagri's test: see apomorphine.

$\mathrm{FeCl}_{3}+\mathrm{Aq}$ : dissolve in few drops very dilute $\mathrm{HCl}$, evaporate to dryness upon water-bath, dissolve in little water and add drop $\mathrm{FeCl}_{3}+$ Aq. Blue color.

Bismuth test: dissolve in conc. $\mathrm{H}_{2} \mathrm{SO}_{4}$ and sprinkle bismuth subnitrate on surface of solution. Dark brown color.

Antipyrine and Caffeine.-Being soluble in ether with some difficulty, but readily soluble in chloroform, these substances may appear in the residue from $\mathrm{D}$, if they have not been previously completely extracted with ether.

Narceine. $-\mathrm{I}_{2}$ test: blue color with $\mathrm{I}_{2}+$ Aq.

Resorcinol- $\mathrm{H}_{2} \mathrm{SO}_{4}$ test: dissolves in resorcinol- $\mathrm{H}_{2} \mathrm{SO}_{4}$, giving intense yellow solution which becomes carmine-red or cherryred, if warmed upon the water-bath and stirred.

Tannin- $\mathrm{H}_{2} \mathrm{SO}_{4}$ test: dissolves in tannin- $\mathrm{H}_{2} \mathrm{SO}_{4}$, giving yellowish brown solution which becomes pure green, if warmed upon the water-bath. 


\section{CHAPTER III \\ METALLIC POISONS \\ Destruction of Organic Matter}

The analyst cannot rely upon tests for poisonous metals, if animal or vegetable matter is present. Consequently complete destruction of interfering organic substances is absolutely essential to success. Description of a few of the more important methods used for this purpose will suffice.

\section{Fresenius-v. Babo Method ${ }^{1}$}

The residue left after removal of volatile poisons by steam distillation may be used in this part of the analysis, as it must contain poisonous metals if any are present.

A portion of the original material, ${ }^{2}$ previously finely chopped and well mixed in a large flask with enough water to produce a fluid mass, may also be used. According to the quantity of material, add Io, 20 or $30 \mathrm{cc}$. of pure concentrated hydrochloric acid. $^{3}$ Finally add I-2 grams of potassium chlorate, shake well and set the flask upon a boiling water-bath. Nascent chlorine should come into contact with the material as intimately as possible.

When the mixture is hot enough, add $0.3-0.5$ gram of potassium chlorate at 5 minute intervals and shake the flask frequently. Continue in this manner, until most of the organic matter is dissolved and the solution is pale yellow. Further

${ }^{1}$ Annalen der Chemie und Pharmazie 49, 306 (1844).

${ }^{2}$ Cadaveric material should be divided as finely as possible, then brought to a thin mixture by stirring with $\mathbf{2} 2.5$ per cent., arsenic-free hydrochloric acid and heated with frequent shaking with $\mathrm{I}-2$ grams of potassium chlorate as directed above. If the material is heated on the water-bath in a porcelain dish, it should be stirred constantly.

In laboratory experiments 5 -10 cc. conc. hydrochloric acid is usually sufficient. A large excess of hydrochloric acid should be avoided. 
addition of potassium chlorate and longer heating should produce no real change. Fat especially resists the action of chlorine.

When organic matter is completely destroyed, dilute with hot water, adding a few drops of dilute sulphuric acid to precipitate possible barium, shake and pour the liquid through a wetted filter. If the excess of free hydrochloric acid is not too large, saturate the filtrate direct with hydrogen sulphide as directed on page 152 . Otherwise, evaporate the solution in a porcelain dish upon the water-bath nearly to dryness to remove most of the free hydrochloric acid. This step frequently gives rise to a dark brown color which a few crystals of potassium chlorate will discharge. In testing for lead, cadmium and copper, it is advisable to evaporate, because hydrogen sulphide precipitates the first two metals incompletely, or not at all, from solutions containing too much hydrochloric acid.

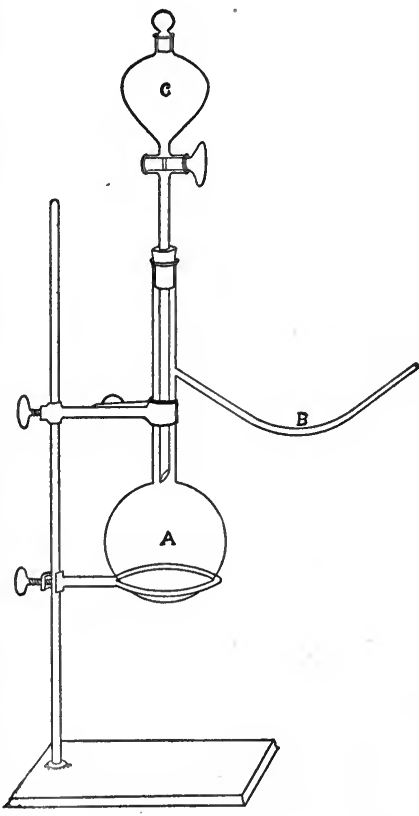

FIG. I2.

An alternative procedure consists in removing part of the free hydrochloric acid from the filtrate, obtained after treatment with hydrochloric acid and potassium chlorate, by first evaporating to smaller volume and then adding ammonium hydroxide solution until alkaline. Add dilute nitric acid until the solution is faintly acid and saturate with hydrogen sulphide (see page 152 ). 
The residue upon the filter may contain silver chloride, barium sulphate and lead sulphate in addition to fat. Examine as directed under "Metallic Poisons IV" (see page I 70).

H. Thoms ${ }^{1}$ destroys organic matter in the apparatus shown in Fig. I2. Oxidation is carried on in an ordinary fractioning flask (A) with the tubulus (B) bent upward. A separating funnel (C), held in the neck of the flask by a stopper, contains an aqueous solution of potassium chlorate ( $\mathrm{r}: 20)$ saturated at room temperature. The organic matter is in the flask as a thin mixture with I 2.5 per cent. hydrochloric acid. Add about I gram of solid potassium chlorate and warm the flask on a boiling water-bath. When the mass in the flask is warm, let the potassium chlorate solution run in drop by drop and shake constantly. Care must be taken not to add too much of this solution at once; otherwise the procedure is identical with that previously described.

Notes.-Potassium chlorate and hydrochloric acid evolve chlorine ( $\alpha$ and $\beta$ ), part of which acts upon the organic material and part in contact with water forms oxygen and oxygen-acids of chlorine $(\mathrm{HOCl})(\gamma$ and $\delta$ ) which are strong oxidizing agents:

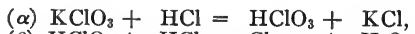

(B) $\mathrm{HClO}_{3}+{ }_{5} \mathrm{HCl}={ }_{3} \mathrm{Cl}_{2}+{ }_{3} \mathrm{H}_{2} \mathrm{O}$,

(r) $\mathrm{Cl}_{2}+\mathrm{H}_{2} \mathrm{O}={ }_{2} \mathrm{HCl}+\mathrm{O}$,

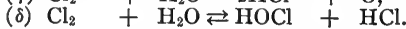

White Arsenic $\left(\mathrm{As}_{2} \mathrm{O}_{3}\right)$ in a mixture probably cannot be volatilized as arsenic trichloride $\left(\mathrm{AsCl}_{3}\right)$ in the procedure described but is oxidized to non-volatile arsenic acid $\left(\mathrm{H}_{3} \mathrm{AsO}_{4}\right)$ :

$$
\begin{aligned}
\mathrm{As}_{2} \mathrm{O}_{3}+{ }_{2} \mathrm{H}_{2} \mathrm{O}+{ }_{2} \mathrm{Cl}_{2} & =\mathrm{As}_{2} \mathrm{O}_{5}+{ }_{4} \mathrm{HCl} \\
\mathrm{As}_{2} \mathrm{O}_{5}+{ }_{3} \mathrm{H}_{2} \mathrm{O} & ={ }_{2} \mathrm{H}_{3} \mathrm{AsO}_{4} .
\end{aligned}
$$

There always remains, even after the most thorough treatment with hydrochloric acid and potassium chlorate, an insoluble white residue wholly unaffected by the action of chlorine. This is the case, especially after the oxidation of vegetable substances or cadaveric material. This treatment converts a portion of the organic matter into volatile compounds (chloranil?) which have a sharp odor and attack the mucous membranes. For this reason destruction of organic matter should take place in a hood with a good draft.

Treatment as described with hydrochloric'acid and potassium chlorate converts metallic poisons into inorganic salts, usually chlorides and sulphates. These either remain in solution or appear as precipitates $\left(\mathrm{AgCl}\right.$ and $\left.\mathrm{BaSO}_{4}\right)$. Protein substances, present in all animal and vegetable organisms, precipitate many heavy metals, as mercury, silver, lead, copper and zinc, from solutions of their

${ }^{1} \mathrm{H}$. Thoms, "Einführung in die praktische Nahrungsmittel Chemie," Leipzig, 1899. Published by S. Hirzel, Leipzig, I899. Figure 64, page 153 . 
salts. These metals are then in the form of metallic albuminates, some of which dissolve in water with great difficulty and are very stable. Usually these metallic protein compounds must receive further treatment before it is possible to detect the metal. Many organic acids, as tartaric acid, and carbohydrates interfere more or less with the detection of heavy metals. In combination with these organic substances heavy metals are like copper in potassium cuprocyanide $\left(\mathrm{K}_{4} \mathrm{Cu}_{2}(\mathrm{CN})_{6}\right)$, which neither sodium hydroxide nor hydrogen sulphide will precipitate because it is electrolytically dissociated in solution in part as follows:

$$
\mathrm{K}_{4} \mathrm{Cu}_{2}(\mathrm{CN})_{6} \rightleftarrows 4 \mathrm{~K}^{\cdot}+\mathrm{Cu}_{2}(\mathrm{CN})_{6}{ }^{\prime \prime \prime \prime} \text {. }
$$

In other words, the solution does not contain cuprous ions. If potassium cuprocyanide is heated with hydrochloric acid and potassium chlorate, copper passes into solution as cupric chloride. The reagents mentioned above now precipitate copper, for cupric chloride ionizes as follows:

$$
\mathrm{CuCl}_{2} \rightleftarrows \mathrm{Cu}{ }^{*}+{ }_{2} \mathrm{Cl}^{\prime} \text {. }
$$

And the solution now contains cupric ions.

The detection, therefore, of these metallic poisons by the usual ionic reactions requires a procedure which permits the analyst to bring about complete destruction of interfering organic substances. The metals in question are thus converted into inorganic salts.

Potassium chlorate acts best only in strong hydrochloric acid solution. Consequently this acid should always be in excess. If the mass becomes too thick at any time during heating, it should be diluted with water or dilute hydrochloric acid. Also the contents of the flask should be well shaken during treatment with potassium chlorate, to prevent a large quantity of this salt from collecting upon the bottom of the flask. Such an occurrence may cause an explosion due to formation of the exceedingly unstable dioxide of chlorine $\left(\mathrm{ClO}_{2}\right){ }^{1}$

The author employs in such analyses 12.5 per cent. hydrochloric acid (sp. gr. r.o6r), saturated with hydrogen sulphide and kept in a loosely stoppered bottle. This insures precipitation of the final traces of arsenic sometimes present even in the purest commercial acid. ${ }^{2}$ Before being used, this acid is filtered through ashfree paper to remove precipitated sulphur which may contain arsenic sulphide.

Cadaveric material, heated with hydrochloric acid and potassium chlorate, is dissolved rather easily. An experiment, in which roo grams of stomach and duodenum, 20 grams of stomach contents, 75 grams of kidney and 200 grams of liver (in all 395 grams) were treated as described, required about $\mathrm{x}$ hour for complete solution. The insoluble part was collected upon a filter and washed. It was amorphous, gummy, yellowish white and greasy. After being dried upon a porous earthen plate, it weighed 52 grams. Dried at $100^{\circ}$, it weighed only 32 grams.

\section{Sonnenschein-Jeserich Method}

This method requires the use of pure free chloric acid instead of potassium chlorate. Place the finely divided material in a

${ }^{1}(\alpha) \mathrm{KClO}_{3}+\mathrm{HCl}=\mathrm{HClO}_{3}+\mathrm{KCl}$,

( $\beta)_{3} \mathrm{HClO}_{3}=\mathrm{HClO}_{4}+{ }_{2} \mathrm{ClO}_{2}+\mathrm{H}_{2} \mathrm{O}$.

2 The use of electrolytic hydrochloric acid (see page 24I) avoids the necessity of this purification. $\mathrm{Tr}$. 
large flask and dilute with water. Add a few cc. of chloric acid and warm slowly and cautiously upon the water-bath. As soon as the mass swells and becomes porous, gradually add small portions of hydrochloric acid. Even a considerable quantity of cadaveric material will dissolve in $2-3$ hours. Water lost by evaporation should be replaced occasionally, otherwise the reaction may take place with explosive violence. In other respects, the product of the reaction should be treated as already described.

\section{C. Mai's Method ${ }^{1}$}

Mix the finely divided material with dilute hydrochloric acid ( 1 : I2) until thin. Add a little potassium chlorate and heat over a free flame, adding from time to time small quantities of potassium chlorate ( 0.2 gram). Cool as soon as liquefaction of the mass is complete. Fat separates and usually can be removed easily from the liquid. Heat this fat once or twice with very dilute nitric acid, filter and add the filtrate to the main part of the liquid. Continue heating the latter, adding small quantities of ammonium persulphate, $\left(\mathrm{H}_{4} \mathrm{~N}\right)_{2} \mathrm{~S}_{2} \mathrm{O}_{8}$, until the liquid is clear and light yellow. Filter and saturate the filtrate as usual with hydrogen sulphide. Ammonium persulphate is a powerful oxidizing agent and also adds nothing non-volatile to the liquid.

\section{Examination of Filtrate for Metallic Poisons}

\section{Precipitation by Hydrogen Sulphide}

A solution properly prepared according to the Fresenius-v. Babo or any other method, freed from excess of hydrochloric acid and filtered, should have only a faint yellow color. ${ }^{2}$ Heat such a solution in a flask upon the water-bath and saturate with arsenic-free hydrogen sulphide. ${ }^{3}$ Pass hydrogen sulphide

\footnotetext{
${ }^{1}$ Zeitschrift für Untersuchung der Nahrungs- und Genussmittel 5, I ro6 (1902).

${ }^{2}$ Chromium in not too small quantity imparts more or less of a green color both to the solution and the filtrate from the hydrogen sulphide precipitate, owing to the presence of chromic chloride $\left(\mathrm{CrCl}_{3}\right)$.

${ }^{3}$ Prepare arsenic-free hydrogen sulphide by saturating dilute sodium hydroxide solution with hydrogen sulphide from crude iron sulphide and commercial hydro-
} 
for $0.5^{-I}$ hour or longer ${ }^{1}$ into the hot solution and continue this treatment after the solution has been removed from the water-bath and is cold.

Allow the solution saturated with hydrogen sulphide to stand in the loosely stoppered flask for several hours or until the next day. If the solution then smells of hydrogen sulphide and blackens a piece of lead acetate paper held over it, the next step

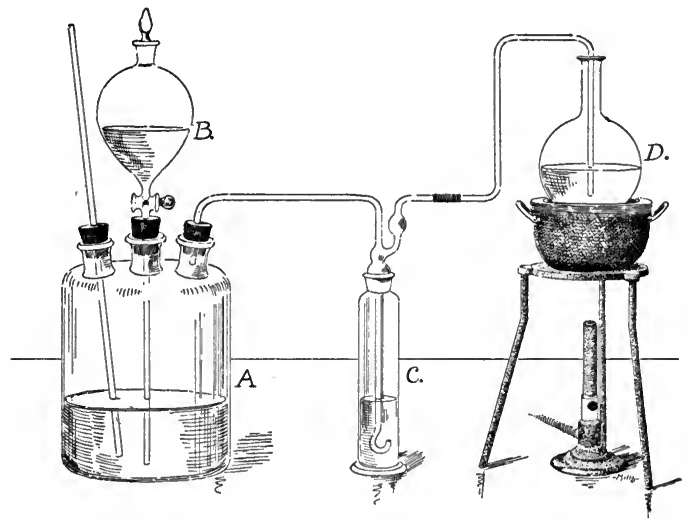

Fig. 13.-Apparatus for Generating Arsenic-free Hydrogen Sulphide. (a) Generator with dilute sulphuric acid; (b) Separating funnel with NaSH; (c) Washbottle; (d) Solution to be saturated with $\mathrm{H}_{2} \mathrm{~S}$.

in the process may be taken. Otherwise warm the solution once more upon the water-bath and again saturate with hydrogen sulphide. Finally collect the hydrogen sulphide precipitate upon a small paper and wash with hydrogen sulphide water.

Examine the precipitate for arsenic, antimony, tin, mercury, lead, copper, bismuth and cadmium (Metallic Poisons I and II)

chloric acid. Pour this sodium hydro-sulphide (NaSH) solution into a separating funnel and add slowly to dilute sulphuric acid ( $\mathrm{r}: 4)$. The generation of the gas can be carried on in the apparatus shown in Fig. 13 .

1 In laboratory experiments treatment with hydrogen sulphide may be shortened somewhat. A Kipp generator in which the gas is prepared from iron sulphide and hydrochloric acid may be used. 
and the filtrate from this precipitate for chromium and zinc (Metallic Poisons III).

Vegetable and animal substances, after treatment with hydrochloric acid and potassium chlorate, frequently give liquids yielding colored precipitates ${ }^{1}$ with hydrogen sulphide even in the absence of the metals mentioned above. Such precipitates consist largely of organic sulphur compounds. Consequently, if hydrogen sulphides produces such a colored precipitate in acid solution, it is not final proof of the presence of a metallic poison. Also without further examination it is impossible to decide from the color of the hydrogen sulphide precipitate as to the presence of a particular metal.

Complete Precipitation.-Before testing for chromium and zinc in the filtrate from the hydrogen sulphide precipitate, add about to times the volume of strong hydrogen sulphide water to a small portion of the solution, stir well and let stand several minutes. Unless a colored precipitate appears, the metals in question (Metallic Poisons I and II) have been completely removed and the filtrate may then be further tested for chromium and zinc (Metallic Poisons III). Otherwise, first dilute the entire filtrate from the hydrogen sulphide precipitate with water and again saturate with hydrogen sulphide. In presence of much hydrochloric acid, lead and cadmium are incompletely precipitated by hydrogen sulphide (see above).

Treatment of Hydrogen Sulphide Precipitate with Ammonia and Yellow Ammonium Sulphide.-Extract the thoroughly washed hydrogen sulphide precipitate, while still moist, upon the filter with a hot mixture of approximately equal parts of ammonia and yellow ammonium sulphide. Heat about 5-10 cc. of the mixture of ammonia and yellow ammonium sulphide to boiling and drop the solution over the precipitate upon the filter. Reheat the filtrate and again pour over the precipitate. Repeat this operation several times. Finally wash the filter

${ }^{1}$ Repeated treatment with potassium chlorate and hydrochloric acid dissolves thoroughly washed casein and fibrin almost completely and gives a filtrate from which hydrogen sulphide precipitates dirty yellow to brownish substances. These products are amorphous and contain organic sulphur compounds together with much free sulphur. 
with a few cc. of a fresh mixture of ammonia and yellow ammonium sulphide. Test the entire filtrate for arsenic, antimony, tin and copper ${ }^{1}=$ Metallic Poisons I. Test the residue upon the filter for mercury, lead, copper, bismuth and cadmium $=$ Metallic Poisons II.

\section{METALLIC POISONS I}

\section{Examination of the Part of the Hydrogen Sulphide Precipitate Soluble in Ammonia-Ammonium Sulphide}

\section{Arsenic, Antimony, Tin, Copper}

Use the solution prepared as described by treating the hydrogen sulphide precipitate with a hot mixture of ammonia and yellow ammonium sulphide. This solution is usually dark brown owing to dissolved organic substances. ${ }^{2}$ Evaporate the solution to dryness in a porcelain dish upon the water-bath. Moisten the cold residue with fuming nitric acid and again evaporate. Then intimately mix the residue with about 3 times its volume ${ }^{3}$ of a mixture of 2 parts of sodium nitrate and I part of dry sodium carbonate. Thoroughly dry this mixture upon the water-bath and introduce small portions at a time into a porcelain crucible containing a little fused sodium nitrate heated to redness. After the final addition, heat the crucible

${ }^{1}$ Copper sulphide (CuS) is somewhat soluble in hot yellow ammonium sulphide. An ammonium sulphide solution containing copper, treated as described on page 156 , yields copper oxide which gives the melt a more or less gray or black appearance. If the melt is extracted with water, the residue contains black copper oxide with stannous oxide and sodium pyro-antimonate. To detect copper, dissolve the black residue in a little hot dilute hydrochloric acid and divide the solution into two parts. Add ammonia to r part until alkaline. The solution is blue, if copper is present. Add potassium ferrocyanide solution to the other part. A brownish red precipitate of cupric ferrocyanide $\left(\mathrm{Cu}_{2} \mathrm{Fe}(\mathrm{CN})_{6}\right)$ appears, if copper is present.

${ }^{2}$ In absence of metals the appearance of dark colored precipitates (see above), when the hydrochloric acid solution is treated with hydrogen sulphide, should not be misunderstood. Such precipitates are due to organic substances soluble with a dark brown color in a hot mixture of ammonia and yellow ammonium sulphide.

${ }^{3}$ In most laboratory experiments 3 grams of a mixture of 2 grams of sodium nitrate and I gram of sodium carbonate are sufficient. A large excess of sodium nitrate should be avoided. 
a short time, introducing possibly a little more sodium nitrate, until the fused mass is colorless. In presence of copper the melt is gray or grayish black from copper oxide. Sodium arsenate, sodium pyro-antimonate, sodium stannate, as well as stannic oxide and copper oxide, may also be present. Soften the cold melt with hot water and wash into a flask. Add a little acid sodium carbonate to the clear or cloudy liquid to decompose the small quantity of sodium stannate possibly in solution and precipitate all the tin as stannic oxide and then filter.

The filtrate (A) contains any arsenic present as sodium arsenate $\left(\mathrm{Na}_{2} \mathrm{HAsO}_{4}\right)$ and the residue upon the filter (B) may contain ${ }^{1}$ sodium pyro-antimonate $\left(\mathrm{Na}_{2} \mathrm{H}_{2} \mathrm{Sb}_{2} \mathrm{O}_{7}\right)$, stannic and copper oxides.

\section{Examination of Filtrate A for Arsenic}

Arsenic is isolated as the element. Positive proof of the presence of the poison is thus afforded. Two methods are in use for this purpose, namely, the Marsh-Berzelius and the Fresenius-v. Babo method. Both are very accurate and exclude any confusion of arsenic and antimony.

Chapter V gives the details for detecting arsenic by the very delicate biological test, which requires the use of certain moulds, and also for the electrolytic separation of arsenic at the cathode as arsine.

\section{Marsh-Berzelius Method}

Principle.-Nascent hydrogen converts oxygen compounds of arsenic, arsenious and arsenic acids, as well as arsenites and arsenates into arsine, $\mathrm{AsH}_{3}$ :

$$
\begin{aligned}
& \mathrm{As}_{2} \mathrm{O}_{3}+\mathrm{I} 2 \mathrm{H}=2 \mathrm{AsH}_{3}+{ }_{3} \mathrm{H}_{2} \mathrm{O} \\
& \mathrm{As}_{2} \mathrm{O}_{5 \cdot 3} \mathrm{H}_{2} \mathrm{O}^{2}+16 \mathrm{H}=2 \mathrm{AsH}_{3}+8 \mathrm{H}_{2} \mathrm{O} .
\end{aligned}
$$

At a red heat arsine is decomposed into metallic arsenic and hydrogen:

$$
\mathrm{AsH}_{3}=\mathrm{As}+{ }_{3} \mathrm{H} \text {. }
$$

This reaction represents the formation of the arsenic mirror.

1 Even in the absence of the substances mentioned under B, a small insoluble residue usually appears. This may come from the porcelain crucible, the glazing of which is slightly attacked in the fusion with sodium nitrate and carbonate.

${ }^{2} \mathrm{As}_{2} \mathrm{O}_{5 \cdot 3} \mathrm{H}_{2} \mathrm{O}$ is the dualistic method of writing $2 \mathrm{H}_{3} \mathrm{AsO}_{4}$. 
Also hydrogen containing arsine burns with a bluish white flame (a). Depress a piece of cold porcelain upon such a flame. Hydrogen will burn but a deposition of metallic arsenic takes place $(\beta)$. This is the so-called arsenic spot:
(a) $2 \mathrm{AsH}_{3}+{ }_{3} \mathrm{O}_{2}=\mathrm{As}_{2} \mathrm{O}_{3}+{ }_{3} \mathrm{H}_{2} \mathrm{O}$,
(B) $2 \mathrm{AsH}_{3}+{ }_{3} \mathrm{O}=2 \mathrm{As}+{ }_{3} \mathrm{H}_{2} \mathrm{O}$.

Hydrogen containing arsine precipitates black metallic silver, if passed into dilute silver nitrate solution. The solution contains arsenious acid:

$$
\mathrm{AsH}_{3}+{ }_{3} \mathrm{H}_{2} \mathrm{O}+6 \mathrm{AgNO}_{3}=\mathrm{H}_{3} \mathrm{AsO}_{3}+6 \mathrm{HNO}_{3}+6 \mathrm{Ag} \text {. }
$$

Procedure.-First acidify "Filtrate A," prepared as described (page ${ }_{5} 5^{6}$ ) and possibly containing sodium arsenate, with dilute arsenic-free sulphuric acid. Evaporate this solution in a porcelain dish upon an asbestos plate over a small free flame. Add a few drops of concentrated sulphuric acid, to expel completely any nitric acid possibly present in the residue, and heat until copious white fumes of sulphuric acid appear. The residue ${ }^{1}$ in the porcelain dish is a thick colorless liquid having a strong acid reaction. Arsenic, if present, is in the form of arsenic acid which when cold frequently solidifies to a white crystalline mass. Examine the solution of this residue in the Marsh apparatus for arsenic. The same solution may also be used in testing for arsenic electrolytically (see pages 233 and 237).

Marsh Apparatus.-Place 30-40 grams of pure arsenic-free zinc $^{2}$ (granulated or in small rods) in the reduction flask A of the Marsh apparatus (Fig. I4). Pour cold dilute arsenic-free sulphuric acid upon the metal. This acid should 'contain I5-16 per cent. of $\mathrm{H}_{2} \mathrm{SO}_{4}{ }^{3}$ Control the temperature of the solution, which should not rise much during the analysis, by

${ }^{1}$ To insure complete removal of nitric acid, test a few drops of this residue with ferrous sulphate and sulphuric acid.

${ }^{2}$ The passage of hydrogen from $15^{-20}$ grams of zinc, treated with dilute arsenic-free sulphuric acid, through the strongly heated ignition-tube $\mathrm{C}$ of the Marsh apparatus should not give a trace of arsenic after I hour. The metal is then pure enough for use. Bertha spelter from the New Jersey Zinc Company will meet such a test.

${ }^{3}$ Add I volume of pure arsenic-free concentrated sulphuric acid to 5 volumes of distilled water. This diluted acid when cold is suitable for use in the Marsh test. 
generating hydrogen slowly. Otherwise, there is danger of partial reduction of sulphuric acid to sulphur dioxide and then to hydrogen sulphide which interferes more or less with the detection of arsenic. Place the reduction flask $\mathrm{A}$ in a dish of cold water, if the acid becomes too warm.

Certain precautions are necessary in using the Marsh apparatus.

I. Have the apparatus absolutely tight.

2. Expel air completely before igniting hydrogen. To tell when this point is reached, collect hydrogen in a dry test-tube

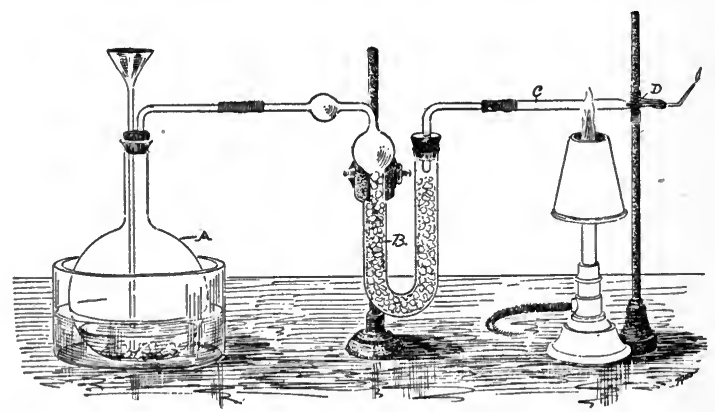

FIG. I4.-Marsh Apparatus. (a) Hydrogen-generator; (b) Chloride of calcium drying-tube; (c) Hard glass tube; (d) Arsenic mirror.

until it ignites without detonation when carried to a flame. If the hydrogen stands this test, ignite the gas at the end of ignition-tube C. There is no danger of an explosion within the apparatus. If the apparatus is tight and the evolution of hydrogen is not too rapid, it requires about 8 minutes to expel the air.

3. Test the hydrogen to insure its entire freedom from arsenic. Neither the arsenic mirror nor spot appears.

If the hydrogen is arsenic-free, gradually introduce the perfectly cold sulphuric acid solution, containing arsenic as arsenic acid (page I 57), in small portions into reduction-flask A. At the same time heat ignition-tube $\mathrm{C}$ to redness just back of 
the capillary tube. If the solution contains arsenic, the gas generated consists of a mixture of arsine $\left(\mathrm{AsH}_{3}\right)$ and hydrogen. A shining mirror of metallic arsenic appears, often in a few minutes, just beyond the point of ignition. Traces of arsenic require considerable time before a brown or brownish black film appears. A piece of white paper held behind the tube brings out clearly even a minute arsenic mirror.

Remove the flame from ignition-tube C. If arsenic is present, the hydrogen flame becomes bluish white. At the same time white fumes of arsenious oxide $\left(\mathrm{As}_{2} \mathrm{O}_{3}\right)$ arise from the flame.

To produce the lustrous, brownish black spot (arsenic spot), depress a cold porcelain dish upon the hydrogen flame.

Arsine has an exceedingly characteristic, garlic-like odor. Extinguish the hydrogen flame and allow. the gas to escape. ${ }^{1}$ The odor is evident even when the hydrogen contains traces of arsine.

A third method of detecting arsenic by the Marsh apparatus consists in extinguishing the hydrogen flame and passing the gas into dilute silver nitrate solution. Arsine darkens this solution, producing a black precipitate of metallic silver. The solution contains arsenious acid and free nitric acid. (See reaction, page ${ }^{5} 57$.)

Filter through a double paper to remove silver and carefully neutralize the filtrate with a few drops of very dilute ammonium hydroxide solution. If the solution is neutral, it is possible to obtain a yellowish white precipitate of silver arsenite $\left(\mathrm{Ag}_{3} \mathrm{AsO}_{3}\right)$ but this compound dissolves easily in ammonium hydroxide solution and in nitric acid.

Extinguish the flame at the end of reduction-tube $\mathrm{C}$ and hold over the tube a strip of paper moistened with concentrated silver nitrate solution ( $\mathrm{I}: \mathrm{I}$ ). A yellow stain appears, if the hydrogen contains arsine. A drop of water added to this yellow spot changes the color to black. This is Gutzeit's arsenic test (see page ${ }_{16}$ ).

${ }^{1}$ The detection of arsine by other tests is so easy that it seems somewhat superfluous to confirm its presence in this way in view of its very poisonous properties. Tr. 


\section{Differences Between Arsenic and Antimony Spots and Mirrors}

Nascent hydrogen reduces various antimony compounds $\left(\mathrm{SbCl}_{3}, \mathrm{Sb}_{2} \mathrm{O}_{3}\right.$, $\mathrm{HSbO}_{3}, \mathrm{KSbOC}_{4} \mathrm{H}_{4} \mathrm{O}_{6}$, etc.) producing the colorless gas stibine $\left(\mathrm{SbH}_{3}\right)$. The behavior of this compound in the Marsh apparatus closely resembles that of arsine, for it gives a spot and mirror, and precipitates black silver antimonide $\left(\mathrm{Ag}_{3} \mathrm{Sb}\right)$ but not metallic silver, if passed into silver nitrate solution.

The procedure employed in preparing material for the Marsh test (see page r55) separates arsenic from antimony and excludes the possibility of the two metals appearing in the Marsh test at the same time. Since the identification of arsenic minors and spots by other tests is important, the differences between arsenic and antimony should be pointed out. The suspicion that antimony is present often necessitates other confirmatory tests (see Antimony, page 164). Introduce the solution into the Marsh apparatus and produce the antimony spot and mirror.

The differences between arsenic and antimony spots and mirrors are:

I. The arsenic mirror has a high metallic luster. It is brownish black and volatile. Owing to this latter property, it sublimes easily when heated in a stream of hydrogen. In the case of the antimony mirror, which appears on both sides of the flame, the metal in contact with the heated glass fuses and is silver white. But in those places removed from the flame it is almost black and has hardly any luster. Stibine decomposes at a temperature much below that required for arsine. This fact explains the deposition of this metal on both sides of the flame. Antimony volatilizes at a high temperature and consequently sublimes with difficulty.

2. The arsenic spot, if not too heavy, is brownish black or brown and lustrous. It dissolves readily in sodium hypochlorite solution, forming arsenious acid:

$$
{ }_{3} \mathrm{H}_{2} \mathrm{O}+2 \mathrm{As}+3 \mathrm{NaOCl}={ }_{2} \mathrm{H}_{8} \mathrm{AsO}_{3}+3 \mathrm{NaCl} \text {. }
$$

The antimony spot is dull, velvet-black and without luster. A thin film of antimony is never brown but has a dark, graphite-like appearance. It is insolúble in sodium hypochlorite solution. Only a freshly prepared solution, however, should be used in this test, since Vaubel and Knocke ${ }^{1}$ have shown that an old solution will dissolve the antimony spot owing to presence of sodium chlorite, formed as shown in the reaction from sodium hypochlorite:

$$
2 \mathrm{NaOCl}=\mathrm{NaClO}_{2}+\mathrm{NaCl}
$$

3. A drop of concentrated nitric acid, or moist chlorine, at once dissolves the arsenic spot forming arsenic acid. Neutralize with ammonia and add silver nitrate solution. A reddish precipitate of silver arsenate $\left(\mathrm{Ag}_{3} \mathrm{AsO}_{4}\right)$ appears.

Nitric acid, or moist chlorine, also dissolves the antimony spot but silver nitrate does not produce a colored precipitate.

4. Gently heat the ignition-tube and pass a stream of dry hydrogen sulphide over the arsenic mirror. Yellow arsenic trisulphide $\left(\mathrm{As}_{2} \mathrm{~S}_{3}\right)$ appears. The antimony mirror becomes brownish red to black $\left(\mathrm{Sb}_{2} \mathrm{~S}_{3}\right)$.

5. Arsine passed into silver nitrate solution precipitates black metallic silver and the filtrate from such a precipitate contains arsenious acid. But stibine precipitates black silver antimonide $\left(\mathrm{Ag}_{3} \mathrm{Sb}\right)$ and the filtrate does not contain a

${ }^{1}$ Chemical Abstracts ro, II47 (I9r6). 
trace of antimony since the precipitation of black $\mathrm{Ag}_{3} \mathrm{Sb}$ is complete. To detect antimony, collect the black precipitate upon paper, wash and heat for some time in Io-15 per cent. tartaric acid solution. Antimony dissolves, whereas silver remains as a grayish white residue. Add dilute hydrochloric acid to this solution and then treat with hydrogen sulphide. Antimony appears as orange-red antimony trisulphide.

\section{Fresenius-von Babo Method}

Principle.-Fusion of oxygen and sulphur compounds of arsenic with a mixture of sodium carbonate and potassium cyanide causes reduction with formation of an arsenic mirror. As a result potassium cyanide changes to potassium cyanate (KCNO) or potassium sulphocyanate (KSCN):

$$
\begin{aligned}
& \mathrm{As}_{2} \mathrm{O}_{3}+3 \mathrm{KCN}=\mathrm{As}_{2}+3 \mathrm{KCNO}, \\
& \mathrm{As}_{2} \mathrm{~S}_{3}+{ }_{3} \mathrm{KCN}=\mathrm{As}_{2}+{ }_{3} \mathrm{KSCN} .
\end{aligned}
$$

Procedure.-Use for this test the sulphuric acid solution prepared by the method already described and containing arsenic in the form of arsenic acid (page 157). To reduce arsenic

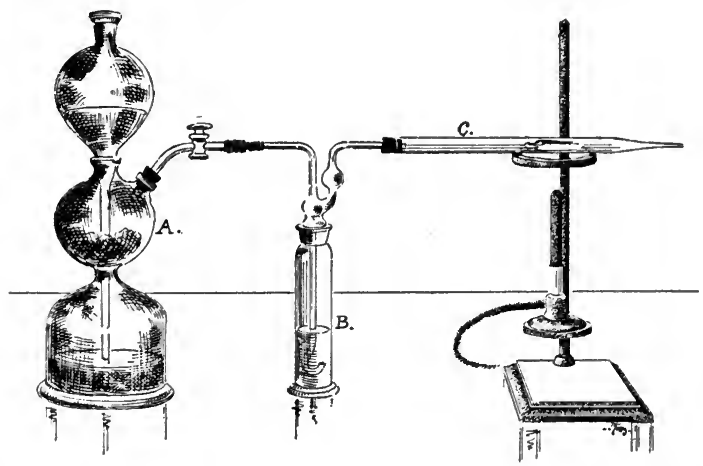

FIG. 15.-Fresenius-Von Babo Apparatus. (a) Carbon dioxide generator; (b) Drying-bottle with pure, concentrated sulphuric acid; (c) Ignition-tube and boat.

acid to arsenious acid, add a few cc. of sulphurous acid to the solution and heat until the odor of this acid has disappeared. Dilute this solution with water and treat with hydrogen sulphide. Collect the precipitate of arsenic trisulphide $\left(\mathrm{As}_{2} \mathrm{~S}_{3}\right)$ upon a small filter and wash thoroughly. Dissolve the precipitate upon the filter in a little hot ammonium hydroxide solu- 
tion. Evaporate this solution in a porcelain dish upon the water-bath and heat the residue with concentrated nitric acid. Expel the latter completely by evaporation, moisten the residue with a little water and add enough dry sodium carbonate to render the mixture distinctly alkaline. Dry thoroughly upon the water-bath and triturate the residue in a mortar with several times the quantity of a mixture of 3 parts of dry sodium carbonate and I part of pure potassium cyanide.

Transfer this mixture to a porcelain boat and place in an ignition-tube of hard glass. Heat in a stream of carbon dioxide (Fig. I5) dried by means of arsenic-free sulphuric acid.

To expel moisture, first heat the ignition-tube gently where the boat is and then ignite at a bright red heat. A mirror of arsenic appears upon the cooler part of the tube, if arsenic is present.

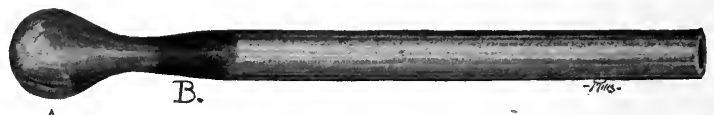

A.

Fig. 16.- $(A)$ Substance and fusion-mixture; $(B)$ Arsenic mirror.

A simpler method of detecting arsenic by means of potassium cyanide is often used. Heat the thoroughly dried material containing arsenic $\left(\mathrm{As}_{2} \mathrm{O}_{3}, \mathrm{As}_{2} \mathrm{~S}_{3}\right)$ in a bulb tube with a dry mixture of sodium carbonate and potassium cyanide until fusion takes place. If the tube is smaller above the bulb, the arsenic mirror will form in the constricted area (Fig. r6).

\section{Other Arsenic Tests}

I. Bettendorff's Test.-Concentrated stannous chloride solution precipitates metallic arsenic from arsenious acid cold and from arsenic acid with heat or after long standing. This test requires the use of a special stannous chloride solution. ${ }^{1}$ The solution is red to brownish red, if only traces of arsenic are present. More than traces of arsenic produce a black precipitate of arsenic:

$$
\begin{aligned}
& \mathrm{As}_{2} \mathrm{O}_{3}+6 \mathrm{HCl}+{ }_{3} \mathrm{SnCl}_{2}={ }_{2} \mathrm{As}+{ }_{3} \mathrm{H}_{2} \mathrm{O}+{ }_{3} \mathrm{SnCl}_{4} \\
& \mathrm{As}_{2} \mathrm{O}_{6}+{ }_{10 H C l}+{ }_{5} \mathrm{SnCl}_{2}+{ }_{3} \mathrm{H}_{2} \mathrm{O}=2 \mathrm{As}+8 \mathrm{H}_{2} \mathrm{O}+{ }_{5} \mathrm{SnCl}_{4} \text {. }
\end{aligned}
$$


Use for this test the sulphuric acid solution obtained as described above (page 157) which contains arsenic in the form of arsenic acid. Bettendorff's test is not as delicate as the Marsh test.

2. Gutzeit's Test. ${ }^{1}$-This test permits the detection of minute traces of arsenious and arsenic acids, as well as their salts, with certainty. Generate hydrogen in a test-tube from arsenic-free zinc and pure dilute hydrochloric acid. To remove any sulphurous acid or hydrogen sulphide, add a few drops of iodine solution until the liquid is yellow. Then add the solution to be tested and place a loose cotton plug in the neck of the test-tube. If arsenic is present, the silver nitrate spot becomes lemon-yellow.

$$
\mathrm{AsH}_{3}+6 \mathrm{AgNO}_{3}=\left({ }_{3} \mathrm{AgNO}_{3} \cdot \mathrm{Ag} 3 \mathrm{As}\right)+{ }_{3} \mathrm{HNO}_{3} .
$$

Gradually a brownish black border forms around the yellow spot. A drop of water at once turns the spot black from separation of metallic silver.

$$
\left({ }_{3} \mathrm{AgNO}_{3} \cdot \mathrm{Ag}_{3} \mathrm{As}\right)+{ }_{3} \mathrm{H}_{2} \mathrm{O}=6 \mathrm{Ag}+\mathrm{H}_{3} \mathrm{AsO}_{3}+{ }_{3} \mathrm{HNO}_{3} .
$$

This is a very delicate arsenic test. One drop of o.I per cent. potassium arsenite solution produces a distinct yellow color upon the silver paper. Gutzeit's test is positive with even 0.05 milligram of $\mathrm{As}_{2} \mathrm{O}_{3} .{ }^{2}$ The sulphuric acid solution containing arsenic as arsenic acid (see page ${ }_{57}$ ) may be used for this test.

But Gutzeit's test is not as characteristic of arsenic as the Marsh test. Stibine, phosphine from phosphorus in zinc and even hydrocarbons color the silver nitrate paper. Dry hydrogen sulphide also produces a yellow or yellowish green spot upon paper moistened with concentrated silver nitrate solution. The latter spot has a black border which gradually extends until the entire spot becomes black. Poleck gives this yellow compound the composition $\left(\mathrm{AgNO}_{3} \cdot \mathrm{Ag}_{2} \mathrm{~S}\right)$.

\section{Detection of Antimony, Tin and Copper in Residue B}

Residue B (see page ${ }_{5} 56$ ), insoluble in water and obtained from the fusion, may contain sodium pyro-antimonate, stannic

1 Gutzeit, Pharmazeutische Zeitung 1879, 263; and Poleck and Tümmel, Berichte der Deutschen chemischen Gesellschaft 16, 2435 (1883).

${ }^{2}$ See page 240 for the application of this method to the quantitative estimation of arsenic. Tr. 
and cupric oxides. Treat this residue upon the filter with a little hot dilute hydrochloric acid (equal parts of concentrated acid and water). Pass this acid repeatedly through the paper until most of the residue is dissolved. If the original color of residue B and of the melt was gray or black, first examine a portion of the hydrochloric acid solution for copper. Excess of ammonia produces a blue color. Potassium ferrocyanide solution gives a brownish red precipitate, or only a coloration with traces of copper.

Concentrate the remainder of the hydrochloric acid solution to a few drops in a porcelain dish upon the water-bath, and put 2 drops of this solution upon platinum foil in contact with zinc. Antimony produces a black, tin a grayish and copper a dark reddish brown spot upon platinum. There is little chance of confusing the tin or copper spot with that given by antimony.

Dilute the remainder of the hydrochloric acid solution with water and introduce a piece of zinc. Keep the zinc in the solution, as long as hydrogen is evolved. Collect the black, metallic flocks from this operation upon a small filter. Wash thoroughly, and gently warm with a little concentrated hydrochloric acid. Finally, filter the solution. Antimony does not dissolve, whereas tin passes into solution as stannous chloride $\left(\mathrm{SnCl}_{2}\right)$, and is in the filtrate. Apply the tests described under tin to this solution.

\section{TIN}

The solution, treated as described in the above analytical procedure, contains tin as stannous chloride $\left(\mathrm{SnCl}_{2}\right)$. Apply the following tests for this metal:

(a) Mercury Test.-Add to a portion of the filtrate a few drops of mercuric chloride solution. Tin precipitates white mercurous chloride (calomel). Heat produces in addition gray, metallic mercury, if there is a large excess of stannous chloride.

(b) Prussian Blue Test.-Add to a second portion of the filtrate a few drops of a dilute mixture of ferric chloride and 
potassium ferricyanide solutions. Tin produces a precipitate of Prussian blue.

This test is not characteristic of tin, as many other substances capable of reducing ferri-ferricyanide to ferri-ferrocyanide, that is to say, to Prussian blue act in the same way.

To identify antimony further, dissolve the black flocks, insoluble in hydrochloric acid, in a few drops of hot aqua regia. Expel excess of acid upon the water-bath and dilute the residue with water. If the quantity of antimony is not too small, water precipitates white antimony oxychloride $(\mathrm{SbOCl})$. Redissolve this precipitate in a little dilute hydrochloric acid. Test a portion of this solution for antimony with hydrogen sulphide. Introduce the remainder into the Marsh apparatus and produce the antimony spot and mirror, or test for stibine with silver nitrate solution as described (see page I60).

\section{METALLIC POISONS II}

Detection of Metals Whose Sulphides are Insoluble in Ammonium Sulphide

Mercury

Bismuth

Lead

Copper

Cadmium

That portion of the hydrogen sulphide precipitate, insoluble in ammonium sulphide solution, may contain mercury, lead, bismuth, copper and cadmium sulphides. Examine this precipitate according to the methods employed in qualitative analysis. Treat a small precipitate repeatedly upon the filter with a few cc. of warm, rather dilute nitric acid (I volume of concentrated acid and 2 volumes of water). Mercuric sulphide does not dissolve, but the other sulphides pass into solution as nitrates.

\section{Detection of Mercury in the Residue Insoluble in Nitric Acid}

Always examine that portion of the hydrogen sulphide precipitate, insoluble in nitric acid, for mercury, even when not black! Treat this residue upon the filter with a little hot, 
somewhat diluted hydrochloric acid, containing in solution a few crystals of potassium chlorate and pass the acid through the paper several times. Evaporate the filtrate to dryness in a porcelain dish upon the water-bath, and dissolve the residue in 2-3 cc. of water containing hydrochloric acid. Filter this solution, and examine the filtrate for mercury.

(a) Stannous Chloride Test.-Add to a portion of the filtrate a few drops of stannous chloride solution. A white precipitate of mercurous chloride (calomel) appears, if mercury is present. Excess of stannous chloride, especially if heat is applied, reduces this precipitate to gray, metallic mercury.

(b) Copper Test.-Put a few drops of the filtrate upon a small piece of bright copper. Mercury immediately deposits a gray spot which has a silvery luster when rubbed. Wash the copper, upon which mercury has been deposited, successively in water, ethyl alcohol and ether. Dry thoroughly and heat in a small bulb-tube of hard glass. Mercury sublimes and collects in small, metallic globules on the cool sides of the tube. A trace of iodine vapor, introduced into the tube, immediately transforms the gray sublimate into scarlet mercuric iodide $\left(\mathrm{HgI}_{2}\right)$.

(c) Phosphorous Acid Test.-Add to another portion of the filtrate some phosphorous acid and warm gently. A white precipitate of mercurous chloride (calomel) appears, if mercury is present:

$$
{ }_{2} \mathrm{HgCl}_{2}+\mathrm{H}_{2} \mathrm{O}+\mathrm{H}_{3} \mathrm{PO}_{3}=\mathrm{Hg}_{2} \mathrm{Cl}_{2}+{ }_{2} \mathrm{HCl}+\mathrm{H}_{3} \mathrm{PO}_{4} \text {. }
$$

(d) Precipitation of Mercuric Iodide.-Add I-2 drops of very dilute potassium iodide solution to the remainder of the filtrate. A red precipitate $\left(\mathrm{HgI}_{2}\right)$, readily soluble in excess of potassium iodide, shows mercury:

$$
\begin{aligned}
& \text { I. } \mathrm{HgCl}_{2}+2 \mathrm{KI}=\mathrm{HgI}_{2}+{ }_{2} \mathrm{KCl}, \\
& \text { 2. } \mathrm{HgI}_{2}+2 \mathrm{KI}=\mathrm{K}_{2} \mathrm{HgI}_{4} .
\end{aligned}
$$

\section{Examination of the Nitric Acid Solution}

The nitric acid solution may contain lead, bismuth, copper and cadmium nitrates. Evaporate this solution in a porcelain dish nearly to dryness and dissolve the residue in a little hot 
water. If the solution contains lead, dilute sulphuric acid produces a heavy white precipitate of lead sulphate. Test the filtrate from this precipitate for bismuth, copper and cadmium.

(a) Copper and Bismuth Tests.-Excess of ammonium hydroxide solution, added to most of this filtrate, produces a blue color if copper is present. A white precipitate at the same time may be bismuthous hydroxide ${ }^{1}\left(\mathrm{Bi}(\mathrm{OH})_{3}\right)$. To detect bismuth, wash the precipitate and dissolve upon the filter in a few drops of hot dilute hydrochloric acid. Pour this solution into considerable water. A white precipitate of bismuthous oxychloride $(\mathrm{BiOCl})$ proves the presence of bismuth. As an alternative test, add stannous chloride to the hydrochloric acid solution and then excess of sodium hydroxide solution. A black precipitate of metallic bismuth appears.

(b) Potassium Ferrocyanide Test.-Potassium ferrocyanide solution precipitates copper as brownish red cupric ferrocyanide

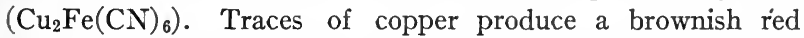
color. There is a deposit of cupric ferrocyanide after some time.

(c) Precipitation of Metallic Copper.-A bright knife blade, or a bright iron nail, immersed for a short time in a copper solution, becomes red from a coating of metallic copper.

To detect cadmium in presence of copper, add solid potassium cyanide to the blue solution, produced by ammonium hydroxide, until the blue color is discharged. Then pass hydrogen sulphide through the solution. Cadmium is precipitated as the yellow sulphide (CdS), whereas copper remains in solution as $\mathrm{K}_{4} \mathrm{Cu}_{2}(\mathrm{CN})_{6}$, potassium cuprocyanide.

When copper is absent, test for cadmium by passing hydrogen sulphide at once into the ammoniacal solution. If a reddish or brownish instead of a yellow precipitate appears, filter, dry the precipitate upon the paper and heat upon charcoal in the blow-pipe flame. Cadmium gives a brown coating.

${ }^{1}$ Ammonium hydroxide solution does not precipitate pure bismuthous hydroxide $\left(\mathrm{Bi}(\mathrm{OH})_{3}\right)$ from solutions of bismuth salts but a basic salt, the composition of which depends upon the temperature and concentration of the particular solutions. 


\section{METALLIC POISONS III}

\section{Detection of Chromium and Zinc}

Test the filtrate from the hydrogen sulphide precipitate for chromium and zinc. Concentrate the filtrate to about onethird its original volume and divide this solution into two parts.

\section{Detection of Zinc}

Add enough ammonium hydroxide solution to render onehalf the concentrated filtrate alkaline. This treatment usually gives the solution a dark color. Then add ammonium sulphide solution in excess. This reagent almost always produces a precipitate even when zinc is not present, since solutions, prepared from animal and vegetable materials, usually contain iron compounds and phosphates of the metals of the earths and of the alkaline earths. When this precipitate has settled, add acetic acid until the solution has a faint acid reaction. Stir the mixture thoroughly, and allow it to stand for some time. The color of the precipitate becomes lighter, because acetic acid dissolves sulphide of iron. Moreover, the phosphates are partly dissolved, except ferric phosphate $\left(\mathrm{FePO}_{4}\right)$ which is insoluble in acetic acid. Collect the precipitate upon a filter. Wash, dry and ignite precipitate and filter in a porcelain crucible. Before ignition, moisten the filter with concentrated ammonium nitrate solution. Extract the residue from ignition with $3 \mathrm{cc}$. of boiling, dilute sulphuric acid. Filter, and divide the filtrate into two parts.

(a) Add sodium hydroxide solution in excess to one portion of the filtrate and shake thoroughly. Filter, to remove the white precipitate of ferric phosphate which usually appears, and add a few drops of ammonium or hydrogen sulphide solution to the clear filtrate and heat. Zinc, if present, gives a white, flocculent precipitate of zinc sulphide.

(b) Add ammonium hydroxide solution in considerable excess to the second part of the filtrate. Filter, to remove ferric phosphate, and acidify the filtrate with acetic acid. Warm 
the solution and treat with hydrogen sulphide. Zinc, if present, appears as the white sulphide.

(c) Test further for zinc by dissolving the precipitate produced by ammonium or hydrogen sulphide (as described above in $a$ and $b$ ), after it has been collected upon a filter and thoroughly washed, in a few drops of hot dilute hydrochloric acid. Boil until hydrogen sulphide is expelled, and filter to remove precipitated sulphur. Add potassium ferrocyanide solution to the clear, cold filtrate. This precipitates $\mathrm{Zn}_{2} \mathrm{Fe}(\mathrm{CN})_{6}$, zinc ferrocyanide, which is white, slimy and nearly insoluble in dilute hydrochloric acid. ${ }^{1}$

\section{Detection of Chromium}

To test for chromium ${ }^{2}$ in the second part of the filtrate from the hydrogen sulphide precipitate, concentrate the solution in a porcelain dish to a small volume. Then add twice the quantity of potassium nitrate and sodium carbonate, until the reaction is decidedly alkaline. Finally, heat this mixture until perfectly dry. Add this dry residue in small portions to a little potassium nitrate fused in a crucible. In fusing a large quantity of material, it is advisable to use a large, bright, nickel crucible which is especially adapted for this operation. When fusion is complete, cool thoroughly, boil crucible and contents with water in a porcelain dish and filter the solution. Chromium colors the filtrate more or less yellow. Even mere traces ${ }^{3}$ of chromium color the filtrate yellow. When the solution of the melt is colorless, it is unnecessary to test for chromium. To detect chromium when the filtrate is yellow, divide the solution into two portions and make the following tests:

1 Excess of potassium ferrocyanide combines with zinc ferrocyanide, which is first precipitated, and forms insoluble potassium zinc ferrocyanide:

$$
{ }_{3} \mathrm{Zn}_{2} \mathrm{Fe}(\mathrm{CN})_{6}+\mathrm{K}_{4} \mathrm{Fe}(\mathrm{CN})_{6}={ }_{2} \mathrm{~K}_{2} \mathrm{Zn}_{3}\left(\mathrm{Fe}(\mathrm{CN})_{6}\right)_{2} \text {. }
$$

${ }^{2}$ In testing for metallic poisons, chromic oxide $\left(\mathrm{Cr}_{2} \mathrm{O}_{3}\right)$, which is insoluble in acids, may be disregarded as it is not poisonous.

${ }^{3}$ Two drops of ro per cent. potassium chromate solution $(=0.01$ gram of $\mathrm{K}_{2} \mathrm{CrO}_{4}$ ) in $500 \mathrm{cc}$. of water produce a marked yellow color. Fifty cc. of this solution contain $0.00 \mathrm{I}$ gram of $\mathrm{K}_{2} \mathrm{CrO}_{4}$ which can still be recognized by the yellow color. 
(a) Chrome Yellow Test.-Add acetic acid in excess to one portion of the filtrate, and boil for some time to expel carbon dioxide and nitrous acid as completely as possible. Then add a few drops of lead acetate solution. A yellow precipitate of lead chromate $\left(\mathrm{PbCrO}_{4}\right.$, "Chrome Yellow") appears, if chromium is present. When the precipitate is mixed with considerable lead sulphate or chloride, the color is only yellowish. A white precipitate is due to $\mathrm{PbSO}_{4}, \mathrm{PbCl}_{2}$ or $\mathrm{Pb}_{3}\left(\mathrm{PO}_{4}\right)_{2}$. When the aqueous solution of the melt is colorless, such a precipitate is usually obtained.

Potassium nitrate, always present in the melt, lead acetate and acetic acid, brought together in solution, produce a distinct yellow color, in which a white precipitate may appear yellow. To eliminate this source of error, allow the precipitate to settle, collect upon a filter and wash thoroughly. If it is pure white, chromium is absent.

(b) Reduction Test.-Add sulphurous acid to the second portion of the yellow filtrate. The yellow color changes to green, or greenish blue, with formation of chrome alum. This is not as delicate as the preceding test.

\section{METALLIC POISONS IV}

Detection of Barium, Lead and Silver in the Residue from Hydrochloric Acid and Potassium Chlorate

Wash the residue left undissolved in the treatment with hydrochloric acid and potassium chlorate thoroughly with water and dry in the air-closet or upon a porous plate. Then add three times the quantity of a mixture of 2 parts of potassium nitrate and I part of sodium carbonate and triturate in a mortar with the filter. Gradually introduce this mixture into a hot porcelain crucible. In this operation organic substances (fats, fatty acids, etc.) are oxidized by potassium nitrate with considerable deflagration. Finally when all the material is in the crucible, add $0.25-0.5$ gram more of potassium nitrate. Cool the melt, soften with water, wash into a flask and pass carbon dioxide through the turbid liquid for several minutes. This treatment converts caustic alkali into carbonate and completely precipitates lead which may be in solution. Then heat the 
solution to boiling and let settle for some time. Collect upon paper the sediment ${ }^{1}$ which may contain barium carbonate, basic lead carbonate and metallic silver. Silver gives the sediment a gray color. Thoroughly wash the precipitate with hot water and dissolve upon the paper in hot, rather dilute nitric acid, ${ }^{2}$ passing the acid through the paper several times. Evaporate this solution to dryness. Dissolve the residue in water, heat the entire solution to boiling, and precipitate silver with dilute hydrochloric acid. Filter, to remove silver chloride, and pass hydrogen sulphide through the filtrate to precipitate lead. To test for barium in the filtrate from lead sulphide, first boil to expel hydrogen sulphide and filter to remove insoluble matter. Then add dilute sulphuric acid which precipitates barium sulphate.

To identify silver further, dry the hydrochloric acid precipitate and fuse in a porcelain crucible with a little potassium cyanide. Extract the melt with hot water. Metallic silver remains undissolved.

To confirm the presence of lead, dissolve the hydrogen sulphide precipitate in hot nitric acid and evaporate the solution to dryness.' Dissolve the residue in water, filter and test the solution for lead with sulphuric acid or potassium chromate.

To identify barium further, collect the sulphuric acid precipitate upon paper, thoroughly wash and test upon a clean platinum wire in a non-luminous flame. Barium imparts a yellowish green color to the flame. To avoid any mistake, examine this flame with the spectroscope. These reactions of identification are always necessary in toxicological analysis.

\section{SYNOPSIS OF GROUP III}

Heat the residue from distillation of volatile poisons, or a portion of original material, in a glass flask or porcelain dish

${ }^{1}$ Even in the absence of barium, lead and silver, such a sediment nearly always appears. In that case it usually consists of the material of the porcelain crucible, the glazing of which is partially attacked in the fusion process.

${ }^{2}$ Use 5-6 cc. of an acid prepared by mixing I volume of concentrated nitric acid and 2 volumes of water. 
upon the water-bath with dilute hydrochloric acid (I 2.5 per cent.) and potassium chlorate and shake frequently or stir. When most of the material is dissolved and the solution is yellow, dilute with water. Add a few drops of sulphuric acid and filter the cold solution.

Material. Treated with $\mathrm{HCl}$ and $\mathrm{KClO}_{3}$. Dilute $\mathrm{H}_{2} \mathrm{SO}_{4}$. Filter.

Filtrate. ${ }^{1}$ Saturated warm with $\mathrm{H}_{2} \mathrm{~S}$.

Residue. Tested for "Metallic Poisons IV."

Precipitate. Treated with hot $\left(\mathrm{H}_{4} \mathrm{~N}_{2} \mathrm{~S}_{x}\right.$ and $\left(\mathrm{H}_{4} \mathrm{~N}\right) \mathrm{OH}$.

\begin{tabular}{l|l}
\hline Filtrate. Tested & Residue. Tested \\
for "Metallic & for "Mertallic \\
Poisons I." & Poisons II." \\
As, Sb, Sn, Cu. & $\mathrm{Hg}, \mathrm{Pb}, \mathrm{Bi}, \mathrm{Cu}, \mathrm{Cd}$.
\end{tabular}

$$
\begin{aligned}
& \text { Filtrate. Tested } \\
& \text { for "Metallic } \\
& \text { Poisons III." } \\
& \text { Cr, Zn. }
\end{aligned}
$$$$
\mathrm{Ag}, \mathrm{Pb}, \mathrm{Ba} \text {. }
$$

\section{Action of Heavy Metals}

Most salts of heavy metals, as lead, copper, mercury, silver, uranium and bismuth, precipitate proteins. These compounds are metallic salts of albumins (albuminates). In combining with the oxides of these metals, proteins behave like acids. If the metal albuminates first formed are insoluble, or only slightly soluble in the body fluids, they are non-toxic or only slightly toxic. But a soluble albuminate is transported throughout the organism and exerts a toxic action. Every cell in contact with the dissolved metal may be poisoned. Mercury albuminate is an example of the latter class of metal albuminates. Being soluble in sodium chloride and protein solutions, it acts as a powerful poison. Copper albuminate on the other hand is not appreciably soluble in solutions of sodium chloride, hydrochloric acid or proteins. Not entering the circulation, it is as good as non-toxic. Lead and silver albuminates are like copper albuminate as regards solubility in the solvents mentioned. But if a heavy metal, which forms a difficultly soluble albuminate, finds its way into the organism in organic combination so that it cannot be precipitated by proteins, for example, copper in union with tartaric acid, it then is as poisonous, or nearly as poisonous, as

${ }^{1}$ If this filtrate contains much free hydrochloric acid, remove most of the acid by evaporation. Then add ammonia until alkaline and finally acidify with dilute nitric acid. 
mercury in corrosive sublimate. Administration intravenously of $20 \mathrm{mg}$. of such copper causes the death of an adult rabbit.

Consequently precipitation takes place wherever the salt of a heavy metal comes in contact with proteins. The term corrosion is applied to such an occurrence. There is always present the metallic oxide, protein and the acid originally combined with the metal. As a rule the acid is loosely held by the precipitate and is washed away by the circulating blood. The corrosive action of salts of heavy metals is due both to the union of the metallic oxide with protein, living protein being changed to dead metal albuminate, and to the caustic action of the free acid. Therefore the intensity of the action of the salt of the heavy metal depends upon the nature of the given metal albuminate. The degree of solubility is especially important (see above), also the quantity and strength of the free acid. Salts of heavy metals not only may affect the place of application but they may give rise to serious changes where eliminated, as in the intestines or kidneys. Before elimination they may also seriously harm parenchymatous organs like the liver, as well as the circulatory organs. Finally salts of heavy metals have an important action upon the blood. R. Kobert and his collaborators have found that white as well as red blood-corpuscles may combine with metals and thus act as antidotes. Kobert has shown that the substance of red blood-corpuscles is capable of taking up a considerable quantity of a heavy metal. A chemical compound (metal hæmoglobin) is formed and the oxyhæmoglobin spectrum is not changed. Thus lead speedily impairs the vitality of red bloodcorpuscles. Consequently red blood-corpuscles are killed in large quantity in lead poisoning.

\section{Fate, Distribution and Elimination of Metals in the Human Body}

Arsenic.-Elimination of arsenic takes place mainly through the urine. It begins several hours (7-12) after administration and, after a single dose, usually lasts 4-7 days. A great many experiments have shown the duration of arsenic elimination in urine to vary from a few days to several weeks. Some observers have found arsenic in urine 80 , and even 90 days, after poisoning. Consequently in suspected arsenic poisoning first examine the urine! In arsenic poisoning the urine is usually diminished in volume and contains albumin and blood-corpuscles.

As regards retention of arsenic by different organs, large quantities of the poison are usually found in the liver. Examine also the stomach and intestines with contents, since most of the poison will obviously be in these organs in case of recent administration. The spleen, kidneys and muscles usually contain arsenic. But the brain rarely shows more than traces of the poison. On the other hand, the bones in many instances have contained arsenic. This fact has given rise to the hypothesis that arsenic is capable of replacing phosphoric acid in the bones.

During the first stage in elimination of arsenic from the organism the poison appears to be deposited in the bones as calcium arseniate. If large doses of difficultly soluble arsenic compounds, as white arsenic, or small doses of soluble compounds have been taken, the liver alone appears to arrest and retain the poison. Probably the quite stable arseno-nucleins are formed in that organ.

From experiments where the organism was deluged with easily soluble arsenic 
compounds, Chittenden concluded that the brain can arrest and retain the poison and that arsenic then accumulates there. But after administration of white arsenic or Schweinfurt green, only traces of the poison can be detected in the brain. Two instances of arsenical poisoning are in favor of this view. In the first case a woman died 9 hours after eating a highly arsenical soup. The liver weighing 1259 grams, contained $76 \mathrm{mg}$. of arsenious oxide; the kidneys and bladder $0.6 \mathrm{mg}$.; and half the brain only a faint trace of the poison. In the second case a young woman diẹd a day after taking Schweinfurt green. In this instance too the brain contained only traces of the poison. In experiments upon animals most of the arsenic was always found in the liver.

\section{Normal Arsenic ${ }^{1}$}

The view that certain organs of the body may contain arsenic as an essential constituent has led to the use of the term "normal" to distinguish such arsenic from that entering the organism in food or drugs. The introduction of this term into chemical literature is unfortunate, because it suggests the possibility of two kinds of arsenic. Such a notion has no foundation whatever. Arsenic is arsenic and no test capable of showing more than one kind is known. A committee of the French Academy of Sciences ${ }^{2}$ after carefully investigating this matter came to the conclusion that arsenic never occurs normally in the human body. But within recent years A. Gautier ${ }^{3}$ after making many analyses of different materials has come to the opposite opinion. Gautier thus summarizes his results in one of his papers: ${ }^{4}$

"Speaking from a medico-legal point of view, I would state that arsenic, aside from the thyroid, mammary and thymus glands, never cccurs in the human body except in the skin, hair, bones, milk and sometimes in the fæces and then only in traces which are often infinitesimal. Excepting the brain, the other organs and fluids, especially those forming the bulk of the body, as muscular tissue, liver, spleen, kidneys, lungs, blood, urine, etc., fail to show the slightest trace of arsenic . . . . . . If a chemist therefore examines individually these arsenicfree organs by my method or by one less delicate and finds traces, especially appreciable traces, of this metalloid, such arsenic has been absorbed during life either medicinally or criminally."

Gautier found the largest quantity of arsenic in the thyroid gland $(0.75 \mathrm{mg}$. in 100 grams of gland) but this result has not yet been confirmed. Other chemists to be sure have found arsenic in the thyroid gland but in much smaller quantity. These same chemists have also found arsenic in organs which Gautier says are non-arsenical. The following is a summary of their results but the original papers should be consulted for full details:

${ }^{1}$ The brief account of "normal arsenic" in the German edition seems insufficient. After thoroughly examining the literature, the translator has therefore decided to treat this subject more fully. Tr.

${ }^{2}$ Comptes rendus de l'Academie des Sciences I 2, 1076-1 109 (I84I).

${ }^{3}$ Ibid., 1 29, 929-936 (1899).

‘ Ibid., r30, 284-29I (1900). 


\begin{tabular}{|c|c|c|c|}
\hline Observer & Material & Arsenic found & $\begin{array}{l}\text { Remarks and } \\
\text { conclusions }\end{array}$ \\
\hline Gautier $^{1}$ & $\begin{array}{l}\text { Human and ani- } \\
\text { mal organs and } \\
\text { other material }\end{array}$ & $\begin{array}{l}0.75 \text { mgr. in } 100 \\
\text { grams of human } \\
\text { thyroid gland }\end{array}$ & $\begin{array}{l}\text { Used } 6 \text { glands and } \\
\text { assumed uniform } \\
\text { distribution of As }\end{array}$ \\
\hline Bertrand $^{2}$ & $\begin{array}{l}\text { Only animal ma- } \\
\text { terial }\end{array}$ & $\begin{array}{l}\text { 0.0I } 5 \text { mgr. per Ioo } \\
\text { grams of dried } \\
\text { sponge }\end{array}$ & $\begin{array}{l}\text { Concludes As is a } \\
\text { normal constitu- } \\
\text { ent of protoplasm }\end{array}$ \\
\hline Schaefer ${ }^{3}$ & Human organs & $\begin{array}{l}0.007 \text { mgr. per Ioo } \\
\text { grams of human } \\
\text { thyroid }\end{array}$ & $\begin{array}{l}\text { Concludes As may } \\
\text { occur in all organs } \\
\text { but found many } \\
\text { free from As }\end{array}$ \\
\hline Pagel $^{4}$ & $\begin{array}{l}\text { Human and ani- } \\
\text { mal organs }\end{array}$ & $\begin{array}{l}\text { Positive but not } \\
\text { quantitative }\end{array}$ & $\begin{array}{l}\text { Found testes ar- } \\
\text { senical but Gautier } \\
\text { says they are not }\end{array}$ \\
\hline
\end{tabular}

On the contrary several chemists have carefully analyzed human and animal organs, either finding no arsenic or detecting this metalloid in mere traces, which are inconstant in occurrence and confined to no special organ. The table on the opposite page briefly summarizes their results.

The results set forth in this table place "normal arsenic" in a doubtful position at least. If it is a reality and not a fancy, the quantity of arsenic, compared with that obtained in an analysis actually dealing with this metalloid, is so minute that the toxicologist need feel no concern. If he has conducted his analysis with every precaution as regards reagents and method and obtained a distinct mirror, he may dismiss the "normal arsenic" chimera and accept the result as due to arsenic that has entered the body from some external source. Kunkel ${ }^{5}$ has summed up the matter in these words.

"The so-called normal arsenic, if there is such a thing, does not affect the results of forensic chemistry, because the so-called normal quantities are so exceedingly small (o.or or even $0.00 \mathrm{r} \mathrm{mg}$. in an organ) that the quantities necessary to furnish a satisfactory forensic proof, which are a hundred or even a thousand times greater, must be regarded as an entirely different and much higher order of magnitudes."

Antimony.-Elimination of antimony takes place largely through the urine. The rest of the metal is found chiefly in the liver and gastro-intestinal tract, as

${ }^{1}$ Loc. cit.

2 Annales de l'Institut Pasteur I7, I-ro (r903); Comptes rendus de l'Academie des Sciences $135,809-812$ (1902); and Bulletin de la Société Chimique de Paris (3), 27, 1 233-1236 (r902).

${ }^{3}$ Annales de Chimie Analytique 12, 52-58 and 97-10I( 1907).

4 Dissertation, University of Nancy, I900.

${ }^{5}$ Zeitschrift für physiologische Chemie 44,5 I I-529 (I905). 


\begin{tabular}{|c|c|c|c|}
\hline Observer & Material & Results & Conclusion \\
\hline$\cdot$ & & & \\
\hline Hödlmoser ${ }^{1}$ & $\begin{array}{l}\text { Human thyroid } \\
\text { gland and liver }\end{array}$ & $\begin{array}{l}20 \text { analyses } \\
\text { Negative or few } \\
\text { traces }\end{array}$ & $\begin{array}{l}\text { Liver gives positive } \\
\text { results as often as } \\
\text { thyroid gland }\end{array}$ \\
\hline Cernýz ${ }^{2}$ & $\begin{array}{l}\text { Human and ani- } \\
\text { mal thyroid and } \\
\text { thymus glands. } \\
\text { Human liver }\end{array}$ & $\begin{array}{l}28 \text { analyses } \\
\text { Negative or faint } \\
\text { traces }\end{array}$ & $\begin{array}{l}\text { Minute traces may } \\
\text { appear but not } \\
\text { constant }\end{array}$ \\
\hline Ziemke $^{3}$ & $\begin{array}{l}\text { Various human } \\
\text { organs }\end{array}$ & $\begin{array}{l}\text { Over } 40 \text { analyses. } \\
\text { Negative. One } \\
\text { case doubtful }\end{array}$ & $\begin{array}{l}\text { Not a normal con- } \\
\text { stituent of human } \\
\text { organs }\end{array}$ \\
\hline Wieser ${ }^{4}$ & $\begin{array}{l}\text { Various human } \\
\text { and animal or- } \\
\text { gans }\end{array}$ & $\begin{array}{l}32 \text { analyses. Mostly } \\
\text { negative but few } \\
\text { traces }\end{array}$ & $\begin{array}{l}\text { Traces inconstant } \\
\text { and due to chance } \\
\text { contamination }\end{array}$ \\
\hline Kunkel $^{5}$ & $\begin{array}{l}\text { Various human } \\
\text { and animal or- } \\
\text { gans }\end{array}$ & Negative & $\begin{array}{l}\text { No such thing as } \\
\text { normal As }\end{array}$ \\
\hline Bloemendal ${ }^{6}$ & $\begin{array}{l}\text { Various human } \\
\text { and animal or- } \\
\text { gans }\end{array}$ & $\begin{array}{l}\text { Mostly negative } \\
\text { but few traces }\end{array}$ & $\begin{array}{l}\text { No such thing as } \\
\text { normal As }\end{array}$ \\
\hline Warren ${ }^{7}$ & $\begin{array}{l}\text { Human thyroid } \\
\text { gland }\end{array}$ & $\begin{array}{lr}32 \text { analyses. Nega- } \\
\text { tive except two } \\
\text { slight traces }\end{array}$ & $\begin{array}{l}\text { No such thing as } \\
\text { normal As }\end{array}$ \\
\hline
\end{tabular}

well as in the kidneys and brain. Pouchet found antimony in bones, skin, hair and chiefly in the intestinal tract. A large part of the ingested poison may be eliminated by emesis. A part is apparently retained in the body for some time, since antimony has been detected in the liver and in the bones months after the last administration.

Lead.-Lead is eliminated in urine and fæces. Elimination by the fæces always exceeds that by the urine, even when the lead has not been taken by the mouth. Mann ${ }^{8}$ (see R. Kobert, Intoxikationen), for example, in the case of two patients was never able to find more than 0.6 milligram of lead in the urine collected during 24 hours, whereas the fæces during the same period contained 2-3 milligrams of lead. In lead poisoning the metal has been found in the

${ }^{1}$ Zeitschrift für physiologische Chemie $33,329-344$ (Igor).

2 Ibid., 34, 408-4I6 (1902).

${ }^{3}$ Vierteljahrsschrift für gerichtliche Medizin (3) 23, 5I-60 (I902).

1 Dissertation, University of Würzburg (1903).

${ }^{5}$ Zeitschrift für physiologische Chemie 44, 5 II-529 (I905).

- Dissertation, University of Leyden ( 1908 ).

7 W. H. Warren, analyses not published.

${ }^{8}$ Zeitschrift für physiologische Chemie 6, 6 (1882). 
saliva, bile and in both red and white blood-corpuscles. In animals relatively most of the lead has been found in the kidneys, after which come bones, liver, testes and finally the brain and blood. In experiments with sheep Ulenberger and Hofmeister obtained the following results:

\begin{tabular}{|c|c|}
\hline Organs and fluids & $\begin{array}{c}\mathrm{Pb} \text { in grams per rooo } \\
\text { grams }\end{array}$ \\
\hline Kidneys & $0.44^{-0.47}$ \\
\hline Liver & $0.3-0.6$ \\
\hline Pancreas & 0.54 \\
\hline Salivary glands & $0.4^{2}$ \\
\hline Bile & $0 . \mathrm{rr}-0.40$ \\
\hline Bones & 0.32 \\
\hline Fæces & 0.22 \\
\hline Spleen & $0 . r_{4}$ \\
\hline Blood & $0.05-0.12$ \\
\hline Urine & $0.06-0.08$ \\
\hline
\end{tabular}

Lead is eliminated especially by the bile and in acute poisoning this secretion may contain more lead than any of the other organs or secretions. Oliver ${ }^{1}$ has given the following results for human material:

Organs

Liver

Spleen

Large brain

Small brain

Kidneys

Heart
$\mathrm{Pb}$ in grams per 1000 grams

0.0416

0.039

$0.02 \times 6$

0.0086

0.013

0.0005

Elimination of lead by the urine is said, in the case of man, not always to be uniform. The urine is free from lead for a long time and later, without further administration, again contains the metal. This behavior is in harmony with the fact that lead can be retained in the organism many months. In chronic lead poisoning the brain has frequently been found to contain much lead. In this kind of poisoning elimination of the metal is always more abundant by the fæces than by the urine.

Chromium.-Chromic acid and soluble chromates and dichromates are quite toxic. The mucous membranes absorb alkaline chromates rapidly and severe, acute poisoning occurs. The poison causes intense pain in the stomach and intestines, collapse and kidney derangement which may terminate fatally in a few hours. Other symptoms are nausea, vomiting of yellow matter which later is tinged with blood, diarrhœa and even bloody stools, intense thirst, emaciation, great anxiety, severe pain in the abdomen, faint and quickened pulse- "the cholera picture." (Kunkel, Toxikologie.) The statements regarding the quantity of an alkaline chromate, capable of producing acute poisoning, agree fairly well. Even a few decigrams ( 0.2 gram) may cause very serious symptoms which

1 The Lancet, March, r8gr. 
sometimes terminate fatally. Chromic acid is eliminated mainly by the urine but partly by the intestines. Elimination takes place rapidly and the body is soon free of the poison. Four days after administration of quite large quantities of a chromate, the urine and fæces are said to contain only traces of this metal.

Copper.--Only a small amount of copper, varying with different compounds, is absorbed by the intestines and carried into the circulation. Sodium cupric tartrate and copper salts of fatty acids are absorbed most easily. Copper poisoning rarely occurs from introducing a copper compound into the stomach. Copper compounds in large amounts act as local caustics and occasion severe pain in the stomach. Vomiting and the sense of taste make it impossible to take much of a copper compound. Foods containing copper are unpalatable. The sense of taste as well as after-taste prevent one from swallowing such in any quantity. Food, containing 0.5 gram of copper per kilogram, has a marked taste. Irresistible nausea, steadily increasing, soon makes it impossible to take more of the food containing copper. Elimination of copper by the urine is very slight. Copper absorbed from the intestines is arrested by the liver where it accumulates. Traces of copper have frequently been found in the human liver. The author in toxicological analyses has repeatedly found weighable quantities of copper in the livers of adults who had not taken copper salts beforehand, except possibly for suicidal purposes. The liver is the most important organ for the detection of copper, next to which come the bile, kidneys and the gastro-intestinal mucosa. Copper is said to be in the liver as a nuclein compound. In the case of blood, copper is located not in the serum but in the corpuscles.

Mercury.-Distribution of mercury in the body is said to be always the same, no matter what the method of administration is. It is immaterial whether it is introduced by the mouth, hypodermically or from an abrasion. Elimination of mercury takes place through the saliva, sweat, bile, gastro-intestinal mucosa and urine. Elimination in the saliva seems to be constant, since mercury can always be detected in the saliva during use of mercurials in lues. A relatively large quantity of this metal is said to be eliminated in the sweat. Opinions differ as to the relative quantities eliminated by the urine and intestines. Usually elimination by the intestines exceeds that by the kidneys. Recent experiments appear to show that mercury is eliminated in the urine regularly and in slowly increasing quantity and then slowly diminishes. Elimination of mercury ceases after 6-9 months and even later. In a most favorable case the total quantity of mercury eliminated in the urine amounts to about 50 per cent. of the total quantity taken but is frequently much less. In mercury poisoning the kidneys, of all the organs persistently contain most of the poison even for weeks. Then follow the liver, spleen, bile and intestinal mucosa. In toxicological analysis the urine should also be examined, though in acute poisoning it always contains only a fraction of a milligram of mercury in a liter. In severe mercurial poisoning the metal may be said to occur in all organs and secretions.

\section{Electrolytic Separation of Mercury from Urine}

Heat a liter of urine upon the water-bath about 2 hours with 5-6 grams of potassium chlorate and ro cc. of concentrated hydrochloric acid and shake frequently. Evaporate to $300 \mathrm{cc}$. and use this solution for the electrolytic separa- 
tion of mercury. Use a Bunsen battery of 3-4 cells, or any other galvanic apparatus having the same strength of current. A thin sheet or rod of gold $2 \mathrm{~mm}$. thick and 6-ro $\mathrm{cm}$. long serves as the cathode, and a piece of platinum wire of about the same thickness as the anode. Place the electrodes in the solution 2-4 cm. apart and allow the experiment to run $24-48$ hours. Wash and dry the gold cathode, upon which the mercury is deposited, and place in a glass tube $(20 \mathrm{~cm}$. long and 4-5 cm. in diameter). This tube is sealed at the bottom and reduced at the top to smaller size. Apply heat until all the mercury is expelled from the gold. Deposit the sublimate $3-4 \mathrm{~cm}$. beyond the top of the gold rod. Then seal the tube below the sublimate. Introduce a small crystal of iodine into the tube and seal the other end. 'Heat the iodine carefully over a small flame to bring it into contact with the mercury. The two elements combine to form red mercuric iodide.

Sometimes it is convenient to precipitate mercury from urine upon other metals, for example, copper, gold, brass and zinc dust. Witz heats to boiling with hydrochloric acid and concentrated potassium permanganate solution to destroy organic matter. Use ro cc. of concentrated hydrochloric acid and I5-20 cc. of potassium permanganate solution for $500 \mathrm{cc}$. of urine. Slowly pass the decolorized liquid through a glass tube over a copper spiral. Wash the copper with potassium hydroxide solution, then with absolute alcohol and cleanse with filter paper. Finally dry at $70-80^{\circ}$ and heat in a glass tube. Treat the mercury sublimate with iodine as described. Satisfactory deposition of mercury upon other metals (gold, copper) requires previous destruction of organic matter in the urine by hydrochloric acid and potassium chlorate. Otherwise, organic substances deposited upon the metal interfere with the iodine test for mercury.

Silver.-In severe poisoning silver has been found in bile, fæces and in many organs. In acute poisoning the urine is usually free from silver, whereas the contents of the intestines may contain the metal even after subcutaneous injection. Absorbed silver salts appear to be reduced in all parts of the body. Examination of the bodies of persons who have suffered from argyria (chronic silver poisoning) has disclosed precipitation of metallic silver in the organs. Silver salts, added to albumin solutions, form very stable compounds usually amorphous. Silver may evince even greater affinity for albumin than for chlorine. All observers agree that only very little of the silver reaching the intestines is absorbed. After silver medication the stools are black from silver sulphide. Frequent quantitative estimations of silver in argyrotic organs have been made. In one case the liver gave 0.047 per cent. of silver and the kidneys $0.06 \mathrm{r}$ per cent. In the condition called argyria, or argyrosis, the skin is black. Internal administration of silver salts causes this color to develop gradually. By'degrees it may become quite marked even causing disfigurement.

\section{Quantitative Estimation of Silver in Organs and Urine}

V. Lehmann, ${ }^{1}$ in determining silver in organs (liver, kidneys, spleen, brain), first thoroughly dries the finely ground material and then fuses with sodium carbonate and potassium nitrate. Extract the melt with water and dissolve the insoluble residue of metallic silver in hot nitric acid. Evaporate this solution

${ }^{1}$ Zeitschrift für physiologische Chemie 6, I9 (1882). 
to dryness upon the water-bath and precipitate silver as chloride. Avoid a large excess of hydrochloric acid.

Mix urine with sodium carbonate and potassium nitrate, evaporate to dryness, fuse the residue and treat the melt as described.

Uranium.-Experiments made by $R$. Kobert have shown that uranium, administered subcutaneously or intravenously, is the most toxic of all metals. Uranyl acetate is an excellent precipitant of albumins and the other uranyl salts must behave in much the same way. Consequently internal administration of concentrated solutions of uranyl salts destroys the mucous surfaces they touch, for example, that of the stomach, changing the living stomach wall to dead uranyl-albuminate. Uranyl salts therefore must be classed among the powerful caustic poisons. In addition to acting as local corrosives, uranium salts resemble hydrocyanic acid in partially arresting internal oxidation in the organs and occasioning the severest disturbances of metabolism.

Bismuth.-This metal becomes quite toxic when it reaches the blood. Bismuth solutions, prepared by dissolving bismuthous hydroxide in tartaric or citric acid and then neutralizing with sodium or ammonium hydroxide solution, have been repeatedly administered to animals subcutaneously and intravenously. The smallest lethal dose of these double bismuth salts, injected subcutaneously, was found to be but $6 \mathrm{mg}$. per kilogram for a dog or cat and $24 \mathrm{mg}$. per kilogram for a rabbit. Bismuth salts insoluble in water produce entirely different results when administered internally. Bismuth subnitrate and similar salts dissolve very slightly in the highly diluted hydrochloric acid of the gastric juice. Consequently very little bismuth is conveyed to the blood. Most of the bismuth taken by the mouth reaches the intestines. Instead of being absorbed, it is changed to bismuthous sulphide by the hydrogen sulphide always present. Absorbed bismuth is eliminated by the saliva, bile, urine, mucous lining of the mouth, stomach, small and large intestine and also the milk. If an animal is poisoned by bismuth, the metal can be detected in the urine, bile, liver, kidneys, spleen, walls of the intestines as well as in the bones. Different observers have found the metal in especially large amount in the milk but very little in the kidneys and liver.

Zinc.-There is no doubt that zinc salts reaching the intestinal tract are absorbed in very small quantity. As yet there is no satisfactory explanation of the fate of absorbed zinc. In zinc poisoning large amounts of the metal have been found repeatedly in the liver and bile. This may mean that zinc is arrested by the liver and eliminated in the bile. Lehmann ${ }^{1}$ after 335 days killed a dog that had been fed for a considerable time upon zinc carbonate. The following organs, arranged according to the quantity of metal in each, contained zinc: liver, bile, large intestine, thyroid gland, spleen, pancreas, urine, kidneys, bladder, muscle, brain, lymphatic gland, stomach, small intestine, lungs, blood. Occasionally considerable quantities of zinc may be taken with articles of food and drink. All acids dissolve metallic zinc very freely. Even water containing carbon dioxide is a solvent. Consequently it may be in drinking water from galvanized pipes. All kinds of food and drink, kept in zinc vessels or vessels coated with zinc, may contain more or less of this metal. Plants grown upon

1 Archiv für Hygiene 28, 29r (1896). 
soil containing zinc take up the metal. Zinc has also been found repeatedly in parts of human cadavers under circumstances precluding all possibility of poisoning by this metal. Even considerable quantities of zinc have been found in the human liver.

Tin.-The cases of tin poisoning thus far observed resemble those of copper and zinc. What knowledge there is regarding the toxic action of absorbed tin has been gained from experiments upon animals. These experiments show that small quantities of tin are absorbed and eliminated in the urine, when ordinary tin compounds are brought into the stomach. But thus far distinct symptoms of poisoning by such quantities of the metal have not been confirmed. (Kunkel, Toxikologie.)

White ${ }^{1}$ failed to produce poisoning by bringing tin into a dog's stomach. The animal received sodium stannous tartrate in increasing doses for 22 days, the daily amount being $0.02-0.06 \mathrm{gram}$. Yet the animal absorbed tin. In the urine, during an experiment lasting 8 days, White found 0.02 gram of tin. But the tin salt mentioned, introduced directly into the circulation of the animal, was quite toxic in its action. Stannous chloride, administered for a very long time to a dog, produced symptoms of poisoning. The urine in this case contained small quantities of tin. Kunkel (Toxikologie) states that tin has a very slight poisonous action. Apparently it is eliminated very rapidly by the kidneys. Quite probably this prevents accumulation of the metal in the body and consequent poisoning. The fact that White did not observe toxic symptoms, after feeding a dog for 22 days with relatively large quantities of easily absorbable sodium stannous tartrate; and that Ungar and Bodlander ${ }^{2}$ failed to produce derangements with the same compound, until it had been administered for a year, prove that tin is quite free from toxic properties. Hence, tin vessels may be used and preserved articles of food containing tin have practically no deleterious action upon health.

${ }^{1}$ Archiv für experimentelle Pathologie und Pharmakologie $13,53$.

${ }^{2}$ Zeitschrift für Hygiene, 2, 24I. 


\section{CHAPTER IV}

\section{POISONS NOT IN THE THREE MAIN GROUPS}

\section{MINERAL ACIDS}

\section{Hydrochloric, Nitric and Sulphuric Acids}

To detect free mineral acid, extract a portion of material with cold water, filter and test as follows, if the solution is strongly acid:

I. Methyl Violet Test.-Add a few drops of an aqueous (o.I : rooo) or alcoholic ( $\mathrm{x}: \mathrm{ro0}$ ) solution of methyl violet ${ }^{1}$ to a small portion of filtrate. A free mineral acid produces a blue or green color.

2. Methyl Orange Test.-Add a few drops of a dilute aqueous solution of methyl orange ${ }^{2}$ to the filtrate. A red color indicates free mineral acid.

3. Congo Paper Test.-Even very dilute solutions of free mineral acids turn "Congo paper" blue.

4. Günzburg's Test.-Mix a few drops of the filtrate with 3-4 drops of Günzburg's reagent ${ }^{3}$ and 'evaporate to complete dryness upon the water-bath, or over a small flame. Frée hydrochloric or sulphuric acid gives a fine red or reddish yellow residue. Nitric acid gives more of a yellowish red residue.

${ }^{1}$ Methyl violet is the hydrochloride of hexa-methyl-para-rosaniline:

or
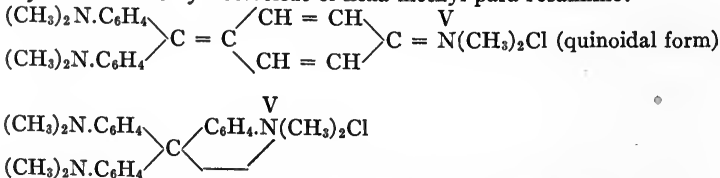

${ }^{2}$ Methyl orange $=$ Dimethyl-amino-azobenzene-4-sulphonic acid:

$$
\left(\mathrm{CH}_{3}\right)_{2} \stackrel{4}{\mathrm{~N}} \cdot \mathrm{C}_{6} \mathrm{H}_{4} \cdot \stackrel{\mathrm{I}}{\mathrm{N}}=\stackrel{\mathrm{I}}{\mathrm{N}} \cdot \mathrm{C}_{6} \mathrm{H}_{4} \cdot \stackrel{4}{\mathrm{~S}} \mathrm{O}_{2} \mathrm{OH} \text {. }
$$

The sodium salt of this sulphonic acid also appears in commerce under the name "methyl orange."

${ }^{3}$ See page $32 \mathrm{I}$ for the preparation of this reagent. 
5. Sulphocyanate Test.-Add a little potassium sulphocyanate solution to ferric acetate solution and dilute with water until yellow. Then add the solution to be tested. Free mineral acid produces a blood-red color. Traces of free mineral acid, especially if considerably diluted, do not give a red color until several minutes have elapsed.

One or more of these general tests, which furnish evidence of a free mineral acid, must always accompany the special tests to be described later. Not only free mineral acids give the special tests but in certain cases their salts. Chlorides, sulphates and nitrates are normal constituents of nearly all vegetable and animal materials. As a rule an examination of cadaveric material for mineral acids is necessary only when the autopsy points conclusively to such poisoning. That is to say, when there are characteristic corrosions and discolorations about the face, mouth, œsophagus and stomach. If general tests show the presence of free mineral acid, make special tests for the particular acid.

\section{Hydrochloric Acid}

I. Chlorine Test.-Warm a little of the aqueous extract, if not too dilute, with finely powdered manganese dioxide. Free hydrochloric acid yields chlorine, recognized by its color and odor, or by passing the gas into potassium iodide solution and liberating iodine. Hydrochloric acid exclusively does not give this test. A chloride $(\mathrm{NaCl})$ and free sulphuric acid give chlorine under the same conditions.

2. Distillation.-If possible, separate hydrochloric acid from other substances by distillation. The concentration of the acid is especially important, since dilute hydrochloric acid upon distillation at first yields only water. Hydrochloric acid ${ }^{1}$ itself does not begin to distil until the concentration is about ro per cent. Since a dilute hydrochloric acid is usually examined, distil the material mixed with water, or preferably a

${ }^{1}$ In the distillation of roo cc. of I per cent. hydrochloric acid, the first $90 \mathrm{cc}$. of distillate will contain only traces of hydrochloric acid, whereas the last portion will contain most of the acid. 
filtered aqueous extract, nearly to dryness. In such a distillation apply heat by means of an oil-bath. To detect hydrochloric acid in the distillate, acidify with dilute nitric acid and add silver nitrate solution. Frequently a quantitative estimation of hydrochloric acid is required. In the absence of other acids, titrate the distillate with o.I n-potassium hydroxide solution, using phenolphthalein as indicator. Otherwise, estimate the acid gravimetrically, precipitating with silver nitrate and weighing silver chloride, or volumetrically by Volhard's method. In the latter case, precipitate hydrochloric acid with o.I $\mathrm{n}$-silver nitrate solution in excess and subsequently estimate that excess by titration with $0.1 \mathrm{n}$-ammonium sulphocyanate solution, using ferric alum as indicator. Since the human stomach normally contains $0.1-0.6$ per cent. of free hydrochloric acid, an examination of stomach contents for this acid must always include a quantitative estimation.

\section{Nitric Acid}

The human body normally contains only a very small amount of nitrates. When present, they are due usually to vegetable foods which contain small quantities of nitrates. Human urine almost always shows traces of the salts of nitric and nitrous acids. The chemical examination of cadaveric material need not include tests for nitric acid, unless the autopsy affords evidence of poisoning by this acid, as distinct signs of corrosion about the lips, mouth, œsophagus and stomach and sometimes perforation. These parts are more or less yellow or yellowish brown. A yellow froth is said to exude from the mouth and nose of the cadaver. Also the stomach contents are yellow in concentrated nitric acid poisoning. If the concentration of the acid is less than 20 per cent., these specific changes may not appear in the gastro-intestinal tract. Nitric acid taken internally, dilute or concentrated, appears at once in the urine.

\section{Detection of Nitric Acid}

I. Distillation.-If possible, extract the material direct with water, filter and test the filtrate for nitric acid in the usual way. When the quantity of nitric acid is large, separate it from other substances by distilling the filtered aqueous extract. Apply heat by means of an oil-bath. Nitric acid ${ }^{1}$ does not distil, until

${ }^{1}$ If roo cc. of I per cent. nitric acid are mixed with bread crumbs and distilled, most of the acid will be in the final ro cc. of distillate. 
it reaches a definite concentration. At the same time a large part of the acid combines with organic substances, if any are present, forming nitro-derivatives, xanthoproteic acid, etc. Nitric acid may also cause oxidation. Consequently the distillate does not contain all the acid originally present. The residue from such a distillation is usually distinctly yellow. Toward the end of distillation brown vapors of nitrogen peroxide often appear. Such a distillate, added to starch paste and potassium iodide, produces an immediate blue color in presence of dilute sulphuric acid.

To detect nitric acid in the distillate, employ the following tests:

2. G. Fleury's Procedure. ${ }^{1-}$-Extract the finely divided material with absolute alcohol, filter and add slaked lime in excess to the filtrate. To decompose any nitric acid ester present, let the mixture stand I 2 hours, filter and evaporate the filtrate to dryness. Dissolve the residue in 95 per cent. alcohol, expel alcohol from the filtered solution and finally test an aqueous solution of the residue for nitric acid. Fleury has obtained by means of this method about 20 per cent. of the nitric acid from animal material. This procedure converts the acid into its calcium salt which is soluble in alcohol. But sodium nitrate is also quite soluble in 95 per cent. alcohol $\left(\mathrm{I}: 5^{\circ}\right)$. Therefore, if the final residue gives a faint test for nitric acid, the proof of free acid in the original material is not conclusive. The following method obviates this difficulty.

3. Baumert's Procedure. ${ }^{2}$-Neutralize the material itself, or its aqueous extract, with milk of lime, dry and extract with alcohol. Or, after neutralization with milk of lime or calcium carbonate, evaporate to a syrup, stir and mix with alcohol. Distil the filtered alcoholic extract obtained in either way, dissolve the residue in water, filter and evaporate the solution. Dissolve the residue again in alcohol and allow this solution to stand for several hours in a closed flask with about. the

1 Annales de Chimie analytique appliquée 6, I 2.

${ }^{2}$ Lehrbuch der gerichtlichen Chemie, second edition (I907). 
same volume of ether. Filter this alcohol-ether solution, evaporate the solvent and dissolve the residue in a little water. Apply the following nitric acid tests to this solution:

(a) Diphenylamine and Sulphuric Acid Test.-Blue color. Add a few drops of diphenylamine sulphate solution ${ }^{1}$ to the aqueous extract, or distillate, and carefully pour this mixture upon pure concentrated sulphuric acid free from nitric acid. If nitric acid is present, a blue zone appears where the two liquids meet.

(b) Brucine and Sulphuric Acid Test.-Red color.

Mix the liquid to be tested with the same volume of brucine sulphate solution ${ }^{2}$ and carefully pour this mixture upon pure concentrated sulphuric acid. If nitric acid is present, a red zone appears where the two liquids meet.

(c) Ferrous Sulphate and Sulphuric Acid Test.-Saturate the liquid to be tested with pure ferrous sulphate and carefully pour this solution upon pure concentrated sulphuric acid. If nitric acid is present, a black zone appears where the two liquids meet.

(d) Copper Test.-Place a small piece of clean copper (wire or sheet) in nitric acid and heat. Red-brown vapors of nitrogen peroxide $\left(\mathrm{NO}_{2}\right)$ appear.

\section{Sulphuric Acid}

Nearly all animal and vegetable substances normally contain sulphates. Consequently an examination for free sulphuric acid must exclude its salts. There is no need of examining cadaveric material for the free acid, unless marked corrosion and discoloration of lips, mouth, œsophagus and stomach indicate its presence. There are eschars upon the lips and the mucous lining of the mouth is grayish white. The white coating on the back of the tongue may have been dissolved exposing the firm, brownish muscular tissue beneath. The tongue often looks as if it had been boiled. The mucous lining of the osophagus is

\footnotetext{
${ }^{1}$ Prepare this solution by dissolving I gram of diphenylamine, $\left(\mathrm{C}_{6} \mathrm{H}_{5}\right)_{2} \mathrm{NH}$, in 5 grams of dilute sulphuric acid and roo cc. of water.

${ }^{2}$ Prepare this solution by dissolving $\mathrm{x}$ gram of brucine in 5 grams of dilute sulphuric acid and roo cc. of water. The sulphuric acid used must give none of the tests for nitric acid. If it does not meet this requirement, heat in a platinum dish to expel interfering nitrous substances. Or distil the acid from a small retort, rejecting the first part of the distillate.
} 
much wrinkled and coated gray. Externally the stomach is usually brown or slate-gray and its contents black. Frequently in sulphuric acid poisoning there is perforation of the stomach wall and brownish black masses find their way into the abdominal cavity. There may be black spots in the stomach, due according to R. Kobert (Intoxikationen) not to charring, as previously supposed, but to brown-black hæmatin. Acids decompose the blood-pigment oxyhæmoglobin mainly into hæmatin and protein (globulin). Methæmoglobin and hæmatoporphyrin may also be formed. Acids produce the latter from hæmatin and in the change there is loss of iron. All three of these decomposition products of the red blood-pigment, namely, methæmoglobin, hæmatin and hæmatoporphyrin may be formed successively and then appear in the urine. The blood in the stomach walls is often acid and then contains chiefly methæmoglobin and hæmatin. The mucosa of the intestines even far down may be grayish white and strongly acid.

\section{Detection of Sulphuric Acid}

I. Extract the finely divided material, if strongly acid, with cold absolute alcohol and after some time filter. The solution contains sulphuric acid but not sulphates. Evaporate the alcoholic filtrate upon the water-bath, or, if the volume is large, distil the alcohol. Dissolve the residue in a little water (Io cc.) and heat the solution to boiling to saponify ${ }^{1}$ ethyl sulphuric acid. Filter and test the filtrate with barium chloride or lead acetate solution. To prove that the precipitate is a sulphate, mix with sodium carbonate and fuse upon charcoal. The sodium sulphide formed blackens metallic silver in presence of water, or gives hydrogen sulphide with acids.

2. Extract the finely divided material with water and apply the following tests to the filtrate:

(a) Sugar Test.-Evaporate some of the filtered extract in a porcelain dish with a small particle of sugar. Free sulphuric acid produces a black, carbonaceous residue.

(b) Sulphur Dioxide Test.-Concentrate the filtered extract upon the water-bath and heat in a test-tube with a few pieces of copper. Free sulphuric acid generates sulphur dioxide, recognized by its stifling odor. Distil the sulphur dioxide (preferably in an atmosphere of carbon dioxide) into a little water and test the distillate as follows:

${ }^{1} \mathrm{HO} . \mathrm{SO}_{2} \cdot \mathrm{OC}_{2} \mathrm{H}_{6}+\mathrm{H}_{2} \mathrm{O}=\mathrm{C}_{2} \mathrm{H}_{5} \cdot \mathrm{OH}+\mathrm{H}_{2} \mathrm{SO}_{4}$. 
$\alpha$. Warm some of the liquid with a little stannous chloride solution. A yellow precipitate of stannic sulphide ${ }^{1}$ appears.

$\beta$. Add iodo-potassium iodide solution drop by drop. The color of the iodine disappears and at the same time sulphuric acid is formed:

$$
\mathrm{H}_{2} \mathrm{SO}_{3}+\mathrm{H}_{2} \mathrm{O}+\mathrm{I}_{2}=\mathrm{H}_{2} \mathrm{SO}_{4}+{ }_{2} \mathrm{HI} \text {. }
$$

Barium chloride then precipitates barium sulphate insoluble in dilute hydrochloric acid.

To estimate sulphuric acid quantitatively, either precipitate and weigh barium sulphate in the usual way, or titrate with o.I n-potassium hydroxide solution, using phenolphthalein as indicator.

I000 cc. of 0.1 n-potassium hydroxide solution $=0.1$ gramequivalent of sulphuric acid $=4.9$ grams of $\mathrm{H}_{2} \mathrm{SO}_{4}$.

\section{Detection of Sulphurous Acid}

Sulphur dioxide acts most injuriously when inhaled. It is very irritating to the respiratory organs and also changes the blood-pigment. After death the respiratory organs are found to be profoundly altered as when acted upon by strong mineral acids. After severe poisoning by vapors containing sulphur dioxide, the blood is dirty brownish red ${ }^{2}$ and usually gives the hæmatin spectrum. Human beings experience discomfort, if there are $0.015-0.02$ volumes of sulphur dioxide per rooo volumes of air. Many persons become quite ill in a few minutes, when there are 0.03 volumes of sulphur dioxide in rooo volumes of air. The gas produces a sharp, stinging sensation in the nostrils, sneezing and coughing. In experiments upon mice, rabbits and guinea-pigs, Lehmann observed marked toxic symptoms from air containing 0.04 volume per cent. of sulphur dioxide; death ensued in 6 hours from 0.06 per cent.; and in 20 minutes from 0.08 per cent. Articles of food and drink, preserved by means of sulphurous acid or its salts, may injure the health, causing especially gastro-intestinal catarrh and other chronic derangements. For this reason it is prohibited to preserve articles of food and drink by means of sulphurous acid, sulphites and hyposulphites.

If the quantity of sulphur dioxide in air is not too small, its presence may be recognized by its characteristic stifling odor. A strip of paper, moistened with

${ }^{1}$ Sulphurous acid and sodium sulphite, added to stannous chloride solution not too strongly acid, precipitate stannous sulphite, $\mathrm{SnSO}_{3}$, white and readily soluble in hydrochloric acid. Warmed in presence of hydrochloric acid, sulphur dioxide acts upon a stannous salt as an oxidizing agent. A precipitate of $\mathrm{Sn}_{6} \mathrm{O}_{10} \mathrm{~S}_{2}$ is formed, or $\mathrm{H}_{2} \mathrm{~S}$ is evolved and $\mathrm{SnCl}_{4}$ formed, depending upon the amount of bydrochloric acid present. (Prescott and Johnson, Qualitative Chemical Analysis. Fifth edition, page 86.)

${ }^{2}$ Neutral sulphites cause the blood to become brick-red. 
a solution of pure potassium iodate $\left(\mathrm{KIO}_{3}\right)$ and starch, turns blue in air containing sulphur dioxide owing to the formation of a compound of iodine and starch. This reaction serves as a preliminary test for the detection of sulphurous acid and hyposulphites in chopped meat, sausage meat and other meat products. Shake the meat in an Erlenmeyer flask with phosphoric acid, suspend in the neck of the flask from the stopper (see Fig. I, page 3 ) a paper strip prepared as described and heat the flask upon the water-bath. The paper should not turn blue.

Explanation.-Sulphur dioxide reduces potassium iodate $(\alpha)$. Sulphuric acid thus formed liberates hydriodic and iodic acids from their salts $(\beta$ and $\gamma)$. The iodine set free by the interaction of these two acids $(\delta)$ finally turns the starch blue.

$$
\begin{aligned}
& \text { (a) } \mathrm{KIO}_{3}+{ }_{3} \mathrm{H}_{2} \mathrm{SO}_{3}=\mathrm{KI}+{ }_{3} \mathrm{H}_{2} \mathrm{SO}_{4} \text {, } \\
& \text { (ß) }{ }_{2} \mathrm{KI}+\mathrm{H}_{2} \mathrm{SO}_{4}={ }_{2} \mathrm{HI}+\mathrm{K}_{2} \mathrm{SO}_{4} \text {, } \\
& \text { ( } \gamma{ }_{2} \mathrm{KIO}_{3}+\mathrm{H}_{2} \mathrm{SO}_{4}={ }_{2} \mathrm{HIO}_{3}+\mathrm{K}_{2} \mathrm{SO}_{4} \text {, }
\end{aligned}
$$

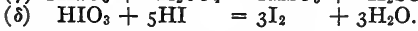

The official directions ${ }^{1}$ for the detection and quantitative estimation of sulphur dioxide in meat are as follows. Mix 30 grams of finely chopped meat with 200 cc. of boiled water in a $500 \mathrm{cc}$. distilling flask. ${ }^{2}$ Add sodium carbonate solution until the reaction is faintly alkaline.

Let the mixture stand for an hour and then completely expel air from the apparatus by passing carbon dioxide through the tube extending to the bottom of the flask. Then introduce into the Peligot tube (see below) $50 \mathrm{cc}$. of iodine solution ( 5 grams of pure iodine and 7.5 grams of potassium iodide in a liter of water). Raise the stopper of the distilling flask and, without stopping the flow of carbon dioxide, add ro cc. of ro per cent. phosphoric acid solution. Then carefully heat the contents of the flask and distil half the liquid, maintaining all the while a current of carbon dioxide. Transfer the contents of the Peligot tube, which should be brown, to a beaker, rinsing it out with water to prevent loss of solution. Add a little hydrochloric acid, heat and by means of barium chloride solution completely precipitate the sulphuric acid formed from the oxidation of sulphurous acid by iodine.

$$
\mathrm{H}_{2} \mathrm{SO}_{3}+\mathrm{H}_{2} \mathrm{O}+\mathrm{I}_{2}=\mathrm{H}_{2} \mathrm{SO}_{4}+{ }_{2} \mathrm{HI} \text {. }
$$

If this test is positive, then the meat examined contains either free sulphurous acid, sulphites or hyposulphites. In the quantitative estimation the barium sulphate should be weighed in the usual manner.

\section{OXALIC ACID}

Oxalic acid and its salts, for example, salt of sorrel, are quite toxic substances. Administration of oxalic acid has terminated fatally in the case of adults in a few

1 Measures for putting into effect the law of the German Empire of June 3, I900, relating to the inspection of beef-cattle and meats.

${ }^{2}$ The apparatus prescribed for official examinations is a distilling flask, having a capacity of 400-500 cc. and provided with a two-hole stopper for two glass tubes entering the flask. One tube extends to the bottom of the flask and the other only into the neck. The latter is connected with a Liebig condenser to which a Peligot tube is fastened at the other end by a tight stopper. 
minutes. Oxalic acid is very abundant in the vegetable kingdom in the form of its acid potassium salt, $\mathrm{KHC}_{2} \mathrm{O}_{4}$, and calcium salt. Sorrel, wood-sorrel and rhubarb are especially rich in salts of oxalic acid. Hence this acid may find access to the body through food and drugs of vegetable origin. Moreover, oxalic acid is a normal constituent in small quantity of human urine, 2-6 milligrams being excreted in the course of a day. Consequently in examining animal material it is often necessary to supplement a positive qualitative test by a quantitative estimation of oxalic acid.

Toxic Action.-An important difference between mineral acids and oxalic acid is the toxicity of salts of the latter. Not only do free oxalic acid and its acid potassium salt, salt of sorrel, show poisonous properties but even very dilute solutions of neutral sodium oxalate, $\mathrm{Na}_{2} \mathrm{C}_{2} \mathrm{O}_{4}$, act in the same way. Therefore in oxalic acid poisoning it is necessary to distinguish between local corrosion, occurring at the point of application and also in part upon elimination, and remote action due to absorption. Local action at the point of application is corrosive like that of all acids. Local action at the place of elimination depends upon the formation and insolubility of calcium oxalate. On account of the ease with which the organism takes up oxalic acid and its alkali salts, the action or the absorbed poison is rapid. The effects caused by its presence may be attributed 'to the fact that this acid removes in part from organs, as the heart, and from body fluids (blood) the calcium they' require for their life processes, converting it in part into insoluble calcium oxalate. Oxalates diminish the coagulating power as well as the alkalinity of blood. On the other hand they increase the quantity of sugar in the blood. In oxalic acid poisoning there is a depression of the entire metabolism. This is also the case as regards taking up oxygen and giving off carbon dioxide. The body temperature falls as the processes of metabolism are retarded. Owing to withdrawal of calcium from the heart, that organ is weakened and finally paralyzed. Local action upon the kidneys is due to clogging of the injured urinary tubules by deposits of calcium oxalate. The flow of urine may wholly cease in consequence of total impairment of the urinary tubules and death may ensue from anuria and uræmia. Fatal poisonings from large doses of oxalic acid are usually of short duration. R. Kobert (Intoxikationen) describes a case where death occurred within ro minutes.

Bischoff $^{1}$ has made statements with regard to the distribution of oxalic acid in the different organs of persons poisoned by this substance. In a case, which terminated fatally in less than ís minutes, the quantity of oxalic acid in each organ was determined separately and found to be:

\begin{tabular}{rlcc} 
Weight & \multicolumn{1}{c}{ Organ } & \multicolumn{2}{c}{ Oxalic Acid } \\
2240 grams & $\begin{array}{l}\text { Stomach, œsophagus, intestine and } \\
\text { contents }\end{array}$ & 2.28 & grams. \\
770 grams & Liver & 0.285 grams. \\
290 grams & Kidneys & 0.0145 grams. \\
r80 grams & Blood from the heart & 0.0435 grams. \\
40 grams & Urine & 0.0076 grans.
\end{tabular}

'Berichte der Deutschen chemischen Gesellschaft, I6, I350 (1883). 
The quantity of oxalic acid in the liver is noticeably large. The kidneys and urine contain only a little of the poison, owing to the short duration of life after poisoning. A striking thing about the urine excreted during oxalic acid poisoning is the abundant deposition of crystallized calcium oxalate.

\section{Detection of Oxalic Acid}

To detect oxalate without discriminating between the free acid, acid potassium salt or calcium oxalate, employ the following method:

Add to the finely divided material 3-4 volumes of alcohol and acidify with dilute hydrochloric acid. Stir frequently and let the mixture digest $\mathrm{I}-2$ hours cold. Then filter through a plaited paper moistened with alcohol and wash the residue with alcohol. To prevent formation of ethyl oxalate during evaporation, add about $20 \mathrm{cc}$. of water to the total filtrate. Evaporate upon the water-bath until all alcohol is expelled. Pass the aqueous residue through a small filter. Extract the filtrate in a separating funnel $3-4$ times with $50-60 \mathrm{cc}$. portions of ether. Let the total ether extract stand for some time in a dry flask, then pass through a dry filter and distil. Dissolve the residue in $2-3 \mathrm{cc}$. of water and pass the solution, if necessary, through a moist filter. Add ammonium hydroxide solution until alkaline and then saturated calcium sulphate solution. If there is a precipitate, acidify with acetic acid and let solution and precipitate stand over night in a covered beaker. If

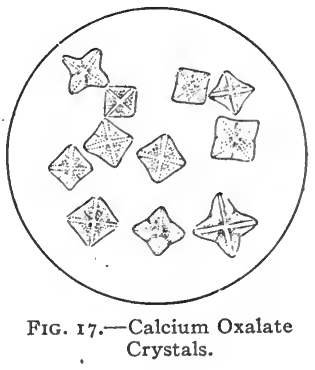
there is still a crystalline precipitate, it can be only calcium oxalate. A microscopic examination of this precipitate is advisable. Calcium oxalate forms characteristic octahedrons having the so-called envelope-shape (Fig. I7). When thoroughly washed, calcium oxalate may be converted by ignition into calcium oxide which may be weighed.

$$
\underset{(56)}{\mathrm{CaO}}: \underset{(126)}{\mathrm{H}_{2} \mathrm{C}_{2} \mathrm{O}_{4} \cdot 2 \mathrm{H}_{2} \mathrm{O}}=\underset{\text { Weight of } \mathrm{CaO}: \mathrm{x}}{\text { found }}
$$


Calculation.-Since the quotient $56:$ 126 $=0.444$, multiply the weight of calcium oxide found by 0.444 to get the corresponding amount of crystallized oxalic acid.

\section{FREE ALKALIES}

\section{Potassium, Sodium and Ammonium Hydroxides}

Free Alkalies.-The same general principles used in detecting mineral acids are applicable also to the alkalies. Since potassium and sodium compounds are normal constituents of animal and vegetable organisms, and since ammonia is a decomposition product of nitrogenous organic matter, the examination must always show that the alkalies are in the free state, for they alone and their carbonic acid salts decompose and corrode animal tissues and not their neutral salts.

Poisonings due to caustic alkalies resemble those caused by corrosive acids. If taken internally, their corrosive action gives rise to pain in the mouth, throat, œsophagus, stomach and abdomen. Mineral acid corrosions are dry and brittle, whereas those from caustic alkalies are soft and greasy. The alkali albuminates formed become gelatinous, swell and may partly dissolve in presence of much water. The destructive action of the caustic alkalies extends deep and affects the parts around the corroded places. In caustic alkaline solutions gelatinous tissues, horny substances, hair and skin swell considerably and finally dissolve The stomach in alkali poisoning is softened in places, corroded and decidedly bright red in color.

\section{Detection of Alkalies}

\section{Ammonia}

Free ammonia is usually recognized by its odor. A piece of moist red litmus paper, held over the material, becomes blue. A paper moistened with mercurous nitrate solution is blackened.

Distillation.-If the material is strongly alkaline, extract several times with absolute alcohol. Use a flask with a glass stopper and distil the combined extracts. Collect the distillate in a little dilute hydrochloric acid and evaporate the solution to dryness in a porcelain dish upon the water-bath. Dissolve the residue in water and test the solution for ammonia, using Nessler's reagent and chloroplatinic acid.

\section{Fixed Alkalies}

The residue from the above distillation may contain potassium and sodium hydroxides. If the residue is strongly alkaline, first add a few drops of phenolphthalein and then excess of barium chloride solution. The red color and the alkaline reac- 
tion, if due to carbonates, disappear, because two neutral salts are formed:

$$
\mathrm{K}_{2} \mathrm{CO}_{3}+\mathrm{BaCl}_{2}=\mathrm{BaCO}_{3}+{ }_{2} \mathrm{KCl} \text {. }
$$

But if alkaline hydroxides are present, the alkaline reaction and red color remain, for soluble barium hydroxide is formed:

$$
{ }_{2} \mathrm{KOH}+\mathrm{BaCl}_{2}=\mathrm{Ba}(\mathrm{OH})_{2}+{ }_{2} \mathrm{KCl} \text {. }
$$

And the solution of this compound reddens phenolphthalein.

To distinguish potassium from sodium hydroxide, neutralize the residue from distillation with dilute hydrochloric acid and test for potassium and sodium as follows:

I. Add solution of chloroplatinic acid $\left(\mathrm{H}_{2} \mathrm{PtCl}_{6}\right)$ which causes the precipitation of potassium in the form of the double chloride of potassium and platinum (potassium chloroplatinate, $\mathrm{K}_{2} \mathrm{PtCl}_{6}$ ).

2. Add de Konink's reagent ${ }^{1}$ which is a solution of sodium cobaltic nitrite, $6 \mathrm{NaNO}_{2} \cdot \mathrm{Co}_{2}\left(\mathrm{NO}_{2}\right)_{6}$. This reagent produces a yellow precipitate of potassium cobaltic nitrite, $6 \mathrm{KNO}_{2}$. $\mathrm{Co}_{2}\left(\mathrm{NO}_{2}\right)_{6}+\mathrm{xH}_{2} \mathrm{O}$, in a solution containing a potassium salt. To hasten the reaction, add a few drops of acetic acid.

3. Test for sodium in a neutral solution by adding a few drops of freshly prepared acid potassium pyro-antimonate solution, $\mathrm{K}_{2} \mathrm{H}_{2} \mathrm{Sb}_{2} \mathrm{O}_{7}$. At first the solution is turbid but, if stirred, deposits a white crystalline precipitate of sodium pyro-antimonate, $\mathrm{Na}_{2} \mathrm{H}_{2} \mathrm{Sb}_{2} \mathrm{O}_{7}$.

Vitali's procedure in testing for caustic alkalies consists in shaking the alcoholic extract of the material, prepared as far as possible with exclusion of air (see above), with freshly precipitated and well-washed mercurous chloride. Free alkali blackens this compound. The solubility of mercurous oxide $\left(\mathrm{Hg}_{2} \mathrm{O}\right)$, the black compound formed, in dilute nitric acid distinguishes it from mercuric sulphide.

Quantitative Estimation of Hydroxides and Carbonates of Alkalies.-To determine both the free caustic alkali and that

1 Prepare sodium cobaltic nitrite by dissolving Io grams of pure sodium nitrite and 4 grams of cobaltous nitrate separately in sufficient water. Mix the solutions, add 2 cc. of acetic acid and dilute to roo cc. with water. 
converted into carbonate, first determine total alkalinity by titrating a portion of the distillation residue with normal or o.I n-hydrochloric acid, using methyl orange as indicator. Then precipitate carbonate in a second portion of the distillation residue with barium chloride solution and determine free caustic alkali in the filtrate. If the examination shows only alkaline carbonate, this does not exclude the possibility of caustic alkali having been originally present.

\section{POTASSIUM CHLORATE}

Toxic Action.-Large doses (4-10 grams) of potassium chlorate, $\mathrm{KClO}_{3}$, are decidedly toxic. During the first stage of intoxication, alteration in the shape of the red corpuscles and conversion of oxyhæmoglobin in the intact corpuscles into brown methæmoglobin take place. Then the red blood-corpuscles, at least in a case of severe poisoning, change their form, becoming shriveled and undergoing decomposition. Toxicologists (see R. Kobert, Intoxikationen) ascribe change of blood pigment and red blood-corpuscles to specific salt action possessed in high degree by potassium chlorate. This explanation also accounts for salt diuresis, ${ }^{1}$ appearing at the beginning of potassium chlorate poisoning, whereby the blood is much thickened. But most notable is the high alkalinity of the urine, resulting in decreased alkalinity of the blood plasma. In severe chlorate poisoning so much oxyhæmoglobin is changed to methæmoglobin that the amount of oxygen in the blood may drop to I per cent. As a result human beings or animals thus poisoned may become asphyxiated from lack of oxygen. Potassium chlorate through the action of potassium weakens the heart. In chlorate poisoning the blood has a characteristic chocolate-brown color (see above).

Potassium chlorate taken by the mouth is quite rapidly eliminated by the kidneys. After administration of 0.1 gram of potassium chlorate, chloric acid appears in the urine in an hour. Most of the potassium chlorate passes into the urine unchanged, only a little of the salt being reduced to potassium chloride. During chlorate poisoning, the urine is usually very dark, even black, and may contain hæmoglobin and methæmoglobin. It is frequently opaque and strongly alkaline. Upon long standing a dark brown sediment gradually deposits. The urine also contains considerable albumin.

In suspected chlorate poisoning, the urine should if possible receive a thorough chemical and microscopical examination. An anuria lasting several days may precede death and render an examination of the urine quite impossible.

\section{Detection of Chloric Acid}

To isolate potassium chlorate from organic material, use a dialyzer which should be as flat as possible, because the thinner the layer in the inner container and the larger the volume of

${ }^{1}$ Diuresis $=$ increased secretion of urine. 
water in the outer vessel, the more rapid the diffusion. Place the material to be examined, as parts of organs and stomach or intestinal contents, in the inner container of a flat dialyzer and pure water in the outer vessel. Allow dialysis to take place 5-6 hours without changing the water in the outer vessel. Then evaporate the dialysate (contents of the outer vessel) to dryness in a porcelain dish upon the water-bath. Dissolve the residue in a little water and examine the filtered solution for chloric acid as follows:

I. Indigo Test.-Add dilute sulphuric acid and a few drops of indigo solution, until there is a distinct blue color. Then introduce sulphurous acid drop by drop. If chloric acid is present, the blue color changes to yellow or greenish yellow. This is a delicate test for chloric acid, given even by o.or gram of potassium chlorate.

2. Silver Nitrate Test.-Add silver nitrate solution in excess. If there is a precipitate $(\mathrm{AgCl})$, filter and add a few drops of sulphurous acid to the clear filtrate. A chlorate will cause the precipitation of more silver chloride. Silver chloride differs from silver sulphite in being insoluble in hot dilute nitric acid. Sulphurous acid reduces silver chlorate to chloride:

$$
\mathrm{AgClO}_{3}+{ }_{3} \mathrm{H}_{2} \mathrm{SO}_{3}=\mathrm{AgCl}+{ }_{3} \mathrm{H}_{2} \mathrm{SO}_{4} \text {. }
$$

3. Free Chlorine Test.-A solution containing a chlorate, heated with concentrated hydrochloric acid, gives free chlorine. The gas passed into potassium iodide solution liberates iodine. Shake the solution with chloroform which dissolves iodine with a violet color. This test indicates chloric acid only in the absence of substances like chromic acid and dichromates which also give chlorine with hydrochloric acid.

If the material is a powder, dissolve in water and filter if necessary. A direct test for chloric acid is usually possible with such a solution.

\section{Quantitative Estimation of Chloric Acid}

To estimate potassium chlorate quantitatively in urine, dialysates and other liquids, reduce with zinc dust, or employ Scholtz's method. 
I. Zinc Dust Method.-Divide the solution into two equal parts. Determine chloride gravimetrically in one portion by precipitating and weighing $\mathrm{AgCl}$, or volumetrically by titrating according to Volhard's method.

Determine chloride and chlorate together in the second portion. Add 5-Io grams of zinc dust and a little dilute sulphuric or acetic acid, and heat the mixture $0.5^{-1}$ hour upon a boiling water-bath. Filter and wash the residue with boiling water. Acidify the filtrate with nitric acid and precipitate chloride with silver nitrate. More chlorine appears in the second than in the first determination. Calculate the percentage of potassium chlorate from the difference between the two chlorine determinations. One molecule of $\mathrm{KClO}_{3}$ upon reduction yields I molecule of $\mathrm{KCl}$ and therefore I atom of chlorine.

Zinc dust in presence of sulphuric or acetic acid reduces potassium chlorate to chloride:

( $\alpha) \mathrm{KClO}_{3}+3 \mathrm{Zn} \quad=\mathrm{KCl}+3 \mathrm{ZnO}$,

( $\beta) \mathrm{ZnO}+{ }_{2} \mathrm{CH}_{3} \cdot \mathrm{COOH}=\mathrm{H}_{2} \mathrm{O}+{ }_{\mathrm{Zn}}\left(\mathrm{CH}_{3} \cdot \mathrm{COO}\right)_{2}$.

2. Method of M. Scholtz. ${ }^{1}$ - This method makes use of the reducing action of nitrous acid upon chloric acid:

$$
\mathrm{HClO}_{3}+{ }_{3} \mathrm{HNO}_{2}=\mathrm{HCl}+{ }_{3} \mathrm{HNO}_{3} .
$$

Add to the solution Io cc. of nitric acid (sp. gr. I.2 $=32$ per cent.) and ro cc. of ro per cent. sodium nitrite solution. Let the mixture stand for $5_{5}$ minutes at room temperature. Then add $30-50 \mathrm{cc}$. of $0.1 \mathrm{n}$-silver nitrate solution and $5 \mathrm{cc}$. of saturated iron alum solution, $\left(\mathrm{H}_{4} \mathrm{~N}\right)_{2} \mathrm{SO}_{4} \cdot \mathrm{Fe}_{2}\left(\mathrm{SO}_{4}\right)_{3} \cdot 24 \mathrm{H}_{2} \mathrm{O}$. Titrate excess of silver with $\mathrm{O} . \mathrm{I} \mathrm{n}$-ammonium sulphocyanate solution. Iooo cc. of $0 . \mathrm{I} \mathrm{n}-\mathrm{AgNO}_{3}=0 . \mathrm{I} \mathrm{KClO}_{3}$ gram $=$ I 2.245 grams of $\mathrm{KClO}_{3}$.

The slight excess of nitrous acid has no effect upon the delicacy of the reaction. Liquids like dialysates of stomach contents and organs always contain chloride. In that case first

${ }^{1}$ Archiv der Pharmazie 243, 353 (1905). 
determine the amount of chloride in another portion by Volhard's method.

H. Hildebrandt ${ }^{1}$ has adapted Scholtz's method to the examination of urine. First completely precipitate chloride in a measured volume of urine with silver nitrate in presence of nitric acid.' Add sodium nitrite solution to the clear, chloridefree filtrate, as well as more silver nitrate solution, until there is no longer a precipitate. Determine as usual the weight of silver chloride obtained.

In the case of urine a larger quantity of nitrous acid is decomposed by the urea : $\mathrm{CO}\left(\mathrm{NH}_{2}\right)_{2}+{ }_{2} \mathrm{HNO}_{2}=\mathrm{CO}_{2}+{ }_{2} \mathrm{~N}_{2}+{ }_{2} \mathrm{H}_{2} \mathrm{O}$.

Consequently do not use too little sodium nitrite.

\section{Behavior of Potassium Chlorate in Putrefaction}

C. Bischoff states that potassium chlorate, mixed with moist, organic substances, especially blood, is very soon reduced to chloride! Bischoff describes several cases, in which poisoning by potassium chlorate had undoubtedly occurred, and yet chloric acid could not be detected chemically in parts of the cadaver.

In an experiment, too grams of blood, 0.5 gram of potassium chlorate and 100 grams of water were allowed to stand for 5 days at room temperatures. Not a trace of chloric acid could be detected in the dialysate. Bischoff concludes from this experiment that potassium chlorate, mixed with moist organic substances, especially with blood, is soon reduced. Consequently, chloric acid may not be detected, even in cases of rapidly fatal poisoning by potassium chlorate.

\section{Detection of Chlorate in Meat}

The German law of June 3, I900, relating to the inspection of beef-cattle and meat, forbids the use of chlorates in preserving meat, sausage and fat. The official directions prescribed for the chemical examination of meat and fats are as follows:

Let 30 grams of finely divided meat stand $\mathrm{I}$ hour in the cold with roo cc. of water and then heat to boiling. Filter when cold and add silver nitrate solution

1 Vierteljahrsschrift für gerichtliche Medizin 32, 8I (1906). 
in excess to the filtrate. Add 2 cc. of ro per cent. sodium sulphite solution and $2 \mathrm{cc}$. of concentrated nitric acid to $50 \mathrm{cc}$. of the clear filtrate from the silver precipitate and then heat to boiling. If there is a precipitate, insoluble in more hot water and consisting of silver chloride, chlorate is present.

\section{SANTONIN, SULPHONAL AND TRIONAL}

These substances do not find a place in the Stas-Otto process on account of their behavior toward cold tartaric acid solution and ether. Use the following method for their detection.

Extract the material, neutral or faintly acid with tartaric acid, under a reflux condenser with boiling absolute alcohol. Filter hot and evaporate the filtrate to dryness upon the waterbath. Dissolve the residue in hot water. If the solution is colored, digest for some time upon the water-bath with boneblack and stir frequently. Filter the hot solution. All of the above substances, if present in considerable quantity, crystallize in part as the solution cools. -Extract the filtrate and any crystals thoroughly with chloroform several times. Pass the chloroform extract through a dry filter. The residue from chloroform may contain santonin, sulphonal and trional, as well as acetanilide and phenacetine.

The chloroform residue may also contain those substances extracted in the Stas-Otto process from the acid solution by ether. Chloroform completely extracts substances like antipyrine, caffeine, acetanilide, phenacetine and salicylic acid. As a rule they are purer from this solvent than from ether. The chloroform residue may also contain the weak base narcotine.

\section{SANTONIN}

Santonin, $\mathrm{C}_{16} \mathrm{H}_{18} \mathrm{O}_{3}$, crystallizes in colorless, inodorous, shining leaflets which are bitter and melt at $r 70^{\circ}$. Santonin dissolves in 5000 parts of cold and 250 parts of boiling water; in 44 parts of ethyl alcohol; and in 4 parts of chloroform. All these solutions are neutral. It is slightly soluble in ether $\left(\mathrm{I}: \mathrm{I}_{50}\right)$. Light turns these crystals yellow. Upon evaporation, an alcoholic solution of the yellow modification deposits white santonin.

Constitution.-Santonin is the internal anhydride (lactone) of santonic acid, $\mathrm{C}_{15} \mathrm{H}_{20} \mathrm{O}_{4}$. Caustic alkalies, as well as calcium and barium hydroxides, dissolve santonin forming salts of this acid. In this case, as with all lactones, the lactone ring is broken as follows: 


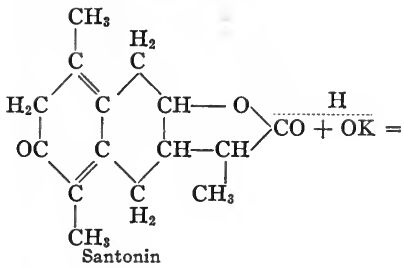

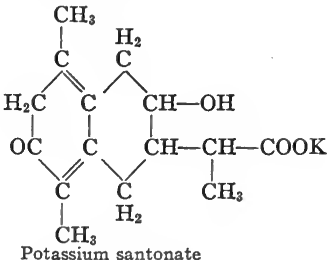

A solution of a santonate, acidified with hydrochloric acid, first gives free santonic acid. To isolate this compound from the mixture, extract at once with ether. Otherwise, the acid loses I molecule of water upon standing and passes into its internal anhydride, santonin.

Santonin is also a ketone. As such it forms a hydrazone, $\mathrm{C}_{15} \mathrm{H}_{18} \mathrm{O}_{2}=\mathrm{N}$ NH. $\mathrm{C}_{6} \mathrm{H}_{5}$, with phenylhydrazine and an oxime, $\mathrm{C}_{15} \mathrm{H}_{18} \mathrm{O}_{2}=\mathrm{NOH}$, with hydroxylamine.

According to the structural formula above, santonin is a derivative of hexahydro-dimethyl-naphthalene. Fused with potassium hydroxide, santonic acid gives hydrogen, propionic acid and a naphthalene derivative, namely, dimethyl- $\beta$-naphthol.

Behavior in the Organism.-Santonin seems to be incompletely absorbed in the body. M. Jaffél has administered quite large quantities of santonin to dogs and rabbits. He obtained a new substance, called $\alpha$-oxysantonin $\left(\mathrm{C}_{15} \mathrm{H}_{18} \mathrm{O}_{4}\right)$, from the urine of the dog, amounting to 5 or 6 per cent. of the santonin administered. He extracted with chloroform considerable quantities of unaltered santonin from the fæces of the dog. Rabbits can usually tolerate being fed with santonin for weeks, and $\alpha$-oxysantonin is formed only in very small quantity. In the ether extract of the rabbit's urine, Jaffé found a second santonin derivative, $\beta$-oxysantonin, isomeric with $\alpha$-oxysantonin, with considerable unaltered santonin. In these experiments only about half the santonin administered was absorbed by the rabbit.

After administration of santonin, a red pigment called santonin red appears in human urine. Even after medicinal doses santonin urine is red, or becomes at least scarlet-red to purple on addition of potassium or sodium hydroxide solution. Urine containing santonin also becomes carmine-red on addition of calcium hydroxide solution.

\section{Detection of Santonin}

Ether, benzene, or better chloroform, extract santonin only from acid solutions. The organic solvent fails to remove this compound from an alkaline solution, as it is then in the form of a santonate. Santonin is not an alkaloid and forms no pre-

${ }^{1}$ Zeitschrift für physiologische Chemie 22, 537 (1896-r897). 
cipitates with the general alkaloidal reagents, but it gives several more or less characteristic color reactions.

I. Alcoholic Potassium Hydroxide Test.-Pure santonin, heated with an alcoholic solution of potassium hydroxide, gives a fine carmine-red color, which gradually changes to reddish yellow and finally fades entirely. In this test yellow santonin dissolves at once with a yellowish red color.

2. Sulphuric Acid-Ferric Chloride Test.-Heat santonin with concentrated sulphuric acid and add a drop of ferric chloride solution. The mixture becomes violet. Use about r cc. of sulphuric acid to o.or gram of santonin.

3. Furfural-Sulphuric Acid Test.-Mix 2-3 drops of alcoholic santonin solution with $\mathrm{I}-2$ drops of 2 per cent. alcoholic furfural solution and $2 \mathrm{cc}$. of pure concentrated sulphuric acid. Warm this mixture in a small porcelain dish upon the waterbath. A purple-red color appears and changes with continued heating to crimson-red, blue-violet and finally to dark blue (Thäter ${ }^{1}$ ).

Only a few alkaloids and glucosides give distinct color reactions with furfural and sulphuric acid. Substances behaving similarly are veratrine, picrotoxin (violet) and piperine (green to blue-green, finally indigo-blue). The colors given by $\alpha$ - and $\beta$-naphthol with furfural and sulphuric acid are also characteristic.

\section{SULPHONAL}

Sulphonal, $\mathrm{C}_{7} \mathrm{H}_{16} \mathrm{O}_{4} \mathrm{~S}_{2}$, crystallizes in colorless, inodorous and tasteless prisms, melting at $125^{-1} 26^{\circ}$ and distilling with slight decomposition at $300^{\circ}$. It is soluble<smiles>CCO[Sb](=O)C(C)(C)OCC</smiles>
in 500 parts of cold and 15 parts of boiling water; in 135 parts of ether; and in 65 parts of cold and 2 parts of boiling ethyl alcohol. Sulphonal is freely soluble in chloroform. Especially characteristic of this compound are the ease with which it crystallizes and its great stability in presence of chemical reagents. The halogens, halogen acids, alkaline hydroxides and carbonates, concentrated sulphuric and nitric acids are without action in the cold.

Preparation.-The condensation of ethyl mercaptan (2 molecules) with acetone (I molecule) by means of dry hydrogen chloride gas, or concentrated sulphuric acid, results in the formation of the ethyl-mercaptole of acetone. The latter com-

${ }^{1}$ Archiv der Pharmazie 235, 4 IO (1897). 
pound, shaken with a saturated solution of potassium permanganate in presence of dilute sulphuric acid, undergoes oxidation with formation of sulphonal: ${ }^{1}$

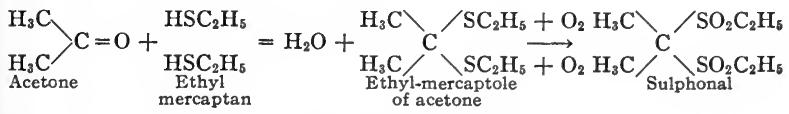

\section{Detection of Sulphonal}

Ether, or better chloroform, extracts sulphonal from acid, neutral and alkaline solutions. Test the residue left upon evaporating these solutions as follows:

I. Melting-point Test.-Determine the melting point ( $125^{-}$ $\mathrm{I} 26^{\circ}$ ) of the perfectly pure substance. Crystallization from boiling water with the use of a little bone-black easily gives a pure product. A mixture of these crystals with pure sulphonal should also melt at $125^{-1} 26^{\circ}$.

2. Reduction Test.-Sulphonal heated in a test-tube with powdered wood charcoal gives the characteristic odor of ethyl mercaptan.

3. Detection of Sulphur.-(a) With Sodium. Fusion of sulphonal in a dry test-tube with a little metallic sodium produces sodium sulphide. Dissolve cautiously (unaltered metallic sodium!) the cold melt in a little water, filter and test the filtrate with sodium nitroprusside solution for sulphide (see page 23).

(b) With Potassium Cyanide.-Fuse I part of sulphonal and about 2 parts of pure potassium cyanide in a dry testtube. Note the penetrating odor of ethyl mercaptan $\left(\mathrm{C}_{2} \mathrm{H}_{5} . \mathrm{SH}\right)$. Potassium sulphocyanate is also a product of the reaction. An aqueous solution of the melt, acidified with dilute hydrochloric acid, becomes deep red with I-2 drops of ferric chloride solution.

(c) With Powdered Iron.-Sulphonal heated with pure powdered iron free from sulphur gives a garlic-like odor. Add

${ }^{1}$ Sulphur in the sulphone group $=\mathrm{SO}_{2}$ is most likely sexivalent, corresponding to the atomic grouping I, and not quadrivalent, as in II:
I. $=\mathrm{VI} / \mathrm{V}$
II. $=$ IV $S_{O}^{O}$ 
hydrochloric acid to the residue. Hydrogen sulphide evolved blackens lead acetate paper.

\section{Detection of Sulphonal in Urine}

Sulphonal is cumulative in its action. Therefore continuous administration for a long time of large doses may result in the collection of a considerable quantity of this compound in the organism. Most of the sulphonal taken appears in the urine as ethyl-sulphonic acid, $\mathrm{C}_{2} \mathrm{H}_{5}-\mathrm{SO}_{2} \mathrm{OH} .{ }^{1}$ The formation of this acid causes an increase of ammonia in the urine during sulphonal intoxication, as does administration of mineral acids.

Sulphonal occurs in urine in detectable quantity only following considerable doses, especially when they have been taken without interruption. Such urine is often dark red to garnet-brown from hæmatoporphyrin. But this decomposition product of blood pigment appears in urine only succeeding severe poisoning by sulphonal, and even then its occurrence is rare.

To isolate sulphonal from urine, evaporate rooo cc. to one-tenth its volume, and extract several times with large quantities of ether. Pass the ether extracts, after they have settled in a dry flask for several hours, through a dry filter and distil. Evaporate the residue with 20-30 cc. of yo per cent. sodium hydroxide solution to dryness upon the water-bath. This will remove coloring matter, extracted from urine by ether, but will not affect the sulphonal. Extract sulphonal from the alkaline residue with ether. Evaporate the solvent, and sulphonal will remain pure and almost colorless. Determine the melting-point of this residue, and make the other tests for sulphonal.

\section{Detection of Hæmatoporphyrin in Urine}

Coloring matters have been observed in red, brownish red to cherry-red urines, which quite probably are identical with hæmatoporphyrin. The spectroscopic examination of such urine is made in the following manner. Add sodium hydroxide solution, drop by drop, to about $500 \mathrm{cc}$. of urine, until the reaction is strongly alkaline, and then add a little barium chloride solution. Filter after a while, and wash the precipitate well. Extract the precipitate upon the filter with hot alcohol, containing a few drops of dilute sulphuric acid. A spectroscopic examination of this filtrate can be made directly with a Browning pocket spectroscope. Acid hæmatoporphyrin solutions are violet; when more concentrated, they have a cherry-red color, and show the characteristic spectrum with two absorption-bands (see page 306 ). If the acid, alcoholic solution is saturated with a few drops of ammonium or sodium hydroxide solution, the spectrum of alkaline hæmatoporphyrin solution with its four absorption-bands appears. Traces of hæmatoporphyrin very frequently appear

${ }^{1}$ The structural formula of ethyl-sulphonic acid is: $\mathrm{C}_{2} \mathrm{H}_{5} \cdot \mathrm{S}=\frac{\mathrm{OH}}{\mathrm{O}}$.

It should not be confused with ethyl-sulphuric acid: $\mathrm{C}_{2} \mathrm{H}_{5}-\mathrm{O}-\mathrm{S} / \mathrm{Z}_{\mathrm{O}}^{\mathrm{O}}$. 
in normal urine. It has been observed more abundantly, at times, in urine during chronic sulphonal poisoning.

\section{TRIONAL}

Trional crystallizes in colorless, shining leaflets melting at $76^{\circ}$. It is soluble in 320 parts of cold, but more easily soluble in hot water. It is also soluble in ethyl<smiles>CCOS(=O)C(C)(CC)CC</smiles>
alcohol, ether and chloroform. The aqueous solution is neutral and bitter. In the latter respect it differs from sulphonal which is tasteless. Trional gives the sulphonal reactions. Trional is completely decomposed in the organism and the danger of cumulative action is much less than in the case of sulphonal. Moreover, hæmatoporphyrin has almost never been observed, even following considerable doses of trional and after uninterrupted use for weeks.

\section{Active Organic Substances ${ }^{1}$ Rarely Occurring in Toxicological Analysis}

\section{CANTHARIDIN}

Cantharidin, $\mathrm{C}_{10} \mathrm{H}_{12} \mathrm{O}_{4}$, is the active vesicating principle of Spanish fly (Lytta vesicatoria) and is present to the extent of $0.8^{-}$I per cent. Cantharidin<smiles>CC12C(=O)OC(=O)C1(C)C1CCC2O1</smiles>
forms colorless, shining, neutral, rhombic leaflets, melting at $2 \mathrm{I} 8^{\circ}$ and subliming at higher temperature in white needles. ' It is almost insoluble even in boiling water. Acids, as tartaric acid, increase its solubility in water, though cantharidin is not a base. It dissolves with difficulty in cold ethyl alcohol (0.03: 100 at $18^{\circ}$ ) and in ether (0.01 I:IOO). Chloroform (r.52:100), acetone and acetic ether are its best solvents. It is as good as insoluble in petroleum benzine.

Constitution.-According to Gadamer ${ }^{2}$ cantharidin has the structural formula shown above. Treated with potassium or sodium hydroxide, it loses its anhydride character and passes into solution as the alkali salt of dibasic cantharidic acid, $\mathrm{C}_{10} \mathrm{H}_{14} \mathrm{O}_{5}$ :

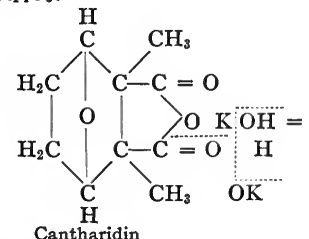<smiles>CC1(C(=O)O)C2CCC(O2)C1(C)C(=O)OO</smiles>

Potassium cantharidate

Potassium cantharidate, $\mathrm{C}_{10} \mathrm{H}_{12} \mathrm{O}_{5} \mathrm{~K}_{2} .2 \mathrm{H}_{2} \mathrm{O}$, recently recommended for phthisis, and sodium cantharidate, $\mathrm{C}_{10} \mathrm{H}_{12} \mathrm{O}_{5} \mathrm{Na}_{2.2} \mathrm{H}_{2} \mathrm{O}$, are well crystallized

1 The toxic substances considered in this place have been arranged in alphabetical order.

2 Chemical Abstracts I2, 806 (rgr 8 ). 
salts. Mineral acid first sets cantharidic acid free from these salts. The latter soon loses a molecule of water, passing into its internal anhydride, cantharidin.<smiles>CC1(C(=O)O)C2CCC(O2)C1(C)C(=O)O</smiles>

Cantharidic acid<smiles>CC12C(=O)OC(=O)C1(C)C1CCC2O1</smiles>

Cantharidin

Cantharidin, heated with hydriodic acid at $100^{\circ}$, or treated at room temperature with chlorosulphonic acid, $\mathrm{Cl}-\mathrm{SO}_{2}-\mathrm{OH}$, changes into the isomeric cantharic acid, $\mathrm{C}_{10} \mathrm{H}_{12} \mathrm{O}_{4}$, crystallizing in colorless needles melting at $275^{\circ}$ and having the following structure:<smiles>CC12CCCC(C)(C1)C(C)(C(=O)O)CCC2</smiles>

This acid is not a vesicant. Heated for 3 hours at $135^{\circ}$ in sealed tube with acetyl chloride, cantharic acid yields another isomer of cantharidin which Gadamer ${ }^{1}$ has shown to be acetyl-hydrato-cantharic anhydride having the following structure:

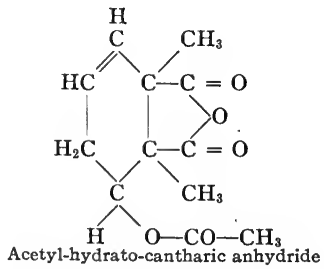

The latter crystallizes from alcohol in large colorless leaflets melting at $76^{\circ}$. There is a close relationship between o-xylene and cantharidin, for the latter, heated at $400^{\circ}$ with calcium hydroxide, gives a dihydro-o-xylene, $\mathrm{C}_{8} \mathrm{H}_{12}$, called cantharene, and also o-xylene, $\mathrm{C}_{6} \mathrm{H}_{4}\left(\mathrm{CH}_{3}\right)_{2}$, and xylic acid. Finally, cantharidin, heated with an excess of phosphorus pentasulphide and distilled, gives pure o-xylene. (J. Piccard.)2

${ }^{1}$ Chemical Abstracts 12, 806 (19r8).

${ }^{2}$ Berichte der Deutschen chemischen Gesellschaft 12, 577 (1879). 


\section{Detection of Cantharidin}

Evaporate a liquid, or material containing much moisture (organs, stomach or intestinal contents, etc.), to dryness upon the water-bath. Dragendorff directs repeated extraction of the finely divided material with alcohol containing sulphuric acid. Filter the extracts, add one-sixth their volume-of water and distil the alcohol. Extract the residue 2-3 times with chloroform and shake the chloroform extracts with water to remove adherent acid. Finally separate the chloroform from water, distil and examine the residue for cantharidin. Since this compound gives no characteristic chemical reactions, employ the physiological test for identification. Dissolve the chloroform residue, unless fatty substances are present, in a few drops of hot almond oil. Bind a cloth, saturated with this solution, upon the upper arm or breast by means of adhesive plaster. Cantharidin reddens the skin and sometimes raises blisters. Even 0.I4 mg. of cantharidin causes blistering. Salts of cantharidic acid also have a vesicating action.

To detect cantharidin in blood, brain, liver and other material rich in protein, E. Schmidt boils with dilute potassium hydroxide solution ( $\mathrm{I}$ gram of $\mathrm{KOH}$ in $\mathrm{I}_{5} \mathrm{cc}$. of water), until the mass is homogeneous, acidifies with dilute sulphuric acid and extracts thoroughly with hot alcohol. The procedure in other respects is as described above.

Cantharidin is said to resist putrefaction.

\section{CYTISINE}

Cytisine, $\mathrm{C}_{11} \mathrm{H}_{14} \mathrm{~N}_{2} \mathrm{O}$, occurs in the ripe seeds of Golden chain (Cytisus Laburnum) which contain about $\mathrm{I} .5$ per cent. Cytisine and the alkaloid originally called ulexine, isolated from the seeds of Ulex europaeus, are identical (A. Partheil).

Preparation.-Extract powdered ripe laburnum seeds with 60 per cent. alcohol containing acetic acid. Distil the alcohol from the extracts, pour the residue through a moist filter and precipitate extractive and tannin substances with lead acetate solution. Filter, add potassium hydroxide solution to the clear filtrate and extract cytisine with chloroform. Distil the chloroform which usually deposits cytisine as a radiating crystalline mass. If purification is necessary, recrystallize the residue from absolute alcohol or boiling ligroin. Sublimation in a partial vacuum also purifies crude cytisine. 
Cytisine crystallizes in large, colorless, tasteless prisms, melting at $15^{2-153^{\circ}}$ and subliming at a higher temperature, if carefully heated. It dissolves freely in water, alcohol, chloroform and acetic ether; less easily in commercial ether, benzene and acetone; and is insoluble in petroleum ether and absolute ether.

Cytisine is a strong secondary base and very toxic. Although capable of combining with I or 2 molecules of hydrochloric acid, this compound behaves in other respects like a monacid base. Only the salts containing one equivalent of acid crystallize well. Nitrous acid converts this secondary base into nitrosocytisine, $\mathrm{C}_{11} \mathrm{H}_{13} \mathrm{ON}-\mathrm{NO}$, which crystallizes in needles. Nitrous fumes appear, if cytisine is warmed upon the water-bath with twice the amount of concentrated nitric acid, and the solution at once becomes reddish yellow to brown. This solution poured into water gives a precipitate of nitro-nitroso-cytisine, $\mathrm{C}_{11} \mathrm{H}_{12} \mathrm{ON}$ $\left(\mathrm{NO}_{2}\right) \mathrm{N}-\mathrm{NO}$. This compound crystallizes from water in pale yellow scales melting at $242-244^{\circ}$.

Toxic Action.-Cytisine produces convulsions, its action in this respect being very similar to that of strychnine. But unlike the latter alkaloid it also irritates the gastro-intestinal mucosa even causing bloody inflammation. Cytisine also differs from strychnine in stimulating the vomiting center. Consequently, after doses of cytisine or laburnum preparations, human beings and animals capable of emesis thus rid the organism of a large part of the poison. Like strychnine, cytisine stimulates the respiratory and vaso-motor centers. Finally as in strychnine intoxication death results from paralysis of these two centers. A part of the cytisine leaves the organism unchanged and appears in the urine (R. Kobert).

\section{Detection of Cytisine}

Prepare an aqueous tartaric acid solution of stomach contents, vomitus or parts of organs, following the general procedure for alkaloids. To remove final traces of fatty acids and fat, shake this solution well with ether. Withdraw the aqueous solution, make alkaline with sodium hydroxide solution and extract thoroughly with chloroform or isobutyl alcohol. Evaporate the chloroform or isobutyl alcohol extracts and test the residue as follows for cytisine:

I. Van der Moer's ${ }^{1}$ Test.-Ferric chloride solution colors cytisine and its salts blood-red. Dilution with water, or acidification, discharges this color. Hydrogen peroxide also produces the same result. The solution containing hydrogen peroxide, warmed upon the water-bath, becomes intensely blue.

\section{A. Ramverda's ${ }^{2}$ Test.-A little nitrobenzene, containing}

${ }^{1}$ Berichte der Deutschen pharmazeutischen Gesellschaft 5, 267 (I 895 ).

${ }^{2}$ Chemisches Zentral-Blatt, I900, II, 268. 
dinitro-thiophene, poured upon cytisine gives a fairly stable, brilliant red-violet color.

A similar color given by coniine is very unstable.

3. Nitro-Nitroso-Cytisine Test.-Nitro-nitroso-cytisine (see above), formed by concentrated nitric acid, serves to detect small quantities of this alkaloid. Nitro-nitroso-cytisine dissolves with difficulty in 94 per cent. alcohol and crystallizes from this solvent in microscopic prisms. Flat, tabular crystals form from $5 \circ$ per cent. alcohol which is a better solvent. The solubility of nitro-nitroso-cytisine in concentrated hydrochloric acid indicates basic properties, but they are feeble, for dilution with water precipitates this compound unchanged.

\section{THE DIGITALIS GLUCOSIDES}

The digitalis plant (Digitalis purpurea $L$.) contains in all its parts, but especially in the leaves and seeds, medicinally useful substances belonging to the glucoside group. Thus far three digitalis glucosides have been isolated as well characterized, crystalline compounds of homogeneous composition. These are digitalin in a narrower sense (= Digitalinum verum crystallisatum Kiliani) $\mathrm{C}_{35} \mathrm{H}_{56} \mathrm{O}_{14}$; digitoxin, $\mathrm{C}_{34} \mathrm{H}_{54} \mathrm{O}_{11}$; and digitonin, $\mathrm{C}_{55} \mathrm{H}_{94} \mathrm{O}_{28}$ or $\mathrm{C}_{54} \mathrm{H}_{92} \mathrm{O}_{28}$. A fourth glucoside called digitalein seems not to have been obtained wholly pure as yet.

Digitonin, $\mathrm{C}_{55} \mathrm{H}_{94} \mathrm{O}_{28}$ or $\mathrm{C}_{54} \mathrm{H}_{92} \mathrm{O}_{28},{ }^{1}$ occurs almost exclusively in digitalis seeds, the leaves containing at most only traces. Digitonin, classified at present with the saponins (see page 220), crystallizes from alcohol in fine needles soluble in $5 \circ$ parts of 50 per cent. alcohol. Even very dilute hydrochloric acid hydrolyzes digitonin into digitogenin, dextrose and galactose: ${ }^{2}$

$$
\underset{\text { Digitonin }}{\mathrm{C}_{55} \mathrm{H}_{94} \mathrm{O}_{28}}+{ }_{2} \mathrm{H}_{2} \mathrm{O}=\underset{\text { Digitogenin }}{\mathrm{C}_{33} \mathrm{H}_{50} \mathrm{O}_{6}}+\underset{\text { Dextrose }}{2 \mathrm{C}_{6} \mathrm{H}_{12} \mathrm{O}_{6}}+\underset{\text { Galactose }}{\mathrm{C}_{6} \mathrm{H}_{12} \mathrm{O}_{6}} \text { (?). }
$$

Digitonin crystallizes from alcohol in fine needles which soften at $235^{\circ}$ and become yellow. Digitonin is not a cardiac poison. Pure digitonin and concentrated sulphuric acid, upon addition

${ }^{1}$ The results obtained by $\mathrm{A}$. Windhaus (Berichte der Deutschen chemischen Gesellschaft 42,238 (1909) favor the formula $\mathrm{C}_{55} \mathrm{H}_{94} \mathrm{O}_{28}$ for digitonin.

${ }^{2} \mathrm{H}$. Kiliani, Berichte der Deutschen chemischen Gesellschaft 24, 340 (I89I). 
of a little bromine water, give a color which becomes intensely red.

Digitoxin, $\mathrm{C}_{34} \mathrm{H}_{54} \mathrm{O}_{11}$, occurs almost exclusively in digitalis leaves. This very active and highly toxic compound is almost wholly insoluble in water and ether but soluble in alcohol and chloroform. Consequently ether precipitates it from chloroform solution. Digitoxin crystallizes from 85 per cent. alcohol in leaflets melting at $145^{\circ}$. Alcoholic hydrochloric acid hydrolyzes it forming digitoxigenin and digitoxose:

$$
\underset{\text { Digitoxin }}{\mathrm{C}_{34} \mathrm{H}_{54} \mathrm{O}_{11}}+\mathrm{H}_{2} \mathrm{O}=\underset{\text { Digitoxigenin }}{\mathrm{C}_{22} \mathrm{H}_{32} \mathrm{O}_{4}}+\underset{\text { Digitoxose }}{2 \mathrm{C}_{6} \mathrm{H}_{12} \mathrm{O}_{4}}
$$

Digitoxin dissolves in concentrated sulphuric acid with a brownish or greenish brown color unchanged by bromine.

Kiliani's Digitoxin Test. ${ }^{1}$-Dissolve a trace of digitoxin in $3-4 \mathrm{cc}$. of glacial acetic acid containing iron (IOO cc. of glacial acetic acid and I cc. of 5 per cent. ferric sulphate solution). Cautiously add sulphuric acid containing iron (roo cc. of sulphuric acid and I cc. of 5 per cent. ferric sulphate solution) in about the same quantity as an under layer. A dark zone appears where the two solutions meet, above which after a few minutes a blue band is visible. After some time the entire acetic acid layer becomes deep indigo-blue.

Digitalin, $\mathrm{C}_{35} \mathrm{H}_{56} \mathrm{O}_{14}$, according to Kiliani occurs only in digitalis seeds. It is soluble in water ( $\mathrm{I}: \mathrm{I}, 00)$ and very active. Boiling with very dilute hydrochloric acid hydrolyzes its alcoholic solution into digitaligenin and two sugars, namely, dextrose and digitalose : ${ }^{2}$

$$
\underset{\text { Digitalin }}{\mathrm{C}_{35} \mathrm{H}_{56} \mathrm{O}_{14}}+\underset{\text { Digitaligenin }}{\mathrm{H}_{2} \mathrm{O}} \underset{\text { Dextrose }}{\mathrm{C}_{22} \mathrm{H}_{30} \mathrm{O}_{3}}+\underset{\text { Digitalose }}{\mathrm{C}_{6} \mathrm{H}_{12} \mathrm{O}_{6}}+\underset{2}{\mathrm{C}_{7} \mathrm{H}_{14} \mathrm{O}_{5}}
$$

Test for digitalin as follows:

I. Concentrated sulphuric acid colors pure digitalin orangeyellow. This solution soon becomes blood-red, changing upon addition of a little bromine water to cherry and blue-red. A drop of nitric acid or ferric chloride solution will do as well as bromine water. This test after $I_{-2}$ hours is surer and more

${ }^{1}$ Archiv der Pharmazie 234, 273-277 (r896).

${ }^{2}$ H. Kiliani, Berichte der Deutschen chemischen Gesellschaft 3r, 2454 (1898). 
permanent, if a trace of digitalin is dissolved direct in concentrated sulphuric acid and nothing else is added.

2. Concentrated hydrochloric acid dissolves digitalin with a golden yellow color, changing with heat to garnet or violet-red.

At present nothing definite is known regarding the fate of digitalis glucosides in the human organism, or the products into which they are changed or the forms in which they are eliminated. In the case of human beings elimination of the three active substances has never been observed. Moreover, R. Kobert has not been able to detect anything active in the urine of animals except in isolated cases. Thus far it has not been possible to find any of the digitalis compounds mentioned above in blood or animal organs. In a toxicological analysis especial attention would have to be given to vomitus and the contents of the gastro-intestinal tract. But there is slight chance of detecting the digitalis bodies in such material.

\section{ERGOT}

Officinal ergot (Secale cornutum) is the sclerotium (compact mycelium or spawn) of Claviceps purpurea collected from rye shortly before the fruiting period and dried at gentle heat. Ergot is commonly known as an abortifacient and intoxications have occurred from its use. Consequently examinations for legal purposes may require its detection in powders and other mixtures. Our knowledge of the constituents of ergot is still very defective notwithstanding several exhaustive investigations. Ergot alkaloids, as ergotine, ergotinine, cornutine, picro-sclerotine, were described long ago. But, with the possible exception of ergotinine (Tanret, C. C. Keller), the preparations were not entirely pure. Ergot contains in addition to alkaloids other peculiar chemical substances which have received but little attention. They have not the characteristic physiological action of ergot but, like the pigment sclererythrin, are useful for purposes of identification. Among these substances belong sphacelic acid and sclerotic acid, according to $\mathrm{R}$. Kobert a very poisonous resin having acid properties.

Alkaloids. - The most recent ${ }^{1}$ researches upon ergot mention as well characterized bases ergotinine, $\mathrm{C}_{35} \mathrm{H}_{39} \mathrm{~N}_{5} \mathrm{O}_{5}$, and hydro-ergotinine, $\mathrm{C}_{35} \mathrm{H}_{41} \mathrm{~N}_{5} \mathrm{O}_{6}$. Barger calls the latter ergotoxine. Ergotinine crystallizes from alcohol in long needles melting at about $229^{\circ}$ when heated rapidly. This compound dissolves in 52 parts of boiling alcohol; in I.I parts of ether; and is readily soluble in chloroform. Crystalline salts of this base have not yet been prepared. Hydro-ergotinine (= hydrate of ergotinine), obtained as a crystalline phosphate from ergotinine mother liquors by means of alcohol and phosphoric acid, is a white powder softening at $155^{\circ}$ and melting at ${ }_{1} 6{ }_{2}-164^{\circ}$. Though freely soluble in alcohol, it dissolves but slightly in ether. As a rule the salts of hydro-ergotinine (ergotoxine) crystallize well. ${ }^{2}$ By preparing a cold methyl alcohol solution of hydro-ergotinine and boiling this solution for several hours under a return condenser, $\mathrm{F}$.

${ }^{1}$ F. Kraft, Archiv der Pharmazie 244, 336 (1906) and G. Barger, Journal of the Chemical Society 9I, 337 .

${ }^{2}$ G. Barger and F. H. Carr, Proceedings of the Chemical Society 23,27 . 
Kraft has converted this substance completely into ergotinine. On the other hand, ergotinine in dilute acetic acid solution passes back almost entirely into hydro-ergotinine within ro days. As an indication of purity, a solution of hydroergotinine in 2 parts of cold methyl alcohol after several days standing should not deposit crystals (ergotinine) nor become green. Solutions of both alkaloids are fluorescent. According to Keller the play of colors with sulphuric acid and ferric chloride is characteristic of ergotinine (see below).

Physiological Action of the Alkaloids.-Ergotinine and hydro-ergotinine according to $\mathrm{A}$. Jaquet produce convulsions and gangrene. They are not, however, the cause of the specific uterine contraction characteristic of ergot. Keller's cornutine according to Kraft is identical with ergotinine, according to G. Barger and H. H. Dale ${ }^{1}$ with ergotinine, which is impure from ergotoxine (hydroergotinine). The English investigators believe that the physiological effects observed with ergotinine are due to adhering ergotoxine. The latter is readily formed when the difficultly soluble ergotinine is brought into solution by means of glacial acetic acid, phosphoric acid, or a little sodium hydroxide solution. Ergotoxine according to Barger and Dale produces the effects typical of ergot, causing powerful contraction of the uterus and later abortion.

Sclererythrin.-This is the pigment of the outer coat of ergot. E. Schmidt gives the following directions for its isolation. Extract freshly powdered ergot with ether to remove fat. Then moisten the powder with water containing tartaric acid, dry and extract with 95 per cent. alcohol. Filter and distil the alcohol. Extract the residue with ether. This solvent now dissolves sclererythrin which can be precipitated by means of petroleum ether.

Sclererythrin is an amorphous red powder which can be sublimed. It is insoluble in water but soluble in absolute alcohol and glacial acetic acid. This substance behaves like an acid, dissolving in caustic alkalies, ammonia, and alkaline carbonate and bicarbonate solutions with a red or red-violet color. Owing to presence of sclererythrin, ether, if shaken with powdered ergot moistened with tartaric acid solution, becomes red. If such an ether solution of sclererythrin is shaken with sodium hydroxide solution, the pigment dissolves in the latter which then becomes red. Solutions of this pigment show characteristic absorption-bands in the spectrum. Moreover the pigment gives blue-violet precipitates with solutions of calcium hydroxide, barium hydroxide and lead acetate. The precipitate with stannous chloride is currant-red; with copper sulphate a pure violet; with ferric chloride a deep green; and with chlorine or bromine water a lemon-yellow.

\section{Detection of Ergot in Flour, Bread and Powders}

This examination usually consists in undertaking to detect by chemical and physical means the red pigment sclererythrin which is characteristic of ergot. The property possessed by this substance of passing from ether into a solution of an alkaline hydroxide or bicarbonate is especially valuable for purposes of identification.

${ }^{1}$ Bio-Chemical Journal 2, 240. 
I. Detection of Sclererythrin.-Shake frequently and let ro grams or more of flour stand for a day in a closed flask with 20 cc. of ether and about ${ }_{5} 5$ drops of dilute sulphuric acid ( $1: 5$ ). Then pass the ether through a dry paper, wash the residue with a little ether and shake the filtrate thoroughly with ro- 5 drops of cold saturated sodium bicarbonate solution. If the flour contains ergot, the aqueous layer separates with a violet color.

R. Palm extracts the flour at $30-40^{\circ}$ with ro-I5 times its volume of 40 per cent. alcohol containing a few drops of ammonia. Express the liquid, filter and add basic lead acetate solution to the filtrate. Press the precipitate between filter paper and warm while still moist with a little cold saturated borax solution. A red-violet color appears, if the flour contains ergot.

2. Spectroscopic Examination.-This test gives a positive result, if the material (powdered ergot, flour, bread) contains more than o.I per cent. of ergot. Examine spectroscopically the alkaline and acid solution of the pigment. The red solution, prepared in Test I by means of ether containing sulphuric acid, shows two absorption-bands. One lies in the green between, $\mathrm{E}$ and $\mathrm{F}$ but nearer $\mathrm{E}$ and a second broader band in the blue midway between $\mathrm{F}$ and $\mathrm{G}$. Then render the solution alkaline with ammonia. Three absorption-bands should appear. The first lies between $\mathrm{D}$ and $\mathrm{E}$, the second at $\mathrm{E}$ somewhat to the right and the third to the left of $\mathrm{F}$.

3. Choline.-Ergot powder, warmed with dilute potassium hydroxide solution, gives the characteristic odor of trimethylamine, $\left(\mathrm{CH}_{3}\right)_{3} \mathrm{~N},{ }^{1}$ due to decomposition of choline in ergot.<smiles>C[14C](O)(O)CCO</smiles>

Occasionally flour that does not contain ergot may give an odor when heated with potassium hydroxide solution.

4. Detection and Quantitative Estimation of Ergotinine (C.C. Keller).-Dry finely powdered ergot over lime, place 25 grams

${ }^{1}$ The so-called corn smut (Ustilago Maidis), said to cause effects similar to those of ergot, also gives the trimethylamine odor when warmed with potassium hydroxide solution, for it contains appreciable quantities of choline. 
in a Soxhlet tube and completely extract fat with petroleum ether. Dry the powder at gentle heat, add roo grams of ether and after Io minutes shake well with milk of magnesia (I gram of $\mathrm{MgO}$ and $20 \mathrm{cc}$. of water). Shake repeatedly during an hour and then pass 80 grams of the ether solution $(=20$ grams of ergot) through a covered folded filter into a separating funnel. Shake the ether in succession with 25 , I 5 , Io and $5 \mathrm{cc}$. of 0.5 per cent. hydrochloric acid. Pour the hydrochloric acid extracts, now containing the ergot alkaloids, through a small moistened filter. ${ }^{1}$ Add ammonia until alkaline and extract the solution twice with about half its volume of ether. Let the ether extract settle in a dry flask, then filter into a dry weighed flask and wash the filter with a little ether. Distil the ether and dry flask and residue at $100^{\circ}$ to constant weight. Good German ergot contains 0.13-0.I6 per cent. and Russian ergot 0.220.25 per cent. of the alkaloid.

To detect ergot alkaloid qualitatively, proceed as follows:

(a) Dissolve a part of the residue in I cc. of concentrated sulphuric acid and add a trace of ferric chloride solution. The mixture is orange-red and becomes at once deep red but the margin appears bluish to bluish green.

(b) Dissolve a part of the residue in about $4 \mathrm{cc}$. of glacial acetic acid and add a trace of ferric chloride solution. Cautiously add this mixture to concentrated sulphuric acid as an upper layer. If ergotinine is present, a brilliant violet color appears where the two liquids meet.

\section{OPIUM}

\section{Detection of Meconic Acid and Meconine}

Since it is comparatively easy to procure small quantities of opium preparations, especially the tincture, poisoning from this source is possible. Consequently, it is often desirable to recognize the presence of opium itself. Detection of the alkaloids narcotine and morphine, always present in opium in

${ }^{1}$ Clarify the filtrate from these hydrochloric acid extracts, if not clear, by agitation with a little talcum powder, previously treated with hydrochloric acid and thoroughly washed with water. Then filter again. 
considerable quantity, affords partial evidence of the presence of this substance. Moreover, opium always contains two nonbasic substances, meconic acid and meconine. Detection of these two compounds in conjunction with narcotine and morphine definitely determines the presence of opium.

Meconic Acid, $\mathrm{C}_{7} \mathrm{H}_{4} \mathrm{O}_{7}=\mathrm{C}_{5} \mathrm{HO}_{2}(\mathrm{OH})(\mathrm{COOH})_{2}$, is an oxypyrone-dicarboxylic acid (II) and therefore a derivative of pyrone (I):
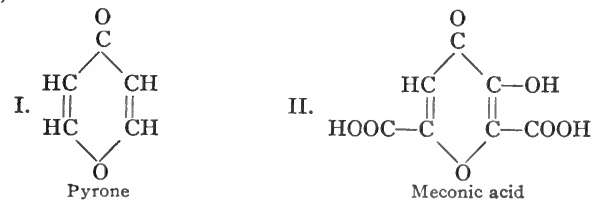

Meconic acid crystallizes in plates or prisms with 3 molecules of water. It is easily soluble in hot water and alcohol. A solution of a ferric salt turns a meconic acid solution dark red.

To detect meconic acid, extract a portion of the material with alcohol containing a few drops of hydrochloric acid. Filter and evaporate the filtrate upon the water-bath. Dissolve the . residue in a little water and heat the filtered solution to boiling with excess of calcined magnesium oxide. The solution contains magnesium meconate. Filter hot to remove undissolved magnesium oxide, evaporate the filrtate to a small volume and acidify faintly with dilute hydrochloric acid. Add a few drops of ferric chloride solution. A blood-red color appears, if meconic acid is present. Warming with hydrochloric acid does not discharge this red color, in which respect it differs from the red color caused by acetic acid. This color differs from that caused by sulphocyanic acid in not being affected upon addition of 'gold chloride. But stannous chloride reduces ferric to ferrous oxide and discharges the color. Nitrous acid, however, at once restores it.

These tests permit the identification of meconic acid in an extract from only 0.05 gram of opium.

Meconine, $\mathrm{C}_{10} \mathrm{H}_{10} \mathrm{O}_{4}$.- Opium contains only $0.05-0.08$ per cent. of this compound. It forms small prisms, melting at I02 $^{\circ}$ 
and subliming at higher temperature without decomposition. Meconine dissolves freely in alcohol, ether, benzene and chloroform, but less easily in water. Alkalies convert meconine into easily soluble salts of meconinic acid, $\mathrm{C}_{10} \mathrm{H}_{12} \mathrm{O}_{5}$. This monobasic acid cannot exist free but changes to meconine when liberated from its salts by a mineral acid. Meconine, formed by abstracting a molecule of water from meconinic acid, is therefore the internal anhydride (lactone) of meconinic acid:
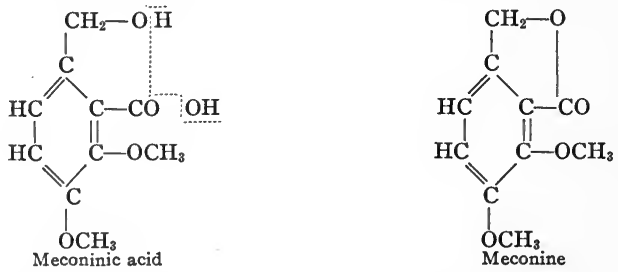

To detect meconine, extract the material with alcohol containing sulphuric acid. Filter and evaporate the filtrate to a syrup upon the water-bath. Dissolve the residue in water and - extract meconine from this acid solution with benzene. Evaporation of the solvent frequently gives crystals of meconine. To detect meconine, dissolve in a little concentrated sulphuric acid. The solution is green but turns red within two days. If the green sulphuric acid solution, or that which has turned red upon standing, is carefully warmed, a fine emerald-green color appears, passing through blue, violet and finally back to red.

\section{Selenious-Sulphuric Acid Reagent for Opium Alkaloids ${ }^{1}$}

Prepare the reagent used in these tests by dissolving 0.5 gram of selenious acid $\left(\mathrm{H}_{2} \mathrm{SeO}_{3}\right)$ in 100 grams of pure concentrated sulphuric acid. This reagent is especially delicate for opium alkaloids, detecting even traces of morphine and codeine ( 0.05 milligram), as well as of papaverine (o.I milligram). Selenious-sulphuric acid gives the following color reactions with the commoner opium alkaloids:

\footnotetext{
${ }^{1}$ Mecke, Zeitschrift für öffentliche Chemie 5, 350 (1899) and Zeitschrift für analytische Chemie 39, 468 (1900).
} 


\begin{tabular}{|c|c|c|}
\hline & Cold & Hot \\
\hline Morphine....... & $\begin{array}{l}\text { Blue; then permanent blue- } \\
\text { green to olive-green. }\end{array}$ & Brown. \\
\hline Apomorphine.... & Dark blue-violet. & Gradually dark brown. \\
\hline Codeine.......... & $\begin{array}{l}\text { Blue quickly changing to } \\
\text { emerald-green and later to } \\
\text { permanent olive-green. }\end{array}$ & Steel-blue; then brown. \\
\hline Narceine........ & $\begin{array}{l}\text { Faint greenish yellow; then } \\
\text { violet. }\end{array}$ & Dark violet. \\
\hline Narcotine... . & $\begin{array}{l}\text { Greenish steel-blue; later } \\
\text { cherry-red. }\end{array}$ & Cherry-red. \\
\hline Papaverine....... & $\begin{array}{l}\text { Greenish, dark steel-blue; } \\
\text { then deep violet. }\end{array}$ & Intense dark violet. \\
\hline Thebaine... & $\begin{array}{l}\text { Deep orange gradually fad- } \\
\text { ing. }\end{array}$ & Dark brown. \\
\hline
\end{tabular}

\section{PAPAVERINE}

Papaverine, $\mathrm{C}_{20} \mathrm{H}_{21} \mathrm{NO}_{4}$, constitutes about $0.5^{-1}$ per cent. of opium. When crude it is usually mixed with narcotine. To remove the latter, prepare the acid<smiles>COc1ccc(Cc2nccc3cc(OC)c(OC)cc23)cc1OC</smiles>
oxalate of papaverine which dissolves with difficulty in water. Crystallize this salt from boiling water until it dissolves in concentrated sulphuric acid without color. Convert papaverine oxalate into the hydrochloride by treatment with calcium chloride and then liberate the alkaloid with ammonia. This product crystallized from alcohol is pure papaverine.

Papaverine crystallizes in colorless prisms melting at $147^{\circ}$. This alkaloid is insoluble in water; soluble with difficulty in ether ( $1: 260$ ), cold alcohol and benzene; but freely soluble in hot alcohol, acetone and chloroform. These solutions are neutral, not bitter, and optically inactive. Papaverine is a weak base which dissolves in but does not neutralize acetic acid. Ether partially extracts it from an aqueous tartaric acid solution and completely extracts it from alkaline solution. Consequently this alkaloid appears in the Stas-Otto process in ether extract B. Chloroform extracts papaverine with almost as much ease from an acid solution as from one that is alkaline.

Constitution.-Papaverine is a monacid, tertiary base which combines with alkyl iodides forming crystalline addition products. As it forms no acetyl deriva- 
tive with acetic anhydride, free hydroxyl is not present. But there are probably four methoxyl groups, for it loses four methyl groups when treated with hydriodic acid according to Zeisel's method. Consequently all the oxygen atoms in papaverine are present as methoxyl groups. The researches of Guido Goldschmiedt, extending from 1883 to 1898 , have completely explained the constitution of papaverine. Moderate oxidation with potassium permanganate and sulphuric acid gives papaveraldine, $\mathrm{C}_{20} \mathrm{H}_{19} \mathrm{NO}_{5}$, without breaking the carbon chain. $\mathrm{Fu}$ sion with potassium hydroxide breaks the latter into nitrogen-free veratric acid and the nitrogenous base dimethoxy-isoquinoline: ${ }^{1}$

${ }^{1}$ Isoquinoline (II) is isomeric with quinoline (I) and like the latter is a monacid, tertiary base:

I.<smiles>O=c1cccccc1</smiles><smiles>COc1ccc(C(=O)O)cc1C(=O)O</smiles>

Papaveraldine
II.<smiles>c1ccc2ccccc2c1</smiles><smiles>COc1cc2ccncc2cc1OC</smiles>

Dimethoxy-isoquinoline<smiles>COc1ccc(C(=O)O[Na])cc1OC</smiles>

\section{Detection of Papaverine}

The following general reagents precipitate papaverine in a dilution of I : I0,000: phospho-molybdic acid, potassium bismuthous iodide and iodo-potassium iodide.

The following still give precipitates in a dilution of I : 5000: tannic acid, gold chloride and potassium mercuric iodide. 
The following special tests should be made:

I. Concentrated Sulphuric Acid.-The cold colorless solution of papaverine in this acid becomes dark violet upon gentle warming. But even a cold solution of impure papaverine in this acid is violet.

2. Froehde's Test.-The solution of pure papaverine in this reagent is green. Blue, violet and finally a brilliant cherry-red color appear upon warming the solution.

3. L. E. Warren's ${ }^{1}$ Test.-Crush a very small crystal of potassium permanganate with a glass rod and intimately mix about 0.0005 gram of papaverine with the powder. Stir this mixture into about 0.2 cc. of Marquis' reagent. A green color, almost instantly changing to blue, appears. The latter color deepens into an intense violet-blue which after some time becomes bluish green, green and finally a dirty brown.

Of thirty-nine alkaloids tested, the only one in any way simulating papaverine was an unnamed alkaloid separated from sanguinaria.

4. Solutions of this alkaloid in concentrated nitric acid, or Erdmann's reagent, are dark red.

Heat to boiling a solution of I part of papaverine with ro parts of nitric acid (sp. gr. $1.06=$ Io per cent. $\mathrm{HNO}_{3}$ ). As the solution cools, yellow crystals of the nitrate of nitro-papaverine, $\mathrm{C}_{20} \mathrm{H}_{20}\left(\mathrm{NO}_{2}\right) \mathrm{NO}_{4} \cdot \mathrm{HNO}_{3} \cdot \mathrm{H}_{2} \mathrm{O}$, appear. Yellow prisms of nitro-papaverine, $\mathrm{C}_{20} \mathrm{H}_{20}\left(\mathrm{NO}_{2}\right) \mathrm{NO}_{4} \cdot \mathrm{H}_{2} \mathrm{O}$, may be obtained from this nitrate by means of ammonia.

5. Ammonia colors the greenish solution of papaverine in chlorine water deep red-brown which becomes later almost black-brown.

6. Selenious-Sulphuric Acid Test.-See page 215 for the color changes given by pure papaverine dissolved in this reagent.

\section{PILOCARPINE}

Pilocarpine, $\mathrm{C}_{11} \mathrm{H}_{16} \mathrm{~N}_{2} \mathrm{O}_{2}$, occurs with isopilocarpine and probably also with pilocarpidine in the leaves of jaborandum (Pilocarpus pennatifolius ${ }^{2}$ ). The

${ }^{1}$ Journal of the American Chemical Society 37, 2402 (1915).

2 According to Jowett, jaborine, which has been described as another alkaloid peculiar to jaborandum leaves, is a mixture of isopilocarpine, pilocarpidine, a little pilocarpine and pigment. 
<smiles>CCC1C(=O)OCC1Cc1cncn1C</smiles>

free base as usually obtained is semi-liquid, viscous, nonvolatile and alkaline. It dissolves but slightly in water; is freely soluble in alcohol, ether and chloroform; and insoluble in benzene. Solutions of pilocarpine and its salts are dextro-rotatory. This alkaloid is a strong base neutralizing acids and forming salts that are usually crystalline. Caustic alkalies, added to concentrated solutions of pilocarpine salts, precipitate the free base which redissolves in an excess of the precipitant. Solutions of sodium hydroxide, or sodium ethylate $\left(\mathrm{C}_{2} \mathrm{H}_{5} . \mathrm{ONa}\right)$, cause a molecular rearrangement of pilocarpine. This reaction runs more smoothly, if pilocarpine hydrochloride is heated for half an hour at $200^{\circ}$. The product of this change is isopilocarpine, $\mathrm{C}_{11} \mathrm{H}_{16} \mathrm{~N}_{2} \mathrm{O}_{2}$, isomeric and very likely stereo-isomeric with pilocarpine. Both isomeric pilocarpines differ in melting-points, solubilities and particularly in specific rotation. Isopilocarpine is less dextro-rotatory than pilocarpine and crystallizes in deliquescent prisms easily soluble in water and alcohol. The salts of the two bases also show similar differences:

Pilocarpine nitrate, $\quad \mathrm{C}_{11} \mathrm{H}_{16} \mathrm{~N}_{2} \mathrm{O}_{2} \cdot \mathrm{HNO}_{3}$; mpt. $\mathrm{I}_{7} 8^{\circ}$; $[\alpha] \mathrm{D}=+82.90^{\circ}$.

Isopilocarpine nitrate, $\mathrm{C}_{11} \mathrm{H}_{16} \mathrm{~N}_{2} \mathrm{O}_{2} \cdot \mathrm{HNO}_{3}$; mpt. $159^{\circ}$; $[\alpha] \mathrm{D}=+35.68^{\circ}$.

Jowett ${ }^{1}$ has succeeded in converting isopilocarpine into pilocarpine by means of the same reagent used in converting pilocarpine into isopilocarpine. Pure isopilocarpine, heated with pure alcoholic potassium hydroxide, gives a mixture of unaltered isopilocarpine and pilocarpine. The identity of the latter with pure pilocarpine was established by preparing the hydrochloride and nitrate (mpt. $178^{\circ}$ ). This reciprocal conversion of one alkaloid into the other strongly supports the idea of the stereo-isomerism of pilocarpine and isopilocarpine. Pinner was the first to show that the two nitrogen atoms of the two isomeric bases belong to a glyoxaline ring. ${ }^{2}$ In 1905 Jowett proposed for pilocarpine and isopilocarpine the following formulæ:<smiles>CC[C+]1COC[C]1Cc1cncn1C</smiles>

Pilocarpine

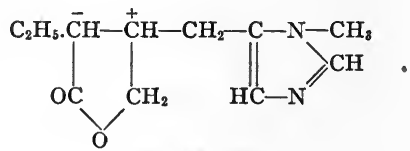

Isopilocarpine

1 Proceedings of the Chemical Society 2r, I72 (r905).

${ }^{2}$ Glyoxaline, or imidazole $\left(\mathrm{C}_{3} \mathrm{H}_{4} \mathrm{~N}_{2}\right)$, is obtained by the action of ammonia upon glyoxal in presence of formaldehyde. It is a strong base and crystalline

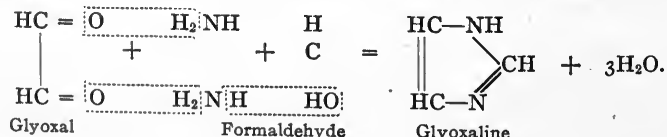




\section{Detection of Pilocarpine}

Ether, chloroform or benzene extracts pilocarpine from aqueous solutions alkaline with sodium hydroxide or carbonate. Evaporation of these solutions leaves a thick, non-crystalline, alkaline syrup. The general reagents especially delicate for pilocarpine are: iodo-potassium iodide, phospho-molybdic acid, phospho-tungstic acid and potassium bismuthous iodide.

I. Place a particle of potassium dichromate and $\mathrm{I}-2$ cc. of chloroform in a test-tube. Then add pilocarpine itself, or its solution and I cc. of 3 per cent. hydrogen peroxide. Shake for several minutes. The mixture yellowish at first gradually darkens and in 5 minutes is dark brown. Depending upon the amount of pilocarpine, the chloroform is blue-violet, dark or indigo-blue. But the upper aqueous solution gradually fades. The chloroform mixture is an intense blue from 0.01 gram of pilocarpine and blue-violet from $0.00 \mathrm{I}$ gram and less. The color lasts from an hour to a day (H. Helch $\left.{ }^{1}\right)$.

Apomorphine (o.or gram) colors chloroform blue-violet even without bydrogen peroxide. Strychnine gives a barely perceptible bluish tint which changes completely within a few minutes. There is a color with antipyrine only after acidification of the hydrogen peroxide.

2. Mandelin's reagent dissolves pilocarpine with a golden yellow color which gradually changes to bright green and finally to light brown.

3. The solution of pilocarpine in formalin-sulphuric acid becomes yellow, yellowish brown and blood-red, if warmed.

Thus far fatal poisonings from this alkaloid have not occurred and nothing is known as to the possibility of its detection in the cadaver.

\section{PTOMAINES}

Ptomaines are basic substances containing nitrogen and may be toxic or non-toxic. They are produced during putrefaction' of cadavers under the influence of bacteria. They are to be regarded to some extent as products of bacterial metabolism and are nearly always present in cadavers, especially in those parts which are in an advanced state of putrefaction. Many ptomaines closely resemble alkaloids. Like alkaloids they give precipitates with the general reagents, and certain ptomaines resemble well-defined alkaloids even with special

${ }^{1}$ Pharmazeutische Post 35, 289, 498 (1902) and 39, 3 I3 (rgo6). 
reagents. Hence ptomaines are of very great importance in forensic chemistry, since their presence may easily lead to mistakes and false conclusions. These putrefactive products also resemble vegetable bases in their behavior with solvents. Ether extracts some of them from acid solution and others from alkaline solution, whereas certain ptomaines are removed from alkaline solution only by amyl alcohol or chloroform. Most of the ptomaines are strong reducing agents, for example, they will immediately convert potascium ferricyanide into ferrocyanide. Consequently, they give the Prucsian blue test with a dilute mixture of solutions of ferric chloride and potassium ferricyanide. Many of the alkaloids like morphine resemble the ptomaines in this respect.

The resemblance of a ptomaine to a definite vegetable base is frequently confined to some one reaction, and never extends to all the reactions characteristic of the particular alkaloid. In a legal-chemical investigation no precaution, which guards against mistaking a ptomaine for a vegetable base, should be omitted. It is an invariable rule to make every test characteristic of the suspected alkaloid, and not to be satisfied with possibly one positive test. A determination of the physiological action of the substance should supplement the chemical examination. A ptomaine may resemble a vegetable base chemically, and yet the two substances may differ very decidedly in physiological action. Thus far, ptomaines have been found which show certain resemblances to coniine, nicotine, strychnine, codeine, veratrine, delphinine, atropine, hyoscyamine, morphine and narceine. Selmi has described a putrefactive product which resembles morphine. Ether failed to extract it, either from asid or alkaline solution, whereas amyl alcohol removed it with ease from an alkaline or ammoniacal solution. It liberated iodine from iodic acid, but failed to give the tests which are characteristic of morphine alone, namely, Husemann's, Pellagri's and the ferric chloride tests!

The object in such cases must be to get a result about which there can be no doubt. Every possible means must be used to isolate the alkaloid in a perfectly pure state. When this can be accomplished, the nature of the poison can always be established beyond question.

\section{SAPONINS}

The term saponins, or saponin substances, includes a large number of glucoside-likc bodies of widespread occurrence in the vegetable kingdom and having in common certain chemical, physical and especially physiological properties. Their aqueous solutions when shaken foam readily. In this respect they resemble the soaps. Many saponin substances have a sharp, harsh taste. In powdered form they excite violent sneezing. They are capable of holding many finely divided substances in a state of emulsion. They dialyze incompletely and salts precipitate them from solution. Excepting the gluco-alkaloid solanine, which contains nitrogen and is alkaline, the saponins may be classified chemically as nitrogen-free glucosides. Most saponins are neutral and only a few are faintly acid. Neutral saponins and alkali salts of acid saponin substances dissolve in water and hot aqueous alcohol but are insoluble in absolute alcohol and ether. Barium hydroxide and lead acetate (neutral and basic) precipitate saponins from concentrated aqueous solution. The former gives baryta saponins. 
Basic lead acetate precipitates all saponins but the neutral salt precipitates only acid saponins. Ammonium sulphate is capable of salting saponin substances from solution as it does proteins. Solutions of saponins in concentrated sulphuric acid are yellow, gradually becoming red and sometimes violet and blue-green. The detection thus far of saponin substances in more than 50 plant families having over 200 monocotyledenous and dicotyledenous species shows the wide occurrence of these substances in the vegetable kingdom. Saponins occur in roots (Senega, Saponaria), tubers (Cyclamen), barks (Quillaja, Guaiacum), fruits (Sapindus, Saponaria), seeds (Æsculus, Agrostemma, Thea), stems (Dulcamara) and leaves (Guaiacum). In fact almost any part of the plant organism may contain saponins. The plant families, producing saponin substances in greater abundance, are the sapindaceæ, caryophyllaceæ, colchicaceæ polygalaceæ, sileneæ and solanaceæ. Quite considerable quantities of saponins may occur in the particular part of the plant.

Saponin solutions, heated with dilute hydrochloric or sulphuric acid, are hydrolyzed into sugars and a non-toxic substance insoluble in water called sapogenin. The sapogenins have not been extensively investigated but they are not entirely identical.

The following saponins have been more closely studied:

Digitonin: in the seeds of Digitalis pupurea.

Saponin: in the root of Saponaria officinalis ( $4-5$ per cent.).

Githagin: in the seeds of the corn cockle, Agrostemma githago (6.5 per cent.).

Senegin: in Senega root, the root of Polygala senega.

Struthiin: in levantine soap root, the root of Gypsophila struthium (I4 per cent.).

Quillaja-Sapotoxin: in the bark of Quillaja saponaria (8.8 per cent.).

Sapindus-Sapotoxin: in the fruit of Sapindus saponaria.

Sarsaparilla-Saponin: in the sarsaparilla root, the root of various kinds of smilax.

Physiological Action of Saponins.-Almost without exception saponin substances are highly toxic, if introduced directly into the blood. Most saponins are absorbed with difficulty. Consequently healthy individuals may take dilute saponin solutions by the mouth in considerable quantities without ill effects. Toxic saponins act in common as protoplasmic irritants. In larger doses saponin substances kill protoplasm. They manifest in various ways their power of acting as protoplasmic poisons. Saponins act upon blood-corpusclès for the same reason. R. Kobert and his collaborators have shown defibrinated blood, diluted Ioo times with physiological salt solution (see below), to be the best and most convenient reagent for saponin substances. Saponins cause hæmolysis and the blood solution becomes laky. Agglutination and formation of methæmoglobin do not occur. The freer the blood is of serum, the more pronounced the hæmolytic action of saponin substances upon blood-corpuscles. Recent investigations have shown that saponins act more vigorously upon blood-corpuscles isolated from serum, because blood serum contains cholesterin which has a protective influence and retards hæmolysis. Most likely the hæmolytic action of saponins is due to removal of cell membrane lecithin, the chief constituent of the cell wall, from red blood-corpuscles, for lecithin-saponins are formed. Saponins 
also combine with cholesterin, as well as with lecithin, forming cholesterinsaponins. The affinities of a saponin having been satisfied by cholesterin, it no longer acts upon the lecithin of the membrane of blood-corpuscles. Thus cholesterin prevents hæmolysis, which a saponin may produce, and so acts as an antidote to saponin substances. Ransam ${ }^{1}$ has made the important discovery that addition of cholesterin checks the solvent action of a saponin upon bloodcorpuscles. At first it was not known whether this antidotal action was due to a chemical reaction, or to absorption, that is to say, to a physical process. $\mathrm{R}$. Kobert $^{2}$ as well as Madsen and Noguchi $^{3}$ were able to dissolve cholesterin, which is insoluble in water, in an aqueous saponin solution. They assumed that this physiologically inactive solution contained a labile saponin-cholesterin compound no longer having hæmolytic power. Recently A. Windhaus ${ }^{4}$ has definitely proved that saponin-cholesterides exist. Digitonin-cholesteride, $\mathrm{C}_{55} \mathrm{H}_{94} \mathrm{O}_{28} \cdot \mathrm{C}_{27} \mathrm{H}_{46} \mathrm{O}$, crystallizes in fine needles, when a hot alcoholic solution of digitonin (I molecule) is poured into a similar solution of cholesterin (I molecule). This cholesteride is formed without elimination of water. Hence in this reaction between digitonin and cholesterin we are dealing most probably with the formation of a molecular compound.

Saponin solutions also dissolve white blood-corpuscles but only at higher concentrations. A physiological action characteristic of many saponins is exhibited in the stupefaction and killing of fish, even in water containing only I: 200,000 of saponin substance (R. Kobert).

\section{Detection of Saponins}

The matter of solubility is especially important in isolating saponin substances from mixtures. All saponins are soluble in water and some in alcohol, but they are practically insoluble in ether, benzene, chloroform and petroleum ether. Employ neutral or basic lead acetate (see above) in isolating saponins. Decompose the washed precipitate with hydrogen sulphide, filter and evaporate the filtrate upon the water-bath. Precipitate the saponin with absolute alcohol and ether from the concentrated solution. Solutions of most saponins in concentrated sulphuric acid are red or yellowish red, gradually becoming violet. Saponin substances give various colors with Froehde's reagent and vanadic-sulphuric acid: brown, redbrown, blue, green and violet (see solanin). A saponin solution heated with dilute hydrochloric acid, undergoes hydrolysis

1 Deutsche medizinische Wochenschrift. I90r, 194.

2 R. Kobert, Die Saponine, Stuttgart, I904.

${ }^{3}$ Chemisches Zentralblatt, I905, I, 1265.

4 Berichte der Deutschen chemischen Gesellschaft 42, 238 (1909). 
and then, owing to formation of sugar, reduces Fehling's solution with heat.

\section{Detection in Foaming Beverages (Beer, Wine, Effervescing Lemonade) ${ }^{1}$}

Treat the beverage to be tested for saponin with excess of basic magnesium carbonate, evaporate to about roo cc. and mix with 2 volumes of 96 per cent. alcohol. Filter after 30 minutes and evaporate the alcohol from the filtrate. Filter the residue hot and extract the cold filtrate with sufficient liquid carbolic $\operatorname{acid}^{2}$ to leave about $5 \mathrm{cc}$. undissolved. Add ammonium sulphate to hasten the separation of the carbolic acid layer. Then shake the latter with water and a mixture of 2 volumes of ether and I volume of petroleum ether. Evaporate the aqueous solution to dryness upon the water-bath. Wash the residue with cold absolute alcohol, in case of wine, and with acetone, in case of beer. The residue fails to give the saponin reaction well, that is to say, a red color with concentrated sulphuric acid, unless treated as described. E. Schaer dissolves the residue in concentrated aqueous chloral hydrate solution and adds the latter to concentrated sulphuric acid as an upper layer. A saponin produces a yellow, then purple-red and finally mallowblue zone.

\section{Detection of Githagin (Corn Cockle Saponin) in Flour}

Heat 500 grams of flour with I liter of alcohol (sp. gr. 0.8496 $=85$ per cent. by volume). Filter hot, distil most of the alcohol, add absolute alcohol as well as ether to the residue and let stand $12-24$ hours. Collect the precipitate upon a filter and dry for a short time at $100^{\circ}$ to coagulate possible protein. Dissolve in a little cold water, filter and precipitate githagin from the filtrafe with absolute alcohol, best with addition of ether. Githagin thus obtained is a yellowish white powder having a sharp, harsh taste.

To prove the presence of a saponin substance, agitate its

${ }^{1}$ K. Brunner, Zeitschrift für Untersuchung der Nahrungs- und Genussmittel 5, 1197 (1902).

${ }^{2}$ Acidum carbolicum liquefactum of the German Pharmacopœia. 
aqueous solution which should foam. Then heat the solution with dilute hydrochloric acid and test its reducing power with Fehling's solution. Finally, if possible, perform the physiological test with blood. Dilute defibrinated ox blood with roo volumes of 0.9 per cent. sodium chloride solution and add the solution of supposed githagin in 0.9 per cent. sodium chloride solution. The blood solution at once becomes laky, if githagin is present. According to J. Brandl, ${ }^{1}$ Agrostemma-Sapotoxin (githagin) produces hæmolysis in very great dilution $(\mathrm{r}: 50,000)$. But after previous treatment with cholesterin, even $0.0 \mathrm{r}$ gram shows no hæmolytic action whatever.

\section{Physiological Salt Solution and Hæmolysis}

To prevent red blood-corpuscles from changing volume in experiments requiring dilution of blood, an isotonic salt solution must be used. What is an isotonic solution? If $n$ grammolecules of a body $A$ are dissolved in a definite volume of solvent and $n$ gram-molecules of a body $B$ are dissolved in an equal volume of the same solvent, certain properties of the original solvent are changed equally in both cases. The freezingpoint of the solutions is lowered and the boiling-point raised equally. The two solutions have the same vapor tension and the same osmotic pressure. In other words they are isotonic. Blood-corpuscles retain their volume unchanged, if brought into a salt solution having the same osmotic pressure as the blood serum. Such a salt solution is isotonic with blood serum. In the case of human and mammalian blood an isotonic solution of sodium chloride hàs a concentration of 9 per thousand = physiological salt solution. Such a solution formerly contained 0.6 per cent. of sodium chloride. Blood-corpuscles give up water to solutions of higher concentration than 0.9 per cent. $\mathrm{NaCl}$ (hyperisotonic solutions) until osmotic equilibrium is established. They shrivel and hence have a smaller volume. On the other hand, blood-corpuscles in salt solutions of lower concentration (hypisotonic solutions) take up water and become distended. In diluting blood with water, this swelling

${ }^{1}$ Archiv für experimentelle Pathologie und Pharmakologie, 54, 245. 
may go far enough to cause hæmoglobin to separate from the stroma and pass into the aqueous solution. This process is called hæmolysis. Alternate freezing and thawing of blood may produce hæmolysis. Various chemical substances, which act as protoplasmic poisons, cause the same result. Such substances are ether, alcohol, chloroform, alkalies, gallic acids, solanine, etc. The saponins described above are also powerful hæmolytic agents. Finally, those globulicidal substances, or. hæmolysins, normally occurring in blood sera. as well as those produced in immunization, belong in this class.

\section{SOLANINE}

Solanine, $\mathrm{C}_{52} \mathrm{H}_{93} \mathrm{NO}_{18}$, at the same time an alkaloid and a glucoside (glucoalkaloid) occurs in the potato plant (Solanum tuberosum) and in other Solanaceæ as Solanum nigrum, Solanum dulcamara and Solanum lycopersicum (tomato). It has been found also in Scopoliacex, as in Scopolia orientalis and Scopolia atropoides. Solanine is not uniformly distributed in all parts of the potato plant but is most abundant in the berry-like fruit and in the chlorophyll-free sprouts appearing in the spring upon potatoes that lie in a cellar. Schmiedeberg and Meyer found 0.024 gram of solanine per kilogram of peeled potatoes in January and February but 0.044 gram in unpeeled potatoes. Potato peelings gave $0.7 \mathrm{I}$ gram of solanine per kilogram and potato sprouts I cm. long even 5.0 grams. The appearance of solanine according to $\mathrm{R}$. Werk is due to the life processes of Bacterium solaniferum (?).

Solanine crystallizes. in white needles having a bitter taste and melting at $244^{\circ}$. Even boiling water dissolves only a little of this alkaloid (about $\mathrm{r}: 8000$ ). It is soluble in 500 parts of cold and I25 parts of boiling alcohol; and in about 4000 parts of ether. These solutions are faintly alkaline. Hot saturated solutions of solanine in alcohol and amyl alcohol gelatinize upon cooling. Ether, chloroform and benzene do not extract solanine either from acid or alkaline solution. But hot amyl alcohol extracts solanine from acid solution and from solutions alkaline with sodium hydroxide or ammonia. Solanine is a weak base, readily dissolving in acids, as acetic acid, and forming crystalline salts. Dilute hydrochloric or sulphuric acid hydrolyzes solanine to solanidine, $\mathrm{C}_{40} \mathrm{H}_{61-}$ $\mathrm{NO}_{2}$, galactose and rhamnose. Hydrolysis is very slow in the cold but rapid upon heating. The hydrochloride or sulphate of solanidine separates as a difficultly soluble, crystalline powder. A good yield of solanidine is obtained, according to Wittmann, by heating solanine under a return-condenser with ro times the quantity of 2 per cent. sulphuric acid, until the liquid is yellowish and the filtrate upon further boiling no longer deposits solanidine sulphate. Solanidine, precipitated from its sulphate with ammonia and recrystallized from ether, forms colorless, silky needles, melting at $207^{\circ}$ and dissolving with difficulty in water but readily in ether or hot alcohol. Solanidine is a stronger base than solanine and the salts it forms with acids are usually crystalline and difficultly 
soluble in water. Solanine and solanidine are highly toxic substances having an action similar to that of the saponin substances (see above).

Toxic Action.--Solanine taken internally is usually very imperfectly absorbed. As a glucoside its action is local and as a saponin-like substance strongly hæmolytic, rendering the blood laky. A solanine solution even in a dilution of $x: 8300$ causes complete hæmolysis. Internal administration of solanine usually produces emesis and larger doses cause gastro-enteritis (gastro-intestinal catarrh). The latter also follows intravenous and subcutaneous injection of doses not rapidly fatal. At the same time a hæmoglobinuria may appear. (R. Kobert, Intoxikationen.)

\section{Detection of Solanine and Solanidine}

Since very dilute mineral acids hydrolyze solanine, these acids cannot be used to detect this alkaloid. E. Schmidt ${ }^{1}$ suggests the following procedure. Extract the material with cold water containing tartaric acid. Neutralize the filtered extract with calcined magnesia and evaporate to dryness upon the waterbath. Extract the residue with alcohol and filter hot. If the quantity of solanine is not too small, the alcoholic extract gelatinizes upon cooling. Otherwise, evaporate the alcoholic solution and examine the residue for solanine. L. Kobert extracts solanine from alkaline solution with isobutyl alcohol. Phospho-molybdic acid is the only general reagent giving a precipitate with a solanine solution and that is yellow. But solanidine, that is to say, a solanine solution that has been boiled with excess of hydrochloric acid, being a stronger base, gives precipitates with most of the other general reagents.

\section{Special Tests for Solanine and Solanidine}

I. A solution of solanine in selenic-sulphuric acid² is raspberry-red. Gentle heat favors the appearance of this color. Solanidine gives the same result.

2. Solutions of solanine and solanidine in vanadic-sulphuric acid $^{3}$ are orange-yellow, soon becoming red and finally blueviolet. Solanine may be dissolved first in sulphuric acid and a drop of vanadic-sulphuric acid added to this solution.

1 Pharmazeutische Chemie, Organischer Teil.

${ }^{2} \mathrm{~A}$ mixture of $\mathrm{r} .3$ grams of sodium selenate $\left(\mathrm{Na}_{2} \mathrm{SeO}_{4}\right.$. $\left.\mathrm{IO}^{\mathrm{H}} \mathrm{H}_{2} \mathrm{O}\right), 8 \mathrm{cc}$. of water and $6 \mathrm{cc}$. of concentrated sulphuric acid.

${ }^{3}$ Dissolve o.I gram of ammonium vanadate $\left(\mathrm{H}_{4} \mathrm{~N} . \mathrm{VO}_{3}\right)$ in roo grams of concentrated sulphuric acid. 
3. Solutions of solanine and solanidine in ethyl sulphuric$\operatorname{acid}^{1}$ are red. An alcoholic solution of solanine, carefully added to concentrated sulphuric acid as an upper layer, produces a red zone where the two liquids meet (E. Schmidt).

4. A solution of solanine in concentrated sulphuric acid is orange but becomes brownish red on longer standing or gentle warming. Red streaks appear, if bromine water is added drop by drop to a solution of solanine in concentrated sulphuric acid.

5. A solution of solanine in Froehde's reagent is first yellowish red then evanescent cherry-red and finally red-brown.

The methods for estimating solanine quantitatively in potatoes are described in Chapter VI (see page 29I).

\section{THEBAINE}

Thebaine, $\mathrm{C}_{19} \mathrm{H}_{21} \mathrm{NO}_{3}=\mathrm{C}_{17} \mathrm{H}_{15}\left(\mathrm{OCH}_{3}\right)_{2} \mathrm{NO}$, constitutes about 0.15 per cent. of opium. This alkaloid crystallizes from dilute alcohol in leaflets having a

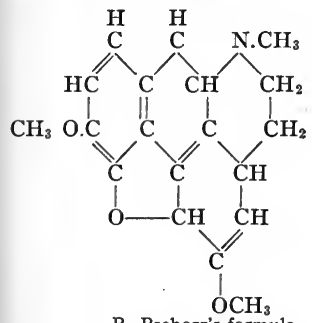

R. Pschorr's formula silvery glitter and from absolute alcohol in prisms melting at $193^{\circ}$. It is nearly insoluble in water, rather easily soluble in hot alcohol, ether, benzene and chloroform. It differs from morphine in being nearly insoluble in caustic alkalies. Its solutions are tasteless and lævorotatory.

Constitution.-Thebaine is a strong tertiary base, forming as a rule well crystallized salts with acids. But excess of acid, especially mineral acid, usually decomposes these salts with ease. Being a tertiary base, it easily combines with methyl iodide, forming thebaine iodomethylate, $\mathrm{C}_{19} \mathrm{H}_{21} \mathrm{NO}_{3} \cdot \mathrm{CH}_{3} \mathrm{I}$, crystallizing in prisms. Two of the three oxygen atoms in thebaine are methoxyl-groups $\left(-\mathrm{OCH}_{3}\right)$ and the third probably forms an ether-like combination, a so-called bridge-oxygen. The thebaine molecule appears not to contain hydroxyl.

Heated with acetic anhydride, thebaine gives the acetyl derivative of the phenol thebaol, $\mathrm{C}_{16} \mathrm{H}_{14} \mathrm{O}_{3}$, and a nitrogenous product, methyl-oxy-ethylamine, $\mathrm{CH}_{3} \cdot \mathrm{NH} . \mathrm{CH}_{2} \cdot \mathrm{CH}_{2} \cdot \mathrm{OH}$. R. Pschorr has synthesized thebaol, or the methyl ether of thebaol, and shown by this synthesis that thebaol is 3,6-dimethoxy4-oxy-phenanthrene (see below). Pschorr assigns to thebaine the structural formula given above which is analogous to that of apomorphine and of morphine (see pages 127 and 131 ). Thebaol has the following structural formula:

${ }^{1}$ Add $6 \mathrm{cc}$. of concentrated sulphuric acid to $9 \mathrm{cc}$. of absolute alcohol. 


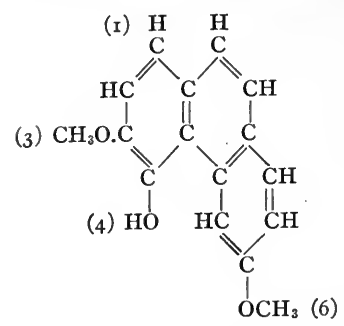

\section{Detection of Thebaine}

Ether and chloroform extract thebaine from an alkaline aqueous solution and consequently this alkaloid appears in ether extract B, if the Stas-Otto procedure is followed. The general reagents, phospho-tungstic acid, iodo-potassium iodide, potassium mercuric iodide and potassium bismuthous iodide precipitate thebaine even from very dilute solutions. Thebaine gives the following color reactions:

I. Concentrated Sulphuric Acid gives a deep red color with thebaine and the solution gradually becomes yellowish red. Froehde's reagent gives the same result.

2. Concentrated Nitric Acid dissolves thebaine with a yellow color. With Erdmann's reagent the color varies from dark red to orange.

3. Chlorine Water dissolves thebaine and ammonia turns the solution an intense red-brown.

\section{Toxalbumins}

Toxalbumins are toxic, protein-like substances either already formed in the plant or animal organism, or produced in the metabolism of pathogenic microorganisms. These substances as yet have not been isolated pure as individual chemical compounds. The chemical and physiological properties of such vegetable toxalbumins as abrin, ricin, robin and crotin are given as a matter of fact by substances obtained from some particular part of the plant by a definite method. The vegetable toxalbumins mentioned possess the common property of clumping, agglutinating and precipitating red blood-corpuscles. Therefore R. Kobert classifies them as "vegetable agglutinines." A trace of one of these agglutinines, added to defibrinated blood in a test-tube, causes clumping into a mass resembling sealing-wax. Abrin, ricin and crotin also cause coagulation of milk. 


\section{Abrin}

This toxalbumin occurs in jequirity seeds from Abrus precatorius. Remove the seed envelopes and extract the finely divided seeds with 4 per cent. sodium chloride solution. Concentrate the filtered liquid in vacuo and acidify with acetic acid. Precipitate abrin from this solution by addition of sodium chloride and finally purify by dialysis. Abrin is an amorphous, highly toxic powder not entirely free from ash. Though abrin and ricin are alike in some respects, they are not identical.

\section{Ricin}

This intensely toxic toxalbumin constitutes 2.8-3 per cent. of the castor bean. Remove the seed envelopes and subject the seeds to powerful pressure to remove as much oil as possible. Then extract with ro per cent. sodium chloride solution. Saturate the filtered extract at the same time with magnesium and sodium sulphate and keep for some time in the cold at room temperature. Place the precipitate, which contains ricin, in a parchment paper dialyzing tube and dialyze for several days. Finally dry the residual ricin in vacuo over sulphuric acid. Ricin is an amorphous, highly toxic powder containing ash and easily soluble in ro per cent. sodium chloride solution. This toxalbumin, dissolved in sodium chloride solution, gives the protein reactions. Ricin possesses in high degree the power of agglutinating blood-corpuscles. Use defibrinated blood for this test-tube experiment, not diluted blood or blood mixed with physiological salt solution. Ricin, according to Elfstrand, agglutinates the red blood-corpuscles of the guinea-pig even in a dilution of $r ; 600,000$. Ricin agglutinates the blood of all mammals but not to the same degree. Removing serum from the blood and substituting physiological salt solution strengthens rather than weakens the agglutinating action of ricin. The inference is that serum must have a certain anti-agglutinating action. Separation of red blood-corpuscles into stroma and hæmoglobin ${ }^{1}$ shows that ricin has not changed hæmoglobin in the least. But the stromata have been altered just as the blood-corpuscles have been.

To detect ricin in castor bean press-cake, or in feeds containing castor beans, extract the finely divided material with physiological salt solution at room temperature, filter and make the agglutination test in a test-tube with undiluted, defibrinated blood and with blood diluted with physiological salt solution.

\section{Crotin}

Crotin is a substance obtained from the seeds of Croton Tiglium. Remove the seed envelopes, express the oil and treat as described for abrin and ricin. Chemically crotin is very similar to ricin. Abrin and ricin agglutinate the blood-corpuscles of all warm-blooded animals thus far tested but crotin does not behave the same with all kinds of blood. (See R. Kobert, Intoxikationen.)

\section{Coagulation of Blood and Defibrinated Blood}

Blood is a transparent fluid, the blood-plasma, suspended in which is a very large number of solid particles, the red and white blood-corpuscles. Outside

1 The two principal components of blood-corpuscles are the stroma, which constitutes the true protoplasm, and the intraglobular contents, the chief constituent of which is hæmoglobin. 
the organism blood coagulates even in a few minutes after being drawn. In the clotting of blood a very difficultly soluble protein, called fibrin, separates. If the blood is still, the clot is a solid mass which gradually contracts and exudes a clear liquid, usually yellow, the blood-serum. The coagulum, thus formed and enveloping the blood-corpuscles, is called the crassamentum (Placenta sanguinis). But if the blood is whipped during coagulation, fibrin separates in threads. The fluid separated from the latter is defibrinated blood which consists of blood-corpuscles and blood-serum. To obtain defibrinated blood, whip with twigs the fresh blood removed from a vein and fibrin will separate on these. Or run the fresh blood into an Erlenmeyer flask, containing iron filings, and shake vigorously for several minutes. Fibrin is precipitated on the filings.

There are several ways to retard coagulation of blood, among which the following may be mentioned:

1. Cool blood suddenly to low temperature.

2. Draw blood direct from the vein into a neutral salt solution, for example, magnesium sulphate solution ( $\mathrm{I}$ volume of salt solution and 3 volumes of blood) and stir. This mixture of blood and salt will not coagulate for a day. .

3. Add blood to sufficient dilute potassium oxalate solution to give a mixture containing 0.1 per cent. of oxalate. The soluble calcium salts of the blood are precipitated by the oxalate and the blood loses its power of coagulating.

4. To prepare a non-coagulating blood-plasma, pour blood into sodium fluoride solution until it contains 0.3 per cent. of $\mathrm{NaF}$. 


\section{CHAPTER V}

\section{SPECIAL QUALITATIVE AND QUANTITATIVE METHODS}

\section{Quantitative Estimation of Phosphorus in Phosphorated Oils}

I. W. Straub's Method.-Straub has found that his test ${ }^{1}$ with dilute copper sulphate solution, recommended for the qualitative detection of phosphorus, may also be used to determine phosphorus in a phosphorated oil. If such an oil is shaken with 3 per cent. copper sulphate solution, there is first a brownish black emulsion in which each individual oil drop is coated with a film of copper phosphide, $\mathrm{PCu}_{3}$ (?). After $4^{-5}$ hours shaking, this brownish black color disappears and the mixture separates into two layers. All the phosphorus in the oil is now in the aqueous solution as phosphoric acid. This method has the further advantage that the decolorization of the emulsion serves as an indicator of the completion of the oxidation.

Procedure.-Put $25 \mathrm{cc}$. of 3 per cent. copper sulphate solution (taken as $\mathrm{CuSO}_{4} \cdot 5 \mathrm{H}_{2} \mathrm{O}$ ) in a separatory funnel. Add $5 \mathrm{cc}$. of the phosphorated oil ${ }^{2}$ and agitate the mixture vigorously for a long time. If a shaking machine is available, place the mixture in a thick-walled glass bottle with a tight glass stopper and shake $3-5$ hours, or until the original brown emulsion has disappeared and become clear and bright blue. Separate the aqueous solution in a 'separatory funnel and precipitate phosphoric acid at once by the molybdate method and finally weigh as magnesium pyrophosphate, $\mathrm{Mg}_{2} \mathrm{P}_{2} \mathrm{O}_{7}$.

1 Zeitschrift für anorganische Chemie 35, 460 (I903).

2 To prepare a phosphorated oil suitable for such determinations, dissolve about o.I gram of yellow phosphorus in the smallest possible quantity of warm carbon disulphide and dilute this solution to roo cc. with olive oil. Although carbon disulphide does not affect the determination of phosphorus, it may be removed by warming the phosphorated oil on the water-bath. 
Remarks.-The accuracy of this method is shown by the results of Straub's determinations. Instead of 0.005 gram of phosphorus, dissolved in $5 \mathrm{cc}$. of oil, he found 0.0047 and 0.00468 gram. Even very considerable dilutions of the phosphorated oil do not affect the accuracy of the determination. In the case of the more concentrated phosphorated oils, shaking with copper sulphate solution must be kept up much longer.

\section{A. Frankel's ${ }^{1}$ and C. Stich's ${ }^{2}$ Method.-Dissolve the oil} in acetone and precipitate phosphorus with hot alcoholic silver nitrate solution. Oxidize the phosphorus in the precipitate to phosphoric acid and finally determine the latter in the usual way.

Procedure.-Dissolve 20-50 cc. of the phosphorated oil, as phosphorated cod liver oil, in Ioo cc. of acetone or ether and completely precipitate with hot alcoholic silver nitrate solution. ${ }^{3}$ First wash the precipitate of silver phosphide with ether-acetone mixture and then with alcohol. Treat next with hot 25 per cent. nitric acid, containing a little fuming acid. Expel excess of nitric acid from the filtrate on the water-bath and precipitate silver with hydrochloric acid. Finally filter. from silver chloride and determine phosphoric acid in the filtrate.

Remarks.-Since sodium hypophosphite and phosphite are soluble in acetone and also precipitated by acetone-silver nitrate, it is advisable first to extract a test portion of the phosphorated oil with water and then test the aqueous extract for these first oxidation products of phosphorus, hypophosphorous and phosphorous acids. If they are present, all the phosphorated oil should first be extracted with water in the same manner.

Phosphorus in phosphorated oils, especially phosphorated cod liver oil slowly disappears. C. Stich found that a phosphorated cod liver oil, containing 0.05 per cent. of phosphorus, with the usual daily removal of 5 grams, lost in 3 weeks only 3-5 milligrams of phosphorus. Such a decrease in the amount of phosphorus in phosphorated oils is only of slight significance. Dilute oily solutions of phosphorus ( $\mathrm{r}: \mathrm{r000}$ ), when kept in tightly stoppered bottles and protected from light, are constant as regards their phosphorus content for a long time, even 5-6 months. Moreover, phosphorus much diluted as vapor or in solution, is oxidized with corresponding difficulty. The same is also true of phosphorus in the animal organism. Therefore it is possible sometimes to detect free phosphorus in the excretory organs, as the liver, even several weeks after phosphorus poisoning.

The distillation method is inapplicable in the quantitative estimation of phos-

1 Pharmazeutische Post 34 , I 7 .

2 Pharmazeutische Zeitung 37, 500 (1902).

${ }^{8}$ Silver nitrate dissolves in about so parts of alcohol. 
phorus in oils, as cod liver oil, since only about 40 per cent. of the phosphorus present is found in the receiver, even when the strongest oxidizing agent and the best absorbent for phosphorus are used. To place the phosphorus-content of the cod liver oil residue at the amount of the distilled phosphorus is not admissible, because cod liver oil as such contains about 0.02 per cent. of combined phosphorus.

\section{Special Methods for the Detection of Arsenic}

\section{Isolation of Arsenic as Arsenic Trichloride ${ }^{1}$}

- This depends upon the volatility of arsenic as chloride, $\mathrm{AsCl}_{3}$, in concentrated hydrochloric acid solution and in presence of ferrous chloride. The latter serves $(\alpha)$ to reduce any arsenic acid possibly present in the material to arsenious acid which with concentrated hydrochloric acid then forms arsenic trichloride $(\beta)$ :

(a) $\mathrm{H}_{3} \mathrm{AsO}_{4}+{ }_{2} \mathrm{HCl}+{ }_{2} \mathrm{FeCl}_{2}=\mathrm{H}_{3} \mathrm{AsO}_{3}+\mathrm{H}_{2} \mathrm{O}+{ }_{2} \mathrm{FeCl}_{3}$,

(及) $\quad \mathrm{H}_{3} \mathrm{AsO}_{3}+{ }_{3} \mathrm{HCl}=\mathrm{AsCl}_{3}+{ }_{3} \mathrm{H}_{2} \mathrm{O}$.

Procedure.-Comminute the material and mix with very concentrated hydrochloric acid (about 40 per cent.) until rather thin. Then add 5 grams of 20 per cent. arsenic-free ferrous chloride solution or saturated ferrous sulphate solution and put the mixture into a capacious retort, the neck of which is directed obliquely upward and connected with a Liebig cooler by an obtuse angle tube, and carefully distil. Distil about a third to a half of the original mixture. Dilute the distillate with water and test for arsenic in the Marsh apparatus, using hydrochloric acid for the evolution of hydrogen.

If a tubulated retort is used for the distillation, hydrochloric acid gas can be passed in during distillation so that the liquid is kept saturated with this acid.

\section{Electrolytic Detection of Arsenic}

To detect arsenic electrolytically, put the liquid, as the sulphuric acid solution obtained according to the general procedure which contains arsenic as arsenic acid (see page 156 ), or urine or stomach contents, in a sufficienly wide U-tube with platinum electrodes (Fig. I8). Pass the current through the liquid acidified with sulphuric acid, and arsine, $\mathrm{AsH}_{3}$, together with

${ }^{1}$ H. Beckurts, Archiv der Pharmazie 222, 653 (1884). 
hydrogen will appear at the cathode, if the liquid contains arsenic. First test the hydrogen for arsenic by the Gutzeit arsenic test (see page $r_{3}$ ). If a yellow spot appears on the paper moistened with saturated silver nitrate solution, arsenic is present. That this is actually arsenic may be shown by connecting the U-tube as shown in the sketch with a chloride of calcium tube and a Marsh reduction-tube; an arsenic mirror then appears in

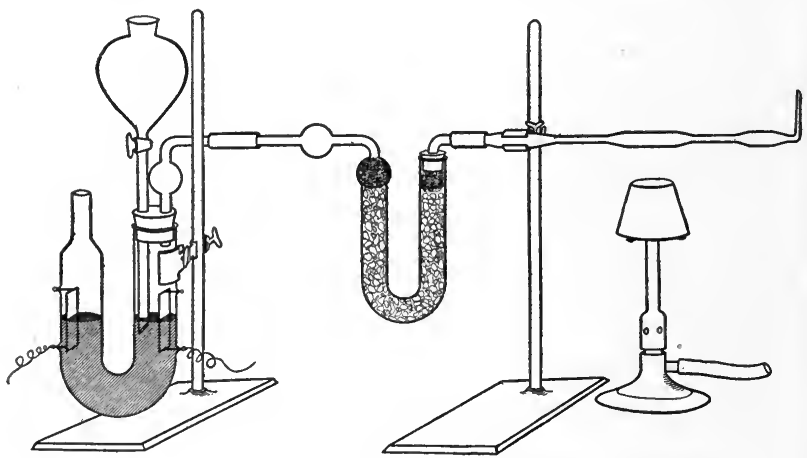

FIG. I8.-Apparatus for the Electrolytic Detection of Arsenic.

the latter when heated to redness. Use a current having an electromotive force of $7-8$ volts. The electrolytic method is especially adapted for the detection of arsenic in inorganic compounds present in secretions, as the urine, but not for arsenic in organic combination as cacodyl compounds and arrhenal. An exception among these organic compounds of arsenic is atoxyl, or the anilid of meta-arsenic acid, $\mathrm{AsO}_{2} \cdot \mathrm{NH} \cdot \mathrm{C}_{6} \mathrm{H}_{5}$. The arsenic being rather loosely bound is broken up by the electric current with formation of arsine.

\section{Destruction of Organic Matter and Detection of Arsenic}

(According to A. Gautier ${ }^{1}$ and G. Lockemann²)

The purpose of this method is to increase the delicacy of the Marsh-Berzelius test for arsenic, and to exclude as far as possible sources of error connected with

${ }^{2}$ Bulletin de la Société chimique de Paris, 29, 639 (1903).

${ }^{2}$ Zeitschrift für angewandte Chemie 18, 416, 491 (1905); also 19, r362 (rgo6). 
the destruction of organic matter, the precipitation of arsenic with hydrogen sulphide and the evolution and drying of hydrogen gas. Organic matter is destroyed without the use of hydrochloric acid, and arsenic is detected without precipitation as arsenic sulphide. Lockemann recommends the following procedure and uses finely divided meat as a test experiment:

Place 20 grams of finely chopped meat in a porcelain dish and add a few cc. of a mixture of ro parts of fuming nitric acid and I part of concentrated sulphuric acid. Warm upon the water-bath. The action of the acid mixture is so vigorous that, even after the addition of about $5 \mathrm{cc}$., the entire mass, which puffs up considerably at first, changes to a yellowish, homogeneous, thick, oily liquid. If too much acid is added at once during warming upon the water-bath, the action may be violent enough to cause sudden charring of the whole mass with copious evolution of smoke. Such an occurrence may result in loss of arsenic. Consequently, it is advisable to add the acid mixture, amounting in all to about $20 \mathrm{cc}$, , to the meat in $\mathrm{I}^{-2} \mathrm{cc}$. portions, not adding a fresh portion of acid until brown fumes cease coming off. The mass is dark yellow and finally becomes brown after long heating upon the water-bath. Stir with a concentrated aqueous solution of 20 grams of a mixture of potassium and sodium nitrate $(I+I)$ and evaporate upon the water-bath. There remains a yellow, crystalline residue which still contains organic matter. Gradually introduce this mixture in small portions into a platinum crucible containing ro grams of fused potassium and sodium nitrate $(I+I)$. Having added all the mixture, heat the crucible for a short time over a free flame. Dissolve the cold melt in water, add sulphuric acid and heat upon the water-bath until nitrous fumes have been expelled. Test a cold solution of the residue for arsenic in the Marsh apparatus.

Lockemann formerly precipitated arsenic with aluminium hydroxide, $\mathrm{Al}(\mathrm{OH})_{3}$. Add Io cc. of a 12 per cent. solution of crystallized aluminium sulphate, $\mathrm{Al}_{2}$ -

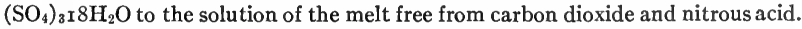
Render the solution alkaline with ammonia and heat about 30 minutes upon the water-bath. Collect the precipitate upon a paper, wash with water containing ammonia and dissolve in about $30 \mathrm{cc}$. of ro per cent. sulphuric acid. Heat the solution in a porcelain dish upon the water-bath until it no longer gives a test for nitric acid with diphenylamine-sulphuric acid. ${ }^{1}$ Then examine this solution for arsenic in the modified Marsh apparatus ${ }^{2}$ devised by Lockemann (Fig. I9).

Lockemann's latest results have shown that ferric hydroxide is much more effective than aluminium hydroxide as a precipitant of small quantities of arsenic. Render the water solution of the melt (see above) slightly acid with sulphuric acid, add a few cc. of iron alum solution, then in the cold, best after cooling with - ice, add just enough ammonia to precipitate all the iron. Filter after 30 minutes, wash the precipitate with cold water to remove nitrates completely, then dissolve in dilute sulphuric acid and test the solution for arsenic in the Marsh apparatus. Iron salts do not interfere with the delicacy of the Marsh test for arsenic.

${ }^{1}$ Dissolve I gram of diphenylamine in Ioo grams of concentrated sulphuric acid. A drop of the liquid with a drop of this diphenylamine solution in a porcelain dish should not give a blue color.

${ }^{2}$ O. Pressler, 30 Bruederstrasse, Leipzig, Germany, supplies this apparatus and also the ignition-tubes. 
Zinc in sticks ${ }^{1}$ and sulphuric acid are used in the preparation of hydrogen. Copper is the best activator of zinc in the Marsh apparatus. Break the zinc sticks into pieces weighing about $\mathrm{r} .2-\mathrm{r} .8$ grams, place for a minute in 0.5 per cent. copper sulphate solution, wash with water, dry with filter paper and preserve carefully in a closed bottle. This procedure does not interfere with the formation of the mirror, whereas addition of copper sulphate to the reduction flask causes retention of arsenic. Copper sulphate used for this purpose should be carefully purified by several recrystallizations. The basic properties of fused and granulated calcium chloride, which are not entirely removed even by hydrogen chloride and carbon dioxide, make this an unsuitable drying agent for hydro-

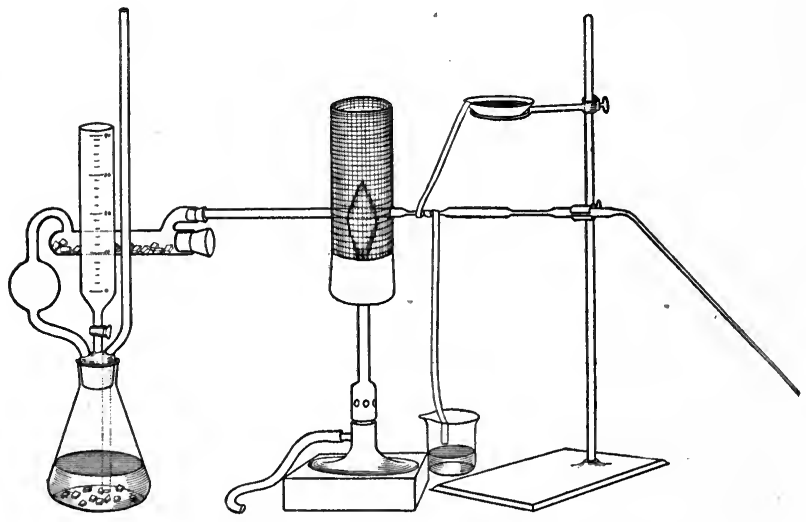

Fig. 19.-Marsh Apparatus Modified by Lockemann.

gen. Lockemann found that potassium carbonate, phosphorus pentoxide and concentrated sulphuric acid cause a noticeable decomposition of arsine, and the same is true of glass wool and cotton. Crystallized calcium chloride in pieces about I cc. in volume is the best drying agent, because it is entirely indifferent to arsine. Lockemann's special drying tube (see sketch) is adapted for the use of this substance. Bohemian glass, having a wall thickness of $1 \mathrm{~mm}$. and an internal diameter of $4 \mathrm{~mm}$., is used for ignition-tubes. 'These are drawn out in two places to a length of $4 \mathrm{~cm}$. The outer diameter of the constriction is $\mathrm{r} .5$ $\mathrm{mm}$. and the inner about $0.5 \mathrm{~mm}$. The reduction flask contains $4^{-6}$ pieces of coppered zinc and about $15 \mathrm{cc}$. of ${ }_{5} 5$ per cent. sulphuric acid are added from the dropping funnel. After hydrogen has been passing through the apparatus for 30 minutes, heat is applied in front of the first constriction of the ignition-tube. If the materials are arsenic-free after $\mathrm{I} \cdot 5^{-2}$ hours heating, place the flame in

'Lockemann has found Kahlbaum's stick zinc always arsenic-free. The same may be said of Bertha spelter from the New Jersey Zinc Company. 
front of the second constriction of the ignition-tube. The solution of the iron hydroxide precipitate, prepared as described above, is added to the reduction flask from the dropping funnel which is washed with a little water or dilute sulphuric acid. In testing for very small quantities of arsenic, it is advisable to cool the place where the mirror is deposited by keeping the cotton thread wet (see sketch).

By means of the apparatus described Lockemann has detected even $0.000 \mathrm{Img}$. of arsenic distinctly.

Moist air gradually oxidizes the arsenic mirror, but in an absolutely dry atmosphere even when exposed to light there is no change. In a'closed tube containing a little phosphorus pentoxide arsenic mirrors may be kept unchanged even for months.

Glass wool, or cotton, noticeably decomposes arsine. The decomposition of arsine in aqueous solution is also hastened by the presence of fine filamentary bodies. This reaction is probably catalytic in character.

\section{Electrolytic Estimation of Minute Quantities of Arsenic}

(C. Mai and H. Hurt ${ }^{1}$ )

By this method minute amounts of arsenic (fractions of a milligram) are separated quantitatively at the cathode from an arsenical electrolyte as arsine. The latter then reacts quantitatively with silver nitrate as follows:

$$
\mathrm{AsH}_{3}+{ }_{3} \mathrm{H}_{2} \mathrm{O}+6 \mathrm{AgNO}_{3}=\mathrm{H}_{3} \mathrm{AsO}_{3}+6 \mathrm{HNO}_{3}+6 \mathrm{Ag} \text {. }
$$

The advantages of the electrolytic detection of arsenic are first the avoidance of traces of arsenic that sometimes come from zinc in the Marsh test and second that destruction of organic matter is often unnecessary. T. E. Thorpe ${ }^{2}$ has shown the latter to be the case in the examination of beer worts and malt extracts for arsenic. To reduce arsenic acid and its salts, a few drops of zinc sulphate solution should be added to the sulphuric acid acting as the electrolyte. The cathode is said to have a higher tension and the hydrogen to be very active.

Apparatus and Procedure.-The apparatus used by Mai and Hurt is shown in Fig. 20.

$A$ is the reduction-tube and $B$ a bulb-tube with $5-6$ bulbs containing o.or n-silver nitrate solution. $A$ and $B$ are connected

11 Zeitschrift für Untersuchung der Nahrungs- und Genussmittel 9, I93 (I905) and also Pharmazeutische Zeitung, 1905.

${ }^{2}$ Proceedings of the Chemical Society I9, I83 (IgO3). 
by a small tube $g$ containing pieces of pumice stone saturated with an alkaline lead solution, or glass wool, to retain any traces of hydrogen sulphide. Anode $a$ and cathode $e$ are lead strips about $\mathrm{I}-2 \mathrm{~mm}$. thick. Their upper ends about $5 \mathrm{~mm}$. thick are luted into glass tubes $b$ which pass through the stopper of the $\mathrm{U}$-tube and are tight. The dropping funnel $d$ holds about $25 \mathrm{cc}$. and its capillary end dips about $2 \mathrm{~cm}$. into the solution to be electrolyzed. Tube $c$ for the escape of oxygen from the anode chamber contains a little water.

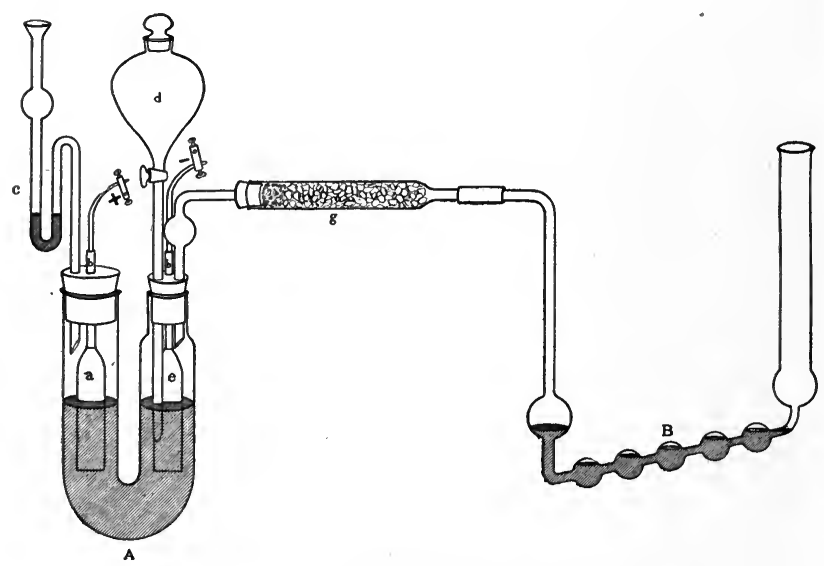

FIG. 20.-Apparatus for the Electrolytic Estimation of Arsenic.

Fill U-tube $A$ up to the mark with 2 per cent. arsenic-free sulphuric acid and bulb-tube $B$ with ro cc. of $0.0 \mathrm{I}$ n-silver nitrate solution. Turn on the current and keep at $2-3$ amperes. If the silver nitrate solution remains unchanged after hydrogen has been running I hour, the lead cathode and sulphuric acid are arsenic-free. Without stopping the current, introduce from the dropping funnel the solution to be 'tested for arsenic, the quantity of which should not be more than ro cc. Add this solution as slowly as possible and wash the last traces in with a little water. If the solution contains arsenic, or arsenic acid, 
the silver nitrate solution will become dark in a few minutes and the reaction will be at an end in 3 hours. Pour the contents of the bulb-tube through a small asbestos filter, wash with $3-4 \mathrm{cc}$. of water and titrate excess of o.or n-silver nitrate with 0.01 n-potassium sulphocyanate according to Volhard's method.

Calculation.-The reaction above shows that 6 molecules of silver nitrate correspond to I atom of arsenic $(=75)$. Therefore I gram-molecule of silver nitrate $=\frac{1}{6}$ gram-atom of arsenic $=\frac{75}{6}=\mathrm{I} 2.5$ grams of arsenic and rooo cc. of $0.01 \mathrm{n}$-silver nitrate $=0.125$ gram of arsenic.

Notes.-Electrodes of platinum (foil or gauze) cannot be used in the electrolytic separation of arsenic as arsine, because either solid arsine or elementary arsenic is formed. Mai and Hurt also found that gold, silver and tin cathodes gave unsatisfactory results and carbon electrodes were not much better. Pure lead alone meets all the requirements as a material for the electrodes. Oxygen compounds of arsenic are quickly and completely reduced to gaseous arsine only upon cathodes of absolutely pure lead. The attachment of a platinum wire to a lead electrode was sufficient to cause incomplete reduction of arsenic compounds. For this reason the electrodes consist of one piece of lead ${ }^{1}$ without soldering on wire of another metal. The best electrolyte is $\mathrm{r} 2$ per cent. sulphuric acid. A stronger acid easily causes the formation of hydrogen sulphide and a weaker acid has the disadvantage of lower conductivity and lower specific gravity. The electrolyte should be specifically heavier than the solution to be tested to keep the latter from passing at once to the bottom of the reduction-tube.

Mai and Hurt found the following amounts of As:

$\begin{array}{lll} & \text { As taken } & \text { As found } \\ \mathrm{As}_{2} \mathrm{O}_{3} & 0.25 \mathrm{mg} . & 0.223 \mathrm{mg} . \\ \mathrm{As}_{2} \mathrm{O}_{3} & 0.10 \mathrm{mg} . & 0.099 \mathrm{mg} . \\ \mathrm{As}_{2} \mathrm{O}_{5} & 0.10 \mathrm{mg} . & 0.105 \mathrm{mg} .\end{array}$

For qualitative tests the bulb-tube may be replaced by the drying and ignition-tubes of the Marsh apparatus.

According to Mai and Hurt the statements of Thorpe and Trotmann, that every solution can be electrolyzed without previously destroying organic matter, do not always hold. In the examination of beer containing arsenic the results were fairly satisfactory, but in the case of urine the results were far too high.

1 Kahlbaum's purest lead. 
Quantitative Estimation of Arsenic and Antimony by the Gutzeit Method

Using a special apparatus and paper sensitized with mercuric chloride, Sanger and Black ${ }^{1}$ have found that the Gutzeit test can be employed to determine small amounts of arsenic quantitatively. The process is very simple and requires only a short

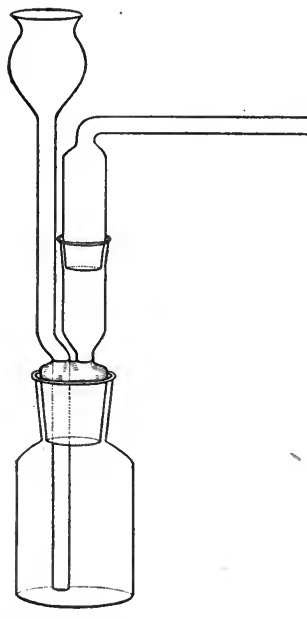

Fig. 21.-Apparatus for the Quantitative Gutzeit Method. ${ }^{4}$ time for completion. Sanger and Riegel $^{2}$ have extended this method to the estimation of antimony.

Sensitized Paper.-Paper strips ${ }^{3}$ uniformly $4 \mathrm{~mm}$. wide are sensitized by being soaked in 5 per cent. solution of recrystallized mercuric chloride. These are dried, cut into $7 \mathrm{~cm}$. lengths and protected from light and moisture in a stoppered bottle containing calcium chloride, or soda lime, covered with cotton.

Apparatus.-A $30 \mathrm{cc}$. bottle (Fig. 2I) for the reduction is closed by a glass stopper provided both with a thistle-tube, constricted to $2 \mathrm{~mm}$. at the end and extending nearly to the bottom of the bottle, and with an exit-tube widened to about $\mathrm{I}_{5} \mathrm{~mm}$. just above the stopper. Connected with this exit-tube by a ground joint and at a right angle is a tube exactly $4 \mathrm{~mm}$. inside diameter and approximately $9 \mathrm{~mm}$. in length from the bend.

Procedure.-Place 3 grams of uniformly granulated zinc $^{5}$ in the bottle and a strip of sensitized paper in the $4 \mathrm{~mm}$. deposition

1 Proceedings of the American Academy of Arts and Sciences 43, 297-324 (1907).

${ }^{2}$ Ibid., 45, 21-27 (r9o9).

${ }^{3} \mathrm{~A}$ cold pressed paper made by Whatman has been found to give the best results.

4 This all glass apparatus, suggested by Mr. W. A. Boughton, is now in use in the Harvard laboratory and is a modification of Sanger's original apparatus.

${ }^{5}$ Bertha spelter from the New Jersey Zinc Company, New York, has been proved free from arsenic. 


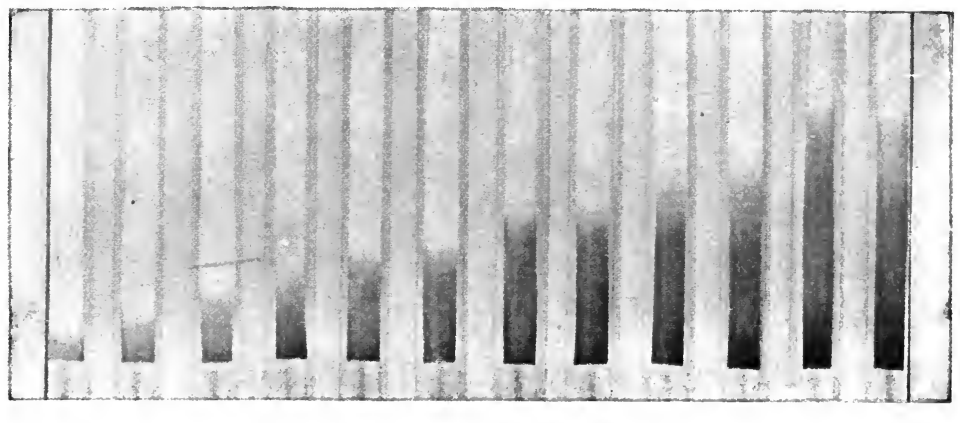

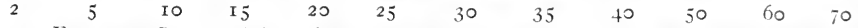

FIG. 22.- Standard Arsenic Bands in Micromilligrams of $\mathrm{A}_{s_{2}} \mathrm{O}_{3}$ (Initial).

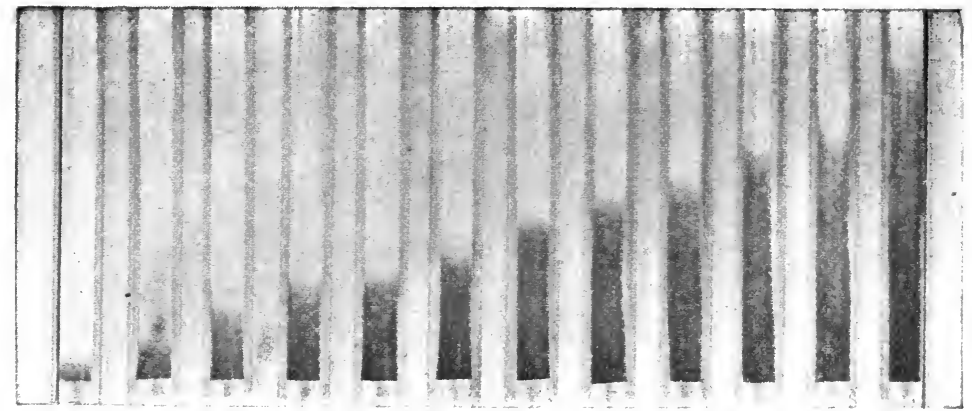
$5 \quad$ IO
I 5
20
$25 \quad 30 \quad 35$
$40 \quad 50$
6070

FIg. 2 2a.-Standard Arsenic Bands in Micromilligrams of $\mathrm{As}_{2} \mathrm{O}_{3}$ (Hydrochloric Acid Development).

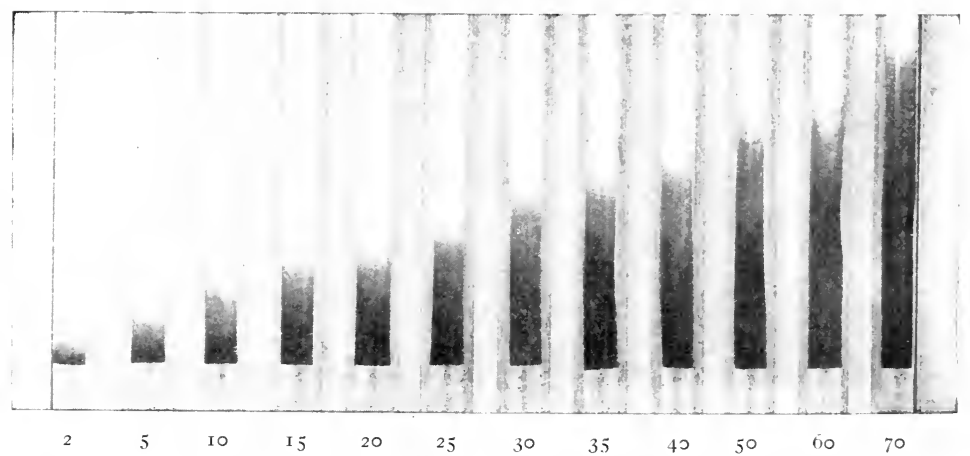

Fig. 22b.-Standard Arsenic Bands in Micromilligrams of $A s_{2} \mathrm{O}_{3}$ (Ammonia I) evelopnent . 

tube. In estimating arsenic, place in the enlargement of the exit-tube a loose plug of lean absorbent cotton that has been kept over sulphuric acid; an hour's preliminary run is necessary to mo:sten the cotton partially. In the case of antimony substitute for cotton a disc of filter paper that has been moistened with normal lead acetate, dried and kept in a well stoppered bottle. Before inserting this disc moisten it with a drop of water. Next add I $_{5}$ cc. of diluted hydrochloric $\operatorname{acid}^{1}$ ( $1: 6$ ) and let the hydrogen run so minutes to make sure the reagents cause no stain. Then add the whole, or an aliquot part of the solution to be tested. Arsenic will produce a color on the paper in a few minutes which will reach a maximum within 30 minutes. Antimony produces no visible effect on the sensitized paper, unless the amount is above $70 \mathrm{mmgr}$. (= $0.070 \mathrm{mg}$.) when a gray color may appear. If there is any color, another trial should be made with a smaller portion of solution. In the determination of arsenic a disc of lead acetate paper should be inserted beneath the cotton as a precaution against the possible formation of hydrogen sulphide.

Standard Bands.- (a) Arsenic. Dissolve I gram of resublimed arsenious oxide in a little arsenic-free sodium hydroxide, acidify with sulphuric acid and make up to a liter with recently boiled water. Dilute ro cc. of this solution (I) to a liter with freshly boiled water which gives a solution (II) containing o.or mg. of arsenious oxide per cc. Using definite volumes of solution II, measured from a burette, prepare a series of color bands (Fig. 22), taking a fresh charge of zinc and acid for each portion. The color ranges from lemon-yellow through orangeyellow to reddish brown.

(b) Antimony.-Dissolve 2.3060 grams of pure, recrystallized tartar emetic in a liter of water. This solution (I) contains I.o mg. of antimonious oxide per cc. By dilution of (I) solutions containing $0.0 \mathrm{I} \mathrm{mg}$. (II) and $0.00 \mathrm{I} \mathrm{mg}$. (III) are prepared and used in making sensitized bands.

${ }^{1}$ Synthetic hydrochloric acid, made from electrolytic hydrogen and chlorine by the Hooker Electrochemical Company, New York, is said to be entirely free from arsenic. Tr. 
These bands will eventually fade but they may be preserved longer by being sealed in glass tubes in the bottom of which is phosphorus pentoxide covered with cotton. The color of the arsenic bands may be developed (I) by placing the band in hydrochloric acid (I : I) for 2 minutes at a temperature not over $60^{\circ}$, washing thoroughly, drying and sealing as before; (2) by treating for a few minutes with ammonium hydroxide, which gives a dense, coal-black color, washing, drying and sealing in a tube over quicklime.

To develop the antimony band; let it stand in a test-tube covered with normal ammonium hydroxide 5 minutes. A black band is slowly developed. These bands may be protected as described, or placed between glass plates cemented together and bound with passepartout paper.

The more dilute standard solutions must be freshly made up within a few hours of use.

Notes.-Solutions should be as free as possible from sulphur compounds yielding hydrogen sulphide; interfering organic matter; and metals retarding formation of arsine and stibine. The cotton in the exit-tube should be replaced after ro-r 2 runs, and the lead acetate disc after each run. If the solution contains arseniate, reduce with ro cc. of arsenic-free sulphurous acid and expel the excess.

The absolute delicacy of the method is set at $0.00008 \mathrm{mg}$. of arsenious oxide and $0.0005 \mathrm{mg}$. of antimonious oxide. The practical delicacy, using a band $4 \mathrm{~mm}$. wide, is $0.00 \mathrm{r} \mathrm{mg}$. of arsenious oxide and 0.002 or $0.003 \mathrm{mg}$. of antimonious oxide. By using, however, a band $2 \mathrm{~mm}$. wide in a correspondingly narrow exit-tube, a practical delicacy of $0.0005 \mathrm{mg}$. of arsenious oxide and $0.00 \mathrm{I}$ mg. of antimonious oxide is obtainable. In length of band and density of developed color, the effect of arsine on the sensitized paper is from 2-3 times as great as that of stibine. The authors do not claim a greater accuracy for the method than within ro per cent.

\section{Biological Detection of Arsenic by Penicillium Brevicaule}

B. Gosio ${ }^{1}$ was the first to show that certain moulds, grown upon media containing minute quantities of arsenic, produce volatile arsenic compounds characterized by a garlic-like odor. Seven species of moulds were found to have this power. Penicillium brevicaule, however, which Gosio isolated from

1 "Azione di alcune muffe sui composti fissi d'arsenico," Rivista d'igiene è sanita publica, 1892,201 . 
air, and which was first found upon decaying paper, possessed this property in the highest degree. Gosio states that we are justified in regarding Penicillium brevicaule as a living reagent for arsenic. Even $0.0000 \mathrm{r}$ gram of arsenic can be recognized with certainty by this biological test. The test is so delicate that it should be of great value in toxicological analysis in the preliminary examination for arsenic.

A. Maasen ${ }^{1}$ states that a temperature of 28 to $32^{\circ}$ is most favorable to the growth of the mould. Crumbs of wheat bread were found to make an especially good culture-medium. When this material is used, a vigorous growth of mould is visible even in 48 hours. Sometimes a test for arsenic can be finished in a few hours, and always in 2 or 3 days. The characteristic garlic odor from weak, arsenical cultures can be distinctly recognized even after several months. That these "arsenic moulds" do not produce gases having a garlic odor from sulphur, phosphorus, antimony, boron and bismuth compounds, is an important fact. But Penicillium brevicaule possesses in high degree the power of converting solid selenium and tellurium compounds into volatile substances having a peculiar odor. The odor. especially from tellurium cultures, is like that produced by arsenic cultures, namely, distinctly like garlic! The odor from selenium cultures, however, differs from that arising from arsenic cultures. It is more of a mercaptan odor.

Biginelli $^{2}$ found that the gases, generated from arsenic cultures by Penicillium brevicaule, are completely absorbed by mercuric chloride solution. Colorless crystals, having the composition $\left(\mathrm{AsH}\left(\mathrm{C}_{2} \mathrm{H}_{5}\right)_{2} \cdot 2 \mathrm{HgCl}_{2}\right)$, are formed. This is a double compound of mercuric chloride and diethyl arsine. This compound can easily be decomposed. It then diffuses an intense garlic odor. On the other hand, Klason ${ }^{3}$ has recently shown as a result of an investigation of the gas given off when Penicillium brevicaule subsists upon an arsenical medium that

${ }^{1}$ Arbeiten aus dem Kaiserlichen Gesundheitsamt, I902, 478.

${ }^{2}$ Chemisches Centralblatt (I900), II, I067, and also (I900), II, I 100.

${ }^{3}$ Berichte der Deutschen chemischen Gesellschaft 47, 2634 (I9I4). 
a double compound of ethyl ${ }^{-}$cacodylic oxide and mercuric chloride is formed, the formula of which is $\left(\mathrm{C}_{2} \mathrm{H}_{5}\right)_{2} \mathrm{As}-\mathrm{O}-\mathrm{As}-$ $\left(\mathrm{C}_{2} \mathrm{H}_{5}\right)_{2}+{ }_{4} \mathrm{HgCl}_{2}$.

R. Abel and J. Buttenberg ${ }^{1}$ state that a mould to be of use in the biological detection of arsenic must satisfy the following conditions: "It must grow rapidly, and not generate any odors during growth, except the garlic odor produced from an arsenical medium. It must not be restricted as to culture medium. It must grow in presence of large, or very small quantities of arsenic. Finally, it must demonstrate its specific action in presence of metallic arsenic and all kinds of arsenical compounds."

The best material for these experiments is white or Graham bread, either of which is a favorite culture medium for moulds. The crust is the only part of bread having a specific aromatic odor. When this has been removed, the crumbs may.besaid to be practically odorless.

Procedure.-When the material examined is liquid, absorb it completely by adding bread crumbs, and scatter a small quantity of dry bread over the surface. Solid material should be finely ground, or cut into as small pieces as possible, and placed in not too small a flask. Add at least the same quantity of bread crumbs, thoroughly mix the two substances by shaking, and moisten the mass with a little water. Close the flask with a cotton plug, and sterilize in steam. Sterilization must kill all micro-organisms in the flask. Therefore, heat the flask in an autoclave ro to 30 minutes under a pressure of I to I. 5 atmospheres. There is no danger of volatilizing arsenic during sterilization. Then inoculate the sterilized material when cold. Place in a flask a slice of potato, superficially coated with mould in the spore-forming stage, and agitate it with bouillon (peptone), salt solution or sterilized water, until it is finely disintegrated. Observe all necessary precautions, and add the mould, suspended in water, in sufficient quantity to impregnate the entire surface of the material suspected of containing arsenic. There should not be more liquid, how-

${ }^{1}$ Zeitschrift für Hygiene, 32, 440 (I899). 
ever, than the culture medium will absorb. Too much moisture retards the growth of the mould. Finally, draw a tight rubber cap over the mouth of the flask and cotton plug. Flasks thus closed may stand in the room, but it is better to keep them at a higher temperature, for example, in an incubator at $37^{\circ}$, since these conditions are most favorable to the growth of the mould. As soon as a growth of mould is distinctly visible to the naked eye upon the medium, the first indication is given that a test of the culture for volatile arsenic compounds may prove successful. In a very favorable case, this is possible in 24 hours. There is always a luxuriant growth of mould in 48 to 72 hours, so that a decision can be reached. If there is no odor, the flask is closed, and the test is repeated once or twice daily on the following days.

Sulphuric, hydrochloric and other strong mineral acids prevent the growth of the mould. This preventive action may be overcome by neutralization with calcium carbonate, which may be present in excess without ill effect. Alkalies also interfere with the growth of the mould. They may be removed by neutralization with tartaric or citric acid, either of which may be present in excess. The great advantage of the biological over the purely chemical method lies in the fact that less time is required to get a result. The tedious and unavoidable destruction of organic matter in the material is rendered unnecessary Moreover, a number of tests for arsenic may be made at the same time.

Abel and Buttenberg (loc. cit.) speak as follows, regarding this method: "The biological method of detecting arsenic has so many advantages, that it deserves to be recommended for the most varied purposes. Its application is very general, and the method of procedure is simple. The culture of the mould can be kept a long time, even a year or more, without being revived. The test is very delicate, and the odor is readily recognized. The generation of the odor, in the case of cultures containing only $0.000 \mathrm{r}$ gram of arsenic, can be demonstrated for a week."

Besides being practically unlimited in application, the biological method is extraordinarily delicate. In this respect, it exceeds the best known chemical methods for detecting arsenic. It is, for example, considerably more delicate than Bettendorff's test, and it might equal in delicacy the Marsh and Gutzeit tests.

\section{Detection of Arsenic in Organic Arsenic Compounds}

\section{Cacodylic Acid, Arrhenal, Atoxyl ${ }^{1}$}

The ordinary reagents usually fail to show arsenic in an organic arsenic compound dissolved in water. Several of these

${ }^{1}$ C. E. Carlson, Zeitschrift für physiologische Chemie 49, 410 (Igo6). 
compounds persistently resist the most powerful oxidizing and reducing agents.

Cacodylic Acid, $\left(\mathrm{CH}_{3}\right)_{2} \mathrm{AsO}-\mathrm{OH}$, and its salts have been used of late as drugs. A 2 per cent. solution of sodium cacodylate, $\left(\mathrm{CH}_{3}\right)_{2} \mathrm{AsO}-\mathrm{ONa} \cdot 3 \mathrm{H}_{2} \mathrm{O}$, conducts the electric current very feebly but no arsine appears at the cathode. Bettendorff's reagent (stannous chloride-hydrochloric acid) does not cause separation of arsenic from cacodylic acid even after evaporation with hydrochloric acid and potassium chlorate. If heated with stannous chloride-hydrochloric acid, cacodylic acid is reduced to the foul-smelling cacodylic oxide, $\left[\left(\mathrm{CH}_{3}\right)_{2} \mathrm{As}\right]_{2} \mathrm{O}$, recognized by its odor. Distillation of sodium cacodylate by Schneider's method with the strongest hydrochloric acid gives no arsenic trichloride in the distillate. The arsenic changes to another form, not precipitable by hydrogen sulphide. Evaporation of the distillate upon the water-bath with nitric acid leaves solid, non-volatile cacodylic acid in which arsenic may be detected by reduction with sodium carbonate-potassium cyanide mixture. Even fuming nitric acid does not oxidize cacodylic acid to arsenious or arsenic acid.

Arrhenal, Sodium Methyl-Arseniate $\left(\mathrm{CH}_{3}\right) \mathrm{AsO}(\mathrm{ONa})_{2.5} \mathrm{H}_{2} \mathrm{O}$, forms white crystals very soluble in water. Possibly owing to partial hydrolysis, an aqueous solution of this compound is alkaline and conducts the electric current feebly. Only traces of arsine appear at the cathode after electrolysis in presence of a good conductor. In arrhenal the arsenic is not held as strongly as in the cacodyl compounds. Hydrogen sulphide precipitates yellow arsenic trisulphide. Distillation with strong hydrochloric acid gives arsenic trichloride in the distillate. Bettendorff's reagent gives a red-brown precipitate, if considerable arrhenal is present.

Atoxyl, the Anilide of Metarsenic Acid, $\mathrm{AsO}_{2} \cdot \mathrm{NH} \cdot \mathrm{C}_{6} \mathrm{H}_{5}$, forms white, odorless crystals readily soluble in water and having a faint, saline taste. As compared with cacodylic acid, arsenic in atoxyl is less firmly bound. Electrolysis gives arsine abundantly at the cathode. Hydrogen sulphide precipitates sulphide of arsenic. Arsenic trichloride passes over, upon dis- 


\section{tillation with concentrated hydrochloric acid. Bettendorff's reagent gives a lemon-yellow precipitate.}

Urine.-In suspected arsenic poisoning first examine the urine, since arsenic is very slowly eliminated by this channel. Carlson in experiments upon himself was able to detect arsenic direct in the urine by the electrolytic method and also by the Gutzeit and Marsh tests. He took ro drops of Fowler's solution ${ }^{1}$ daily. Five days after the last dose Carlson could still get a distinct test for arsenic in concentrated urine. The urine was not wholly free from arsenic until I4 days had passed. He then experimented with sodium cacodylate, taking daily 29 drops of a I per cent. solution. He could not detect a trace of arsenic in the urine by the electrolytic method. Therefore the salt of cacodylic acid had passed through the organism unaltered. But cacodylic acid can be detected easily in the urine, upon treating the latter with hypophosphorous acid (sp. gr. I.15). ${ }^{2}$ Cacodylic oxide is formed and can be recognized by its odor. Sometimes the mixture must stand several hours in a closed test-tube. Arrhenal, in daily doses of about 30 drops of 1 per cent. solution, behaved like the cacodyl compound. Arsenic could not be detected in the urine by electrolysis Consequently neither arsenious nor arsenic acid had been formed within the organism. Hypophosphorous acid immediately precipitated arsenic from arrhenal and gave the cacodyl odor.

To detect cacodylic acid in urine, phosphorous acid, as well as zinc or tin and hydrochloric acid, may be used instead of hypophosphorous acid. Frequently it is advisable to oxidize most of the organic matter in the urine beforehand. Boil $25 \mathrm{cc}$. of urine with $25 \mathrm{cc}$. of water, 5 per cent. potassium permanganate solution and ro cc. of 25 per cent. sodium hydroxide solution, until the filtrate is odorless and nearly colorless. Excess of hydrochloric acid (sp. gr. I.rg) and zinc filings, added to this filtrate, produce with heat the odor of cacodyl, if the urine contains cacodylic acid. Arsenic from atoxyl can be isolated at the cathode in the form of arsine by electrolysis. Therefore arsenic can be detected in the urine electrolytically after administration of atoxyl.

\section{Quantitative Estimation of Minute Amounts of Arsenic} (Karl Th. Mörner ${ }^{3}$ )

This method is said to be useful in estimating arsenic quantitatively in various kinds of fabrics and in urine in cases of poisoning. It is a titration method devised especially for quantities of arsenic not exceeding $0.5 \mathrm{mg}$. Arsenic is first precipitated as trisulphide with thioacetic acid, $\mathrm{CH}_{3} \cdot \mathrm{CO} . \mathrm{SH}$. Under the conditions arsenious as well as arsenic acid is thus precipitated. In alkaline

${ }^{2}$ Fowler's solution contains I per cent. of $\mathrm{As}_{2} \mathrm{O}_{3}$ as potassium arsenite.

${ }^{2}$ Instead of free hypophosphorous acid, prepare Engel and Bernard's arsenic reagent (Comptes rend. de l'Acad. des sciences I22, 399), or J. Bougault's (I. Pharm. Chim. (6), 15, 527). Dissolve 20 grams of sodium hypophosphite in $20 \mathrm{cc}$. of water and add $200 \mathrm{cc}$. of hydrochloric acid (sp. gr. 1.17). Filter through a cotton plug to remove $\mathrm{NaCl}$ and use the filtrate.

${ }^{3}$ Zeitschrift für analytische Chemie 4I, 397 (I902). 
solution potassium permanganate readily oxidizes arsenic trisulphide completely to arsenic acid and sulphuric acid:

$$
\mathrm{As}_{2} \mathrm{~S}_{3}+{ }_{14} \mathrm{O}=\mathrm{As}_{2} \mathrm{O}_{5}+{ }_{3} \mathrm{SO}_{3} .
$$

Potassium permanganate solution, added to an alkaline solution of arsenic trisulphide, immediately loses its color, being decomposed in the proportion of 9 molecules to 1 molecule of arsenic trisulphide. ${ }^{1}$ Since 2 molecules of potassium permanganate in sulphuric acid solution yield 5 atoms of oxygen for oxidation, 9 molecules according to the proportion

$$
2: 5=9: x(x=22.5)
$$

should give 22.5 atoms of oxygen. But according to the reaction above, only I4 atoms of oxygen are used to oxidize I molecule of arsenic trisulphide, whereas the remaining 8.5 atoms are stored up in the precipitate as hydrated manganese dioxide $\left(\mathrm{MnO}_{2} \cdot \mathrm{H}_{2} \mathrm{O}\right)$. But if the reaction mixture is heated with oxalic acid in presence of dilute sulphuric acid, these oxygen atoms become active:

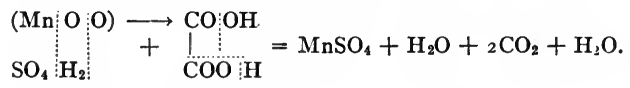

Since 2 molecules of $\mathrm{KMnO}_{4}$ yield 5 atoms of oxygen and since $\mathrm{I}_{4}$ atoms of oxygen are necessary for $\mathrm{I}$ molecule of $\mathrm{As}_{2} \mathrm{~S}_{3}$, according to the following proportion

$$
\begin{aligned}
& \text { Atoms : Mols. } \mathrm{KMnO}_{4} \\
& 5: 2 \quad 2=14: \times(x=5.6)
\end{aligned}
$$

5.6 molecules of potassium permanganate are required for I molecule of $\mathrm{As}_{2} \mathrm{~S}_{3}$ $\left(=21_{4}\right)$, or 2 atoms of arsenic $(=150)$.

I000 cc. of $0.01 \mathrm{n}$-potassium permanganate $\left(=0.3162 \mathrm{gram} \mathrm{KMnO}_{4}\right)$ contain in solution $\frac{2 \mathrm{KMnO}_{4}}{\mathrm{IO} \times 10 \times 10}=0.002$ gram-molecule of $\mathrm{KMnO}_{4}$ which according to the proportion

$$
\begin{aligned}
& \text { Gram-mols. } \mathrm{KMnO}_{4} \text { : Grm. As } \\
& 5.6: \text { I } 50=0.002: \mathrm{x}(\mathrm{x}=0.0536)
\end{aligned}
$$

represents 0.0536 gram of arsenic. Hence rooo cc. of o.or n-potassium permanganate solution correspond to $0.053^{6}$ gram of arsenic.

Procedure.-Dissolve arsenic trisulphide in 0.5 per cent. potassium hydroxide solution ${ }^{2}$ and run this solution into a small flask containing $25 \mathrm{cc}$. of $0.0 \mathrm{I}$-potassium permanganate solution. Mix the contents and add $5 \mathrm{cc}$. of 5 per cent. sulphuric acid, as well as the quantity of o.or n-oxalic acid solution found necessary by special titration. Warm until the color is discharged and finally titrate with o.or n-potassium permanganate solution.

${ }^{1}$ Since 2 mols. $\mathrm{KMnO}_{4}$ give in alkaline solution 3 atoms of available oxygen $\left(2 \mathrm{KMnO}_{4}={ }_{2} \mathrm{MnO}_{2}+3 \mathrm{O}+\mathrm{K}_{2} \mathrm{O}\right)$, I mol. of $\mathrm{As}_{2} \mathrm{~S}_{3}$, according to the proportion: $3: 2=14: x(x=9.33)$, requires not 9 mols, but more exactly 9.33 mols. of $\mathrm{KMnO}_{4}$.

${ }^{2}$ Ammonium hydroxide cannot be substituted for potassium or sodium hydroxide solution. 
Prelimnary Titration.-Add the same quantity of 0.5 per cent. potassium hydroxide solution used to dissolve arsenic trisulphide, as well as $5 \mathrm{cc}$. of 5 per cent. sulphuric acid, to $25 \mathrm{cc}$. of $0.0 \mathrm{r}$ n-potassium permanganate solution. Heat the mixture to boiling and add.oxalic acid solution in slight excess so that the liquid becomes colorless. Titrate back with $0.01 \mathrm{n}$-potassium permanganate. This titration shows how much oxalic acid solution, in conjunction with traces of reducing substances that may be present in the potassium hydroxide solution or sulphuric acid, is needed in a regular titration for the exact reduction of 25 cc. of $0.0 \mathrm{or}$-potassium permanganate.

Example.-Suppose that $25.5 \mathrm{cc}$. of oxalic acid solution were required to decolorize the boiling liquid. Titration required $0.3 \mathrm{cc}$. of $0.0 \mathrm{n}$-potassium permanganate solution. Therefore $25+0.3=25.3 \mathrm{cc}$. of $0.0 \mathrm{r} \mathrm{n}$-potassium permanganate correspond to $25.5 \mathrm{cc}$. of oxalic acid solution and $25 \mathrm{cc}$. of the former correspond to $25.2 \mathrm{cc}$. of the latter solution. Consequently in a regular titration 25.2.cc. of $0.01 \mathrm{n}$-oxalic acid solution must be used.

The amount of potassium permanganate in $25 \mathrm{cc}$. of $0.0 \mathrm{r}$ normal solution is sufficient for all amounts of arsenic up to $0.5 \mathrm{mg}$. Mörner's method of determining arsenic gives very reliable results, if arsenic is in the form of the trisulphide and free from every other substance soluble in 0.5 per cent. potassium hydroxide solution and capable of reducing permanganate.

Using the strongest hydrochloric acid, Mörner first distils arsenic as arsenic trichloride by the Schneider-Fife method. About $200 \mathrm{sq} . \mathrm{cm}$. of carpet, 100 sq. $\mathrm{cm}$. of other woven and paper materials and 15 grams of sealing wax, stearine of wax candles and dried apples were used for each determination of arsenic by this method. According to Mörner, the distillate from such materials by the Schneider-Fife method always contains organic matter, even when caught in dilute nitric acid. To remove this organic matter before precipitating arsenic with thio-acetic acid, collect the distillate in a receiver containing dilute nitric acid and evaporate to dryness in a porcelain dish. Add to the small residue in the dish upon the water-bath successively $2 \mathrm{cc}$. of potassium hydroxide solution ( 0.5 per cent. $\mathrm{KOH}$ ) heating I minute, then $2 \mathrm{cc}$. of potassium permanganate solution (5 per cent. $\mathrm{KMnO}_{4}$ ) heating about 3 minutes, and finally $\mathrm{r}$ cc. of tartaric acid solution (20 per cent. $\left.\mathrm{H}_{2} \cdot \mathrm{C}_{4} \mathrm{H}_{4} \mathrm{O}_{6}\right)^{1}$ heating until the color is discharged. Filter into a porcelain dish, wash the filter with a little water and set the dish upon a boiling water-bath. Add after I minute I cc. of thio-acetic acid (5 per cent. $\left.\mathrm{CH}_{3} \cdot \mathrm{COSH}\right)^{2}$ and warm the mixture 3 minutes. Arsenic is precipitated as arsenic trisulphide. After cooling for 5 minutes, collect the precipitate upon a filter and wash first 5 times with $2 \mathrm{cc}$. portions of 0.5 per cent. sulphuric acid and then 3 times with 2 cc. portions of water. Place under the funnel a

${ }_{1}^{1}$ Tartaric acid readily dissolves the precipitate of manganese peroxide. To reduce the latter, Mörner used oxalic and lactic acids, sodium sulphite and also thio-acetic acid. But tartaric acid proved to be bettter than any of these substances.

2 Prepare thio-acetic acid solution by shaking $5 \mathrm{cc}$. of thio-acetic acid with roo cc. of water. Filter and keep this solution in a dark flask. This solution gradually decomposes with evolution of hydrogen sulphide:

$$
\mathrm{CH}_{3} \cdot \mathrm{COSH}+\mathrm{H}_{2} \mathrm{O}=\mathrm{CH}_{3} \cdot \mathrm{COOH}+\mathrm{H}_{2} \mathrm{~S} \text {. }
$$


small flask containing $25 \mathrm{cc}$, of $\circ .0 \mathrm{r} \mathrm{n}$-potassium permanganate solution and pour over the filter 3 portions of 0.5 per cent. potassium hydroxide solution, using $2 \mathrm{cc}$. each time. The alkaline solution of arsenic trisulphide thus drops directly into the permanganate solution. Otherwise, proceed as described. Subtract $0.3 \mathrm{cc}$. of 0.0 I n-permanganate solution from the volume of this solution used. This correction is necessary because even the finer qualities of filter paper contain traces of substances which dissolve in 0.5 per cent. potassium hydroxide solution and reduce permanganate. ${ }^{1}$

Note.-The procedure described separates arsenic trisulphide from every other substance soluble in 0.5 per cent. potassium hydroxide solution and capable of reducing potassium permanganate. This method is accurate to $0.02 \mathrm{mg}$. of arsenic.

\section{Detection of Salicylic Acid in Foods and Beverages}

Wine. ${ }^{2}-$ Place $50 \mathrm{cc}$. of wine in a cylindrical separating funnel with $50 \mathrm{cc}$. of a mixture of equal parts of ether and petroleum ether. Shake frequently, taking care not to form an emulsion but yet to mix the liquids thoroughly. Remove the etherpetroleum ether layer, pour through a dry filter, evaporate upon the water-bath and add a few drops of ferric chloride solution to the residue which becomes red-violet if salicylic acid is present. But if the color is black or dark brown, add a few drops of hydrochloric acid, dissolve in water, extract with ether-petroleum ether and proceed with the extract as just described.

Meat and Meat Products. ${ }^{3-}$-For experimental purposes add about 0.0 I gram of salicylic acid to some chopped meat. Extract the finely divided material with $5 \circ$ per cent. alcohol and add some milk of lime to the filtered alcoholic solution. Evaporate to dryness upon the water-bath and stir the residue with a slight excess of dilute sulphuric acid. Shake with ether without filtering, pass the ether extract through a dry filter and evapo-

'After passing through the entire process in several blank experiments, Mörner never obtained higher results for permanganate used. Consequently the method of washing described completely removes tartaric and thio-acetic acids.

2 "Official Directions for the Chemical Examination of Wine" of June 25th, r896. (German.)

3 "Agreements with regard to uniformity in inspecting and testing foods, household supplies and other articles used in the German Empire" Heft I, 36 . 
rate. Dissolve the residue in hot water and test the filtered solution for salicylic acid with very dilute ferric chloride solution.

Milk. ${ }^{1-M i x ~} 100 \mathrm{cc}$. of milk with $100 \mathrm{cc}$. of water at $60^{\circ}$. Precipitate with acetic acid and mercuric nitrate solution, using 8 drops of each, shake and filter. Extract the filtrate with 50 cc. of ether, evaporate the ether, dissolve the residue in $5 \mathrm{cc}$. of hot water and test the filtered solution for salicylic acid with dilute ferric chloride solution (sp. gr. r.005-r.oro).

\section{Maltol}

Maltol, $\mathrm{C}_{6} \mathrm{H}_{6} \mathrm{O}_{3},{ }^{2}$ is formed in the preparation of caramel from malt, possibly from maltose or isomaltose. Ether or chloroform extracts this substance from the condensed vapors given off during caramelization and also from beer-wort. Maltol crystallizes in monoclinic prisms and plates from a cold saturated solution in 50 per cent. alcohol (Osann). Chloroform gives denser crystals. This substance dissolves with difficulty in cold water or benzene; more readily in hot water, alcohol, ether or chloroform; and is insoluble in petroleum ether. It dissolves in caustic alkaline solutions but is reprecipitated by carbon dioxide. Maltol sublimes in shining leaflets and is volatile with water vapor. It reduces silver solution in the cold and Fehling's solution with heat. An aqueous maltol solution resembles salicylic acid in becoming intense violet with ferric chloride solution, but differs from carbolic and salicylic acids in not turning red with Millon's reagent.

Maltol, shaken with benzoyl chloride and sodium hydroxide solution, gives a mono-benzoyl derivative and consequently must contain one hydroxyl group.

\section{Aqueous Chloral Hydrate Solution as a Solvent for Alkaloids, Glucosides and Bitter Principles and Its Use in Toxicological Analysis Richard Mauch}

(Communication from Professor E. Schaer's Institute, Strassburg)

One part of water at $\mathrm{I} 7.5^{\circ}$ dissolves 4 parts of chloral hydrate, forming a very mobile solution which is easily filtered and capable of being kept for a long time without decomposition. This 80 per cent. solution of chloral hydrate easily dissolves relatively large quantities of alkaloids and glucosides without altering

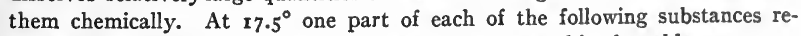
quires for solution the number of parts of solvent stated in the table:

${ }^{1}$ Method of Ch. Girard, Zeitschrift für analytische Chemie 22, 277 (1883) and the above "Agreements" Heft I, 62.

2 J. Brand, Berichte der Deutschen chemischen Gesellschaft 27,806 (1894), H. Kiliani and M. Bazlen, Ibidem 27,3115 (1894). 


\begin{tabular}{|c|c|c|c|c|}
\hline $\begin{array}{r}\text { Chlor } \\
\text { S }\end{array}$ & $\begin{array}{l}\text { ral Hydr } \\
\text { Solution } \\
(80 \%)\end{array}$ & Water & Ether & Chloroform \\
\hline Atropine.............. & 5 & 600 & 50 & $3 \cdot 5$ \\
\hline 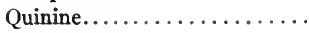 & 6 & 2000 & freely scluble & 2 \\
\hline Cocaine................ & 5 & 700 & freely soluble & freely soluble \\
\hline Morphine............. & 5 & 5000 & 1250 & roo \\
\hline Santonin $\ldots \ldots \ldots \ldots \ldots$ & 4 & 5000 & 125 & 4 \\
\hline Strychnine............. & 6.5 & 6600 & 1300 & 6 \\
\hline Brucine................ & 6.5 & $\ldots$ & $\ldots$ & $\ldots \ldots$ \\
\hline Veratrine.............. & $7 \cdot 5$ & $\ldots$ & $\ldots$ & $\ldots \ldots$ \\
\hline
\end{tabular}

Caffeine is the only alkaloid which forms with chloral hydrate a molecular compound soluble in water. If a chloral hydrate solution of an alkaloid, which has been freshly prepared in the cold, is diluted with considerable water, the unchanged alkaloid is precipitated almost quantitatively, for instance, morphine, strychnine and quinine. Substances like picrotoxin, santonin and acetanilide behave similarly. But when such solutions stand for a long time at ordinary temperatures, or are heated for $\mathrm{I}^{-2}$ hours, chloral hydrate is decomposed by the vegetable base into chloroform and formic acid. Since the alkaloidal salts of formic acid are soluble in water, dilution with this solvent does not precipitate the alkaloids. R. Mauch has shown clearly that atropine, brucine, quinine, cocaine, morphine, narcotine, strychnine and veratrine behave as just described.

In the tests ordinarily made with the ether or chloroform residue, $\mathrm{R}$. Mauch recommends dissolving the residue in 80 or 60 per cent. chloral hydrate solution. The "chloral solution" should prove of great value in color tests which depend upon the use of pure sulphuric acid or sulphuric acid containing iron or molybdic acid. These solutions contain so little water that it cannot modify the action of sulphuric acid upon the substance in solution. Such a "chloral solution" is also well adapted for zone tests. An aqueous solution forms an upper layer with the "chloral solution," and the latter forms an upper layer with concentrated sulphuric acid.

Specific gravity of 80 per cent. chloral hydrate solution $=\mathrm{r} .5 \mathrm{I} 4$.

Specific gravity of 60 per cent. chloral hydrate solution $=1.3535$.

In the tests ordinarily performed in test-tubes, it is best to use small tubes ( 6 or $7 \mathrm{~cm}$. high; $\mathbf{r} \mathrm{cm}$. in diameter) holding $6 \mathrm{cc}$. They should not be made of too thin glass. The chloral solution cannot be used in detecting picrotoxin, because chloral hydrate itself produces the same reduction changes caused by picrotoxin. The same is true of the test for strychnine, where sulphuric acid and potassium dichromate, or any other oxidizing agent, are used. Coniine and nicotine also belong to the class of alkaloids which cannot be detected in chloral hydrate solution. Concentrated chloral hydrate solutions cannot be used directly in making tests with general alkaloidal reagents, because precipitates do not appear until the solutions have been diluted with 6-8 volumes of very dilute hydrochloric or sulphuric acid.

In using the "chloral hydrate method" in toxicological analysis, the ether, chloroform or amyl alcohol extract should be evaporated with gentle heat upon a 
watch-glass of medium size (about $5 \mathrm{~cm}$. diameter) and not too flat. Add to the residue, depending upon the quantity, about $3 \mathrm{cc}$. of 75 per cent. chloral hydrate solution. Cover the glass and let it stand for some time. Occasionally tilt the glass and bring the solution thoroughly in contact with the residue. Pass the solution through a very small filter, if necessary, and wash both watch-glass and filter with a few drops of pure chloral hydrate solution. Use this chloral hydrate solution for the individual tests. In testing for strychnine, evaporate a part of the chloral solution to dryness upon the water-bath. Warm, until the residue does not smell of chloral, and then test for strychnine with sulphuric acid and potassium dichromate.

To recover from the chloral hydrate solution most of the alkaloids and substances like picrotoxin, acetanilide and phenacetine, add excess of sodium hydroxide solution and extract thcroughly with a little chloroform. The "chloral hydrate method" is conducive to very neat work and this is a great advantage. The use of metallic utensils like knives and spatulas is entirely unnecessary.

\section{ESTIMATION OF ALKALOIDS}

\section{Picrolonate Method of $\mathrm{H}$. Matthes ${ }^{1}$}

$\mathrm{Knorr}^{2}$ gave the name picrolonic acid to I-p-nitrophenyl-3methyl-4-isonitro-5-pyrazolone. This compound is formed by the action of nitric acid upon methyl-phenyl-pyrazolone. Picrolonic acid resembles picric acid in its properties and is characterized by forming crystalline salts with many organic bases, as the alkaloids. As a rule these salts dissolve with difficulty and are yellow or red. Heat causes their decomposition. Picrolonic acid is frequently of service in characterizing bases. Hydrochloric acid precipitates this compound from a solution of its sodium salt as a yeliow, mealy powder, melting when rapidly heated at about $\mathrm{I} 28^{\circ}$, becoming dark in color and undergoing decomposition with rapid evolution of gas. Knorr first gave picrolonic acid formula $\mathrm{I}$ but formula $\mathrm{II}^{3}$ is preferred:
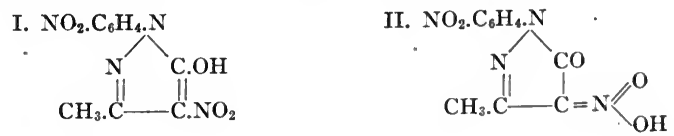

\section{H. Matthes has estimated many alkaloids quantitatively by}

${ }^{1} \mathrm{H}$. Matthes and O. Rammstedt, Zeitschrift für analytische Chemie 46, 565 (1907) and Archiv der Pharmazie 245, II 2 (1907).

2 Berichte der Deutschen chemischen Gesellschaft 30, 914 (1897).

${ }^{8}$ R. Zeine, Inaugural Dissertation, Jena, 1906. 
means of picrolonic acid. Collect the precipitated alkaloidal picrolonate in a weighed Gooch crucible, wash, dry and weigh. Estimation of alkaloids is possible by this method, because the picrolonates are constant in composition. Morphine, hydrastine, codeine, strychnine, brucine, pilocarpine and stypticine ${ }^{\mathfrak{l}}$ can be quantitatively estimated by this method.

\section{Estimation of Morphine, Codeine and Stypticine in Solutions, Tablets and Sugar Triturations}

Dissolve the weighed trituration or tablet in the smallest quantity of water possible and add picrolonic acid solution (about o.I n-solution in alcohol) in slight excess. The picrolonate separates at once; or very soon, as yellow crystals or a crystalline meal. Cool I $_{5}-30$ minutes in ice water and collect the precipitate in a weighed Gooch crucible. Wash with a little ice water, dry 30 minutes at $110^{\circ}$ and weigh. Morphine picrolonate usually separates in ro- 30 minutes. Cooling aids precipitation.

\begin{tabular}{llcc} 
precipitation. & \multicolumn{1}{c}{ Formula } & Mol. Wt. & Decomposition-point \\
Morphine picrolonate: & $\mathrm{C}_{17} \mathrm{H}_{19} \mathrm{NO}_{3} \cdot \mathrm{C}_{10} \mathrm{H}_{8} \mathrm{~N}_{4} \mathrm{O}_{5 .}$ & 549 & $200-210^{\circ}$ \\
Codeine picrolonate: & $\mathrm{C}_{18} \mathrm{H}_{21} \mathrm{NO}_{3} \cdot \mathrm{C}_{10} \mathrm{H}_{8} \mathrm{~N}_{4} \mathrm{O}_{5}$. & 563 & about $225^{\circ}$ \\
Cotarnine picrolonate: & $\mathrm{C}_{12} \mathrm{H}_{15} \mathrm{NO}_{4} \cdot \mathrm{C}_{10} \mathrm{H}_{8} \mathrm{~N}_{4} \mathrm{O}_{5}$. & 501 & $205^{-210^{\circ}}$
\end{tabular}

Notes.-Practice Analyses: Morphine powder: 0.01-0.02 gram morphine hydrochloride, $\mathrm{C}_{17} \mathrm{H}_{19} \mathrm{NO}_{3} . \mathrm{HCl}_{3} \mathrm{H}_{2} \mathrm{O}+0.5$ gram sugar. $0.2-0.5$ gram codeine phosphate, $\mathrm{C}_{18} \mathrm{H}_{21} \mathrm{NO}_{3} \cdot \mathrm{H}_{3} \mathrm{PO}_{4.2} \mathrm{H}_{2} \mathrm{O}+0.5$ gram sugar. Stypticine tablets $\mathrm{E}$. Merck.

Do not use too dilute solutions of the alkaloids in these determinations and do not wash the picrolonate precipitates with too much water. Dissolve the powdered morphine and sugar mixture in about 5-ro cc. of water. Matthes and Rammstedt in examining the morphine powder obtained the following results:

Weights taken: 0.019 morphine hydrochloride +0.5 gram sugar. Results obtained:

I. 0.0273 gram morphine picrolonate $=0.0187$ gram morphine hydrochloride.

II. 0.0274 gram morphine picrolonate $=0.0187$ gram morphine hydrochloride.

In a second experiment every ro cc. of an aqueous solution contained 0.0104 gram of morphine hydrochloride.

Results obtained:

I. 0.0147 gram morphine picrolonate $=0.0101$ gram morphine hydrochloride.

II. 0.0146 gram morphine picrolonate $=0.0099$ gram morphine hydrochloride. ${ }^{2}$

${ }^{1}$ Stypticine $=$ cotarnine hydrochloride, $\mathrm{C}_{12} \mathrm{H}_{15} \mathrm{NO}_{4} \cdot \mathrm{HCl} \cdot \mathrm{H}_{2} \mathrm{O}$.

${ }^{2}$ Professor Matthes has kindly stated that this picrolonate method gives less satisfactory results with smaller quantities of morphine (0.005 and less). 
The application of the picrolonate method to the estimation of hydrastine in hydrastis root and extract, of nux vomica alkaloids in nux vomica and extract and of pilocarpine in jaborandum leaves is described in Chapter VI (see pages 282, 296 and 286.

\section{Estimation of Alkaloids by Means of Potassium Bismuthous Iodide}

(H. Thoms ${ }^{1}$ )

Dissolve the particular alkaloid in sulphuric acid and precipitate completely with potassium bismuthous iodide prepared as described by Kraut. $^{2}$ Decompose the precipitate with a mixture of sodium carbonate and hydroxide, extract the free alkaloid with ether and weigh. By this method Thoms has recovered atropine, hyoscyamine, scopolamine, strychnine, quinine, caffeine and antipyrine from their potassium bismuthous iodide precipitates unaltered and nearly quantitatively. He has also used this method with success in estimating quantitatively the alkaloids in belladonna extract.

Procedure.-Dissolve the alkaloidal salt, or 2 grams of belladonna extract, in $50 \mathrm{cc}$. of water. Add first Iocc. of ro per cent. sulphuric acid, stir and precipitate with $5 \mathrm{cc}$. of potassium bismuthous iodide solution. Collect the precipitate upon a dry filter and wash twice with $5 \mathrm{cc}$. portions of ro per cent. sulphuric acid. Transfer the thoroughly drained precipitate and paper to a wide-mouth extraction cylinder having a tight glass stopper. Add 0.3 gram of sodium sulphite, then $30 \mathrm{cc}$. of 15 per cent. sodium hydroxide solution and shake. Add quickly 15 grams of sodium chloride and roo cc. of ether. Shake frequently and let stand for 3 hours. The ether contains the alkaloid and settles well. Remove with a pipette $50 \mathrm{cc}$. of the ether solution (= half the solution of the alkaloidal salt, or I gram of belladonna extract) and titrate this ether solution in a flask with $0.0 \mathrm{I}$ n-hydrochloric acid, using iodeosine as indicator.

After titrating the belladonna alkaloids, use in the calculation the equivalent weight of atropine-hyoscyamine, $\mathrm{C}_{17} \mathrm{H}_{23} \mathrm{NO}_{3}=$

1 Berichte der Deutschen pharmazeutischen Gesellschaft r3, 240 (r903); r 5,85 (r905); r6, r30 (rgo6) (D. Jonescu).

2 Annalen der Chemie und Pharmazie 2ro, 3 ro (1882). See "Preparation of Reagents," page 318. 
289. I000 cc. of $0.01 \mathrm{n}$-hydrochloric acid correspond to 2.89 grams of atropine-hyoscyamine. Atropine and hyoscyamine being isomeric, monacid bases, their formula weight and equivalent weight are the same.

Quinine, caffeine and antipyrine were also recovered unaltered from potassium bismuthous iodide precipitates. After decomposition of the precipitates, they were obtained almost quantitatively but were estimated gravimetrically.

Dissolve 2 grams of quinine ${ }^{1}$ in $50 \mathrm{cc}$. of water acidified with sulphuric acid and precipitate with potassium bismuthous iodide. Filter the precipitate with suction and wash with 5 per cent. sulphuric acid. Transfer precipitate and paper to an extraction cylinder and shake thoroughly with a mixture of 20 grams of crystallized sodium carbonate and $40 \mathrm{cc}$. of Io per cent. sodium hydroxide solution. The yellowish red precipitate gradually becomes white. Add $30 \mathrm{cc}$. of ether and shake well for 30 minutes. Pipette off $25 \mathrm{cc}$. of the clear ether solution, evaporate in a weighed glass dish, dry the residue at $100^{\circ}$ and weigh. The weight of quinine was 0.9405 instead of I gram.

Caffeine was estimated in the same way, except that this alkaloid was extracted with chloroform after decomposition of the potassium bismuthous iodide precipitate with alkaline hydroxide and carbonate. The weight of caffeine was 0.9546 instead of I gram.

The precipitate obtained by adding potassium bismuthous iodide to a solution of antipyrine in sulphuric acid (Io per cent. $\mathrm{H}_{2} \mathrm{SO}_{4}$ ) is not decomposed as easily as are those of quinine and caffeine. The precipitate from 2 grams of antipyrine must be shaken I hour with 20 grams of sodium carbonate and $60 \mathrm{cc}$. of to per cent: sodium hydroxide solution. Antipyrine must be extracted with chloroform. The weight of antipyrine was 0.9273 instead of I gram.

Notes.-Potassium bismuthous iodide precipitates fixed and volatile alkaloids but not ammonium salts. If the estimation of volatile bases is unnecessary, as in the examination of belladonna extract, evaporate the $50 \mathrm{cc}$. of ether extract (see above) upon the water-bath. Warm the residue and in a few minutes

${ }^{1}$ According to experiments of D Jones cu (loc. cit.). 
the strong narcotic odor of volatile bases will disappear. Dissolve the residue in a little acid-free alcohol and dilute with ether. Before using a flask for titrations carefully test it beforehand for alkalinity. If a positive test is obtained, alkalinity must be removed. An odor like iodoform, probably due to the action of sodium hypo-iodite upon the alkaloid, has been observed when sodium hydroxide solution acts upon potassium bismuthous iodide precipitates. Addition of sodium sulphite may prevent this action. After addition of sodium chloride ether takes up the alkaloid more readily. But vigorous shaking is always needed to cause complete transfer of alkaloid to the ether.

\section{Estimation of Alkaloids by H. M. Gordin ${ }^{1}$}

Gordin has found that periodides of the alkaloids, whatever be their composition, when precipitated from aqueous solution by iodo-potassium iodide in presence of acids, always contain one equivalent of combined acid for every molecule of monacid alkaloid. These periodides have the general formula (Alkaloid, $\mathrm{HI})_{\mathrm{m}} \mathrm{I}_{\mathrm{n}}$. Iodo-potassium iodide, added to a solution of a monacid alkaloid acidified with hydrochloric acid, first gives an alkaloid hydrochloride, changed by potassium iodide to alkaloid hydriodide and finally precipitated as insoluble periodide by taking up iodine:
( $\alpha$ ) Alkaloid
$+\mathrm{HCl}=$ Alkaloid. $\mathrm{HCl}$,
( $\beta$ ) Alkaloid. $\mathrm{HCl}+\mathrm{KI}=$ Alkaloid. $\mathrm{HI}+\mathrm{KCl}$,
$(\gamma) \mathrm{m}$ (Alkaloid.HI) $+\mathrm{I}_{\mathrm{n}}=$ (Alkaloid.HI) $\mathrm{mI}_{\mathrm{n}}=$ Precipitate.

In the precipitation of an alkaloid in acid solution with iodo-potassium iodide, one equivalent of acid goes with the precipitate and disappears from solution. In many cases potassium mercuric iodide may be substituted to advantage for iodo-potassium iodide. Gordin has found that the composition of the precipitate changes only as regards mercuric iodide and not as far as acid is concerned, for in this case also the precipitate contains one equivalent of acid for a monacid alkaloid.

Use in the titration $0.05 \mathrm{n}$-hydrochloric acid and $0.05 \mathrm{n}$-potassium hydroxide solution. Prepare a solution containing a weighed quantity of pure alkaloid, for example, pure morphine. Dissolve about 0.2 gram of chemically pure morphine, previously completely dehydrated at $120^{\circ}$, in $30 \mathrm{cc}$. of $0.05 \mathrm{n}$-hydrochloric acid in a $100 \mathrm{cc}$. volumetric flask. Shake and add gradually iodo-potassium iodide to this solution, until precipitation ceases and the supernatant liquid is dark red. Dilute to the roo cc. mark with water and shake vigorously until the liquid above the precipitate is entirely clear. Pass $50 \mathrm{cc}$. of solution through a dry filter, decolorize the filtrate with a few drops of sodium thiosulphate solution and titrate excess of $0.05 \mathrm{n}$-hydrochloric acid with $0.05 \mathrm{n}$-potassium hydroxide solution, using phenolphthalein as indicator. Calculate from the result how many grams of morphine have been neutralized by $\mathrm{I}$ cc. of the acid. Comparison of the equivalent weight of morphine with that of any other monacid alkaloid gives the corresponding factor to be used in the calculation. For example, Gordin found in his experiments that I cc. of approximately $0.05 \mathrm{n}$-hydrochloric acid neutralized

${ }^{1}$ Berichte der Deutschen chemischen Gesellschaft 32, 2871 (1899). Archiv der Pharmazie 238, 335 (1900). Gordin and A. B. Prescott, Archiv der Pharmazie 237,380 (r899). 
0.0137 gram of anhydrous morphine, $\mathrm{C}_{17} \mathrm{H}_{19} \mathrm{NO}_{3}$. The factor $(\mathrm{x})$ for strychnine, $\mathrm{C}_{21} \mathrm{H}_{22} \mathrm{~N}_{2} \mathrm{O}_{2}(=334)$, which is also a monacid base, is as follows:

Morphine : Strychnine

$$
285: 334=0.0137: x \quad(x=0.0160)
$$

and that for the monacid base cocaine, $\mathrm{C}_{17} \mathrm{H}_{21} \mathrm{NO}_{4}(=303)$, according to the proportion is:

$$
\begin{gathered}
\text { Morphine : Cocaine } \\
285
\end{gathered} 3^{\circ} 3=0.0137: x \quad(x=0.0146) .
$$

If potassium mercuric iodide is used to precipitate an alkaloid, the method is the same as with iodo-potassium iodide except that there is no need of treating the $50 \mathrm{cc}$. of filtered solution with thiosulphate.

Berberine and colchicin cannot be estimated by Gordin's method.

\section{Quantitative Estimation of Strychnine and Quinine Together}

E. F. Harrison and D. Gair ${ }^{1}$

Occasionally a small amount of strychnine must be estimated in presence of a relatively large quantity of quinine, as in certain pharmaceutical preparations. ${ }^{2}$ Separation of the two alkaloids is possible by means of Rochelle salt. Quinine tartrate, $\left(\mathrm{C}_{20} \mathrm{H}_{24} \mathrm{~N}_{2} \mathrm{O}_{2}\right)_{2} \cdot \mathrm{C}_{4} \mathrm{H}_{6} \mathrm{O}_{6.2} \mathrm{H}_{2} \mathrm{O}$, being difficultly soluble in water, forms a white crystalline precipitate, whereas strychnine tartrate remains in solution.

Procedure.-Render the solution of the mixed alkaloids in about 40 .cc. of water faintly acid with sulphuric acid. Add enough ammonia to cause a slight turbidity, then 5 grams of solid Rochelle salt and more ammonia, still leaving the liquid acid to litmus paper. Heat for $\mathrm{I}_{5}$ minutes upon the water-bath and then set aside for 2 hours until entirely cold. Filter precipitated quinine tartrate by suction and wash with aqueous Rochelle salt solution ( ${ }_{5}$ grams of salt in 45 cc. of water) containing $I^{-2}$ drops of dilute sulphuric acid. To determine strychnine, add sodium hydroxide solution to the combined filtrate and wash water from quinine tartrate until the reaction is alkaline. Extract 2-3 times with chloroform, pour the chloroform extract through a dry filter and distil in a weighed flask to about $4 \mathrm{cc}$. Add ro cc. of absolute alcohol and evaporate to dryness upon the water-bath. To remove quinine still adhering to the strycknine, extrast the dry residue $2-3$ times with I cc. portions of ether, ${ }^{3} \mathrm{dry}$ at $100^{\circ}$ and weigh. This residue consists of pure, quinine-free strychnine.

\section{Estimation of Toxicity of Chemical Compounds by Blood Hæmolysis}

\section{(A. J. J. Vandevelde ${ }^{4}$ )}

Vandevelde originally used living cells of a variety of Allium cepa (red Brunswick onion), the cell membrane of which is rich in anthocyan. The presence of this substance obviated the necessity of using a special coloring matter in determining plasmolytically the toxicity of alcohols, ethereal oils and other substances. ${ }^{5}$ Vandevelde has recently recommended determining the toxicity of chemical compounds by blood hæmolysis, using for this purpose defibrinated ox

1 Pharmaz. Journ. (4) I7, I 55.

${ }^{2}$ Compound Syrup of Hypophosphites, U. S. P.

${ }^{3}$ More ether dissolves a weighable quantity of strychnine.

${ }^{4}$ Chemiker Zeitung 29, 565 (1905).

- Bulletin de l'Association Belgée de Chimie r 7, 253. 
blood (see pages 229 and 230). To establish the toxicity of different alcohols, the concentration at which hæmolysis just ceases is determined. A solution, in which blood-corpuscles are not hydrolyzed after a definite time but are hydrolyzed upon addition of the slightest trace of the substance being examined, is a nontoxic solution for blood-corpuscles, called by Vandevelde a "critical solution."

The estimation requires:

I. A solution of 0.9 per cent. sodium chloride in 50 per cent. alcohol by volume. ${ }^{1}$

2. An aqueous 0.9 per cent. sodium chloride solution.

3. A suspension of 5 per cent. defibrinated ox blood in 0.9 per cent. aqueous sodium chloride solution.

Experiments are made in test-tubes. Place first in each of several tubes $2.5 \mathrm{cc}$. of the mixtures (of different concentration) of alcoholic and aqueous sodium chloride solution and then $2.5 \mathrm{cc}$. of the suspended blood. The end-point for the appearance of hæmolysis was set at three hours.

Vandevelde's experiments with ethyl alcohol gave the following results:

$\begin{array}{ccccc}\text { Cc. of sus- } & \begin{array}{c}\text { Cc. of alcoholic Cc. of aqueous } \\ \text { NaCl solu- } \\ \text { tion }\end{array} & \begin{array}{c}\text { Alcoholic con- } \\ \text { centration of } \\ \text { pended blood }\end{array} & \begin{array}{c}\text { After } 3 \\ \text { mixture in vol. } \\ \text { per cent. }\end{array} & \text { hours } \\ 2.5 & 2.20 & 0.30 & 22.0 & \text { Hæmolysis } \\ 2.5 & 2.15 & 0.35 & 21.5 & \text { Hæmolysis } \\ 2.5 & 2.10 & 0.40 & 21.0 & \text { Hæmolysis } \\ 2.5 & 2.05 & 0.45 & 20.5 & \text { Hæmolysis } \\ 2.5 & 2.00 & 0.50 & 20.0 & \text { Hæmolysis } \\ 2.5 & 1.95 & 0.55 & 19.5 & \text { No hæmolysis } \\ 2.5 & 1.90 & 0.60 & 19.0 & \text { No hæmolysis }\end{array}$

Consequently the critical solution of ethyl alcohol is one containing $19.5 \mathrm{cc}$. of absolute alcohol $\left(\mathrm{C}_{2} \mathrm{H}_{6} \mathrm{O}\right)$ in $100 \mathrm{cc}$., or 15.489 grams of $\mathrm{C}_{2} \mathrm{H}_{6} \mathrm{O}$ in $100 \mathrm{cc}$. The specific gravity of absolute alcohol being 0.7943, I 9.5 cc. weigh $19.5 \times 0.7943-$ I 5.489 .

According to Vandevelde's experiments addition of methyl alcohol diminished the toxicity of ethyl alcohol, whereas the higher alcohols were found to be more toxic than the latter. If the toxicity of 100 parts by weight of ethyl alcohol is taken as 100, then 47 parts by weight of isopropyl, 29 parts of isobutyl and 12.5 parts of amyl alcohol are isotoxic with that quantity of ethyl alcohol. Using the plasmolytic method with onion cells, Vandevelde obtained the following results with the same series:

$$
\text { I00, } 36.8,21.2 \text {, } 12.6 \text {. }
$$

The hæmolytic method is easily performed in test-tubes and does not require the use of the microscope. The form of the tube, especially its diameter, is quite important in these experiments. The speed of hæmolysis increases with the diameter of the tube. The quantity of the blood-corpuscles is of slight influence except in narrow tubes and at the beginning of the reaction. Vandevelde applies the term "critical coefficient" to the number giving the concentration of a substance necessary to kill the cells.

1 The specific gravity of such an alcohol at $15^{\circ}$ is 0.9348 . 


\section{CHAPTER VI}

\section{QUANTITATIVE ESTIMATION OF ALKALOIDS AND OTHER PRINCIPLES}

\section{Estimation of Alkaloids in Drugs and Pharmaceutical Preparations}

(German Pharmacopœia)

Alkaloids are nitrogenous bases occurring in plants. The term "plant base" is synonymous with alkaloid. The plant families especially rich in alkaloids are berberideæ, cinchonaceæ, papaveraceæ, solanaceæ and strychnaceæ. Alkaloids as a rule are not uniformly distributed in all parts of plants. They occur most often in roots, fruits and seeds. If the plant is a tree, there is often more alkaloid in the bark than in other parts. The particular part of the plant usually contains only a few per cent. of alkaloid. Quinine bark is an exception, the quantity of alkaloid being $5^{-10}$ per cent. and sometimes more. Plants as a rule do not contain free alkaloids but their salts. They are combined not only with the mineral acids, sulphuric, hydrochloric and possibly phosphoric, but with organic acids, as malic, aconitic, tannic, citric, quinic and meconic. Most free plant bases dissolve only slightly in water but readily in ether and chloroform. The German Pharmacopœia prescribes a mixture of ether and chloroform for the extraction of free alkaloids. The finely powdered drug should first be treated with a solution of sodium hydroxide, ammonia or sodium carbonate to liberate alkaloids from their salts:

$$
\begin{aligned}
& \mathrm{C}_{20} \mathrm{H}_{24} \mathrm{~N}_{2} \mathrm{O}_{2} \\
& \underset{\text { Quinine quinate }^{1}}{\mathrm{C}_{6} \mathrm{H}_{7}(\mathrm{OH})_{4} \mathrm{COOH}}+\underset{\text { Quinine }}{\mathrm{NaOH}}=\underset{{ }_{2} \mathrm{H}_{24} \mathrm{~N} \mathrm{O}_{2}}{\mathrm{NaOdium}_{4}}+\underset{\mathrm{C}_{6} \mathrm{H}_{7}(\mathrm{OH})_{4} \mathrm{COONa}}{\mathrm{C}_{2}}+\mathrm{H}_{2} \mathrm{O} \text {. }
\end{aligned}
$$

The ether-chloroform mixture removes not only alkaloids from the drug but varying amounts of other substances, as fat,

${ }^{1}$ Cinchona bark contains quinine in the form of this salt. 
resin, wax and pigments. To free the alkaloid from such impurities, shake the ether-chloroform extract with a measured excess of 0.1 or 0.01 n-hydrochloric acid. The alkaloid passes into aqueous solution as hydrochloride:

$$
\underset{\text { Quinine }}{\mathrm{C}_{20} \mathrm{H}_{24} \mathrm{~N}_{2} \mathrm{O}_{2}}+\mathrm{HCl}=\underset{\text { Quinine hydrochloride }}{\mathrm{C}_{20} \mathrm{H}_{24} \mathrm{~N}_{2} \mathrm{O}_{2} \cdot \mathrm{HCl}}
$$

But the impurities remain in the ether-chloroform mixture. Finally, determine excess of hydrochloric acid by titration with o.r or 0.0 I n-potassium hydroxide solution, employing usually iodeosine as indicator. Calculate the amount of alkaloid in the drug from the difference between the original quantity of acid and the excess.

The estimation of alkaloids in drugs and pharmaceutical preparations, according to directions given by the German Pharmacopœia, requires the following steps:

I. Liberation of alkaloids from salts by means of stronger bases, as potassium and sodium hydroxides, ammonia and sodium carbonate.

2. Extraction of free alkaloids with ether-chloroform mixture.

3. Transference of alkaloids from ether-chloroform to aqueous o.I or o.or n-hydrochloric acid solution.

4. Determination of excess of hydrochloric acid in an aliquot volume, usually $50 \mathrm{cc}$. of the hydrochloric acid solution of the alkaloid diluted to I00 cc., by titration with o.I or 0.0 I n-potassium hydroxide solution.

\section{Alkaloids in Aconite Root}

Officinal aconite root is the root of Aconitum Napellus collected at the end of flowering. Two alkaloids are present, namely, aconitine, $\mathrm{C}_{34} \mathrm{H}_{47} \mathrm{NO}_{11}$, and picraconitine, $\mathrm{C}_{31} \mathrm{H}_{47} \mathrm{NO}_{10}$ (?), characterized by its very bitter taste. Both alkaloids are combined with aconitic acid,

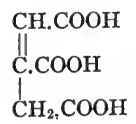


Boiled with water, or alcoholic potassium hydroxide solution, aconitine yields a new base, aconine, benzoic and acetic acids: ${ }^{1}$

$$
\underset{\text { Aconitine }}{\mathrm{C}_{34} \mathrm{H}_{47} \mathrm{NO}_{11}}+{ }_{2} \mathrm{H}_{2} \mathrm{O}=\underset{\text { Aconine }}{\mathrm{C}_{25} \mathrm{H}_{41} \mathrm{NO}_{9}}+\underset{\substack{\text { Acetic } \\ \text { acid }}}{\mathrm{C}_{4} \mathrm{O}_{2}}+\underset{\text { Benzoic acid }}{\mathrm{C}_{6} \mathrm{H}_{5} . \mathrm{COOH}}
$$

This reaction presents aconitine as acetyl-benzoyl-aconine,

$$
\mathrm{C}_{26} \mathrm{H}_{3} \mathrm{NO}_{3}<\begin{aligned}
& \mathrm{COCH}_{3} \\
& \mathrm{COC}_{6} \mathrm{H}_{5} .
\end{aligned}
$$

Since aconitine has been shown to contain four methoxyl groups, the formula of this alkaloid may be written:

$$
\mathrm{C}_{21} \mathrm{H}_{27}\left(\mathrm{OCH}_{3}\right)_{4} \mathrm{NO}_{5}<<\begin{aligned}
& \mathrm{COCH}_{3} \\
& \mathrm{COC}_{6} \mathrm{H}_{5}
\end{aligned}
$$

Aconine, therefore, is $\mathrm{C}_{21} \mathrm{H}_{27}\left(\mathrm{OCH}_{3}\right)_{4}(\mathrm{OH})_{2} \mathrm{NO}_{3}$; and picraconitine must be regarded as benzoyl-aconine, having the formula $\mathrm{C}_{21} \mathrm{H}_{21}\left(\mathrm{OCH}_{3}\right)_{4}(\mathrm{OH}) \mathrm{NO}_{4}\left(\mathrm{COC}_{6} \mathrm{H}_{5}\right)$.

\section{Estimation of Aconitine}

(German Pharmacopœia)

Place 12 grams of rather finely powdered aconite root dried at $100^{\circ}$ in an Erlenmeyer flask and add 90 grams of ether and 30 grams of chloroform. Shake well and add ro cc. of a mixture of 2 parts of sodium hydroxide solution and I part of water. Let the mixture stand 3 hours, shaking vigorously at frequent intervals. Then add ro cc. of water, or enough to cause the powder to gather into balls after vigorous shaking and leave the supernatant ether-chloroform solution perfectly clear. After an hour pass roo grams of the clear etherchloroform solution through a dry filter kept well covered and receive the filtrate in a small flask. Distil about half the solvent and pour the remainder into a separating funnel. Wash the flask with three $5 \mathrm{cc}$. portions of a mixture of 3 parts of ether and I part of chloroform, and thoroughly shake the combined solutions with $25 \mathrm{cc}$. of $0.01 \mathrm{n}$-hydrochloric acid. When the liquids have separated perfectly clear, add enough ether to bring the ether-chloroform solution to the surface. Pass the acid solution through a small filter moistened with water and collect the filtrate in a $100 \mathrm{cc}$. flask. Make three more extractions of the ether-chloroform solution with ro cc. portions of water, and pass these extracts through the same filter. Wash the latter with water and dilute the total solution with water to roo cc. Place $50 \mathrm{cc}$. of this solution in a $200 \mathrm{cc}$. flask with $50 \mathrm{cc}$ of water and enough ether to make a layer about $1 \mathrm{~cm}$. thick. Add 5 drops of iodeosine solution and run in, while shaking, enough $0.01 \mathrm{n}$-potassium hydroxide solution to turn the aqueous solution pale red.

1 Freund and Beck, Berichte der Deutschen chemischen Gesellschaft 27 , 433, 720 (I894); 28, I92, 2537 (1895). 
Calculation.-Dissolve the aconite alkaloids set free from their salts by sodium hydroxide solution in 120 grams of ether-chloroform. Weigh roo grams of this solution ( $=$ alkaloids from ro grams of aconite root). Dissolve the alkaloids with $25 \mathrm{cc}$. of $0.0 \mathrm{I} \mathrm{n-hydrochloric} \mathrm{acid,} \mathrm{bringing} \mathrm{the} \mathrm{volume} \mathrm{to} \mathrm{roo} \mathrm{cc.}$ Determine excess of acid in $50 \mathrm{cc}$. of this solution (= alkaloids from 5 grams of root). If, for example, this requires $8.5 \mathrm{cc}$. of $0.0 \mathrm{r}$-potassium hydroxide solution, then the alkaloids have combined with $\mathrm{I} 2.5-8.5=4 \mathrm{cc}$. of 0.0 . $\mathrm{n}$-acid. Since the equivalent weight of aconitine $\mathrm{C}_{34} \mathrm{H}_{47} \mathrm{NO}_{11}=645$, roo cc. of o.or $\mathrm{n}$-hydrochloric acid unite with 6.45 grams of aconitine. The proportion

$$
1000: 6.45=4: x \quad(x=0.0258)
$$

shows that 5 grams of aconite root contain 0.0258 gram of alkaloids, corresponding to 0.5 I per cent. The German Pharmacopoia demands this quantity of aconitine in aconite root as a minimum. Using a different method, the United States Pharmacopœia has the same limit.

\section{Estimation of Cantharidin in Spanish Flies}

\section{(German Pharmacopœia)}

Place 25 grams of Spanish flies ground mediumly fine in an Erlenmeyer flask and add roo grams of chloroform and 2 cc. of hydrochloric acid. ${ }^{1}$ Shake the mixture frequently during 24 hours. Then pour 52 grams of the chloroform solution through a dry filter kept well covered, and collect the filtrate in a weighed flask holding 80-roo cc. Distil the chloroform, and add $5 \mathrm{cc}$. of petroleum ether to the residue. Stopper the flask, and let the mixture stand $1_{2}$ hours, with occasional agitation. Dry at $100^{\circ}$ and weigh a filter $(5 \mathrm{~cm}$. in diameter). Pass the liquid through this filter, having first moistened it with petroleum ether. Treat the undissolved residue twice with petroleum ether, each time using ro cc. and shaking. Pass this solvent through the same filter, and disregard crystals adhering to the side of the flask. Dry the filter and flask, and wash both with a little water, containing a drop of ammonium carbonate solution to every ro cc., until this solvent is only faintly yellow. Finally, wash once with $5 \mathrm{cc}$. of water, and dry both flask and filter. Place filter and contents in the flask, and dry at $100^{\circ}$ to constant weight. The crystalline residue should weigh at least 0.1 gram.

Notes.-Additional information about cantharidin is given on page 203. Spanish flies contain cantharidin partly free and partly as an alkali salt of cantharidic acid (cantharidate). Hydrochloric acid sets cantharidic acid free and the latter then passes at once into cantharidin, its internal anhydride. Consequently hydrochloric acid is essential to the determination of that cantharidin present in Spanish flies as cantharidate. Chloroform not only dissolves cantharidin but fatty_substances

${ }^{1}$ Specific gravity I.I $24=25$ per cent. $\mathrm{HCl}$. 
in the flies. To isolate pure cantharidin from these impurities, distil the chloroform and let the residue stand for $\mathrm{I} 2$ hours in the cold with petroleum benzene. Fat readily dissolves but cantharidin is as good as insoluble in this solvent. The German Pharmacopœia finally directs weighing the cantharidin from I2.5 grams of powdered Spanish flies. The quantity should be at least 0.I gram, corresponding to 0.8 per cent. of cantharidin as a minimum. With sufficient care, white crystalline cantharidin may be isolated from Spanish flies.

Baudin obtained from good flies 1.06 per cent. of cantharidin, of which 0.72 per cent. was free and 0.34 per cent. combined as cantharidate. Dieterich found only 0.3 per cent. of free cantharidin.

\section{Estimation of Cinchona Alkaloids}

\section{(German Pharmacopœia)}

I. In Cinchona Bark.-To determine total alkaloids, pour 90 grams of ether and 30 grams of chloroform upon 12 grams of finely ground cinchona bark, dried at $100^{\circ}$ and placed in an Erlenmeyer flask. Add ro cc. of sodium hydroxide solution. Shake vigorously at frequent intervals during 3 hours. Then add ro cc. of water, or enough to cause the powdered cinchona to gather into lumps after vigorous shaking, thus leaving the supernatant ether-chloroform solution perfectly clear. Let the ether-chloroform solution stand an hour, and then pass roo grams through a dry filter, kept well covered. Collect the filtrate in a flask, and distil half the solvent. Pour the remaining ether-chloroform solution into a separating funnel, and wash the flask three times with $5 \mathrm{cc}$. portions of a mixture of 3 parts of ether and I part of chloroform. Thoroughly extract the total ether-chloroform solution with $25 \mathrm{cc}$. of $0.1 \mathrm{n}$-hydrochloric acid. When the contents of the separating funnel are perfectly clear, add enough ether to bring the ether-chloroform solution to the surface. Pass the acid solution through a small filter moistened with water, and receive the filtrate in a roo cc. flask. Make three more extractions of the ether-chloroform solution with ro cc. portions of water, and pass these extracts through the same filter. Wash the filter with water, and bring the volume of the filtrate to roo cc. Finally, measure $50 \mathrm{cc}$. of this solution with a pipette, and add freshly prepared hæmatoxylin solution, made by dissolving a small particle of this substance in I cc. of alcohol. Shake and add enough o.I n-potassium hydroxide solution to give the mixture a yellowish color, which quickly changes after vigorous agitation to bluish violet. ${ }^{1}$

Notes and Calculation.-Both quinine and quinidine have the formula $\mathrm{C}_{20} \mathrm{H}_{24} \mathrm{~N}_{2} \mathrm{O}_{2}$ and cinchonine and cinchonidine the

1 The German Pharmacopœia prescribes that not more than $4.3 \mathrm{cc}$. of 0.1 n-potassium hydroxide should be required. 
formula $\mathrm{C}_{19} \mathrm{H}_{22} \mathrm{~N}_{2} \mathrm{O}$. These are the most important alkaloids in cinchona bark. They are present in all true cinchona barks as salts of quinic acid, $\mathrm{C}_{17} \mathrm{H}_{12} \mathrm{O}_{6}$, and quino-tannic acid. Fuller information regarding the chemistry of quinine and cinchonine is given on page II 9 .

Quinic acid is widespread in the vegetable kingdom. This monobasic, pentatomic acid, having the formula, $\mathrm{C}_{6} \mathrm{H}_{7}(\mathrm{OH})_{4}$. $\mathrm{COOH}$, is a hexahydro-tetroxy-benzoic acid. It crystallizes in large monoclinic prisms melting at $162^{\circ}$. As far as the chemical behavior of quinic acid is concerned, either of the following formulas is possible:
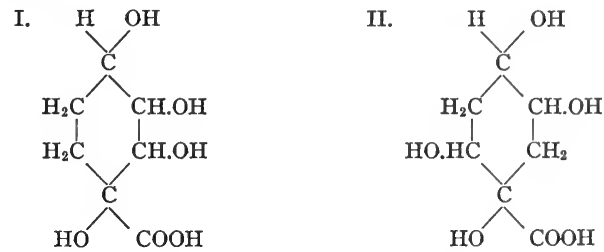

The formation of tetra-acetyl-quinic acid, $\left(\mathrm{CH}_{3} \mathrm{COO}\right)_{4}$ $\mathrm{C}_{6} \mathrm{H}_{7} \mathrm{COOH}$, and tetra-benzoyl-quinic acid, $\left(\mathrm{C}_{6} \mathrm{H}_{5} \mathrm{COO}\right)_{4} \mathrm{C}_{6} \mathrm{H}_{7}$ $\mathrm{COOH}$, shows that quinic acid contains four alcoholic hydroxyl groups. Addition of sodium hydroxide solution to cinchona powder sets the alkaloids free from their salts:

$$
\underset{\substack{\mathrm{C}_{20} \mathrm{H}_{24} \mathrm{~N}_{2} \mathrm{O}_{2} \\ \text { Quinine quinate }}}{\mathrm{H}_{7}(\mathrm{OH})_{4} \mathrm{COOH}}+\underset{\text { QuOHine }}{\mathrm{NaOH}_{20} \mathrm{H}_{24} \mathrm{~N}_{2} \mathrm{O}_{4}}+\mathrm{H}_{2} \mathrm{O}+\underset{\text { Sodium quinate }}{\mathrm{C}_{6} \mathrm{H}_{7}(\mathrm{OH})_{4} \mathrm{COONa}}
$$

Only 100 grams of the original $\mathrm{I} 20$ grams of ether-chloroform mixture (=I 2 grams of cinchona powder) are in the filtrate. This solution contains the alkaloids in to grams of bark. These roo grams are extracted with $25 \mathrm{cc}$. of $0.1 \mathrm{n}$-hydrochloric acid, the alkaloids passing into aqueous solution as hydrochlorides, and the volume is brought to roo cc. Finally, excess of o.I $\mathrm{n}$-hydrochloric acid in $50 \mathrm{cc}$. (= alkaloids in 5 grams of bark) of this hydrochloric acid solution is determined by titration. In these determinations with very dilute hydro- 
chloric acid, cinchona alkaloids behave as monacid bases, ${ }^{1}$ quinine forming $\mathrm{C}_{20} \mathrm{H}_{24} \mathrm{~N}_{2} \mathrm{O}_{2} \cdot \mathrm{HCl}$ and cinchonine $\mathrm{C}_{19} \mathrm{H}_{22} \mathrm{~N}_{2} \mathrm{O}$.$\mathrm{HCl}$.

The mean of the equivalent weight of quinine (324) and cinchonine (294), that is to say, $(324+294)$ divided by $2=309$, may be taken as the equivalent weight. This value agrees approximately with the actual quantities of these alkaloids in cinchona bark. Consequently rooo cc. of o.I n-hydrochloric acid are equivalent to 30.9 grams of cinchona alkaloids.

Example.-Titration of $50 \mathrm{cc}$. of the hydrochloric acid solution of alkaloids, in preparing which I $2.5 \mathrm{cc}$. of $0 . \mathrm{I} \mathrm{n}$-hydrochloric acid were used, required $2.6 \mathrm{cc}$. of $0.1 \mathrm{n}$-potassium hydroxide solution, equivalent to the volume of $0.1 \mathrm{n}$-hydrochloric acid in excess. I2.5-2.6 $=9.9 \mathrm{cc}$. of O.I $\mathrm{n}$-hydrochloric acid have combined with the alkaloids in 5 grams of cinchona bark. The proportion

Cc. O.I n-HCl:Grams of Alkaloids

$$
\text { I000 : } 30.9=9.9: x(x=0.3059 \mathrm{I})
$$

shows that 5 grams of bark contain 0.30591 gram of alkaloids. Consequently roo grams of bark contain $20 \times 0.3059 \mathrm{I}=6.1 \mathrm{I}$ grams of alkaloids.

Titrate the filtered ether-chloroform solution of cinchona alkaloids at once. The solution should not be exposed for any length of time to direct sunlight. Otherwise chloroform may give free hydrochloric acid

$$
\mathrm{CHCl}_{3}+\mathrm{O}=\mathrm{COCl}_{2}+\mathrm{HCl}
$$

which will neutralize alkaloids. The decomposition of 0.05 gram of chloroform would give enough hydrochloric acid to neutralize 0.25 gram of cinchona alkaloids. Panchaud ${ }^{2}$ has shown that such chloroform solutions of cinchona alkaloids after standing $\mathrm{I} 2$ hours yield only 80 per cent. of the total quantity of alkaloids originally present.

Hæmatoxylin, $\mathrm{C}_{16} \mathrm{H}_{14} \mathrm{O}_{6.3} \mathrm{H}_{2} \mathrm{O}$, occurs in logwood, the heart-wood of Hæmatoxylon campechianum. It usually crystallizes in colorless, shining, quadratic prisms containing 3 molecules of water, more rarely in rhombic crystals with I molecule of water. It dissolves only slightly in cold water but freely in boiling water, alcohol or ether. In contact with air hæmatoxylin gradually becomes reddish.

2. In Aqueous and Alcoholic Cinchona Extracts.-To determine total alkaloids in these preparations, dissolve 2 grams of the given extract in an Erlenmeyer flask, using 5 grams of water and 5 grams of absolute alcohol. Add 50 grams of

${ }^{1}$ Quinine dihydrochloride, $\mathrm{C}_{20} \mathrm{H}_{24} \mathrm{~N}_{2} \mathrm{O}_{2} \cdot 2 \mathrm{HCl}$, is formed by passing gaseous hydrogen chloride over quinine and also by dissolving the monohydrochloride, $\mathrm{C}_{20} \mathrm{H}_{24} \mathrm{~N}_{2} \mathrm{O}_{2} \cdot \mathrm{HCl}$, in strong hydrochloric acid with gentle heat. An aqueous solution of the dihydrochloride has an acid reaction.

${ }^{2}$ Schweizer Wochenschrift für Pharmazie $44,580$. 
ether and 20 grams of chloroform, and, after vigorous shaking, ro cc. of sodium carbonate solution $(\mathrm{r}: 3)$. Shake frequently, and let the mixture stand an hour. Then pass 50 grams of the ether-chloroform solution through a dry filter, kept well covered. Receive the filtrate in a flask, and distil half the solvent. Pour the remainder into a separating funnel, wash the flask three times with $5 \mathrm{cc}$. portions of a mixture of 3 parts of ether and I part of chloroform, and then shake the total ether-chloroform solution with ro cc. of o.I n-hydrochloric acid. When the contents of the separating funnel are perfectly clear, add enough ether to bring the ether-chloroform solution to the surface. Pass the acid solution through a small filter moistened with water, and receive the filtrate in a roo cc. flask. Make three more extractions of the ether-chloroform solution with ro cc. portions of water, and pass these extracts through the same filter. Wash the filter with water and bring the volume of the filtrate to roo cc. Finally, measure $50 \mathrm{cc}$. of this solution with a pipette, and add freshly prepared hæmatoxylin solution, made by dissolving a small particle of this substance in I cc. of alcohol. Shake and add enough o.I $\mathrm{n}$-potassium hydroxide solution to give the mixture a yellowish color, which quickly changes upon vigorous agitation to bluish violet.

Notes and Calculation.-The alkaloids in the two cinchona extracts are set free from their salts by sodium carbonate:

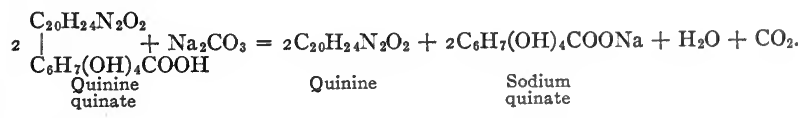

The alkaloids from 2 grams of extract are dissolved in 75 grams of alcohol-ether-chloroform mixture. Two-thirds of this solution, or 50 grams (= alkaloids in $\mathrm{I} .33$ grams of extract) are used in the determination. The free alkaloids in this portion pass into aqueous solution as hydrochloride, $\mathrm{C}_{20} \mathrm{H}_{24} \mathrm{~N}_{2} \mathrm{O}_{2}$.$\mathrm{HCl}$, upon extraction with Io cc. of o.I n-hydrochloric acid. The excess of hydrochloric acid in half of this solution diluted to $100 \mathrm{cc}$, that is to say, in $50 \mathrm{cc}$. (= alkaloids in $0.666 \mathrm{gram}$ of extract), is finally determined by titration. If $3.7 \mathrm{cc}$. of o.I n-potassium hydroxide solution are required, $5-3.7=$ I. 3 cc. of 0.1 n-hydrochloric acid have combined with the alkaloids in 0.666 gram of cinchona extract. The mean equivalent weight of quinine and cinchonine $(=309)$, used in the proportion:

$$
\text { I000: } 30.9=\mathrm{I} .3: \mathrm{x}(\mathrm{x}=0.04017)
$$

shows that I.3 cc. of $0.1 \mathrm{n}$-hydrochloric acid correspond to 0.04017 gram of alkaloids, or 6.03 per cent. The German 
Pharmacopœia demands this quantity as a minimum for the aqueous extract of cinchona bark.

In the determination of alkaloids in alcoholic cinchona extract, titration of excess of $0.1 \mathrm{n}$-hydrochloric acid in $50 \mathrm{cc}$. should not require more than $2.3 \mathrm{cc}$. of 0.1 n-potassium hydroxide solution. Then $5-2.3=2.7 \mathrm{cc}$. of o.I n-hydrochloric acid represent the alkaloids in this solution. This extract at the minimum must contain $\mathrm{r} 2.55$ per cent. of alkaloids.

\section{Sulphate Method of Estimating Quinine in Mixtures of Cinchona Alkaloids}

$(\mathrm{J} . \text { Carles })^{1}$

This method is especially recommended for practical purposes because of its accuracy and simplicity. Differences in the solubilities of the sulphates of cinchona alkaloids in ammonium sulphate solutions form the basis of the method. E. Schmidt has found that these sulphates have the following solubilities in water at $15^{\circ}$ :

Quinine sulphate $\mathrm{I}: 800$

Cinchonine sulphate $\mathrm{I}: 65$

Quinidine sulphate I : Io0

Cinchonidine sulphate $\mathrm{r}: 97$.

Guareschi has found quinine sulphate practically insoluble in an ammonium sulphate solution, a result which Hille ${ }^{2}$ has confirmed. An addition of 0.0078 gram to the quantity of quinine sulphate obtained is necessary on account of the quinine sulphate in the $20 \mathrm{cc}$. of wash water used.

I. Cinchona Bark.-Place I 2 grams of finely powdered cinchona bark dried at $100^{\circ}$ in an Erlenmeyer flask and add 90 grams of ether and 30 grams of chloroform. Add ro cc. of sodium hydroxide solution. Shake vigorously at frequent intervals for 3 hours. Then add ro cc. of water, or enough to cause the powdered cinchona to gather into balls after thorough shaking and leave the supernatant ether-chloroform layer perfectly clear. After I hour pass roo grams of the ether-chloroform solution through a dry filter kept well covered. Collect the filtrate in a dry weighed flask, distil the ether-chloroform and dry the flask at $110^{\circ}$ to constant weight. The increase in the weight of the flask corresponds to the total alkaloids in ro grams of bark.

${ }^{1}$ Zeitschrift für analytische Chemie 9, 467 (1870).

${ }^{2}$ W. Hille (Archiv der Pharmazie 24I, 54 (1903), has reviewed critically the various methods that have appeared thus far for the estimation of quinine in presence of other cinchona alkaloids. 
Warm the alkaloidal residue in the flask with water and dilute sulphuric acid and filter the solution. Wash the flask 3 times with water containing sulphuric acid and pour the wash water through the same filter. Dilute the filtrate to about $50 \mathrm{cc}$., heat to boiling and exactly neutralize with ammonia. Cool and after 6 hours collect upon a weighed filter, or better in a Gooch crucible, the flocculent precipitate of quinine sulphate, wash with $20 \mathrm{cc}$. of cold water, dry at $\mathrm{r} \mathrm{r}^{\circ}$ and weigh.

Add 0.0078 gram to the weight of quinine sulphate found and calculate the quantity of quinine in ro grams of cinchona bark as follows:

$$
\mathrm{C}_{20} \mathrm{H}_{24} \mathrm{~N}_{2} \mathrm{O}_{2} \cdot \mathrm{H}_{2} \mathrm{SO}_{4}: \underset{(646)}{\mathrm{C}_{20} \mathrm{H}_{24} \mathrm{~N}_{2} \mathrm{O}_{2}}=\text { Quinine sulphate }+0.0078: \mathrm{x} \text {. }
$$

2. Cinchona Extract.-Dissolve 3 grams of aqueous cinchona extract in 5 grams each of water and absolute alcohol and place in a measuring cylinder. Add $50 \mathrm{cc}$. of ether, ro cc. of chloroform and, after shaking vigorously, ro cc. of sodium carbonate solution $(\mathrm{r}: 3)$. Shake at frequent intervals during 3 hours. When two layers have formed, bring the ether layer to the $75 \mathrm{cc}$. mark with more ether. Rotate the container carefully and evaporate $50 \mathrm{cc}$. of the clear ether-chloroform layer in a dry weighed flask. Dry $\mathrm{r}$ hour at $105^{\circ}$ and weigh when cold. The increase in weight corresponds to the total alkaloids in 2 grams of cinchona extract. There should be at least 0.12 gram, or 6 per cent. of alkaloid.

To determine quinine, pour very dilute sulphuric acid over the weighed alkaloidal residue in the flask, warm and filter. Rinse the flask several times with very dilute sulphuric acid, bring the volume to about $50 \mathrm{cc}$. with water and proceed as directed above under cinchona bark. Collect the quinine sulphate in 2 hours upon a weighed filter, or Gooch crucible. The calculation is the same as for cinchona bark.

\section{Estimation of Colchicin in Colchicum Seed and Corms}

(J. Katz and G. Bredemann ${ }^{1}$ )

Exhaust colchicum seed or corms with 60 per cent. alcohol and evaporate 50 grams of this extract to $20 \mathrm{cc}$. Add 0.5 gram of solid paraffine and $20 \mathrm{cc}$. of water. Warm until the paraffine is melted and the alcohol has been completely expelled. Cool the liquid evaporated to ro- 5 cc. and pass through a moist filter. Melt the paraffine cake upon the water-bath with Io cc. of ro per cent. acetic acid and pour the cold liquid through the same filter. Wash the latter, the paraffine cake and the dish with water. Saturate the total filtrate with sodium chloride and extract first with $20 \mathrm{cc}$. of chloroform and then with ro cc. portions until a few drops of the aqueous liquid show scarcely any turbidity with 0.05 n-iodine solution. Pass the chloroform solution through a filter moistened with this solvent

1 Pharmazeutsche Zentral-Halle 42, 289 and Apotheker-Zeitung I8, 8I 7. 
and evaporate. To expel chloroform retained by the colchicin, dissolve the residue in a little water and filter. Evaporate the solution in a weighed dish and dry the residue over sulphuric acid to constant weight.

Note.-Using this method, Bredemann obtained the following quantities of colchicin:

$\begin{array}{lll}\text { In seed } & 0.46-0.13 \text { per cent. } \\ \text { In corms } & 0.032-0.06 \text { per cent. } \\ \text { In fresh flowers } & 0.6 & \text { per cent. } \\ \text { In dry flowers } & 1.8 & \text { per cent. }\end{array}$

\section{Alkaloids in Pomegranate Bark}

Pomegranate bark, the bark of Punica Granatum, contains the following four alkaloids:

Pelletierine, $\mathrm{C}_{8} \mathrm{H}_{15} \mathrm{NO}$, Isopelletierine, $\mathrm{C}_{8} \mathrm{H}_{15} \mathrm{NO}$,
Methyl-pelletierine, $\mathrm{C}_{9} \mathrm{H}_{17} \mathrm{NO}$, Pseudo-pelletierine, $\mathrm{C}_{9} \mathrm{H}_{15} \mathrm{NO}$.

According to Piccini there is still another alkaloid in the bark of pomegranate root isomeric with methyl-pelletierine and therefore called isomethyl-pelletierine.

Ciamician and Silber have determined the structure of pseudo-pelletierine which they call n-methyl-granatonine. Pseudo-pelletierine (I) is a ketone which, upon treatment with sodium amalgam or with sodium and alcohol, adds two atoms of hydrogen and passes into the corresponding secondary alcohol, n-methyl-granatoline (II). Chromic and sulphuric acids oxidize the latter to n-methyl-granatic acid (III). Nitrogen can be eliminated by exhaustive methylation and the final product is normal suberic acid (IV):

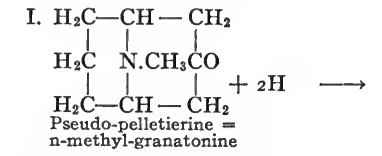

III. $\mathrm{H}_{2} \mathrm{C}-\mathrm{CH}-\mathrm{COOH}$
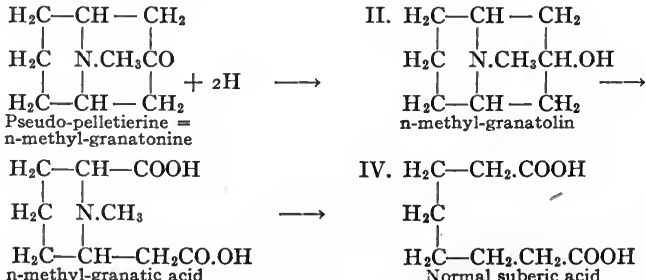

IV. $\mathrm{H}_{2} \mathrm{C}-\mathrm{CH}_{2} \cdot \mathrm{COOH}$
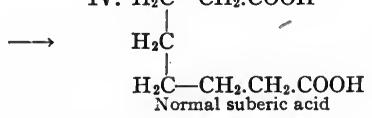


\section{Estimation of Alkaloid in Pomegranate Bark}

\section{(German Pharmacopœia)}

To determine total alkaloids, pour 90 grams of ether and 30 grams of chloroform upon 12 grams of rather finely ground pomegranate bark, dried at $100^{\circ}$ and placed in an Erlenmeyer flask. Shake vigorously and add ro cc. of a mixture of 2 parts of sodium hydroxide solution and I part of water. Let the mixture stand 3 hours, shaking vigorously at frequent intervals. Then add ro cc. of water, which will cause the powder to gather into balls after vigorous shaking, and leave the supernatant ether-chloroform solution perfectly clear. After an hour, pass roo grams of the clear ether-chloroform solution through a dry filter, kept well covered and receive the filtrate in a separating funnel. Extract this filtrate with $50 \mathrm{cc}$. of $0.0 \mathrm{I} \mathrm{n}$-hydrochloric acid and pass this acid solution, when perfectly clear, through a small filter moistened with water into a roo cc. flask. Make three more extractions with ro cc. portions of water, and pass these extracts through the same filter. Wash the filter with water and dilute the total solution with water to roo cc. Place $50 \mathrm{cc}$. of this solution in a $200 \mathrm{cc}$. flask with $50 \mathrm{cc}$. of water and enough ether to make a layer about $\mathrm{x} \mathrm{cm}$. thick. Add 5 drops of iodeosine solution and enough $0.01 \mathrm{n}$-potassium hydroxide solution, shaking vigorously after each addition, to give a pale red color to the lower aqueous solution.

Calculation.-The roo grams of filtered ether-chloroform solution correspond to ro grams of bark. The alkaloids are transferred from this ether-chloroform solution to $50 \mathrm{cc}$. of $0.0 \mathrm{r} \mathrm{n}$-hydrochloric acid which are diluted with water to roo cc. The excess of hydrochloric acid in $50 \mathrm{cc}$. of this solution (= alkaloids from 5 grams of bark) is determined by titration. If, for example, II cc. of 0.0 I n-potassium hydroxide solution are used, then 25 II $=14 \mathrm{cc}$. of $0.0 \mathrm{r}$ n-hydrochloric acid have combined with the alkaloids in $50 \mathrm{cc}$. of the solution. If the mean of the equivalent weights of pelletierine (I4I) and pseudo-pelletierine ( $\left.{ }_{53}\right)$, or $\mathrm{r}_{47}$, is used in the calculation, rooo cc. of o.or $\mathrm{n}$-hydrochloric acid neutralize r.47 grams of the mixed alkaloids. According to the proportion

$$
r 000: r .47=r_{4}: x\left(x=0.0205^{8}\right)
$$

5 grams of pomegranate bark contain $0.0205^{8}$ gram of alkaloids which corresponds to an alkaloid content of $20 \times 0.02058$, or $0.4 \mathrm{I}$ per cent. The German Pharmacopœia demands this quantity of alkaloids in pomegranate bark as a minimum.

\section{Estimation of Caffeine in Coffee, Tea, Cola Nuts and Guarana}

\section{(Literature)}

A. Hilger and A. Juckenack.-Zur Bestimmung des Kaffeins in Kaffee und Tee. Forschungsberichte über Lebensmittel und ihre Beziehungen zur $\mathrm{Hy}$ giene 4, 49-50; $\mathrm{C}^{1}$ I 897 I, 775 and also 4, r45-154 and $\mathrm{C}$ I $897 \mathrm{Il}, 233$.

H. Trillich and H. Göckel.-Beiträge zur Kenntniss des Kaffees und der Kaffeesurrogate. Forschungsberichte über Lebensmittel und ihre Beziehungen zur Hygiene 4, 78-88 and C r897 I, I 248.

${ }^{1} \mathrm{C}=$ Chemisches Zentralblatt. 
L. Graf.-Ueber Zusammenhang von Kaffeīngehalt und Qualität bei chinesischen Tee. Forschungsberichte ueber Lebensmittel und ihre Beziehungen zur Hygiene 4, 88-89, and C I 897 I, r 249.

A. Forster and R. Riechelmann.-Zur Bestimmung des Kaffeïns im Kaffee. Zeitschrift für öffentliche Chemie 3, I29-13I and $\mathrm{C}_{1897} \mathrm{I}$, I259.

C. C. Keller.-Die Bestimmung des Kaffeĩns im Tee. Berichte der Deutschen pharmaceutischen Gesellschaft 7, 105-II2 (I897) and C I897 I, II 34.

A. Forster and A. Riechelmann.-Zur Bestimmung des Kaffeỉns im Kaffee. (Entgegnung.) Zeitschrift für öffentliche Chemie $3,235^{-236}$ and $\mathrm{C}$ r897 II, 436 .

E. Tassily.-Ueber ein neues Verfahren zur Bestimmung des Kaffeins im Kaffee. Bulletin de la Société chimique, Paris, (3) $17,766-768$ and C 1897 11, 644 .

K. Dieterich.-Ueber die Werthbestimmung der Kolanuss und des Kolaextraktes. Vortrag auf der Naturforscherversammlung in Braunschweig gehalten. Pharmaceutische Zeitung 42, 647-650 and C 1897 II, 977.

H. Brunner and H. Leins.-Ueber die Trennung und quantitative Bestimmung des Kaffeïns und Theobromins. Schweizer Wochenschrift für Pharmacie $36,301-303$ and $\mathrm{C} 1898 \mathrm{II}, 5 \mathrm{I} 2$.

J. Gadamer.-Ueber Kaffeīnbestimmungen in Tee, Kaffee und Kola. Archiv der Pharmacie 237, 58-68 and $\mathrm{C}_{1} 899 \mathrm{I}, 713$.

E. Katz.-Ueber die quantitative Bestimmung des Kaffeïns. Berichte der Deutschen pharmaceutischen Gesellschaft I2, 250 (I902).

I. C. C. Keller's Method.-Pour 'I 20 grams of chloroform upon 6 grams of dry, unbroken tea leaves ${ }^{1}$ in a wide-mouth separating funnel. In a few minutes, add $6 \mathrm{cc}$. of ammonium hydroxide solution (Io per cent. $\mathrm{H}_{3} \mathrm{~N}$ ), and at frequent intervals shake vigorously during 30 minutes. Then let the separating funnel stand at rest, until the solution is perfectly clear and the tea leaves have absorbed all the water. This may require 3-6 hours, or even longer, depending upon the variety of tea. Pass 100 grams of clear chloroform extract, representing 5 grams of tea, through a small filter moistened with chloroform. Receive the filtrate in a small, weighed flask and distil the chloroform upon the water-bath. Pour 3-4 cc. of absolute alcohol over the residue. Heat upon the water-bath to remove alcohol and expel alcohol vapor with a hand bellows. In a few minutes

${ }^{1}$ When a wide-mouth separating funnel cannot be obtained, triturate the tea leaves somewhat, solely to facilitate their removal from the separating funnel after extraction. Finely powdered tea is not only unnecessary, but even objectionable, because the extracts have a much deeper color, and the yield of caffeine is not increased. 
the caffeine will be dry and at the same time free from imprisoned chloroform. In a measure also, this treatment with alcohol separates caffeine from extraneous chlorophyll. The latter adheres to the bottom and side of the flask, whereas caffeine forms a white incrustation upon it. Caffeine thus obtained is usually impure from small quantities of ethereal oil, fat, vegetable-wax and principally chlorophyll. Consequently, it must be purified. Set the flask upon a boiling waterbath and pour a mixture of $7 \mathrm{cc}$. of water and $3 \mathrm{cc}$. of alcohol over the crude caffeine, which upon being shaken will pass into solution almost immediately. Then add $20 \mathrm{cc}$. of water, stopper the flask and shake vigorously. The chlorophyll will form lumps and the solution will filter easily. Pass the caffeine solution through a small filter moistened with water, wash flask and filter with ro cc. of water, evaporate the total filtrate in a weighed glass dish to dryness upon the water-bath and weigh the residue of nearly pure caffeine. The weight of this residue multiplied by 20 will give the percentage of caffeine in the tea.

Notes.-Ammonia causes tea leaves to swell considerably, and at the same time combines with the tannic acid present. Caffeine is set free and dissolved by chloroform. The color of the chloroform extract depends upon the variety of tea. Black teas (Pekoe, Souchong and Congo) give clear, pale green to yellowish green solutions. Teas not so black, or green teas, give darker and more brownish green solutions. In assaying those varieties of tea, which probably contain a small quantity of caffeine, take I 2 grams and extract with I 50 grams of chloroform. C. C. Keller has shown that the best and most expensive varieties of tea contain most caffeine. The average percentage of caffeine, based upon 50 assays of tea, was found to be 3.06. A green tea gave the smallest yield, namely, I.78 per cent. of caffeine; and a Pekoe tea the highest yield, namely, 4.24 per cent. of caffeine.

J. Gadamer states that Keller's method of estimating caffeine in tea is applicable also to coffee and cola preparations. Keller's method is especially useful for roasted coffee. The caffeine, though somewhat brown, is aways sufficiently pure. 
2. Hilger-Juckenack Method.-Macerate 20 grams of finely ground coffee, or triturated tea, with 900 grams of water for several hours in a large beaker at room temperatures. Then boil thoroughly and replace the water lost by evaporation. Raw coffee requires 3 hours and roasted coffee and tea 1.5

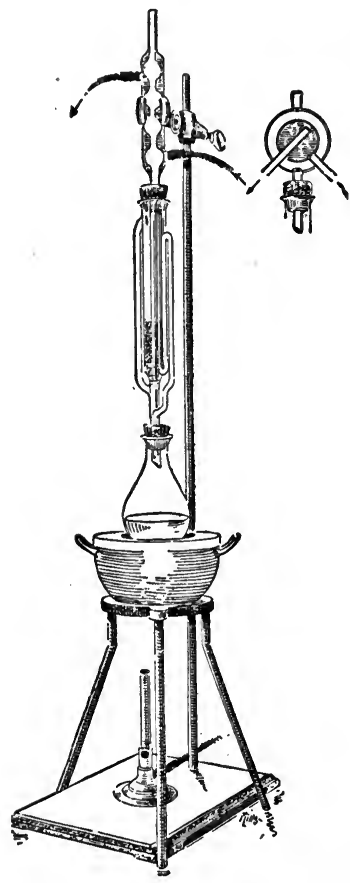

FIG. 23.-Soxhlet Apparatus. hours. Cool somewhat $\left(60\right.$ to $\left.80^{\circ}\right)$ and add 75 grams of aluminum acetate solution (see note, page 289) and gradually, while stirring, I.9 grams of acid sodium carbonate. Boil about 5 minutes and bring the total weight when cold to I020 grams. Filter 750 grams (= I 5 grams of original material) and add to the clear filtrate ro grams of precipitated and powdered aluminum hydroxide and some filter paper made into a magma by agitation with water. Stir frequently and evaporate upon the water-bath. Thoroughly dry the residue in an air-closet at I00, and extract for 8-ro hours with pure tetrachloromethane $\left(\mathrm{CCl}_{4}\right)$, using a Soxhlet apparatus (Fig. 23). Tetrachloromethane, which is always colorless, is finally distilled and the residue of perfectly white caffeine is dried at $100^{\circ}$ and weighed. The results thus obtained are usually accepted without question. But if an absolutely accurate result is required, nitrogen in the crude caffeine may be determined by Kjeldahl's method. The quantity of anhydrous caffeine is calculated on the basis of this analysis. One cc. of o.I n-oxalic acid represents 0.00485 gram of anhydrous caffeine. Commercial tetrachloromethane is usually impure and cannot be used 
directly in the extraction. It should be shaken 3-4 times with sodium carbonate solution, then several times with water, dried over fused calcium chloride and distilled fractionally. It boils at $76-77^{\circ}$.

\section{Trillich-Goeckel Modification of Hilger's Method.-} Exhaust to grams of finely ground coffee with water. This will require 3 extractions with boiling water, using $200 \mathrm{cc}$. portions and heating each for 30 minutes. Combine the filtered extracts, cool and dilute to $495 \mathrm{cc}$. Add $5 \mathrm{cc}$. of basic lead acetate solution, shake thoroughly, filter $400 \mathrm{cc}$. and pass hydrogen sulphide through the filtrate. Dilute this filtrate to $500 \mathrm{cc}$., shake thoroughly and again filter $400 \mathrm{cc}$. This filtrate will represent 6.4 grams of coffee. Concentrate this filtrate $(400$ cc.) upon the water-bath, and, after addition of I gram of magnesium oxide and sand, evaporate to dryness. Triturate the residue and extract for 30 hours with acetic ether, using a Soxhlet apparatus. Evaporate the acetic ether extract in a $\mathrm{Kjeldahl} \mathrm{flask,} \mathrm{or} \mathrm{distil} \mathrm{the} \mathrm{solvent,} \mathrm{and} \mathrm{determine} \mathrm{nitrogen} \mathrm{in}$ the residue by Kjeldahl's method. One gram of nitrogen represents 3.4643 grams of caffeine. Crude caffeine is easily decomposed by the acid used in the Kjeldahl process and by mercuric oxide. Roasted coffee may easily give too much caffeine by this method, because the bases formed by roasting coffee, pyridine for example, are also extracted by acetic ether.

4. Trillich-Goeckel Modification of Socolof's Method.Put to grams of finely ground, dried coffee into a separating funnel, provided with a plug of glass wool for a filter, and moisten with ammonium hydroxide solution. Let the mixture stand for 30 minutes, extract for 12 hours with $200 \mathrm{cc}$. of acetic ether and shake frequently. Filter, and wash three times with $50 \mathrm{cc}$. portions of acetic ether. Distil the acetic ether upon the water-bath and boil the residue with milk of magnesia. Filter, and evaporate the filtrate to dryness upon the water-bath. Dissolve the residual caffeine in acetic ether or chloroform. Filter this solution into a weighed dish or Kjeldahl flask. Evaporate the solvent and weigh the caffeine, or calculate it from the percentage of nitrogen. The latter method is the more 
accurate. According to $\mathrm{C}$. Wolf ${ }^{1}$ the residue from the acetic ether or chloroform extract should not be accepted as pure caffeine. Determination of nitrogen in this residue by Kjeldahl's method is the most reliable way of estimating caffeine in the extract.

5. E. Katz's Method of Estimating Caffeine.-This method is based upon the fact that chloroform will extract caffeine quantitatively from a solution which is ammoniacal, or faintly acid with hydrochloric acid.

Shake ro grams of powdered coffee, or tea, for 30 minutes with 200 grams of chloroform and 5 grams of ammonium hydroxide solution. When the liquid has settled, filter I50 grams of the chloroform solution through a Sander's filter which will give a perfectly bright filtrate free from water. Distil the chloroform completely and dissolve the residue with gentle heat in about $6 \mathrm{cc}$. of ether. Add $20 \mathrm{cc}$. of 0.5 per cent. hydrochloric acid and, in an assay of coffee, also $0.2-0.5$ gram of solid paraffine. Evaporate the ether and filter the cold, aqueous solution. Wash the flask and filter paper a few times with small portions of 0.5 per cent. hydrochloric acid. Finally extract the total aqueous hydrochloric acid solution four times with $20 \mathrm{cc}$. portions of chloroform. Distil the filtered chloroform extract, dry the residue and weigh. This residue will consist of nearly pure caffeine. J. Katz found the following percentages of caffeine:

Caffeine

Raw Coffee Beans........ 0.9-1.27 per cent.

Dried Cola Nuts......... I.5I-I.94 per cent.

Black Tea........... 2.5 $1-3.56$ per cent.

Guarana............ 2.83-4.74 per cent.
Average

I.I4 per cent.

I.68 per cent.

3.07 per cent.

. 4.08 per cent.

J. Katz recommends the following method for estimating caffeine in maté or Paraguay tea:

Treat finely triturated tea with ammonium hydroxide solution and chloroform, as described above, and dissolve the chloroform residue in ether. Add water to the ether and evaporate. Warm the aqueous solution ro minutes upon the water-bath

${ }^{1}$ Zeitschrift für öffentliche Chemie I2, I86. 
with 2 cc. of lead hydroxide suspended in water ( $1: 20$ ). If it is very difficult to get a clear filtrate from this liquid, add a little calcined magnesium oxide. This treatment usually gives a filtrate, which is perfectly clear when cold, and but slightly colored. Chloroform extracts quite pure caffeine from this solution. By this method maté yields $0.3-1.6$ per cent. of caffeine, the average being 0.7 I per cent.

\section{K. Dieterich's Method of Estimating Total Alkaloids} (Caffeine and Theobromine) in Cola Nuts.-Moisten ro grams of finely grațed cola nuts with a little water, mix with io grams of granulated, unslaked lime and extract with chloroform in a Soxhlet apparatus for 45 minutes. Evaporate the extract almost to dryness and dissolve the residue with gentle heat in $20 \mathrm{cc}$. of normal hydrochloric acid. Filter and dilute to roo cc. Add ammonium hydroxide solution in large excess to this filtrate, shake at frequent intervals during 15 minutes and extract three times with $20 \mathrm{cc}$. portions of chloroform. Evaporate this chloroform solution in a weighed flask and dry the residue, which usually consists of perfectly pure caffeine, at $100^{\circ}$ to constant weight.

This method may also be used in estimating caffeine in Paraguay tea. Mix the finely ground material with unslaked lime, and extract with chloroform, in a Soxhlet apparatus. Tea gives pure, white caffeine free from chlorophyll.

\section{Estimation of Alkaloids in Ipecac}

Ipecac has been shown ${ }^{1}$ to contain three alkaloids:

Cephaeline, $\mathrm{C}_{28} \mathrm{H}_{40} \mathrm{~N}_{2} \mathrm{O}_{4}$, Emetine, $\mathrm{C}_{30} \mathrm{H}_{44} \mathrm{~N}_{2} \mathrm{O}_{4}$, Psychotrine.

The composition of the last alkaloid is unknown. This drug acts as an expectorant and emetic, because of cephæline and emetine. Psychotrine is said not to possess thèse properties. Therefore, in assaying ipecac for medicinal purposes, only the percentage of the first two alkaloids need be estimated. The equivalent weights of these two alkaloids (cephæline 234 and

${ }^{1}$ Frerichs and de Fuentas Tapis, Archiv der Pharmacie, 1902, Heft 5 and 6. 
emetine 248) are so nearly the same, that the mean of the two (24I) may be used as the factor.

Procedure.-Put 6 grams of finely powdered root in a dry Erlenmeyer flask and shake with 60 grams of ether. Then add $5 \mathrm{cc}$. of ammonium hydroxide solution, or $5 \mathrm{cc}$. of sodium carbonate solution ( $1: 3$ ), and shake frequently during an hour. Add to cc. of water and, after shaking vigorously, filter 50 grams of the ether extract into a small flask. Evaporate half the ether upon the water-bath, and extract the remainder in a separating funnel with ro cc. of o.I n-hydrochloric acid. Pass the acid solution through a small filter into a $200 \mathrm{cc}$. flask. Make two more extractions of the ether with ro cc. portions of water, and pass these through the same filter. Bring the volume of the acid solution to roo cc., and then add enough ether to form a layer about I $\mathrm{cm}$. thick after thorough agitation. Add 5 drops of iodeosine solution ( $1: 250$ ), and titrate excess of hydrochloric acid with o.I n-potassium hydroxide solution. The number of cc. of o.I n-hydrochloric acid, combined with the alkaloids, multiplied by $0.024 \mathrm{I}$ gives the quantity of emetine and cephæline in 5 grams of ipecac.

To estimate these alkaloids gravimetrically, shake vigorously the ether solution of the alkaloids ( $5 \circ$ grams $=5$ grams of root) in a separating funnel with $5 \mathrm{cc}$. of dilute hydrochloric acid and Io cc. of water. Transfer the acid solution to another separating funnel. Make two more extractions of the ether with ro cc. portions of water and add these to the acid extract. Add 5 cc. of ammonium hydroxide solution to the acid extract and shake vigorously with 50 grams of ether. Remove the aqueous layer and filter 40 grams of the ether solution into a weighed flask. Evaporate the ether and weigh the flask after drying for an hour at $100^{\circ}$. This will give the quantity of emetine and cephæline in 4 grams of root.

Test for Cephæline.- This reaction is very characteristic of this alkaloid. Froehde's reagent dissolves pure cephæline, as the free base, almost without color. A trace of hydrochloric acid, or better sodium chloride, added to this solution produces an intense blue color. Pure emetine gives no color with 
Froehde's reagent, nor when sodium chloride is added. This test for cephæline may be made with the ether residue.

The method of estimating alkaloids in ipecac, prescribed by the German Pharmacopœia, is the same as that for cinchona bark. Use I 2 grams of finely powdered root dried at $100^{\circ}$, but in ascertaining excess of acid use iodeosine, and not hæmatoxylin, as the indicator. Finally, measure with a pipette 50 cc. of the proper solution having a volume of roo cc., place in a $200 \mathrm{cc}$. flask and add about $5 \circ \mathrm{cc}$. of water and enough ether to make a layer $\mathrm{r} \mathrm{cm}$. thick. Add 5 drops of iodeosine solution and enough $0.01 \mathrm{n}$-potassium hydroxide solution, shaking thoroughly after each addition, to give the lower aqueous layer a pale red color. This should require not more than $20 \mathrm{cc}$. of alkaline solution.

\section{Estimation of Nicotine in Tobacco}

I. R. Kissling's ${ }^{1}$ Method.-First remove the ribs and then cut the tobacco leaves into small pieces. Dry $\mathrm{I}-2$ hours $\left(50-60^{\circ}\right)$, and then reduce to a uniform, coarse powder. Triturate 20 grams of this powder with ro cc. of dilute, alcoholic sodium hydroxide solution (6 grams of sodium hydroxide dissolved in $40 \mathrm{cc}$. of water and $60 \mathrm{cc}$. of 95 per cent. alcohol). Transfer this moist powder to a paper thimble and extract 2-3 hours with ether in a Soxhlet apparatus. Carefully distil the ether solution so that a portion of the solvent remains. Add $50 \mathrm{cc}$. of very dilute sodium hydroxide solution (4 grams of sodium hydroxide in I000 cc. of water) to the residue.and distil with steam. Begin introducing steam after the nicotine solution has been boiling several minutes. Collect about $400 \mathrm{cc}$. of distillate, that is to say, continue distilling until the distillate is no longer alkaline. Mix well, add a few drops of rosolic acid solution to the distillate, and titrate nicotine with $0.1 \mathrm{n}$-sulphuric or oxalic acid until the red color has just disappeared.

Calculation.-Although nicotine, $\mathrm{C}_{10} \mathrm{H}_{14} \mathrm{~N}_{2}$ ( ${ }^{6} 62$ ), as a di-acid base can combine with two equivalents of acid, it behaves upon titration, with rosolic acid or iodeosine as indicator, as if it were a monacid base with the equivalent weight I62. I000 cc. of 0.1 n-acid consequently correspond to 16.2 grams of nicotine.

2. C. C. Keller's ${ }^{2}$ Method.-Pour 60 grams of ether and 60 grams of petroleum ether over 6 grams of dry tobacco in a 200

${ }^{1}$ Zeitschrift für analytische Chemie $34, \mathrm{r} 73 \mathrm{I}$ and $2 \mathrm{r}, 76$.

${ }^{2}$ Berichte der Deutschen pharmazeutischen Gesellschaft 8, 145 (r898). 
cc. Erlenmeyer flask. Add ro cc. of 20 per cent. aqueous potassium hydroxide solution and let the mixture stand half an hour, shaking vigorously at frequent intervals. After the liquid has stood at rest $3-4$ hours, pour roo grams of ether solution through a small, plaited filter and receive the filtrate in a $200 \mathrm{cc}$. Erlenmeyer flask. Nicotine is in solution together with a little ammonia, which must be removed before titration. By means of a hand bellows and a glass tube reaching to the bottom of the flask force a current of air through the solution, so that there is considerable agitation. It requires about a minute and a half to expel all ammonia. At the same time 8-Io grams of ether evaporate. Add ro cc. of alcohol, a drop of I per cent. iodeosine solution and ro cc. of water to the ammoniafree solution. Stopper the flask and shake vigorously. Nicotine and iodeosine dissolve in the water which has a red color. Add a slight excess of o.I n-hydrochloric acid, enough to discharge the color, and titrate excess of acid with o.I n-potassium hydroxide solution. The quantity of nicotine in tobacco shows a wide variation and ranges from 0.6 to 4.8 per cent.

3. J. Toth's ${ }^{1}$ Method.-According to Toth two sources of error in C. C. Keller's method lead to low results. An aqueous potassium hydroxide solution retains variable quantities of nicotine and a current of air passed through an ether solution of nicotine volatilizes some of this alkaloid. Therefore Toth recommends the following procedure:

Mix 6 grams of air-dried tobacco with Io cc. of 20 per cent. sodium hydroxide solution in a porcelain dish. Add gypsum until the mixture is like powder. Extract thoroughly with roo cc. ether-petroleum ether mixture (I:I) and after I hour pipette off as quickly as possible $25 \mathrm{cc}$. of the solvent. Add 40-50 cc. of water, I drop of iodeosine solution and an excess of 0.1 n-sulphuric acid. Determine excess of acid by titration with o.I n-sodium hydroxide solution. The ether-petroleum ether mixture takes up at most 0.0005 gram of ammonia.

${ }^{1}$ Chemisches Zentralblatt, I90I, I, 973. 


\section{Estimation of Hydrastine in Fluid Extract of Hydrastis}

(German Pharmacopœia)

Evaporate I 5 grams of fluid extract of hydrastis to about 5 grams in a weighed dish upon the water-bath, and wash the residue into an Erlenmeyer flask with about Io cc. of water. Add ro grams of petroleum ether, 50 grams of ether and 5 grams of ammonium hydroxide solution. Let the mixture stand an hour, shaking vigorously at frequent intervals. Then pass 50 grams of the clear ether solution through a dry filter into a separating funnel. Add ro cc. of a mixture, composed of I part of hydrochloric acid and 4 parts of water, and shake the solution vigorously several minutes. When the liquids have separated clear, run the acid solution isto an Erlenmeyer flask. Make two more extractions of the ether with 5 cc. portions of water containing a few drops of hydrochloric acid, and add these to the first extract. Add to the total extract excess of ammonium hydroxide solution and 50 grams of ether. Let the mixture stand an hour, shaking vigorously at frequent intervals. Pass 40 grams of the clear ether solution through a dry filter and collect the filtrate in a weighed, dry flask. Distil the ether, dry the residue at $100^{\circ}$ and weigh when cold. The residue should weigh at least 0.2 gram.

Notes.-Additional information about hydrastine is given on page ri6. Ammonia, added to an aqueous solution of the residue from hydrastis extract ( 5 grams), sets the alkaloids, hydrastine and berberine, free from their salts. The etherpetroleum benzine mixture dissolves hydrastine but not berberine, the latter being nearly insoluble in this mixed solvent. But phytosterin, which is always present in hydrastis extract, is dissolved. Only 50 grams (= hydrastine in 12.5 grams of extract) of the original 60 grams of ether-petroleum benzine mixture are used. Hydrastine is extracted from the solvent by agitation with dilute hydrochloric acid and dissolved in the acid solution as hydrochloride. The alkaloid is then precipitated from the acid solution by ammonia and dissolved in $5^{\circ}$ grams of ether:

$$
\underset{\substack{\text { Hydrastine } \\ \text { hydrochloride }}}{\mathrm{C}_{21} \mathrm{H}_{21} \mathrm{NO}_{6} \cdot \mathrm{HCl}}+\left(\mathrm{H}_{4} \mathrm{~N}\right) \mathrm{OH}=\mathrm{C}_{21} \mathrm{C}_{21} \mathrm{H}_{21} \mathrm{NO}_{6}+\mathrm{H}_{2} \mathrm{O}+\left(\mathrm{H}_{4} \mathrm{~N}\right) \mathrm{Cl} \text {. }
$$

The hydrastine in 40 grams of the ether solution (= Io grams of original extract) is finally weighed. Good extract of hydrastis should contain $2-2.5$ per cent. of hydrastine.

When the ether-petroleum benzine solution of hydrastine and phytosterin is extracted with dilute hydrochloric acid, the alkaloid passes into the acid solution free of phytosterin. 
Estimation of Berberine.-This alkaloid has only a slight physiological action. To determine approximately the quantity present in hydrastis extract, add 20 grams of dilute sulphuric acid ( $\mathrm{r}: 5)$ to ro grams of the extract and let the mixture stand for 24 hours at as low a temperature as possible. Crystallization of berberine as the difficultly soluble acid sulphate, $\mathrm{C}_{20} \mathrm{H}_{17} \mathrm{NO}_{4} \cdot \mathrm{H}_{2} \mathrm{SO}_{4}$, is almost complete. Filter in a Gooch crucible with suction, washing. first with a little water containing sulphuric acid and then with pure water. Dry at $100^{\circ}$ to constant weight. (E. Schmidt.)

W. Meine $\mathrm{e}^{\mathrm{l}}$ has found that the crystalline deposit, frequently seen in hydrastis extract, consists mostly of berberine mixed with a little phytosterin. This deposit is said to contain only traces of hydrastine.

\section{Picrolonate Method of Estimating Hydrastine in Hydrastis Root and Extract}

(H. Matthes and O. Rammstedt) ${ }^{2}$

The German Pharmacopœia requires the estimation of hydrastine but not of the physiologically inert substances, berberine and phytosterin, also present in hydrastis preparations. Ether-petroleum benzine mixture, used as a solvent, dissolves phytosterin and hydrastine but not berberine. Dilute hydrochloric acid extracts hydrástine but leaves phytosterin in the ether mixture.

Estimation of hydrastine by means of picrolonic acid appears simpler than by the method of the Pharmacopœia, because picrolonic acid does not precipitate phytosterin and therefore hydrastine is not mixed with this impurity. Matthes and Rammstedt obtained nearly pure hydrastine picrolonate from hydrastis extract, melting at $220-225^{\circ}$. The picrolonate prepared from pure hydrastine, $\mathrm{C}_{21} \mathrm{H}_{21} \mathrm{NO}_{6} \cdot \mathrm{C}_{10} \mathrm{H}_{8} \mathrm{~N}_{4} \mathrm{O}_{5}$, melts at $225^{\circ}$.

I. In Fluid Extract of Hydrastis.-Evaporate I 5 grams of fluid extract to about 5 grams in an Erlenmeyer flask upon the water-bath. Add Io cc. of water; ro grams of petroleum benzine, $5 \circ$ grams of ether and 5 grams of ammonium hydroxide (Io per cent. $\mathrm{NH}_{3}$ ). Shake vigorously for Io minutes. After the mixture has stood for 20 minutes, pour 40 grams of the ether-

1 Zeitschrift des allgemeinen österreichischen Apotheker-Vereins 55, 494.

${ }^{2}$ Further information about picrolonic acid and its use in precipitating alkaloids is given on page 253 . 
benzine extract through a double, creased filter and evaporate about one-half in a beaker. Then add ro cc. of o.I n-picrolonic acid solution. After 24 hours collect the hydrastine picrolonate in a weighed Gooch crucible, wash with $2 \mathrm{cc}$. of an alcohol-ether mixture ( $1: 3$ ), dry for 30 minutes at $105^{\circ}$ and weigh.

2. In Hydrastis Root.-Shake 6 grams of powdered root vigorously for 30 minutes with 50 grams of ether, ro grams of petroleum ether and 6 grams of ammonium hydroxide (Io per cent. $\mathrm{NH}_{3}$ ). Then add 6 grams of water and shake until the upper layer of liquid is clear. Quickly filter 50 grams of the ether-petroleum benzine extract and evaporate about one-half in a beaker. Then add $5 \mathrm{cc}$. of o.I n-picrolonic acid. After 24 hours filter the picrolonate precipitate and wash with I cc. of alcohol-ether mixture $(I: 3)$. Otherwise the procedure is the same as described in $\mathrm{I}$.

In the calculation use the formula of hydrastine picrolonate (Mol. Wt. 647) given above.

\section{Estimation of Morphine in Opium and Pharmaceutical Preparations}

\section{(German Pharmacopœia)}

In Opium.-Triturate 6 grams of rather finely powdered opium with 6 grams of water. Wash the mixture into a weighed, dry flask with water and add enough more of this solvent to bring the weight to 54 grams. Shake frequently and let the mixture stand an hour. Pour upon a piece of dry linen and express the liquid. Pass 42 grams of this extract through a dry, plaited filter (10 cm. in diameter) into a dry flask. Add 2 grams of sodium salicylate solution $(1: 2)$ to this filtrate and shake vigorously. Filter 36 grams of the clear solution through a dry, plaited filter (ro cm. in diameter) into a small flask. Mix this filtrate by gentle agitation with ro grams of ether, and add also 5 grams of a mixture consisting of I 7 grams of ammonium hydroxide solution and 83 grams of water. * Stopper the flask, shake vigorously for ro minutes and let the mixture stand at rest 24 hours. Then decant the ether layer as completely as possible upon a smooth filter ( $8 \mathrm{~cm}$. in diameter). Add ro grams more of ether to the residual, aqueous liquid in the flask, shake gently for a few minutes and again pour the ether layer upon the filter. Then after all the ether solution has passed through, pour the aqueous solution upon the filter, and disregard crystals adhering to the side of the flask. Wash filter and flask three times with $5 \mathrm{cc}$. portions of water saturated with ether. When the filter has drained thoroughly, dry the morphine crystals and dissolve in $25 \mathrm{cc}$. of $0.1 \mathrm{n}$-hydrochloric acid. Pour this solution into a roo cc. flask, carefully wash filter and flask with water and finally dilute the solution to $100 \mathrm{cc}$. Measure $50 \mathrm{cc}$. of this solution into a $200 \mathrm{cc}$. flask, add 
$50 \mathrm{cc}$. of water and enough ether to form a layer $\mathrm{x} \mathrm{cm}$. thick. Add 5 drops of iodeosine solution and enough o.I n-potassium hydroxide solution, shaking vigorously after each addition, to produce a pale red color in the lower aqueous layer. ${ }^{1}$

Notes and Calculation.-Most of the opium alkaloids are combined with meconic (see page $2 \mathrm{1} 3$ ) and sulphuric acids. Ammonium hydroxide, added to an aqueous opium extract, sets the alkaloids free from their salts:

$$
\begin{aligned}
& \left(\mathrm{C}_{17} \mathrm{H}_{19} \mathrm{NO}_{3}\right)_{2} \quad \mathrm{OH} \\
& \mathrm{C}_{5} \mathrm{HO}_{2}(\mathrm{OH})(\mathrm{COOH})_{2}+{ }_{2}\left(\mathrm{H}_{4} \mathrm{~N}\right) \mathrm{OH}={ }_{2} \mathrm{C}_{17} \mathrm{H}_{19} \mathrm{NO}_{3}+{ }_{2} \mathrm{H}_{2} \mathrm{O}+\mathrm{C}_{5} \mathrm{HO} \\
& \text { II }
\end{aligned}
$$

The ether used dissolves all opium alkaloids except morphine which having once become crystalline is insoluble in this solvent. Saturated sodium salicylate solution precipitates resinous and greasy substances from the filtered aqueous opium extract and also narcotine which next to morphine is present in opium in largest quantity.

The morphine from 6 grams of opium is in 54 grams of filtered aqueous extract. After the second filtration only 36 grams of this extract are used (= morphine from 4 grams of opium). Morphine, precipitated by ammonium hydroxide from these 36 grams of extract, is dissolved in $25 \mathrm{cc}$. of $0.1 \mathrm{n}$-hydrochloric acid as hydrochloride, $\mathrm{C}_{17} \mathrm{H}_{19} \mathrm{NO}_{3} . \mathrm{HCl}$. This solution is then diluted to $100 \mathrm{cc}$. and excess of acid in $50 \mathrm{cc}$. of this hydrochloric acid solution (= morphine from 2 grams of opium) is determined.

Morphine being a monacid base has the same molecular and equivalent weights $=\mathrm{C}_{17} \mathrm{H}_{19} \mathrm{NO}_{3}=285$. Therefore $1000 \mathrm{cc}$. of $0.1 \mathrm{n}$-hydrochloric acid $=28.5$ grams of morphine.

Example.-Titration with o.I n-potassium hydroxide solution has shown that there are 4.I cc. of $0.1 \mathrm{n}$-hydrochloric acid in $50 \mathrm{cc}$. of the hydrochloric acid solution of morphine. There remain therefore $12.5-4 . \mathrm{I}=8.4 \mathrm{cc}$. of the $0 . \mathrm{I}$ n-acid originally present now combined with the morphine from 2 grams of opium. According to the proportion

Cc. o.I n-HCl : Grams morphine

$$
\text { 1000 : } 28.5 \quad=8.4: \mathrm{x} \quad(\mathrm{x}=0.2394)
$$

1 The German Pharmacopœia demands that not more than $5.4 \mathrm{cc}$. nor less than 4.I cc. of 0.I n'-alkaline hydroxide solution, corresponding to a morphine-content of 10-12 per cent., shall be used to produce this color. 
8.4 cc. of 0.1 n-acid correspond to 0.2394 gram of morphine. Consequently the opium contains $50 \times 0.2394=$ II.97 per cent. of morphine. This is the maximum quantity of morphine allowed in opium by the German Pharmacopoia.

2. In Extract of Opium.- "Dissolve 3 grams of opium extract in 40 grams of water, add 2 grams of sodium salicylate solution ( $1: 2$ ), shake vigorously, pass 30 grams of clear solution through a dry filter (ro $\mathrm{cm}$. in diameter) and collect in a dry flask. Mix this filtrate with ro grams of ether by rotating the flask and add also 5 grams of a mixture of 17 grams of ammonium hydroxide and 83 grams of water." Continue the assay as directed above in I (Opium) from the point marked with an asterisk.

Calculation.- Only 2 of the 3 grams of opium extract weighed are used in the determination, since only 30 grams of the original 45 grams of solution ( 3 grams of extract +2 grams of sodium salicylate solution +40 grams of water) are filtered. The morphine obtained from these 2 grams of extract is dissolved in 25 cc. of $0.1 \mathrm{n}$-hydrochloric acid and the volume is then brought to roo cc. The titration uses $50 \mathrm{cc}$. of this solution which contains the morphine from I gram of opium extract.

Example.-If $5.5 \mathrm{cc}$. of $0.1 \mathrm{n}$-potassium hydroxide solution were required to neutralize the excess of $0.1 \mathrm{n}$-hydrochloric acid in the $50 \mathrm{cc}$. of solution, then I $2.5-5.5=7$ cc. of $0.1 \mathrm{n}$-hydrochloric acid are combined with morphine. According to the proportion

Cc. o.I n-HCl : Grams morphine $1000: 28.5 \quad=7: x \quad(x=0.1995)$

7 cc. of 0.1 n-acid correspond to 0.1995 gram of morphine. Therefore this quantity of alkaloid is in I gram of extract. Consequently the opium extract contains 19.95 per cent. of morphine.

The German Pharmacopœia requires that not more than $6.5 \mathrm{cc}$. nor less than $5.5 \mathrm{cc}$. of $0.1 \mathrm{n}$-potassium hydroxide solution shall be used to produce a pale red color in the aqueous layer, corresponding to a morphine content of I7.I I to r 9.95 per cent.

3. In Wine of Opium and Tincture of Opium.- "Evaporate about 50 grams of either preparation in a weighed dish to 15 grams, add water until the weight is 38 grams and also 2 grams of sodium salicylate solution $(1: 2)$. Shake vigorously and pass 32 grams of clear solution through a dry creased filter (ro cm. in diameter) into a dry flask. Mix this filtrate with ro grams of ether by rotating the flask and add also 5 grams of a mixture of 17 grams of ammonium hydroxide solution and 83 grams of water." Continue the assay as directed above in I (Opium) from the point marked with an asterisk.

Calculation.- Only 32 grams of the 40 grams of clear liquid ( 38 grams of evaporated opium tincture +2 grams of sodium salicylate solution) are used for the morphine determination. These correspond to 40 grams of the original opium preparation. The morphine from this quantity of solution is dissolved in 25 cc. of $0.1 \mathrm{n}$-hydrochloric acid and the volume brought to roo cc. Excess of acid in $50 \mathrm{cc}$. of this solution is determined by titration. These $50 \mathrm{cc}$. contain the morphine from 20 grams of the opium preparation.

Example.-If $4.2 \mathrm{cc}$. of $0.1 \mathrm{n}$-potassium hydroxide solution are required for $50 \mathrm{cc}$. of morphine hydrochloride solution, then $\mathrm{I} 2.5-4.2=8.3 \mathrm{cc}$. of $0.1 \mathrm{n}$ - 
hydrochloric acid have combined with morphine. According to the proportion Cc. o.I n- $\mathrm{HCl}$ : Grams morphine

$$
\text { roo0 : } 28.5 \quad=8.3: x \quad(x=0.23655)
$$

20 grams of the opium preparation contain 0.23655 gram of morphine, corresponding to a morphine content of 1.18 per cent.

The German Pharmacopœia requires that not more than $5.55 \mathrm{cc}$. nor less than 4.2 cc. of $0.1 \mathrm{n}$-potassium hydroxide solution shall be used to produce a pale red color in the aqueous liquid, corresponding to a morphine content in Wine of Opium and Tincture of Opium of r.O to r.I8 per cent.

\section{Estimation of Pilocarpine in Jaborandum Leaves ${ }^{1}$}

I. G. Fromme's ${ }^{2}$ Method.-Extract I 5 grams of rather finely powdered jaborandum leaves with 150 grams of chloroform and I 5 grams of ammonium hydroxide solution (Io per cent. $\mathrm{NH}_{3}$ ), shaking frequently for 30 minutes. Filter this mixture through a large, smooth paper, covering the funnel with a glass plate. As soon as the chloroform drops slowly, add a little water and filtration will become more rapid. After collecting a full roo grams of filtrate, add about I gram of water, shake vigorously and set aside. The water takes up fine particles of powder that may have passed through the paper, leaving the chloroform solution quite clean. After I hour weigh Ioo grams of chloroform solution ( $=$ alkaloids in ro grams of jaborandum leaves).

Fromme directs extracting these roo grams of chloroform solution successively with 30,20 and ro cc. of I per cent. hydrochloric acid which dissolves pilocarpine (and isopilocarpine) as hydrochlorides. Extract this acid solution first with $20 \mathrm{cc}$. of ether, to remove fat and resin. Then add an excess of ammonia and extract the free alkaloids successively with 30,20 and ro cc. of chloroform. Pour the combined chloroform extracts through a dry filter, evaporate in a weighed flask, dry the residue at $100^{\circ}$ and weigh.

2. Matthes and Rammstedt's ${ }^{3}$ Method.-Evaporate - Ioo grams of chloroform solution obtained above in a beaker to

1 Further information about pilocarpine is given on page 217 .

${ }^{2}$ Caesar and Loretz, Geschäftsbericht' r $901,27$.

${ }^{3}$ See page 253. 
about $20 \mathrm{cc}$. Add first $3 \mathrm{cc}$. of $0.1 \mathrm{n}$-picrolonic acid and then $60 \mathrm{cc}$. of ether. After 24 hours collect the precipitate of pilocarpine picrolonate in a weighed Gooch crucible, wash with I cc. of alcohol-ether mixture ( $1: 3$ ), dry at $110^{\circ}$ and weigh. Pilocarpine picrolonate thus obtained (= pilocarpine from ro grams of jaborandum leaves), $\mathrm{C}_{11} \mathrm{H}_{16} \mathrm{~N}_{2} \mathrm{O}_{2} \cdot \mathrm{C}_{10} \mathrm{H}_{8} \mathrm{~N}_{4} \mathrm{O}_{5}$ (Mol. Wt. 472 ) melts at $200-205^{\circ}$.

\section{Piperine in Pepper}

Black pepper is the dried, unripe fruit of the pepper plant, Piper nigrum L., whereas the ripe fruit deprived of its outer covering is the white pepper of commerce. The actual constituents of pepper are piperine, an ethereal oil (oil of pepper) and a resin called chavicine. In rather large doses pepper is toxic. ${ }^{1}$

Preparation of Piperine.-Extract finely divided white pepper with $9 \circ$ per cent. alcohol and distil the latter from the extracts. Treat the residue with cold potassium hydroxide solution which dissolves the resin but not the piperine. Wash the residual piperine with water and crystallize from hot alcohol, using animal charcoal to remove color. White pepper contains 7-8 per cent. of piperine.

Piperine, $\mathrm{C}_{17} \mathrm{H}_{19} \mathrm{NO}_{3}$, crystallizes in colorless, shining, rectangular, monoclinic prisms melting at $\mathrm{r} 28-\mathrm{r} 29^{\circ}$. When pure it is almost tasteless but impure piperine has a sharp, burning taste. It is nearly insoluble in water, freely soluble in alcohol and also soluble in ether, benzene and chloroform. Piperine is a very weak base, dissolving in dilute mineral acids with almost as much difficulty as in pure water. A solution of piperine in concentrated sulphuric acid has a ruby color, soon changing to dark brown and gradually to greenish brown and fading upon addition of water. Concentrated nitric acid converts piperine into an orangered resin soluble with blood-red color in dilute potassium hydroxide solution. The constitution of piperine is known.

Prolonged heating with alcoholic potassium hydroxide solution decomposes piperine into the potassium salt of piperic acid and piperidine:

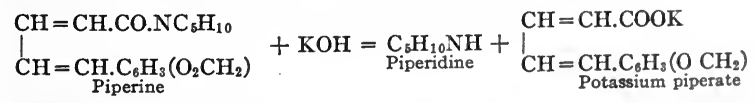

Rügheimer ${ }^{1}$ synthesized piperine by putting together these two products. Piperic acid was first converted into its chloride by means of phosphorus penta-

'R. Kobert ("Intoxikationen") mentions a case where a teaspoonful of pepper was given to each of three young pigs. There was severe inflammation of the gastro-intestinal tract in all three animals and two died. The toxic action of pepper is attributed to piperine, since the ethereal oil according to Kobert does not take part in the toxic action due to absorption.

2 Berichte der Deutschen chemischen Gesellschaft 15, 1390 (x882). 
chloride. Piperyl chloride was then condensed in benzene solution with piperidine:

$$
\begin{aligned}
& \begin{array}{l}
\mathrm{CH}=\mathrm{CH} . \mathrm{COOH} \\
\underset{\mathrm{CH}}{1}=\mathrm{CH} \cdot \mathrm{C}_{6} \mathrm{H}_{3}\left(\mathrm{O}_{2} \mathrm{CH}_{2}\right)
\end{array}+\mathrm{PCl}_{5} \quad \stackrel{\mathrm{CH}=\mathrm{CH} \cdot \mathrm{COCl}}{\mathrm{CH}=\mathrm{CH} \cdot \mathrm{C}_{6} \mathrm{H}_{3}\left(\mathrm{O}_{2} \mathrm{CH}_{2}\right)}+\mathrm{POCl}_{3}+\mathrm{HCl}
\end{aligned}
$$

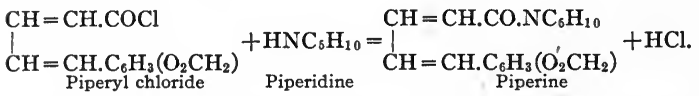

On the basis of the known structure of piperic acid and piperidine, piperine must have the following constitution:

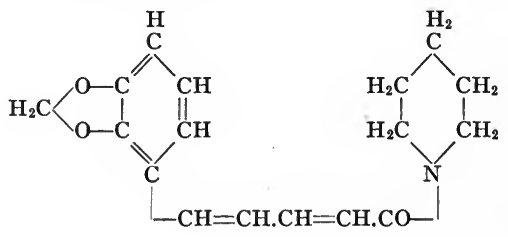

\section{Estimation of Piperine in Pepper}

I. J. Koenig's Method.-Exhaust Io-20 grams of pepper, ground as finely as possible, in a Soxhlet apparatus with strong ethyl or methyl alcohol, or petroleum ether. Distil the alcohol or petroleum ether. The residue consists of piperine and resin. Shake this residue with cold potassium or sodium carbonate solution to dissolve the resin. Filter from undissolved piperine and wash the latter with cold water. Dissolve in alcohol or petroleum ether, evaporate the filtered solution in a weighed flask or dish and dry the residue at $100^{\circ}$ to constant weight.

To determine the resin in pepper at the same time, filter the potassium or sodium carbonate solution from crude piperine and add hydrochloric acid to the filtrate. Filter the precipitated resin, redissolve in alcohol, evaporate the solvent and dry the residue to constant weight.

2. Cazeneuve and Caillot's Method.-Add enough water to make a thin mixture of powdered pepper with twice its weight of slaked lime and stir well. Boil in a porcelain dish, dry thoroughly upon the water-bath and then extract with ether in a Soxhlet apparatus. Distil the ether in a weighed flask and 
dry the residue of piperine at $100^{\circ}$ to constant weight. To obtain pure crystalline piperine, dissolve the residue from the ether distillation in the least possible volume of boiling alcohol, surround the solution with ice, collect the piperine upon a weighed filter and dry at $100^{\circ}$ to constant weight. This purification of piperine is attended with more or less loss and consequently the result is only approximately correct.

\section{Estimation of Santonin in Wormseed ${ }^{1}$}

I. K. Thaeter's ${ }^{2}$ Method.-Extract Io grams of crushed wormseed in a Soxhlet apparatus with ether for I2 hours. Distil the ether and boil the residue for an hour with 5 grams of lime and about $300 \mathrm{cc}$. of water. Replace water lost by evaporation. Filter while hot and wash the residue with water. Faintly acidify the filtrate with sulphuric acid and warm gently until santonin crystals begin to form. Then add roo grams of aluminium acetate solution, ${ }^{3}$ heat the mixture to boiling and finally evaporate to dryness upon the waterbath. Mix the finely powdered residue with 3 grams of magnesium oxide, moisten this mixture with a little water and again bring quickly to dryness. Powder the residue as finely as possible, dry at $105^{\circ}$ and extract in a Soxhlet apparatus with anhydrous, acid-free ether for 5 hours. Santonin is deposited upon distilling the ether as a faintly yellowish residue which is then dried at $100^{\circ}$ to constant weight.

Remarks.-When wormseed is heated with lime, santonin passes into solution as calcium santonate, and at the same time resinous substances are.saponified.

1 Wormseed (Flores cinæ) consists of the unexpanded flower-heads of Artemisia cina which are $3-4 \mathrm{~mm}$. in length.

2 Archiv der Pharmacie 237, 626-632 (1899) and 238, 383-387 (1900).

3 Dissolve 300 parts of aluminium sulphate in 800 parts of water; add acetic acid (sp. gr. 1.04I) 360 parts; triturate calcium carbonate 130 parts with 200 parts of water, and add this mixture slowly and with continued stirring to the first solution; set the whole aside for 24 hours without applying heat, and stir occasionally; then strain, press the precipitate without washing it and filter the liquid. It is a clear, colorless liquid, having the sp. gr. 1.044 to 1.046, a faint odor of acetic acid, an acid reaction, and a sweetish, astringent taste. National Dispensatory. 
Dilute sulphuric acid liberates first santonic acid which passes at once into its inner anhydride, santonin. Basic aluminium acetate, produced by boiling, precipitates resinous and colored substances. Finally, magnesium oxide serves to neutralize free acetic acid. Under the conditions, practically no magnesium santonate is formed. Thaeter obtained 88 to 92 per cent. of the santonin present. Wormseed contains about 2.5 per cent. of santónin.

2. J. Katz's ${ }^{1}$ Method.-Extract Io grams of coarsely powdered wormseed in a Soxhlet apparatus with ether for 2 hours. Distil the ether. There usually remains a dark green resin weighing $1.5^{-2}$ grams. Boil this residue I $^{-3}-30$ minutes, under a reflux condenser, with 5 grams of crystallized barium hydroxide dissolved in Ioo cc. of water. Cool and, without filtering, render the solution acid to litmus with carbon dioxide. Filter immediately with a pump to remove barium carbonate, and wash the precipitate twice with $20 \mathrm{cc}$. portions of water. Evaporate the pale yellow solution to about $20 \mathrm{cc}$. in a dish upon the water-bath. Add Io cc. of I2.5 per cent. hydrochloric acid, continue heating upon the water-bath exactly 2 minutes longer and pour the solution into a separating funnel. Dissolve santonin crystals left in the dish in about $20 \mathrm{cc}$. of chloroform. Pour this solution into the separating funnel and shake thoroughly. When the solutions have separated clear, withdraw the chloroform solution and pass it through a dry filter. Wash dish, separating funnel and filter 2-3 times with ro cc. portions of chloroform. Distil the chloroform and boil the residue ro minutes, under a reflux condenser, with $50 \mathrm{cc}$. of I 5 per cent. alcohol. Filter while hot into a weighed flask, and wash flask and filter twice with I $_{5} \mathrm{cc}$. portions of I $_{5}$ per cent. boiling alcohol. Cover the flask and set aside in the cold 24 hours. Weigh flask and contents and pass the latter through a weighed filter, disregarding the milky appearance of the filtrate caused by minute, resinous drops. Wash flask and filter once with ro cc. of 15 per cent. alcohol, dry the filter in the flask and weigh both. Finally, apply a correction on account of the solubility of santonin in the alcohol used. Every ro grams of filtrate contain $6 \mathrm{mg}$. of santonin. Santonin by this method is crystalline,

${ }^{1}$ Archiv der Pharmacie 237, 25 I (1899). 
and usually faintly yellow. J. Katz found the quantity of santonin in wormseed to vary between I.2I and 3.I6 (average 2.42) per cent. This method is based upon the fact that the santonin in to grams of wormseed is easily soluble in $50 \mathrm{cc}$. of hot I 5 per cent. alcohol, whereas only a very little resin is dissolved by this dilute alcohol. As this dilute, alcoholic solution cools, santonin crystallizes out almost quantitatively.

Troches of Santonin.-To estimate the quantity of santonin in troches, made from this substance and sugar, directly extract the finely ground mixture with hot chloroform. The santonin can usually be weighed without further purification.

Chocolate Troches of Santonin.-In a somewhat simpler form, the method described above may be used to estimate santonin in chocolate troches. Weigh 3 or 4 troches and boil 15 minutes under a reflux condenser with 5 grams of barium hydroxide and $100 \mathrm{cc}$. of water. Saturate the liquid when cold with carbon dioxide. Filter, wash the residue with water and evaporate the brownish filtrate to Ioo cc. Warm the liquid and add Io cc. of dilute hydrochloric acid. Three extractions with chloroform yield nearly pure santonin. To get santonin crystals almost white and ready for weighing, evaporate the chloroform solution and expel the last traces of chloroform by adding a few cc. of ether. If santonin is impure from traces of fatty acids, boil once with ro cc. of petroleum ether and filter when cold. Santonin is nearly insoluble in cold petroleum ether.

Santonin can be detected and estimated in toxicological analysis in a similar manner. Acidify the material with hydrochloric acid, extract with chloroform and treat the chloroform residue with barium hydroxide solution as described above.

\section{Estimation of Solanine in Potatoes ${ }^{1}$}

r. O. Schmiedebèrg and G. Meyer's Method.-Mix 500 grams of finely grated potatoes with water and press out the liquid. Decant the liquid from the deposit of starch. Again mix the starch with water and decant the latter when the starch has settled. Neutralize the entire liquid with ammonia and evaporate to the consistency of an extract. In the meantime mix the press-cake with several times its volume of boiling alcohol. Press out the alcohol completely after several hours. Make two such extractions. Filter the combined alcoholic extracts and wash the residue (starch) upon the filter with alcohol. The aqueous liquid from the potatoes contains very

${ }^{1}$ See page 225 . 
little solanine. To isolate this small quantity, use the alcoholic filtrate to extract the residue from the aqueous extract and again filter. Wash the insoluble part with hot alcohol. The alcoholic filtrate after half an hour usually deposits some crystals of asparagine. ${ }^{1}$ Separate the supernatant liquid from these crystals and evaporate upon the water-bath to the consistency of an extract. Dissolve the residue in water containing sulphuric acid, filter and wash. Warm the clear liquid very gently, saturate with ammonia and set aside for a day. Solanine appears in small crystals. Collect the deposit upon a weighed filter, wash first with water and then with ether, dry at $100^{\circ}$ and weigh.

2. F. von Morgenstern's ${ }^{2}$ Method.-Express as much liquid as possible from 200 grams of finely grated potatoes by means of a press. Make two separate extractions of the press-cake with water and express the liquid thoroughly each time. Precipitate protein substances from the combined liquid by adding $0.5 \mathrm{cc}$. of acetic acid and warming for I hour upon the water-bath. Filter, evaporate the filtrate to a syrup, stir and add gradually hot 96 per cent. alcohol until cloudiness ceases. ${ }^{3}$ Decant the solution after I 2 hours and extract the residue containing sugars and dextrins twice with hot alcohol. Evaporate the combined alcoholic extracts upon the water-bath, warm the residue with some water containing acetic acid and filter. Heat the filtrate to boiling and add ammonia drop by drop to precipitate solanine. After standing for 5 minutes upon the water-bath, the base separates in flocks that are easily filtered. Wash the precipitate with water containing ammonia, dissolve in boiling alcohol and treat this solution as follows. Evaporate

${ }^{1}$ Asparagine is the amide of aspartic acid, or mono-amino-succinic acid, $\mathrm{H}_{2} \mathrm{~N}$. CH $-\mathrm{COOH}$<smiles>CCC(N)=O</smiles>

$+\mathrm{H}_{2} \mathrm{O}$. It appears in shining, rhombic crystals that dissolve rather easily in hot water but less easily in alcohol or ether. Lævoasparagine is widespread in the plant kingdom in seeds.

${ }^{2}$ Landwirtschaftliche Versuchsstation 65, 301 ( 1907 ).

${ }^{8}$ To extract those parts of the potato plant, which can be dried at $100^{\circ}$ and reduced to a fine powder, heat to boiling several times with water containing acetic acid and filter each time. 
upon the water-bath and dissolve the residue in water containing acetic acid. Filter, heat the filtrate to boiling and precipitate solanine with ammonia. Collect the pure white flocks of solanine from this second precipitation upon a filter that has been dried at $90^{\circ}$ and weighed. Wash with 2 per cent. ammonia and dry at $90^{\circ}$ to constant weight.

Notes. - v. Morgenstern obtained on the average by this method 0.0125 per cent. of solanine in table potatoes and $0.095^{8}$ per cent. in those used as forage. The yield of solanine from yellow tubers upon the average was less than from red. Tubers grown upon sandy soil were richer in solanine than were those from humus soil. Moisture and abundance of humus appear to diminish the quantity of solanine. A nitrogenous fertilizer increased the quantity of solanine, a potash fertilizer lowered it and a phosphate fertilizer appeared to have little effect. There was less solanine in large than in small potatoes of the same variety. Solanine first appears to increase during the process of germination. Passing into sprouts, without wholly disappearing from the tubers, solanine increases with the growth of the plant. As growth advances the distribution of solanine in the different parts of the plant is indication of a tendency on the part of the plant to withdraw solanine from the older sprouts and spread it throughout the young organs. Consequently solanine may serve first of all as the natural protector of the plant, especially of the growing parts.

\section{Estimation of Alkaloids in Nux Vomica}

$$
\text { (C. C. Keller }{ }^{1} \text { ) }
$$

Remove fat from nux vomica by treating 15 grams of the well-dried and finely powdered drug in a $25^{\circ} \mathrm{cc}$. Erlenmeyer flask two or three times with $30 \mathrm{cc}$. portions of ether. Shake thoroughly for 5 minutes. Pour these ether washings into a flask, and, since they contain a little alkaloid, extract dissolved alkaloid with $5 \mathrm{cc}$. of $0 . \mathrm{I} \mathrm{n}$-hydrochloric acid and ro cc. of water. Repeat the extraction of the ether layer, separated from the aqueous solution, using water instead of acid. Add roo cc. of ether, 50 grams of chloroform and Io grams of to per cent. ammonium hydroxide solution to the powdered nux vomica free from fat. Shake thoroughly for 30 minutes and add to this mixture the hydrochloric acid solution used in extracting alka-

1 Festschrift presented at the fiftieth anniversary of the founding of the Swiss Pharmaceutical Association. Abstract in Zeitschrift für analytische, Chemie, 23, 49 I (1894). 
loid from the first ether washings. Again shake thoroughly and, when the liquids have separated clear, pour too grams of ether-chloroform solution through a small filter into a weighed Erlenmeyer flask. Distil the chloroform and ether as completely as possible. The alkaloids usually appear as colorless varnishes which persistently retain chloroform. To remove the latter, pour a few cc. of absolute alcohol upon the residue and expel completely upon the water-bath. Repeat this treatment 2-3 times. This will give crystalline alkaloids which can be dried at $100^{\circ}$ to constant weight.

\section{Method of the German Pharmacopœia}

I. In Nux Vomica.-Place I5 grams of nux vomica, ground mediumly fine and dried at $100^{\circ}$, in an Erlenmeyer flask and add 100 grams of ether and 50 grams of chloroform. Shake vigorously and add ro cc. of a mixture of 2 parts of sodium hydroxide solution and $r$ part of water. Shake at frequent intervals and let the mixture stand for 3 hours. Then add 15 cc. more water, or enough to cause the powder after vigorous shaking to gather into balls and leave the supernatant ether-chloroform solution perfectly clear. After I hour filter roo grams of the clear ether-chloroform solution through a dry filter kept well covered. Collect the filtrate in a small flask and distil about half the solvent. Transfer the residual ether-chloroform solution to a separating funnel, rinse the flask 3 times with $5 \mathrm{cc}$. portions of a mixture of 3 parts of ether and $\mathbf{r}$ part of chloroform. Extract the combined solvent with ro cc. of o.r n-hydrochloric acid. Add enough ether to cause the ether-chloroform solution to rise to the top of the acid liquid and pass the latter through a small filter moistened with water into a roo cc. flask. Then extract the' ether-chloroform solution with 3 additional ro cc. portions of water. Pass these extracts through the same filter, wash the latter with water and dilute the total liquid to roo cc. Finally measure $50 \mathrm{cc}$. of this solution into a flask holding about $200 \mathrm{cc}$., add about $50 \mathrm{cc}$. of water and sufficient ether to make a layer $\mathrm{I} \mathrm{cm}$. deep. Add 5 drops of iodeosine solution and run in enough o.or n-potassium hydroxide solution, shaking vigorously after each addition, to turn the aqueous layer a permanent pale red.

Calculation.- Ioo grams ( = alkaloids from ro grams of nux vomica) of the original r 50 grams of ether-chloroform mixture were used. The alkaloids were dissolved by ro cc. of o.r n-hydrochloric acid and the volume was brought to $100 \mathrm{cc}$. The excess of acid in $50 \mathrm{cc}$. of this solution ( $=5$ grams of nux vomica) was determined by titration with o.or n-potassium hydroxide. If strychnine and brucine are present in nux vomica in equal amount, the average equivalent weight of the two alkaloids is 364 . Therefore rooo cc. of $0.1 \mathrm{n}$-hydrochloric acid correspond to 36.4 grams of alkaloids.

Example.-Suppose that the titration of the excess of acid in $5 \circ \mathrm{cc}$. of solution required $15.6 \mathrm{cc}$. of $0.0 \mathrm{r}$ n-potassium hydroxide $=1.56 \mathrm{cc}$. of $0.1 \mathrm{n}$-alkali. Then 
$5-r .56=3.44 \mathrm{cc}$. of $0.1 \mathrm{n}$-hydrochloric acid are combined with the alkaloids in 5 grams of nux vomica. According to the proportion

Cc. O.I n-HCl: Grams alkaloid

$$
\text { roo0: } \quad 36.4=3.44: x(x=0.12522)
$$

3.44 cc. of 0.1 n-acid are combined with 0.12522 gram of alkaloid, corresponding to an alkaloid content of $20 \times 0.12522=2.50$ per cent. The German Pharmacopœia places this percentage as the minimum for total alkaloids in nux vomica.

2. In Extract of Nux Vomica.-Dissolve I gram of extract in an Erlenmeyer flask in 5 grams of water and 5 grams of absolute alcohol, and add 50 grams of ether and 20 grams of chloroform to this solution. Shake vigorously and add ro cc. of sodium carbonate solution $(\mathrm{r}: 3)$. Let the mixture stand and agitate at frequent intervals for an hour.* Then pass 50 grams of the clear ether-chloroform solution through a dry filter kept well covered, and receive the filtrate in a flask. Distil half the solvent and pour the remainder into a separating funnel. Wash the flask three times with $5 \mathrm{cc}$. portions of a mixture of 3 parts of ether and I part of chloroform. Thoroughly extract the total ether-chloroform solution with $50 \mathrm{cc}$. of $0.0 \mathrm{r} \mathrm{n}$-hydrochloric acid. When the liquids have separated clear, if necessary, after addition of enough ether to bring the ether-chloroform solution to the surface, pass the acid solution through a small filter moistened with water and receive the filtrate in a $200 \mathrm{cc}$. flask. Make three extractions of the ether-chloroform solution with ro cc. portions of water, and pass these washings through the same filter. Finally, wash the filter with water and bring the entire solution to roo cc. Add enough ether to make a layer I $\mathrm{cm}$. thick and 5 drops of iodeosine solution. Run in o.or n-potassium hydroxide solution, shaking vigorously after each addition, until the aqueous solution is pale red.

Calculation.--Only 5o grams, or two-thirds of the original 75 grams of alcoholether-chloroform mixture, were used. The alkaloids in 0.666 gram of nux vomica extract were in this volume of solvent. Alkaloids were dissolved in $5^{\circ}$ cc. of o.or $\mathbf{n}$-hydrochloric acid and excess of acid determined by titration with o.or n-potassium hydroxide solution.

Example.-Suppose that $18 \mathrm{cc}$. of $0.0 \mathrm{I}$ n-potassium hydroxide solution were used in this titration. Then $50-18=32 \mathrm{cc}$. of 0.01 n-acid were combined with the alkaloids in 0.666 gram of extract. According to the proportion

$$
\begin{aligned}
& \text { Cc. o.or } \mathrm{n}-\mathrm{HCl} \text { : Grams alkaloids } \\
& 1000: 3.64=32: x(x=0.11648)
\end{aligned}
$$

0.666 gram of extract contains 0.1 I 648 gram of alkaloids, corresponding to $17 \cdot 47$ per cent. The German Pharmacopœia places this percentage as the minimum for total alkaloids in extract of nux vomica.

3. In Tincture of Nux Vomica.-Evaporate 50 grams of tincture of nux vomica in a weighed dish to ro grams. Wash this residue into an Erlenmeyer flask and rinse with 5 grams of absolute alcohol. Add 50 grams of ether and 20 grams of chloroform and shake vigorously. Then add ro cc. of sodium carbonate solution $(\mathrm{r}: 3)$ which has been previously employed in rinsing the dish used in evaporating the tincture. Let this mixture stand an hour, shaking vigorously at fre- 
quent intervals. Filter 50 grams of the clear ether-chloroform solution. To extract alkaloids, use $40 \mathrm{cc}$. of $0.0 \mathrm{n}$-hydrochloric acid. In other respects, the estimation of alkaloids is the same as described for extract of nux vomica.

Calculation.-This is the same as that given for extract of nux vomica. Only two-thirds ( $=33.3$ grams) of the original weight of nux vomica tincture were used. The alkaloids were dissolved in 40 cc. of o.or $\mathrm{n}$-hydrochloric acid and excess of acid was determined by titration with o.or n-potassium hydroxide solution. If $\mathrm{I} 7 \mathrm{cc}$. of the latter solution were used, then $40-\mathrm{I} 7=23 \mathrm{cc}$. of o.or $\mathrm{n}$-hydrochloric acid were combined with the alkaloids in 33.3 grams of the tincture. According to the proportion

\section{Cc. o.or $\mathrm{n}-\mathrm{HCl}$ : Grams alkaloids}

$$
\text { rooo: } \quad 3.64=23: x(x=0.08372)
$$

this weight of tincture contains 0.08372 gram of alkaloids, corresponding to $2.5 \mathrm{I}$ per cent. The German Pharmacopoia places this percentage as the minimum for total alkaloids in tincture of nux vomica. Brucine and strychnine are assumed to be present in equal quantity.

\section{Estimation of Alkaloids in Nux Vomica and Its Preparations by Means of Picrolonic Acid}

(H. Matthes and O. Rammstedt)

Nux vomica upon the average contains strychnine and brucine in equal quantity combined with tannic acid. Alkaline hydroxide or carbonate solutions liberate the alkaloids from their salts. The free bases are then extracted with an etherchloroform mixture. The solvent is reduced to smaller volume by evaporation or distillation and the alkaloids are precipitated with picrolonic acid.

Strychnine picrolonate, $\mathrm{C}_{21} \mathrm{H}_{22} \mathrm{~N}_{2} \mathrm{O}_{2} \cdot \mathrm{C}_{10} \mathrm{H}_{8} \mathrm{~N}_{4} \mathrm{O}_{5}$ (Mol. Wt. 598) melts with decomposition at $286^{\circ}$.

Brucine picrolonate, $\mathrm{C}_{23} \mathrm{H}_{26} \mathrm{~N}_{2} \mathrm{O}_{4} \cdot \mathrm{C}_{10} \mathrm{H}_{8} \mathrm{~N}_{4} \mathrm{O}_{5}$ (Mol. Wt. 658) melts with decomposition at $277^{\circ}$.

I. Extract of Nux Vomica.-Dissolve I gram of extract in 5 grams of absolute alcohol and 5 grams of water. Shake well with 50 grams of ether and 20 grams of chloroform. Add Io cc. of sodium carbonate solution ( $\mathrm{I}: 2$ ) and again shake thoroughly for ro minutes. Let the mixture stand at rest for 20 minutes. Pass 50 grams of the ether-chloroform mixture through a dry, double, creased filter and evaporate half the solvent in a beaker. Add about $5 \mathrm{cc}$. of $0 . \mathrm{I} \mathrm{n}$-alcoholic picrolonic acid to the warm 
solution. A yellow crystalline precipitate of strychnine and brucine picrolonates soon appears. After 24 hours collect the mixed picrolonates in a weighed Gooch crucible. Wash excess of picrolonic acid from the precipitate with $2 \mathrm{cc}$. of an alcohol-ether mixture ( $1: 3$ ), dry 30 minutes at $110^{\circ}$, cool in desiccator and weigh.

Calculation.- Use the mean molecular weight of brucine and strychnine picrolonates $(=628)$ and also the mean molecular weight of brucine and strychnine $(=364)$. The proportion is

$\begin{array}{ccc}\text { Grams picrolonate Grams strychnine } \\ \text { mixture } & : & \text { and brucine } \\ 628 & : & 364\end{array}=$ cipitate $: x$.

Since the quotient $364: 628=0.5798$, the weight of mixed alkaloids is obtained by multiplying the weight of the picrolonate precipitate by this quantity. This precipitate represents total alkaloids in 0.666 gram of extract of nux vomica, for only two-thirds ( $=50$ grams) of the original 75 grams ( 5 grams of alcohol + 50 grams of ether +20 grams of chloroform) of solvent, containing the alkaloids in I gram of extract of nux vomica, were used.

2. Tincture of Nux Vomica.-Evaporate 50 grams of tincture in an Erlenmeyer flask to Io cc. Cool and shake well with $5 \mathrm{cc}$. of absolute alcohol, 50 grams of ether and 20 grams of chloroform. Add ro cc. of sodium carbonate solution ( $1: 2$ ) and shake again for Io minutes. After 20 minutes pass 50 grams (= two-thirds of the original mixture) of the ether-chloroform mixture through a double, creased filter. Evaporate half the solvent in a beaker and add 5 cc. of o.I n-alcoholic picrolonic acid to the warm residue. Treat the picrolonate precipitate as described above.

3. Nux Vomica.-Exhaust I 5 grams of powdered nux vomica, previously dried at Ioo, by thoroughly agitating with roo grams of ether and 50 grams of chloroform. Then add ro cc. of a mixture of 2 parts of $r_{5}$ per cent. sodium hydroxide solution and I part of water and shake again for ro minutes. Add an additional i 5 cc. of water, or enough to cause the powder to gather into balls after vigorous agitation and leave the supernatant ether-chloroform mixture clear. After 30 minutes pass the clear ether-chloroform solution through a dry, double, creased filter. Evaporate $50 \mathrm{cc}$. of the filtrate in a beaker nearly to 
dryness and add a second $50 \mathrm{cc}$. portion of filtrate to the residue, bringing everything into solution (= alkaloids from Io grams of nux vomica). Add 5 cc. of o.r n-alcoholic picrolonic acid and treat the precipitate as previously described.

\section{Estimation of Strychnine in Mixtures of Nux Vomica Alkaloids}

(Gordin's ${ }^{1}$ Modification of Keller's Method)

Strong nitric acid, gently heated with a solution of strychnine and brucine in 3 per cent. sulphuric acid, is without action upon the former alkaloid. But brucine is converted into nonbasic substances not extracted by chloroform from an alkaline solution.

Procedure.-Dissolve the mixed alkaloids ( $0.2-0.3$ gram) upon the water-bath in $15 \mathrm{cc}$. of 3 per cent. sulphuric acid and add $3 \mathrm{cc}$. of a diluted nitric acid (equal parts of 68-69 per cent. acid (sp. gr. I.42) and water) to the cold solution. Pour the mixture into a separating funnel after exactly ro minutes and add sodium hydroxide solution in excess. Extract strychnine 3-4 times with chloroform. Pass the chloroform solution through a double filter into a small weighed flask, wash the filter with a little chloroform, add 2 cc. of pure amyl alcohol and distil to dryness upon the water-bath. Remove the last traces of liquid, consisting mainly of amyl alcohol, by forcing a current of air through the flask warmed upon the water-bath. Finally dry the residue 2 hours at $135^{-}-10^{\circ}$ and weigh when cold. In this way a very pure, white strychnine free from brucine is obtained.

Notes.-According to Gordin, ammonia cannot be substituted for sodium hydroxide, for it gives colored strychnine. Amyl alcohol is added to the chloroform solution to prevent strychnine crystals from being carried by decrepitation into the condenser during distillation.

\section{Estimation of Theobromine and Caffeine in Cacao and Chocolate ${ }^{2}$}

Cacao and its preparations contain only very little caffeine which is usually determined with theobromine.

${ }^{1}$ Archiv der Pharmazie 240, 643 (I902).

${ }^{2}$ H. Beckurts, Archiv der Pharmazie, 244, 486 (1906). 
Boil 6 grams of powdered cacao, or I 2 grams of chocolate, for 30 minutes under a reflux condenser in a weighed liter flask with 200 grams of a mixture of 197 grams of water and 3 grams of dilute sulphuric acid. Then add 400 grams of water and 8 grams of finely powdered magnesia and boil for an hour longer. When the mixture is cold, add exactly enough water by weight to replace what has been lost by evaporation. After the mixture has settled, filter 500 grams of solution $(=5$ grams of cacao or Io grams of chocolate) and evaporate the filtrate to dryness in a dish either by itself or with some quartz sand. Triturate the residue and exhaust in a Soxhlet tube with chloroform.

In case of evaporation without quartz sand, rub the residue with a few drops of water, transfer to a separating funnel with Io cc. of water and extract 8 times with $50 \mathrm{cc}$. portions of hot chloroform. Pass the chloroform extract through a dry filter into a tared flask, distil the chloroform and dry the residue ( = theobromine and caffeine) at $100^{\circ}$ to constant weight.

Carbon tetrachloride is used to separate theobromine from caffeine, the latter alkaloid alone being soluble at room temperature. Let the weighed residue from chloroform stand for I hour with Ioo grams of carbon tetrachloride at room temperature. Shake occasionally and then filter. Distil carbon tetrachloride and extract the residue repeatedly with water. Evaporate the aqueous solution in a weighed dish and dry the residue ( $=$ caffeine) at $100^{\circ}$ to constant weight.

Repeatedly extract the theobromine, insoluble in carbon tetrachloride, and also the filter paper with water. Filter, evaporate the total filtrate and weigh the residue ( $=$ theobromine) dried at $100^{\circ}$.

Notes.-In the method described above, H. Beckurts and Fromme eliminate injurious effects due to concentration by boiling with dilute sulphuric acid. Xanthine bases are set free from combination with organic acid and recombined with sulphuric acid. Magnesia sets these bases free from their sulphates and at the same time holds back coloring matter and fat, thus eliminating these impurities.

Theobromine, 3,7-dimethyl-xanthine, $\mathrm{C}_{7} \mathrm{H}_{8} \mathrm{~N}_{4} \mathrm{O}_{2}$, is a white powder consisting of microscopic needles having a bitter taste. It dissolves in 3282 parts of cold and 148 parts of boiling water; in 422 parts of boiling absolute alcohol; and in I05 parts of boiling chloroform. Theobromine solutions are neutral. This 
alkaloid acts both as an acid and as a base and therefore is soluble in both acid and alkaline solutions. The salts with acids crystallize well but are not very stable. These theobromine salts are partially decomposed into theobromine and acid in presence of much water, or, if the given acid is volatile, by heating at $100^{\circ}$. Theobromine is isomeric with theophylline, or $r, 3$-dimethyl-xanthine, and paraxanthine, or $\mathrm{r}, 7$-dimethyl-xanthine:

(3)<smiles></smiles>

\section{(3)}<smiles>CCn1c(=O)c2[nH]cnc2n(CC)c1=O</smiles>

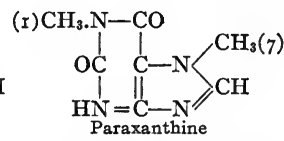

Theophylline occurs in tea leaves and paraxanthine has been isolated from human urine. The latter is therefore called urotheobromine.

\section{Estimation of Alkaloids in Leaves of Atropa Belladonna, Hyoscyamus Niger and Datura Strammonium}

\section{(E. Schmidt's Modification of Keller's Method')}

Shake vigorously ro grams of finely powdered leaves, dried to constant weight over quicklime, in an Erlenmeyer flask with 90 grams of ether and 30 grams of chloroform. Add ro cc. of ro per cent. sodium hydroxide solution and shake vigorously and often for 3 hours. Then add ro cc. of water, or enough to cause the powder to gather into balls when thoroughly shaken. After I hour pass 60 grams of the ether-chloroform extract ( $=5$ grams of leaves) through a dry filter kept well covered. Distil $60 \mathrm{cc}$. of this filtrate to half its volume to remove ammonia, and transfer the deep green solution to a separating funnel, rinsing the flask with three $5 \mathrm{cc}$. portions of ether. Shake the combined extracts well with ro cc. of o.or n-hydrochloric acid. Add enough ether to cause the etherchloroform solution to rise to the top, and pass the acid solution through a moist filter into a $200 \mathrm{cc}$. glass stoppered flask. Shake the ether-chloroform solution 3 times with ro cc. portions of water, pouring these extracts through the same filter and washing the latter with enough water to bring the total volume to roo cc. Add enough ether to make a layer $\mathrm{I} \mathrm{cm}$. deep and 5 drops of iodeosine solution. Having determined beforehand the exact relation of acid to alkali, titrate excess of $0.01 \mathrm{n}$-hydrochloric acid with o.or $\mathrm{n}$-potassium hydroxide solution. The calculation is the same as that for extract of belladonna (see page $30 \mathrm{r}$ ).

Notes.-Using this method, E. Schmidt obtained 0.4 per cent. of alkaloid in wild belladonna leaves but only 0.26 per cent. in cultivated leaves. The average of many determinations gave 0.4 per cent. in strammonium leaves and $0.27-0.28$ per cent. in hyoscyamus leaves without stalks. Alkaloids were calculated as atropine.

Sodium hydroxide solution liberates alkaloids from the acids with which they are naturally combined in the plant, for example:

$$
\left(\mathrm{C}_{17} \mathrm{H}_{23} \mathrm{NO}_{3}\right)_{2} \cdot \mathrm{H}_{2} \mathrm{SO}_{4}{ }^{2}+{ }_{2} \mathrm{NaOH}={ }_{2} \mathrm{C}_{17} \mathrm{H}_{23} \mathrm{NO}_{3}+{ }_{2} \mathrm{H}_{2} \mathrm{O}+\mathrm{Na}_{2} \mathrm{SO}_{4} \text {. }
$$

1 Apotheker-Zeitung r $_{5}, \mathrm{r}_{3}$.

2 The formula of atropine sulphate used in medicine. 


\section{Estimation of Alkaloids in Extract of Belladonna}

(German Pharmacopœia)

Dissolve 2 grams of extract of belladonna in an Erlenmeyer flask in 5 grams of water and 5 grams of absolute alcohol, and add $5 \circ$ grams of ether and 20 grams of chloroform to this solution. Shake vigorously and add ro cc. of sodium carbonate solution $(r: 3)$. Let the mixture stand and agitate at frequent intervals for an hour. Then pass 50 grams of the clear ether-chloroform solution through a dry filter kept well covered, and receive the filtrate in a flask. Distil half the solvent and peur the remainder into a separating funnel. Wash the flask three times with $5 \mathrm{cc}$. portions of ether. Thoroughly extract the total ether-chloroform solution with $20 \mathrm{cc}$. of $0.01 \mathrm{n}$-hydrochloric acid. When the liquids have separated clear, if necessary, after addition of enough ether to bring the etherchloroform solution to the surface, pass the acid solution through a small filter moistened with water and receive the filtrate in a $200 \mathrm{cc}$. flask. Make three extractions of the ether-chloroform solution with Io cc. portions of water, and pass these washings through the same filter. Finally, wash the filter with water and bring the entire solution to roo cc. Add enough ether to make a layer $\mathrm{I} \mathrm{cm}$. thick and 5 drops of iodeosine solution. Run in o.or n-potassium hydroxide solution, shaking vigorously after each addition, until the aqueous solution is pale red.

Calculation.-Sodium carbonate like sodium hydroxide liberates the alkaloids atropine and hyoscyamine, from their salts in belladonna leaves:

$\left(\mathrm{C}_{17} \mathrm{H}_{23} \mathrm{NO}_{3}\right)_{2} \cdot \mathrm{H}_{2} \mathrm{SO}_{4}+\mathrm{Na}_{2} \mathrm{CO}_{3}={ }_{2} \mathrm{C}_{17} \mathrm{H}_{23} \mathrm{NO}_{3}+\mathrm{Na}_{2} \mathrm{SO}_{4}+\mathrm{CO}_{2}+\mathrm{H}_{2} \mathrm{O}$. The free alkaloids dissolve in the alcohol-ether-chloroform mixture. Fifty grams of this solution ( = alkaloids from r. 33 grams of extract) are extracted with $20 \mathrm{cc}$. of $0.0 \mathrm{r}$-hydrochloric acid, the alkaloids passing into the aqueous solution as salts of hydrochloric acid $\left(\mathrm{C}_{17} \mathrm{H}_{23} \mathrm{NO}_{3} \cdot \mathrm{HCl}\right)$. Excess of acid in this solution is determined by titration. If $\mathrm{r}_{3} \mathrm{cc}$. of $0.0 \mathrm{n}$-potassium hydroxide solution are used, then $20-\mathrm{r}_{3}=7 \mathrm{cc}$. of $0.0 \mathrm{r} \mathrm{n}$-hydrochloric acid correspond to the alkaloids in r.33 grams of extract. The equivalent weight of the two isomeric bases, atropine and hyoscyamine, being 289 , 1000 cc. of o.or n-hydrochloric acid correspond to 2.89 grams of alkaloids. The proportion

$$
\text { rooo: } 2.89=7: x(x=0.02023)
$$

shows that r.33 grams of belladonna extract contain 0.02023 gram of alkaloids corresponding to r.5I per cent. The German Pharmacopœia places this percentage as the minimum for total alkaloids in extract of belladonna.

\section{Extract of Hyoscyamus}

The alkaloids in 2 grams of this extract are determined in the manner described for extract of belladonna. Use ro cc. of o.I n-hỳdrochloric acid instead of $20 \mathrm{cc}$. to extract alkaloids. The German Pharmacopoia requires that not more than $6.5 \mathrm{cc}$. of $0.0 \mathrm{I} \mathrm{n}$-potassium hydroxide solution shall be used in titrating the excess of hydrochloric acid. Therefore ro $-6.5=3.5 \mathrm{cc}$. of $0.0 \mathrm{r}$-hydrochloric acid are combined with the alkaloids in 1.3 grams of henbane extract ( $=$ two-thirds of the original extract). The proportion

$$
1000: 2.89=3.5: x(x=0.0101 \mathrm{I})
$$


shows that I.33 grams of extract contain 0.oror gram of alkaloid, corresponding to 0.76 per cent. This percentage is placed as the minimum for total alkaloids in henbane extract.

\section{Assaying Officinal Extracts}

\section{(E. $\operatorname{Merck}^{1}$ )}

With a view to obviating as many sources of error as possible, E. Merck has proposed the following procedures:

Extract of Belladonna.-Dissolve 4 grams of extract in $6 \mathrm{cc}$. of water and wash the solution into a separating funnel with an additional ro cc. Add roo cc. of ether, shaking well, then $10 \mathrm{cc}$. of sodium carbonate solution $(\mathrm{r}: 3)$ and shake at once for 5 minutes. Stopper the funnel and let the mixture stand for 20 minutes. Then pass the ether layer through a dry filter ( $10 \mathrm{~cm}$. in diameter) into a glassstoppered flask. To lessen evaporation of ether as much as possible, cover the funnel with a glass plate. If an emulsion keeps the ether from separating well, add a few grams of powdered tragacanth at the end of the time stated above. Shake until the tragacanth gathers into balls in the aqueous layer. After 15 minutes decant $75 \mathrm{cc}$. of the ether layer. To check results by making more than one assay, use $25 \mathrm{cc}$. of the ether solution ( $=\mathrm{I}$ gram of extract). Test a clean glass-stoppered flask to make sure that it does not give up alkali to the water. Then introduce into such a flask $50-60 \mathrm{cc}$. of water, 5 drops of iodeosine solution and $20 \mathrm{cc}$. of ether. Shake and add $0.0 \mathrm{n}$-hydrochloric acid until the aqueous layer just becomes colorless upon shaking. This procedure obviates a special determination of the alkalinity of the water, since the resulting mixture is brought to the neutral point. Now add $25 \mathrm{cc}$. of the ether solution of the alkaloid and titrate until there is no color. Multiply the number of cc. of 0.0 . n-hydrochloric acid used by $0.00289 .{ }^{2}$ The product is the quantity of alkaloid, calculated as atropine, in I gram of belladonna extract. Upon the average this preparation contains $\mathrm{I} .8$ per cent. of alkaloid.

Extract of Cinchona.-Hæmatoxylin is frequently an unsatisfactory indicator in the titration of cinchona alkaloids, because the color change is slow enough to make it difficult to fix the end-point exactly. Therefore E. Merck makes a gravimetric and volumetric determination at the same time by the following method:

Dissolve 3 grams of aqueous cinchona extract in to cc. of water in a porcelain dish. Pour the solution into a $250 \mathrm{cc}$. shaking flask, rinsing it in with ro cc. of water. Add $150 \mathrm{cc}$. of ether and $10 \mathrm{cc}$. of sodium carbonate solution $(\mathrm{t}: 3)$ to this mixture and shake vigorously for ro minutes. Cork the flask and let the mixture stand at rest for 30 minutes. This extract frequently forms an emulsion. In that case add a few grams of tragacanth powder which has no effect upon the result. Pour the ether solution of cinchona alkaloids as rapidly as possible through a dry creased filter. Use $30 \mathrm{cc}$. (= I gram of cinchona extract) for each determination. Distil the solvent from the $50 \mathrm{cc}$. in a weighed $100 \mathrm{cc}$. flask and

${ }^{1}$ Zeitschrift für analytische Chemie 4I, 584 ( 1902 ) and also Merck's Bericht über das Jahr; 1900 .

${ }_{2}^{2} 29$ = the equivalent weight of the two isomeric bases, atropine and hyoscyamine, $\mathrm{C}_{17} \mathrm{H}_{23} \mathrm{NO}_{3}$. 
dry the residue in an air bath at $100-110^{\circ}$ to constant weight. The alkaloids obtained are nearly colorless or faintly yellow. Having ascertained the weight of the alkaloids, proceed with the titration. Dissolve the residue in the flask in ro cc. of alcohol, adding $50 \mathrm{cc}$. of water, which partially precipitates the alkaloids, and then alcoholic hæmatoxylin solution. ${ }^{1}$ Run in o.r n-hydrochloric acid until the alkaloids again dissolve and the red color of the solution passes through reddish yellow into a pure yellow. The mean equivalent weight of the cinchona alkaloids is 309. Therefore $\mathrm{r}$ cc. of $0.1 \mathrm{n}$-hydrochloric acid $=0.0309 \mathrm{gram}$ of alkaloid. Upon the average, officinal aqueous extract of cinchona contains 9 per cent. of alkaloid.

Extract of Nux Vomica.-Dissolve 0.1 gram of this extract in a flask in 5 grams of absolute alcohol and ro grams of water. Add 95 grams of ether and shake well. Then add 10 cc. of sodium carbonate solution $(\mathrm{r}: 3)$ and shake vigorously at once for about 10 minutes. After 15 minutes pour the ether solution as rapidly as possible through a creased filter. Weigh in a flask 50 grams of this solution $(=0.05$ gram of the original extract), having previously placed in this flask a neutral mixture of $50 \mathrm{cc}$. of water, $20 \mathrm{cc}$. of ether and 5 drops of iodeosine solution. Add $20 \mathrm{cc}$. of $0.0 \mathrm{r} \mathrm{n}$-hydrochloric acid and titrate with $0.0 \mathrm{r}$ $\mathrm{n}$-potassium hydroxide solution until the aqueous layer is just red.

Calculation.- Since strychnine and brucine are present in nux vomica in nearly equal parts, the mean equivalent weight of such a mixture of bases $(334+394): 2$ $=364$. Hence 0.00364 gram of the mixed alkaloids neutralizes $\mathrm{r}$ cc. of $0.0 \mathrm{r}$ n-hydrochloric acid. The officinal extract of nux vomica contains 18 per cent. of alkaloid.

${ }^{1}$ E. Merck advises keeping on hand an alcoholic solution of hæmatoxylin, because a freshly prepared solution usually gives a blue-violet instead of a red color change. 


\section{CHAPTER VII}

\section{DETECTION OF CARBON MONOXIDE BLOOD, BLOOD STAINS AND HUMAN BLOOD}

\section{Carbon Monoxide Blood}

Carbon monoxide ( $\mathrm{CO}$ ) has a direct toxic action upon the blood. This gas passed into blood displaces loosely bound oxygen from oxyhæmoglobin forming the more stable carboxyhæmoglobin. The latter compound is cherry-red, not dichroic and entirely resistant to putrefaction if air is excluded. In carbon monoxide poisoning the cherry-red color of the blood is usually noticed at once.

\section{Detection of Carbon Monoxide Blood}

I. Boiling Test.-Blood containing carbon monoxide gives a brick-red coagulum, if boiled or warmed upon the water-bath. Ordinary blood gives a grayish brown or brownish black precipitate.

2. Sodium Hydroxide Test.-Carbon monoxide blood shaken with I-2 volumes of sodium hydroxide solution (sp. gr. I.3 $=26.8$ per cent.) remains red and in a thin layer is the color of red lead or vermilion. Normal blood similarly treated is almost black and in a thin coating upon a porcelain plate is dark greenish brown. A procedure recommended consists in diluting the blood with 6-ro times its volume of water and using about 5 drops of sodium hydroxide solution to so cc. of diluted blood. Even gentle warming with sodium hydroxide solution (Io per cent. $\mathrm{NaOH}$ ) does not alter the red color of this carboxyhæmoglobin solution, whereas a solution of normal human blood becomes greenish to dark brown.

3. Basic Lead Acetate Test.-Mix 4-5 volumes of basic lead acetate solution in a test-tube with diluted or undiluted carbon monoxide blood and shake vigorously for a minute. 
Such blood remains bright red but normal blood is first brownish and then chocolate to greenish brown.

4. Potassium Ferrocyanide Test.-Mix undiluted blood (I 5 cc.) with an equal volume of 20 per cent. potassium ferrocyanide solution and $2 \mathrm{cc}$. of diluted acetic acid. ${ }^{1}$ Shake the mixture gently and a coagulum will gradually form. That from normal blood is dark brown but from blood containing carbon monoxide bright red. This difference disappears slowly but not entirely for weeks.

5. Tannin Test.-Mix an aqueous blood solution ${ }^{2}$ with 3 times its volume of I per cent. tannin solution and shake thoroughly. A difference in color between normal and carbon monoxide biood can be recognized after several hours, most distinctly after 24 hours. Normal blood is gray but carbon monoxide blood is crimson-red. This difference is apparent even after several months. Ten per cent. of carboxyhæmoglobin can be detected in blood by tests 4 and 5 .

6. Copper Sulphate Test.-A drop of saturated copper sulphate solution added to $2 \mathrm{cc}$. of carbon monoxide blood mixed with the same volume of water gives a brick-red precipitate. The deposit from normal blood is greenish brown. In all these precipitation tests $(4,5$ and 6$)$ the less easily decomposed carbon monoxide blood remains bright red but the more easily decomposed normal blood in presence of the precipitants used and others is off color or dark.

7. Ammonium Sulphide Test.-Mix 0.2 cc. of ammonium sulphide solution and $0.2-0.3 \mathrm{cc}$. of 30 per cent. acetic acid with Io cc. of 2 per cent. aqueous blood solution. Carbon monoxide blood gives a fine rose color but normal blood is greenish gray. The former within 24 hours gives a red flocculent precípitate.

8. Palladous Chloride Test.-Carbon monoxide precipitates black metallic palladium from a neutral aqueous palladous chloride solution:

$$
\mathrm{CO}+\mathrm{H}_{2} \mathrm{O}+\mathrm{PdCl}_{2}=\mathrm{CO}_{2}+{ }_{2} \mathrm{HCl}+\mathrm{Pd} \text {. }
$$

${ }^{1}$ Mix I volume of glacial acetic acid with 2 volumes of water. This acid contains about 30 per cent. acetic acid.

${ }^{2}$ Use I part of blood to 4 parts of water. 
Mix a few drops of potassium hydroxide solution with the blood and warm gently upon the water-bath. By means of a suction pump draw through the solution air that has been washed until pure. Pass the gas evolved first through lead acetate solution to remove possible hydrogen sulphide, then through sulphuric acid to absorb ammonia and finally through a neutral light red palladous chloride solution (I: 500).

9. Spectroscopic Examination.-The detection of carboxyhæmoglobin with the spectroscope is comparatively easy. The

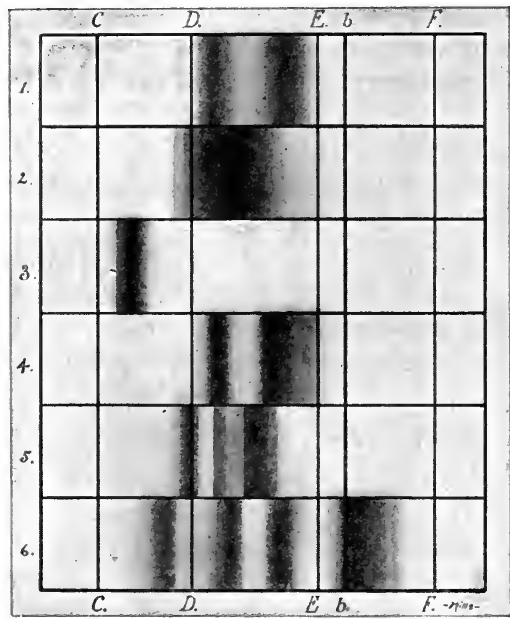

Oxyhæmoglobin.

Hæmoglobin.

Methæmoglobin.

Carboxyhæmoglobin.

Hæmatoporphyrin, very dilute, acid.

Hæmatoporphyrin, not so dilute, alkaline.

FIG. 24--Absorption-Spectra.

two absorption-bands of this compound are quite similar to those of oxyhæmoglobin but they lie somewhat nearer together and more toward the violet. The main difference, however, between the absorption-bands of these compounds is that those of carboxyhæmoglobin are not extinguished by reducing agents. To prepare the blood solution for spectroscopic examination, dilute I-I.5 parts of blood with Ioo parts of water and make the 
observations through a layer $\mathrm{r} \mathrm{cm}$. thick. To reduce $\mathrm{I}$ per cent. blood solution, mix thoroughly with a few drops of ammonium sulphide solution and add $4^{-6}$ drops more of the same reagent as a surface layer to exclude air. Reduction begins in about 6-8 ininutes. A solution of tartaric acid and ferrous sulphate in presence of an excess of ammonium hydroxide solution will also reduce oxyhæmoglobin.

Oxyhæmoglobin under these conditions is changed to reduced hæmoglobin. The two absorption-bands characteristic of the former disappear and a broad diffuse absorption-band occupies the previous bright space between the two bands. The spectrum of carboxyhæmoglobin remains unchanged only when 27 per cent. at least of the hæmoglobin is saturated with carbon monoxide. If allowed to stand in an open vessel, blood will lose carbon monoxide within 8 days. But carbon monoxide blood sealed in glass tubes is said to keep for years. Carbon monoxide has been detected in blood of a cadaver after 18 months.

Tollens ${ }^{1}$ recommends adding sóme formaldehyde to the blood solution. This reagent has not the slightest effect upon the two oxyhæmoglobin bands. Warming the mixture very gently with ammonium sulphide solution develops a third and nearly as distinct black band almost midway between the original bands which gradually disappear. Finally only this band will remain. This is a far more satisfactory test than that given by the indefinite band of blood alone. If the solution is cooled and agitated with air, this third band will disappear and the two original oxyhæmoglobin bands will return.

If carbon monoxide is present, formaldehyde does not have this action.

\section{Detection of Blood Stains}

The detection of blood in dry stains upon fabrics, wood, knives, weapons, etc., ${ }^{2}$ is more certain and less open to question, if hæmin crystals (Teichmann's blood crystals) are prepared from the blood pigment. If hæmin crystals are obtained, the stain in question may be regarded with certainty as due to blood. Fresh blood when dry is bright red and has a smooth surface.

${ }^{1}$ Berichte der Deutschen chemischen Gesellschaft 34, I426 (1901).

${ }^{2}$ Blood mixed with iron oxide as, for example, blood upon rusty knives and weapons usually fails to give hæmin crystals. 
Flakes of such blood scraped from any material are garnet-red by transmitted light. A solution of fresh blood stains in potassium or sodium hydroxide is dichroic, being red by transmitted and green by reflected light. Later dried blood becomes brownish red or dark brown. These color changes are due to conversion of oxyhæmoglobin into methæmoglobin and then into hæmatin. The first two substances are soluble in water but the last is not. But hæmatin is soluble in alkalies and in alcohol containing sulphuric acid. This change of the blood pigment depends not only upon the age of the stain but really upon the action of air (oxygen), light,. heat and moisture upon the blood before it is dry. If the blood is in a thin layer, hæmoglobin will sometimes change into methæmoglobin even in 3-10 days. Boiling water causes immediate insolubility. The action is also very rapid in direct sunlight. Washing in alkaline solutions (boiling solutions of potassium or sodium soap, sodium carbonate solution, ammonia and sewage) also causes rapid decomposition. But acids, nitric and hydrochloric, as well as putrefaction, act more slowly, giving the blood a laked appearance and even making it clear and colorless. If, however, the blood has once dried, these injurious agencies, even putrefaction, act less easily.

Preparation of Hæmin Crystals.-Prepare a cold aqueous extract of the stain as free as possible from fibers and evaporate the solution upon a watch-glass away from dust. Add a trace of sodium chloride ${ }^{1}$ to the residue, also 8-ro drops of glacial acetic acid, and stir with a glass rod. Heat just for an instant over a small flame, then evaporate the solution gradually upon a moderately warm water-bath and examine the residue with a microscope magnifying 300-500 times. If hæmin

${ }^{1}$ Strzyzowski (Chemisches Centralblatt, r 897, I, 295) advises using sodium iodide instead of sodium chloride. Place a small particle of material suspected of containing blood upon a glass slide and add a drop of sodium iodide solution ( $1: 500)$. Evaporate and cover with a cover-glass. Heat for $3^{-6}$ seconds with concentrated acetic acid which is allowed to run under the cover-glass. The test with this modification is said to be more delicate, owing to the darker color of the hæmatin hydriodide crystals. The crystals are usually obtained in less time and with as small a quantity as 0.000025 gram of fresh blood. Tr. 
crystals fail to appear, repeat the evaporation several times, using in each instance 8-ro drops of glacial acetic acid, and examine the residue each time under the microscope. Hæmin crystals are brownish red to dark brown and form rhombic scales which frequently lie crossed (Fig. 25). Usually glacial acetic acid is the only solvent that will extract the pigment from old blood stains. Brucke heats the stains or scrapings to boiling in a test-tube with ro-20 drops of glacial acetic acid. The decanted or filtered solution, after addition of a trace of sodium chloride, is evaporated upon a watch-glass to dryness at $40-80^{\circ}$ and the residue is examined under the microscope. By this

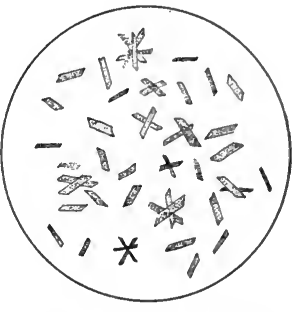

FIG. 25.-Hæmin Crystals. method it is immaterial whether the blood has coagulated or not.

Cold water is without effect upon blood stains, if they have previously been treated with hot water. Protein substances in the blood are thus coagulated and rendered insoluble. In such a case treat the stain with water containing a few drops of sodium hydroxide solution. If the stains are upon wool, use very dilute sodium hydroxide solution since alkalies dissolve wool. Water containing ammonium hydroxide will extract stains and this alkali does not act upon wool. Use the alkaline aqueous extract to prepare hæmin crystals. Evaporate the solution to dryness in a watch-glass upon the water-bath and mix the residue intimately with 8-10 drops of glacial acetic acid. Add a trace of sodium chloride and again evaporate. Sometimes it is advisable, after acidifying the extract of the stain with acetic acid, to add tannic acid, or zinc acetate, and prepare Teichmann's crystals from the precipitate.

Occasionally it is necessary to extract suspected stains with hot alcohol containing sulphuric acid. Hæmatin formed from the blood pigment dissolves. If this compound is present, the solution has a brown color. Excess of sodium hydroxide solution will produce the dichroism characteristic of an alkaline 
hæmatin solution, namely, red by transmitted and green by reflected light. Obviously, hæmatin should be identified by the spectroscope both in acid and alkaline solution.

Blood mixed with iron oxide (blood upon rusty weapons) usually fails to give hæmin crystals but the extract with dilute sodium hydroxide solution frequently shows the dichroism of hæmatin solution. Since iron oxide or rust forms an insoluble compound with hæmatin, warm such stains for some time upon the water-bath with sodium hydroxide solution to dissolve any hæmatin present.

Hæmatin.-Warming an aqueous blood solution to about $70^{\circ}$ decomposes the blood pigment oxyhæmoglobin into a protein substance called globin and hæmatin, a pigment containing iron. Acids, alkalies and several metallic salts decompose oxyhæmoglobin in the same way. If this decomposition takes place in the absence of oxygen, another pigment appears. Hoppe-Seyler gave the latter the name hæmochromogen and other experimenters have called it "reduced hæmatin." Oxygen and consequently air rapidly oxidizes this pigment to hæmatin. On the other hand reducing agents like ammonium sulphide convert hæmatin into hæmochromogen. Different formulæ are given for hæmatin. W. Küster and others now give it the formula $\mathrm{C}_{34} \mathrm{H}_{34} \mathrm{~N}_{4} \mathrm{FeO}_{5}$. Hæmatin is amorphous and has a dark brown or blue-black color. In water, dilute acids, alcohol, ether and chloroform it is insoluble but soluble in alcohol or ether containing acid. In even very dilute solutions of caustic alkalies it is freely soluble. Alkaline hæmatin solutions are dichroic. In rather thick layers the color appears red by transmitted light and greenish in thin layers. Acid solutions are always brown. Alkaline hæmatin solutions are precipitated by calcium or barium hydroxide solution.

Hæmin is the hydrochloric ester of hæmatin. Very probably hæmin has the empirical formula $\mathrm{C}_{34} \mathrm{H}_{33} \mathrm{~N}_{4} \mathrm{FeO}_{4} \mathrm{Cl}$.

Note.-If the blood stain is perfectly fresh, it may be recognized by observing blood-corpuscles with the microscope. Human blood can be differentiated from animal blood by comparing blood-corpuscles with those of animal blood as to size, only when the corpuscles are still intact.

\section{Spectroscopic Detection of Blood}

If the extract of a blood stain with cold water is already brown, a third fainter and narrower band will appear in addition to the two oxyhæmoglobin bands. This lies in the orange between $\mathrm{C}$ and $\mathrm{D}$ and is the methæmoglobin band. Cold 
water will dissolve most of the methæmoglobin from fresh dried blood stains.

Acetic acid will discharge these two bands, if the oxyhæmoglobin solution is not too dilute. At the same time the solution will become mahogany-brown from formation of hæmatin in acid solution. This solution has a characteristic spectrum, namely, four absorption-bands in the yellow and green. If excess of ammonium hydroxide is added to this solution, the alkaline solution contains hæmatin, recognizable by a broad faint absorption-band lying between the red and yellow. A few drops of ammonium sulphide solution will extinguish this band and bring out two broad bands, namely, one in the green and the other in the light blue. These bands lie farther to the right than do those of oxyhæmoglobin and are of about the same width. This is the spectrum of reduced hæmatin (hæmochromogen). All these spectroscopic tests are very characteristic, especially the spectra of oxyhæmoglobin, hæmoglobin and, in the case of old blood stains, that of reduced hæmatin.

Up to the present time no red solution has been found which, upon abstraction and addition of oxygen, will give the same spectroscopic phenomena as blood.

When the quantity of blood is very small, or when the blood pigment has undergone further decomposition, so that the bands of oxyhæmoglobin are no longer visible, it is advisable to extract the stains for several hours with concentrated potassium cyanide solution. Blood will give a light red or yellowish brown solution containing the cyano-compound of hæmatin. The spectrum of hæmatin in alkaline solution will appear as a broad, faint band.

The investigations of Kratter and Hammerl have shown that charred blood, which no longer responds to any of the other blood reactions, will still give the hæmatoporphyrin spectrum upon treatment with concentrated sulphuric acid (E. v. Hofmann, Lehrbuch der gerichtlichen Medizin, 1903).

Ammoniacal carmine solution gives two absorption-bands similar to those of oxyhæmoglobin but they do not change upon addition of acetic acid or ammonium sulphide. A band 
given by fuchsine analogous to that of hæmoglobin remains unchanged after agitation with air.

\section{Other Blood Tests}

I. Schönbein-Van Deen Ozone Test.-A mixture of ozonized turpentine ${ }^{1}$ and alcoholic tincture of guaiac resin, shaken with a little blood, produce a light blue color. Separated from the turpentine, the tincture is deep blue. Though very delicate, this test is not characteristic of blood, for many inorganic and organic substances under the same conditions produce "guaiac blue." Nitrous acid, chlorine, bromine and iodine, chromic and permanganic acids, ferric and cupric salts produce blue solutions direct with guaiac resin. In examining blood stains usually it is possible to exclude these substances beforehand. But other substances like cell contents or hæmoglobin, having the power of transferring ozone, may attach false significance to the guaiac-blue reaction. Enzymes (diastases), hydrolytic ferments (enzymes in the narrower sense), as well as the so-called oxidation ferments (oxidases), are organic substances of this character. They occur in different parts of plants, especially in fungi and in seeds. Saliva, extracts of certain organs, contents of white blood-corpuscles and pus cells are animal products of similar nature. E. Schaer ${ }^{2}$ states that these animal and vegetable substances differ from hydrogen dioxide in being catalytic in action and carriers of oxygen at the same time. And also that a temperature of $100^{\circ}$, or contact with hydrocyanic acid, completely destroys their power of transferring oxygen, or at least greatly diminishes it, in which respect they are essentially different from hæmoglobin. Neither high temperature $\left(100^{\circ}\right)$ nor hydrocyanic acid has any restraining influence upon hæmoglobin so far as transference of oxygen is concerned. Consequently an extract, containing one of these ferment-like substances but no blood, placed even for a

${ }^{3}$ Turpentine always contains ozone, if exposed to light for a long time in a loosely stoppered bottle.

${ }^{2}$ Forschungsberichte über Nahrungsmittel, etc., 3, I (1896) and Archiv der Pharmazie 236, 57I (I898). 
short time in a hot water-bath, loses the power of giving the "guaiac blue" test. In absence of blood, the result will also be negative, if the extract of the suspected stain is treated with hydrocyanic acid. For these reasons great care is necessary in interpreting a positive guaiac test given by the extract of a supposed stain. The guaiac test is certainly very useful as a delicate preliminary test and in many instances as a check upon blood. The three forms of the blood pigment entering into such an examination, namely, hæmoglobin, methæmoglobin and hæmatin, are alike in the guaiac test, at least qualitatively, as far as transference of oxygen is concerned. The examination and extraction of the stain may, therefore, be conducted in neutral, acid or alkaline solution, depending upon the nature of the substance, and either hot or cold. Render an alkaline extract faintly acid with acetic acid before adding guaiac tincture. In many instances it is advisable to extract the blood stain with hot alcohol containing sulphuric acid. Treat such an acid, alcoholic hæmatin solution with guaiac tincture direct. Addition of water will precipitate the resin with the adherent blood pigment.

(a) Vitali's Procedure.-Extract the stain with water containing carbon dioxide, or old stains with very dilute sodium hydroxide ${ }^{1}$ solution free from nitrite and nitrate. F lter the extract and add a little alcoholic guaiac tincture to a portion of the filtrate after acidification with acetic acid, if necessary. If the milky liquid is not blue in I 5 minutes, interfering oxidizing agents are absent. Then add a few drops of old turpentine and shake. The milky liquid will turn blue at once, or in a short time, if blood pigment is present. Very.gentle warming upon the water-bath increases the delicacy of the reaction. Even putrid blood 2 months old is said to give a positive test.

(b) E. Schaer's Procedure.-Blood stains upon linen, though quite old, dissolve completely when treated for some time with 70 per cent. chloral hydrate solution. Moistening the stains beforehand with glacial acetic acid aids solution. Also prepare an extract of guaiac resin in 70 per cent. chloral hydrate

${ }^{1}$ In this test use sodium hydroxide prepared from metallic sodium. 
solution. Mix the extract of the stain with an equal volume of the latter solution. In absence of nitrites, the color of this mixture is brownish yellow to light brown. If preferred, a contact test for blood may be made by this method. Add to the mixture of blood and guaiac Hünefeld's ${ }^{1}$ turpentine solution, or hydrogen peroxide, as a surface-layer. An intense blue zone will appear where the two solutions meet. Guaiaconic acid in guaiac resin produces "guaiac blue." O. Döbner has suggested substituting a dilute solution of guaiaconic acid for guaiac resin. Blood, or blood pigment, behaves like a ferment and activates the ozonized turpentine or hydrogen peroxide, either of which by itself will not turn the solution of guaiac resin blue.

2. Schaer's Aloin Test.-The same conditions, producing "guaiac blue" from guaiaconic acid, give rise to "aloin red" from aloin. This substance has a stronger coloring power and lasts longer than "guaiac blue." Use the same solution of blood in 70-75 per cent. chloral hydrate solution mixed with a weak chloral hydrate solution of aloin. Add Hünefeld's hydrogen peroxide solution as a surface layer. After some time a violet-red zone will appear and a red color of equal intensity will gradually extend throughout the aloin solution. Another method of making this test consists in first extracting the blood stain with pure water, acetic acid, chloral hydrate solution or alkaline salt solution. Neutralize this solution and add dilute alcoholic aloin solution and hydrogen peroxide. If the suspected stain contains blood pigment, a red color will appear or once and persist for a long time.

\section{Biological Detection of Human Blood ${ }^{2}$}

Injection of bacteria produces specific, bacteriolytic bodies and similarly injection of the blood of one animal species into

${ }^{1}$ See page 321 for the preparation of this reagent.

2 This subject has been introduced for the sake of completeness. If such ${ }^{-}$an investigation is for forensic purposes, the chemist will either decline to undertake it, or conduct the experiment with an associate who has had bacteriological and pathological experience. 
an animal of a different species gives rise to specific, hæmolytic and agglutinating bodies. Rabbit's blood, for example, injected repeatedly into a guinea pig, develops in the serum of such a guinea pig substances capable of agglutinating and dissolving red corpuscles of the rabbit, setting hæmoglobin free and rendering the blood laky. Blood serum from an animal, into which defibrinated blood, or blood serum from a different animal species has been injected intravenously, subcutaneously or intraperitoneally, that is to say, into the peritoneal cavity, has the peculiar property of causing precipitation only in blood serum of this particular animal species. Uhlenhuth, ${ }^{1}$ Wassermann and Schütze, ${ }^{2}$ and others have made independent experiments of this kind with blood serum to find for forensic purposes a test, based upon this biological method, which shall differentiate human blood from the blood of every other animal species. Repeated injection of to cc. of defibrinated human blood, or human blood serum free from cells, into a rabbit, either intraperitoneally or subcutaneously, yields a serum producing a heavy, cloudy precipitate in an aqueous solution of human blood. This coagulin is specific in action, producing a precipitate only in presence of human blood. Wassermann and Schütze tested the blood of 23 different animals, among which were mammals, birds and fishes, and obtained negative results with blood solutions from these very different animal species. By use of blood serum it is possible to differentiate even old human blood, dried for many weeks, from the blood of other animals.

To demonstrate the use of this method, A. Dieudonné ${ }^{3}$ prepares I per cent. blood solutions, placing $2 \mathrm{cc}$. of the clear filtered solution in small test-tubes and adding an equal volume of double physiological salt solution ( $=$ r.8 per cent. $\mathrm{NaCl}$ ). Then add 6 drops of serum to each portion and place the tubes in an incubator at $37^{\circ}$. The serum of the rabbit, treated with

1 Deutsche medizinische Wochenschrift, rgor, No. 6; und Zeitschrift für Medizinalbeamte, 1903 , Heft 5 and 6.

${ }^{2}$ Berliner klinische Wochenschrift, 1901, No. 7 .

${ }^{3}$ Münchener medizinische Wochenschrift, 1901, page 533. 
human blood serum, added to an aqueous solution of 'human blood, produced in a few minutes a distinct flocculent precipitate which gradually became more and more marked. As a check, test also with normal rabbit's serum which will cause no precipitate in a solution of human blood. Dieudonné found also that rabbit's serum; obtained after injecting human blood serum, causes precipitates not only in human blood solutions but in human urine containing albumin, with an exudate from human pleura and with peritoneal exudate. But precipitation in the case of human blood was much more marked than in these other tests. In his experiments Dieudonné used blood expressed from the placenta, repeatedly.injecting it subcutaneously into rabbits in separate doses of ro cc. and at intervals of 3-4 days. The animals were bled several days after the last injection and the blood was kept upon ice.

The antiserum used in detecting blood should above everything else be perfectly clear. To prepare such serum, use a sterile Berkefeld filter attached to a water pump. The antiserum should be active in very dilute solution. Distinct turbidity should appear immediately in a solution diluted I : Io00, or in $\mathrm{I}-2$ minutes at latest. Sera must be of this high efficiency for practical use. Uhlenhuth has shown that the biological method of detecting blood is specific for human albumin. A necessary consequence of this fact is that the material should first of all be shown to be blood. The first question for the expert to answer in such an investigation must always be: "Is there any blood at all present?" If the answer is affirmative, the next question is: "Is it human or animal blood?" Consequently the material should first be examined for blood stains by van Deen's ozone test, Teichmann's hæmin test and by the spectroscope. If the suspected stains are upon a hard surface, as a knife, hatchet, gun-barrel, wood, stone, etc., they should be scraped off for the biological blood test and extracted for several hours in a test-tube with physiological salt solution ( $=0.9$ per cent. $\mathrm{NaCl}$ ). First, filter the extract through paper. If the filtrate is not clear, next use a Berkefeld filter. 


\section{APPENDIX}

\section{PREPARATION OF REAGENTS}

General Alkaloidal Reagents.-A class of reagents, known as general alkaloidal reagents, added to solutions of most of the alkaloids or of their salts, produce precipitates characterized by their color, their amorphous or crystalline appearance and their insolubility or sparing solubility in water. But these reagents do not precipitate alkaloids exclusively. Several members of this class, for example, the chlorides of gold, platinum and mercury, phospho-molybdic and phospho-tungstic acids, react similarly with ammonia and many ammonium derivatives. An explanation of this similarity in behavior is found in the fact that most of the alkaloids, being secondary or tertiary bases, are themselves ammonium derivatives. Nearly all the general alkaloidal reagents also precipitate proteins, albumoses, peptones, creatinine and the nuclein bases, adenine, guanine, hypoxanthine and xanthine.

The general alkaloidal reagents are especially useful in detecting the presence, or absence, of alkaloids and other basic compounds. If there is only a slight residue from the ether extract of the alkaline solution in the Stas-Otto method, test first with the general alkaloidal reagents and then, if necessary, for individual alkaloids. To perform these tests, dissolve the given residue in very dilute hydrochloric or sulphuric acid, distribute the filtered solution upon several watch-glasses and add to each portion a drop of the more sensitive reagents. If an alkaloid or any other basic substance is present, distinct precipitates or at least decided cloudiness will appear in all or in nearly all of the tests.

The most important general alkaloidal reagents are the following:

Gold Chloride dissolved in water $(\mathrm{x}: 30)$ produces white, yellow or brown precipitates which are amorphous or crystal- 
line. These precipitates decompose to some extent with separation of metallic gold.

Platinum Chloride dissolved in water ( $1: 20$ ) produces yellowish white to yellow precipitates which are usually granular and crystalline. These precipitates are usually analogous in composition to ammonium chloroplatinate, $\left(\mathrm{H}_{4} \mathrm{~N}\right)_{2} \mathrm{PtCl}_{6}$.

Mercuric Chloride dissolved in water $(\mathrm{I}: 2,0)$ produces white to yellowish precipitates which are usually amorphous but gradually become crystalline.

Iodo-potassium Iodide, prepared by dissolving 5 parts of iodine and ro parts of potassium iodide in roo parts of water, produces brown precipitates which are usually flocculent.

Potassium Cadmium Iodide, prepared by dissolving 20 grams of potassium iodide in $20 \mathrm{cc}$. of boiling water, adding ro grams of cadmium iodide and diluting to roo cc., produces white or yellowish precipitates with sulphuric acid solutions of most of the alkaloids, even when these solutions are very dilute. These precipitates, at first amorphous but later crystalline, dissolve in an excess of the reagent and also in alcohol.

Potassium Bismuthous Iodide may be prepared according to Kraut $^{1}$ by dissolving 80 grams of bismuth subnitrate in 200 grams of nitric acid (sp. gr. I.I $8=30$ per cent. $\mathrm{HNO}_{3}$ ) and pouring this solution into a concentrated solution of 272 grams of potassium iodide in water. Allow the potassium nitrate to crystallize and dilute the solution with water to rooo cc. This reagent produces orange-red precipitates with sulphuric acid solutions of many alkaloids. By shaking these precipitates with sodium hydroxide and carbonate solution, it is often possible to recover the alkaloids unchanged and sometimes almost quantitatively.

Potassium Mercuric Iodide, prepared by dissolving I.35 grams of mercuric chloride and 5 grams of potassium iodide in roo cc. of water, produces white or yellowish precipitates with hydrochloric acid solutions of most of the alkaloids. These precipitates, at first amorphous, gradually become crystalline.

${ }^{2}$ Annalen der Chemie und Pharmazie, 2 10, 310 (1882) und Archiv der Pharmazie, 235, I52 (x897). 
Potassium Zinc Iodide is prepared by dissolving to grams of zinc iodide and 20 grams of potassium iodide in $100 \mathrm{cc}$. of water.

Phospho-molybdic Acid may be prepared by either of the following thethods:

(a) Saturate sodium carbonate solution with pure molybdic acid, add I part of crystallized disodium phosphate $\left(\mathrm{Na}_{2} \mathrm{HPO}_{4}\right.$ I $2 \mathrm{H}_{2} \mathrm{O}$ ) to 5 parts of the acid and evaporate to dryness. Fuse the residue in a porcelain crucible and dissolve the cold melt in water. Prepare ro parts of solution from I part of this residue. Add enough nitric acid to the filtered solution to produce a golden yellow color.

(b) If molybdic acid is not at hand, completely precipitate at $40^{\circ}$ with excess of sodium phosphate solution the nitric acid solution of ammonium molybdate used in testing for phosphoric acid. Thoroughly wash the yellow precipitate, add water and dissolve in warm concentrated sodium carbonate solution. Evaporate this solution to dryness and fuse the residue until ammonia is completely expelled. If there is any reduction (blue or black color), moisten the residue with nitric acid and fuse again. Dissolve this residue in hot water and add nitric acid in large excess. Prepare ro parts of solution from I part of residue. The golden yellow solution should be protected from ammonia vapor.

Phospho-molybdic acid produces yellowish, amorphous precipitates with sulphuric acid solutions of most of the alkaloids. After a while these precipitates are frequently greenish or bluish from reduction of molybdic acid to molybdic oxide.

Phospho-tungstic Acid, prepared by adding a little 20 per cent. phosphoric acid to an aqueous solution of sodium tungstate, produces precipitates similar to those given by phosphomolybdic acid.

Tannic Acid is a 5 per cent aqueous solution of tannin. This reagent produces whitish or yellowish, flocculent precipitates partially soluble in hydrochloric acid. Alkaloids may be recovered in part from these precipitates by treating them with lead or zinc carbonate, evaporating to dryness and extracting the residue with ether, alcohol or chloroform. 
Picric Acid is a concentrated aqueous solution of picric acid which produces yellow crystalline precipitates, or amorphous precipitates which soon become crystalline.

Picrolonic Acid is used as 0.I normal alcoholic solution by dissolving 26.4 grams of solid picrolonic acid $\left(\mathrm{C}_{10} \mathrm{H}_{8} \mathrm{~N}_{4} \mathrm{O}_{5}\right)$ in a liter of alcohol. With most of the alkaloids this solution produces salts called picrolonates which are crystalline, difficultly soluble and yellow to red in color. Picrolonic acid behaves toward bases like a monobasic acid. ${ }^{1}$

\section{B. Other Reagents and Solutions}

Erdmann's Reagent.-Sulphuric acid containing nitric acid, prepared by adding to $20 \mathrm{cc}$. of pure concentrated sulphuric acid ro drops of a solution of 6 drops of concentrated nitric acid in $100 \mathrm{cc}$. of water.

Froehde's Reagent.-A solution of molybdic acid in sulphuric acid, prepared by dissolving $5 \mathrm{mg}$. of molybdic acid, or sodium molybdate', in I cc. of hot, pure concentrated sulphuric acid. This solution, which should be colorless, does not keep long.

Fehling's Solution.-The two following solutions, which should be kept separate, are used in preparing this reagent:

I. Copper Sulphate Solution.-Dissolve 34.64 grams of pure crystallized copper sulphate $\left(\mathrm{CuSO}_{4} \cdot \mathrm{H}_{2} \mathrm{O}\right)$ in sufficient water to make $500 \mathrm{cc}$.

2. Alkaline Rochelle Salt Solution.-Dissolve 173 grams of Rochelle salt (K.Na. $\mathrm{C}_{4} \mathrm{H}_{4} \mathrm{O}_{6} \cdot 4 \mathrm{H}_{2} \mathrm{O}$ ) and 50 grams of sodium hydroxide in hot water and dilute this solution when cold to $500 \mathrm{cc}$.

These two solutions, mixed volume for volume, constitute Fehling's solution which should be prepared just before being used. Fehling's solution, which has been made up and kept, should always be tested before being used. The solution should not be used, if it gives a red precipitate of cuprous oxide when warmed by itself.

${ }^{1}$ L. Knorr, Berichte der Deutschen chemischen Gesellschaft, 30, 914 (1897); H. Matthes and O. Rammstedt, Zeitschrift für analytische Chemie 46, 565 and Archiv der Pharmazie 245, II 2 (1907). 
Formaldehyde-sulphuric Acid.-Add 2-3 drops of aqueous formaldehyde solution (formalin) to $3 \mathrm{cc}$. of pure concentrated sulphuric acid just before using.

Günzturg's Reagent. ${ }^{1-D i s s o l v e ~ I ~ p a r t ~ o f ~ p h l o r o g l u c i n o l ~ a n d ~}$ I part of vanilline in 30 parts of alcohol. This reagent is used to detect free mineral acid, especially hydrochloric acid, but it does not react with free organic acids.

Hünefeld's Solution.-Add $25 \mathrm{cc}$. of alcohol, $5 \mathrm{cc}$. of chloroform and I.5 cc. of glacial acetic acid to $15 \mathrm{cc}$. of old turpentine which has been exposed for some time to air and light. The turpentine used should not produce a blue color with guaiac tincture direct nor with ${ }_{5} \mathrm{cc}$. of $3-5$ per cent. hydrogen peroxide free from acid. This solution is used in the detection of blood.

Iodic Acid Solution.-Prepare a Io per cent. aqueous solution of iodic acid $\left(\mathrm{HIO}_{3}\right)$.

Magnesia Mixture.-Dissolve II grams of crystallized magnesium chloride $\left(\mathrm{MgCl}_{2} \cdot 6 \mathrm{H}_{2} \mathrm{O}\right)$, and $\mathrm{I}_{4}$ grams of ammonium chloride in $\mathrm{r} 30 \mathrm{cc}$. of water and add 70 grams of allmonium hydroxide solution (sp. gr. $0.96=$ Io per cent. of $\mathrm{NH}_{3}$ ). This mixture should be clear. It is used to detect arsenic and phosphoric acids.

Mandelin's Reagent.-Dissolve I part of ammonium metavanadate $\left(\mathrm{H}_{4} \mathrm{~N} . \mathrm{VO}_{3}\right)$ in 200 parts of pure concentrated sulphuric acid.

Millon's Reagent.-Dissolve I part of mercury in I part of cold fuming nitric acid. Dilute with twice the volume of water and decant the clear solution after several hours.

Nessler's Reagent.-Dissolve separately in the cold 3.5 grams of potassium iodide in Io cc. of water and I.7 grams of mercuric chloride in $30 \mathrm{cc}$. of water. Add mercuric chloride solution to potassium iodide solution until there is a permanent precipitate. Dilute with 20 per cent. sodium hydroxide solution until the volume is roo cc. Add mercuric chloride solution, until there is again a permanent precipitate and let the solution

1 It is advisable to prepare this reagent as required. Keep two, separate alcoholic solutions $(\mathrm{r}: \mathrm{r}$ ) of phloroglucinol and vanilline and mix volume for volume as needed. Tr. 
settle. Decant the clear solution and keep in small bottles in the dark. This reagent improves upon standing.

Mecke's Reagent. ${ }^{1}$-Dissolve 0.5 gram of selenious acid in Io grams of pure concentrated sulphuric acid.

Stannous Chloride Solution.-Mix 5 parts of crystallized stannous chloride with I part of hydrochloric acid and completely saturate with dry hydrochloric acid gas. Let this solution settle and filter through asbestos. It is a pale, yellowish, refractive liquid (sp. gr. at least r.9). This solution is used to detect arsenic (Bettendorff's Arsenic Test).

\section{The Indicator Iodeosine}

Iodeosine, or erythrosine, $\mathrm{C}_{20} \mathrm{H}_{8} \mathrm{I}_{4} \mathrm{O}_{5}$, is a tetra-iodo-fluoresceine, formed by treating fluoresceine with iodine, and has the formula:

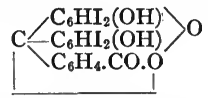

The commercial preparation usually contains as impurities small quantities of substances almost insoluble in ether. To obtain a pure product, ${ }^{2}$ dissolve commercial iodeosine in aqueous ether and extract iodeosine from the filtered ether solution by means of dilute sodium hydroxide solution. Strong sodium hydroxide solution, added to this aqueous alkaline solution, precipitates the sodium salt of iodeosine. Filter, wash with cold alcohol and crystallize from hot alcohol. Well formed, almost rectangular plates having a green color on the surface are obtained. Hydrochloric acid precipitates pure iodeosine from the aqueous solution of the sodium salt. Pure iodeosine dried at $120^{\circ}$ is markedly lighter than the commercial preparation. It is almost insoluble in absolute ether, benzene and chloroform; more easily soluble in acetone, alcohol and aqueous ether. The tone of the purified pigment dissolved in aqueous alkali is yellower than that of the crude product. Iodeosine is a scarlet crystalline powder which dissolves in alcohol with a deep red and in ether with a yellowish red color. Iodeosine is said to be insoluble in water containing a trace of hydrochloric acid. To prepare iodeosine solution for use as an indicator, dissolve $\mathrm{I}$ gram of the pigment in 500 grams of alcohol.

${ }^{1}$ Zeitschrift für offentliche Chemie 5, 350 (1899).

${ }^{2}$ Fr. Mylius and F. Foerster, Berichte der Deutschen chemischen Gesellschaft 24,1482 (r89r). 


\section{INDEX OF NAMES}

A

Abel, R., Biological arsenic test, 244 . Mould for same, 245 .

Archangelsky, Chloral hydrate estimation, 4r. Acetone on dogs, 55 .

Autenrieth, W., Phenacetine test, 76 . Morphine in putrefaction, 137 . Destruction of organic matter, $\mathrm{r}_{5} \mathrm{x}$. Copper in organs, $\mathrm{x} 78$.

\section{B}

v. Babo, Destruction of organic matter, 148. Detection of arsenic, $\mathrm{r} 6 \mathrm{r}$.

Barger, Ergotoxine, 209.

Baudin, Cantharidin in Spanish flies, 264 .

Baumann, E., Carbolic acid in putrefaction, 27.

Baumert, Nitric acid detection, 185 .

Bazlen, M., Maltol, 25 r.

Beck, Aconine, 262.

Beckurts, H., Carbolic acid estimated, 3r. Arsenic isolated as trichloride, 233. Theobromine and caffeine in cacao and chocolate, 298.

Beissenhirtz, Chromic acid test for aniline, 45 .

Benedikt, R., Compound of carbolic acid and bromine, 29.

Bernard, Arsenic reagent, 247.

Berthelot, M., Ethyl alcohol test, 50.

Bertrand, Normal arsenic, 175.

Berzelius, Arsenic test, ${ }_{5} 6$.

Bettendorff, Arsenic test, 162. Preparation of reagent, 322 .

Beuttel, F., Carbolic acid determination, 31 .

Biggs, Methyl alcohol test, 53 .

Biginelli, Gas from biological arsenic test, 243 .
Bischoff, C., Carbolic acid in the body, 27. Oxalic acid in the body, 190 . Potassium chlorate in putrefaction, 197 .

Black, Gutzeit estimation of arsenic, 240.

Bloemendal, Normal arsenic, 176 .

Blondlot, Phosphorus test, 8.

Bodlander, Tin poisoning, $\mathrm{r} 8 \mathrm{r}$.

Borsche, Composition of isopurpuric acid, $7 \mathbf{r}$.

Bougault, J., Arsenic reagent, 247 .

Boughton, W. A., Apparatus for Gutzeit test, 240 .

Brand, J., Maltol, $25 \mathrm{r}$.

Brandl, J., Haemolysis by githagin, 224.

Bredemann, Colchicin estimation, 269.

Brucke, Acetic acid for blood stains, 309.

Bruger, P., Composition of picrotoxin, 67.

Brunner, H., Caffeine and theobromine estimation, 272 .

Brunner, K., Saponin in beverages, 223.

Büdinger, Chloroform in mucus, 35 .

Buttenberg, Biological arsenic test, 244. Mould for same, 245.

\section{C}

Caesar, Estimation of pilocarpine, 286.

Caillot, Piperine in pepper, 288

Carles, J., Quinine estimation, 268.

Carlson, C. E., Arsenic in organic compounds, 245 .

Carr, F. H., Ergotoxine, 209.

Cazeneuve, Piperine in pepper, 288.

Cerný, Normal arsenic, $\mathbf{1} 76$.

Chancel, Solubility of carbon disulphide, 46. 
Chittenden, Arsenic in the brain, I74.

Ciamician, Structure of pseudo-pelletierine, 270.

Ckindi, Solubility of carbon disulphide, 46 .

Couerbe, Narcotine test, Ir5.

Dale, H. H., Ergotinine, 2 ro.

Denigès, Methyl alcohol test, 54 . Cocaine test, rog.

Dieterich, K., Cantharidin in Spanish flies, 264. Analysis of cola nuts and extracts, 272 .

Dieudonné, A., Biological blood test, 315.

Dilthey, A., Veronal preparation, 80.

Döbner, O., Guaiaconic acid in blood test, 3 I4.

Doepmann, Morphine in putrefaction, 137 .

Dragendorff, G., Phosphorus in cadavers, r6. Narcotine test, Ir5. Detection of cantharidin, 205 .

Dusart, Phosphorus test, 8.

\section{E}

Eichwede, Compound of carbolic acid and bromine, 29.

Elfstrand, Blood agglutinated by ricin, 229.

Elvers, Phosphorus in cadavers, $\mathrm{I} 6$.

Engel, Arsenic reagent, 247.

v. Engelhardt, R., Aniline black in the organism, 44.

Erdmann, Narcotine test, Ir5. Narceine test, I39. Thebaine test, 228. Reagent prepared, 320 .

\section{F}

Faust, Morphine in the organism, I37.

Fehling, Reagent prepared, 320.

Fihlene, Nitrobenzene absorptionband, 42.

Fischer, A., Interference with phosphorus test, 8 .
Fischer, B., Chloroform estimation, 38 . Distribution of same in cadavers, 38. Distribution of ethyl alcohol in organism, 49 .

Fischer, E., Veronal preparation, 8o. Excretion of same, 8r. Recovery from urine, 82 . Murexide reaction, 85 .

Fleury, G., Morphine test, r35. Nitric acid detection, 185 .

Flückiger, F. A., Carbolic acid test, 29. Foerster, F., Preparation of pure iodeosine, 322 .

Forster, A., Estimation of caffeine in coffee, 272.

Fränkel, A., Estimation of phosphorus in phosphorated oils, 232 .

Frerichs, Alkaloids in ipecac, 277.

Frerichs, G. and H., Toxicological examination for veronal, $8 \mathrm{I}$.

Fresenius, C. R., Phosphorus test, ro. Destruction of organic matter, r48. Detection of arsenic, r6r. Freund, M., Structure of veratrine alkaloids, 95. Narcotine structure, Ir4. Identity of pseudo-narceine and narceine, $\mathrm{r}_{3} 8$. Aconine, 262. Froehde, Test for codeine, II2. For narcotine, Ir5. For hydrastine, ri8. For apomorphine, I28. For morphine, 134. For papaverine, 217. For saponins, 222. For solanine and solanidine, 227. For thebaine, 228. For cephaeline, 278. Preparation of reagent, 320 .

Fromme, G., Pilocarpine in jaborandum, 286. Theobromine and caffeine in cacao and chocolate, 299. de Fuentas Tapis, Alkaloids in ipecac, 277.

Fühner, H., Thalleioquin reaction, $\mathbf{2} 2 \mathbf{r}$. Fujiwara, Chloroform test, 37 .

G

Gadamer, J., Cantharidin structure, 203. Structure of cantharic acid and acetyl-hydrato-cantharic an- 
hydride, 204. Caffeine in tea, coffee and cola, 272 .

Gair, D., Estimation of strychnine and quinim together, 258 .

Gautier, A., Normal arsenic, I 74. Destruction of organic matter, 234.

Girard, Ch., Detection of salicylic acid in milk, $25 x$.

Goeckel, H., Caffeine, 275.

Goldschmiedt, G., Structure of papaverine, 216.

Gordin, H. M., Estimation of alkaloids, 257. Of strychnine, 298.

Gosio, B., Moulds on arsenical media, 242.

Graf, L., Caffeine in tea, 272.

Grandeau, Veratrine test, 96 .

Guareschi, Solubility of quinine sulphate, 268.

Guglialmelli, Pyramidone test, I25.

Gunning, Acetone differentiated from ethyl alcohol, 56 .

Günzburg, Mineral acid test, I82. Reagent for mineral acid, $32 \mathrm{I}$.

Gutzeit, Arsenic test, I $_{3}$.

\section{$\mathrm{H}$}

Halász, Z., Modification of BlondlotDusart phosphorus test, 13. Organic phosphorus compounds, I3.

Hammerl, Examination of charred blood, 3 II.

Harmsen, Carbon disulphide on lipoids, 46 .

Harrison, E. F., Estimation of quinine and strychnine, 258.

Helch, H., Pilocarpine test, 219.

Hildebrandt, H., Chlorate in urine, 197.

Hilger, Phosphorus test, II. Reduction of phosphorous acid to phosphine, 14. Apparatus to estimate phosphorus, 15. Sensitiveness of Mitscherlich test, 16. Caffeine in coffee and tea, $27 \mathrm{I}$.

Hille, W., Solubility of quinine sulphate, 268.

Hinkel, Methyl alcohol test, 54 .
Hinsberg, Phenacetine test, 76 .

Hirschsohn, Quinine test, I 22.

Hödlmoser, Normal arsenic, 176 .

Hofmann, A. W., Sensitiveness of phenylisocyanide test, 36. Exhaustive methylation, II 7 .

v. Hofmann, E., Haematoporphyrin spectrum, 3 II.

Hofmeister, Lead in sheep, 177.

Holmes, Isolation of methyl alcohol, 53.

Hoppe-Seyler, F., Haemochromogen, 3ro.

Huchard, Toxic action of nicotine, 9I. Hünefeld, Preparation of blood reagent, 32r.

Hurt, H., Electrolytic estimation of arsenic, 237. Electrodes for same, 239. Organic matter in arsenic determinations, 239.

Husemann, Morphine test, 'I33.

Ipsen, Resistance of atropine to putrefaction, 103 .

$\mathrm{J}$

Jaffé, Rubazonic acid, r24. Santonin in dogs, 199 .

Jaquet, A., Toxic action of ergotinine and hydro-ergotinine, 2 ro.

Jaworowski, Chloral hydrate differentiated from chloroform, 39.

Jeserich, Destruction of organic matter, $15 \mathrm{I}$.

Jonescu, D., Antipyrine in the organism, r23. Alkaloids estimated by potassium bismuthous iodide, 255 .

Jowett, Pilocarpine, 217. Formula of same, 218.

Juckenack, Caffeine in coffee and tea, 27 I.

\section{$\mathrm{K}$}

Katz, E., Estimation of caffeine, 272, 276.

Katz, J., Estimation of colchicin, 269. Caffeine in plant products, 276 . Santonin in wormseed, 290. 
Keller, C. C., Ergotinine, 209. Detection and estimation of same, 2 II. Caffeine in tea, 272. Nicotine in tobacco, 279. Alkaloids in nux vomica, 293.

Kiliani, H., Digitalinum verum crystallisatum, 207. Hydrolysis of digitonin, 207. Test for digitoxin, 208. Digitalin, 208. Digitalose, 208. Maltol, 25 I.

Kippenberger," C., Extraction of morphine, I3o.

Kissling, R., Nicotine in tobacco, 279 .

Klason, Arsenic and mercuric chloride compound in biological test, 243.

Kleist, Excretion of veronal, 8ז. Recovery from urine, 82 .

Knocke, Solubility of antimony spot, I60.

Knorr, L., Picrolonic acid, 253, 320.

Kobert, L., Solanine extracted with isobutyl alcohol, 226.

Kobert, R., Toxic action of hydrocyanic acid, 2o. Chloroform converted in organism to chloride, 35 . Chloral hydrate in brain, $4 \mathrm{I}$. Physiological action of nitrobenzene, 43. Urine in aniline poisoning, 44. Carbon disulphide on lipoids, 46. Ethyl alcohol in blood, 49. Acetone in urine, 55 . Picric acid in urine, 70. Acetanilide poisoning, 73. Strychnine in the body, 97. Cocaine physiological test, rog. Metals and red blood corpuscles, r73. Toxicity of uranium, I80. Sulphuric acid poisoning, I87. Toxicity of oxalic acid, rgo. Potassium chlorate in blood, I94. Cytisine in urine, 206. Digitalis glucosides and urine, 209. Toxic ergot resin, 209. Saponins on blood, 22r. Saponins solvents of cholesterin, 222. Toxicity of saponins, 222. Extraction of s solanine, 226. Toxic action of solanine, 226 . Vegetable agglutinines, 228 .
Crotin, 229. Toxic action of piperine, 287.

Koenig, J., Piperine in pepper, 288.

Koenigs, W., Structure of quinine, I I 9. de Konink, Detection of potassium, I93.

Koppeschaar, Estimation of carbolic acid, 3 r.

Kossler, Estimation of carbolic acid, 33.

Kraft, F., Ergotinine, 209.

Kratter, Examination of charred blood, 3 II.

Krauss, L., Iodine on potassium xanthogenate, 49 .

Kraut, Preparation of potassium bismuthous iodide reagent, 255,3 I 8 .

Kreis, H., Cholesterine and phytosterine with Melzer's reagent, 68.

Küster, W., Formula of haematin, 310. Kunkel, Normal arsenic, I75. Chromium poisoning, 177. Tin poisoning, $\mathrm{I} 8 \mathrm{I}$.

L

Langley, Picrotoxin test, 68.

Lautenschlaeger, Morphine test, I36. Laves, E., Furfural test for veratrine, 96:

Leach, Estimation of methyl alcohol, 55 .

Legal, Acetone test, 56 .

Lehmann, V., Determination of silver in organs, 179. Zinc in the dog, r8o. Toxic effect of sulphur dioxide in air, 188 .

Leins, H., Separation and estimation of caffeine and theobromine, 272.

Le Nobel, Modification of Legal's acetone test, 56 .

Lieben, Iodoform test for ethyl alcohol, 50. Same for acetone, 56 .

Link, Limit of delicacy of Prussian blue test for hydrocyanic acid, 22.

Lloyd, J. U., Morphine test, I34.

Lockemann, G., Destruction of organic matter, 234. Precautions in Marsh test, 236. Delicacy of Marsh test, 237. 
Loretz, Estimation of pilocarpine, 286. Ludwig, Estimation of chloroform, 38 . Lustgarten, Naphthol test for chloroform, $3^{f}$. Iodoform test, 42.

Lythgoe, Estimation of methyl alcohol, 55 .

\section{M}

Maassen, A., Biological arsenic test, 243.

Madsen, Solution of cholesterin in saponin solutions, 222.

Magnin, Alkaloids extracted from animal matter, 62 .

Mai, C., Destruction of organic matter, I52. Electrolytic estimation of arsenic, 237. Electrodes in arsenic determinations, 239. Destruction of organic matter in arsenic determinations, 239.

Mandelin, Test for strychnine, 99. For hydrastine, I 8 . For pilocarpine, 219. Preparation of reagent, 321 .

Mann, Lead in urine and faeces, 176. Marquis, Morphine test, 134. Morphine in the organism, 137 .

Marsh, Arsenic test, ${ }_{5} 6$.

Matthes, H., Estimation of alkaloids as picrolonates, 253. Hydrastine, 282. Pilocarpine, 286. Nux vomica alkaloids, 296.

Mauch, R., Chloral hydrate in alkaloid tests, 88. In brucine test, I02. In codeine test, I I 2. In toxicological analysis, $25 \mathrm{I}$.

Mecke, Codeine test, II3. Reagent for opium alkaloids, 214 . Preparation of reagent, 214,322 .

Meine, W., Berberine, 282.

Melzer, H., Carbolic acid test, 30. Picrotoxin, 67. Nicotine, 92.

Mensching, J., Interference with phosphorus test, 6.

Merck, E., Assaying officinal extracts, $3 \circ 2$.

v. Mering, J., Conjugated chloral hydrate in urine, 4I. Excretion of veronal, $8 \mathrm{I}$. Isolation of same from urine, 82 .

Messinger, Estimation of carbolic acid; 33.

Meyer, G., Solanine in potatoes, $29 \mathrm{I}$.

Meyer, H., Chloroform in the body, 35 .

Action of chloral hydrate, 40 .

Meyer, R., Composition of picrotoxin, 67. Percentage of solanine in potatoes, 225.

Miller, W. V., Structure of quinine, I 20.

Millon, Carbolic acid test, 28. Salicylic acid test, 78 . Preparation of reagent, $32 \mathrm{I}$.

Mitscherlich, Phosphorus test, 5. Estimation of phosphorus, 15.

Möckel, Limit of delicacy of Prussian blue test for hydrocyanic acid, 22.

Molle, B., Excretion of veronal, 8I. Isolation from urine, 82 .

v. Morgenstern, F., Solanine in potatoes, 292.

Mörner, Karl Th., Estimation of minute amounts of arsenic, 247.

Moufang, Structure of brucine, ror.

Mulliken, Methyl alcohol test, 53.

Musculus, Conjugated chloral hydrate in urine, $4 \mathrm{I}$.

Mylius, Fr., Preparation of pure iodeosine, 322 .

\section{$\mathrm{N}$}

Nattermann, Phosphorus test, II. Reduction of phosphorous acid to phosphine, 14. Apparatus for estimating phosphorus, 15. Sensitiveness of Mitscherlich test, 16.

Nessler, Chloral hydrate test, 39. Preparation of reagent, $32 \mathrm{I}$.

Neubauer, Phosphorus test, 10.

Neumann, Detection of phosphorus in cadavers, 16 .

Nietzki, Composition of isopurpuric acid, $7 \mathrm{r}$.

Noguchi, Solution of cholesterin in saponin solutions, 222. 
O

Oliver, Lead in the organism, 177 .

Osann, Maltol, 25 I.

P

Page, Solubility of carbon disulphide, 46.

Pagel, Normal arsenic, 175 .

Pagenstecher, Hydrocyanic acid test, $2 \mathrm{r}$.

Palet, Pyramidone test, I26. Apomorphine test, I 29.

Palm, R., Detection of ergot in flour, 2 II.

Panchaud, Estimation of cinchona alkaloids, 266.

Parmentier, Solubility of carbon disulphide, 46 .

Partheil, A., Identity of cytisine and ulexine, 205 .

Péligot, Solubility of carbon disulphide, 46.

Pellagri, Codeine test, rr2. Apomorphine, r28. Morphine, I34.

Penny, Estimation of carbolic acid, 33 .

Penzoldt, Acetone test, 57.

Petri, Composition of isopurpuric acid, $7 \mathrm{I}$.

Piccard, J., Ortho-xylene from cantharidin, 204.

Piccini, Isomethyl-pelletierine, 270.

Pictet, A., Nicotine synthesis, 9 r.

Pinner, Nicotine formula, 9 r. Nitrogen in pilocarpine and isopilocarpine, 2 r8.

Pisani, Cocaine test, rog.

Pohl, Chloroform in blood and in the body, 35 .

Polacci, E., Procedure in thalleioquin test, $\mathrm{r} 2 \mathrm{r}$.

Poleck, Arsenic test, r63.

Polstorff, K., Interference with phosphorus test, 6.

Pouchet, Antimony in the body, I 76 .

Prescott, A. B., Estimation of alkaloids, 257.

Proells, Cocaine in the cadaver, ro7.
Pschorr, R., Apomorphine structure, r27. Morphol synthesis, $\mathbf{r}_{32}$. Morphine structure, r33. Thebaine structure, 227. Thebaol synthesis, 227.

\section{$\mathrm{R}$}

Radulescu, D., Morphine test, $\mathrm{r}_{36} 6$.

Rammstedt, O., Estimation of alkaloids as picrolonates, 253. $\mathrm{Hy}$ drastine, 282. Pilocarpine, 286. Nux vomica alkaloids, 296.

Ramverda, Cytisine test, 206.

Ransam, Influence on saponins of cholesterin in blood, 222.

Reichard, C., Cocaine test, rog.

Reynolds, Acetone test, 57 .

Riechelmann, Estimation of caffeine in coffee, 272 .

Riegel, Estimation of antimony by Gutzeit method, 240.

Roser, Narcotine structure, $\mathrm{rr}_{4}$. Pseudo-narceine from narcotine, 138 .

Rossi, Conversion of nitrobenzene in organism to aniline, 43.

Roussin, Nicotine test, 92.

Rügheimer, Piperine synthesis, 287 .

Rupp, E., Iodine on xanthogenate, 49.

Russanow, A., Formula of compound from phenol and benzaldehyde, 30.

\section{$\mathrm{S}$}

Sanger, C. R., Estimation of arsenic and antimony by Gutzeit method, 240.

Schaefer, Normal arsenic, I75.

Schaer, E., Saponin test, 223. Animal and vegetable catalysts and oxygen carriers, 3 I2. Blood stain test, 3 r3. Aloin blood test, 3 r4.

Schaffer, Conjugated sulphuric acid in urine in carbolic acid poisoning, 27.

Scherer, Phosphorus test, 3. Estimation of same, $\mathrm{r} 5$.

Schiff, Reagent for aldehydes, 54 .

Schindelmeiser, Nicotine test, 93. 
Schmidt, E., Crystalline veratrine, 94. Apomorphine tests, I29. Detection of cantharidin, 205. Isolation if schlererythrin, 210. Detection of solanine and solanidine, 226. Test for solanine and solanidine, 227. Solubilities of sulphates of cinchona alkaloids, 268. Estimation of berberine, 282. Estimation of alkaloids in belladonna, etc., 300 .

Schmiedeberg, O., Percentage of solanine in potatoes, 225. Solanine in potatoes, 291 .

Schmitt, R., Preparation of salicylic acid, 77 .

Schneider, Sodium cacodylate not decomposed by hydrochloric acid, 246.

Schönbein, Hydrocyanic acid test, 21. Ozone test for blood, 3 I 2.

Scholtz, M., Detection of potassium chlorate, 196 .

Schütze, Biological blood test, 315.

Schwarz, Resorcinol test for chloroform, 36 .

Scudder, Methyl alcohol test, 53.

Selmi, Organic phosphorus compounds in phosphorus test, I3. Ptomaine resembling morphine, 220.

Silber, Structure of pseudo-pelletierine, 270.

Simmonds, Methyl alcohol test, 54 .

Skraup, Quinine structure, 120.

Socoloff, Caffeine in coffee, 275.

Sonnenschein, Destruction of organic matter, I5I.

Stas-Otto, Process for extracting alkaloids, 63 .

Stich, C., Estimation of phosphorus in phosphorated oils, 232 .

Strassmann, Ethyl alcohol eliminated by lungs, 49 .

Straub, W., Test for phosphorus in phosphorated oils, 14. Estimation of phosphorus in same, $23 \mathrm{I}$. Accuracy of method, 232 .

Strzyzowski, Sodium iodide in haemin test, 308 .
$\mathrm{T}$

Tafel, Strychnine structure, 97. Bichromate test for same explained, 99. Structure of brucine, ior.

Tanret, Ergotinine, 209.

Tassily, E., Caffeine in coffee, 272.

Teichmann, Haemin blood test, 309.

Thäter, K., Santonin test, 200. Santonin in wormseed, 289.

Thiele, Compound of bromine and phenol, 29.

Thoms, H., Separation of quinine from mixtures, 122. Destruction of organic matter, r5o. Alkaloids estimated by potassium bismuthous iodide, 255 .

Thorpe, T. E., Isolation of methyl alcohol, 53. Arsenic in beer worts, 237.

Tollens, B., Formaldehyde in blood test, 307 .

Toth, J., Nicotine in tobacco, 280.

Trillich, H., Caffeine, 275 .

Trotmann, Arsenic determined in beer worts, 237.

Tümmel, Arsenic test, 163 .

$\mathrm{U}$

Uhlenhuth, Biological blood test, 315. Ulenberger, Lead in sheep, 177 .

Ungar, Tin poisoning, 18I.

\section{V}

Van Deen, Ozone blood test, 312.

Van der Moer, Cytisine test, 206.

Vandevelde, Toxicity estimated by blood haemolysis, 258 .

Vaubel, Solubility of antimony spot, I6o.

Vitali, Carbon disulphide test, 47 Ethyl alcohol test, 51. Veratrine test, 96. Atropine test, ro4. Detection of caustic alkalies, 193 Blood stain test, $3 \mathrm{I} 3$. 
Volhard, Estimation of hydrochloric Werk, R., Cause of solanine in potaacid, 184 .

Von Pohl, Methyl alcohol in the or- White, Tin poisoning, I8r. ganism, 52.

Vortmann, Hydrocyanic acid test, 23. Estimation of carbolic acid, 33.

W

Wangerin, Narcotine test, I 5 . Apomorphine test, I 28 . Lloyd's morphine test, 135. Narceine test, I40.

Warren, L. E., Papaverine test, 217.

Warren, W. H., Alkaloids purified by picrolonic acid, 89. Normal arsenic, 176 .

Wassermann, Biological blood test, 24 .

Weehuizen, Hydrocyanic acid test, 24.

Weiss, R. S., Alkaloids purified by picrolonic acid, 89 .

Weppen, Veratrine test, 95 .

Wieser, Normal arsenic, 176 .

Willstätter, Cocaine structure, 106.

Windhaus, Digitonin, 207. Saponin cholesterides, 222.

Wittmann, Solanidine from solanine, 225.

Witz, Determination of mercury in the body, 179.

Wolff, C., Caffeine in coffee, 276.

$\mathrm{Z}$

Zappi, Extraction of alkaloids from animal matter, 62 .

Zeine, Formula of picrolonic acid, 253.

Zeisel, Colchicin test, 69. Determination of methoxyl groups in alkaloids, ror.

Ziemke, Normal arsenic, I76. 


\section{INDEX OF SUBJECTS}

A

Abortifacients, Ergot, 209. Nitrobenzene, 42 .

Abrin, 229.

Absorption-spectra, blood, 306, 3 Io.

Carmine, 3rr. Codeine, Ir2.

Ergot, 2II. Fuchsine, 3I2.

Nitrobenzene, 42. Physos-

tigmine, Iro.

Acetanilide, 66, 73, r4r.

Acetic ether (see Ethyl acetate).

Acetone, 55.

Acetonuria, 55 .

Acid acetic, Aconitine, 262.

aconitic, 26r.

amino-acetic, 58 .

$\alpha$-amino-isobutyl-acetic, I 7 .

angelic, 94 .

arsenic, 150.

atropic, I03.

benzoic, 58. Aconitine, 262.

Cocaine, 106.

brucic, ror.

cacodylic, 246. Electrolysis, 234.

cantharic, 204 .

cantharidic, $203,263$.

carbolic, 26. Aniline, 34. Estimation, 31. Urine, 34 .

chloric, Organic matter, I5I. Reduction, I 96.

chloroplatinic, 318. Potassium, 193.

chromic, Alcohol, 51. Aniline, 45. Cocaine, Io8.

cincholoiponic, 119 .

cinchonic, 119.

ethyl-sulphonic, 202.

ethyl-sulphuric, Solanine and solanidine, 227 .
Acid formic, Chloral hydrate, 39. Phosphorus, 4 .

glycuronic, 4r, 44. Aceto-pamino-phenol, 74. Morphine, I37. Organism, 123.

guaiaconic, 314 .

hippuric, 58 .

hydrastic, 117 .

hydrochloric, 183 . Colchicin, 69 .

Digitalin, 209. Electrolytic,

24I. Organic matter, I5I.

Veratrine, 95 .

hydrocyanic, r9. Blood, 312.

Estimation, 25. Oil of bitter

almonds, 58. Patassium

ferrocyanide, 25. Prelimi-

nary test, $2 \mathrm{I}$.

hydro-p-cumaric, I 7, 28.

hypophosphorous, 8 .

iodic, 321 . Morphine, r34.

isopurpuric, $7 \mathrm{r}$.

lactic, Hydrocyanic acid, 20.

loiponic, I I9.

mandelic, 105.

meconic, 2 r 3 .

meconinic, 2 I 4 .

n-methyl-granatic, 270.

Acid nicotinic, 9r.

nitric, I84. Antipyrine, 83. Apomorphine, 128 . Cocaine, 107. Codeine, III. Colchicin, 69. Cytisine, 207. Morphine, I33. Narceine, 139. Papaverine, 217. Phenacetine, 76. Physostigmine, Iro. Pyramidone, 125. Thebaine, 228. Veratrine, 95.

nitrous, Antipyrine, 83 .

nor-meta-hemipinic, $x 17$. 
Acid opianic, Hydrastine, II7. Narcotine, II4.

oxalic, 189 . p-amino-phenyl-sulphuric, 44 . p-hydroxy-benzoic, 28. p-oxy-phenyl-acetic, I 7, 28. p - oxy - phenyl - $\alpha$ - amino - propionic, 17.

p-oxy-phenyl-propionic, 17,28 . phenyl-glycuronic, 26 . phospho-molybdic, 3 rg. phosphoric, Reduction, 8. phosphorous, 8, I4. Mercury, r66.

phospho-tungstic, 3 I9.

picramic, Organism, 70. Picric acid, 24,72 .

picric, 66, 70, I4I. Hydrocyanic acid, 24. Reagent, 320.

picrolonic, 320. Alkaloids estimated, 253; purified, 89. Hydrastine, 282. Nux vomica, 296. Pilocarpine, 286.

piperic, 287.

quinic, 119,265 .

quino-tannic, 265 .

rubazonic, 124 .

salicylic, 66, 77, 142. Foods and beverages, 250. Phenols, 28, 78.

salicyluric, 79 .

santonic, 198, 290.

sarcolactic, 17 .

sclerotic, 209

selenious, Ir3.

sphacelic, ${ }^{200}$.

strychnic, 97 .

suberic, 270 .

sulphanilic, 136 .

sulphocyanic, 19.

sulphuric, 186. Apomorphine, 128. Cocaine, 107. Codeine, III. Colchicin, 69. Conjugations, 74. Digitalin, 208. Hydrastine, Ir 8. Meconine, 214. Narceine, 139. Narcotine, 115. Ṕapaverine, 217. Physostigmine, Iro.
Preformed and conjugate, 26. Santonin, 200. Saponins, 223. Solanine, 227. Thebaine, 228. Veratrine, 95 . sulphurous, 188.

tannic, 319. Antipyrine, 83. Blood, 305. Caffeine, 85. Colchicin, 69. Narceine, I40.

thioacetic, 249 .

tiglic, 94 .

trichlor-ethyl-glycuronic, $4 \mathrm{I}$.

trimethyl-colchicinic, 69 .

tropic, 103, ro6.

uric, 84 .

urochloralic (see Trichlor-ethylglycuronic).

vanadic-sulphuric, 226.

veratric, 216 .

Acid xanthoproteic, 185 .

Acids, mineral, 182 .

Aconine, 262.

Aconitine, 26r.

Agglutinines, 228.

Air, Carbon disulphide, 48. Sulphur dioxide, $\mathrm{r} 88$.

Albuminates, metallic, ${ }_{5} \mathrm{I}, \mathrm{r} 72$.

Alcohol, ethyl, 49. Differentiation, 57. Purification, 6r. Toxicity, 259 .

methyl, 52. Cocaine, ro6. Colchicin, 68. Estimation, 55 . Toxicity, 25.9 .

trichlor-ethyl, $4 \mathrm{I}$.

Alkalies, 192.

Alkaloidal reagents (see Reagents, alkaloidal).

Alkaloids, Aconite, 26r. Alkaloidal reagents, 87. Belladonna, 30r. Cinchona, 264. Estimation, 253, 255, 257, 296. Extraction, 86. Extracts, 266. Hyoscyamus, 301. Ipecac, 277. Nux vomica, 293-295. Physiological test, 88. Pomegranate, 270 . Purification, 88. Selenious-sulphuric acid, 215. Viscera, 62. 
Aloes, Quinine test, I22.

Aloin, Blood test, 3 r4.

Aluminium acetäte, 289 .

Ammonia, 192. Fapaverine, 217.

Physostigmine, I Io.

Ammoniacal copper, Picric acid, 72.

Ammoniacal silver, Morphine, I35.

Picrotoxin, 67.

Ammonium magnesium phosphate, 7 . molybdate, 7 .

persulphate, $\mathrm{I}_{52}$.

sulphide, Blood, 305. Sulphides, 154.

Anhydro-ecgonine, ro6.

Aniline, 44, 93, 143. Carbolic acid, 34.

Antimony, 155. Detection, I63, 165. Distribution, I75. Estimation, 240. Differentiation of spot, $\mathrm{r} 60$.

Antipyrine, 66, 82, 87, г 23, r42, I45, 147. Extract, 87. Thalleioquin, r21. Urine, 83 , r23.

Antipyryl-urea, I 25.

Apomorphine, 127, I46. SeleniousArgyria, I 79 .

sulphuric acid, 2 I5.

Arrhenal, 246. Electrolysis, 234. Urine, 247.

Arsenic, 155. Beer, 237. Bettendorff, I62. Biological, 242. Bougault's reagent, 247 . Bulb-tube, r62. Differentiation, 160 . Distribution, 173 . Electrolysis, 233, 237. Elimination, I73. Fresenius-v. Babo, I6r. Gutzeit, I63, 240. Isolation, 233. Lockemann, 234. Marsh-Berzelius, I56. Mörner, 247. Normal, r74. Organic compounds, 245. Spot, $x_{57}$. Urine, 247.

Arsenic trichloride, 150. Arsenic isolated, 233.

Arseno-tungstic reagent, 125 . Arseno-tungsto-molybdic reagent, 125. Arsine, ${ }_{5} 6$.
Asparagine, 292.

Atoxyl, 246. Electrolysis, 234. Urine, 247.

Atropa belladonna, Alkaloids, 300 .

Atropine, 87, ro2, 144. Estimation, 300,302 . Extract, 87. General reagents, 105 . Physiological test, IO4. Putrefaction, I03.

Barbital, 79

Barium, I 70 .

Baumert's nitric acid test, 185 .

Beer, Arsenic, 237. Picrotoxin, 68. Saponins, 223.

Benzaldehyde, 57. Phenol, 30. o-nitro, 57.

p-dimethyl-amino, 104.

Benzaurine, 31 .

Benzoyl cocaine test, ro8.

Benzoyl-ecgonine, ıo6.

Berberine, II6. Estimation, 282 .

Berthelot's alcohol test, $5 \circ$.

Bettendorff's reagent, 322 . Test, I62. Biological test, Arsenic, 242. Blood, 314.

Bile, Elimination of lead, I 77 .

Bismuth, $16_{5}$. Elimination, 180 . Morphine, I35. Physiolog. ical action, I72. Toxicity, I80.

Bitter almond water, 57 .

Blondlot-Dusart test, 8 .

Blood, Acetone, 55. Aniline, 44. Carbon disulphide, 46. Carbon monoxide, 304. Chloral hydrate, 40. Chloroform, 35. Copper, r78. Defibrinated, 229. Heavy metals, I73. Hydrocyanic acid, 20. Lead, I77. Nitrobenzene, 42. Oxalates, rgo. Phosphorus, 17. Picric acid, 70. Potassium chlorate, 194. Saponins, 221. Stains, 307 , 313. Sulphurous acid, 188. 
Tests, 306, 3ro, 3r2, 3r4. Toxalbumins, 228 .

Boiling test for blood, 304 .

Bread, Ergot, 2 rr.

Bromine test, Aniline, 45. Digitonin, 208. Phenol, 28. Pyramidone, r25. Salicylic acid, 78 .

Brucine, 86, Ioo, r43. Estimation, 296. Extract, 86. General reagents, ror. Nitric acid test, I86. Picrolonate, 296 . Tests, ro2.

Bulb-tube test, Arsenic, 162 .

\section{C}

Cacao, Caffeine and theobromine, 298. Cadmium, 167 .

Caffeine, 66, 83, 87, r22, r42, r45, $\mathrm{r}_{47}$ Estimation, 27I, 274, 298. Extract, 87. Extraction, 275. Fate, 84. Tests, 85. Thalleioquin test, I2I.

Calabar bean, iro.

Canadine, i 16 .

Cantharene, 204.

Cantharic anhydride, acetyl-hydrato, 204.

Cantharidin, I42, 203. Estimation, 263.

Carbolic urine, 26.

Carbon disulphide, 46. Air, 48 . Tests, 47.

Carbon monoxide blood, 304 .

Carbon tetrachloride, Caffeine, 274. Purification, 275. Separation of theobromine, 299 .

Carboxyhæmoglobin, 304 .

Carmine, Absorption-bands, $3 \mathrm{rr}$.

Castor bean, 229.

Cephæline, 277. Test, 278.

Cevadine, 93 .

Cevine, 94.

Chavicine, 287.

Chloral hydrate, 38 . Blood, $3 \times 3$. Decomposition, 39. Estimation, 4r. Fate, 4o. Physiological action, 40. Powders,
40. Saponin test, 223. Solvent, 25I. Tests, 39 . Toxicological analysis, 40 .

Chlorine-ammonia test, Narceine, $\mathbf{r}_{39}$.

Chlorine test, Hydrochloric acid, 183 .

Potassium chlorate, I95.

Chlorine water, Preparation, 85. Thebaine, 228.

Chloroform, 35. Estimation, 38 . Tests, 36 .

Chocolate, Caffeine, 298.

Cholesterine, Hæmolysis, 222, 224. Melzer's reagent, 68. Saponins, $22 \mathrm{I}$.

Choline, Ergot, 2 I I.

Chrome yellow test, I 70 .

Chromium, 169, 177 .

Cicutoxine, 66.

Cinchona, Alkaloids, 264. Quinine, 268.

Cinchonidine, 265 .

Cinchonine, rIg.

Claviceps purpurea, 209.

Cocaine, I05, I44. Organism, ro7. Physiological test, rog. Relation to atropine, ro6. Tests, I07.

Cocculus indicus, 66 .

Codeine, 87 , III, I44. Estimation, 254. Extract, 87. Selenious acid, $2 r_{5}$. Tests, rII.

Coffee, Caffeine, 27 I.

Cola nuts; Caffeine, 273, 276.

Colchicein, 68.

Colchicin, 65, 68, I4r. Estimation, 269. Hydrolysis, 68. Purification, 70. Structure, 69. Tests, 69 .

Colchicum autumnale, 68. Colchicin, 269.

Congo paper, 182 .

Conhydrine, 89 .

$\gamma$-Coniceine, 89 .

Coniine, 89, r42. Diazonium test, I37. Tests, 90 .

Conium maculatum, 89 .

Conjugated aceto-p-amino-phenol, 74 . p-Amino-phenol, 44. Anti- 
pyrine, 123. Benzoic acid, 58. Carbolic acid, 26. Chloral hydrate, 4r. Morphine, 137. Salicylic acid, 79.

Constitution, Acetanilide, 73. Antipyrine, 82. Apomorphine, 127. Atropine, ro3. Caffeine, 83. Cantharidin, 203. Cocaine, r05. Codeine, III. Coniine, 89. Homatropine. I05. Hydrastine, II 7 , Morphine, 131. Narceine. 138. Narcotine, I I3. Nico, tine, 91. Papaverine, 215Phenacetine, 75. Picric acid, 70. Piperine, 288. Pseudopelletierine, 270. Quinine, I19. Salicylic acid, 77 . Santonin, 198. Thebaine, 227. Veratrine, 94. Veronal, 79 .

Copper, 155, 165, 167. Elimination, I78. Fusion, 155. Mercury test, I66. Nitric acid test, 186. Physiological action, 172, 178. Tests, 155, 167. Toxicity, 172 .

oxide, 156.

sulphate, Blood test, 305. Phosphorus, 14 .

sulphide, Solubility, 155 .

Corn smut, 2 Ir.

Cornutine, 209.

Corrosion, 173.

Cotarnine, I14, I18.

Couerbe's test, Narcotine, II5.

Creatinine, 87 .

Cresols, 28.

Crotin, 229.

Croton Tiglium, 229.

Crystallization test, Coniine, 90 . Nicotine, 92. Oxalic acid, Igr.

Cyanogen, 24.

Cyano-hæmoglobin, 20.

Cystine, 17.

Cytisine, 205. Tests, 206.

Cytisus Laburnum, 205.
D

Datura strammonium, Alkaloids, 300 .

Denigès test, Cocaine, rog.

Destruction of organic matter, $\mathbf{1 4 8}$, 150-152.

Dextrose, Digitalin, 208. Digitonin, 207. Hydrocyanic acid, 20. Phosphorus, 17.

Dialysis, Potassium chlorate, $194^{\circ}$

Diazonium test, Morphine, I36.

Dichromate test, Strychnine, 98.

Digitalis glucosides, 207.

Dimethoxy-isoquinoline, 216.

p-Dimethyl-amino-benzaldehyde, ro4.

Dionine, 136 .

Diphenylamine test, Nitric acid, 186. Reagent, I86, 235.

Distillation test, Ammonia, 192. Hydrochloric acid, 183 . Nitric acid, $\mathbf{r} 84$.

Distribution, Antimony, 175. Arsenic, 173. Carbolic acid, 27. Chloroform, 35, 38 . Ethyl alcohol, 49. Heavy metals, 173. Hydrocyanic acid, 20. Mercury, 178 . Oxalic acid, 190. Tin, 18r. Zinc, 180 .

Diuresis, 194 .

Dyeing test, Picric acid, 72.

E

Ecgonine; 106.

Electrolysis, Arsenic compounds, 233, 237.

Elimination, Antimony, 175. Arsenic, 173. Bismuth, 180. Chromium, 178. Coćaine, 107. Copper, 178 . Heavy metals, 173. Hydrocyanic acid, 19. Lead, I76. Mercury, 178 . Picric acid, to. Potassium chlorate, r94. Strychnine, 97. Tin, I8I. Veronal, 8r.

Emetine, 277. Diazonium test, 137. Epichlorohydrin, 92. 
Erdmann's reagent, 320. Cocaine, 107. Colchicin, 69. Narceine, 139. Narcotine, II5. Papaverine, 217. Strychnine, 98. Thebaine, 228 . Veratrine, 95 .

Ergot, 209.

Ergotine, 209.

Ergotinine, 209. Estimation, 21r. Test, 2 I 2.

Ergotoxine, 209.

Erythrosine (see Iodeosine).

Eserine (see Physostigmine).

Ether extracts, evaporation, 64 .

Ethyl acetate, Alcohol, 5r. Caffeine, 275.

Exhaustive methylation, Ir7. nMethyl-granatic acid, 270.

Extract belladonna, Alkaloids, 3or, 302.

cinchona, Alkaloids, 266, 302 . Quinine, 269.

hydrastis, Hydrastine, 28r, 282.

Berberine, 282.

hyoscyamus, 3 or.

nux vomica, Alkaloids, 295, 296, 303.

opium, Morphine, 285.

Fæces, lead in, $x 76$.

Fat, Phosphorus, $\mathrm{r} 7$.

Fate, Acetanilide, 74. Benzaldehyde, 58. Caffeine, 84: Chloral hydrate, 40. Cocaine, ro7.

Digitalis glucosides, 209 . Ethyl alcohol, 49. Heavy metals, 173 .

Fehling's solution, 320 . Chloroform,

37. Githagin, 224. Maltol, 25r. Picrotoxin, 67. Saponins, 224 .

Ferments, Hydrocyanic acid, 20.

Ferric chloride, Antipyrine, 83. Apomorphine, r29. Benzoic acid, 58. Carbolic acid, 29. Codeine, Ir2. Cytisine, 206.
Ergotinine, 2I 2. Maltol, 25I. Meconic acid, 213. Morphine, I34. Pyramidone, I25. Salicylic acid, 77, 250, 251. Santonin, 200.

Ferric hydroxide, Arsenic, 235 .

Ferrous sulphate test, Nitric acid, 186. Fleury's test, Morphine, I35. Nitric acid, 185 .

Flour, Ergot, 21 r. Githagin, 223.

Fluorescence test, Hydrastine, Ir8. Quinine, 120.

Formaldehyde, Blood, 307. Phosphorus, 4.

Formaldehyde-sulphuric acid, 321. Codeine, Ir2. Morphine, r34. Pilocarpine, 2 19.

Formamide test, Cocaine, rog.

Fowler's solution, 247 .

Fresenius-v. Babo, Arsenic, r6r. Organic matter, $\mathrm{I}_{4} 8$.

Froehde's reagent, 320. Apomorphine, 128 . Cephæline, 278 . Cocaine, ro7. Codeine, II 2. Emetine, 279. Hydrastine, I18. Morphine, I34. Narceine, r39. Narcotine, I15. Papaverine, 217. Saponins, 222. Solanine, 227. Strychnine, 98. Thebaine, 228 . Veratrine, 95 .

Fuchsine, Absorption-band, 3 I 2.

Furfural, Codeine, II 2. Santonin, 200.

Fusion of heavy metals, 155 .

\section{G}

Galactose, Digitonin, 20\%. Solanine, 225.

General alkaloidal reagents (see $\mathrm{Re}-$ agents, general).

German Pharmacopœia (see Pharmacopœia, German).

Githagin in flour, 223.

Glucose (see Dextrose).

Glycocoll (see Acid, amino-acetic).

Glycosuria, 17 . 
Glycurone, 44 .

Glyoxaline, 218.

Gold chloride, 3 I 7 .

Golden chain, 205.

Grandeau's test, Veratrine, 96 .

Guaiac-copper paper, 2 I.

Guaiac resin, Blood, 312.

Guarana, Caffeine, 276.

Guglialmelli's test, Pyramidone, I25.

Günzburg's reagent, 32r. Mineral acid, 182 .

Gutzeit's test, 163,240 .

\section{$\mathrm{H}$}

Hæmatin, 3 ro.

Hæmatin, reduced (see Hæmochromogen).

Hæmatoporphyrin, Spectrum, 3 II. Urine, 202.

Hæmatoxylin, 266, 303.

Hæmin, 3 ro.

Hæmin crystals, 308 .

Hæmochromogen, 310.

Hæmoglobin with metals, 173 .

Hæmoglobinuria, 226.

Hæmolysis, 224. Cholesterin, 222. Saponins, 22 I.

Herapathite test, Quinine, 120.

Heroine, 136.

Hirschsohn's test, Quinine, x22.

Homatropine, I05.

Hünefeld's solution, 32r.

Husemann's test, Morphine, 133.

Hydrastal, II 7 .

Hydrastine, II6, I44. Estimation, 28 I.

Hydrastinine, I 7 .

Hydrastis canadensis, II6.

Hydrocotarnine, II 4 .

Hydro-ergotinine, 209.

Hydrogen sulphide, arsenic-free, 152. Phosphorus, 3 .

Hydrolysis, Aconitine, 262. Atropine, 103. Cocaine, 106. Colchicin, 68. Digitalin, 208. Digitonin, 207. Digitoxin, 208. Narcotine, I14. Piperine,
287. Saponins, 221. Solanine, 225 .

Hydroquinol, 26.

Hyoscyamine, 103.

Hyoscyamus niger, Alkaloids, 300 .

Hyper-isotonic solutions, 224.

Hyp-isotonic solutions, 224.

Hypochlorite test, Acetanilide, 74. Aniline, 45. Carbolic acid, 29.

Ignatius beans, 96 .

Indigo test, Chloric acid, 195 .

Indigotine test, Acetone, 57.

Indophenol test, Acetanilide, 73. Phenacetine, 76.

Iodeosine, 322 .

Iodine test, Narceine, I39. Pyramidone, 125 .

Iodoform, 4I.

Iodoform test, Acetone, 56 .

Iodo-potassium iodide, 318. Alkaloids, 257.

lpecac, Alkaloids, 277, 279.

Isomethyl-pelletierine, 270 .

Iso-pelletierine, 270.

Iso-pilocarpine, 217 .

Isopurpuric acid test, Picric acid, $7 \mathrm{I}$.

Iso-quinoline, 216.

Isotonic solutions, 224.

$\mathrm{J}$

Jaborandum, Alkaloids, 217. Pilocarpine, 286.

Jaborine, 217.

Jequirity seeds, 229.

K

Kiliani's test, Digitoxin, 208.

Kjeldahl, Caffeine estimation, 274 .

de Konink's reagent, 193.

L

Langley's test, Picrotoxin, 68.

Lead, 167. Blood, 173. Elimination, 
I76. Sheep, I77. Physiological action, $\mathbf{I} 72$.

Lead acetate test, Blood, 304. Carbon disulphide, 47 .

Lead paper, Phosphorus, 3 .

Lecithine-saponins, 221.

Leucine, Phosphorus, 17.

Leucocytosis, 46.

Lieben's test, Alcohol, 50. Acetone, 56.

Liver, Copper, 178.

Lloyd's test, Morphine, I34.

Lustgarten's test, Iodoform, 42.

Lytta vesicatoria, 203 .

\section{M}

Magnesia mixture, 7, $32 \mathrm{I}$.

Mai's method, Organic matter, 152.

Maltol, 25I.

Mandelin's reagent, 32 I. Hydrastine, I 8. Pilocarpine, 2 I9. Strychnine, 99.

Marquis' reagent, I34.

Marsh apparatus, I34.

Marsh-Berzelius test, $1_{56} 6,236$.

Maté (see Paraguay tea).

Material to be examined, Arsenic, I 73. Bismuth, I80. Carbolic acid, 26, 27. Chloroform, 38. Copper, I78. Digitalis glucosides, 209. Ethyl alcohol, 49. Hydrocyanic acid, acid, 2I. Mercury, I 78 . Potassium chlorate, I94. Veronal, 81. Zinc, r 80.

Mauch's solvent, 25I. Brucine, I02.

Meadow saffron, 68.

Meat, Potassium chlorate, 197. Salicylic acid, 250. Sulphur dioxide, 189 .

Mecke's reagent, 322. Codeine, II3. Meconine, 2 I3.

Melting-point test, Salicylic acid, 78 . Sulphonal, 201.

Melzer's reagent, 67. Carbolic acid, 30. Picrotoxin, 67 .

Melzer's test, Nicotine, 92 ,
Menispermum cocculus, 66 .

Mercuric chloride, 318.

cyanide, 25,65 .

iodide, Mercury, 166.

Mercury, I65. Distribution, 178. Physiological action, 172. Tests, I66. Tin, I64. Toxicity, I72. Urine, 178.

Meroquinene, $\mathbf{I} 20$.

Metallic poisons (see Poisons, metallic).

Metals, heavy, 772.

Methæmoglobin, 308. Absorptionband, 3 ro.

n-Methyl-coniine, 89 .

n-Methyl-granatoline, 270.

n-Methyl-granatonine (see Pseudopelletierine).

Methyl orange, 182 .

Methyl-pelletierine, 270.

Methyl violet, I82.

Milk, Salicylic acid, 25I. 'Toxalbumins, 228.

Millon's reagent, $32 \mathrm{I}$. Carbolic acid, 28. Maltol, 25I. Salicylic acid, 78 .

Mitscherlich's phosphorus test, 5, I5. Morphenol, I32.

Morphine, I3I, I46. Estimation, 254. General reagents, I37. Opium, 283. Organism, 137 . Preliminary test, 130. $\mathrm{Pu}$ trefaction, 137. Selenioussulphuric acid, 2 I5.

Morphol, I32.

Murexide reaction, 85 .

\section{N}

Naphthol test, Chloroform, 36.

Narceine, 138, I47. Selenious-sulphuric acid, 2 I 5 .

Narcotine, I13, I45. Selenious-sulphuric acid, 215.

Nessler's reagent, 321 . Ammonia, I92. Chloral hydrate, 39 .

Nicotine, 90, r43. Diazonium test, I37. Tobacco, 279.

Nitrite test, Carbolic acid, 30. 
Nitrobenzene, 42. Differentiation, 58.

Nitro-phenacetine, 76 .

Nitroprusside test, Acetone, 56. $\mathrm{Hy}$ drocyanic acid, 22.

Non-volatile poisons (see Poisons, nonvolatile).

Normal arsenic, I 74 .

Nux vomica, 96. Alkaloids, 293-296.

\section{O}

Odor test, Atropine, ro4.

Oil of bitter almonds, Hydrocyanic acid, 58 .

Oils, phosphorated, Phosphorus, I4, 231.

Opium, 212. Morphine, 283. Morphine in tincture, 285. Morphine in wine, 285 .

Organic arsenic compounds, 245. Electrolysis, 234.

Organic matter, Destruction, 148 , I5I, 152, 234.

Oxalates in plants, rgo.

Oxidation test, Methyl alcohol, 53-55. Caffeine, 85. Codeine, III. Phenacetine, 76. Picrotoxin, 67 .

Oxy-dimorphine, 133 .

Oxy-hæmoglobin, 304. Reduction, 307.

Oxy-santonins, 199 .

\section{$\mathrm{P}$}

Palet's test, Apomorphine, I 29. Pyramidone, 126.

Palladous chloride test, Blood, 305.

Papaveraldine, 216.

Papaverine, 145, 215.

Paracholesterine, 68.

Paraguay tea, Caffeine, 276.

Paraxanthine, 84, 300.

Pellagri's test, Apomorphine, I 28. Codeine, II2. Morphine, 134 .

Pelletierine, 270.
Penzoldt's test, Acetone, 57.

Pepper, 287. Piperine, 288.

Peptones, Phosphorus, 17. Animal matter, 87 .

Peptonuria, 17 .

Peronine, 136 .

Pharmacopœia, German, Aconitine, 262. Alkaloids, 260. Belladonna, 3or. Cantharidin, 263. Cinchona, 264. Hydrastine, 28I. Hyoscyamus, 30r. Ipecac, 279. Nux vomica, 294, 295. Opium, 283. Pomegranate, $27 \mathrm{I}$.

Phenacetine, 66, 75, I4I.

Phenanthrene, 132 .

Phenol (see Acid, carbolic).

Phenol, aceto-p-amino, 74 .

Phenolphthalein, 24.

Phenolphthalin test, Hydrocyanic acid, 24.

Phenylisocyanide test, Acetanilide, 73. Aniline, 45. Chloroform, 36 .

Phosphine, 8.

Phosphorous acid (see Acid, phosphorous).

Phosphorus, yellow, 5. Antidote, I4. Blondlot-Dusart, 8. Fresenius, Neubauer, ro. HilgerNattermann, II. Magnesium test, 7. Metabolism, I6. Mitscherlich test, 5. Molybdate test, 7. Oils, 14, 23I. Organic compounds, 13 . Scherer's test, 3. Spectrum, 12. Urine, 17 .

Physiological action, Acetanilide, 73. Acetone, 55. Alkalies, 192. Berberine, 282. Bismuth, 172. Cantharidin, 205 . Carbolic acid, 26. Chloral hydrate, 40. Chloroform, 35. Chromium, 177. Copper, 172, 178. Ergot, 210 . Heavy metals, 172 . Homatropine, ro5. Hydrocyanic acid, 19. Lead, 172 . Mercury, 172. Morphine, 
137. Nicotine, 91. Nitric acid, 184 . Nitrobenzene, 42 . Oxalic acid, Igo. Phosphorus, r6. Picric acid, 70. Pyramidone, r24. Santonin, 199. Saponins, 22r. Silver, I72, I 79. Strychnine, 97. Sulphuric acid, r86. Sulphurous acid, $\mathrm{r} 88$. 'Tin, $\mathrm{r} 8 \mathrm{r}$. Uranium, I72, I80. Veronal, 8 o.

Physiological salt solution, 224.

Physiological test, Alkaloids, 88. Atropine, ro4. Cantharidin, 205. Cocaine, rog. Nicotine, 93. Physostigmine, I 10. Strychnine, 99. Veratrine, 94 .

Physostigma venenosum, iro.

Physostigmine, rro, r45. Diazonium test, 137 .

Phytosterine, 68, 28r.

Picraconitine, 26I.

Picro-sclerotine, 209.

Picrotin, 66.

Picrotoxin, 65, 66, r 40 .

Picrotoxinin, 66.

Pilocarpidine, 2 I 7.

'Pilocarpine, 2I 7. Estimation, 286.

Pilocarpus pennatifolius (see Jaborandum).

Piperidine, 287. Diazonium test, 137 .

Piperine, 287. Estimation, 288.

Pisani's test, Cocaine, rog.

Platinum chloride, 3 I 8 .

Poisons, metallic, I48.

non-volatile, $6 \mathrm{r}$.

volatile, 3 . Distillation, 18.

Pomegranate bark, Alkaloids, 270.

Potassium arsenate, III.

bismuthous iodide, 3r8. Alkaloids, 255 .

cadmium iodide, 3 r8.

chlorate, I94. Meat, I97. Organic matter destroyed, $\mathrm{I}_{4} 8$. Putrefaction, 197. Urine, I97. ferrocyanide, 25. Blood, 305. Copper, 167 .
Potassium hydroxide, r92. Santonin, 200.

mercuric iodide, 3 I8. Alkaloids, $25^{8}$.

permanganate, Cocaine, ro8. pyro-antimonate, Sodium, r93. zinc iodide, 3 I9.

Potatoes, Asparagine, 292. Solanine, $29 \mathrm{r}$.

Precipitation test, Cocaine, ro7. Copper, 167.

Proteins, Phosphorus, I7. Chloroform, 35. Heavy metals, I 72.

Prussian blue test, Hydrocyanic acid, 22. Morphine, 135. Tin, r64.

Pseudo-conhydrine, 89.

Pseudo-morphine (see Oxy-dimorphine).

Pseudo-narceine, 138 .

Pseudo-pelletierine, 270.

Psychotrine, 277.

Ptomaines, 2 I9.

Putrefaction, Carbolic acid, 27. Atropine, ro3. Cantharidin, 205. Morphine, 137. Potassium chlorate, r97. Phosphorus, I6.

Pyramidone, 87, r24, I45. Extract, 87 .

Pyridine, Chloroform, 37. Coffee, 275 .

Pyrocatechol, 26.

Pyrone, 213.

\section{Q}

Quinidine, 264.

Quinine, 87, I19, I45. Estimation, 258,268 . Extract, 87 .

Quinoline, 2r6.

$\mathbf{R}$

Radulescu's test, Morphine, 136 .

Ramverda's test, Cytisine, 206.

Reagents, general alkaloidal, 317 . 
Resorcinol test, Chloroform, 36. Narceine, I 40.

Reynold's test, Acetone, 57.

Rhamnose, 225.

Ricin, 229.

Roussin's test, Nicotine, 92.

Rubreserine test, Physostigmine, Iro.

\section{S}

Santonin, 198. Estimation, 289.

Sapogenins, 221.

Saponins, 220. Beer, 223. Cholesterides, 222.

Schaer's test, Blood, 313, 3I4.

Scherer's test, Phosphorus, 3 .

Schindelmeiser's test, Nicotine, 93.

Schlererythrin, 209. Test, 21r.

Schmidt's test, Apomorphine, 129.

Schönbein-Pagenstecher test, Hydrocyanic acid, 21.

Schönbein-Van Deen test, Blood, 312.

Scopolamine, ro4.

Secale cornutum, 209 .

Selenic-sulphuric acid test, Solanine, 226.

Selenious-sulphuric acid, 214. Narcotine, II6. Papaverine, 217.

Selenium, Moulds, 243.

Silver, 17r. Estimation, 179. Organism, 179. Physiological action, $\mathbf{x} 72$.

Silver nitrate test, Hydrocyanic acid, 23. Potassium chlorate, 195 .

Silver phosphide, 3,9 .

Sodium arsenate, 156 .

hydroxide, 192. Blood, 304 .

iodide, Hæmin crystals, 308.

nitroprusside, 56 .

perchlorate, Cocaine, rog.

pyro-antimonate, 156 .

stannate, 156 .

thiosulphate, Chloral hydrate, 39 .

Solanidine, 225. Test, 226.

Solanine, 225. Estimation, 291 . Test, 226.

Solanum tuberosum, 225.

Solubility test, Coniine, $9 \circ$.
Sonnenschein-Jesserich, Destruction of organic matter, 151 .

Spanish flies, 263.

Sparteine, $x 37$.

Spectroscopic test, Barium, 17 I. Blood, 306, 310. Hydrogen, I2. Phosphorus, 12.

Spotted hemlock, 89 .

Stannic oxide, 156 .

Stannous chloride, 102, 322. Mercury, $\mathbf{r} 66$.

Starch test, Sulphur dioxide, 1899 .

Stas-Otto process, 63.

Stibine, 160 .

Straub's test, Phosphorus, I4.

Strychnine, 86, 96, 143. Estimation, 258, 298. Extract, 86. Picrolonate, 296.

Stypticine, 254.

Sugar test, Sulphuric acid, 187 .

Sulphocyanate test, Carbon disulphide, 47. Hydrocyanic acid, 22. Mineral acid, 183 .

Sulphonal, 200. Extraction, 198.

Sulphur dioxide test, Sulphuric acid, 187. Meat, 189 .

Synopsis of Group I, 59.

Group 1I, I40.

Group III, 17 I.

Synthesis, Acetanilide, 73. Antipyrine, 82. Nicotine, 9r. Phenacetine, 75. Piperine, 287. Pyramidone, I 24. Salicylic acid, 77. Sulphonal, 20I. Veronal, 80.

$\mathrm{T}$

Tea, Caffeine, 272, 274, 276. Theophylline, 300 .

Teichmann's crystals (see Hæmin crystals).

Tellurium, Moulds, 243.

Tetra-chloro-methane (see Carbon tetrachloride).

Thalleioquin test, Quinine, 120.

Thebaine, 146, 227. Selenious-sulphuric acid, 215 . 
Thebaol, 227.

Theine (see Caffeine).

Theobromine, 84, 299, 300. Estimation, 277,298 .

Theophylline, 84, 300 .

Thyroid gland, Arsenic, I 74 .

Tin, I55. Distribution, I8r. Tests, 164 .

Tincture of nux vomica, Alkaloids, 295, 297.

opium, Morphine, 285.

Tobacco, Nicotine, 278,280 .

Toxalbumins, 228.

Toxicity, Acetone, 55. Aniline, 44. Bismuth, s8o. Carbolic acid, 26. Carbon disulphide, 46. Chloral hydrate, 40. Chromium, I77. Copper, I72. Cytisine, 206. Estimation by hæmolysis, 258 . Ethyl alcohol, 259. Homatropine, ro5. Hydrocyanic acid, I9. mercury, I 72 . Metallic albummates, I72. Methyl alcohol, 52, 259. Nicotine, 9r. Nitrobenzene, 42. Oxalic acid, rgo. Pepper, 287. Phosphorus, I6. Picrotoxin, 66. Potassium chlorate, 194. Saponins, 221 . Solanine, 226. Sulphurous acid, I88. Tin, I8I. Toxalbumins, 229. Uranium, 180.

Tribromophenol, 28 .

Trichlor-ethyl alcohol (see Alcohol, trichlor-ethyl).

Trimethyl-amine, 2 I I.

Trional, 203.

Triphenyl-methane, p-dihydroxy, 30 .

Troches, Santonin, 29r.

Tropidine, ro6.

Tropine, 103, 106.

Turpentine, ozonized, Blood, 3 I 2.

Tyrosine, $x 7,28$.

U

Ulex europæus, 205.

Ulexine (see Cytisine).
Uranium, I 72, 180.

Urobilin, I 7.

Urotheobromine, 300 .

Vanadic-sulphuric acid test, Solanine, 226.

Van der Moer's test, Cytisine, 206.

Veratridine, 94.

Veratrine, 87,93, I43. Extract, 87. Veronal, 66, 79, 142 .

Vitali's test, Alcohol, 5I. Alkalies, r93. Atropine, ro4. Blood, 313. Carbon disulphide, 49. Veratrine, 96 .

Vortmann's test, Hydrocyanic acid, 23.

\section{W}

Wangerin's test, Apomorphine, I 28 .

Narcotine, II 5 .

Warren's test, Papaverine, 2 I 7.

Weehuizen's test, Hydrocyanic acid, 24.

Weppen's test, Veratrine, 95 .

White arsenic, I5o.

Wine of opium, Morphine, 285.

Wine, Salicylic acid, 250. Saponins, 223.

Wormseed, Santonin, 28 g.

\section{$\mathrm{X}$}

Xanthine bases, Urine, 84 .

Xanthogenate test, Carbon disulphide, 47.

o-Xylene, 204 .

\section{Y}

Yellow phosphorus, 5.

\section{Z}

Zeisel's test, Colchicin, 69. Methoxyl, ror.

Zinc, r68, r 80 . 




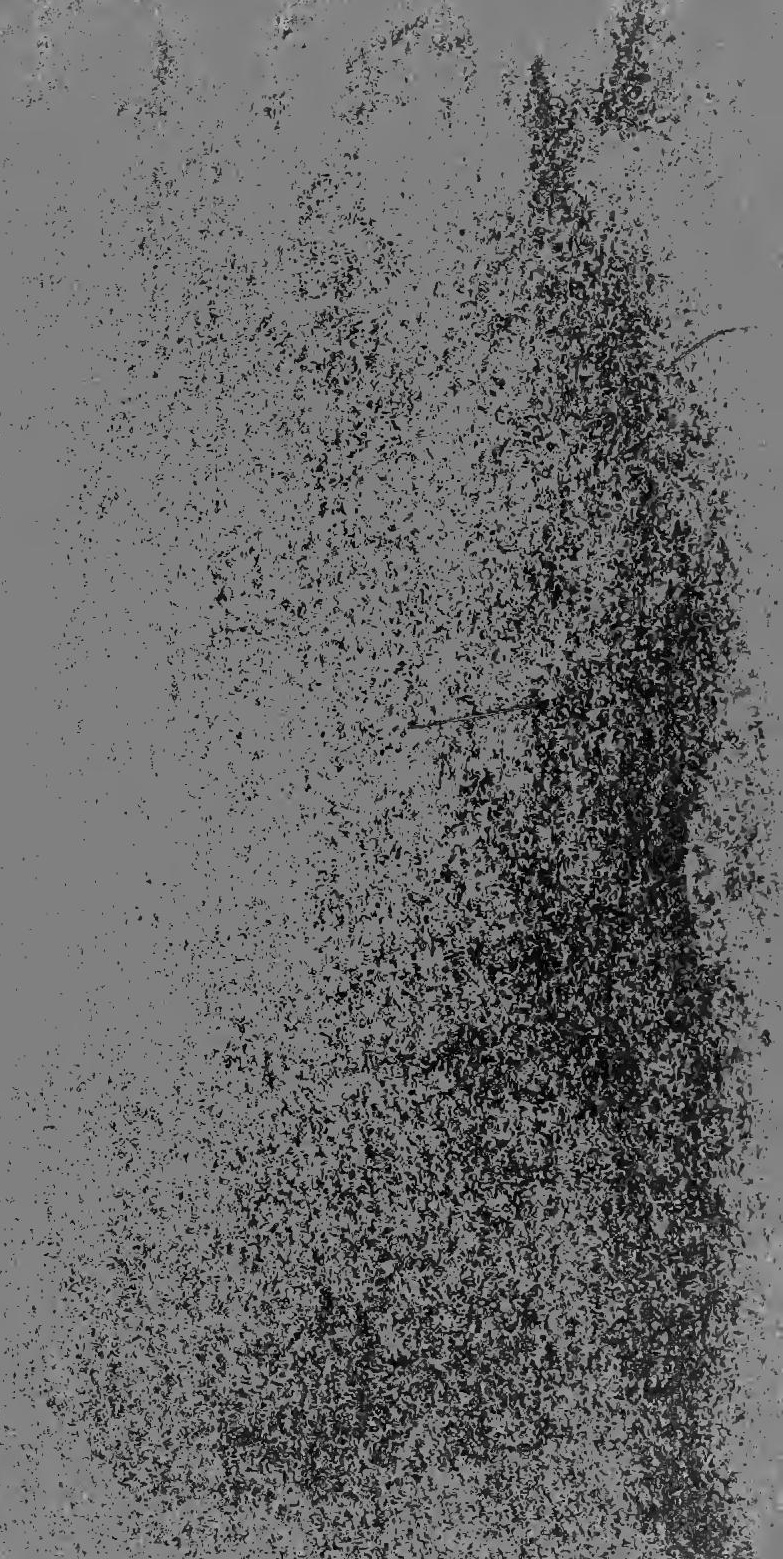




\section{UNIVERSITY OF CALIFORNIA LIBRARY}

Los Angeles

This book is DUE on the last date stamped below.

Form L9-25m-9,'55 (B4283s4) 444
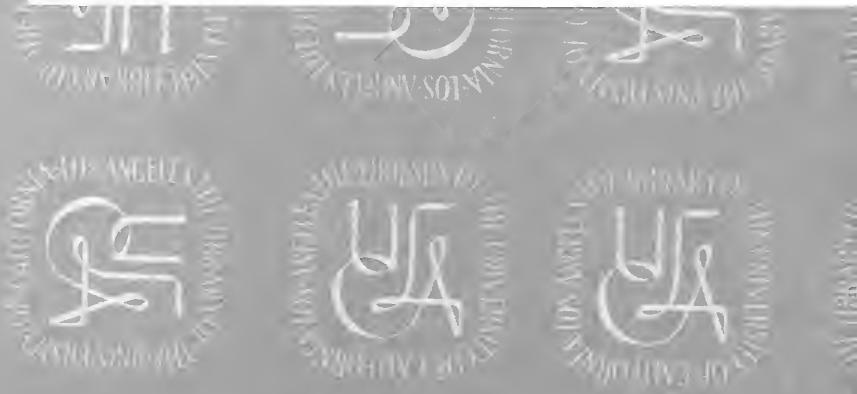


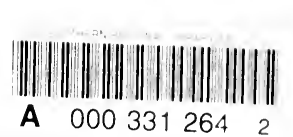


8) (6)

F.

ais

(3)

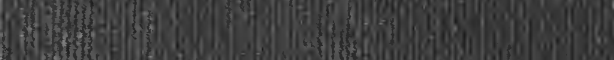

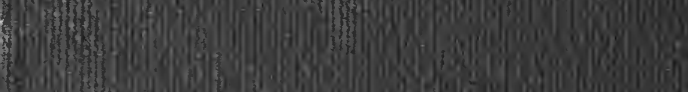
a 4) 5. If a 35 (3)

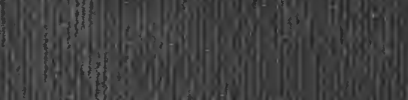

